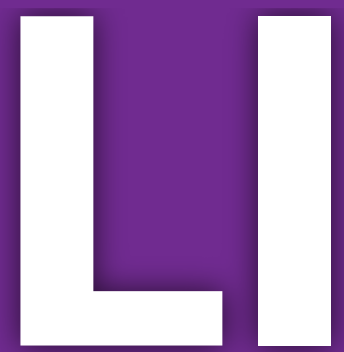

\title{
LABORATORY INVESTIGATION
}

THE BASIC AND TRANSLATIONAL PATHOLOGY RESEARCH JOURNAL

VOLUME 98 | SUPPLEMENT 1 | MARCH 2018

\section{※USCAP 2018}
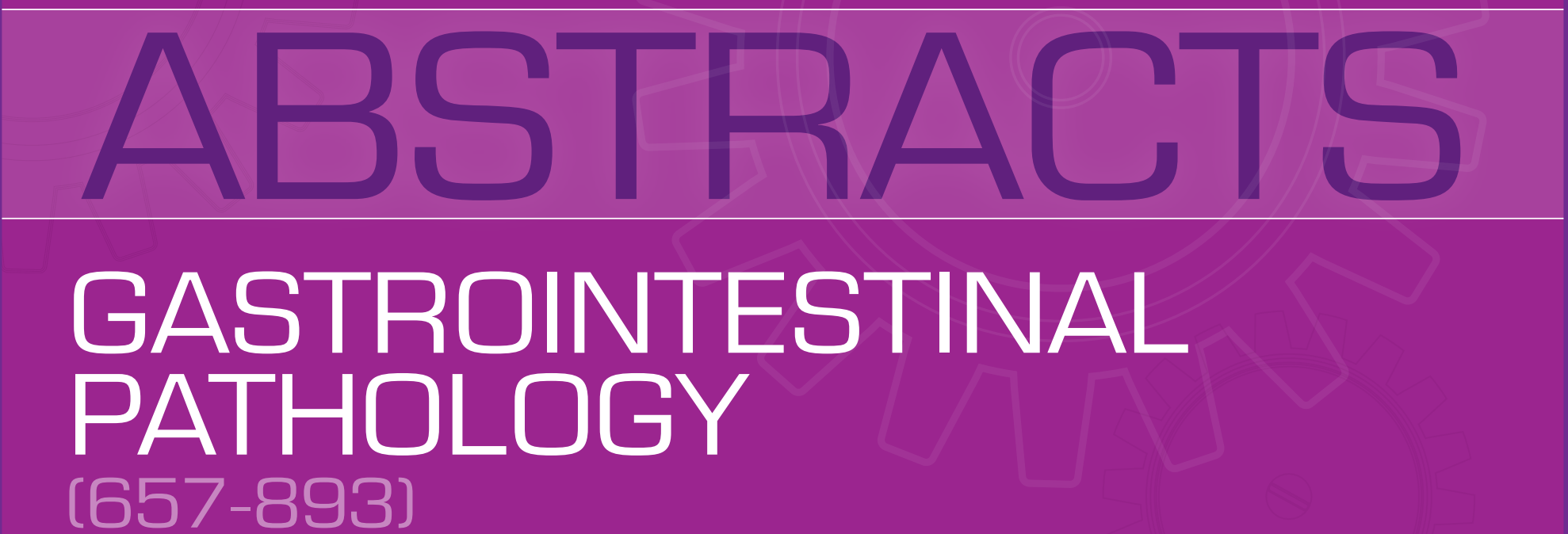

107TH ANNUAL MEETING

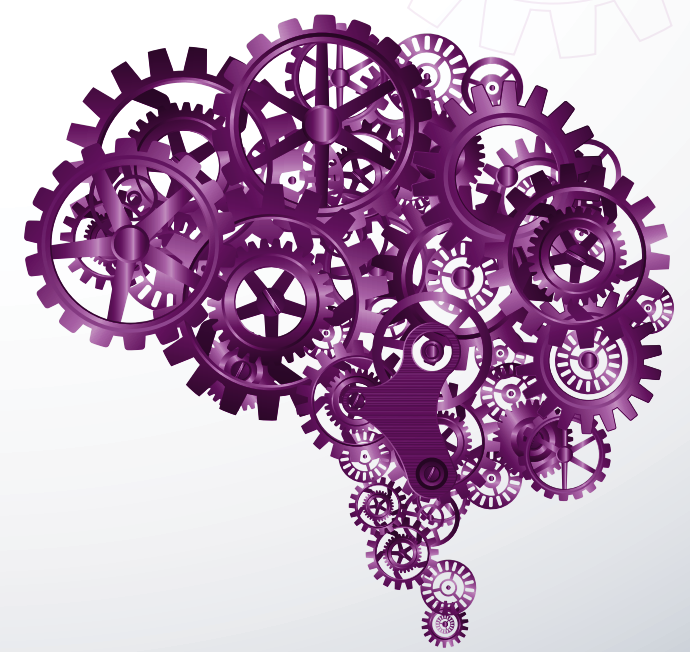

MARCH 17-23, 2018 Vancouver Convention Centre 


\section{EDUCATION COMMITTEE}

Jason L. Hornick, Chair

Rhonda Yantiss, Chair, Abstract Review Board and Assignment Committee

Laura W. Lamps, Chair, CME Subcommittee

Steven D. Billings, Chair, Interactive Microscopy

Shree G. Sharma, Chair, Informatics Subcommittee

Raja R. Seethala, Short Course Coordinator Ilan Weinreb, Chair, Subcommittee for Unique Live Course Offerings

David B. Kaminsky, Executive Vice President (Ex-Officio)

Aleodor (Doru) Andea

Zubair Baloch

Olca Basturk

Gregory R. Bean, Pathologist-in-Training Daniel J. Brat
Amy Chadburn

Ashley M. Cimino-Mathews

James R. Cook

Carol F. Farver

Meera R. Hameed

Michelle S. Hirsch

Anna Marie Mulligan Rish Pai

Vinita Parkash

Anil Parwani

Deepa Patil

Lakshmi Priya Kunju

John D. Reith

Raja R. Seethala

Kwun Wah Wen, Pathologist-in-Training

\section{ABSTRACT REVIEW BOARD}

Narasimhan Agaram

Christina Arnold

Dan Berney

Ritu Bhalla

Parul Bhargava

Justin Bishop

Jennifer Black

Thomas Brenn

Fadi Brimo

Natalia Buza

Yingbei Chen

Benjamin Chen

Rebecca Chernock

Andres Chiesa-Vottero

James Conner

Claudiu Cotta

Tim D'Alfonso

Leona Doyle

Daniel Dye

Andrew Evans

Alton Farris

Dennis Firchau

Ann Folkins

Karen Fritchie

Karuna Garg

James Gill

Anthony Gill

Ryan Gill

Tamara Giorgadze

Raul Gonzalez

Anuradha Gopalan

Jennifer Gordetsky

Ilyssa Gordon

Alejandro Gru
Mamta Gupta

Omar Habeeb

Marc Halushka

Krisztina Hanley

Douglas Hartman Yael Heher

Walter Henricks

John Higgins

Jason Hornick

Mojgan Hosseini

David Hwang

Michael Idowu

Peter Illei

Kristin Jensen

Vickie Jo

Kirk Jones

Chia-Sui Kao

Ashraf Khan

Michael Kluk

Kristine Konopka

Gregor Krings

Asangi Kumarapeli

Frank Kuo

Alvaro Laga

Robin LeGallo

Melinda Lerwill

Rebecca Levy

Zaibo Li

Yen-Chun Liu

Tamara Lotan

Joe Maleszewski

Adrian Marino-Enriquez

Jonathan Marotti

Jerri McLemore
David Meredith

Dylan Miller

Roberto Miranda

Elizabeth Morgan

Juan-Miguel Mosquera

Atis Muehlenbachs

Raouf Nakhleh

Ericka Olgaard

Horatiu Olteanu

Kay Park

Rajiv Patel

Yan Peng

David Pisapia

Jenny Pogoriler

Alexi Polydorides

Sonam Prakash

Manju Prasad

Bobbi Pritt

Peter Pytel

Charles Quick

Joseph Rabban

Raga Ramachandran

Preetha Ramalingam

Priya Rao

Vijaya Reddy

Robyn Reed

Michelle Reid

Natasha Rekhtman

Michael Rivera

Mike Roh

Marianna Ruzinova

Peter Sadow

Safia Salaria

Steven Salvatore
Souzan Sanati

Sandro Santagata Anjali Saqi

Frank Schneider

Michael Seidman

Shree Sharma

Jeanne Shen

Steven Shen

Jiaqi Shi

Wun-Ju Shieh

Konstantin Shilo

Steven Smith

Lauren Smith

Aliyah Sohani

Heather Stevenson-Lerner

Khin Thway

Evi Vakiani

Sonal Varma

Marina Vivero

Yihong Wang

Christopher Weber

Olga Weinberg

Astrid Weins

Maria Westerhoff

Sean Williamson

Laura Wood

Wei Xin

Mina Xu

Rhonda Yantiss

Akihiko Yoshida

Xuefeng Zhang

Debra Zynger

To cite abstracts in this publication, please use the following format: Author A, Author B, Author C, et al. Abstract title (abs\#). Laboratory Investigation 2018; 98 (suppl 1): page\# 

to 7.6 in normal colon $(p=0.02)$. MSI-H status was associated with increased IEL density, with values of 28.8, 27.7, and 37 for SSA-LD SSA-HD, and CRC; respectively $(p=0.03)$. Average PD-1 lymphocytic

\section{Interobserver Agreement in the Diagnosis of Anal Dysplasia}

Sohaib Abu-Farsakh1, Michael Drage ${ }^{2}$, Aaron Huber ${ }^{1}$, Bradley M. Turner ${ }^{3}$, Sharlin Varghese ${ }^{4}, X_{i}$ Wang $^{5}$, Christa Whitney-Miller ${ }^{1}$, Raul $S$ Gonzalez ${ }^{6}$. ${ }^{1}$ Rochester, NY, 2 University of Rochester, Rochester, NY, ${ }^{3}$ University of Rochester Medical Center, Rochester, NY, ${ }^{4}$, Fairport, NY, ${ }^{5}$ Univ of Rochester, Rochester, NY, ${ }^{6}$ University of Rochester Medical Center, Rochester, NY

Background: Management of anal dysplasia relies on the accurate diagnosis of anal tissue biopsy and anal cytology specimens, as lowgrade squamous intraepithelial lesion (LSIL) is generally managed with observation, while high-grade squamous intraepithelial lesion (HSIL) often requires ablation. Previous studies have shown that anal dysplasia can be subjective, with significant interobserver variability despite existing histologic criteria. As institutions move toward subspecialty signout (SSSO), decisions must be made regarding whether to assign anal biopsies to the gastrointestinal (GI) or gynecologic (GYN) pathology service. We investigated interobserver agreement in the diagnosis of anal dysplasia, comparing GI and GYN pathologists.

Design: We identified 200 archival tissue biopsies of anal mucosa and circulated them among three GI pathologists and three GYN pathologists. Each pathologist separately scored each biopsy as normal, atypical, LSIL, or HSIL. The GI pathologists then convened to establish a consensus diagnosis on cases with discordant individual interpretations; the GYN pathologists did the same. Weighted kappa coefficients were calculated to reflect the agreement between 1) each Gl pathologist and the GI consensus diagnoses, 2) each GYN pathologist and the GYN consensus diagnoses, and 3) the GI consensus diagnoses and the GYN consensus diagnoses.

Results: The GI pathologists agreed diagnostically on $97(49 \%)$ cases prior to consensus; the GYN pathologists agreed on 33 (17\%). Weighted kappa coefficients for agreement between each GI pathologist and the $\mathrm{GI}$ consensus diagnoses ranged from 0.529 to 0.668; for the GYN pathologists with the GYN consensus diagnoses, they ranged from 0.104 to 0.719 . The weighted kappa coefficient for the agreement between $\mathrm{Gl}$ and GYN consensus diagnoses was 0.633 . The GI pathologists diagnosed 14 cases as HSIL, with four $(29 \%)$ agreed upon prior to consensus. The GYN pathologists diagnosed 14 cases as HSIL, with $13(93 \%)$ agreed upon prior to consensus; 11 cases were called HSIL by both groups.

Conclusions: In general, interobserver agreement on the diagnosis of anal dysplasia was moderate to good, but noticeable variability existed. In our study, the Gl pathologists had a tighter range of interobserver variability, but the GYN pathologists had more consistent individual interpretations of HSIL. Institutions with SSSO will likely need to weigh their own individual practice characteristics in determining whether to assign anal biopsies to Gl or GYN pathologists.

\section{Immune Environment in Serrated Lesions of the Colon: Intraepithelial-Lymphocyte Density, PD- 1, and PD-L1 Expression Correlate with Serrated Neoplasia Pathway Progression}

Gabriel Acosta-Gonzalez', Madhu Ouseph², Kara A Lombardo3, Shaolei Lu', Jonathan Glickman ${ }^{5}$, Murray Resnick ${ }^{3}$. 'Brown University Warren Alpert Medical School, Providence, RI, ${ }^{2}$ Johnston, RI, ${ }^{3}$ Rhode Island Hospital, Providence, RI, ${ }^{4}$ Brown University, Providence, RI, ${ }^{5}$ Beth Israel Deaconess Medical Center, Boston, MA

Background: The serrated neoplasia pathway accounts for $20-30 \%$ of colorectal carcinomas (CRCs). Sessile serrated adenomas (SSAs) are molecularly driven by $\mathrm{MLH} 1$ promoter methylation and Microsatellite instability (MSI) in their progression to CRC. MSI-high (MSI-H) lesions accumulate mutations and neoantigens, leading to intraepithelial lymphocyte (IEL) infiltration and immune checkpoint expression upregulation. Our study's aim was to determine how the kinetics of this immune environment relates to SSAs in their progression through low-grade (SSA-LD) to high-grade dysplasia (SSA-HD), and CRC.

Design: We retrospectively searched our pathology databases for cases of CRC with serrated precursor lesions, SSA SSA-LD, and SSA-HD. Total 84 cases were analyzed (16 CRCs, 14 SSA-HD, 44 SSALD, and 10 SSAs). MSI status, IEL density, and immune checkpoint expression were assessed by immunohistochemistry (IHC) for mismatch repair proteins, CD3, and PD-1/PD-L1; respectively. IEL density and lymphocyte PD-1/PD-L1 expression were scored per 200 epithelial cells. Epithelial PD-L1 was scored positive if expressed in over $25 \%$ of cells. Adjacent normal colon was scored for internal control.

Results: IEL density, lymphocyte PD-1, and epithelium PD-L1 expression correspondingly increased with lesion progression from SSA to SSA-LD/HD to CRC. Average IEL density was 15.0, 19.1, 26.5, expression was $17,29,4.5$ and 12.4 in SSA SSA-LD, SSA-HD and CRC; respectively, compared to 0.68 in normal crypts ( $p=0.03)$. PDexpression increased to 6.2 and 18.2 in MSI-H SSA-HD and CRCs; respectively. PD-L1 epithelial expression increased from $70 \%$ positivity in SSAs to $86 \%$ in $\mathrm{CRC}$, and $93 \%$ in $\mathrm{MSI}-\mathrm{H}$.

Conclusions: Increased IELs and PD-1/PD-L1 expression correlate with sequential progression of SSAs, through development of cytologic dysplasia, to $\mathrm{CRC}$ and MSI-H status. This finding supports the hypermutator phenotype mechanism of MSI-H lesions, which stimulates lymphocytic infiltration and upregulation of immune checkpoint expression.

\section{Comparison of HIF-2 $\alpha$ and NF-kB Proteins during Development of Acute Reflux Esophagitis in Patients with and without Long-Segment Barrett's Esophagus}

Agoston (Tony) Agoston 1 , Kerry B Dunbar ${ }^{2}$, Rhonda Souza ${ }^{3}$, Stuart $J$ Spechler ${ }^{4}$, Robert Odze'. 'Brigham \& Women's Hospital, Boston, MA ${ }^{2}$ UT Southwestern Med Ctr, Dallas, TX, ${ }^{3}$ Baylor University Medical Center, Dallas, TX, ${ }^{4}$ Baylor University Med Ctr, Dallas, TX

Background: Our previous, prospective study on acute GERD induced by discontinuing PPI therapy in 12 patients who had severe reflux esophagitis (RE) without long-segment Barrett's esophagus (BE) suggested that hypoxia-inducible factor (HIF)-2 $\alpha$ and NF-kB signaling play a pathogenetic role in RE. However, the development of acute GERD had not been studied prospectively in patients with long segment BE. Now, we have explored the effects of discontinuing PPIs on histologic features of esophagitis and on HIF- $2 \alpha$ and NF-kB protein levels in the squamous esophagus of patients with long-segment BE.

Design: Ten patients with long-segment BE without endoscopic esophagitis on PPI therapy had their PPIs discontinued, and endoscopy with procurement of esophageal biopsies [8 across the squamocolumnar junction (SCJ) 8 of squamous mucosa $1-2 \mathrm{~cm}$ proximal to the SCJ] was performed at baseline (on PPIs), and at weeks 1 and 2 after stopping PPIs. Histopathologic features of RE were evaluated, and immunostaining for HIF-1 $\alpha$, HIF-2 $\alpha$, phospho-p65, and total-p65 was performed and compared with similar esophagea biopsies from patients without long-segment BE in our earlier study on acute GERD.

Results: All 10 patients with long-segment BE developed histologic evidence of RE after stopping PPIs (4 with ulceration by week 1). From baseline to weeks 1 or 2, there was a significant increase in basa cell hyperplasia, spongiosis, and papillary hyperplasia (all $p<0.05$ ) in the squamous and SCJ biopsies. Between baseline and week 2 there was significantly increased staining of phospho-p65 ( $p=0.02)$ and total-p65 $(p=0.05)$; HIF- $2 \alpha$ also increased, but not significantly $(p=0.11)$. Compared to the patients in our earlier study, our patients with BE showed significantly elevated staining for HIF-2 $\alpha$, p65, and phospho-p65 at all time points $(p<0.05)$, including at baseline on PPIs.

Conclusions: Within 2 weeks of stopping PPIs, patients with long segment BE develop acute, erosive RE associated with increases in HIF-2 $\alpha$, p65, and phospho-p65. Their histologic pattern of acute reflux esophagitis development is similar to that of patients without long segment $B E$, but long-segment $B E$ patients have markedly higher levels of HIF-2 $\alpha$, p65, and phospho-p65 at baseline (when there is no endoscopic evidence of esophagitis), suggesting a potential role fo HIF- $2 \alpha$ and NF-kB signaling in the development or progression of BE.

\section{Distinction Between Ductal Involvement anc Invasive Nests in Esophageal Squamous Cell Carcinoma}

Junko Aida', Kaiyo Takubo ${ }^{2}$, Naoshi Ishikawa ${ }^{3}$, Yoko Matsuda ${ }^{4}$ Tomio Arai ${ }^{5}$, Toshiyuki Ishiwata ${ }^{6}$. ${ }^{1}$ Tokyo Metropolitan Institute of Gerontology, Tokyo, ${ }^{2}$ Tokyo, Japan, ${ }^{3}$ Tokyo Metropolitan Geriatric MC, Tokyo, Japan, ${ }^{4}$ Tokyo Metropolitan Geriatric Hospital, ${ }^{5}$ Tokyo Metropolitan Geriatric Hospital, Tokyo, ${ }^{6}$ Tokyo Metropolitan Institute of Gerontology

Background: Esophageal squamous cell carcinoma (ESCC) often involves the ducts of the esophageal glands proper. Such ducta involvement is not invasive to blood vessels or the lymphatic system and remains confined within the ducts and glands even if the tumo nests are present in the submucosa. Histopathologic determination of tumor depth in endoscopically resected (ER) specimens is importan when considering additional therapies. However, it is often difficult to determine the tumor nest is a ductal involvement or an invasive nest. In the present study, therefore, we attempted to clarify the histopathologic differences between ductal involvement and invasive nests. However, it is often difficult to differentiate a submucosal nest that is a focus of ductal involvement from stromal invasion. 
Design: In 10 ER specimens of superficial ESCC (40 blocks), we examined the histopathologic features of the ducts and terminal portions, including ductal involvement and invasive nests, using histological staining ( $\mathrm{HE}, \mathrm{PAS}-\mathrm{AB}, \mathrm{EVG})$ and immunohistochemistry (CK 5/6, 7, CAM5.2, CK10, 18, 19, P63, CEA, Ki-67, S-100, laminin, $\alpha$-SMA).

Results: In the ducts of esophageal glands proper, the luminal cells were positive for PAS-AB, CK5 $6,7,19$ and CEA, whereas the basal cells were positive for $\mathrm{CK} 5 / 6$, CAM5.2, CK19, P63 and Ki-67. In the terminal portion, the acinar cells were positive for PAS-AB and CK18. The myoepithelial cells were positive for CK5/6, 19, P63 and SMA. ESCC showed CAM5.2 expression, and various degrees of positivity for CK 5/6, 10 and 19. Many nuclei in ESCC were positive for P63 and Ki-67. Carcinoma cells in areas of ductal involvement expressed CK5/6, 19, CAM5.2, CK 7, 18 and CEA. The invasive nests showed positivity for CK5/6, 19, CAM5.2, CK 18 and CEA. PAS-AB and laminin were positive in the basal membrane, but the degree of positivity varied from case to case. Elastic fibers and SMA-positive fibers were seen in only the periductal region, and not in invasive nests.

Conclusions: Normal ducts and those with ESCC involvement can be easily distinguished on the basis of CK $5 / 6,7$, and 18 expression. P63 and $\mathrm{Ki}-67$ are considered good markers for distinguishing hyperplastic normal ducts from ducts with ESCC involvement because of their high frequency of positivity. Staining methods for epithelial cells are not useful for distinguishing ductal involvement from invasive nests, and such distinction can easily be made from the periductal presence of elastic fibers and SMA-positive fibers.

\section{Pancreatic Acinar Metaplasia in Distal Esophageal and Esophagogastric Junction Biopsies is Significantly Associated with proton pump inhibitors and NSAID use}

Suhair Al Salihi', Vanya Jaitly², David Saulino ${ }^{3}$, Andrew W DuPont ${ }^{3}$, Atilla Ertan ${ }^{3}$, Jamie Everett ${ }^{3}$, Mamoun Younes ${ }^{3}$. 'University of Texas Health Science Center at Houston - McGovern Medical School, Houston, TX, ${ }^{2}$ University of Texas Health Science Center at Houston McGovern Medical School, Houston, TX, ${ }^{3}$ University of Texas Health Science Center at Houston McGovern Medical School

Background: The pathogenesis of acinar metaplasia (PAM) at the esophagogastric junction (EGJ) and distal esophagus biopsies is still unclear. While some authors believe it is congenital, present at birth, others believe it is acquired due to mucosal inflammation of the gastric cardia. More recently, experimental studies in mice suggested that PAM may be caused by chronic proton pump inhibitors (PPI). The aim of this study was to determine whether there is correlation between PPI use and PAM in humans.

Design: Four hundred and forty-four consecutive patients who underwent upper GI endoscopy and EGJ or distal esophagus biopsy at a single institution were entered in the study. The presence of PAM on histopathologic examination was correlated with several clinical and pathologic findings using the chi-square Test.

Results: PAM was more likely to be found in association with chronic carditis $(p=0.015)$, in younger patients $(p=0.002)$, chronic PPI use ( $p$ $=0.007$ ), and chronic nonsteroidal anti-inflammatory drugs (NSAID) use $(p<0.001)$. PAM did not correlate with patient gender, presence of intestinal metaplasia (Barrett's esophagus), clinical history of chronic gastroesophageal reflux disease (GERD), history of cholecystectomy, current or previous Helicobacter pylori gastritis, or history of bariatric surgery.

Conclusions: Our results confirm the significant association between PAM and chronic inflammation of the gastric cardia (chronic carditis), and the association with chronic PPI use previously proposed from a mouse model. The strong association with chronic NSAID use has not been previously reported, and warrants further studies.

\section{Clinicopathologic Features of Viral Esophagitis Can Guide Judicious Use of Immunohistochemical Stains}

Said Albahra', Elena Lucas', Amit G Singal', Suntrea Hammer', Purva Gopal'. 'University of Texas Southwestern Medical Center, Dallas, TX

Background: Immunohistochemical stains (IHC) for viral esophagitis (VE), specifically herpes virus $1 / 2$ (HSV) and cytomegalovirus (CMV), are frequently applied on esophageal biopsies. The aim of our study was to identify clinical and histologic features of VE that could trigger more judicious use of IHC.

Design: We conducted a case-control study including patients who underwent an upper endoscopy with esophageal biopsy at a single academic center between January 2006 and July 2017. Cases included patients diagnosed with HSV and/or CMV esophagitis, and controls were defined as patients with negative IHC stains for HSV and CMV. All biopsy slides were reviewed and histologic features, including type of inflammatory cells, presence and type of reactive changes, and patterns of inflammation were recorded. Clinical data of interest included indication for biopsy, endoscopic findings, pre-test clinical concern for $\mathrm{VE}$, and immunocompromised status.

Results: We included 58 cases of VE $(32 \mathrm{HSV}, 21 \mathrm{CMV}$, and 5 $\mathrm{HSV}+\mathrm{CMV}$ ), and 60 controls. Of VE cases, 31 were diagnosed with HSV, 13 with CMV, 2 with both HSV and CMV on H\&E, and 12 were only diagnosed on IHC. IHC was still performed although vira inclusions were present on H\&E in $13 \mathrm{HSV}$ cases and $5 \mathrm{CMV}$ cases. VE patients were more likely to have an endoscopic ulcer $(p=0.03)$ and be immunocompromised $(\mathrm{p}<0.001)$ than controls. Pre-test clinical concern for VE was common compared to controls $(p=0.012)$ On biopsy, VE patients were significantly more likely to have ulcer and granulation tissue $(86 \%$ vs. $63 \%, \mathrm{p}=0.004)$, ulcer exudate rich in neutrophils and histiocytes ( $84 \%$ vs. $55 \%, p=0.001)$, neutrophils in the squamous epithelium ( $88 \%$ vs. $41 \%, \mathrm{p}=0.0001)$, histiocyte aggregates composed of $>15$ histiocytes $(69 \%$ vs. $11 \%, p<0.001)$ and spongiosis (96\% vs. $74 \%, p=0.001)$. Control cases had more frequent eosinophils, either alone $(p=0.009)$ or admixed with other types of inflammatory cells $(p=0.0001)$. Features of sloughing or pill esophagitis were seen in $7 \%$ and $8 \%$ of controls respectively, but not in any VE cases $(p=0.045$ and $p=0.025$ respectively). There was no significant difference in basal cell hyperplasia, apoptotic squamous cells, keratosis, or parakeratosislike changes.

Conclusions: IHC is overused in VE patients with definite viral inclusions on $\mathrm{H} \& \mathrm{E}$ as well as in biopsies from patients without concerning features for VE. A combination of clinical information, endoscopic findings and histologic features on H\&E stain can be used to better target IHC staining for VE.

\section{The Utility of Random Colon Biopsy in Investigating} Non-specific Lower Gastrointestinal Symptoms

Mohammed Alghamdi', Endashaw M Omer', Mostafa Fraig ${ }^{2}$, Houda Alatassi ${ }^{3}$, Nemencio Ronquillo4. 'University of Louisville, Louisville, $\mathrm{KY}$, ${ }^{2}$ Univ. of Louisville Hosp, Louisville, KY, ${ }^{3}$ University of Louisville, ${ }^{4} U$ CSD, San Diego, CA

Background: Patients often present with non-specific lowe gastrointestinal signs and symptoms prompting colonoscopy and biopsy. We hereby present our experience with random colonic biopsies to investigate cases with mainly unexplained chronic diarrhea in addition to other presentations.

Design: The surgical pathology database was searched for "Random Colon Biopsy" in adult patients with endoscopically unremarkable mucosa between January 2016 to September 2017. We excluded patients with history of inflammatory bowel disease, HIV, and bone marrow transplant.

Results: The search yielded 69 cases $(\mathrm{M}=28, \mathrm{~F}=41)$ ranging in age from 43-78 (average $=60.8$ ) with 3 distinct clinical indications, as follows:

\begin{tabular}{|c|c|}
\hline Indication & Frequency \\
\hline Diarrhea & $41(59.4 \%)$ \\
\hline IBS & $17(24.6 \%)$ \\
\hline Abd. Pain & $11(15.9 \%)$ \\
\hline
\end{tabular}

Seven out of 69 cases (10\%) showed pathological findings as follows:

\begin{tabular}{|c|c|}
\hline $2 / 7(28.5 \%)$ & Microscopic colitis \\
\hline $5 / 7(71.5 \%)$ & Acute colitis with variable severity \\
\hline
\end{tabular}

Additionally, 11 cases (15.9\%) were found to have incidental polyps on colonoscopy, and subsequently diagnosed as tubular adenoma. Ten out of 11 cases $(91 \%)$ were above the age 50 years.

Conclusions: Our results showed that $10 \%$ of the random colon biopsies explained the nonspecific clinical presentations which represented a high utility of random biopsies in this population. Also $15.9 \%$ of the colonoscopy attempts showed incidental colonic polyps diagnosed as tubular adenoma. The latter finding is consistent with the US Preventive Services Task Force (USPSTF) recommendations of screening colonoscopy in patients age $50-75$ years old, since most tubular adenomas were found within this age group.

\section{Immunohistochemical Expression for the Mismatch Repair Proteins is Similar in Precursor Lesions to Associated Invasive Colorectal Carcinomas}

Mohammed Alsomali', Taha Sachak', Rachel Pearlman'1, Michael Markow ${ }^{1}$, Wei Chen ${ }^{1}$, 'Christina A Arnold", Debbie Knight' ${ }^{1}$, Heather Hampel', Wendy L Frankel'. 'The Ohio State University Wexner Medical Center, Columbus, $\mathrm{OH}$

Background: Microsatellite-unstable (MSI) colorectal carcinoma (CRC) occurs in approximately $15 \%$ of all CRCs, with Lynch syndrome (LS) accounting for 3\%. Conventional adenomas in those with LS do not always display loss of mismatch repair protein (MMRP) for the 
affected gene, since the second allele may not be altered; reported concordance ranges from $73 \%$ to $100 \%$. Conventional adenomas with high grade dysplasia are more likely to show loss of the associated MMRP. We studied MMRPs in presumed precursor lesions $(P L)$ at the edge of invasive carcinomas (IC) to determine how often they showed comparable expression.

Design: We reviewed biopsy and resection slides available from CRC patients enrolled in our statewide study who had undergone tumor screening with MMRP immunohistochemistry (IHC) by 2-stain method (PMS2, MSH6) with reflex to partner (MLH1, MSH2), if absent. MMRP was considered present with staining $>5 \%$. We assessed presence and type of PL (conventional adenomas and serrated lesions) and evaluated MMRP staining in PL and IC.

Results: Of 1692 CRC patients with MMRP slides available for review, 1493 CRCs were microsatellite stable by IHC (88.2\%) and 199 were MSI by IHC (11.8\%). 251 PLs were identified at the edge of ICs including 243 conventional adenomas ( 51 with high grade dysplasia) and 8 serrated lesions including 4 sessile serrated adenomas (SSAs), 1 traditional serrated adenoma, and 3 serrated polyps (classification difficult). Two of the 4 SSAs contained cytologic dysplasia. MMRP IHC in all conventional adenomas was concordant with IC. Of serrated lesions, MMRP IHC was concordant with IC in 6/8 (75\%). The discordant cases showed loss of MLH1/PMS2 (2 SSAs with dysplasia) in IC and cytologic dysplasia, and presence in SSA. One had methylation of the $M L H 1$ promoter, while no germline mutation or methylation was found in the other. All PLs in the MSS cases showed presence of MMRP IHC in IC and PL.

Table 1. Precursor and Invasive Cancer Mismatch Repair Protein Concordance

\begin{tabular}{|l|l|l|l|}
\hline Precursor lesion & \# MSS IC (\%) & \# MSI IC (\%) & \# Concordant IC and PL (\%) \\
\hline $\begin{array}{l}\text { Conventional } \\
\text { adenoma }\end{array}$ & $174 / 192(90.6)$ & $18 / 192(9.4)$ & $192 / 192(100)$ \\
\hline $\begin{array}{l}\text { Conventional } \\
\text { adenoma, high } \\
\text { grade dysplasia }\end{array}$ & $50 / 51(98.1)$ & $1 / 51(1.9)$ & $51 / 51(100)$ \\
\hline SSA & $2 / 2(100)$ & 0 & $2 / 2(100)$ \\
\hline SSA, dysplasia & 0 & $2 / 2(100)$ & $0 / 2(0)$ \\
\hline Serrated polyp & $3 / 3(100)$ & $0 / 3(0)$ & $3 / 3(100)$ \\
\hline TSA & $1 / 1(100)$ & $0 / 1(0)$ & $1 / 1(100)$ \\
\hline Total & $230 / 251(91.6)$ & $21 / 251(8.4)$ & $249 / 251(99.2)$ \\
\hline
\end{tabular}

$\mathrm{IC}=$ invasive carcinoma, $\mathrm{PL}=$ precursor lesion, $\mathrm{SSA}=$ sessile serrated adenoma, TSA = traditional serrated adenoma

Conclusions: MMRP IHC was concordant between presumed PL and associated IC in all but 2 of 251 cases. This concordance is better than the rate previously reported in conventional adenomas in those with LS likely due to the more advanced nature of adenomas at the edge of IC. MMRP stains on conventional adenomas, found at the edge of IC predict the MMRP status of the CRC. Occasionally, serrated PLs will be discordant with the ICs, but the areas with cytologic dysplasia are more likely to be concordant, supporting the molecular progression of SSAs in patients without LS.

\section{Low Tissue Expression of Amphiregulin in Human Intestinal Acute Graft-Versus-Host Disease}

Khalid Amin ${ }^{1}$, Usman Yaqoob ${ }^{1}$, Byron Vaughn ${ }^{1}$, Colleen Forster ${ }^{1}$, Bruce Blazar', Angela Panoskaltsis-Mortari', Shernan Holtan'. 'University of Minnesota, Minneapolis, MN

Background: Hematopoietic cell transplantation (HCT) is frequently associated with a potentially fatal donor T-cell-mediated complication, acute graft-versus-host disease (aGVHD). Increasing evidence suggests that poor tissue repair correlates with shortened survival after aGVHD. Amphiregulin (AREG) is a weak epidermal growth factor receptor (EGFR) ligand involved in intestinal epithelial repair and regeneration. The expression of these EGFR ligands in human aGVHD target tissues, and their association with clinical outcomes, is not known.

Design: We analyzed tissue expression of AREG and EGF by immunohistochemistry (IHC) from intestinal tissues of 48 biopsyproven aGVHD patients compared to 11 patients with previously untreated inflammatory bowel disease (IBD) and 10 non-HCT patients. A semi-quantitative score of $0-3$ was used for IHC staining intensity. An additional $8 \mathrm{HCT}$ recipients diagnosed with aGVHD clinically (negative biopsy results) were evaluated as a separate group. In addition, we tested the serum of patients for AREG and EGF.
Results: Tissue expression of AREG was significantly lower in aGVHD biopsies and in IBD than normal colon $(p=0.004$ and $p=0.03$, respectively). Tissue expression of EGF in aGVHD was not significantly different than normal colon (95\% in mucosa and localized to nucleus) although it was higher in aGVHD than IBD $(p=0.01)$. However, overall tissue EGF expression was significantly lower in higher histologic grade aGVHD (grade 2-4) than lower histologic grade aGVHD (grade $0-1, p=0.03$ ). There was no association of tissue AREG or EGF with clinical severity of aGVHD. The AREG/EGF ratio was the lowest in patients with aGVHD at $0.33(p=0.003$ vs normal and $p=0.007$ versus IBD). Eight HCT recipients with "negative" pathologic biopsies treated for a clinical diagnosis of aGVHD had similar IHC results to biopsy-proven aGVHD. In addition, serum AREG was higher in histologic grade 2-4 aGVHD compared to histologic grade 0-1 $(p=0.04)$

Conclusions: Our results suggest that AREG expression is weak in intestinal tissues involved with aGVHD. AREG may be released into the circulation from damaged intestinal tissues as indicated by its higher serum levels in more severe aGVHD. Low GI AREG expression by IHC may be helpful for pathologic aGVHD diagnosis in the absence of classic apoptotic changes.

\section{Recurrent Ileal Strictures are Histologically Simila to Primary Strictures in Crohn's Disease Patients}

Karan Amlani', Ruiqi Huang ${ }^{1}$, Carolina Palmela², Joana Torres ${ }^{3}$, Russell B McBride ${ }^{4}$, Xiaofei Zhang ${ }^{1}$, Nikki Vyas ${ }^{1}$, Judy Cho ${ }^{1}$, Jean-frederic Colombel ${ }^{4}$, Alexandros D Polydorides ${ }^{1}$, Hongfa Zhu', Qingqing Liu ${ }^{1}$ Noam Harpaź, Mayte Suarez-Farinas", Huaibin Mabel Ko'. "Icahn School of Medicine at Mount Sinai, New York, NY, ${ }^{2}$ Hospital Beatriz Ângelo, Loures, Portugal, Loures, Lisboa, ${ }^{3}$ Beatriz Ângelo, Loures, Portugal, ${ }^{4}$ The Mount Sinai Hospital, ${ }^{5}$ Mount Sinai Med. Center, New York, NY

Background: Ileal strictures in Crohn's disease (CD) are pathologically heterogeneous, comprising variable combinations of smooth muscle fibrous, neural and lymphoid tissue, granulomas, edema and lymphatic dilatation. Ileal strictures affect $25 \%$ of CD patients, and the risk of post-operative recurrence is high, 44-55\% after 10 years. It is not known whether any histologic features impact on the risk of stricture recurrence. Hitherto, there has been no attempt to determine whether the tissue composition of recurrent strictures recapitulates that of primary strictures.

Design: Patients with Crohn's ileitis who underwent 2 resections for strictures $>6$ months apart were identified from our archives. Representative H\&E slides of the strictures were reviewed. The tissue composition of each layer of the intestinal wall [mucosa, muscularis mucosae (MM), submucosa (SM), muscularis propria inner (MP-I) and outer layer (MP-O), and subserosa (SS)], was evaluated semiquantitatively. The parameters assessed included layer thickness, expansion of smooth muscle, neural hypertrophy, adipose tissue, fibrosis, lymphatic dilatation, inflammatory cell infiltration, granulomas, edema, and lymphoid aggregates. All analyses were performed by an expert Gl pathologist who was blinded to the clinical information. The presence of each feature was graded on a scale of $0-3+$. Statistical analysis of the paired data was performed by determining weighted Cohen's Kappa tests with correlation ( $r$ ) values $>0.4$ considered meaningful. Additionally, cluster analysis was used to identify groups of variables with similar results with $p<0.05$ considered significant.

Results: 44 ileal resection specimens from 22 patients were retrieved ( $64 \%$ male; mean age at 1 st surgery $32 \pm 13 y$ ). All of the specimens featured expansion of the MM and MP-I. Table 1 shows the histological features with high intraclass correlation coefficients (ICC), of which the highest are highlighted. Of note, most of the similarities between strictures involved the deep layers of the intestinal wall.

Table 1.

\begin{tabular}{|l|l|l|}
\hline Histological Variables, N=22 pairs & ICC & P-value \\
\hline Inner MP active inflammation (neutrophils) & 0.508 & 0.006 \\
\hline Inner MP dilated lymphatics & 0.589 & 0.001 \\
\hline Outer MP active inflammation (neutrophils) & 0.808 & $<0.001$ \\
\hline Outer MP dilated lymphatics & 0.525 & 0.004 \\
\hline Presence of granulomas (any layer) & 0.607 & $<0.001$ \\
\hline Outer MP granulomas & 0.455 & 0.037 \\
\hline Subserosal granulomas & 0.476 & 0.009 \\
\hline Submucosal lymphoid aggregates & 0.494 & 0.007 \\
\hline Subserosal lymphoid aggregates & 0.408 & 0.024 \\
\hline
\end{tabular}

To our knowledge this study is the first systematic analysis of histologic correlation between paired ileal resections in CD patients. Of the numerous parameters that were examined, deep inflammation, granulomas and lymphoid aggregates yielded the highest correlations. Despite the heterogeneity of strictures among different individual patients, strictures resected from the same patient at different times retain certain distinctive pathological similarities. 
667 Morphologic Features Predictive of Invasive Adenocarcinoma in Colonic Endoscopic Biopsies in the Absence of Definite Stromal Desmoplasia

Joshua Anderson', Sarah Umetsu², Melike Pekmezcỉ, Sanjay Kakar ${ }^{3}$, Linlin Wang4. 'University of California, San Francisco, San Francisco, CA, ${ }^{2}$ UCSF, San Francisco, CA, ${ }^{3}$ Univ. of California, San Francisco, San Francisco, CA, ${ }^{4}$ UCSF

Background: The diagnosis of invasive colorectal adenocarcinoma requires the presence of submucosal invasion or definitive stromal desmoplasia. Evaluation for these features can be difficult in endoscopic biopsies, which are often superficial and fragmented. The distinction between invasive adenocarcinoma and high grade dysplasia/intramucosal adenocarcinoma is clinically important. The aim of this study is to identify any additional histologic features which may assist in the diagnosis of invasive adenocarcinoma.

Design: 88 colorectal biopsy specimens with diagnoses which were suspicious but not definitive for invasive adenocarcinoma (e.g. at least intramucosal adenocarcinoma, at least high-grade dysplasia (HGD)) were collected from 2000 to 2017. Of these cases, $65(74 \%)$ had invasive adenocarcinoma on the subsequent resection specimens (termed positive cases). $23(26 \%)$ cases were either did not have invasive adenocarcinoma on the subsequent resection or had negative subsequent endoscopy (termed negative cases). Morphologic features were reviewed by two pathologists. Statistical significance of the findings was analyzed using the Fisher's exact test.

Results: $96 \%$ (65/68) of biopsies with diagnoses suspicious but not definitive for invasive adenocarcinoma in the context of an endoscopic mass demonstrated invasive adenocarcinoma on the subsequent resection. Common morphologic features included HGD in association with granulation tissue, fibroblastic proliferation without myxoid change (not diagnostic for desmoplasia), single cell infiltration, confluent HGD (HGD more than 2 high power field), and eosinophilic luminal necrosis (Table 1). No submucosal invasion or definitive desmoplasia was identified in any of the cases. The positive predictive value (PPV) for HGD in association with granulation tissue for invasive adenocarcinoma was 95\%, and the PPV for fibroblastic proliferation without myxoid change for invasive adenocarcinoma was $93 \%$.

\begin{tabular}{|l|l|l|l|l|l|}
\hline & $\begin{array}{l}\text { HGD in } \\
\text { associa- } \\
\text { tion with } \\
\text { granulation } \\
\text { tissue }\end{array}$ & $\begin{array}{l}\text { Fibroblastic } \\
\text { proliferation } \\
\text { without } \\
\text { myxoid } \\
\text { change }\end{array}$ & $\begin{array}{l}\text { Single cell } \\
\text { infiltration }\end{array}$ & $\begin{array}{l}\text { Confluent } \\
\text { HGD }\end{array}$ & $\begin{array}{l}\text { Eosinophilic } \\
\text { luminal } \\
\text { necrosis }\end{array}$ \\
\hline $\begin{array}{l}\text { positive } \\
\text { cases }\end{array}$ & $60 \%(39 / 65)$ & $57 \%(37 / 65)$ & $28 \%(18 / 65)$ & $20 \%(13 / 65)$ & $29 \%(19 / 65)$ \\
\hline $\begin{array}{l}\text { negative } \\
\text { cases }\end{array}$ & $9 \%(2 / 23)$ & $13 \%(3 / 23)$ & $9 \%(2 / 23)$ & $17 \%(4 / 23)$ & $30 \%(7 / 23)$ \\
\hline P value & $\mathrm{p}<0.0001$ & $\mathrm{p}<0.001$ & $\mathrm{p}=0.08$ & $\mathrm{p}=1$ & $\mathrm{p}=1$ \\
\hline
\end{tabular}

Conclusions: Morphologic features of HGD in association with granulation tissue and fibroblastic proliferation without myxoid change are significantly associated with invasive adenocarcinoma. In superficial colonic biopsy of a mass lesion, in the absence of submucosal invasion or definitive desmoplasia, the presence of these features can add additional support for diagnosis of invasive adenocarcinoma.

\section{Low Mucosal Gland Density in Barrett's Esophagus is Associated with Progression to High Grade Dysplasia and Adenocarcinoma}

Juliana Andrici', Matthew Stachler', William Jeck ${ }^{2}$, Vikram Deshpande ${ }^{3}$, Robert Odze1, Agoston (Tony) Agoston'. 'Brigham and Women's Hospital, Boston, MA, ${ }^{2}$ Boston, MA, ${ }^{3}$ Massachusetts General Hospital, Boston, MA

Background: Barrett esophagus (BE) is recognized as the most important risk factor in the development of esophageal adenocarcinoma (EAC), but only a small minority of BE patients go on to develop high-grade dysplasia (HGD) or EAC, and the risk factors of progression remain poorly understood. Specifically, our focus was on the mucous and oxyntic mucosal glands, as little is known about their role in disease progression. Anecdotally, we have noticed that neoplastic biopsies in BE have few if any glands, and we aimed to test the hypothesis that loss of mucous glands plays a role in $\mathrm{BE}$ progression in a large cohort of patients with non-dysplastic $B E$ undergoing endoscopic surveillance.

Design: The pathology archives of two large academic hospitals were searched for all BE cases that went on to progress to HGD or EAC. The control (non-progressor) group consisted of BE patients who had at least two biopsies on two separate occasions and who had not developed HGD or EAC after at least 10 years of follow up. For all cases, we evaluated the index BE biopsy and a BE biopsy just prior to progression or for non-progressors, a time point prior to last follow up biopsy. The morphologic variables evaluated were the percentage of the biopsy specimen occupied by mucosal glands, and the relative percentage of mucous and oxyntic mucosal glands.

Results: We identified $24 \mathrm{BE}$ progressors and $32 \mathrm{BE}$ non-progressor (controls) (overall: mean age $=60$ years; $54 \%$ male). For the progressors, the median time between index biopsy and last nondysplastic biopsy was 51 months (range 0.1-207), and for the nonprogressors it was 152 months (range 128-325). The progressors were more likely to be over 65 years old (odds ratio (OR) 9.45, $p<0.001$ ). The mean percentage of the mucosa with glands was $33 \%$ and $39 \%$ in the index biopsy of progressor and non-progressor groups, respectively, and $27 \%$ and $30 \%$ in the post-index biopsy of progressor and non-progressor groups, respectively. The presence of mucosal glands occupying less than $20 \%$ of the mucosa in the index biopsy was significantly associated with an increase in risk of progression (OR 5.40, $p=0.008$ ). Furthermore, having less than $40 \%$ of the glands comprised of oxyntic glands was also associated with increased risk of progression (OR $3.64, \mathrm{p}=0.03$ ), and there was a trend towards increased risk when more than $50 \%$ of the glands were mucous glands (OR 2.77, $\mathrm{p}=0.10$ ).

Conclusions: A low density of mucosal glands, and specifically a low proportion of oxyntic glands relative to mucous glands, are assoc

\section{Identifying Knowledge Gaps in the Appendix: A Multi-Institutional Study from Six Academic Centers}

Christina A Arnold", Rondell Graham², Dhanpat Jain ${ }^{3}$, Sanjay Kakar ${ }^{4}$ Dora Lam-Himlin ${ }^{5}$, Bita V Naini ${ }^{6}$, Tsung-Teh W ${ }^{2}$, Matthew $M Y^{2}{ }^{7}{ }^{7}$ Michael Torbenson'. 'The Ohio State University Wexner Medical Center, Columbus, $\mathrm{OH},{ }^{2}$ Mayo Clinic, Rochester, MN, ${ }^{3}$ Yale Univ./ Medicine, New Haven, CT, ${ }^{4}$ Univ. of California, San Francisco, San Francisco, CA, ${ }^{5}$ Mayo Clinic, Scottsdale, AZ, ${ }^{6}$ UCLA Medical Center, Santa Monica, CA, 7 Univ of Washington Med Ctr, Seattle, WA

Background: Extra-mural consults represent clinically important diagnostic challenges and opportunities to advance the field.

Design: This study describes the findings of 1,203 consecutive $\mathrm{GI}$, Appendix, Liver, Pancreas, and Biliary surgical pathology consultations submitted to 6 academic centers between 2016-2017. Consults were aggregated by organ: Liver 40\%, Upper GI 32\%, Lower GI 22\% (TI 7\%, Colon $91 \%$, Anus $2 \%$ ), Appendix 3\%, Pancreas/Biliary Tree $=5 \%$.

Results: For the appendix $(n=32)$, the majority were resections $(84 \%)$, and most were pathologist-directed $(94 \%)$. The majority were finalized without additional studies $(63 \%$; additional studies included levels $25 \%$, NOS $13 \%$ ). Epithelial-based queries predominated $191 \%$; mesenchymal $3 \%)$, with major categories including classification of tumors $(79 \%)$, mucinous lesions $(55 \%)$, invasion $(10 \%)$, serrated lesions $(10 \%)$, müllerian structures $(3 \%)$, and acute appendicitis $(3 \%)$. For tumors, the most frequent query was related to low-grade appendiceal mucinous neoplasms (LAMN, 44\%), but adenocarcinoma ex-goblet cell carcinoid (13\%), goblet cell carcinoid $(9 \%)$, high-grade appendiceal mucinous neoplasms (HAMN, 9\%), and neuroendocrine tumors $(4 \%)$ were also seen. For mucinous lesions, most were finalized as LAMN (59\%), but HAMN $(12 \%)$, diverticulum $(12 \%)$ appendicitis $(6 \%)$, benign, unremarkable appendix $(6 \%)$, and SSA $(6 \%)$ were also seen. For serrated lesions, most were finalized as SSA ( $n=2$ of $3,67 \%$; the other one was descriptively diagnosed as "serrated/ hyperplastic change"). Disagreements with the contributor were noted in $48 \%$ of cases; all were major $(91 \%)$ and most were downgraded $(90 \%)$. Downgraded major diagnoses included reclassifying a gastrointestinal stromal tumor to prominent fibrous obliteration of the tip; restaging a LAMN from pT3 to pTis; revising LAMN diagnoses to acute appendicitis, ruptured diverticulum, or SSA. The one majo disagreement that was upgraded was revising a diagnosis of LAMN to HAMN. Missed diagnoses included granulomatous appendicitis and endometriosis. Of the two clinician-driven consults (6\%), one was in agreement and the other was a major disagreement (revising a diagnosis of adenocarcinoma to LAMN).

Conclusions: This study highlights knowledge gaps in the appendix as classification of tumors and mucinous lesions, specifically LAMN. Consult diagnoses were often in major disagreement with the contributor and usually downgraded.

\section{Knowledge Gaps in the Lower Gastrointestinal Tract (GIT): A Multi-Institutional Study from Six Academic Centers}

Christina A Arnold", Rondell Graham², Dhanpat Jain ${ }^{3}$, Sanjay Kakar ${ }^{4}$ Dora Lam-Himlin ${ }^{5}$, Bita V Naini ${ }^{6}$, Tsung-Teh W ${ }^{2}$, Matthew $M Y^{2} h^{7}$ Michael Torbenson'. 'The Ohio State University Wexner Medical Center, Columbus, $\mathrm{OH},{ }^{2}$ Mayo Clinic, Rochester, MN, ${ }^{3}$ Yale Univ./ Medicine, New Haven, CT, ${ }^{4}$ Univ. of California, San Francisco, San Francisco, CA, ${ }^{5}$ Mayo Clinic, Scottsdale, AZ, ${ }^{6}$ UCLA Medical Center, Santa Monica, CA, ${ }^{7}$ Univ of Washington Med Ctr, Seattle, WA

Background: Extra-mural consults represent clinically important diagnostic challenges. They serve as an opportunity to advance the field and improve patient care by directing future research projects, guideline recommendations, and education. 
Design: This study describes the findings of 1,203 consecutive $\mathrm{Gl}$, Appendix, Liver, Pancreas, and Biliary surgical pathology consultations submitted to 6 academic centers between 2016-2017 (range 75-250 cases per site). Consults were aggregated by organ: Liver $40 \%$, Upper GI 32\%, Lower GI 22\% (TI 7\%, Colon 91\%, Anus 2\%), Appendix 3\%, Pancreas/Biliary Tree, including Gallbladder $=5 \%$.

Results: For the lower GI tract, $64 \%$ were biopsies, and most were pathologist-directed $(84 \%)$. The majority were finalized without additional studies $161 \%$; additional studies included levels $17 \%$, IHC $9 \%$, special stains $2 \%$, molecular $1 \%$ ). Epithelial-based queries predominated the study (83\%; mesenchymal $9 \%$, hematolymphoid $3 \%$, melanoma $0.3 \%)$. Major categories included polyps $(47 \%)$, dysplasia $(25 \%)$, ileitis/colitis (19\%), and tumors (11\%). For polyps, the majority were conventional adenomas $(40 \%)$, followed by serrated $(21 \%)$, mesenchymal $(18 \%)$, lymphoid $(12 \%)$, neuroendocrine $(5 \%)$, prolapse (4\%); common queries included dysplasia $(53 \%)$ and invasion $(51 \%)$. Most TI biopsies had ileitis $(56 \%)$, and $89 \%$ of these were IBDrelated consults. Of the colitis consults, most were IBD-related $(44 \%)$, but colitis NOS $(23 \%)$, microscopic $(7 \%)$, medication $(7 \%)$, ischemia $(5 \%)$, and diverticular colitis $(1 \%)$ were also seen. For IBD-related consults, common questions related to establishing/classifying an IBD diagnosis (69\%) and dysplasia (36\%). Atypia in ulcers constituted $3 \%$ of cases. Many consults were finalized in disagreement with the contributor $(44 \%)$ : of the disagreements, most were major $(92 \%$; downgraded $75 \%$, upgraded $25 \%$ ) and $8 \%$ were minor (neoplastic $60 \%$, nonneoplastic $40 \%$ category). Clinician-driven consults were similar: $43 \%$ disagreement with the contributor, $89 \%$ major (downgraded $75 \%$, upgraded $25 \%$ ), minor $11 \%$ (neoplastic 100\%)]. Missed diagnoses included the following: Kayexalate, low-grade dysplasia, high-grade dysplasia, Cellcept colitis ( $n=1$ each).

Conclusions: This study highlights knowledge gaps in the lower GIT as classification of polyps, dysplasia, and colitis. Consults were often in major disagreement with the contributor, and the finalized disagreements were usually downgraded from the submitting diagnosis.

\section{Assessment of Molecular Alterations in Recurrent Gastrointestinal Stromal Tumors Compared to Matched Primary Lesions by Next Generation Sequencing}

Naziheh Assarzadegan', Gregory Olsen², Michael Chang ${ }^{3}$, Kimberly Newsom ${ }^{4}$ Michael Feely'. 'Gainesville, FL, ${ }^{2}$ University of Florida, Gainesville, FL, ${ }^{3}$ Malcom Randall VA Medical Center, ${ }^{4}$ University of Florida

Background: Due to well defined molecular alterations and the availability of medications which take advantage of these changes, gastrointestinal stromal tumors (GISTs) are particularly well suited to targeted therapy. However, tumor recurrence is not uncommon and the development of resistance to these therapies has been implicated in this process to some degree. While previous studies have evaluated the presence of secondary mutations in KIT and PDGFRA in these resistant tumors, a broader evaluation of the genomic changes in recurrent GISTs is lacking. In this study, we examined the genomic alterations in matched pairs of primary and recurrent GISTs by next generation sequencing to more comprehensively evaluate this process.

Design: From our institutional archives, 8 instances of primary GIST were identified which also had subsequent recurrent material. The corresponding pathology reports and clinical charts were reviewed. DNA from each specimen was sequenced on the Illumina NextSeq using our institutional NGS panel targeting the exons of 76 genes. Sequence data was processed using a customized analysis pipeline designed to detect base substitutions and insertions/deletions using hg19 as the reference.

Results: A total of 8 matched pairs, equaling 16 individual samples, of primary GIST and subsequent recurrence were retrieved. Of these samples, 15 had sufficient coverage to report meaningful data (Table 1). Most patients received a tyrosine kinase inhibitor prior to excision of their recurrence ( 6 of 8 patients) and mean time to the evaluated recurrence was 40 months (range: 5-75). All resulted cases demonstrated a KIT mutation in the primary tumor with 6 of 7 recurrences showing the same alteration. A minority of cases had novel alterations identified in the recurrent tumor with variations in KIT exon 17, NF1, TET2 and $B C R$ being identified.

\begin{tabular}{|l|l|l|l|}
\hline Case & Interval Therapy & Alterations in Primary & Alterations in Recurrence \\
\hline 1 & imatinib & KIT (exon 9) & KIT (exon 9) \\
\hline 2 & none & KIT (exon 11) & KIT (exon 11) \\
\hline 3 & none & KIT (exon 11) & NF1 \\
\hline 4 & imatinib/sunitinib & KIT (exon 11) & KIT (exon 11) \\
& & KIT (exon 17) \\
\hline 5 & imatinib & KIT (exon 11) & KIT (exon 11) \\
\hline 6 & imatinib & insufficient coverage & KIT (exon 11) \\
\hline & & & KIT (exon 11) \\
7 & imatinib & KIT (exon 11) & TET2 \\
& & & BCR \\
\hline 8 & imatinib & KIT (exon 11) & KIT (exon 11) \\
\hline
\end{tabular}

Conclusions: Secondary mutations in recurrent GIST can involve KIT but alterations in BCR and TET2 may also occur. The significance of these findings warrants further investigation. Also, while NF1 mutations have been implicated in a subset of GISTs, alterations in these gene are not thought to coexist with $K I T$ mutations. The significance of this alteration in one of our recurrent cases is unknown.

\section{Microsatellite Status and Key Cancer-Related Gene Mutation Profile in Colonic Signet Ring Cel Carcinoma Using Next Generation Sequencing Approach}

Andrew Bandy', Audrey Deeken-Draisey², Juehua Gao², Katrina Krogh $^{3}$, Ryan Jones ${ }^{4}$, Maryam Pezhouh ${ }^{5}$, Guang-Yu Yang ${ }^{1} .{ }^{1}$ Chicago, IL, ${ }^{2}$ Northwestern Memorial Hosp., Chicago, IL, ${ }^{3}$ Northwestern Memorial Hospital, ${ }^{4}$ Naperville, IL, ${ }^{5}$ Northwestern University, Feinberg School of Medicine, Chicago, IL

Background: While colorectal adenocarcinoma as an overall group continues to decline in incidence, the less common subtype of colonic signet ring cell carcinoma (CSRCC) remains steady. CSRCC is generally diagnosed at an advanced stage with worse prognosis compared to the conventional type of colonic adenocarcinoma. However there are many reports of improved survival in SRCC in the setting of microsatellite instability (MSI). Although CSRCC histology is a presumed feature of MSI-high tumors, their cancer-related gene mutation profile and microsatellite status is not well characterized.

Design: 26 cases of CSRCC were retrieved from our institution from 2012-2017. Clinical history, histology, staging and follow up data of al cases were reviewed. The IHC expression of MLH1, MSH2, MSH6 and PMS2 mismatch repair (MMR) proteins were studied on representative sections of each tumor as a surrogate for microsatellite status. Next second generation sequencing was also performed on 15 tumors with available/sufficient tissue for analysis of hotspot mutations in key cancer related genes including KRAS, EGFR, BRAF, PIK3CA, AKT1, ERBB2, PTEN, NRAS, STK11, MAP2K1, ALK, DDR2, CTNNB1, MET, TP53, SMAD4, FBX7, FGFR3, NOTCH1, ERBB4, FGFR1/2.

Results: In this cohort, $16 / 26(62 \%)$ of the CSRCC were right-sided and only $10 / 26(38 \%)$ displayed defective staining for MMR proteins (8/10 with loss of both MLH1/PMS2 and 2/10 with loss of both MSH2/ MSH6), with $8 / 10(80 \%)$ of the defective MMR protein cases being right-sided. The median age of patients was 65 years of age (range $18-86)$ and $25 / 26(96 \%)$ of patients were clinical stage III-IV. Of the 14 cases with tissue sufficient for sequencing analysis, $3 / 14(21 \%)$ demonstrated a BRAF mutation (2/3 with MMR protein defect), 4/14 demonstrated KRAS \pm PIK3CA mutations (2/4 with MMR protein defect) and 3/14 displayed TP53 mutations (none with MMR protein defect). The remainder of cases showed no distinct hotspot mutations in the genes tested ( $1 / 5$ with MMR protein defect).

Conclusions: Although CSRCC were mainly right-sided in our cohort, the association with MSI status appears to be only one of the major alterations seen ( $38 \%$ for MSI). Of cases with abnormal MMR proteins and NGS data, $40 \%$ demonstrated BRAF mutation suggestive of CIMP pathway. An additional $40 \%$ displayed KRAS/ PIK3CA mutations suggestive of an alternative pathways to CIMP or $\mathrm{MSI}$. Key gene mutation profiles in this study indicate Braf/Kras/p53 mutations appear to be major genetic events $(9 / 14,64 \%)$ in CSRCC carcinogenesis.

\section{Clinicopathologic Analysis of Benign Lipomatous Lesions of the Colon}

Sam L Barron', Raul S Gonzalez'. 'University at Albany, Albany, NY, ${ }^{2}$ University of Rochester Medical Center, Rochester, NY

Background: Benign lipomatous lesions of the colon are relatively common. Most are small and clinically silent, detected during 
colonoscopy for screening or unrelated symptoms. A few histologic subtypes are appreciable, and intramucosal lipomas have been linked to Cowden syndrome. Overall, however, subtype relationships with clinical features have not been well established.

Design: We categorized 404 benign colonic lipomatous lesions from 395 patients as angiolipoma (more than $30 \%$ of the lesion composed of large or ectatic blood vessels), fibrolipoma (more than $30 \%$ fibrous bands), intramucosal lipoma (benign fat predominantly or entirely involving the mucosa) or lipoma not otherwise specified (NOS) (benign adipose tissue without distinguishing features). Available clinical data were recorded. We compared the following across groups: patient age and sex; tumor site and size; and whether the lesion was symptomatic, was flat or pedunculated endoscopically, and/or showed another overlying polyp microscopically.

Results: Findings are summarized in the Table. Approximately $11 \%$ of the lesions were symptomatic, usually causing bleeding, obstruction, or a visible mass on imaging. Flat lesions more often showed an overlying polyp than pedunculated ones (55\% vs. $6 \%, P<0.0001)$. Symptomatic lesions were larger on average than non-symptomatic ones (mean $2.83 \mathrm{~cm}$ vs. $1.23 \mathrm{~cm}, P<0.0001$ ). Unlike other lesion types, lipoma NOS had a female predominance $(P=0.041)$, was more commonly right-sided $(P<0.001)$, was more often flat $(P=0.013)$, and was more commonly associated with an overlying polyp $(P=0.0056)$. Compared to other lesion types, angiolipomas were larger (mean $1.93 \mathrm{~cm}, P<0.0001)$ and more commonly symptomatic $(P=0.038)$. Intramucosal lipomas were smallest on average (mean $0.48 \mathrm{~cm}$ ), and no patient with this lesion type carried a diagnosis of any genetic syndrome. Fibrolipomas were most commonly left-sided (77\%). Only 4 cases showed associated reactive change; 3 of these were $\geq 4 \mathrm{~cm}$.

\begin{tabular}{|l|l|l|l|l|l|}
\hline & $\begin{array}{l}\text { Angio- } \\
\text { lipoma } \\
\text { (n=24) }\end{array}$ & $\begin{array}{l}\text { Fibrolipoma } \\
\text { (n=57) }\end{array}$ & $\begin{array}{l}\text { Intramuco- } \\
\text { sal lipoma } \\
\text { (n=24) }\end{array}$ & $\begin{array}{l}\text { Lipoma NOS } \\
\text { (n=299) }\end{array}$ & P-value \\
\hline $\begin{array}{l}\text { Average age } \\
\text { (years) }\end{array}$ & 64 & 62 & 60 & 63 & 0.34 \\
\hline Sex & $15 \mathrm{M}: 9 \mathrm{~F}$ & $32 \mathrm{M}: 25 \mathrm{~F}$ & $14 \mathrm{M}: 10 \mathrm{~F}$ & $126 \mathrm{M}: 173 \mathrm{~F}$ & 0.041 \\
\hline Site (right/left) & $6 \mathrm{R}: 16 \mathrm{~L}$ & $13 \mathrm{R}: 43 \mathrm{~L}$ & $12 \mathrm{R}: 12 \mathrm{~L}$ & $181 \mathrm{R}: 107 \mathrm{~L}$ & $<0.001$ \\
\hline Symptomatic & $\begin{array}{l}5 \text { yes, } 16 \\
\text { no }\end{array}$ & 1 yes, 38 no & 0 yes, 8 no & 24 yes, 189 no & 0.038 \\
\hline $\begin{array}{l}\text { Average size } \\
\text { (cm) }\end{array}$ & 1.93 & 0.91 & 0.48 & 1.24 & $<0.0001$ \\
\hline $\begin{array}{l}\text { Appearance } \\
\text { (flat/peduncu- } \\
\text { lated) }\end{array}$ & $2 \mathrm{f}, 7 \mathrm{p}$ & $9 \mathrm{f}, 7 \mathrm{p}$ & $1 \mathrm{f}, 1 \mathrm{p}$ & $50 \mathrm{f}, 19 \mathrm{p}$ & 0.013 \\
\hline $\begin{array}{l}\text { Overlying } \\
\text { polyp }\end{array}$ & 0 yes, 24 & 4 yes, 53 no & 1 yes, 23 no & 65 yes, 234 no & 0.0056 \\
\hline
\end{tabular}

Conclusions: A sizable minority ( 11\%) of colonic lipomas are nonincidental. Angliolipomas are more often large and symptomatic, while lipomas NOS are more often flat with an associated overlying polyp. These lesions are generally sporadic and do not appear consistently linked to syndromes, including intramucosal lipomas.

\section{Does Surveillance Colonoscopy in Colorectal Inflammatory Bowel Disease (IBD) Protect Against the Development of High Stage Colorectal Carcinoma (CRC)?}

Mayada Basheer ${ }^{1}$, Mai Iwaya ${ }^{2}$, James Conner ${ }^{2}$, Robert RiddelR ${ }^{1}$ University of Toronto, Toronto, ON, ${ }^{2}$ Mount Sinai Hospital, Toronto, ON

Background: Optimal management of IBD patients includes regular colonoscopic surveillance, to detect IBD-associated dysplasia and carcinoma. We reviewed carcinomas arising in patients under surveillance, and hypothesized that they would have a low pathology stage, that there would be a relationship between the grade of previous dysplasia and the pathology stage, and that longer intervals between the last colonoscopy and diagnosis of CRC would have a higher pathology stage.

Design: 24 patients had undergone prior colonoscopies. These were classified into 3 groups according to the histological grade of dysplasia in prior biopsies (group 1: no prior dysplasia (NPD-8), groups 2: indefinite for dysplasia (1) and low grade dysplasia (LGD-9) group 3: high grade dysplasia (HGD-6). The cohort was subdivided into two categories according to time interval between surveillance $(\leq 2$ years and $>2$ years, the latter not strictly being in surveillance). Carcinomas were classified into pTN stages 1, 2 or 3.

Results: There were 14 men and 10 women. 14 had ulcerative colitis(UC), 9 Crohn's disease(CD) and 1 IBDU. Patients with CD had a higher risk ( $5 / 9$ patients) of having no prior dysplasia compared with $3 / 14$ with UC (not significant). 8 patients had no prior dysplasia on colonoscopic surveillance biopsies (3UC and 5CD), 6 of which had had surveillance colonoscopy within 2 years of the diagnosis of carcinoma ( $3 \cup C$ and $3 C D$ ). In this group there were no stage 1, 3 stage 2 and 3 stage 3 carcinomas. 2 were in the midst of inflammatory polyps, 2 were in the distal rectum, one was a flat/dome-like lesion and one was at the ileocecal valve. Of those with dysplasia in the 2 years prior o the diagnosis of carcinoma there were 4 stage 1 (1 prior LGD and 3 prior HGD) but also 5 stage 3 carcinomas (4LGD and 1HGD). Of those whose prior colonoscopy was $>2$ years prior to the diagnosis of carcinoma, 2 had NPD (stage 1 and stage 2), 5 had prior LGD (2 stage 2 and 3 stage 3 ), and 2 had a diagnosis of HGD ( 1 stage 1 and 1 stage 2). Paradoxically patients with HGD had fewer high stage lesions (4 stage 1 , 1 each stage 2 and stage 3), whereas LGD had a preponderance of high stage CRC (1 stage 1, 2 stage 2 and 7 stage 3 ).

Conclusions: These findings suggest that most patients who develop CRC while under surveillance do not have low stage tumors at time of diagnosis, regardless of the time since last biopsy, or history, or grade of dysplasia.

675 Significance of Clinicopathologic Parameters, Including Margin Distance and Tumor Budding, on Local Disease Recurrence Following Esophageal Endoscopic Mucosal Resection

Phoenix Bell', ILKe Nalbantoglu², Justin Cates ${ }^{3}$, Raul S Gonzalez ${ }^{1}$ ${ }^{1}$ University of Rochester Medical Center, Rochester, NY, ${ }^{2}$ Washington University in St. Louis, Saint Louis, $\mathrm{MO},{ }^{3}$ Vanderbilt University Medical Center, Nashville, TN

Background: Endoscopic mucosal resection (EMR) is widely employed for treatment of esophageal dysplasia and low-stage carcinoma. A detailed, large-cohort analysis evaluating margin status margin clearance, and tumor budding in EMR specimens has not been performed.

Design: We evaluated 270 esophageal EMR specimens with glandular neoplasia from 133 patients. We recorded patient age, patient sex, specimen fragmentation (i.e., 1 or $\geq 1$ tissue fragment per endoscopic lesion), intestinal metaplasia, highest-grade lesion, margin status and clearance, cancer invasion depth, lymphovascular invasion (LVI), and tumor budding (using published guidelines counting one hotspot in a $0.785 \mathrm{~mm}^{2}$ field). These factors were compared to local disease recurrence, defined as the same or a higher-grade lesion recurring within $2 \mathrm{~cm}$ of the prior EMR site.

Results: Adenocarcinoma was the highest-grade lesion in $42 \%$ of specimens (39\% T1a, 3\% T1b), high-grade dysplasia in $36 \%$, and low-grade dysplasia in 22\%. Average age at first EMR was 66 years. Seventeen percent of specimens were fragmented. Age had a minor but significant effect on recurrence risk (hazard ratio [HR] 1.02 per yea older, 95\% confidence interval $[\mathrm{Cl}]$ 1.00-1.04, $\mathrm{P}=0.033$ ). Fragmentation did not significantly influence recurrence $(P=0.072)$, nor did "positive tissue edges" in fragmented specimens $(P=0.52$ for dysplasia, $P=0.49$ for carcinoma). Positive margins increased recurrence risk in intact (non-fragmented) specimens $(P<0.001)$, but in specimens with negative margins, margin clearance did not significantly influence recurrence $(\mathrm{HR}=0.71$ per millimeter clearance, 95\% Cl 0.49-1.03, $\mathrm{P}=0.074)$. Adenocarcinoma recurrence risk was not affected by tumor budding $(P=0.82), L V I(P=0.70)$, or depth of invasion $(P=0.28)$

Conclusions: Positive resection margins increase the risk of recurrence for intact EMR specimens, but not fragmented ones, which supports labeling margin status "not evaluable" in fragmented specimens. As lesional distance from margin was not significantly associated with risk of recurrence, appropriate minimum clearance cannot be recommended based on these data. Tumor budding and lesional depth do not influence local recurrence in malignant EMRs. These results offer some insight into endoscopic management of Barrett's metaplasia-related lesions, but they may be confounded by "field effect," as subsequent development of dysplasia or malignancy may be due to the abnormal local microenvironment rather than the pathologic characteristics of prior EMR specimens.

\section{Extended Immunophenotyping of Small Intestinal Adenocarcinoma Reveals Frequent CK20/CDX2/ CDH17 Expression but Only Rare SATB2-Positivity}

Andrew Bellizzi', Matthew Keeney², Daniel Pelletier'. 'University of lowa Hospitals and Clinics, lowa City, IA, ${ }^{2}$ lowa City, IA

Background: We recently encountered a metastatic carcinoma of occult origin (CUP) with a "lower Gl immunophenotype" (i.e. diffuse, strong CK20/CDX2) subsequently found to be of small intestinal origin; our medical oncologists make treatment decisions in ampullary cancer based on whether we assign an "intestinal (I)" or "pancreatobiliary (PB)" phenotype; and we recently validated the emerging intestinal markers $\mathrm{CDH} 17$ and SATB2. All of these prompted us to reassess the immunophenotype of small intestinal adenocarcinoma (SIA).

Design: Immunohistochemistry for CK7, CK20, CDX2, CDH17 SATB2, and the MMR proteins was performed on tissue microarrays of 93 SIAs. The first 5 markers were assessed for intensity $(0-3+)$ and extent $(0-100 \%)$ with an $\mathrm{H}$-score calculated (intensity*extent) the MMR proteins were assessed as intact or lost. Primary site was recorded and grade (well [WD], moderately [MD], poorly [PD], and undifferentiated[UD]) and histotype (I vs PB) were assigned based on 
H\&E review. Fisher's exact test was used with $\mathrm{p}<0.05$ significant.

Results: Overall, the most frequently/strongly expressed marker was $\mathrm{CDH} 17$ (88\%; mean H-score 246), while SATB2 was infrequently/weakly expressed (13\%; mean $\mathrm{H}$-score 64). Expression of differentiation markers was not influenced by grade (data not shown). $17 \%$ of cases demonstrated loss of $>/=1 \mathrm{MMR}$ protein (dMMR). Detailed expression data are presented in the Table. All combinations of CK7/CK20/CDX2 were encountered (data not shown).

Table: Detailed Expression Data: \% Positive (Mean H-score, if Positive)

\begin{tabular}{|l|l|l|l|l|l|l|l|}
\hline & $\begin{array}{l}\text { Overall } \\
(\mathrm{n}=93)\end{array}$ & I $(\mathrm{n}=55)$ & $\begin{array}{l}\text { PB } \\
(\mathrm{n}=34)\end{array}$ & $\begin{array}{l}\text { Foregut } \\
(\mathrm{n}=79)\end{array}$ & $\begin{array}{l}\text { Midgut } \\
(\mathrm{n}=14)\end{array}$ & $\begin{array}{l}\mathrm{pMMR} \\
(\mathrm{n}=73)\end{array}$ & $\begin{array}{l}\text { dMMR } \\
(\mathrm{n}=15)\end{array}$ \\
\hline CK7 & $\begin{array}{l}65 \% \\
(232)\end{array}$ & $\begin{array}{l}56 \% \\
(200)\end{array}$ & $\begin{array}{l}79 \% \\
(278)\end{array}$ & $\begin{array}{l}70 \% \\
(233)\end{array}$ & $36 \%(221)$ & $62 \%(253)$ & $73 \%(140)$ \\
\hline CK20 & $\begin{array}{l}54 \% \\
(184)\end{array}$ & $\begin{array}{l}73 \% \\
(187)\end{array}$ & $\begin{array}{l}27 \% \\
(159)\end{array}$ & $\begin{array}{l}51 \% \\
(171)\end{array}$ & $71 \%(233)$ & $58 \%(206)$ & $53 \%(65)$ \\
\hline CDX2 & $\begin{array}{l}74 \% \\
(162)\end{array}$ & $\begin{array}{l}93 \% \\
(189)\end{array}$ & $\begin{array}{l}47 \% \\
(85)\end{array}$ & $\begin{array}{l}70 \% \\
(150)\end{array}$ & $\begin{array}{l}100 \% \\
(212)\end{array}$ & $74 \%(175)$ & $93 \%(124)$ \\
\hline CDH17 & $\begin{array}{l}88 \% \\
(246)\end{array}$ & $\begin{array}{l}100 \% \\
(268)\end{array}$ & $\begin{array}{l}71 \% \\
(212)\end{array}$ & $\begin{array}{l}86 \% \\
(239)\end{array}$ & $\begin{array}{l}100 \% \\
(283)\end{array}$ & $88 \%(253)$ & $\begin{array}{l}100 \% \\
(231)\end{array}$ \\
\hline SATB2 & $\begin{array}{l}13 \% \\
(64)\end{array}$ & $\begin{array}{l}20 \% \\
(67)\end{array}$ & $\begin{array}{l}3 \% \\
(27)\end{array}$ & $\begin{array}{l}10 \% \\
(59)\end{array}$ & $29 \%(73)$ & $14 \%(73)$ & $13 \%(16)$ \\
\hline
\end{tabular}

Conclusions: CK20 and CDX2 expression are more frequent in I than in PB tumors (both $\mathrm{p}<0.0001$ ). Despite this, SATB2 is infrequently expressed, indicative of its colorectal-specificity. We will thus employ SATB2 in the setting of a CUP with "Iower GI immunophenotype" to distinguish small intestinal from colorectal origin.

\section{Malignant Colorectal Polyps - Completeness of Pathology Reports And The Direct Impact On Patient Management: Initial Outcomes From A Colon Cancer Screening Program}

Kyra Berg ${ }^{1}$, David Schaeffer ${ }^{2}$, Jennifer Telford ${ }^{3}$, Laura Gentile ${ }^{4}$. 'University of British Columbia, New Westminster, BC, ${ }^{2}$ Vancouver General Hospital, Vancouver, BC, ${ }^{3}$ St Paul's Hospital, ${ }^{4} \mathrm{BC}$ Cancer Agency

Background: With the widespread introduction of colon cancer (CRC) screening programs, there has already been a shift towards detection of CRC at an earlier stage, and pathologists can expect to see increasing numbers of early CRC (malignant polyps). Some of these patients may be treated by polypectomy alone, but $9-24 \%$ have lymph node metastases at the time of presentation. Lack of pathologic poor prognostic factors (high grade differentiation, Iymphovascular invasion, resection margin of $1 \mathrm{~mm}$ or less, and high grade tumor budding) has repeatedly been shown to predict patients at low risk of residual malignancy and lymph node metastases. These criteria have now been included to stratify patients into those who do not require further treatment after polypectomy in the National Comprehensive Cancer Network guidelines, as well as the Japanese guidelines. We assessed if these reporting standards are being met in a large colon cancer screening program, and the resulting effect on patient management.

Design: 289 malignant polyps were identified retrospectively from the colon cancer screening program database by ICD-O v.3 code and pathology reports were screened for appropriate classification, leaving 236 cases. Pathology reports were reviewed for reporting of tumor grade, lymphovascular invasion, tumor budding, and resection margin. Outcome data was collected from subsequent rebiopsy and endoscopic resection reports, surgical pathology reports, oncology consultations, imaging, and autopsy reports.

Results: From the 26,399 patients that underwent colonoscopy following a positive fecal immunochemical test or high risk family history, there have been 236 malignant polyps from 233 patients $(0.9 \%)$. Complete reporting was seen in $41 \%$ of reports. Margin status was reported in $90 \%$, grade in $84 \%$, lymphovascular invasion in $80 \%$, and tumor budding in $44 \%$. Of these 233 patients, 144 patients had a subsequent surgery and only 32 had residual carcinoma identified. 20 patients $(14 \%)$ had lymph node metastases identified. 63 patients had no poor prognostic factors on biopsy, and $26(41 \%)$ of these patients went for surgery. Of those 26 patients, 21 had an incomplete report.

Conclusions: Routine pathologic reporting in malignant polyps is incomplete, with only $41 \%$ complete reporting in this cohort of 236 . This has a major impact on patient care, with $41 \%$ of patients with no identified poor prognostic factor going for subsequent surgery and the majority $(80 \%)$ of those patients having an incomplete report.

\section{Sessile Serrated Adenomas in Young Patients May Have Limited Risk of Malignant Progression}

Mark L Bettington 1 , lan Brown ${ }^{2}$, Christophe Rosty ${ }^{3}$, Neal Walker ${ }^{4}$, Barbara Leggett ${ }^{5}$, Vicki Whitehalf. ${ }^{1}$ Envoi Specialist Pathologists, Brisbane, Queensland, 'Envoi Pathology, Brisbane, Australia, ${ }^{3}$ Brisbane, Australia ${ }^{4}$ Toowong, Qld, Australia, ${ }^{5}$ The Royal Brisbane and Women's Hospital, ${ }^{6}$ QIMR Berghofer Medical Research Institute
Background: Sessile serrated adenomas (SSAs) are the dominant polyp of the serrated neoplasia pathway and as such give rise to approximately $20 \%$ of all colorectal carcinomas. Up to $90 \%$ of these cancers are expected to harbour a BRAF mutation. BRAF mutation is a robust indicator of the serrated neoplasia pathway and the vast majority of BRAF mutated colorectal carcinomas have their origins in a sessile serrated adenoma. Sessile serrated adenomas are being diagnosed with increasing frequency in young patients, placing a significant burden on colonoscopic services. Evidence to direct the surveillance intervals in these young patients is not available. We aimed to provide prelimary evidence that sessile serrated adenomas in young patients may be low risk polyps.

Design: We used two patient cohorts comprising 1) a consecutive series of patients who underwent colonoscopy through a tertiary hospital and 2) a consecutive series of resection specimens for colorectal carcinoma processed through a pathology laboratory. The prevalence of sessile serrated adenomas by age was determined in the patients who underwent colonoscopy and compared to the ages of the patients with BRAF mutated colorectal carcinoma in the pathology series.

Results: The prevalence of SSAs was similar regardless of age (overall prevalence $20.1 \%$ ). In contrast BRAF mutated colorecta carcinomas were very rare at less than fifty years of age $(3.8 \%)$ and uncomon at less than sixty years of age (9.3\% of cases). However in old patients (over eighty years) they were common $(39.8 \%)$.

Table 1. Percentage of BRAF mutated colorectal carcinomas divided by age
\begin{tabular}{|l|l|l|}
\hline Age & All carcinomas & All BRAF mutated carcinomas \\
\hline$<50$ & 40 & $1(2.5 \%)$ \\
\hline $50-59$ & 63 & $3(4.8 \%)$ \\
\hline $60-69$ & 112 & $16(14.3 \%)$ \\
\hline $70-79$ & 131 & $40(30.5 \%)$ \\
\hline $80+$ & 98 & $39(39.8 \%)$ \\
\hline
\end{tabular}

Conclusions: These results suggest that sessile serrated adenomas develop at a young age but have a prolonged dwell time and are unlikely to develop into cancer before sixty years of age. These finding support the concept that sessile serrated adenomas may not require close surveillance in young patients, however further targeted studies are required prior to drawing firm conclusions.

\section{Intestinal C4d Immunohistochemical Staining in Adult Transplant-Associated Intestinal Thrombotic Microangiopathy}

Luke E Blower', Sarah Wall', Qiuhong Zhao', Spero Cataland" Sumithira Vasu', Martha M Yearsley'. 'The Ohio State Wexner Medical Center, Columbus, $\mathrm{OH}$

Background: Thrombotic microangiopathy (TMA) is an uncommon but devastating complication of hematopoietic stem cell transplantation (HSCT). C4d immunohistochemical (IHC) staining in renal arterioles has shown to be both a good marker of complement system activation and a useful diagnostic tool to support a diagnosis of transplant associated TMA (TA-TMA). We sought to investigate C4d IHC deposition in intestinal vasculature as a marker of TA-TMA.

Design: Endoscopic biopsies from 124 adult patients with HSCT and clinical grade 3/4 intestinal Graft-Versus-Host Disease (GVHD) admitted at our institution from 2008 - 2016 were reviewed. Patients were assigned to groups by likelihood of TA-TMA based on presence of transfusion requirements, hemolysis, and renal dysfunction. 16 cases were selected for $\mathrm{C} 4 \mathrm{~d}$ IHC staining, 11 with clinical diagnosis of TMA ( $\geq 2$ clinical criteria) and 5 of clinical GVHD who did not meet requirements for TMA ( $\leq 1$ clinical criteria). The $\mathrm{C} 4 \mathrm{~d}$ IHC staining pattern was reviewed in a blinded fashion and 8 histologic features were recorded.

Results: 10 out of 11 cases (90. 91\%) with $\geq 2$ clinical criteria for TMA had positive linear C4d IHC endothelial staining compared to 3 out of 5 cases $(60 \%)$ with $\leq 1$ clinical criteria. The following staining pattern was present in the positive clinical TMA cases: Strong staining $(80 \%)$ mucosal blood vessels $(80 \%)$, capillaries $(80 \%),>50 \%$ circumferential staining $(90 \%)$, linear staining in $1-25 \%$ of the blood vessels $(70 \%)$ microthrombi staining (27.3\%). Muscularis mucosae, muciphages and periarteriolar staining were disregarded as non-specific. In contrast cases with $\leq 1$ clinical criteria showed strong capillary mucosal staining in $1-25 \%$ of vessels and $<50 \%$ of the circumference in 2 out of 3 cases $(66.7 \%)$. 
Table 1. Pattern of C4d IHC staining in intestinal biopsies

\begin{tabular}{|c|c|c|c|c|}
\hline \multirow[t]{2}{*}{ C4d Staining } & \multicolumn{2}{|c|}{$\geq 2$ Clinical Criteria for TMA } & \multicolumn{2}{|c|}{$\leq 1$ Clinical Criteria for TMA } \\
\hline & $n=11$ & $\%$ & $n=5$ & $\%$ \\
\hline \multicolumn{5}{|c|}{ Linear Endothelial Staining } \\
\hline Present & 10 & 90.91 & 3 & 60 \\
\hline Absent & 1 & 9.09 & 2 & 40 \\
\hline \multicolumn{5}{|l|}{ Stain Intensity } \\
\hline Strong & 8 & 80 & 2 & 66.7 \\
\hline Weak & 2 & 20 & 1 & 33.3 \\
\hline \multicolumn{5}{|c|}{ Blood Vessel Compartment } \\
\hline Mucosal & 5 & 50 & 2 & 66.67 \\
\hline Submucosal & 2 & 20 & 1 & 33.33 \\
\hline Both & 3 & 30 & 0 & 0 \\
\hline \multicolumn{5}{|c|}{$\begin{array}{l}\text { Vessels with positive } \\
\text { staining }\end{array}$} \\
\hline Capillaries & 5 & 50 & 1 & 33.33 \\
\hline Arterioles & 2 & 20 & 1 & 33.33 \\
\hline Both & 3 & 30 & 1 & 33.33 \\
\hline \multicolumn{5}{|c|}{$\begin{array}{l}\text { Percentage of Circumferen- } \\
\text { tial Staining }\end{array}$} \\
\hline$>50 \%$ & 9 & 90 & 1 & 33.3 \\
\hline$<50 \%$ & 1 & 10 & 2 & 66.7 \\
\hline \multicolumn{5}{|c|}{$\begin{array}{l}\text { Percentage of Vessels with } \\
\text { Linear Staining }\end{array}$} \\
\hline$>50 \%$ & 0 & 0 & 1 & 33.3 \\
\hline $25-50 \%$ & 3 & 30 & 0 & 0 \\
\hline $1-25 \%$ & 7 & 70 & 2 & 66.7 \\
\hline \multicolumn{5}{|c|}{ Thrombi Staining } \\
\hline Present & 3 & 27.3 & 2 & 40 \\
\hline Absent & 8 & 72.7 & 3 & 60 \\
\hline \multicolumn{5}{|c|}{ Periarteriolar Staining } \\
\hline Present & 6 & 54.5 & 2 & 50 \\
\hline Absent & 5 & 45.5 & 2 & 50 \\
\hline
\end{tabular}

Conclusions: Our study is the first one to describe the pattern of expression of $\mathrm{C} 4 \mathrm{~d}$ IHC staining in the intestinal mucosa in adult patients with TA-TMA. In our study, strong linear endothelial C4d IHC staining in mucosal capillaries, in $>50 \%$ of the circumference, in at least $1-25 \%$ of the capillaries, and microthrombi staining were features present in patients with $\geq 2$ clinical criteria for TA-TMA. Our study shows that $\mathrm{C} 4 \mathrm{~d}$ IHC staining may be a reliable diagnostic tool to help determine the presence of TA-TMA. Future studies to include a larger cohort with additional control population are needed to further characterize the utilization of C4d staining in TA-TMA.

680 Histologic Features of Adult Intestinal TransplantAssociated Thrombotic Microangiopathy in 124 Patients with Grade 3/4 Intestinal Graft-VersusHost-Disease: A Retrospective Review

Luke E Blower', Sarah Walr', Akwasi Agyeman', Qiuhong Zhao', Spero Cataland', Sumithira Vasu', Martha M Yearsley'. 'The Ohio State Wexner Medical Center, Columbus, $\mathrm{OH}$

Background: Transplant associated thrombotic microangiopathy (TATMA) is an uncommon but devastating complication of hematopoietic stem cell transplantation (HSCT) that has a poor survival rate. TMA involvement of the gastrointestinal tract has no well-established histologic diagnostic criteria. We sought to validate in adults histologic findings that have been associated with pediatric TA-TMA.

Design: Endoscopic biopsies from 124 adult patients with HSCT and clinical grade 3/4 Gastrointestinal Graft-Versus-Host Disease (GVHD) admitted at our institution from 2008-2016 were reviewed retrospectively. Likelihood of clinical TA-TMA (cTA-TMA) was determined based on presence of transfusion requirements, hemolysis, and renal dysfunction. All available biopsies were reviewed in a blinded fashion and 11 histologic features were recorded.

Results: Clinical TA-TMA occurred in 76 patients who had a biopsy performed within 100 days of a GVHD diagnosis. Intraluminal fibrin thrombi were present in 19 of $76(25 \%)$ opatients with cTA-TMA and in 2 of $31(6.5 \%)$ patients with no cTA-TMA $(p=0.03)$. Neutrophilic crypt abscesses were present in 18 of $76(23.7 \%)$ patients with cTATMA and in 14 of $31(45.2 \%)$ patients with no cTA-TMA ( $p=0.03)$. Lamina propria acute inflammation was present in 36 of $76(47.4 \%)$ patients with cTA-TMA and in 22 of $31(71 \%)$ patients with no cTA-TMA $(p=0.03)$. Other histologic features including mucosal hemorrhage, loss of glands, intraluminal schistocytes, endothelial cell separation, denuded mucosa, apoptotic crypt abscess, and ulceration had no statistical significance.

Table 1. Histologic Features in intestinal biopsies from patients with grade 3/4 GVHD and/or clinical TA-TMA

\begin{tabular}{|c|c|c|c|c|c|}
\hline \multirow[t]{2}{*}{ Histologic Features } & \multicolumn{2}{|c|}{ No cTA-TMA $(n=31)$} & \multicolumn{2}{|c|}{ cTA-TMA $(n=76)$} & \multirow[b]{2}{*}{$p$-value } \\
\hline & $\mathbf{n}$ & $\%$ & $\mathbf{n}$ & $\%$ & \\
\hline Mucosal hemorrhage & & & & & 0.76 \\
\hline Absent & 3 & 9.7 & 6 & 7.9 & \\
\hline Present & 28 & 90.3 & 70 & 92.1 & \\
\hline Loss of glands & & & & & 0.32 \\
\hline Absent & 11 & 35.5 & 35 & 46.1 & \\
\hline Present & 20 & 64.5 & 41 & 53.9 & \\
\hline Intraluminal schistocytes & & & & & 0.11 \\
\hline Absent & 31 & 100.0 & 70 & 92.1 & \\
\hline Present & 0 & 0.0 & 6 & 7.9 & \\
\hline Intraluminal fibrin thrombi & & & & & 0.03 \\
\hline Absent & 29 & 93.5 & 57 & 75.0 & \\
\hline Present & 2 & 6.5 & 19 & 25.0 & \\
\hline Endothelial cell separation & & & & & 0.17 \\
\hline Absent & 22 & 71.0 & 63 & 82.9 & \\
\hline Present & 9 & 29.0 & 13 & 17.1 & \\
\hline $\begin{array}{l}\text { Total denudation of areas of } \\
\text { mucosa }\end{array}$ & & & & & 0.15 \\
\hline Absent & 10 & 32.3 & 36 & 47.4 & \\
\hline Present & 21 & 67.7 & 40 & 52.6 & \\
\hline Apoptotic crypt abscesses & & & & & 0.25 \\
\hline Absent & 9 & 29.0 & 31 & 40.8 & \\
\hline Present & 22 & 71.0 & 45 & 59.2 & \\
\hline Neutrophilic crypt abscesses & & & & & 0.03 \\
\hline Absent & 17 & 54.8 & 58 & 76.3 & \\
\hline Present & 14 & 45.2 & 18 & 23.7 & \\
\hline Lamina propria acute inflammation & & & & & 0.03 \\
\hline Absent & 9 & 29.0 & 40 & 52.6 & \\
\hline Present & 22 & 71.0 & 36 & 47.4 & \\
\hline Ulceration & & & & & 0.49 \\
\hline Absent & 23 & 74.2 & 61 & 80.3 & \\
\hline Present & 8 & 25.8 & 15 & 19.7 & \\
\hline $\begin{array}{l}\text { CMV/Adenovirus cytopathic } \\
\text { effect H\&E or +IHC }\end{array}$ & & & & & 0.81 \\
\hline Absent & 29 & 93.5 & 72 & 94.7 & \\
\hline Present & 2 & 6.5 & 4 & 5.3 & \\
\hline
\end{tabular}

Conclusions: In our study of adult patients with cTA-TMA, histologic features overlapped with those of intestinal grade 3/4 GVHD but no cTA-TMA. The presence of intraluminal fibrin thrombi was the most statistically significant histologic feature in differentiating adult intestinal TA-TMA from intestinal GVHD. Presence of neutrophilic crypt abscesses and/or lamina propria acute inflammation favor other entities over intestinal TA-TMA. Further studies of patients with intestinal GVHD to identify other possible histologic features of TATMA are warranted.

681 Assessing the Predictive Value of Anatomic Site, Gross Dimension, Pathologist and Endoscopist in the Diagnosis of Sessile Serrated Adenoma in 31,354 Colorectal Polyp Specimens using Logistic Regression and Funnel Plots

Michael Bonert ${ }^{1}$, Jennifer Dmetrichuk ${ }^{2}$, Sahar Al-Haddad ${ }^{\beta}$, Hamid Kazerouni', Marko Simunovic', Asghar H Naqvi'. 'McMaster University, Hamilton, ON, ${ }^{2}$ Hamilton, Ontario, ON, ${ }^{3}$ Mississauga, ON

Background: Logistic regression (LR) can be used to assess the predictive value of multiple factors simultaneously. Funnel plots (FPs) provide context to raw pathologist call rates (PCRs) and raw endoscopist capture rates (ECRs).

Design: All in house colorectal polyp specimen (CRPS) reports in 2 teaching institutions were extracted for a 6 year period (2011-16). A hierarchical free text string matching algorithm (HFTSMA) coded the diagnoses, anatomic site (AS), found the maximal gross dimension (MAGD) and calculated the PCRs \& ECRs. The PCRs were compared with FPs centered on the group median call rate (GMCR) with funnel edges defined by \pm 2 and \pm 3 standard errors.

Results: 31,354 CRPS were submitted. In $99 \%, 99 \%$ and $81 \%$ of the CRPS, respectively, the diagnosis, AS and MAGD could be coded. An audit of 200 cases showed the HFTSMA had a categorization accuracy of $98 \%$ in the coded cases for the diagnosis, AS and MAGD. 570 sessile serrated adenomas (SSAs) were diagnosed. Raw SSA PCR statistics (mean/median/stdev/min-max) in those interpreting $>200$ CRPS (26 pathologists) were 2.4/1.7/2.3/0.0-7.4\%. In a cleaned subset of cases where all endoscopists/pathologists were involved in 
$>200$ cases $(27,090)$, the pathologist, endoscopist and AS proximal to descending colon were all predictors of SSA $(P<0.0001)$. An analysis of variance (ANOVA) showed the largest deviance was explained by pathologists (535), followed by the AS (241) and then endoscopists (105). The MAGD was non-predictive for SSA; however, MAGD was predictive for hyperplastic polyp (HP) $(\mathrm{P}<0.0001)$. The GMCR \& number of pathologist FP outliers were $52.8 \%$ and $1(P<0.001) / 5(P<0.05)$ of 26 for tubular adenoma (TA), $26.0 \%$ and $12 / 17$ of 26 for HP, and $1.7 \%$ and $15 / 18$ of 26 for SSA. When divided into left, $\mathrm{mid} /$ flexures and right, the GMCRs for SSA (as \% of all polyps in each region) were $0.6 \%, 2.2 \%$ \& $3.6 \%$ respectively. Higher SSA PCR was associated with lower HP PCR; however, HP PCR also varied considerably in relation to normal tissue; a FP for HP+SSA had a GMCR of $27.0 \%$ and $12 / 18$ of 26

Conclusions: LR confirmed the significance of AS in SSAs and suggests polyp dimension is not a strong predictor for SSA. The PCR variation in our practices for SSA is high compared to other diagnoses such as TA. The SSA to SSA+HP ratio is not a useful predictor, as HP PCR varies considerably independent of the SSA PCR. A partial expert review of SSA cases was done, and we plan to prospectively follow PCRs using funnel plots and LR, as part of a continuous quality improvement initiative.

\section{SATB2 Versus CDX2: A Battle Royale for Diagnostic} Supremacy in Mucinous Tumors

Stefan M Brettfeld", Benjamin Ramos², David Martin ${ }^{3}$, Joshua Hanson'. 'University of New Mexico School of Medicine, Albuquerque, NM, ${ }^{2}$ Albuquerque, NM, ${ }^{3}$ Univ. of New Mexico, Albuquerque, NM

Background: Determining the site of origin for metastatic mucinous tumors is challenging as their immunophenotypes often differ from conventional carcinomas of the same organ. CDX2 has been used as a marker of colorectal origin for many years. However, its specificity is imperfect. Recent studies have investigated the use of SATB2 as an alternative marker, though virtually none of these have comparatively assessed these stains in mucinous tumors from a diverse array of primary sites.

Design: We analyzed SATB2 and CDX2 expression in 219 mucinous tumors. Primary sites included: colorectum (44), ovary (60), breast (31), lung (26), uterus (28), pancreas (15) and upper gastrointestinal tract (15). We employed an additive scoring system measuring intensity (0-3 based on the ER/PR grading system in breast cancer) plus amount $(0=$ no staining, $1=<5 \%, 2=5 \%-49 \%, 3=\geq 50 \%)$ to evaluate these tumors.

Results: SATB2 performed well even at low expression levels as its diagnostic accuracy was acceptable with scores of $\geq 1-3$, evident by Youden's indices $>0.7$ (correlated to the Area under the Curve spanned by a single operating point) at these cutoff scores (Table 1). The sensitivity of SATB2, however, dropped significantly at a score of $\geq 4$. Its greatest diagnostic value (evident by the Overall Accuracy, Table 1) was measured at a score of $\geq 3$, meaning one should see either moderate to high intensity staining in $<5 \%$ of tumor cells or weak staining in $\geq 5 \%$ of the tumor to determine an accurate result. In contrast, CDX2 only showed acceptable accuracy with an expression score of $\geq 5$, as its specificity suffered at lower expression levels (Table 1). Because a SATB2 score $\geq 3$ was found to be the optimal test within our cohort, we calculated positivity rates in all mucinous tumors for both markers at or above this expression level and found that SATB2's specificity was significantly better than CDX2 in the majority of mucinous tumors (Table 2).

Table 1: Accuracy of SATB2 and CDX2 at Various Additive Score Cutoffs.

\begin{tabular}{|l|l|l|l|l|l|}
\hline Stain & $\begin{array}{l}\text { Additive } \\
\text { Score }(\geq)\end{array}$ & $\begin{array}{l}\text { Sensitivity } \\
(95 \% \mathrm{Cl})\end{array}$ & $\begin{array}{l}\text { Secificity } \\
(95 \% \mathrm{Cl})\end{array}$ & $\begin{array}{l}\text { Overall } \\
\text { Accuracy } \\
(95 \% \mathrm{Cl})\end{array}$ & $\begin{array}{l}\text { Youden's } \\
\text { Index }\end{array}$ \\
\hline SATB2 & 1 & $\begin{array}{l}88.6(75.4- \\
96.2)\end{array}$ & $\begin{array}{l}92.6(87.6- \\
96.0)\end{array}$ & $\begin{array}{l}91.8(87.3- \\
95.1)\end{array}$ & 0.81 \\
\hline CDX2 & 1 & $\begin{array}{l}100(92.0- \\
100.0)\end{array}$ & $\begin{array}{l}45.1(37.6- \\
52.8)\end{array}$ & $\begin{array}{l}56.2(49.3- \\
62.8)\end{array}$ & 0.45 \\
\hline SATB2 & 2 & $\begin{array}{l}86.4(72.6- \\
94.8)\end{array}$ & $\begin{array}{l}93.1(88.3- \\
96.4)\end{array}$ & $\begin{array}{l}91.8(87.3- \\
95.1)\end{array}$ & 0.79 \\
\hline CDX2 & 2 & $\begin{array}{l}100(92.0- \\
100.0)\end{array}$ & $\begin{array}{l}49.7(42.1- \\
57.4)\end{array}$ & $\begin{array}{l}59.2(53.0- \\
66.4)\end{array}$ & 0.50 \\
\hline SATB2 & 3 & $\begin{array}{l}81.8(67.3- \\
91.8)\end{array}$ & $\begin{array}{l}94.9(90.5- \\
97.6)\end{array}$ & $\begin{array}{l}92.2(87.9- \\
95.4)\end{array}$ & 0.77 \\
\hline CDX2 & 3 & $\begin{array}{l}100(92.0- \\
100.0)\end{array}$ & $\begin{array}{l}57.7(50.0- \\
65.1)\end{array}$ & $\begin{array}{l}66.2(59.5- \\
72.4)\end{array}$ & 0.58 \\
\hline SATB2 & 4 & $\begin{array}{l}68.2(52.4- \\
81.4)\end{array}$ & $\begin{array}{l}97.1(93.5- \\
99.1)\end{array}$ & $\begin{array}{l}91.3(86.8- \\
94.7)\end{array}$ & 0.65 \\
\hline CDX2 & 4 & $\begin{array}{l}100(92.0- \\
100.0)\end{array}$ & $\begin{array}{l}66.9(59.4- \\
73.8)\end{array}$ & $\begin{array}{l}73.5(67.2- \\
79.2)\end{array}$ & 0.67 \\
\hline SATB2 & 5 & $\begin{array}{l}27.3(15.0- \\
42.8)\end{array}$ & $\begin{array}{l}100(97.9- \\
100.0)\end{array}$ & $\begin{array}{l}85.4(80.0- \\
89.8)\end{array}$ & 0.27 \\
\hline CDX2 & 5 & $\begin{array}{l}81.8(67.3- \\
91.8)\end{array}$ & $\begin{array}{l}90.9(85.6- \\
94.7)\end{array}$ & $\begin{array}{l}89.0(84.1- \\
92.9)\end{array}$ & 0.73 \\
\hline
\end{tabular}

Conclusions: In mucinous tumors, SATB2 is a more accurate marke of colorectal origin compared to CDX2. This is primarily due to high specificity even at low expression levels. CDX2 is a more sensitive marker but requires strong staining in a significant number of tumo cells to be considered specific. Therefore, while SATB2 outperform benefit in using both stains in a panel to optimize sensitivity and specificity.

\section{Sartans Can Cause Chronic Gastrointestinal Injury Mimicking Several Common Conditions}

Ian Brown' ${ }^{1}$, Alfred Daveson ${ }^{2}$, Benedict Devereaux ${ }^{2}$, Robyn M Laurie ${ }^{3}$ Rhonda Yantiss ${ }^{4}$, Joel Greenson ${ }^{5}$. 'Envoi Pathology, Brisbane, Australia, 'University of Queensland, 'Dorevitch Pathology, of Michigan Hospitals, Ann Arbor, MI

Background: Sartans are angiotensin 2 receptor agonists that can cause injury to the gastrointestinal tract (GIT), most notably an enteropathy that simulates celiac disease (CD). However distinguishing features of sartan-related injury have not been formally studied, and extra-duodenal injury has not been well-described. This study compares the histological features of sartan enteropathy with in the GIT.

Design: We collected well documented cases of sartan-associated mucosal biopsies were evaluated for intraepithelial lymphocytes (IELs), intraepithelial neutrophils, eosinophils/HPF, sub-epithelia collagen, and crypt apoptosis. Findings in the duodenum were

Results: The study group included 4 men and 18 women (mean age: 70 years, range: $34-88$ ) who presented with diarrhoea and normal TTG levels. Compared with controls, study patients were usually $\geq 60$ years old $(17 \%$ v $90 \%, p<0.01)$. While duodenal biopsies from the study group contained $>25 \mathrm{IELs} / 100$ epithelial cells with frequent $(95 \%$ ) villous blunting, IELs were less than in controls (mean $53 \vee 88, p<0.01$ ). Study cases showed more frequent intraepithelial neutrophils $(52 \%$ v $15 \%, p<0.01)$, $>30$ eosinophils/HPF $(71 \%$ v $4 \%, p<0.01)$ and collagen $\geq 10 \mu \mathrm{m}(33 \% \vee 2 \%, p<0.01)$. $90 \%$ showed crypt apoptosis; $33 \%$ had $>1 / 5$ crypts. Gastric biopsies ( $n=19)$ were normal in $32 \% ; 21 \%$ showed chronic gastritis; lymphocytic $(16 \%)$ lleal biopsies $(n=10)$ demonstrated collagenous $(40 \%)$ or lymphocytic $(20 \%)$ ileitis, often with $>30$ eosinophils/HPF $(30 \%)$. Colon biopsies $(n=15)$ also showed frequent collagenous $(20 \%)$ or lymphocytic $(13 \%)$ colitis with $>30$ eosinophils/HPF (13\%); active chronic colitis was seen in $13 \%$ of patients.

Conclusions: Features suggesting sartan enteropathy rather than CD include normal TTG, age $\geq 60$ years, fewer IELs, intraepithelia neutrophils, $>30$ eosinophils/HPF, subepithelial collagen deposits, and crypt apoptosis. Sartan-associated injury occurs throughou mbination with crypt architectural remodeling and active inflammation. Sartans should be considered as a possible etiologic factor for a range of GIT pathologies.

\section{Autophagy in Tumorigenesis of Colon Cancer}

Megan Brown', Zhiqiang Wang'. 'UFCOM Pathology Department,

(I) Colonic mucosa functions environment and autophagy is one mechanism the cells exploit to adapt to such conditions. While being protective, dysregulated autophagy may bring damage to the cells. This study sets out to investigate one such role that autophagy dysregulation plays in the tumorigenesis of colon cancer by systemically profiling expression of 84 key autophagy genes in low risk colonic adenomas against high GIT injury from 4 institutions. Age, sex, and TTG levels were recorded, 
risk ones.

Design: Two groups with each comprised of two low risk tubular adenomas (TA) and two high risk tubulovillous adenomas (TVA) from rectosigmoid colon were identified. Both groups were age and gender matched. A third group with one TA and one TVA were chosen as a blind group. Formalin fixed paraffin embedded tissue curls from candidate polyps were subject to RNA extraction. $\mathrm{RT}^{2}$ Profiler PCR Human Autophagy Arrays (Qiagen Inc) were used to examine expression of 84 genes encoding key components of molecular machinery and regulators of the autophagy process. Relative quantity of expression was determined according to standard method. Normalized expression levels of a gene of interest (GOI) to a housekeeping gene (HKG) were calculated. The fold change in gene expression was then obtained using the normalized expression of the GOI in TVA group divided by the same GOI in TA group.

Results: A global upregulated gene expression was observed in the TVAs as compared to the TAs. Specifically, expression of the 5 HKGs are high with CT values ranging from $24-29$ in TAs and from 20-25 in TVAs. 11 genes are not expressed. In the TAs, 28 genes show relatively low expression with CT values $>30 ; 45$ genes are highly expressed $(\mathrm{CT}<30)$. Taken the relative quantity of expression against the GAPDH gene, $85 \%(38 / 45)$ of the GOI with high expression are upregulated above $2 x$ levels in TVAs. The most profoundly upregulated gene (10X) is CLN3 in the TVA group. In addition, based on the expression profile as established which is highly duplicable, we were able to reversely designate the two polyps in the blind group as one low risk TA and one high risk TVA.

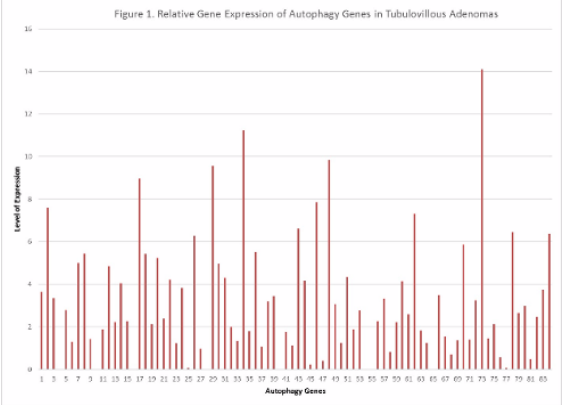

Conclusions: Battenin, the CLN3 gene product, is functionally involved in the digestion and recycling of cellular materials. Its high level of expression and profound dysregulation in high risk TVAs should open research opportunities to further explore its role in the autophagy mediated tumorigenesis in colon cancer and as a potential biomarker to help further stratify risk on patients with adenomatous polyps.

685 Somatic Copy Number Alterations Detected In NonDysplastic Epithelium Are Genomic Biomarkers For Predicting Risk Of Dysplasia And Adenocarcinoma In Barrett's Patients

Diana Bryk ${ }^{1}$, Jorge $L$ Sepulveda ${ }^{1}$, Elena $V$ Komissarova, Sarawut Kongkarnka², Brynn Levy ${ }^{1}$, Armando Del Portillo ${ }^{3}$, Antonia Sepulveda ${ }^{3}$. ${ }^{1}$ Columbia University Medical Center, New York, NY, ${ }^{2}$ Department of Pathology \& Cell Biology, New York, NY, ${ }^{3}$ New York, NY

Background: Patients with Barrett's intestinal metaplasia (BIM) may sequentially progress to low-grade and high-grade dysplasia, and esophageal adenocarcinoma (EAC) and require frequent surveillance endoscopies with biopsy. BIM tissues may harbor genetic alterations that precede the histologic appearance of dysplasia and cancer. Our aim was to identify genomic biomarkers to better predict the risk of development or concurrent dysplasia/EAC by testing routine biopsies of BIM patients for allelic variation of selected genes detected by SNP array analysis.

Design: We tested FFPE biopsies or endoscopic mucosal resection specimens from 57 BIM patients including: 12 with dysplasia/EAC (DAC), 21 BIM samples from never dysplastic BIM patients (BIM-ND) and 21 samples from BIM areas of patients with concurrent dysplasia/ EAC elsewhere (C-BIM). Patients' mean age was $63.9+/-11.8$ and $73 \%$ were male. High-resolution OncoScan FFPE Express 2.0 SNP Arrays (Affymetrix) were used to analyze whole-genome copy number changes and copy neutral loss of heterozygosity (CN-LOH). OncoScan data were analyzed with the Chromosome Analysis Suite application version 3.1 and $\mathrm{R}$ version 3.3.1.

Results: Losses of FHIT exon 5 were seen more frequently in DAC $(67 \%)$ and $\mathrm{C}-\mathrm{BIM}(43 \%)$ than in BIM-ND $(5 \%)(\mathrm{P}=0.01 \mathrm{C}-\mathrm{BIM}$ vs. BIMND). Likewise, deletions or somatic CN-LOH of chromosome $9 p$ involving CDKN2A were more frequent in DAC (58\%) and C-BIM (52\%) than in BIM-ND (19\%)( $\mathrm{P}=0.07 \mathrm{C}-\mathrm{BIM}$ vs. BIM-ND). A copy number gain in 10q11.22 including the PPYR1 gene was more frequently seen in DAC $(33 \%)$ and C-BIM $(19 \%)$ than BIM-ND (5\%). Additional losses in 18q21.1-18q21.2 including BCL2 and SMAD7, 17p13.1 (TP53),
1p15.4(ILK) and 19p13.2(MAP2K7) and gains in 8q24.21(MYC) and 24.2-6q25.1(SAMD5) were seen in DAC and C-BIM but not in BIMND. The fraction of non-diploid genome was low in BIM-ND $(0.01 \%)$ and increased in C-BIM (2.0\%) and DAC $(6.1 \%)$ with higher levels in EAC (10\%) than HGD (1.8\%) ( $p=0.008$ DAC vs. BIM-ND).

Conclusions: The most frequent copy number changes in nondysplastic BIM involved losses of FHIT exon 5 and CDKN2A/B genes which were more frequent in patients who developed dysplasia/EAC than in never dysplastic Barrett's patients. Since these changes can be detected in non-dysplastic epithelium of patients who developed $\mathrm{DAC}$, they represent robust biomarkers to predict risk of dysplasia and adenocarcinoma that may be integrated in the workup of cancer surveillance of Barrett's esophagus patients.

\section{Clinical Associations in Young Patients with Ischemic Colitis}

Kathleen Byrnes', Rifat Mannan', Armen Khararjian², Lysandra Voltaggio'. 'The Johns Hopkins Hospital, ${ }^{2}$ Baltimore, MD

Background: Ischemic colitis (IC) is typically seen in older patients (pts) and associated with hypertension, heart disease, chronic renal failure, and atherosclerosis. In this retrospective study we aimed to characterize the clinical associations of IC in pts younger than 40 years.

Design: All cases of young-onset ischemic colitis from 1984-2017 were retrieved from our files. Slides were reviewed to confirm the diagnosis. Pertinent clinical information and available follow-up was obtained via chart review. Pts were subdivided into the following age groups: $0-20$ and $21-40$.

Results: Of the 60 cases available for re-review, $50 \%(n=30)$ had histologic features of IC. $53 \%$ were female with a mean age of 32 (range, $14-40) .60 \%(n=18)$ were resections. The most common presentations were diarrhea (27\%) and abdominal pain (33\%). In the younger cohort $(n=3)$, IC was associated with surgery, volvulus, and constipation. In the $21-40$ year group, $37 \%(n=10)$ lacked a clinical association. A second subset $(15 \%, n=4)$ had autoimmune disorders (lupus, dermatomyositis, vasculitis). Cocaine use was endorsed by 2 pts. All 5 (18\%) HIV-positive pts were from the pre-HAART era. One pt had severe premature atherosclerosis while another had HMG Co-A lyase deficiency. Histologic evidence of vasculitis was identified in $24 \%$ of the resections and in none of the biopsies. Medication history was available for 5 pts all of whom were on multiple medications, none strongly associated with IC. Microbiology studies were available for 6 pts, all with negative stool cultures. One pt had a positive $C$ difficile toxin but the histologic findings were fitting for IC and an angiogram showed extensive mesenteric vasculitis. Overall, the most common clinical associations were autoimmune disorders (17\%), HIV $(17 \%)$, cocaine use $(7 \%)$, volvulus $(7 \%)$, and smoking $(7 \%)$. On followup, $20 \%$ of the pts died $(n=6)$ from complications of IC, all treated surgically; two pts died of unrelated causes.

Conclusions: IC in children and teenagers is rare and typically associated with prior surgery, volvulus, and constipation. Associations in young adulthood include vasculitides, immune dysregulation, cocaine use, premature atherosclerosis, and smoking. Our cohort had a mortality rate of $33 \%$ in pts treated surgically, which is within the range reported by others in patients with IC. Our findings highlight the importance of accurate diagnosis in these young individuals.

\section{Mast Cell Infiltration and Activation in the Gallbladder Wall: Implications for the Pathogenesis of Functional Gallbladder Disorder in Adult Patients}

Zongxian Cao ${ }^{1}$, Magda Esebua ${ }^{2}$, Arthur Rawlings ${ }^{1}$, Lester Layfield ${ }^{1}$ ${ }^{1}$ University of Missouri, Columbia, MO, ${ }^{2}$ Columbia, $\mathrm{MO}$

Background: Functional gallbladder disorder (FGD) is characterized by recurrent biliary colic with a decreased gallbladder ejection fraction on cholescintigraphy but absence of a visible gallbladder abnormality on ultrasonography. FGD is generally regarded as a primary gallbladder motility disturbance, however, the underlying pathophysiology remains largely unknown. In this study, mast cell infiltration and activation in the gallbladder wall are investigated to determine whether mast cells play a role in the pathogenesis of FGD.

Design: Twenty adult patients with FGD undergoing cholecystectomy were included in the study. Seven patients with no gallbladder disease served as controls who were subject to incidental cholecystectomy during abdominal surgery such as partial hepatectomy. The density of mast cells in the gallbladder wall was assessed by immunohistochemistry and by toluidine blue special stain. Mast cell activation was evaluated by calculating the percentage of degranulated mast cells.

Results: Patients with FGD showed a significant increase in mast cell infiltration in the gallbladder walls compared to the controls $(46.0 \pm$ 1.95 vs $33.02 \pm 0.99, p=0.0004)$. The peak mast cell accumulation was predominantly located in the layer of muscularis mucosae. The percentages of degranulated mast cells were markedly increased in the FGD group $(67.2 \pm 12.5 \%$ vs $38.5 \pm 15.4 \%, p=0.007)$. 
Conclusions: These findings suggest that increased mast cell infiltration and activation in the muscularis mucosae of gallbladder wall might contribute to the compromised gallbladder motility in patient with FGD.

\section{The Utility of CDX2 Loss as a Prognostic Marker in Stage II Colon Cancer}

Matthew J Cecchini', Joanna C Walsh ${ }^{1}$, Jeremy Parfitt ${ }^{1}$, S. Chakrabarti ${ }^{1}$ Rohann Correa', Mary MacKenzie', David Driman². 'London Health' Sciences Centre, Western University, London, ON, ²London, ON

Background: The treatment for colorectal cancer (CRC) is largely surgical followed by adjuvant chemotherapy in high risk cases. In patients with stage II cancer, there is no clear benefit for chemotherapy and the current tools for assessment of risk of relapse and benefit of chemotherapy are inadequate. A recent study identified that decreased expression of genes involved in colonic differentiation was associated with reduced overall survival. With tissue microarrays, it was shown that loss of CDX2 by immunohistochemistry (IHC) resulted in a worse prognosis and that this could be utilized to predict patients that would benefit from chemotherapy.

Design: Having observed that CDX2 expression can be patchy, which could affect tissue microarray evaluation, we elected to validate these prior results for clinical practice by using archival cases of stage II colon cancer. The pathology of all cases was reviewed and three blocks were selected for CDX2 IHC using the mouse monoclonal DAKCDX2 antibody. The mouse monoclonal antibody CDX2-88 was also utilized on a subset of cases. CDX2 expression was scored based on previously published criteria and using a semi-quantitative method. Related markers of colonic differentiation including CDX1, Muc2, GPX2 and villin were also assessed by IHC on a subset of cases.

Results: We studied 122 cases. CDX2 expression was diffusely lost in $11 \%$ and focally lost in $29 \%$ of cases. There was significant variation in CDX2 expression in a given tissue section in $53 \%$ of cases. We did not identify a difference in survival based on CDX2 expression. The CDX2 antibody CDX2-88 had similar results with no difference in overall survival. In a subset of cases we found that Muc2 loss resulted in a reduced survival (HR 4.41; 95\% Cl 1.36-14.22).

Conclusions: Our results with whole slide IHC are different from the previous study, which used tissue microarrays in which only small parts of the tumor were assessed. This suggests that CDX2 may not be a useful prognostic marker in clinical practice. We have identified that loss of Muc2, a downstream transcriptional target of CDX2, is associated with reduced overall survival. This supports the use of colonic differentiation to identify high risk patients.

\section{Histology Can Distinguish Primary Small Intestinal Cancers from Metastatic Neoplasms with Pseudoadenomatous Transformation}

Odise Cenaj', Melanie E Johncilla², Robert Odze1. 'Brigham and Women's Hospital, Boston, MA, ${ }^{2}$ Weill Cornell Medical College, New York, NY

Background: Metastases to the tubal gut may rarely colonize the mucosa in a manner that simulates adenomatous (in situ) change termed "pseudoadenomatous transformation" (PAT). Due to this effect, metastatic tumors may be erroneously interpreted as a primary lesion. The purpose of this study was to determine if there are histologic features in resection specimens that can help distinguish primary from secondary tumors with PAT in the small intestine.

Design: The study group consisted of 23 patients (M/F ratio 1.1, mean age 63 years) with metastatic neoplasm to the small intestine with PAT (primary sites: colon $n=6$, lung $n=3$, pancreas $n=2$, appendix $n=2$, small intestine $n=1$, gallbladder $n=1$, breast $n=1$, kidney $n=1$, uterus $n=1$, and melanoma $n=5$ ), retrieved from institutional pathology files between 2005-2017. In addition to the 23 primary tumors of these cases, 13 additional cases of primary small intestine and 10 cases of primary colon carcinomas from the same period served as a control group $(\mathrm{N}=46)$. Cases and controls were evaluated for a wide variety of histologic parameters in both primary and secondary sites, such as pattern of the leading edge of tumor, paradoxical maturation, features of muscularis mucosae and adjacent non-neoplastic mucosa, as well as growth pattern and relationship of PAT to the invasive component, among others.

Results: Histologically, extension of intramucosal neoplasia beyond the lateral borders of the invasive tumor ("shoulder effect") was present in $100 \%$ of primary tumors vs $0 \%$ of metastatic tumors with PAT $(p<0.01)$. In addition, a "top-down" growth pattern of intramucosal neoplasia was present in $100 \%$ of primary tumors vs $0 \%$ of metastatic tumors with PAT $(p<0.01)$. Other features that were present more frequently, but not exclusively, in PAT vs primary in situ neoplasia were low-grade morphology of the intramucosal neoplastic component $(87 \%$ vs $48 \% ; p=0.02)$, association with ulceration of adjacent non-neoplastic mucosa $(61 \%$ vs $17 \% ; \mathrm{p}<0.01)$, and lack of a polypoid growth pattern ( $4 \%$ vs $48 \%$; $p<0.01$ ). No other features, such as paradoxical maturation, were significantly different between
Conclusions: Lateral extension of intramucosal neoplasia beyond the limits of the mural invasive carcinoma ("shoulder effect") and/ or "top-down" intramucosal neoplasia are highly specific features of primary intestinal carcinoma and can be reliably used to distinguish primary carcinomas from metastatic tumors with PAT in the small intestine.

\section{Detection of ERBB2 Amplification by Next Generation Sequencing Predicts HER2 Expression in Colorectal Carcinoma}

Odise Cenaj', Jason L Hornick', Lynette Sholl'. 'Brigham and Women's Hospital, Boston, MA

Background: ERBB2 (HER2) amplification and overexpression are reported in $2-5 \%$ of colorectal carcinomas (CRC). In treatment-refractory metastatic CRC, HER2 expression predicts response to combination HER2 inhibitor therapy (HERACLES trial). Next generation sequencing (NGS) is increasingly used in clinical practice for biomarker detection however, its utility for selection of patients with advanced CRC who might benefit from HER2 inhibitor therapy has not been established. We hypothesized that detection of ERBB2 amplification by NGS correlates with HER2 overexpression by immunohistochemistry (IHC).

Design: Hybrid capture NGS targeting 300 genes, including the entire coding sequence of $E R B B 2$, was performed on formalin-fixed paraffin-embedded specimens with $\geq 20 \%$ tumor content and $\geq 50 \mathrm{ng}$ DNA. CRC cases were selected retrospectively based on ERBB2 copy number (CN) status, enriching for ERBB2-amplified cases (defined as estimated $\mathrm{CN} \geq 7$ ). Estimated $\mathrm{CN}$ was derived from the log2 ratio of tumor: normal coverage, adjusted for sample tumor content. HER2 IHC (SP3 rabbit monoclonal antibody) was scored based on percentage and intensity of membranous tumor cell staining.

Results: Institutional NGS of 1300 CRC identified $2 \%$ with ERBB2 amplification. Of $102 \mathrm{CRC}$ cases selected for IHC based on known ERBB2 CN status, 15 had ERBB2 amplification (CN 14 to 152, mean 83) 10 had low gain (CN 3-5) and 78 were CN neutral. Using the HERACLES cut-off criteria for HER2 IHC of strong staining in $\geq 10 \%$ of cells $(3+)$ or weak to moderate staining in $\geq 50 \%$ of cells $(2+)$, ERBB2 amplification by NGS was $100 \%$ sensitive $(95 \% \mathrm{Cl} .73-1)$ and $99 \%$ specific $(95 \% \mathrm{Cl}$ .93-.99) for HER2 positivity. One tumor estimated to have $>100$ copies of ERBB2 by NGS showed only weak HER2 expression in $35 \%$ of tumor cells. All cases with $2+$ or $3+$ HER2 expression were amplified by NGS. Of the 10 cases with low ERBB2 CN gain by NGS, 7 showed no HER2 expression and 3 showed weak to moderate staining in $5-20 \%$ of tumor cells. Of CN neutral cases, 11 showed weak HER2 staining in $1-10 \%$ of tumor cells. ERBB2 amplification correlated with lack of RAS/RAF mutations $(p=0.0001)$. Mismatch repair deficiency was detected in 9 of 87 non-amplified cases and none of the 15 ERBB2 amplified cases. No ERBB2 mutations were identified.

Conclusions: A subset of $\mathrm{CRC}$ shows pronounced $E R B B 2$ amplification by NGS. This finding predicts HER2 protein overexpression by IHC NGS may be a suitable approach for selection of CRC patients for antiHER2 targeted therapy.

\section{Epithelial-Mesenchymal Transition Markers ZEB1 and Fibronectin are Associated with Advanced Tumor Stage and Poor Survival in Esophageal Adenocarcinoma}

Odise Cenaj', Robert Odze1, Agoston (Tony) Agoston'. '1Brigham and Women's Hospital, Boston, MA

Background: Epithelial-mesenchymal transition (EMT) is a critical step in cancer invasion and metastasis. However, the prognostic value of biomarkers of tumor progression in esophageal adenocarcinoma (EAC) remains largely unexplored. The aim of this study was to evaluate the prognostic significance of various EMT markers and their association with tumor progression.

Design: Esophagectomy specimens from 44 chemoradiotherapynaive patients with EAC were retrospectively selected for review from the pathology files of a tertiary care academic medical center between 1989-2012. A histologic tissue macroarray was built targeting foci of deep and superficial invasive carcinoma and adjacent dysplasia in each case. Immunohistochemistry was performed and scored for EMT markers Zeb1, Zeb2, fibronectin, E-cadherin, and transcription factors of intestinal differentiation CDX2 and SOX9. Statistical correlational analyses were performed between marker expression, primary tumor stage, lymph node status, and survival outcomes.

Results: The study group male/female ratio was 3.4 with a mean age of 66 years. Zeb1 and Zeb2 expression was seen in $9 \%$ and $2 \%$ of tumors fibronectin expression and $E$-cadherin loss in 23\% and $2 \%$, and CDX2 and SOX9 in $50 \%$ and $93 \%$ of tumors, respectively. Expression of Zeb1 was significantly associated with poor survival (Cox proportional hazards ratio $3.52, \mathrm{p}=0.028$ ). Furthermore, expression of Zeb1 and fibronectin was significantly associated with advanced (T2 or higher) primary tumor stage $(O R>100, p=0.015$ for $Z$ eb1 and $O R=12, p=0.003$ 
for fibronectin), but not with lymph node status. Of note, all cases that expressed Zeb1 also co-expressed fibronectin (OR>100, $p=0.002$ ). No significant associations were noted with regard to expression of Zeb2, CDX2, SOX9 and E-cadherin loss versus tumor stage, lymph node status, and patient survival. Interestingly, the only tumor with E-cadherin loss also expressed fibronectin and Zeb1 (transcriptional repressor of E-cadherin).

Conclusions: Expression of EMT markers Zeb1 and fibronectin is associated with advanced tumor stage in EAC and may play a role in facilitating invasion and metastasis. Zeb1 is also a prognostic marker of poor survival outcome in EAC.

\section{Incidence of Neoplasia in Gastric Hyperplastic Polyps: An Institutional Experience of 459 Polyps}

Joanna M Chaffin', Ashwin S Akki', Michael Feely'. 'University of Florida, Gainesville, FL, ${ }^{2}$ University of Florida

Background: Gastric hyperplastic polyps (HP) are largely regarded as having minimal risk of progression to dysplasia or malignancy. The incidence of neoplasia reportedly increases with polyp size. However, most literature on this matter is derived from Asian populations which may differ from a Western setting. Therefore, we examined the incidence of neoplasia in HPs at our own institution and assessed the relationship of this finding with polyp size and other clinical and pathologic features.

Design: Cases of gastric HPs acquired over a seventeen year period at our institution were retrospectively reviewed. Slides were retrieved for independent histologic assessment by two gastrointestinal pathologists. Polyps were considered HPs if they demonstrated architecturally distorted foveolar epithelium with dilation and elongation along with lamina propria expansion by inflammatory infiltrates and edema. Slides were evaluated for the presence of dysplasia or malignancy as well as other pathologic features, including intestinal metaplasia (IM). Diagnostic discrepancies were resolved by consensus. Following histologic confirmation, patient charts were reviewed for clinical history and endoscopic findings. Patients with known gastrointestinal polyp-producing syndromes were excluded as were HPs arising in the gastroesophageal junction.

Results: A total of 459 polyps obtained from 379 patients, including $169(44.6 \%)$ men and $210(55.4 \%)$ women, were examined. Neoplasia was present in twenty-five HPs $(5.4 \%)$, with low-grade dysplasia, high-grade dysplasia, and carcinoma demonstrated in seventeen $(3.7 \%)$, six $(1.3 \%)$, and two $(0.4 \%)$ polyps, respectively. The rate of neoplasia in polyps measuring $1 \mathrm{~cm}$ or less by endoscopy was $2.1 \%$ $(3 / 143)$ compared to $6.5 \%(6 / 93)$ in HPs greater than $1 \mathrm{~cm}(P=0.1602)$ However, the rate of neoplasia in HPs measuring $3 \mathrm{~cm}$ or less $[2.7 \%$ $(6 / 224)]$ was significantly lower than HPs measuring greater than $3 \mathrm{~cm}$ $[25 \%(3 / 12)](P=0.0072)$. Additionally, the presence of neoplasia was significantly associated with IM, with neoplasia noted in $31 \%(18 / 59)$ of HPs with IM compared to $1.8 \%$ (7/400) of HPs without IM ( $\mathrm{P}<0.0001)$.

Conclusions: The incidence of neoplasia in gastric HPs is $5.4 \%$ Additionally, HPs measuring greater than $3 \mathrm{~cm}$ carry a significant risk of harboring neoplasia, as do polyps containing IM. The presence of either of these findings in HPs should prompt an attentive evaluation.

\section{Gastrointestinal Tract Pathology in Patients with Thymoma}

Vishal Chandan1, Anja Roden ${ }^{2}$, Tsung-Teh Wu $u^{3}$. ${ }^{1}$ Rochester, MN, ${ }^{2}$ Mayo Clinic Rochester, Rochester, MN, ${ }^{3}$ Mayo Clinic, Rochester, MN

Background: Morphological findings of gastrointestinal (GI) tract in thymoma patients have not been studied in detail. They have only been reported as isolated case reports or small case series showing autoimmune enteropathy (AIE) or graft-versus-host disease (GVHD) like enterocolitis. We reviewed the histological findings of Gl tract in a large cohort of thymoma patients to better understand their morphological spectrum.

Design: $84 \mathrm{Gl}$ biopsies (26 colon, 25 stomach, 24 small bowel, 9 esophagus) from 41 patients with a histological diagnosis of thymoma (WHO type A-2 pts; AB-4; B1-6; B2-18; B3-6; unknown type-5) between 1988 and 2016 were included. Various histologic features including architectural distortion, apoptosis, inflammatory infiltrate, small bowel villous atrophy, infection, absence of goblet and/or Paneth cells were noted. Clinical and laboratory findings were reviewed.

Results: The average age was 46 yrs (range 23 to 76 yrs) with 17 males and 24 females. The most common presenting symptom was diarrhea $(n=24)$ followed by dysphagia $(n=8)$. Onset of symptoms ranged from 2 months before the initial diagnosis/recurrence of thymoma to 10 years after the initial diagnosis of thymoma. 6/24 (25\%) patients who presented with diarrhea showed a spectrum of histological changes: $3 / 6$ with AIE-like changes within the small bowel and colon characterized by scarce or absent goblet and/or Paneth cells, prominent crypt apoptosis and villous blunting in the small bowel, the diarrhea in all responded to steroids or stopped after the thymoma was excised ( 1 patient had recurrent AIE-like changes years later, after stopping steroid): 2/6 with GVHD-like changes showing increased crypt apoptosis in colon, duodenum and stomach $1 / 6$ with absence of goblet and Paneth cells in the duodenum. other patient who presented with dysphagia also showed GVHD like change in colon. $16 / 41(40 \%)$ patients showed no significant abnormality within the GI tract. $18 / 41$ (44\%) patients showed unrelated findings like $\mathrm{H}$. Pylori gastritis, peptic type duodenitis, mild increase in intraepithelial lymphocytes of small bowel and tubular adenoma. There was no statistical significance between type of thymoma and findings within the Gl tract.

Conclusions: A subset of patients with thymoma $(7 / 41,17 \%)$ can show AIE-like changes, GVHD-like changes and absence of goblet and Paneth cells in the Gl tract. Awareness of these thymoma associated changes in the GI tract may facilitate its diagnosis.

\section{MSH6 Immunohistochemical Heterogeneity in Colorectal Cancer: Comparative Sequencing from Different Tumor Areas}

Wei Chen ${ }^{1}$, Rachel Pearlman ${ }^{1}$, Michael Markow' ${ }^{1}$, Christina A Arnold ${ }^{1}$ Colin Pritchard ${ }^{2}$, Debbie Knight ${ }^{1}$, Heather Hampel', Wendy L Frankel ${ }^{\prime}$ ${ }^{1}$ The Ohio State University Wexner Medical Center, Columbus, $\mathrm{OH}$ 2University of Washington, Seattle, WA

\section{Disclosures:}

Heather Hampel: Advisory Board, InVitae Genetics

Background: Mismatch repair protein (MMR) immunohistochemistry (IHC) is an important tool to screen colorectal cancer (CRC) patients for Lynch syndrome. Heterogenous MSH6 staining has been reported in $\mathrm{CRC}$ and endometrial cancer. We aimed to better understand MSH6 heterogeneity in CRC patients by using IHC and molecular analysis.

Design: $3346 \mathrm{CRC}$ patients enrolled in our statewide initiative underwent MMR IHC testing. MLH1 methylation was done if absent MLH1/PMS2 on IHC. All cases with available MMR stained slides $(n=1754)$ were reviewed for heterogeneous MSH6 staining Heterogeneous staining was defined as a patchy staining pattern with discrete areas of tumor that show loss of MSH6 staining and areas with retained staining, in the presence of appropriately stained internal control cells in both areas. Next-generation sequencing (NGS) of tumor MMR genes (MLH1, PMS2, MSH2, MSH6, EPCAM) and BRAF V600E were performed using tumor DNA extracted from the retained and lost staining areas, respectively. 3 patients with abnormal staining without $M L H 1$ methylation received germline NGS of the MMR genes.

Results: 9 out of 1754 CRC cases $(0.05 \%)$ demonstrated heterogenous MSH6 staining. Patients' age ranged from 60 to 83 years (average 69) female to male ratio 7:2. The area of the tumor with loss of MSH6 ranged from $5 \%$ to $60 \%$ (average $22 \%$ ). None of the patients received preoperative neoadjuvant therapy. Four cases had enough tissue remaining in both retained and lost MSH6 areas to perform tumo sequencing. Case data are shown in Table 1. Interestingly, two cases (\#3 and \#7) showed MSH2 heterogenous staining with focal loss of $\mathrm{MSH} 2$ staining in the areas that mirrored MSH6 loss. 
Table 1. Clinical, immunohistochemistry, and molecular data of MSH6 heterogenous staining colorectal cancers. (Abbreviations: A, Absent; P, Present; N/A, Not available)

\begin{tabular}{|c|c|c|c|c|c|c|c|c|}
\hline \# & $\begin{array}{l}\text { MSH6 } \\
\text { Lost } \\
\text { Area } \\
(\%)\end{array}$ & $\mathrm{MSH} 2$ & MLH1 & PMS2 & $\begin{array}{l}\text { MLH1 } \\
\text { Meth- } \\
\text { yla- } \\
\text { tion }\end{array}$ & $\begin{array}{l}\text { Germline } \\
\text { Mutation }\end{array}$ & $\begin{array}{l}\text { Tumor MSH6 } \\
\text { Gene Alterations } \\
\text { in MSH6 Stain- } \\
\text { ing Absent vs. } \\
\text { Present Areas }\end{array}$ & $\begin{array}{l}\text { Tumor Oth } \\
\text { er Genetic } \\
\text { Alterations } \\
\text { in Both } \\
\text { Areas }\end{array}$ \\
\hline 1 & 30 & P & A & A & Yes & N/A & $\begin{array}{l}\text { A: } 3 \text { somatic } \\
\text { mutations } \\
\text { P: No mutation }\end{array}$ & $\begin{array}{l}\text { BRAF } \\
\text { V600E } \\
\text { mutation; } \\
\text { MSH2 } \\
\text { mutation }\end{array}$ \\
\hline 2 & 40 & $P$ & A & A & Yes & $\mathrm{N} / \mathrm{A}$ & $\begin{array}{l}\text { A: Biallelic so- } \\
\text { matic mutations } \\
\text { P: } 3 \text { Somatic } \\
\text { mutations }\end{array}$ & No \\
\hline 3 & 10 & $P$ & A & A & Yes & $\mathrm{N} / \mathrm{A}$ & 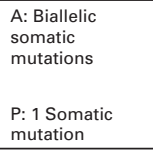 & $\begin{array}{l}\text { BRAF } \\
\text { V600E } \\
\text { mutation; } \\
\text { MSH2 } \\
\text { mutation }\end{array}$ \\
\hline 4 & 60 & $P$ & $P$ & $P$ & No & $\begin{array}{l}\text { PMS2 } \\
\text { likely } \\
\text { patho- } \\
\text { genic } \\
\text { mutation }\end{array}$ & $\begin{array}{l}\text { A: Biallelic so- } \\
\text { matic mutations } \\
\text { P: Biallelic so- } \\
\text { matic mutations }\end{array}$ & $\begin{array}{l}\text { PMS2 } \\
\text { mutation }\end{array}$ \\
\hline 5 & 8 & $P$ & A & A & Yes & $\mathrm{N} / \mathrm{A}$ & $\mathrm{N} / \mathrm{A}$ & $\mathrm{N} / \mathrm{A}$ \\
\hline 6 & 12 & $P$ & A & A & No & $\begin{array}{l}\text { MLH1 } \\
\text { patho- } \\
\text { genic } \\
\text { mutation }\end{array}$ & $\mathrm{N} / \mathrm{A}$ & $\mathrm{N} / \mathrm{A}$ \\
\hline 7 & 15 & $\mathrm{P}$ & A & $\mathrm{A}$ & Yes & $\mathrm{N} / \mathrm{A}$ & N/A & $\mathrm{N} / \mathrm{A}$ \\
\hline 8 & 20 & $P$ & $P$ & A & No & $\begin{array}{l}\text { PMS2 } \\
\text { patho- } \\
\text { genic } \\
\text { mutation }\end{array}$ & $\mathrm{N} / \mathrm{A}$ & $\mathrm{N} / \mathrm{A}$ \\
\hline 9 & 5 & $P$ & A & A & Yes & $\mathrm{N} / \mathrm{A}$ & $\mathrm{N} / \mathrm{A}$ & $\mathrm{N} / \mathrm{A}$ \\
\hline
\end{tabular}

Conclusions: Double somatic MSH6 mutations were identified solely in the tissue with MSH6 IHC loss; they were not identified in the adjoining tissue with retained $\mathrm{MSH} 6 \mathrm{IHC}$, in 2 of 4 cases. MSH6 heterogenous staining is most commonly seen in tumors with loss of MLH1 and/or PMS2 expression on IHC indicating that this could be due to somatic MSH6 mutations caused by deficient MMR. Rarely MSH6 heterogeneous staining occurs in CRC cases without MLH1 and PMS2 loss by IHC. We confirmed that MSH6 germline mutation is not identified in MSH6 heterogenous staining cases.

\section{Overexpression of RAB GTPase 3C Promotes Colorectal Cancer Metastasis by Enhancing IL6- STAT3 Signaling Axis}

Chi-Long Chen ${ }^{1}$, Yu-Chan Chang ${ }^{2}$, Michael Hsiao ${ }^{3}$. 'Taipei Medical Univer, Taipei, Taiwan, ${ }^{2}$ Academia Sinica, Taipei, ${ }^{3}$ Academia Sinica

Background: Distant metastasis and recurrence are the most critical issues in management of colorectal cancer (CRC). Recently, RAB GTPases (RABs) have been reported in the regulation of membrane trafficking and cell movement. However, the detail molecular mechanism of RABs family in colorectal cancer are still unknown.

Design: To identify significant target of RABs family members by immunohistochemistry analysis of tissue array containing 215 CRC. Complementary functional overexpression and knockdown model of $\mathrm{RAB} 3 \mathrm{C}$ in colon cancer cells were performed in vitro and in vivo.

Results: Univariate and multivariate analyses showed high RAB3C expression served as an independent prognostic factor in overall survival and disease-free survival of CRC patients. High RAB3C expression was found to be significantly associated with late pathological stage, distant metastasis event, and poor prognosis in CRC patient cohort. We observed RAB3C promoted migration/ invasion ability in vitro and lung nodule forming ability in vivo. The effect of RAB3C overexpressing cell-conditioned medium was found to significantly promote the migration ability of parental colon cancer cells suggesting the migration-promoting is exocytosis dependent. Analyzing established RAB3C-based transcriptomics and proteomics databases, we found IL-6 pathway was the top pathway with gene expression changes after RAB3C overexpression through Ingenuity Pathway Analysis (IPA). Therefore, blocking IL-6 signaling by IL-6 recombinant protein and knockdown clones significantly inhibited the metastasis potentials in RAB3C overexpression colon cancer cell model. In addition, Ruxolitinib, a JAK2 inhibitor, was found to inhibit the phosphorylation status of STAT3 induced by IL-6.

Conclusions: Our data revealed the molecular mechanism of RAB3C in promoting colorectal cancer metastasis was via IL-6 activation and increased phosphorylation status of STAT3. The results suggested that inhibition of RAB3C-IL6-STAT3 axis by using Ruxolitinib may provide a new therapeutic approach for preventing metastasis of colorectal cancer.
696 Micropapillary Early Gastric Carcinoma with High-risk Lymph Node Metastasis and Worse Clinicopathologic Features

YQ Cheng' ${ }^{1}, X L Z_{\text {Zhou }}^{2}, L$ Chen ${ }^{3}, M Z D u^{4}, Y H W a n W^{5}, L i u T Y^{6}$, XiangShan $\mathrm{Fan}^{7}, \mathrm{LC} \mathrm{Guo}^{4}$, YiFen Zhang, Qin Huang ${ }^{8}$ ' ${ }^{1}$ Changzhou Second Hospital, Changzhou, Jiangsu Province, ${ }^{2}$ Changzhou Second Hospital, Changzhou, Jiangsu, ${ }^{3}$ Nanjing Drum Tower Hospital, Nanjing, Jiangsu, ${ }^{4}$ Soochow University First Hospital, Suzhou, Jiangsu, 5 Jiangsu Provincial Hospital of Traditional Chinese Medicine, Nanjing, Jiangsu, ${ }^{6}$ Nanjing Drum Tower Hospital, Nanjing, Jiangsu, ${ }^{7}$ Nanjing Drum Tower Hospital, Nanjing University Medical School, Nanjing, Jiangsu Province, ${ }^{8}$ VA Boston Healthcare System, West Roxbury, MA

Background: Micropapillary early gastric carcinoma(MEGC) is rare and reported to be at high risk for lymph node metastasis (LNM) in single center studies. We aimed to validate this finding in a large multicenter study on early gastric carcinoma (EGC).

Design: We reviewed pathology reports on 11489 consecutive combined gastric cancer radical resections in 4 tertiary medical centers in Jiangsu Province in China and identified 1890 (16.5\%) qualified EGCs diagnosed with the WHO criteria. On review of tumor slides, $30(1.6 \%)$ were qualified as MEGC with a micropapilary component in $>5 \%$ of tumor volume. HER2 immunoreactivity of MEGC and pancytokeratinimmunostain of lymph nodes diagnosed as the absence of LNM on H\&E stained sections were carried out. Clinicopathological features and risk factors for LNM were statistically analyzed.

Results: MEGCs showed a male predominance (M:F=7:3) with an average age of 63 years. ALL, except for 2 , tumors invaded submucosa(M3=2, $\mathrm{SM} 1=3, \quad \mathrm{SM} 2=25)$. Immunostaining of pancytokeratin in negative lymph nodes revealed 2 additional cases with LNM. Overall, lymphovascular invasion (LVI) and LNM were found in $15(50 \%)$ and $14(46.7 \%)$ cases, respectively. Advanced pathologic stages were demonstrated in $6(20 \%)$. HER2-positive (3+) immunostaining was found in $4 / 28$ cases. Compared to nonMEGCs, MEGCs showed significantly higher frequencies in LNM, LVI, submucosal andperineural invasion, and advanced stages, but not significant in age, gender, gross type, tumor size, and location. By univariate analysis, LVI and mixed papillary/poorly cohesive carcinoma were risk factors of LNM, whereas mixedpapillary/poorly cohesive carcinomacomponents in MEGC was the only independent risk factor for LNM by multi-variant analysis with an odds ratio of 7.1 (95\% confidence interval:1.1-48.2; $<<0.05$ ).

Conclusions: Compared to non-MEGCs, MEGCs demonstrated a high propensity for LNM and LVI withsignificantly worse clinicopathologic features, especially for cases with mixed papillary/poorly cohesive carcinoma components.

\section{Standardization of the histologic criteria for activity in ulcerative colitis leading to improved inter-observer agreement of activity grade and} moderate activity: An institutional study

Kenrry Chiu ${ }^{1}$, Astrid-Jane Greenup ${ }^{2}$, Brian Bressler ${ }^{2}$, David Schaeffer ${ }^{1}$, Wei Xiong ${ }^{3}$. 'Vancouver General Hospital, Vancouver, BC, ${ }^{2}$ University of British Columbia, ${ }^{3}$ St. Paul's Hospital

Background: Histologic activity may predict recurrence in ulcerative colitis (UC). There are various indices for grading histologic activity in ulcerative colitis (UC). Thus, there is an interest in standardizing criteria for grading activity in clinical practice. The aim of this study is to assess the inter-observer agreement for histologic grading of activity in $\mathrm{UC}$ at a tertiary care center before and after standardization of histologic activity.

Design: One training set (20 cases) and one test set (20 cases) were created based on a histologic index comprising of four grades: inactive colitis (chronic colitis with no cryptitis or crypt abscesses), mild activity (cryptitis or crypt abscesses involving $<50 \%$ of crypts), moderate activity (cryptitis or crypt abscesses involving $>50 \%$ of crypts), and severe activity (erosion and/or ulceration). Eight pathologists from a tertiary care center participated in the study. The pathologists scored the training set prior to being informed of the criteria for each grade. Following feedback after the training set, the test set was scored by each pathologist after a period of at least 8 weeks. The inter-observer agreement was assessed for each set and compared between sets.

Results: Overall inter-observer agreement was good with the training set (Fleiss' kappa 0.68, 95\% confidence interval (CI) $0.63-0.73$ ), and increased with the test set (Fleiss' kappa $0.78,95 \% \mathrm{Cl} 0.73-0.82$ ) (Table 1). The most significant improvement was observed in the moderate category: $71 \%$ (range $17-83 \%$ ) with the training set, which increased to $84 \%$ (range $50-100 \%)$ with the testing set $(p=0.04)$. Severe activity had the highest average rate of agreement between individual pathologists and the reference with both the training set $(98 \%$, range $80-100 \%)$ and the test set $(95 \%$, range $80-100 \%)$. 
Table 1. Inter-observer agreement for histologic activity

\begin{tabular}{|l|l|l|l|}
\hline $\begin{array}{l}\text { Grade of histo- } \\
\text { logic activity }\end{array}$ & $\begin{array}{l}\text { Training set Fleiss' } \\
\text { kappa }(95 \% \mathrm{Cl})\end{array}$ & $\begin{array}{l}\text { Test set Fleiss' } \\
\text { kappa }(95 \% \mathrm{Cl})\end{array}$ & $\begin{array}{l}\text { Difference in kappa } \\
\text { between test and } \\
\text { training sets }\end{array}$ \\
\hline Inactive colitis & $0.78(0.69-0.86)$ & $0.92(0.84-1.00)$ & 0.14 \\
\hline Mild activity & $0.57(0.49-0.65)$ & $0.67(0.58-0.75)$ & 0.10 \\
\hline Moderate activity & $0.57(0.49-0.65)$ & $0.60(0.51-0.68)$ & 0.03 \\
\hline Severe activity & $0.80(0.72-0.88)$ & $0.94(0.86-1.00)$ & 0.14 \\
\hline Overall & $0.68(0.63-0.73)$ & $0.78(0.73-0.82)$ & 0.10 \\
\hline
\end{tabular}

Conclusions: Agreement was lowest for moderate activity and highest for severe activity. Standardization of criteria for histologic activity in ulcerative colitis is feasible.

\section{DNA Flow Cytometric Analysis and Outcomes of Serrated Lesions in Inflammatory Bowel Disease}

Won-Tak Choi', Kwun Wah Wen', Peter S Rabinovitch', Danning Huang ${ }^{3}$, Aras Mattis', Ryan Gilß. 'University of California San Francisco, San Francisco, CA, ${ }^{2}$ University of Washington, ${ }^{3}$ SUNY Upstate Medical University

Background: Sporadic serrated lesions (SLs), such as sessile serrated adenoma (SSA) and traditional serrated adenoma (TSA), are important premalignant lesions for colorectal cancers (CRC). However, the clinical significance and natural history of SLs in inflammatory bowel disease (IBD) remain unclear. This study reports DNA flow cytometric analysis and outcomes of SLs in IBD patients using formalin-fixed paraffin-embedded tissue.

Design: DNA flow cytometry was performed on 32 colonic SLs without dysplasia (21 polypoid and 11 flat lesions) from 28 IBD patients. Thirty-one samples of adjacent non-serrated mucosa from the same cohort were utilized as controls. When aneuploidy was detected in the background mucosa (which can rarely occur in IBD), the serrated sample was considered to have aneuploidy, only when it shows a distinct aneuploid peak different from that of the background mucosa. For comparison, flow cytometry was also performed on 48 sporadic SLs with dysplasia (12 SSAs with low-grade dysplasia (LGD), 3 SSAs with high-grade dysplasia (HGD), 30 TSAs, and 3 TSAs with HGD). Three to four 60-micron thick sections were cut from each block, and the area of interest was dissected for analysis.

Results: Four $(14.3 \%)$ of 28 IBD patients with SLs were found to have LGD or HGD within a mean follow-up time of 42 months (range: $4-122)$, of which only 2 patients $(7.1 \%)$ developed HGD. The 6 -month, 5-year, and 12-year detection rates of LGD or HGD for SL patients were $7.4 \%, 16.7 \%$ and $58.3 \%$, respectively, whereas the detection rate of HGD remained stable at $7.4 \%$ within 12 years. Among 32 SLs, only 1 case $(3.1 \%)$ showed aneuploidy at baseline, which was associated with subsequent detection of HGD. No patient developed CRC. By comparison, none of 15 sporadic SSAs with dysplasia, $1(3.3 \%)$ of 30 TSAs, and $2(66.7 \%)$ of 3 TSAs with HGD showed aneuploidy. Only one case of SSA with LGD (2.1\%) developed CRC.

Conclusions: SLs in IBD appear to have low malignant potential, as only two patients $(7.1 \%)$ developed HGD. Similarly, patients with sporadic SLs with dysplasia have a low risk of developing HGD or CRC. The rate of aneuploidy is low $(<5 \%)$ in all SLs regardless of IBD status, except in TSAs with HGD $(66.7 \%)$, suggesting that the majority of SLs may develop dysplasia via alternative pathways to the conventional adenoma-carcinoma model that frequently results in aneuploidy. The lack of aneuploidy may also explain good clinical outcomes, and aneuploidy status could be considered in determining surveillance strategies.

\section{Prognostic Implications of Programmed Death- Ligand 1 and Immunoregulatory Lymphocytes in Various Subsets of Gastric Carcinomas}

Euno Choi', Sun Young Park', Sun-ju Byeon', Mee Soo Chang ${ }^{4}$. 'Seoul National Úniversity Boramae Hospital, Seoul, Seoul, 2Department of Pathology, Seoul National University Boramae Hospital, Seoul National University College of Medicine, Seoul, Korea, ${ }^{3}$ Seoul Nat'l Univ/Medicine, Seoul, SE, ${ }^{4}$ Seoul National University Boramae Hospital, Seoul National University College of Medicine

Background: Programmed death-ligand 1 (PD-L1)/Programmed death-1(PD-1) checkpoint blockade has been emerging as a therapeutic target in diverse human malignancy. However, the hitherto published data show a discrepancy in prognostic value of tumor PD-L1 expression in gastric carcinomas.

Design: We reviewed clinicopathologic features of 528 cases of consecutive surgically-resected gastric carcinomas from 2006 to 2011. Immunohistochemistry for PD-L1, CD3, CD8, FoxP3, PD-1, CD 10, MUC2, MUC5AC, MUC6, CD44, hMLH1 and hMSH2, in situ hybridization for EBV-encoded small RNAs, and microsatellite instability (MSI) analysis

\section{using fluorescent multiplex PCR were performed.}

Results: Among a total of 528 carcinomas, there were $130(25 \%)$ cases of tumor PD-L1-expressing gastric carcinomas. Tumor PD-L1 expression was positively correlated with advanced pT stage, EBVpositive in tumor cells, and higher populations of immunoregulatory lymphocytes such as $\mathrm{CD} 3+, \mathrm{CD} 8+$ and FoxP3+ tumor infiltrating lymphocytes (TILs) $(P<0.05$, respectively). In multivariate analysis, pathological tumor stage (TNM) was an absolute independent prognostic factor in a total of 528 patients. Interestingly, in advanced gastric carcinomas with Lauren intestinal type $(n=119)$, the group with higher populations of CD3+, CD8+, FoxP3+ and PD-1+ TILs revealed better overall survival rate than lower population group $(P<0.05$, respectively). Adding mucin- and stem-cell phenotypes and molecular classifications, there were $158(30 \%)$ cases of gastric mucin-phenotype, $53(10 \%)$ intestinal, $10(2 \%)$ gastrointestinal and $288(55 \%)$ unclassifiable-phenotype. CD44+ stem cell phenotype was observed in $209(40 \%)$ cases. MS-unstable carcinomas were found in $53(10 \%)$ cases, and EBV-positive carcinomas in $33(6 \%)$. It is notable that in EBV-positive gastric carcinomas, tumor PD-L1-expressing group demonstrated an adverse impact on patient survival than PD-L1 negative group $(P<0.05)$, but not in other variable subtypes. Furthermore, EBV-positive gastric carcinomas showed more frequently higher populations of CD3+, CD8+, FoxP3+ or PD-1+ TILs than EBV-negative carcinomas $(P<0.05)$.

Conclusions: PD-L1 may be justified as a prognostic marker in EBVpositive gastric carcinomas, and it has a limited value in other subsets or heterogeneous total gastric carcinomas. The immunoregulatory lymphocyte population in tumor microenvironment may become one of the prognostic factors in advanced gastric carcinoma group with Lauren intestinal type.

\section{Targeted Next-Generation Sequencing as a Primary Screening Tool for Mismatch Repair Deficiency in Upper Gastrointestinal Tract Carcinomas}

Alexander Christakis', David J Papke', Jonathan A Nowak', Matthew B Yurgelun ${ }^{2}$, Agoston (Tony) Agoston ${ }^{1}$ Neal Lindeman', Laura E MacConaill', Lynette Sholl', Fei Dong'. 'Brigham and Women's Hospital, Boston, MA, 'Dana Farber Cancer Institute

Background: Next-generation sequencing has previously been demonstrated to be sensitive and specific in determining mismatch repair protein deficiency (MMR-D) in colorecta adenocarcinomas. Upper gastrointestinal tract (GI) cancers are not routinely screened for MMR-D by immunohistochemistry in the pathology laboratory; however, a small proportin of these neoplasms are known to have MMR-D, which may hold implications in familial cancer predisposition and eligibility for treatment with immune checkpoint inhibitor therapy. This study aims to address whether targeted next- generation sequencing used for the genomic profiling of oncogenic driver mutations is also effective as a primary screening tool for MMR-D in upper GI cancers.

Design: Targeted next-generation sequencing was performed on a series of 644 upper Gl carcinomas. A criterion of at least 3 or more single nucleotide insertion or deletion mutations per megabase pair in mononucleotide repeat regions is used as a biomarker for MMR-D. Sequencing findings were validated in a subset of 39 cases stained by MLH1, MSH2, MSH6, and PMS2 immunohistochemistry.

Results: MMR-D was identified by next generation sequencing criterion in 23 of $644(3.6 \%)$ of upper Gl cancers, including 3 of 23 esophageal/gastroesophageal $(1.3 \%), 10$ of 97 gastric $(10.3 \%), 7$ of 29 small intestine $(24.1 \%), 2$ of 208 pancreatic $(1.0 \%), 1$ of 61 biliary tract $(1.6 \%)$, and 0 of 18 gallbladder carcinomas. In a subset of cancers with immunohistochemistry, including 9 MMR-D carcinomas and 30 mismatch repair proficient carcinomas, next-generation sequencing and immunohistochemistry were concordant in 39 of 39 neoplasms (100\%).

Conclusions: The frequency of MMR-D depends on primary site in upper Gl carcinomas and is seen in a significant number of small intestinal and gastric cancers. Next generation sequencing is an accurate screening tool for the detection of MMR-D in upper GI cancers.

\section{Histologic evidence for acute cellular rejection within colon allografts included during small bowel or multivisceral transplantation}

Jesse Cox ${ }^{1}$, Shaheed Merani', Wendy J Grant ${ }^{1}$, Stanley Radio ${ }^{1}$ Benjamin Swanson'. 'University of Nebraska Medical Center, Omaha, NE

Background: Inclusion of donor colon allograft is one strategy to reduce dehydration and hospital readmissions in patients undergoing isolated bowel or multivisceral transplantation. Although a standardized grading system has been established to characterize acute cellular rejection (ACR) in small bowel allografts, a similar system for colonic allograft rejection has not. 
Design: Records from small bowel transplant patients at one center over the last two years (December 2015 - September 2017) were retrospectively screened to identify subjects who received a colon allograft. Of included subjects, biopsy records were retrieved and cases with concurrent small bowel and colonic allograft biopsies were assessed microscopically. Small bowel allografts were characterized according to guidelines from the $8^{\text {th }}$ International Small Intestinal Transplantation Symposium. Colonic allograft biopsies were characterized descriptively, with emphasis placed upon quantitation of apoptotic cells, increased inflammation, and ulcer formation. Where applicable, sequential biopsies from the same patient were included for assessment.

Results: Thirty-two (32) patients (age 1.3 to 66.1 years) underwent small bowel transplantation at our institution over the included time period. Of these, 17 had a portion of colon included in their allograft. During surveillance screening, 6 of 17 patients had colon allograft biopsied, whereas the remaining patients did not. Four of 6 showed no evidence of rejection nor significant histologic alteration. One patient showed Grade 1 ACR in the small bowel allograft without alteration of the colon allograft. Two of the 6 protocol biopsies showed histologic evidence of apoptotic injury limited to the colon allograft, one of which showed progressive colonic injury over the course of 6 weeks, leading to explantation of the donor colon only. This explanted colon demonstrated diffuse ulceration and crypts with numerous apoptotic bodies. An additional patient underwent explantation of small bowel and colon allograft due to evidence of grade 3 ACR in a small bowel allograft biopsy; while the colon of this patient was not sampled prior to explantation, it did show extensive apoptotic injury in the epithelium.

Conclusions: Our study indicates that cellular damage may occur in colonic allograft mucosa similar to that seen in small bowel ACR. Additional studies correlating histologic findings along with clinical presentations will be necessary to develop a grading scheme for colon allografts.

\section{Overcoming the Diagnostic Challenge of Severe Colonic Acute Graft Versus Host Disease with Diffuse Ulceration}

Ashley Cross', David Lewin', Shaoli Sun ${ }^{3}$. ${ }^{1}$ MUSC Pathology \& Laboratory Medicine, Charleston, SC, ${ }^{2}$ MUSC, Charleston, SC, ${ }^{3}$ Medical University of S Carolina, Mount Pleasant, SC

Background: Conventionally, histologic diagnosis of intestinal acute graft versus host disease (A-GVHD) associated with stem cell transplant is dependent on identification of epithelial cell apoptosis. Clinical management rests upon swift and accurate diagnosis and grading of A-GVHD. Paradoxically, the severe cases of A-GVHD have extensive ulceration with total or focal denudation of the mucosa, which makes diagnosing GVHD extremely difficult, if not impossible. Accordingly, a study to review and summarize the histologic features of severe A-GVHD is both diagnostically essential and of critical clinical impo

Design: This is a retrospective and IRB approved study. De-identified colonic biopsies from 13 allogeneic BMT patients with clinical symptoms of severe A-GVHD with partial or complete mucosal ulceration were compared with 21 patients with ulceration secondary to other causes (negative control group). Hematoxylin and eosin stained slides, along with a subset of Masson Trichrome, were reviewed by pathologists blinded to the etiology.

Results: Ghost crypts were identified in $92 \%$ of A-GVHD cases and absent in negative controls ( $p$ value $<0.0001$ ). A trichrome stain increased ghost crypt recognition in A-GVHD to $100 \%$ ( $p$ value $<0.0001)$. The vessel walls were thinner in A-GVHD cases $(92 \%<10$ $\mu \mathrm{m})$ and thicker in the negative controls $(95 \%>10 \mu \mathrm{m})$. The endothelium was inactive in $77 \%$ of A-GVHD cases and activated in $95 \%$ of negative controls ( $p$ value $<0.0001$ ). Ulcer slough was identified in a minority $(31 \%)$ of A-GVHD cases and $100 \%$ of negative controls ( $p$ value $<0.0001$ ). The majority of A-GVHD cases had low stromal density $(62 \%)$ and low cellularity $(71 \%)$, while negative controls had high stromal density $(92 \%)$ and high cellularity $(71 \%)$ ( $p$ value 0.002). $77 \%$ of A-GVHD cases had lymphocytic inflammation, while $75 \%$ of negative controls were neutrophilic ( $p$ value $<0.0001$ ). The mucosa was thinner in A-GVHD cases $(0.48 \pm 0.32 \mathrm{~mm}$ versus $1.01 \pm$ $0.28 \mathrm{~mm}, \mathrm{p}$ value 0.0003 )

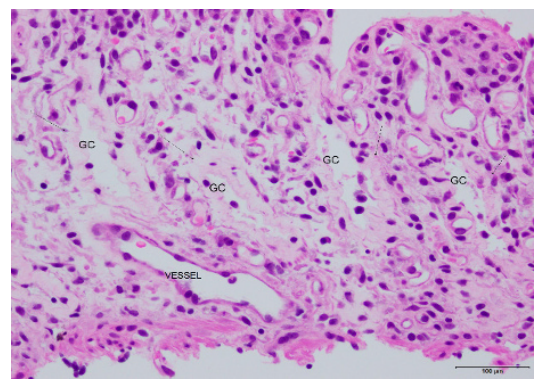

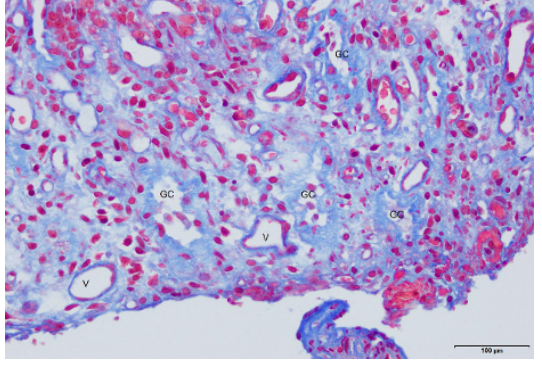

Conclusions: Histomorphological features characteristic of severe A-GVHD related ulceration includes ghost crypts (identification increases with trichrome stain), thin vessel walls, inactive endothelium, lack of ulcer slough, edematous stroma with low cellularity, thin mucosa, and a lymphocytic infiltrate. Findings suggestive of nonGVHD etiologies include the absence of ghost crypts, thick vessel walls, activated endothelium, ulcer slough, dense fibrous stroma with high cellularity, thickened mucosa, and a neutrophilic infiltrate.

\section{A Reappraisal of Mismatch Repair Protein Deficiency versus Microsatellite Instability Testing: Do We Need Both?}

Elizabeth P Crowe 1 , Rhonda Yantiss ${ }^{2}$, Yao-Tseng Chen ${ }^{2} .{ }^{1} \mathrm{New}$ York Presbyterian Weill Cornell Medical Center, New York, NY, ${ }^{2}$ Weill Cornell Medical College, New York, NY

Background: Immunohistochemistry (IHC) for mismatch repair (MMR) proteins and PCR-based assays for microsatellite instability (MSI) are complementary techniques for assessing MMR-deficiency in tumors. Many institutions use one, or both methods to identify colorectal cancer patients with Lynch syndrome, or to facilitate treatment of advanced solid tumors from multiple sites. Each of these methods has high sensitivity and specificity, but well-recognized limitations of both tests have led to widespread concomitant, or serial, utilization of IHC and PCR. We believe the reported discordance between IHC and PCR is overstated, possibly reflecting unfamiliarity with nuances of IHC and/or older PCR panels for MSI testing. The purpose of this study was to assess the frequency of, and reasons for discordance between IHC for MMR proteins and PCR for MSI in a large series of colorectal cancers.

Design: We identified 1014 colorectal cancers during an 8-year period, 813 of which were analyzed with both IHC for MMR proteins and PCR for MSI. Immunostains for MLH1, PMS2, MSH2, and MSH6 were performed using standard techniques. PCR was performed using a commercial panel of 5 mononucleotides and interpreted as microsatellite stable (MSS), MSI-H (instability at $>2$ markers), or MSI-L (instability at 1 marker). IHC and PCR were repeated on two blocks pe case when results between the two methods were discrepant.

Results: $649(79.8 \%)$ cases showed preserved staining for all MMR proteins; $164(20.2 \%)$ showed loss of staining for MLH1/PMS2 ( $n=131$, $16.1 \%)$, PMS2 only ( $n=10,1.2 \%)$, MSH2/MSH6 $(n=17,2.1 \%)$, and MSH6 only $(n=2,0.3 \%) .647(79.6 \%)$ were MSS, $163(20.1 \%)$ showed MSI-H and $3(0.4 \%)$ showed MSI-L; all $3 \mathrm{MSI}-\mathrm{L}$ cases showed preserved staining for MMR proteins by IHC. Discordance between IHC and PCR was observed in only $3(0.4 \%)$ cases (Table).

\begin{tabular}{|l|l|l|l|l|}
\hline & $\begin{array}{l}\text { Original DNA- } \\
\text { PCR }\end{array}$ & $\begin{array}{l}\text { Repeat DNA- } \\
\text { PCR }\end{array}$ & Original IHC & Repeat IHC \\
\hline Case 1 & MSS & MSS & Loss of MSH6 & $\begin{array}{l}\text { All } 4 \text { markers } \\
\text { preserved }\end{array}$ \\
\hline Case 2 & MSS & MSI-H & $\begin{array}{l}\text { Loss of MLH1 } \\
\text { and PMS2 }\end{array}$ & $\begin{array}{l}\text { Loss of MLH1 and } \\
\text { PMS2 }\end{array}$ \\
\hline Case 3 & MSI-H & MSI-H & $\begin{array}{l}\text { All } 4 \text { markers } \\
\text { preserved }\end{array}$ & $\begin{array}{l}\text { All } 4 \text { markers } \\
\text { preserved }\end{array}$ \\
\hline
\end{tabular}

Upon review, the discordance in Case 1 was attributed to an interpretive error due to weak MSH6 staining of rare tumor cells in the setting of neoadjuvant treatment. The discordance in Case 2 likely reflected poor tumor selection for PCR. Only Case 3 showed a persistent discrepancy with preserved MMR protein expression and MSI following repeat testing.

Conclusions: Concordance between IHC for MMR proteins and PCR for MSI is extremely high $(810 / 813,99.6 \%)$; rare discordant cases usually reflect human errors, which can be minimized with education. The added value of performing both IHC and PCR in every case is minimal. Of the two, IHC is the preferred test because it is inexpensive, widely available, and identifies the culprit gene for further testing. 
704 Helicobacter Pylori (H. pylori) infection in Sleeve Gastrectomy with Inactive Chronic Gastritis

Min Cui', Noam Harpaz², Qingqing Liu'. 'Icahn School of Medicine at Mount Sinai, New York, NY, ${ }^{2}$ Mount Sinai Med. Center, New York, NY

Background: The prevalence of $H$. pylori infection may be underestimated in patients with chronic infection as these patients frequently do not have any signs or symptoms. Bariatric surgery for morbid obesity has dramatically increased over the last few decades, producing a large number of sleeve gastrectomies for histopathological evaluation. However, the $\mathrm{H}$. pylori infection rates in sleeve gastrectomy with inactive chronic gastritis have not been well studied, and its assessment using $\mathrm{H}$. pylori immunostains is not routinely performed in the absence of acute inflammation.

Design: One thousand and twenty-four sleeve gastrectomies for morbid obesity (263 male and 761 female, median age 39 years) at our institute (2015-2017) were included in the study. For those specimens with diagnosis of inactive chronic gastritis, H\&E slides were reviewed for the presence of chronic inflammation, and features suggestive of $\mathrm{H}$. pylori infection, including formation of germinal centers and uniform band-like lymphoplasmacytic infiltrates. H. pylori immunostaining was performed to assess $\mathrm{H}$. pylori infection.

Results: H. pylori immunostaining was performed on one hundred and forty-four sleeve gastrectomies diagnosed with inactive chronic gastritis $(144 / 1024,14.1 \%)$. Twenty-nine cases were positive for $\mathrm{H}$. pylori by immunostaining. The overall $\mathrm{H}$. pylori infection rate in sleeve gastrectomy with inactive chronic gastritis was $20.1 \%$ (29/144). Although most of the positive cases demonstrated characteristic histopathologic features of chronic $\mathrm{H}$. pylori infection, five cases $(5 / 29,17.2 \%)$ only showed mild chronic inflammation featuring minimal or focal clusters of plasma cells in lamina propria.

Conclusions: Our study showed a relatively high $\mathrm{H}$. pylori infection rate $(20.1 \%)$ in sleeve gastrectomy diagnosed with inactive chronic gastritis. In addition, up to $17 \%$ of the cases with chronic inactive $\mathrm{H}$. pylori gastritis did not show characteristics histopathologic features of chronic $\mathrm{H}$. pylori infection. Immunohistochemistry for $\mathrm{H}$. pylori is particularly helpful in detecting $\mathrm{H}$. pylori infection in this group of patients. H. pylori immunostaining should be recommended in sleeve gastrectomy with inactive chronic gastritis.

\section{Intestinal Neuroendocrine Tumors in Crohn's Disease and Ulcerative Colitis}

Min Cui', Xiaofei Zhang ${ }^{1}$, Xiaoyan Liao', Qingqing Liu' ${ }^{1}$, Hongfa Zhu ${ }^{2}$, Huaibin Mabel Ko', Alexandros D Polydorides', Noam Harpaz ${ }^{3}{ }^{1}$ Icahn School of Medicine at Mount Sinai, New York, NY, ${ }^{2}$ Mount Sinai Hospital, ${ }^{3}$ Mount Sinai Med. Center, New York, NY

Background: The question whether patients with inflammatory bowel disease (IBD) are at increased risk of developing neuroendocrine tumors has been controversial. We determined the incidence and pathological features of intestinal neuroendocrine tumors in a large series of IBD patients treated at a tertiary care center.

Design: Using a text-based search of our pathology data base we identified all intestinal resections performed at our institution from 2002 to 2017 that referred to neuroendocrine neoplasia and Crohn's disease (CD) or ulcerative colitis (UC). The corresponding demographic and pathologic data were recorded and the available slides were reviewed.

Results: Of 2391 and 842 intestinal resections performed for CD and UC, respectively, we identified 27 neuroendocrine tumors, 14 in CD and 13 in UC for overall incidence rates of $0.6 \%$ and $1.5 \%$, respectively. The Crohn's-associated tumors included 10 well-differentiated NETs (4 small intestinal, 1 cecal, and 5 appendiceal), one appendiceal goblet cell carcinoid (GCC), and 3 mixed adenoneuroendocrine carcinomas, 2 of which were reclassified as adenocarcinoma ex GCC. The UCassociated tumors included 7 well differentiated NETs (6 appendiceal and 1 small intestinal mesenteric), one appendiceal GCC and 5 mixed adenoneuroendocrine carcinomas, 2 of which were reclassified as adenocarcinoma ex GCC.

Conclusions: The incidence of neuroendocrine tumors in this survey, which is the largest single-institution study reported to date, is higher than published incidence rates from the general population. Although such comparisons have been marshalled as evidence for higher risk among IBD patients in the past, they do not take into account such factors as differences in surgery rates and medical surveillance and should be interpreted cautiously.

\section{Histological Classification Based on Quantifiable Parameters Performs Better for Assessing Disease Severity in Adult Celiac Patients}

Prasenjit Das' ${ }^{1}$, Gaurav P Gahlot' ${ }^{1}$ Alka Singh', Govind K Makharia'. . ${ }^{1}$ All India Institute of Medical Sciences, New Delhi, India

Background: The existing histological classifications for assessment of severity of villous flattening in celiac disease (CD) are based n qualitative parameters; hence, are prone to inter-observer disagreements. Histological classification is needed to understand mucosal disease severity and for judging histological improvement on follow-up.

Design: The intra and inter-observer agreements using origina Marsh, modified Marsh, Corazza/ Villanacci, and Ensari classifications were determined by engaging four senior histopathologists, followed by computer assisted image analysis ( $\mathrm{CIA}$ ) of duodenal biopsies (from D2 \& D3) from 147 normal adult controls and 210 patients with CD by using Image ProPlus Software, Media Cybernatics. Crypt to villous ratio, IEL count, villous area, crypt depth, epithelial cell height at villi tip were measured in multiples. To identify histological parameters with good discriminative power, two-sample t test with equal variances, followed by two-sample Wilcoxon rank-sum tests were applied Diagnostic sensitivity and specificity of each parameter was calculated by ROC analysis, followed by univariate and multivariate analyses. Based on findings, a new histological classification was proposed and validated among four senior histopathologists. Institutional ethical clearance was taken.

Results: The intra-observer and inter-observer agreements with existing classifications varied between $39.7 \%-64.5 \%$ and $12.9 \%$ $48.7 \%$, respectively. The normative features in our control cohor included: C:V- 1:2 and normal intra-epithelial lymphocyte (IEL) count $13.4 \pm 8.1 / 100$ epithelial cells (ECs). On multivariable analysis villous height $<33.5 \mu \mathrm{m}$, IEL count $\geq 25 / 100 \mathrm{ECs}$ and villous area $\geq 750 \mu \mathrm{m}^{2}$ were found as best cut-offs for differentiation between biopsies from patients with $\mathrm{CeD}$ and controls. A histological classification was proposed based on these quantifiable parameters [Table], with which higher intra-observer (41.9\%-86.2\%) and inter-observer (27.2\%-54.9\%) agreements were achieved.

Proposed histological classification system for assessment of disease severity on duodenal biopsies from patients with celiac disease

\begin{tabular}{|l|l|l|l|}
\hline $\begin{array}{l}\text { Histological } \\
\text { Classes }\end{array}$ & Criteria & $\begin{array}{l}\text { Corresponding } \\
\text { modified Marsh } \\
\text { types }\end{array}$ & $\begin{array}{l}\text { Figure Cita- } \\
\text { tions }\end{array}$ \\
\hline Type 0 & IEL count $<25 / 100 \mathrm{ECs}+\mathrm{C}: \mathrm{V}<1: 2$ & Type 0 & Figs A \& B \\
\hline Type I & IEL count $\geq 25 / 100 \mathrm{ECs}+\mathrm{C}: \mathrm{V}<1: 2$ & Type I & Fig C \\
\hline Type 2 & IEL count $\geq 25 / 100 \mathrm{ECs}+\mathrm{C}: \mathrm{V}$ ratio $>1: 2$ & Types 3a \& 3b & Figs D \& E \\
\hline Type 3 & $\begin{array}{l}\text { IEL count } \geq 25 / 100 \mathrm{ECs}+\mathrm{C}: \mathrm{V} \text { ratio } \\
>1: 5: 1\end{array}$ & Type 3c & Fig F \\
\hline
\end{tabular}

IEL-intra-epithelial lymphocytes; EC-epithelial cells; C:V-crypt to villous ratio

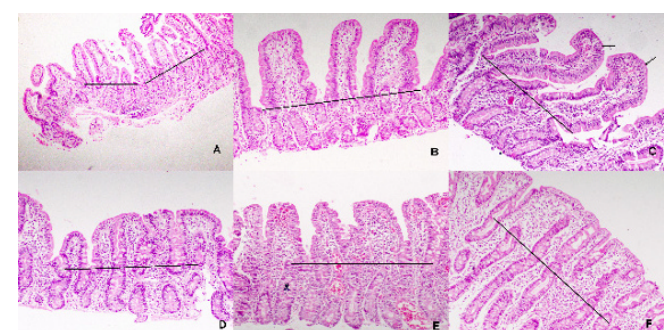

Conclusions: In comparison to existing classifications, newly proposed classification is based on quantifiable histological parameters, simple to use and showed better inter-observer agreements. Normal mucosal dimensions vary in different regions.

\section{Lynch Syndrome Screening in Central Europe: Preponderance of MSH6 and Recognition of $8 \mathrm{New}$ Germline Mutations}

Ondrej Daum¹, Magdalena Dubova², Martin Dusek, Ladislav Hadravsky ${ }^{4}$, Jan Stehlik ${ }^{5}$, Katerina Cerna ${ }^{5}$, Radmila Curcikova ${ }^{6}$, Marian Svajdler ${ }^{7}$, Bohuslava Saskova ${ }^{8}$, Tomas Jirasek ${ }^{9}$, Michal Michal ${ }^{10}$ ${ }^{1}$ Biopticka laborator, PIzen, ${ }^{2}$ Biopticka laborator s.r.o., Plzen, Czech Republic, ${ }^{3}$ Biopticka laborator s.r.o., Plzen, Czech Republic, ${ }^{4}$ Charles University, Praha, Plzensky kraj, Czech Republic, ${ }^{5}$ Biopticka laborator s.r.o., ${ }^{6}$ Liberec Regional Hospital, Liberec, Czech Republic, ${ }^{7}$ Biopticka laborator s.r.o, Plzen, ${ }^{8}$ Biopticka Laborator Plzen, Plzen, Czech Republic, ${ }^{9} \mathrm{Hospital}$ Liberec, Liberec, ${ }^{10}$ Bioptical Laboratory s.r.o., Plzen

Background: To report on special issues of Lynch syndrome (LS) screening in Central Europe and present results of germline analysis which point to the possibility of specific spectrum of founder mutations.

Design: Until 2014, selection of patients for LS screening was based on combination of clinical and morphological criteria (PREDICT score). Since then, all colorectal cancers (CRC) and endometrial cancers (EC) diagnosed in the Faculty Hospital $(\mathrm{FH})$ were investigated by $\mathrm{IHC}$ for MLH1, PMS2, MSH2 and MSH6. Then, in suspicious cases molecular genetic analysis of $B R A F, M L H 1$ promoter methylation status and germline analysis of MMR genes were performed according to current 
guidelines. To evaluate the significance of detected gene variants the following databases were used: HGMD, MMRGVD, InSIGHT, dbSNP NCBI, IGSR, and prediction program Provean.

Results: In total, 5814 patients with CRC (1417 in FH, 4397 in other hospitals) were investigated. Suspicious PREDICT score and/or MMRdeficiency was detected in 225 cases. LS was proven in 20 patients $(0.34 \%)$ with CRC. Of those, 10 were $\mathrm{FH}$ patients $(0.71 \%), 10$ were from other hospitals $(0.22 \%)$. Further, LS was also diagnosed in 3 patients with EC and 1 with duodenal cancer. In 26 cases suspicious of LS $(0.45 \%)$ germline analysis has not been perfomed yet. If only the group of $\mathrm{FH}$ patients is analyzed, potential frequency of LS during 20132016 reaches $1.42 \%$, in 2016 even $2.4 \%$. Age range was $26-80$ years, 5 patients $(21.7 \%)$ were older than 70 years at the time of diagnosis. MLH1 germline mutation or epimutation was detected in 6 cases of CRC $(25 \%), P M S 2$ in 3 cases of CRC (12.5\%), MSH2 in 5 cases of CRC and $1 \mathrm{EC}(25 \%)$, and $M S H 6$ in 6 cases of CRC, 1 duodenal cancer and $1 \mathrm{EC}(37.5 \%) .8$ of the detected variants were not previously regarded as pathogenic (MSH6 in 5 cases, MLH1 in 2 cases, MSH2 in 1 case)

Conclusions: Following introduction of the universal IHC MMR investigation in all CRCs examined in $\mathrm{FH}$ the frequency per year reached $2.4 \%$. The results favor universal IHC screening over a selective approach. Lower LS frequency than reported in the literature may result from low number of cases, technical aspects of investigation, regional differences of genetic background and environmental factors. The latter 2 factors may be involved in the $\mathrm{FH}$ group of patients, as the population of the region is relatively homogenous and known to lead the CRC prevalence worldwide. Finally, the preponderance of MSH6 mutations may mirror region-specific distribution of founder mutations

\section{Morphologic and Endoscopic Characterization of Distinct Sloughing Eosinophilic Esophagitis: A Single Institution Cohort Study}

Audrey Deeken-Draisey', Andrew Bandy², Ryan Jones ${ }^{3}$, Katrina $K_{r o g h}^{4}$, M. Sambasiva Rao ${ }^{5}$, Ikuo Hirano ${ }^{4}$ Guang-Yu Yang ${ }^{2}$. ${ }^{1}$ Northwestern Memorial Hosp., Chicago, IL, ${ }^{2}$ Chicago, IL, ${ }^{3}$ Naperville, IL, ${ }^{4}$ Northwestern Memorial Hospital, ${ }^{5}$ Northwestern University, Chicago, IL

Background: Eosinophilic esophagitis (EoE) is a chronic, immune/ antigen-mediated esophageal disease characterized clinically by symptoms related to esophageal dysfunction and histologically by eosinophil-predominant inflammation. EoE is an entity with increasing recognition and prevalence. Esophagitis dissecans superficialis, also known as sloughing eosphagitis, is a disease of squamous epithelium characterized by superficial epithelial necrosis, parakeratosis, lack of inflammation, and sloughing of necrotic epithelium from viable surface. It appears endoscopically with sloughed mucosa in patches. We present a cohort of patients with morphologic and endoscopic feature defined as sloughing eosinophilic esophagitis.

Design: This study aims to establish diagnosis of sloughing EoE as a unique diagnostic entity defined as extensive superficial epithelial necrosis (sloughing) in the background of eosinophilic esophagitis (>15 intraepithelial eosinophils/HPF). We searched our database since 2007, finding 356 eosinophilic eosphagitis cases, 12 of which diagnosed as sloughing eosinophilic esophagitis. We retrospectively analyzed the endoscopic and morphologic findings and further expand on diagnostic criteria.

Results: Twelve cases of sloughing EoE were identified in the past 10 years, with male to female ratio of $1: 1$ and an average age of 41 years. Endoscopic findings include: white plaques (7/12, Fig.A), dissecans (2/12, Fig. B), furrows (9/12), and edema/congestion (7/12). Morphologic findings include: sloughing necrosis (12/12, Fig, C-F), epithelial spongiosis (12/12), basal cell hyperplasia (12/12), lamina propria fibrosis (9/12), and eosinophilic microabscess formation $(10 / 12$, Fig. E-F). The sloughing necrosis associated eosinophilic microabscess formation ( $>5$ eos) was more diffuse and associated with greater viable epithelial eosinophil count (40 vs 15 eosinophils/ HPF, Fig. F)
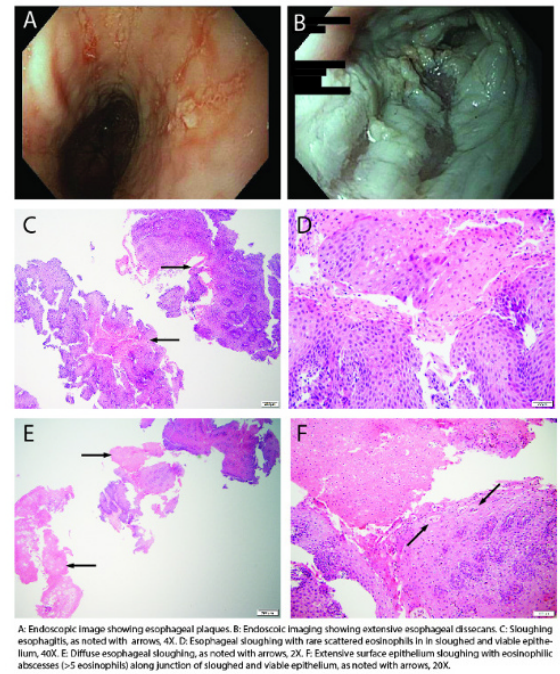

Conclusions: Overall, these findings suggest that sloughing eosinophilic esophagitis is a distinct type of eosinophilic esophagitis The criteria necessary for diagnosis should include: 1) $\geq 15$ eosinophils/ HPF within squamous mucosa, 2) superficial epithelial necrosis with sloughing, 3) eosinophilic microabscess formation between boundary of necrotic and viable epithelium, 4) lamina propria fibrosis, and 5) endoscopic findings including plaques and/or dissecans of the esophagus. The sloughing necrosis associated eosinophilic microabscess formation appears a key pathogenic event in this disease process.

709 Comparison of Barrett's Low Grade Dysplasia Diagnosis Rate and Neoplastic Progression Rate across Independent Subspecialized and General Surgical Pathology Practices: An Attempt to Measure "Expertise"

Christopher Deitrick', Jon Davison'. 'University of Pittsburgh, Pittsburgh, PA

Background: The American Gastroenterological Association (AGA) recommends that all diagnoses of low grade dysplasia (LGD) in Barrett's esophagus (BE) be confirmed by an "expert". To substantiate one's expertise, the AGA recommends monitoring the proportion of LGD diagnosed among surveillance patients (LGD/BE ratio) and the risk of neoplastic progression among patients diagnosed with LGD vs non-dysplastic BE. The aim of this study is to compare independent pathology practices in a multihospital system using these metrics.

Design: We retrospectively identified all esophageal biopsies $(\mathrm{N}=29,188)$ for suspected $\mathrm{BE}$ with histologically confirmed intestinal metaplasia diagnosed from 1999-2016. Diagnoses were rendered in one subspecialized gastrointestinal pathology practice (SSGI), three high BE volume general practices (GSP1-3 with $>200$ BE cases annually) and 15 low volume general practices (LV-GSPs, grouped together for analysis). There were 5111 patients with $>30$ days of total follow up. Each patient was categorized based on the highest grade of neoplasia during surveillance (negative, indefinite or LGD) and assigned to the hospital where the highest grade was first diagnosed.

Results: The annual number of BE biopsies ranged from 8.6 to 835 per year and the annual number of high grade dysplasia (HGD) or adenocarcinoma (EAC) cases ranged from 0.6 to 163 across the practice groups (Table 1). The LGD/BE diagnosis ratio was highest $(6.7 \%)$ in the LV-GSPs (Table 1). LGD diagnosed by the LV-GSPs was not a significant risk for neoplastic progression to HGD or EAC relative to patients with non-dysplastic BE; however, LGD diagnosed by the other groups had a 6.3- to 19.5-fold risk of neoplastic progression (Table 1). LGD diagnosed by any of the general pathology groups had a $50-80 \%$ lower risk of progression compared to LGD diagnosed by the SSG (Table 1). Across practices, there were significant differences in the proportion of patients that progressed to HGD or EAC within 2 years of the first LGD diagnosis (range $2.0 \%-31.8 \%, P<0.001$, Table 1). 
Table 1: Comparison of subspecialist GI (SSGI), high volume (GSP1, 2 and 3) and low volume (LV-GSPs) general surgical pathology practices

\begin{tabular}{|c|c|c|c|c|c|}
\hline & SSGI & GSP1 & GSP2 & GSP3 & LV-GSPs \\
\hline $\begin{array}{l}\text { Annual Case Volume }{ }^{1} \\
\text { All BE } \\
\text { HGD and EAC }\end{array}$ & $\begin{array}{l}835.3 \\
163.3\end{array}$ & $\begin{array}{l}305.4 \\
8.8\end{array}$ & $\begin{array}{l}413.2 \\
18.7\end{array}$ & $\begin{array}{l}226.2 \\
8.8\end{array}$ & $\begin{array}{l}8.6 \\
0.6\end{array}$ \\
\hline LGD/BE Ratio ${ }^{2}$ & $4.5 \%$ & $0.9 \%$ & $4.0 \%$ & $5.4 \%$ & $6.7 \%$ \\
\hline $\begin{array}{l}\text { Risk of progression }{ }^{3} \\
\text { Non-dysplastic BE } \\
\text { LGD BE }\end{array}$ & $\begin{array}{l}\text { Reference } \\
8.0(5.7- \\
11)\end{array}$ & $\begin{array}{l}\text { Reference } \\
19.5 \text { (1.1- } \\
366)\end{array}$ & $\begin{array}{l}\text { Reference } \\
6.3(1.4- \\
28)\end{array}$ & $\begin{array}{l}\text { Reference } \\
8.2(2.8- \\
24)\end{array}$ & $\begin{array}{l}\text { Reference } \\
1.5(0.6- \\
3.6)\end{array}$ \\
\hline $\begin{array}{l}\text { LGD progression risk } \\
\text { vs SSG }\left.\right|^{4}\end{array}$ & Reference & $\begin{array}{l}0.5(0.1- \\
1.9)\end{array}$ & $\begin{array}{l}0.2(0.1- \\
0.3)\end{array}$ & $\begin{array}{l}0.3(0.2- \\
0.5)\end{array}$ & $\begin{array}{l}0.3(0.2- \\
0.7)\end{array}$ \\
\hline $\begin{array}{l}\text { LGD } 2 \text { yr progression } \\
\text { rate }^{5}\end{array}$ & $31.8 \%$ & $16.7 \%$ & $2.0 \%$ & $9.1 \%$ & $10.1 \%$ \\
\hline
\end{tabular}

${ }^{1}$ Annual number of endoscopic biopsy cases per hospital.

2 Ratio of LGD diagnoses to total number of BE cases, 1999-2016 inclusive.

${ }^{3}$ Risk of progression to HGD or EAC in patients diagnosed with LGD compared to patients with only non-dysplastic BE during surveillance ladjusted for age, sex, BE segment length and time to next biopsy after first LGD). Expressed as hazard ratio with $95 \%$ confidence interval in parentheses. Hazard ratio $>1$ are considered higher risk (e.g. 8.0 implies an 8-fold higher risk for LGD compared to non-dysplastic $\mathrm{BE}) ; 95 \%$ confidence interval that does not include 1 is considered significant.

${ }^{4}$ Risk of progression to HGD or EAC in patients diagnosed with LGD during surveillance, comparing patients diagnosed by general surgical pathology practices to patients diagnosed by subspecialized gastrointestinal pathologists (adjusted for age, sex, BE segment length and time to next biopsy after first LGD). Expressed as hazard ratio with $95 \%$ confidence interval in parentheses. Hazard ratio<1 implies lower risk (e.g. 0.5 implies a $50 \%$ lower risk of progression relative to LGD diagnosed by SSGI); $95 \%$ confidence interval that does not include 1 is considered significant.

${ }^{5}$ Proportion of patients who progress to HGD or EAC within 2 years of the first diagnosis of LGD.

Conclusions: General practices with low annual volume of neoplastic and non-neoplastic BE cases underperformed in the diagnosis of LGD as measured by comparative risk of neoplastic progression (vs nondysplastic BE and vs subspecialist diagnosed LGD). LGD/BE ratio, risk of progression vs non-dysplastic $B E$, and 2 year progression rate are metrics that identify differences between practices and allow objective assessment of expertise in the diagnosis of LGD.

\section{Pathology Diagnosis of "At Least Intramucosal Adenocarcinoma" in Colorectal Biopsies of Mass Lesions Correlates with Advanced Tumor Stage}

Armando Del Portillo', Paul Oberstein ${ }^{2}$, Alfred Neugut ${ }^{2}$, David P Horowitz ${ }^{2}$ Steven Lee-Kong ${ }^{2}$, Daniel L' Feingold², Ravi' $P$ Kiran ${ }^{2}$, Antonia Sepulveda'. ${ }^{1}$ New York, NY, ${ }^{2}$ Columbia University

Background: When a mass lesion is found in the colon/rectum, biopsy-proven invasive adenocarcinoma (CRC) may be required before treatment can begin. However, the biopsy may not capture the invasive lesion (due to small/superficial biopsy), and may only show adenocarcinoma in the mucosa. Thus the diagnosis of "at least intramucosal adenocarcinoma" ("at least IMCA") may be warranted to convey that while histologic features of invasion beyond the muscularis mucosae (MM) are not seen (e.g. desmoplasia, tumor cells adjacent to large blood vessel), deeper invasion also cannot be excluded. The treatment implications may significantly differ between a CRC limited to the mucosa (Tis: carcinoma in situ/intramucosal adenocarcinoma) and a CRC that invades beyond the MM (pT1-pT4). Thus, repeated biopsies to prove invasion may result in significant delays in treatment for the patient.

Design: We searched our pathology database and clinical records from 2007 to 2017 to determine how many patients with the diagnosis of "at least IMCA" go on to have a pathologic diagnosis of invasive CRC or CRC metastasis. Follow-up definitive pathology specimens included biopsies, EMR/ESDs or colorectal resections. We recorded location of mass, endoscopic impression, radiologic impression at time of diagnosis, and follow-up pTNM stages.

Results: Eighty-nine patients were found with an age range from 36 to 93 (average 68), 52\% female. Eight had "at least IMCA" in a polyp and 81 had it in a mass/lesion that was suspicious/likely malignant on endoscopy. The locations of the primary lesion were 27 cecum/ ascending, 11 transverse, 4 descending, 20 sigmoid, 8 rectosigmoid,
10 rectum, 1 anal. Of 71 patients who had available radiology records, 28 had an impression of an advanced lesion, where features of invasion or metastasis were suspected or found. Of the 81 patients with endoscopic impression of a mass lesion, $2(2.5 \%)$ had further work-up that showed intramucosal CRC (pTis), in an ESD of a recta lesion and in an ileocolectomy for a cecal mass. The remaining 79 patients showed pathologic invasive CRC or biopsy-proven metastasis (at least pT1=2, pT1=5, pT2=11, pT3=37, pT4=17, pM1=7).

Conclusions: In the context of a colorectal mass with strong clinica suspicion for malignancy, nearly all cases with a biopsy diagnosis of "at least IMCA" had a more advanced lesion that was not sampled. These data suggest that in this context, extensive repeat biopsies to show invasion pathologically may not be warranted in order to start treatment.

\section{Gastroesophageal Adenocarcinoma Biopsies and Resections: Impact of Programmed Death-Ligand 1 (PD-L1) Expression on Patient Management}

Armando Del Portillo', Ladan Fazlollahi², Jonas Heymann ${ }^{3}$, Anjali Saqi $^{2}$. ${ }^{1} \mathrm{New}$ York, NY, ${ }^{2}$ Columbia University Medical Center, New York, NY, ${ }^{3}$ Weill Cornell Medicine

Background: Gastric cancer $(\mathrm{GC})$ is the $5^{\text {th }}$ most common cancer worldwide but represents a major cancer death burden as the $3^{\text {rd }}$ leading cause of cancer death worldwide. Esophagea adenocarcinoma (EAC) is far less common, but incidence rates are increasing in developed countries. Prognoses for these cancers are poor and more therapeutic options are necessary to improve survival outcomes. Earlier this year, pembrolizumab (PEM) was approved for solid tumors that were microsatellite instability-high (MSI-H) or mismatch repair deficient (dMMR). Recently, the FDA approved PEM for recurrent locally advanced or metastatic GC and gastroesophagea (GE) junction adenocarcinomas that are positive for programmed death-ligand 1 (PD-L1). The aim of the study was to determine the impact of the new guidelines regarding PEM as a potential therapeutic option for GE adenocarcinomas.

Design: A retrospective search was performed for $\mathrm{GE}$ adenocarcinomas (including GC, GE junction adenocarcinomas and EACs) that had immunohistochemistry (IHC) using anti-PD-L1 antibody 22C3 (Dako) The tumor proportion score (TPS) representing the percentage of tumor cells with membranous staining was quantified. This was compared against corresponding available MMR or MSI results.

Results: 18 cases of primary GE adenocarcinomas (biopsies $=13$; EMRs = 1; resections = 4) that underwent PD-L1 IHC testing, $10(56 \%)$ male and $8(44 \%)$ female, with an age range of 39 to 93 (median age 71), were identified. Of these cases, $5(28 \%)$ had a TPS $>1 \%$ and none of these 5 were MSI-H or dMMR (all 5 tested by IHC, one tested for MSI).

Conclusions: Based on this limited cohort, at least an additional $28 \%$ of patients with GE adenocarcinomas may have an additional therapy option. Moreover, PD-L1 IHC analysis performed on our cohort analyzed mostly biopsies and only tumor cells (TPS), whereas the FDA approval was based on a combined positive score (CPS) determined by evaluating tumor cells, lymphocytes and macrophages. Thus, ou results may be an underestimation of the number of PEM eligible carcinomas. In conclusion, PD-L1 IHC may markedly increase the number of patients for whom PEM could be a therapeutic option.

\section{HSV Esophagitis: Something Old, Something New}

Santiago Delgado', Andrea L Barbieri², Joanna Gibson³. 'Yale-New Haven Hospital, New Haven, CT, ${ }^{2}$ Yale University, New Haven, CT, ${ }^{3}$ Yale School of Medicine, New Haven, CT

Background: Herpes simplex virus esophagitis (HSVe) commonly occurs in immunocompromised patients with dysphagia and esophageal ulcers. In recent years, we have observed an increase of HSVe in immunocompetent patients. The aim of this study is to describe the clinical and histopathological features of HSVe, and compare any differences in patient presentation and immune status.

Design: All patients with HSVe from 1990 to 2017 were retrieved for review. Data collected after IRB approval included: gender age at diagnosis, symptoms, immune status, endoscopic findings, and biopsy site. H\&E slides were evaluated for presence of inclusions and ulceration. When available, immunohistochemica (IHC) stained slides were assessed for confirmation of HSV.

Results: Thirty-eight patients with HSVe were identified, with 17 patients diagnosed between 2010-2017 (Group A) and 21 patients diagnosed between 1990 and 2009 (Group B). Group A patients were older than Group B patients (average age 68 and 54 years, respectively), showing a trend toward an age difference $(p=0.076)$ Patient gender did not vary between groups. The distal esophagus was the most common site of biopsy ( $67 \%$ in both groups). Presence of ulceration on endoscopy (89\% Group A, 85\% Group B) and on biopsy (94\% Group A, 90\% Group B) was similar in both patient groups. Immunosuppression was more common in Group B patients (88\%) 
than in Group A (71\%), but this was not statistically significant. In $18 \%$ of Group A patients, inclusions were not identified on $\mathrm{H} \& \mathrm{E}$, compared to 1 patient in Group B (5\%) without an inclusion. All patients without H\&E inclusions had positive HSV immunohistochemistry.

Conclusions: Our data demonstrates a trend in the last 8 years of HSVe within older patients without immunocompromise. Additionally, inclusions appear to be less commonly seen on H\&E exam, necessitating a high index of suspicion and use of immunohistochemistry for confirmation of diagnosis. Larger studies are needed to determine if this trend is statistically significant and to further describe the clinicopathological features of HSV esophagitis.

\section{Buried Barrett's Esophagus: Interobserver Study and Outcomes Analysis}

Ryan Demkowicz', Rocio Lopez², Tanmayee Benjamin², Deepa T Pati ${ }^{3}$, Erinn Downs ${ }^{4}$, Jennifer Jeung ${ }^{5}$, Keith Lai ${ }^{3}$, James Lapinski ${ }^{6}$, Erica Savage $^{3}$, John Goldblum ${ }^{3}$, Prashanthi Thota ${ }^{2}$, Ilyssa Gordon ${ }^{3}$. ${ }^{1} \mathrm{Cleveland}$ Clinic, Cleveland Heights, $\mathrm{OH}_{2}{ }^{2} \mathrm{Cleveland} \mathrm{Clinic},{ }^{3} \mathrm{Cleveland}$ Clinic, Cleveland, $\mathrm{OH},{ }^{4} \mathrm{Cleveland}$ Clinic, Cleveland, $\mathrm{OH},{ }^{5} \mathrm{Cleveland}$ Clinic, Willoughby Hills, $\mathrm{OH},{ }^{6} \mathrm{Cleveland}$ Clinic, LaGrange, $\mathrm{OH}$

Background: Radiofrequency ablation (RFA) is a safe and effective therapy for Barrett's esophagus (BE)-related dysplasia. Following RFA, growth of neosquamous epithelium over residual BE results in the development of buried BE. The risk of dysplasia in buried BE is unknown, and there is no formal definition for buried BE. Previous studies also have not sufficiently addressed the adequacy of biopsies to contain sufficient subsquamous tissue to assess for buried BE. We aimed to assess interobserver agreement for buried BE and the association of buried BE with clinical outcome.

Design: Eight gastrointestinal pathologists reviewed 140 biopsies from $28 \mathrm{BE}$ patients undergoing surveillance biopsy at least three years after RFA. Presence or absence of surface BE and buried BE, as well as the presence of sufficient subsquamous tissue, were recorded. Interobserver agreement by slide and by patient was assessed by calculating a kappa value (к). The number of pathologists who agreed on a diagnosis of buried BE was correlated with clinical outcome.

Results: There was moderate agreement $(81 \%)$ on the diagnosis of each slide between pathologists $(\mathrm{k}=0.75,95 \% \mathrm{Cl}: 0.73,0.77)$. There was also moderate agreement $(81 \%)$ on the final diagnosis per patient $(\mathrm{k}=0.70,95 \% \mathrm{Cl}: 0.63,0.78)$. There was insufficient subsquamous tissue to evaluate for buried $B E$ in 47 of 140 biopsies $(33.6 \%)$, although per patient, there were no cases with insufficient subsquamous tissue, as patients typically had multiple segments biopsied. Median follow-up was 77 months, during which time 24 subjects completed treatment, 2 were still undergoing treatment and 2 were lost to follow-up. Of the 24 that completed treatment, $96 \%(n=23)$ had eradication of BE and $4 \%(n=1)$ had eradication of dysplasia. Twenty-one percent of subjects $(n=5)$ had recurrence of $B E$. While the median number of pathologists who agreed on diagnosis of either buried $B E$ only or surface and buried $B E$ was higher in subjects who had recurrent $B E$, this did not reach statistical significance.

Conclusions: There is moderate agreement in identifying surface and buried BE in post-RFA biopsies, despite the lack of a formal definition. In all cases, at least one slide per patient contained sufficient subsquamous tissue to evaluate for buried BE. Additional studies on the correlation between buried BE and clinical outcome are needed.

\section{Traditional Serrated Adenomas- A Longitudinal Clinical Outcome Study}

Fatemeh Derakhshan', David Schaeffer', Douglas Webber'. 'Vancouver General Hospital, Vancouver, BC, Canada

Background: Traditional serrated adenomas (TSA) are the least common of colonic serrated polyps but have recently been recognized to encompass a heterogeneous group. Although predominantly microsatellite stable proximal TSA often show BRAF mutations while distal ones are KRAS driven. The natural history of TSAs is not well defined and longitudinal clinical follow up studies to assess for malignant transformation are lacking.

Design: In a retrospective multi-center cohort study using data from Vancouver Coastal Health's databases, all TSAs diagnosed between 2005 and 2012 were selected, giving a minimum of 6 years followup. In total, there were 103 colorectal polyps diagnosed as TSAs, all without prior history of either CRC or advanced adenoma. An age and location matched control cohort of adenomatous polyps (AP) was identified.

Results: The average age at diagnosis was 64 years, with a M:F ratio of 1.8:1. 81 of the103 included patients have had follow-up colonoscopy. Of these, 9 patients had CRCs present synchronously, 3 patients developed subsequent CRCs (right colon $(n=2)$ and rectum $(n=1))$ and 2 patients developed subsequent high risk AP (high grade dysplasia (HGD))(14.8\% incidence of synchronous and metachronous CRCs). Of the CRCs overall, 5 were in the same segment as the TSA and one CRC at presentation derived directly from a TSA. The time for
列 on average (range: 1.5-5.7 years). Only the right sided CRC showed presence of mutated BRAFV600E protein

Conclusions: Given the high incidence (14.8\%) of both synchronous and metachronous CRC in our cohort of TSA patients, especially if compared to the malignant transformation of patients with high risk polyps $(7.7 \%$ to $9.8 \%)$, TSA lesions deserve to be classified as high risk polyps themselves. Our study further highlights the need to adapt current screening protocols accordingly.

\section{Evaluation of Mcl-1, Bcl-xL, Bcl-2 Expression as Potential Therapeutic Targets for Colorectal Adenocarcinoma}

Josephine Dermawan ${ }^{1}$, Abeba Demelash ${ }^{2}$, Brian Gastman $^{2}$, Cameron Mayelß, Lukas PfannenstieR, Matthew Kalady ${ }^{4}$, Jennifer DeVecchio ${ }^{5}$ Deepa T Patil'. 'Cleveland Clinic, Cleveland, $\mathrm{OH},{ }^{2}$ Cleveland Clinic Lerner Research Institute, ${ }^{3}$ Cleveland Clinic Lerner Research Institute, North Royalton, Ohio, ${ }^{4}$ Cleveland Clinic, ${ }^{5}$ Cleveland Clinic Lerner Research Institute, Cleveland, $\mathrm{OH}$

Background: Solid tumors, including colorectal cancers (CRC) are known to express anti-apoptotic markers belonging to the $\mathrm{Bcl}$ 2 family (Mcl-1, Bcl-xL, Bcl-2). It was recently shown that $\mathrm{Mcl}-1$ prevents chemotherapy-induced senescence (CIS), presumably by translocating to the nucleus to activate anti-senescence genes. In order to understand the role of these markers as potential therapeutic targets in primary and metastatic CRC (mCRC), a systematic analysis was performed to evaluate their expression and correlate these findings with clinicopathologic features.

Design: Primary ( $n=79$; mean age 62 ) and $m C R C$ to liver $(n=61 ;$ mean age 58), 5 normal colon and 7 adenomas were assessed for $\mathrm{Mcl}-1$ (Abcam,1:100), Bcl-xL (Cell Signaling,1:100), and Bcl-2 (LifeSpan, pre-diluted) expression. Staining was categorized as neg/low or moderate/high by generating a composite score based on intensity (weak, moderate, strong) and \% positive tumor cells $(<30 \%, 30-70 \%$ $>70 \%$ ). Nuclear and/or cytoplasmic Mcl-1 and cytoplasmic Bcl-xL and $\mathrm{Bcl}-2$ staining was considered positive. $\mathrm{Chi}^{2}$ or Fisher's exact test were used to correlate marker expression with tumor location, grade, stage, and MSI status.

Results: The grade distribution of 39 (48\%) right-sided and $43(52 \%)$ left-sided CRC was 91 (88\%) low-grade and $13(13 \%)$ high-grade. Moderate/high Mcl-1, Bcl-xL, and Bcl-2 expression was seen in 38 $\%, 63 \%$, and $6 \%$ of primary CRC, and $45 \%, 58 \%$, and $3 \%$ of $\mathrm{mCRC}$ respectively. Mcl-1 expression increased from normal to adenomas and CRC $(0 \%, 29 \%, 42 \% ; p=0.086)$. Nuclear Mcl-1 expression was seen in $14 \%$ primary $\mathrm{CRC}$ and $26 \% \mathrm{mCRC}$. Bcl-xL was highly expressed in $\mathrm{CRC}(61 \%)$ and adenomas $(71 \%)$ compared to normal $10 \%$ $p=0.01)$. Majority of primary CRC $(94 \%)$ and mCRC $(97 \%)$ showed neg/ low Bcl-2, but there was no difference in expression between normal vs. adenoma vs.CRC. Moderate/high Mcl-1 correlated positively with $\mathrm{Bcl}-\mathrm{xL} \quad\left(\mathrm{R}^{2}=0.28 ; p<0.001\right)$, but not with $\mathrm{Bcl}-2$ expression $\left(\mathrm{R}^{2}=0.07\right.$ $p=0.45)$. Bcl-xL $(55 \%)$ was significantly decreased in stage III/IV compared to Stage I/II CRC $(77 \% ; \mathrm{p}<0.014)$. The expression of markers did not vary with grade, location or MSI status.

Conclusions: $\mathrm{Mcl}-1$ expression increases with neoplastic progression and can serve as a potential target to induce $\mathrm{CIS}$ in CRC. As Bcl-xL expression is preferentially expressed in early-stage CRC, anti- Bcl-xL therapy could be an option for treating early-stage CRC. Finally, lack of Bcl-2 expression in the vast majority of primary and mCRC may explain failure of Bcl-2 inhibitors in clinical trials.

\section{Molecular Lymph Node Total Tumor Load Correlates Better With High Risk Factors Than pN Stage in Early colorectal carcinoma. A multicenter study}

Sherley Diaz Mercedes ${ }^{1}$, Iban Aldecoa ${ }^{2}$, Antoni Bombi ${ }^{3}$, Jord Tarragona ${ }^{4}$, Blanca Gonzalez-Farre ${ }^{3}$, Paola Castillo ${ }^{5}$, Iñigo Gorostiaga ${ }^{6}$, Antonio Lacy ${ }^{3}$, Dulce Momblan ${ }^{3}$, Ainitze Ibarzabaß ${ }^{3}$ Nuria Chic ${ }^{3}$, Joan Maure ${ }^{\beta}$, Antoni Castells ${ }^{7}$, Francesc Balaguer ${ }^{8}$, Miriam Cuatrecasas Freixas ${ }^{9}$. ${ }^{1}$ Hospital Clinic of Barcelona, Spain, ${ }^{2} \mathrm{Hospital}$ Clinic of Barcelona, Spain, San Cugat del Valles, Barcelona, ${ }^{3}$ Hospital Clinic of Barcelona, Spain, ${ }^{4}$ Arnau of Vilanova University Hospital, ${ }^{5} \mathrm{Hospita}$ Clinic, Barcelona, ${ }^{6}$ Araba University Hospital, Spain, Vitoria-Gasteiz Álava, ${ }^{7}$ Hospital Clinic, Barcelona, Spain, ${ }^{8}$ Hospital Clinic, Barcelona. Spain, ${ }^{\circ}$ Hospital Clinic, Universitat de Barcelona, Spain

Background: Total tumor load (TTL), or the amount of tumor burden present in regional lymph nodes (LNs) of colorectal carcinomas (CRC) has been described as a high-risk factor. The clinical application of TTL is well established in breast cancer guidelines, but still under investigation in CRC. We aimed to validate previous published results and push forward the power of TTL in early stage CRC.

Design: Between June 2012 and July 2017, freshly dissected LNs from CRC of 3 different hospitals were analyzed by H\&E and RT-LAMP using the pooling method (analysis of multiple LNs per assay). The TTL resulting from LN molecular analysis was correlated with the classica pN staging assessed with $\mathrm{H} \& \mathrm{E}$, and with clinical and pathological factors. 
Results: Analysis of $6609 \mathrm{LNs}$ from $341 \mathrm{CRC}$ was performed (35 In situ, 63 pT1, 72 pT2, 128 pT3, 43 pT4). A median of 15 LNs were freshly dissected per patient. TTL values correlated with $\mathrm{pT}$ stage $(p=0.0004)$, $p N$ stage H\&E-assessed $(p>0.0001)$, high tumor budding $(p<0.0001)$ tumor grade $(p=0.0002)$, vascular and perineural invasion $(p<0.0001$ each), and presence of tumor deposits $(p<0.0019)$. The median TTL values were (Mín/Máx): pN0 cases: 0 (0/380.310) copies/ $\mu \mathrm{L}$, pN1: $8.100(0 / 969.700) \mathrm{c} / \mu \mathrm{L}$, and pN2: $25.955(3.600 / 370.000) \mathrm{c} / \mu \mathrm{L}$.

Conclusions: The TTL present in CRC lymph nodes correlates with $\mathrm{H} \& \mathrm{E}$-assessed $\mathrm{pN}$ stage and with other high risk factors. It arises a feasible way to accurately assess LNs in early CRC patients.

\section{Cytology Smears As A Tool To Enable Whole Molecular Lymph Node Analysis In Colorectal Carcinoma}

Sherley Diaz Mercedes ${ }^{1}$, Iban Aldecoa ${ }^{2}$, Antoni Bombi ${ }^{3}$, Blanca Gonzalez-Farre ${ }^{3}$ Paola Castillo ${ }^{4}$, Iñigo Gorostiaga ${ }^{5}$, Antonio Lacy ${ }^{3}$, Dulce Momblan ${ }^{3}$, Ainitze Ibarzabaß, Jordi Tarragona ${ }^{6}$, Francesc Balaguer ${ }^{7}$, Antoni Castells ${ }^{8}$, Miriam Cuatrecasas Freixas ${ }^{9}{ }^{1} \mathrm{H}$ ospital Clinic of Barcelona, Spain, ${ }^{2}$ Hospital Clinic of Barcelona, Spain, San Cugat del Valles, Barcelona, ${ }^{3} \mathrm{H}$ ospital Clinic of Barcelona, Spain, ${ }^{4}$ Hospital Clinic, Barcelona, ${ }^{5}$ Araba University Hospital, Spain, VitoriaGasteiz, Álava, ${ }^{6}$ Arnau of Vilanova University Hospital, ${ }^{7}$ Hospital Clinic. Barcelona, Spain, ${ }^{8} \mathrm{Hospital}$ Clinic, Barcelona, Spain, ${ }^{9} \mathrm{H}$ ospital Clinic. Universitat de Barcelona, Spain

Background: Current colorectal carcinoma (CRC) guidelines base $\mathrm{pN}$ staging on the gold standard H\&E lymph node (LN) analysis. Due to its low sensitivity, at least 12 LNs must be evaluated for a reliable pNO stage. Nevertheless, the presence of occult tumor within LNs is an important prognostic factor and can be overlooked on conventional histopathology analysis. Molecular methods of LN analysis can be used as an alternative to $\mathrm{H} \& \mathrm{E}$ in early CRC, but often the results are given in different measures than $\mathrm{pN}$. For that reason, most studies have used only part of the LN for molecular analysis, preserving some for conventional $H \& E$ pN staging. We aimed to use the entire LN for molecular analysis and also give the standard pN stage.

Design: From December 2016 to July 2017, analysis of 1001 LNs from 54 CRC was performed. LNs were cut on the long axis, half was used for cytology smear+CK19 immunohistochemistry (IHC), H\&E and CK19 IHC, and the other half was processed with the RT-LAMP molecular assay. The $\mathrm{pN}$ obtained with the three morphology-based methods of LN analysis and the number of positive LNs were compared between them and with the values of the molecular results.

Results: The number of positive LNs per patient and the number of patients with positive LNs was higher with cytology smear+CK19 IHC evaluation (18 patients) than with H\&E alone (15 patients). Cytology smear detected $5,6 \%$ more patients than H\&E $(p<0.0001)$. Additional CK19 IHC on FFPE LNs increased 1,9\% detection (16 patients). RT-PCR detected higher number of patients with presence of tumor burden in LNs than any other morphology-based method (20 patients; $p<0.0001$ ).

Conclusions: Cytology smear + CK19 IHC could replace H\&E and allows to use the entire LN tissue for molecular determination. This approach enables to use the $\mathrm{pN}$ stage according to the current guidelines, as well as obtaining more accurate data based on the complete molecular LN analysis in early-stage CRC patients.

\section{Expression of TIM3 (CD366) and LAG3 (CD223) in Colorectal Carcinoma-associated Inflammatory Infiltrate Suggest Novel Therapeutic Targets for Immune Checkpoint Blockade}

Michael Drage ${ }^{1}$, Max Klapholz ${ }^{2}$, Ana C Anderson², Amitabh Srivastava ${ }^{2}$ 'University of Rochester, Rochester, NY, 'Brigham and Women's Hospital, Boston, MA

Background: Immune checkpoint blockade therapy has shown limited utility in management of colorectal carcinoma (CRC). While microsatellite instability (MSI)-high status is the best predictor of response, only $40 \%$ of $\mathrm{MSI}$-high CRC show an objective response to Pembrolizumab, and microsatellite stable (MSS) CRC show no response. We hypothesize that other costimulatory and coinhibitory receptor/ligand interactions play an important role in immune modulation by CRC.

Design: 113 cases of primary colorectal adenocarcinomas (91 MMR intact; 22 MMR deficient) were reviewed for a predetermined set of histological features. Immunohistochemical staining of PD1, PD-L1, TIM3 (CD366), and LAG3 (CD223) was performed on tissue sections. Manual morphometric analysis of extent and intensity of staining for each of the immune checkpoint proteins was scored in three compartments: invasive carcinoma, intratumoral inflammatory infiltrate, and inflammatory infiltrate at the advancing front. Patient demographics and outcome was obtained by chart review. Association of staining pattern with clinicopathologic features was assessed by Fisher Exact Test.

Results: Both MSS and MMR deficient (MMRd) CRC showed positivity ithin inflammatory infiltrate with all four markers. Relative to MSS CRC, MMRd CRC showed a significantly increased extent $(p<0.001)$ and intensity $(p<0.001)$ of carcinoma PD-L1 staining, and also with intensity of inflammatory PD1 staining within the tumor $(p<0.001)$ and at the advancing front $(p=0.14)$. MMRd CRC was also significantly associated with greater extent $(p=0.016)$ and intensity $(p=0.008)$ o intratumoral inflammatory PD-L1, and with increased staining extent $(p=0.001)$ and intensity $(p<0.001)$ within peritumoral inflammatory infiltrate at the advancing front. TIM3 and LAG3 were uniformly negative in carcinomatous epithelium, yet showed similar increased inflammatory staining both within the tumor and at the advancing front in MMRd CRC compared to MSS CRC.

Conclusions: In addition to the PD1 signaling axis, both MSS and MSI high colorectal carcinoma are associated with inflammatory infiltrates positive for two additional immune checkpoint proteins which may represent redundant mechanisms of immune regulation. Investigation of TIM3 and LAG3 as therapeutic targets for MSS CRC is warranted.

\section{International Consensus Criteria for Tumor Budding (ITBCC 2016) Predict Lymph Node Metastasis and Elastin-Detected Venous Invasion in Colorectal Cancer}

Kai Duan 1 , James Conner², Rossi Tomin ${ }^{3}$, Erin Kennedy ${ }^{3}$, Mantaj Brar $^{3}$, Siham Zerhoun ${ }^{3}$, Richard Kirsch ${ }^{3}$, Aysegul Sari'. ${ }^{1}$ University of Toronto, East York, ON, 'Mount Sinai Hospital, Toronto, ON, ${ }^{3}$ Mount Sinai Hospital, ${ }^{4}$ Mount Sinai Hospital, Toronto, Toronto, ON

Background: Tumor budding (TB) is an independent prognostic factor in colorectal cancer (CRC), with the potential to impact clinical management decisions. Recent international consensus on a standardized method for TB assessment and reporting (International Tumor Budding Consensus Conference [ITBCC], Bern, Switzerland, April 2016) has led to incorporation of TB into the most recent CAP CRC checklist. This study aimed to determine whether TB assessed by ITBCC criteria correlated with two major pathways of tumor dissemination, namely venous invasion (VI) and lymph node metastasis.

Design: 292 Stage I-IV CRC resections (without neoadjuvant therapy) from a single institution were included in this study. TB was assessed according to the ITBCC method. Briefly, H\&E slides were scanned at medium power to identify the 'hotspot' in which tumor bud counts were performed using a $20 x$ objective, and then normalized to a field area of $0.785 \mathrm{~mm}^{2}$. Cut-offs defined by ITBCC were used: Low (Bd1): 0-4 buds, intermediate (Bd2): $5-9$ buds, high (Bd3): $\geq 10$ buds. To facilitate detection of $\mathrm{VI}$, at least 5 tumor blocks were stained with elastin trichrome. The associations between TB and lymph node metastasis, elastin-detected VI (overall VI, extramural VI [EMVI] and number of foci of VI) and other prognostic factors were evaluated.

Results: The study group included 67 stage 1, 105 stage 2, 107 stage 3 , and 13 stage 4 CRC. The median number of tumor buds/hotspot was 8.3 (range 0-45). Overall, 86 (29.5\%) of cases were Bd1, $90(30.8 \%)$ Bd2 and $116(39.7 \%)$ Bd3. Elastin-detected VI was identified in 168 tumors $(58 \%)$ and EMVI in $105(36 \%)$. High-grade tumor budding (Bd3), as well as tumor bud count as a continuous variable were associated with VI EMVI, number of foci of VI, lymph node metastasis, pT, and TNM stage $(p<0.001$ for each variable). Bd3 was independently associated with VI EMVI, and lymph node metastasis in multivariate analysis $(p=0.004$, $\mathrm{p}=0.01$ and $\mathrm{p}<0.001$, respectively).

Conclusions: This is the first study to evaluate the relationship between high-grade TB (Bd3), based on ITBCC criteria, and venous invasion (VI), based on evaluation of elastin stains. Bd3 was independently associated with VI, EMVI and lymph node metastasis. Given the strong association between $\mathrm{Bd} 3$ and $\mathrm{VI}$, its presence should prompt careful evaluation for VI, ideally with elastin stains and particularly in stage II CRC where high-risk features may prompt consideration of adjuvant chemotherapy.

\section{The Impact of Tissue Sampling on the Detection of Venous Invasion in Colorectal Cancer: A Prospective Analysis}

Kai Duan1, Aysegul Sari², Brian Chou ${ }^{3}$, Colin Elliot ${ }^{3}$, Nadia Saito ${ }^{3}$ William Tsui ${ }^{4}$,Emily Almeida ${ }^{3}$, Richard Kirsch ${ }^{3}$, James Conner ${ }^{5}$ ${ }^{1}$ University of Toronto, East York, ON, ${ }^{2}$ Mount Sinai Hospital, Toronto, Toronto, ON, ${ }^{3}$ Mount Sinai Hospital, ${ }^{4}$ Mount Sinai Hospital, Toronto, Ontario, ${ }^{5}$ Mount Sinai Hospital, Toronto, ON

Background: Venous invasion (VI) is an independent predictor of hematogenous spread and mortality in colorectal cancer (CRC), ye remains widely under reported. There is growing evidence that elastin stains can substantially increase VI detection, and that elastin-detected $\mathrm{VI}$ is a superior predictor of outcome compared to that assessed by $\mathrm{H} \& \mathrm{E}$ alone. However, the number of tumor blocks required to optimize VI detection when using elastin stains remains to be determined. We sought to address this question in a prospective cohort of $79 \mathrm{CRC}$ resection specimens. 
Design: A standardized tumor mapping and sampling protocol was developed for this study and applied prospectively to all CRC specimens from a single institution. A minimum of 8 tumor-containing blocks, perpendicularly sectioned starting from the point of deepest invasion and then moving proximally and distally in a stepwise fashion, were submitted for microscopic examination. Tumors that were too small to yield 8 tissue blocks were submitted in their entirety. All sections of tumour were stained with both H\&E and elastin trichrome and assessed for $\mathrm{VI}$, including both intramural venous invasion (IMVI) and extramural venous invasion (EMVI).

Results: The 79 CRC resections (stage 1, $n=10$; stage 2, $n=20$; stage 3 $\mathrm{n}=26$; stage $4, \mathrm{n}=2$; post-neoadjuvant therapy cases, $\mathrm{n}=21$ ) included 26 right sided, 18 left sided and 35 rectal cancers. The mean tumor size was $4.9 \mathrm{~cm}$ (range $0.5-12.7 \mathrm{~cm}$ ). An average of 7.3 blocks were submitted from each specimen. VI was identified in 49 tumors $(62 \%)$, including 31 with EMVI (39\%) and 18 with IMVI only (23\%). The sensitivity of $\mathrm{VI}$ detection increased with the number of tumor-containing blocks submitted (59\% for 2 blocks, $67 \%$ for 3 blocks, $80 \%$ for 4 blocks, $88 \%$ for 5 blocks and $98 \%$ for 6 blocks). Similar increases in sensitivity were noted for EMVI detection (59\% for 2 blocks, $66 \%$ for 3 blocks, $78 \%$ for 4 blocks, $91 \%$ for 5 blocks and $99 \%$ for 6 blocks). Subgroup analyses of tumors $>5 \mathrm{~cm}$ and those receiving neoadjuvant therapy showed a similar increase in sensitivity of VI and EMVI detection with increasing numbers of tumor blocks.

Conclusions: Using a standardized tumor sampling protocol and routine elastin staining, the submission of 6 tumor-containing blocks identified VI and EMVI with a sensitivity of over $97 \%$. The sensitivity of $\mathrm{VI}$ and EMVI detection decreased as the number of tumor blocks decreased. These findings may help inform sampling practices for optimal detection of $\mathrm{VI}$ and $\mathrm{EMVI}$ in $\mathrm{CRC}$.

\section{Decreased Expression of Yes-Associated Protein (YAP) in Mismatch Repair Protein- Deficient (MMR-D) Colorectal Cancer}

Tony El Jabbour', Siddhartha Dalvi², Christine Sheehan ${ }^{3}$, Hwajeong Lee ${ }^{4} .{ }^{1}$ Albany Medical Center, Albany, NY, ${ }^{2}$ Delmar, NY, ${ }^{3}$ Albany Medical College, Albany, NY, ${ }^{4}$ Albany Medical College, Guilderland, NY

Background: YAP, a transcriptional coactivator normally suppressed by the Hippo pathway, is considered as an oncoprotein in a variety of malignancies and YAP overexpression is associated with poor prognosis in multiple solid tumors. Its role in colorectal cancer (CRC) is controversial and its association with MMR status is unknown.

Design: A total of 85 MMR-proficient (MMR-P; $n=53$ ) and MMR-D $(n=32)$ CRCs were retrieved and representative tumor blocks were immunostained using YAP (D8H1X) XP®) rabbit mAb (Cell Signaling). MMR status was determined by immunohistochemistry (MMR-D: any loss of nuclear MLH1, MSH2, MSH6 and PMS2 staining) in 83 and by PCR in 2 cases. Cytoplasmic (cYAP) and nuclear (nYAP) immunoreactivity was semiquantitatively assessed in the tumor and adjacent benign mucosa (present in 73 cases). Scoring was based on staining intensity [weak (1) to strong (3) ] and percentage of positive cells [0: no stain, $1:<=10 \%, 2: 11-50 \%, 3:>50 \%$ ]. A multiplicative index ( $\mathrm{Ml}$; maximum 9) was determined for benign epithelium and tumor. The indices of each case were then assessed as tumor=benign $(T=B)$, tumor $>$ benign $(T>B)$, tumor $<$ benign $(T<B)$, or negative $(N)$. Clinicopathologic parameters including TNM stage (7th AJCC) were reviewed and correlated with MI for CYAP and nYAP.

Results: There were 45 male and 40 female, with the median age of 66 (range 22-93) years. Excluding one biopsy case, there were 13 stage I, 31 stage II, 35 stage III and 5 stage IV. 49 tumors were located in the right and 36 were located in the left colon. Strong and diffuse $(\mathrm{Ml}=9)$ cYAP immunoreactivity was noted in $13 \%(11 / 85)$ of tumors, predominantly in the MMR-P group (MMR-P: $73 \%$, MMR-D: $27 \%$, $\mathrm{p}=0.008)$. When compared to the adjacent benign epithelium, $63 \%$ (46/73) were $T=B, 16 \%(12 / 73) T>B$ and $21 \%(15 / 73)$ were $T<B$. T $>B$ was more frequent in the MMR-P subgroup [MMR-P: $83 \%(10 / 12)$, MMR-D: 17\% (2/12)], and T < B was more frequent in MMR-D [MMR-P: $33 \%(5 / 15)$, MMR-D: $67 \%(10 / 15), p=0.017]$. No significant correlations between cYAP, nYAP, and other clinicopathologic parameters were found.

Conclusions: Decreased expression of cYAP significantly correlates with MMR-D status. This may be due to frequent alterations of the binding partner genes in the Hippo pathway in MMR-D CRC, and may account for overall better prognosis of MMR-D CRC. CYAP protein expression warrants further study as a biomarker with potential to predict high mutation burden and aid in screening patients who may benefit from immune checkpoint inhibitor therapies.

\section{Predictive value of CEA, NLR and intra-epithelial CD8+ T Iymphocytes in biopsy for tumor response to neoadjuvant chemoradiotherapy in rectal cancer}

Xinjuan Fan', Xiaoying Lou', Yaxi Zhu', Yan Huang'. 'Six Affiliated Hospital of Sun Yat-sen University, Guangzhou, Guangdong
(CRT) followed by surgery is the standard treatment for patient with rectal cancer. Here we analyzed the potential predictive factors determining pathologica response to CRT of rectal cancer.

Design: In our study, 156 rectal cancer patients treated with CRT were studied. The patients were classified into two cohort (good response VS poor response) according to the Dworak tumor regression grading system. Using immunohistiochemistry, the density of intra-epithelia and peri-tumor $\mathrm{CD} 4(+)$ and $\mathrm{CD} 8(+)$ T lymphocytes of biopsy samples were measured. Meantime, we investigated the predictive value of clinicopathological factors, including carcinoembryonic antigen (CEA), carbohydrate antigen 199 (CA199), C-reactive protein (CRP) and neutrophil to lymphocyte ratio (NLR).

Results: CEA, NLR, CA199, CRP, intra-epithelial and peri-tumor CD8+ T lymphocytes and peri-tumor CD4+ T lymphocytes were significantly associated with tumour regression $(P=0.031, P=0.129, P=0.028$, $P=0.019, P<0.05, P=0.025$, respectively). CEA, CA199 and CRP were upexpressed in the subset of poor response compared to the subgroup of good response. Whereas, the densities of intra-epithelial and peritumor CD8+ and peri-tumor CD4+ T lymphocytes were higher in the good response cohort. In multivariate analysis, CEA level, NLR, intraepithelial CD8 + T lymphocytes were independent predictive factors for good pathological response to CRT $(P=0.028, P=0.013, P<0.05$, respectively )

Conclusions: In rectal cancer, CEA, NLR combined with intraepithelial CD8+ T lymphocytes in the tumour microenvironment play essential role in tumor response to CRT and can be used as predictors of CRT.

\section{Eosinophilic Esophagitis Versus Proton Pump Inhibitor-Responsive Esophageal Eosinophilia: Comparison of Histology and T Cell Subpopulations}

Ayesha Farooq1, Christopher Hartley², Catherine Hagen ${ }^{3}$. ${ }^{1}$ Medical College of Wisconsin, Milwaukee, WI, ${ }^{2}$ Milwaukee, WI, ${ }^{3}$ Medical College of Wisconsin, Milwaukee, WI

Background: Eosinophilic esophagitis (EOE) and proton pump inhibitor-responsive esophageal eosinophilia (PPI-REE) show endoscopic and histopathologic overlap but respond to different treatment regimens. Histologically, both are characterized by presence of $\geq 15$ eosinophils(eo)/high power field (HPF) on esophageal biopsy, and no distinguishing histologic features are known. The aim of this study was to review biopsies of patients with EoE, PPI-REE and reflux esophagitis (RE) to determine if any distinguishing histologic features or T cell subpopulations can be identified.

Design: Biopsies with esophageal eosinophilia from 1/2016 to 6/2017 were retrieved from our archives. Cases of PPI-REE were defined as having $\geq 15$ eo/HPF on initial biopsy with improvement of eosinophilia $(<15 \mathrm{eo} / \mathrm{HPF}$ and at least $50 \%$ decrease in peak eo count) following a PPI trial. 22 consecutive cases of EOE were selected for comparison. EOE cases were defined as persistence of $\geq 15 e o / H P F$ after PPI trial. 21 consecutive cases of RE with $<15$ eo/HPF were also selected. H\&E stained slides were reviewed blindly for peak eo count, basal cell hyperplasia $(\mathrm{BCH})$, eo microabscess, and superficial layering of eos. Immunohistochemistry for CD3, CD4, CD8, and FoxP3 was performed to characterize $T$ cells. Clinical data was collected from chart review.

Results: 9 patients (M:F 1:3.5; mean age 39.2) with PPI-REE were included in the study. Mean age of the EOE group was 43.1 years (M:F 1.8:1) and 47.2 years (M:F 1.1:1) for RE. The most common presenting symptom for both the EOE and PPI-REE groups was dysphagia $(81.8 \%$ and $66.7 \%$ respectively). $14(63.6 \%)$ EOE patients and $3(33.3 \%)$ PPI-RE patients had esophageal rings on endoscopy $(p=0.23)$. Compared to PPI-REE, EOE cases had higher mean peak eo counts $(67.1$ vs. 30.9 $\mathrm{p}=0.01$ ), more prominent $\mathrm{BCH}$ (marked vs. mild; $86.4 \%$ vs. $33.3 \%$, $\mathrm{p}=0.007)$, more frequent microabscesses $(68.2 \%$ vs $0 \%, \mathrm{p}=0.0008)$ and superficial layering $(50 \%$ vs $0 \%, p=0.01)$. EOE cases had higher CD3 CD8, and FoxP3 lymphocyte counts compared to PPI-RE (Table 1). By ROC curve analysis, the optimal peak eo count to distinguish EOE from PPI-REE was 48/HPF (AUC 82\%) (figure 1).

Table 1 Mean and range of peak lymphocyte counts for all groups

\begin{tabular}{|l|l|l|l|l|}
\hline & EOE & PPI-REE & Reflux & P value \\
\hline CD 3+ & $127.1(76-204)$ & $90.0(62-135)$ & $71.7(17-136)$ & $<0.001$ \\
\hline CD 4+ & $43.9(15-93)$ & $40.3(13-81)$ & $24.4(4-82)$ & 0.06 \\
\hline CD 8+ & $78.7(12-149)$ & $36.7(22-66)$ & $45.4(3-169)$ & 0.001 \\
\hline FoxP3 & $29.7(15-52)$ & $13.7(7-21)$ & $15.4(3-51)$ & $<0.001$ \\
\hline
\end{tabular}




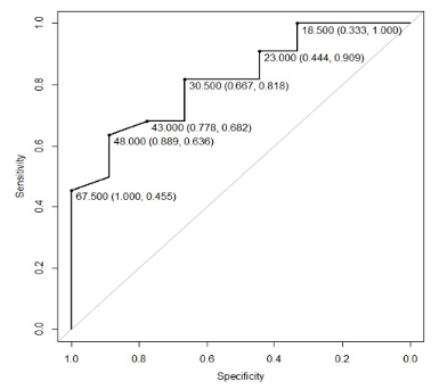

\begin{tabular}{|l|l|l|l|l|l|}
\hline & Esophagus & Ampulla & Duodenum & Stomach & Pancreas \\
\hline $\begin{array}{l}\text { Average Age } \\
\text { (range) }\end{array}$ & $65(45-81)$ & $65(57-73)$ & 77 & $78(59-93)$ & 59 (46-68) \\
\hline Outcome & $1 / 4$ DOD & $2 / 2$ Alive & $1 / 1$ Alive & $1 / 13$ DOD & $1 / 3$ DOD \\
\hline Grade (n) & $2-3(4)$ & $\begin{array}{l}1(1), \text { Med- } \\
\text { ullary (1) }\end{array}$ & $1(1)$ & $2-3(13)$ & $2(3)$ \\
\hline $\begin{array}{l}\text { Stage (n) } \\
\text { (2) }(1), y T 3\end{array}$ & $\begin{array}{l}\text { T1 (1), } \\
\text { T3 (1) }\end{array}$ & T1 (1) & $\begin{array}{l}\text { T1 (3), T2 (1), T3 } \\
(2), \text { T4(1), yT1 } \\
(1), y T 3(1)\end{array}$ & $\begin{array}{l}\text { T1 (1), } \\
\text { T3 (2) }\end{array}$ \\
\hline $\begin{array}{l}\text { N-stage } \\
\text { (N0:>N1) }\end{array}$ & $1: 2$ & $1: 1$ & N/A & $3: 5$ & $1: 2$ \\
\hline $\begin{array}{l}\text { Host in- } \\
\text { flammatory } \\
\text { response }\end{array}$ & $\begin{array}{l}\text { Minimal (1), } \\
\text { Moderate }\end{array}$ & High (2) & $\begin{array}{l}\text { Minimal } \\
(1)\end{array}$ & Moderate/ & $\begin{array}{l}\text { Minimal } \\
(1), \text { High } \\
(2)\end{array}$ \\
\hline
\end{tabular}

Conclusions: Eo microabscesses and superficial layering are features specific to EOE and can be used to distinguish EOE from PPIREE. A higher peak eo count (>48/HPF) has $89 \%$ specificity and $64 \%$ sensitivity for predicting failure of PPI therapy in cases of esophageal eosinophilia.

\section{PREVIOUSLY PUBLISHED}

\section{Clinicopathologic Features of Mismatch Repair Protein-Deficient Non-Colorectal Gastrointestinal, Esophageal and Pancreatic Adenocarcinomas}

Erna Forgo', Greg Charville², Teri Longacre3. 'Stanford University Medical Center, Stanford, CA, ${ }^{2}$ Stanford University School of Medicine, Stanford, CA, ${ }^{3}$ Stanford University, Stanford, CA

Background: Mismatch repair (MMR)-deficient colorectal adenocarcinomas (CRC) have distinct features that portend different prognosis and treatment options compared to MMR-intact CRC. We report the clinicopathologic characteristics of a series of non-CRC, MMR-deficient Gl carcinomas.

Design: The pathology database was searched for MMR-deficient, non-CRC Gl carcinomas from 2012-2017. All cases were reviewed and the following parameters recorded: age, gender, histologic type, grade (G1-3), tumor site, TNM stage, lymphovascular invasion (LVI), large venous invasion, average mitotic count/10HPF (MC), tumor necrosis, tumor budding (according to ITBCC 2016 Consensus), intestinal metaplasia (IM), HER2 expression (where applicable), host inflammatory response, MMR protein expression by IHC, promotor methylation, cancer history and outcome. MMR-deficient gastric adenocarcinomas were compared with 36 consecutive MMR-proficient gastric adenocarcinomas using Fisher's exact test and 2-tailed t-test ( $\mathrm{p}<0.05$ considered significant).

Results: Of 23 MMR-deficient non-CRC tumors, 4 were esophageal, 2 ampullary, 1 duodenal, 13 gastric and 3 pancreatic (Table 1). 4/23 cases showed loss of MSH2 and MSH6 (1 gastric, 3 pancreatic), 2 with confirmed Lynch syndrome. 19/23 showed loss of MLH1 and PMS2 with 10 cases ( 2 ampullary, 8 gastric) having MLH1 promoter hypermethylation. Skewed M:F ratios were seen in esophagus, ampulla, and stomach tumors. All esophageal tumors were G2-3 with low tumor budding. Ampullary tumors had MC of 15 and inverse tumor budding to stage. The duodenal tumor showed high tumor budding, MC of 8 and no tumor necrosis or host response. Gastric tumors were all $\mathrm{G} 2-3$ with varying mitotic activity $(M C=14)$. Tumor budding and host response did not correlate with stage. Pancreatic tumors were all $\mathrm{G} 2$ with consistently low mitotic activity $(\mathrm{MC}=4)$ and high tumor budding correlating with high stage. Overall, LVI correlated with LN metastasis. No significant differences were found between MMRdeficient and MMR-proficient gastric adenocarcinomas ( $p>0.05)$.
Conclusions: MMR-deficient non-CRC GI adenocarcinomas exhibit a spectrum of clinicopathologic features, but are generally high grade and high stage at resection. Most tumors show loss of MLH1 and PMS2 expression secondary to $M L H 1$ promoter hypermethylation. An association with Lynch syndrome may be present ( $10 \%$ in this series). Further characterization of these rare cancers will provide insight into their biology and may aid in diagnosis and treatment.

\section{The Correlation and Upregulation of PD-L1 and CMTM6 in EB virus-associated gastric cancer}

Yao Fu' ${ }^{1}$, Hongyan Wu', Qianyun Shi', XiangShan Fan' ${ }^{1}{ }^{1}$ Affiliated Drum Tower Hospital Nanjing University Medical School, Nanjing, JiangSu

Background: EBV-associated gastric cancer (EBVaGC) is defined as a distinct gastric adenocarcinoma subtype with EBV infection and always over-express programmed death ligand-1 (PD-L1). CKLF-like MARVEL transmembrane domain containing protein6 (CMTM6) is a ubiquitously expressed protein that binds PD-L1 and maintains its cell surface expression. In this study, we examined the expression of PDL1 and CMTM6 in EBVaGC and analyzed the association between the expression status of these markers and the prognosis of patients.

Design: The expressions of PD-L1 (OR-5E3, Roche) and CMTM6 (ab198284, Abcam) were detected by immunohistochemistry in 45 cases of gastric carcinoma Anchor25 cases of EBV-positive GC, 9 MLH1-negative GC, 11 EBV-negative and MLH1-positive GC. The immunoreactivity of PD-L1 at cytomembrane in tumor cells and cytoplasm in stromal cells, and CMTM6 at cytoplasm/cytomembrane in tumor cells and cytoplasm in stromal cells was regarded as positive. Four score levels ( $P$-scores) were classified according to the proportion of PD-L1 and CMTM6 positive cancer cells and stroma cells as follows: score 0 , no stained cancer cells; score $1, \leq 5 \%$ of positive tumor cells; score 2, $5 \%$ of positive tumor cells; and score 3,50\% of positive tumor cells. $\mathrm{P}$-scores of 0 and 1 were regarded as negative, whereas P-scores of 2 and 3 were regarded as positive. The PD-L1 mRNA in situ hybridization (ISH) was performed with RNAscope assay according to the manufacturer's protocols. The correlation of PD-L1 and CMTM6 expression with the clinicopathologic features of gastric carcinoma was analyzed by SPSS version 20.0

Results: Expression of PD-L1 and CMTM6 was frequently detected in cancer cells of EBVaGC (15/25, 60\%; 16/25, 64\%), with infiltration of PD-L1 and CMTM6-positive immune cells in tumor stroma $(17 / 25,68 \% ; 23 / 25,92 \%)$. Moreover, there was a positive correlation of the expression status between PD-L1 and CMTM6 in EBVaGC (Kappa=0.644, P 0.01). In univariate analysis, PD-L1 expression in EBVaGC tumor cells and CMTM6 expression in EBVaGC immune cells were correlated with poor outcomes in overall survival $(P=0.021$ and 0.004 , respectively).

The expression of PD-L1 and CMTM6 in gastric adenocarcinoma

\begin{tabular}{|c|c|c|c|c|c|}
\hline & & EBVaGC & MLH1-negative & $\begin{array}{l}\text { EBV-Neg and } \\
\text { MLH1-Pos }\end{array}$ & $\mathrm{P}$ \\
\hline \multirow{2}{*}{$\begin{array}{l}\text { PD-L1 } \\
\text { in cancer cells }\end{array}$} & Positive & 15 & 4 & 1 & \multirow{2}{*}{0.018} \\
\hline & Negative & 10 & 5 & 10 & \\
\hline \multirow{2}{*}{$\begin{array}{l}\text { PD-L1 } \\
\text { in immune cells }\end{array}$} & Positive & 17 & 6 & 3 & \multirow{2}{*}{0.062} \\
\hline & Negative & 8 & 3 & 8 & \\
\hline \multirow[t]{2}{*}{ СMTM6 } & Positive & 16 & 4 & 2 & \multirow{2}{*}{0.039} \\
\hline & Negative & 9 & 5 & 9 & \\
\hline \multirow[t]{2}{*}{ СMTM6 } & Positive & 23 & 6 & 3 & \multirow{2}{*}{0.000} \\
\hline & Negative & 2 & 3 & 8 & \\
\hline
\end{tabular}


Relationships between PD-L1 and CMTM6 expression in cancer cells and immune cells

\begin{tabular}{|c|c|c|c|c|c|c|c|c|c|}
\hline & & \multicolumn{2}{|c|}{$\begin{array}{l}\text { PD-L1 in } \\
\text { cancer cells }\end{array}$} & \multirow{2}{*}{$\begin{array}{l}\text { Kap- } \\
\text { pa }\end{array}$} & \multirow{2}{*}{$P$} & \multicolumn{2}{|c|}{$\begin{array}{l}\text { PD-L1 in im- } \\
\text { mune cells }\end{array}$} & \multirow{2}{*}{ Kappa } & \multirow{2}{*}{$\mathrm{P}$} \\
\hline & & $\begin{array}{l}\text { Posi- } \\
\text { tive }\end{array}$ & $\begin{array}{l}\text { Neg- } \\
\text { ative }\end{array}$ & & & $\begin{array}{l}\text { Posi- } \\
\text { tive }\end{array}$ & $\begin{array}{l}\text { Nega- } \\
\text { tive }\end{array}$ & & \\
\hline \multirow{2}{*}{$\begin{array}{l}\text { CMTM6 } \\
\text { in cancer cells }\end{array}$} & $\begin{array}{l}\text { Posi- } \\
\text { tive }\end{array}$ & 17 & 5 & \multirow{2}{*}{0.644} & \multirow{2}{*}{0.000} & 15 & 7 & \multirow{2}{*}{0.203} & \multirow{2}{*}{0.167} \\
\hline & $\begin{array}{l}\text { Nega- } \\
\text { tive }\end{array}$ & 3 & 20 & & & 11 & 12 & & \\
\hline \multirow{2}{*}{$\begin{array}{l}\text { CMTM6 } \\
\text { in immune } \\
\text { cells }\end{array}$} & $\begin{array}{l}\text { Posi- } \\
\text { tive }\end{array}$ & 18 & 14 & \multirow{2}{*}{0.321} & \multirow{2}{*}{0.000} & 22 & 10 & \multirow{2}{*}{0.334} & \multirow{2}{*}{0.019} \\
\hline & $\begin{array}{l}\text { Nega- } \\
\text { tive }\end{array}$ & 2 & 11 & & & 4 & 9 & & \\
\hline
\end{tabular}
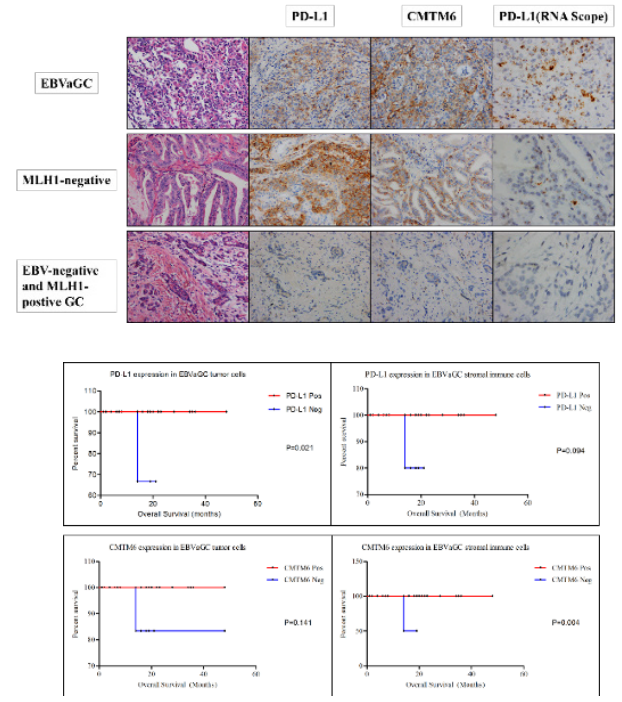

Conclusions: The decreased expression of PD-L1 in EBVaGC tumor cells and CMTM6 in EBVaGC immune cells might contribute to the poor prognosis of EBVaGC may be related to immune evasion. Furthermore, our findings suggest that targeting the PD-L1 and CMTM6 signaling pathways may be a new strategy for cancer immune therapy and targeted therapy for EBVaGC.

\section{PD-L1 expression in EBV-negative gastric adenocarcinoma and clinicopathological analysis}

Yao Fu', Qianyun Shi', Hongyan Wu', XiangShan Fan'. 'Affiliated Drum Tower Hospital Nanjing University Medical School, Nanjing, JiangSu

Background: Programmed death ligand-1(PD-L1), a member of B7 family, is expressed in T cells, B cells, dendritic cells, macrophagocytes, vascular endothelial cells and islet cells. Overexpression of PD-L1 and programmed death-1(PD-1) in tumor cells have proved to play a predominant role by inhibiting the function of $T$ cells in cancer immune surveillance. Our research was to evaluate the prognostic value of PD-L1 and the association between PD-L1 expression and clinicopathological characteristics in gastric cancer.

Design: PD-L1(SP142) expression was carried out in 202 EBV-negative gastric adenocarcinoma by immunohistochemistry. The expressions of tumor cells and stromal cells were evaluated with a cutoff of $1 \%$. The staining pattern of tumor cells was cytomembrane, whereas staining pattern of stromal cells was cytoplasm/cytomembrane. The relationship between PD-L1 expression and clinicopathologic characteristics or prognosis was analyzed via x2-tests, Kaplan-Meier method and Cox regression model.

FOR TABLE DATA, SEE PAGE 317, FIG. 727

Results: Of 202 gastric adenocarcinoma specimens, the median age of the patients at diagnosis was 62.4 years old. $13.86 \%$ had high PD-L1 expression in tumor cells and $42.08 \%$ was positive in stromal cells. There was no relationship between tumor cells PDL1 expression and gender, age, pTNM stage, Lauren classification and histological subtypes. In stromal cells, PD-L1 expression was associated with Lauren classification and histological subtypes, which was more common in mixed subtype and papillary adenocarcinoma ( $P$ value $=0.009$ and 0.005 , respectively). Moreover, distinct PL-L1 expression in stromal cells of infiltrating edge was discovered in $50 \%$ papillary adenocarcinoma. In univariate analysis, there was no significant difference in prognosis between tumor and stromal cells PD-L1 expression ( $P$ value $=0.858$ and 0.198 , respectively). However, poorer tumor differentiation, bigger size of the tumor, advanced pTNM stage, cancer embolus and nerve invasion had significant adverse impact on survival ( $P$ value 0.05 ). Multivariate analyses revealed that only cancer embolus was the independent prognostic factors for the patients with EBV-negative gastric adenocarcinoma.

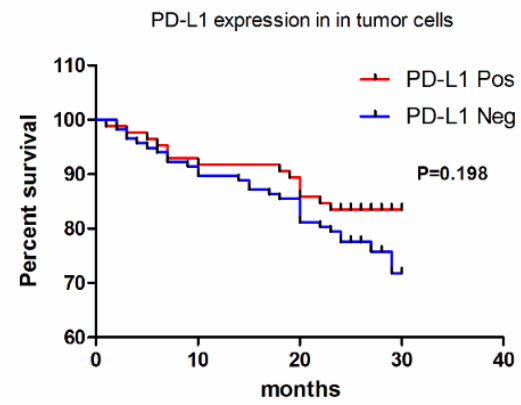

Conclusions: In gastric adenocarcinoma, PD-L1 expression in the stromal cells was associated with histologic subtypes. Neither tumor cells nor stromal cells PD-L1 expression had effect on prognosis in our research, which might attribute to the follow-up and the treatment. Future studies are needed to investigate the value of PD-L1 in gastric adenocarcinoma.

728 Frequency of HER2 overexpression in hepatoid adenocarcinoma and gastric carcinoma with enteroblastic differentiation: analysis of $\mathbf{3 5}$ cases compared to 334 gastric carcinomas of other histological types

Masakazu Fujimoto 1 , Ibu Matsuzaki', Masaru Nishino', Yoshifumi Iwahashi', Kenji Warigaya', Fumiyoshi Kojima', Shin-ichi Murata'. 'Wakayama Medical School, Human Pathology, Wakayama

Background: Alpha-fetoprotein (AFP)-producing gastric carcinoma (AFPGC) is one of the most aggressive subtypes of gastric carcinoma (GC). Frequent expression of HER2 has been reported previously in hepatoid adenocarcinoma (HAC), a major histological subtype of AFPGC originating from intestinal type GC. However, the HER2 expression levels in other histological types of AFPGC are unknown. In this study, we analyzed HER2 expression in other types of GC with a primitive phenotype in addition to HAC.

Design: HER2 test was performed on a representative complete section from each of $16 \mathrm{HACs}$ (Figure 1A), 19 gastric carcinomas with enteroblastic differentiation (GCEDs) (Figure 1B), and 334 GCs of other histological types as controls. The Ruschoff/Hofmann method was used to evaluate HER2 immunohistochemistry. Cases with a HER2 score 2+ were further assessed using FISH. Oncofetal protein (OFP) expression in HAC and GCED was confirmed through immunohistochemical staining of AFP, GPC3, and SALL4.

Results: In total, 13 out of $35(37.1 \%)$ cases of HAC/GCED were determined HER2 positive. Regarding AFP positivity, 11 out of 30 (36.7\%) AFP-positive cases of HAC/GCED were HER2-positive. Among 35 cases of HAC/GCED, 30 cases comprised more than 2 histological patterns. In total, 16 HAC, 26 GCED, and 27 common type GC (CGC) components were evaluated. The CGC components in HAC/GCED also commonly expressed OFP; the positivity rates were $40.7 \%$ for AFP $51.9 \%$ for GPC3, and $88.9 \%$ for SALL4. The HER2 positivity rate of each histological component in HAC/GCED was $25.0 \%$ for HAC (Figure 1C), $34.6 \%$ for GCED (Figure 1D), and $48.1 \%$ for CGC. The results of HER2 test and immunohistochemistry for OFP are summarized in Figure 2. The HER2 positivity rates of HAC, GCED, and CGC components were higher than those of the entire control group $(13.8 \%)$ as well as the intestinal type GC within the control group (24.8\%).

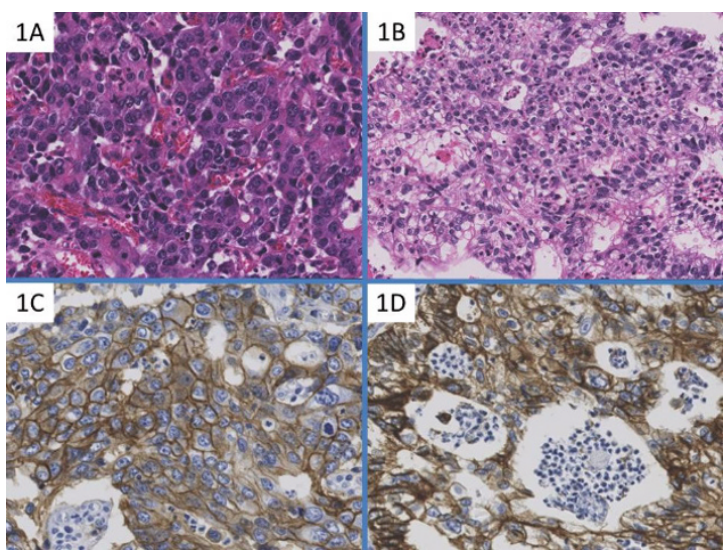




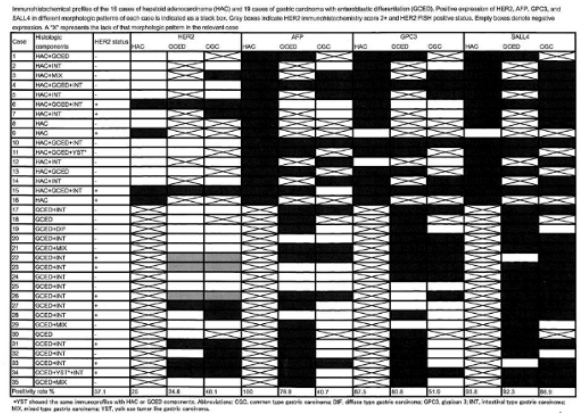

Conclusions: HER2 is frequently overexpressed in GCs with a primitive phenotype, which indicates an association between HER2 and OFP. Since the CGC component of HAC/GCED had higher rates of HER2 overexpression than the control CGC $(p<0.05)$, our findings also suggest that HER2-positive CGC has higher risk for progression to HAC/GCED than HER2-negative GCs.

\section{Pathologic Features Associated with Colonic Intraluminal Metallic Stents}

Clifton G Fulmer', Rhonda Yantiss ${ }^{2}$, Jose Jessurun' ${ }^{2}$ ' ${ }^{1}$ New York Presbyterian Hospital-Weill Cornell Medicine, New York, NY, ${ }^{2}$ Weill Cornell Medical College, New York, NY

Background: Self-expanding metal stents (SEMS) were initially used as a palliative procedure in patients with unresectable colon cancer, but may also be used in decompensated patients who present with acute obstruction, allowing for medical optimization prior to the surgical procedure. SEM placement induces changes in the colonic mucosa that have not been well characterized and which may be misinterpreted at the time of endoscopic examination. This study examines the endoscopic, gross and histological features of the colonic mucosa following stent placement.

Design: From 2009 to 2017, there were 21 surgical resections of the colon or rectum where a SEM was placed prior to the procedure. The clinical information and endoscopic reports were reviewed. Gross descriptions and photos from the pathology report were analyzed and histologic slides of the resection specimen and mucosal biopsies taken from stented colonic mucosa were reviewed.

Results: The patient population consisted of 12 men and 9 women with an average age of 67 years (range 39-90 years) who presented with colonic obstruction. On endoscopy, the stricture was attributed to a malignant tumor in $15(83 \%)$, to an unknown cause in $1(6 \%)$ and to benign processes in $2(11 \%)$ cases. The mean duration from stent placement to surgical resection was 26 days (range 2-150 days). At resection, colorectal tumors were identified in (17) $81 \%$ cases, while diverticular disease accounted for $3(14 \%)$ cases and an anastomotic stricture for 1 case $(5 \%)$. Stented areas exhibited segmental pseudopolyposis (Fig A and B) in all cases and gross perforation in $3(14 \%)$. Stent associated tumor necrosis was seen in $11 / 13(85 \%)$ neoplastic cases. A distinctive type of basophilic necrosis was seen in $14(88 \%)$ cases (Fig C, arrows) as were pseudomembranes $(63 \%)$ (Fig D) and submucosal eosinophil rich inflammation (50\%). Similar findings were present in the reviewed biopsies.

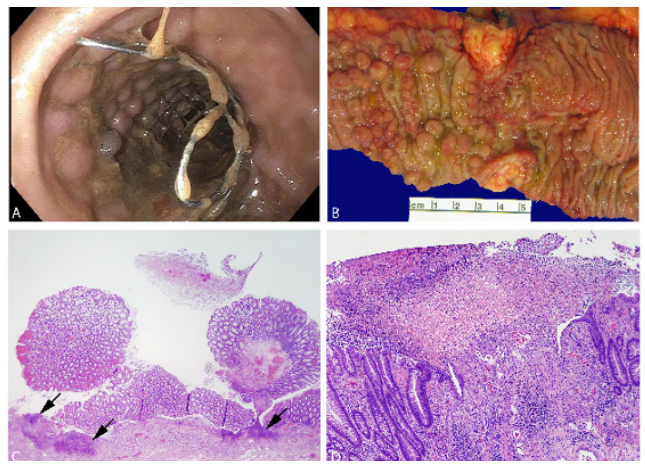

Conclusions: SEM placement is associated with characteristic endoscopic, gross, and histopathologic findings. Segmental pseudopolyposis is a pathognomonic finding on endoscopic and gross examination. Other common findings include basophilic necrosis, pseudomembranes and eosinophil-rich infiltrates. The SEM related changes mimic preneoplastic conditions such as polyposis, and infectious colitis like peudomembranous colitis. Familiarity with these changes is important to prevent misdiagnosis of potentially confusing inflammatory patterns in mucosal biopsy specimen taken prior to surgical resections.<
730 Histological Features of Graft Versus Host Disease in Colonic Biopsies of Bone Marrow Recipients: IBD-like Changes and Role of Mycophelonate Mofetil

Caterina Fumagalli', Maria del Valle Rodríguez Santás, Ignasi Gich ${ }^{2}$ Albert EsquiroR, Silvana Novelli ${ }^{2}$, Ana Mozos Rocafort ${ }^{3}$, Justyna Szfranska4. ${ }^{1} \mathrm{H}$ ospital de la Santa Creu i Sant Pau, Barcelona, ${ }^{2} \mathrm{Hospita}$ de la Santa Creu i Sant Pau, ${ }^{3}$ Hospital Sant Pau, Barcelona, Spain, ${ }^{4}$ Hospital Santa Creu i Sant Pau, Barcelona

Background: Mycophelonate mofetil (MMF) is an immunosuppressive agent used in bone marrow (BM) and solid organ transplant fo preventing allograft rejection. One of the collateral effects is the development of diarrhea, which could clinically and histologically mimic the appearance of graft-versus-host disease (GVHD) Furthermore, IBD-like, infectious-like and ischemic-like changes could coexist in patients (pts) with GVHD, making the diagnosis challenging. The aim of the study is to analyze the histological features of colonic biopsies in BM recipients with GVHD treated with immunosuppressive therapy with or without the addition of MMF

Design: We evaluated 192 colonic biopsies of 107pts (35 females, 72 males) with BM transplant, diagnosed of GVHD between 1992 and 2017. Fifty-four patients were treated with the usual immunosuppressive therapy and MMF (Group 1), while 53 patients did not receive MMF (Group 2). All biopsies were evaluated by two independent reviewers checking the presence of apoptosis, type of stromal and crypt inflammatory infiltrate, crypt distortion, mucin depletion, presence of granulomas, ulcers, Paneth cell metaplasia and aggregates of neuroendocrine cells.

Results: In our study, typical GVHD, IBD-like, infectious-like and ischemic-like patterns were found in both groups, as seen in table 1 Cytomegalovirus (CMV) infection was present in 13 pts, but GVHD histology persisted after directed therapy. In each group, one patient with histological findings simulating IBD was present, consisted of chronic active colitis with plasma cell-rich expansion of the lamina propria and basal lymphoplasmacytosis with granulomas' formation. Both patients had a poor outcome and died. Statistical analysis revealed a more severe inflammation with numerous eosinophils (>15/10hpf) and presence of hypereosinophilic (degenerative) crypts in group 1 (with MMF) compared to group 2 (without MMF) ( $<<0.05$ ).

\begin{tabular}{|l|l|l|l|l|}
\hline & Typical GVHD & $\begin{array}{l}\text { Ischemic-like } \\
\text { pattern }\end{array}$ & $\begin{array}{l}\text { Infectious -like } \\
\text { pattern }\end{array}$ & IBD-like pattern \\
\hline $\begin{array}{l}\text { Group 1 (with } \\
\text { MMF) }\end{array}$ & 44 & 1 & 8 & 1 \\
\hline $\begin{array}{l}\text { Group 2 (with- } \\
\text { out MMF) }\end{array}$ & 46 & 1 & 5 & 1 \\
\hline
\end{tabular}

Conclusions: Histologically, there's overlapping between MMFinduced colitis and GVHD. Our study showed microscopic features, suggesting MMF-related changes, consistent of neutrophilic infiltrates with prominent eosinophils present in lamina propria and degenerative crypts. Furthermore, IBD-like pattern was present in 2 cases raising the clinical suspicion of de-novo IBD appearance in immunosuppressed pts. Clinical, endoscopic and microscopic findings are required to distinguish between GVHD patterns, MMFrelated changes and de-novo IBD in transplant recipients.

\section{Cessation of Reflex Immunohistochemical Staining Does Not Lead to a Significant Decrease in Helicobacter pylori Detection Rate}

Maira Gaffar', Zhongbo Jin', Jennifer R Reppucci', Gregory Olsen ${ }^{1}$ Danielle Harrel/2, Ashwini Esnakula', David Hernandez Gonzalo ${ }^{3}$, Jesse Kresak', Xiuli Liu', Michael Feely'. 'University of Florida, Gainesville, $\mathrm{FL},{ }^{2}$ University of Florida, ${ }^{3}$ University of Florida-Shands, Gainesville, $\mathrm{FL}$

Background: While Helicobacter pylori (HP) infections can often be detected on routine H\&E stain, upfront ancillary testing using histochemical or immunohistochemical methods (IHC) is a common practice at many institutions. This procedure has been recently scrutinized and current studies regarding this topic have reported contradictory results. In this study, we examined our own experience in HP detection rates following termination of reflex IHC at our institution. These rates were further assessed in context of any associated mucosal disease.

Design: Gastric biopsy reports which were collected during the 6 months preceding (reflex period) and 6 months immediately following the cessation of upfront HP IHC staining (selective period) were reviewed. Cases limited to targeted biopsies of masses or polyps were excluded. Data regarding the presence of HP, the use of ancillary IHC and concurrent clinical and histologic findings, including the existence of mucosal disease, were recorded.

Results: Over the entire 12 month interval, a total of 2451 gastric biopsy reports were reviewed. During the reflex period, $99.2 \%$ $(1248 / 1258)$ of cases had HP IHC while $55.8 \%(666 / 1193)$ of cases from the selective period had IHC performed. HP was detected in $8.9 \%(112$ 
of reflex cases and $7.9 \%$ (94) of selective cases, a difference which was not statistically significant $(P=0.3825)$. In terms of the presence of mucosal disease, $57.5 \%(100 / 174)$ of reflex cases with active gastritis had HP while $52.3 \%(81 / 155)$ of selective cases with this finding had HP detected. In cases interpreted as mild chronic inactive gastritis, HP was noted in $1.2 \%(4 / 336)$ of reflex cases and $1.2 \%(4 / 333)$ of selective cases. Of these 8 occurrences of HP, 5 were in pediatric patients. In cases of reactive gastropathy, no instances of HP were reported in either the reflex $(n=323)$ or selective periods $(n=313)$. Despite the minimal occurrences of HP in cases diagnosed as mild chronic inactive gastritis or reactive gastropathy, HP IHC was performed on $90.7 \%(302 / 333)$ of chronic inactive gastritis cases and 50.2\% (157/313) of cases with reactive gastropathy following the cessation of upfront HP IHC.

Conclusions: The detection rate of HP is not significantly affected by the elimination of reflex IHC. Furthermore, the selective use of HP IHC in the setting of biopsies interpreted as mild chronic inactive gastritis or reactive gastropathy is largely unwarranted, perhaps with the exception of pediatric cases.

\section{Primary Gastrointestinal Liposarcoma - A Case Series of a Rare Entity}

David C Gajzer ${ }^{1}$, Mahsa Khanlari², Christopher D Fletcher ${ }^{3}$, Andrew Rosenberg. ${ }^{1}$ University of Miami - Miller School of Medicine/Jackson Memorial Hospital, Miami, FL, ${ }^{2}$ Jackson Memorial Hospital, Miami, FL, ${ }^{3}$ Brigham and Women's Hospital, Boston, MA, ${ }^{4}$ University of Miami Miller School of Medicine, Miami, FL

Background: Liposarcoma is the most common malignant soft tissue tumor in adults and usually occurs in the retroperitoneum, deep soft tissues of the trunk, and lower extremities. Primary gastrointestinal liposarcoma (GIL), however, is very rare. We present five cases of primary GIL and discuss the clinicopathological findings.

Design: Surgical pathology files of the consultations of 2 authors and the Jackson Memorial Hospital were searched for GIL between the years 2008-2014. Five cases were identified and form the study group. Histological sections, immunohistochemical stains, surgical pathology reports and clinical information were reviewed.

Results: The patients were aged from 51-81, median 58 years, and included 4 males and 1 female. The tumors arose in the stomach (1), small bowel (3) and colon (1) and often led to intestinal obstruction. The tumors ranged in size from $6-10 \mathrm{~cm}$ and originated in the submucosa or muscularis and frequently protruded into the bowel lumen resulting in mucosal ulceration. Histologically, 2 tumors were high grade dedifferentiated liposarcoma (DDLPS), 1 was intermediate grade DDLPS and 2 tumors were well differentiated liposarcoma (WDLPS). The DDLPS were composed of cytologically malignant pleomorphic, mitotically active spindle cells. Immunohistochemistry for MDM2 and CDK4 was positive for each tumor. The two WDLPS were the sclerosing and adipocytic subtypes and each contained abundant mature appearing adipocytes with scattered cells having enlarged hyperchromatic nuclei. The tumors were treated by surgical resection. Follow-up shows that one high grade DDLPS recurred and one possibly metastasized.

Conclusions: Primary GIL is rare. The tumors are usually large at the time of diagnosis and cause bowel obstruction. In our series, $60 \%$ of tumors were dedifferentiated and tended to infiltrate the full thickness of the bowel wall. Based on morphology, the differential diagnosis included malignant gastrointestinal stromal tumor, leiomyosarcoma, undifferentiated pleomorphic sarcoma and malignant peripheral nerve sheath tumor. Identification of the well differentiated component and immunohistochemistry are invaluable for accurate diagnosis. These tumors are biologically aggressive and should be excised with widely negative margins, if possible. WDLPS of the bowel is often submucosal and can be distinguished from lipoma by the presence of large atypical cells and further confirmed with immunohistochemistry and cytogenetic studies, if necessary. Complete excision is curative.

\section{Tumour Budding and Poorly Differentiated Clusters are Stage-Independent Prognostic Markers in Mismatch Repair Deficient Colorectal Cancer}

Robert Geraghty', Yi Ling Khaw², Eanna Ryan³, Ben Creavin', David Gibbons ${ }^{4}$, Elizabeth Ryan ${ }^{5}$, Ronan O'Connel/ ${ }^{6}$, Sean Martin ${ }^{6}$, Des Winter $^{3}$, Kieran Sheahan ${ }^{7}$. 'Dublin, ${ }^{2}$ Dublin, Ireland, ${ }^{3}$ St. Vincent's University Hospital, ${ }^{4}$ St. Lukes Hospital, Dublin, Ireland, ${ }^{5}$ Centre For Colorectal Disease, Dublin, Ireland, ${ }^{6} 2$. Centre for Colorectal Disease, St. Vincent's University Hospital, Elm Park, Dublin, Ireland, ${ }^{7}$ St. Vincent Hospital, Dublin

Background: Tumour budding (TB) and poorly differentiated clusters (PDCs) in colorectal adenocarcinoma (CRC) may predict metastatic potential. TB and PDC assessment may be more reproducible than the evaluation of other histological parameters. This may be useful in CRCs with microsatellite instability (MSI), due to the association with poor histological grade yet improved prognosis compared to microsatellite stable CRC. The aim of this study was to correlate nventional adverse pathological features including WHO grade, TB and PDC grade in MSI CRC with the risk of lymph node metastasis (LNM) and long-term outcome.

Design: We investigated the predictive value of WHO grade, TB, PDC grade and other histological parameters on the presence of LNM and clinical outcome in $238 \mathrm{MSI}$ CRCs. MSI status was determined by immunohistochemistry for MLH1, PMS2, MSH2 \& MSH6.

Results: TB and PDC were highly correlated (Pearson correlation $0.734 ; \mathrm{p}<0.000)$. Both TB and PDC grade in MSI CRC were significantly associated with WHO grade, peri-neural, lympho-vascular and extramural vascular invasion, and nodal metastases. Independent predictors of LNM were PDC grade (HR; 4.12;95\% Cl; $1.69-10.04$ $\mathrm{p}=0.011$ ) and $\mathrm{EMVI}$ (HR; 3.81, 95\% Cl; $1.56-9.19, \mathrm{p}<0.000$ ). TB $(p=0.003)$ and PDC grade $(p=0.012)$ but not conventional grading significantly correlated with recurrence. Only TB (HR; 2.99, 95\% C $1.72-5.19, p<0.000)$ \& T stage (HR; 4.11; 95\% Cl; $1.48-11.36 ; p=0.007$ ) were associated with a shorter DFS in multivariable analysis. If TB was excluded from the model PDC grade became significant for DFS (HR; $2.34,95 \% \mathrm{Cl} ; 1.34-4.09, \mathrm{p}=0.003)$.

Conclusions: WHO Grade is not correlated with clinical outcome in MSI CRC. Tumour budding and tumour stage are the most powerfu independent predictors of poor clinical outcome. If TB cannot be assessed (i.e. when inflammation obscures small clusters of cancer cells) PDC grade may be used as a prognostic surrogate.

\section{CDX2 is Not of Prognostic Significance In Mismatch Repair Deficient Colorectal Cancer}

Robert Geraghty', Eanna Ryan², Ben Creavin², Yi Ling Khaw, David Gibbons, Elizabeth Ryan ${ }^{5}$, Ronan O'Connell ${ }^{4}$, Sean Martin ${ }^{6}$, Rory Kennelly ${ }^{6}$, Des Winter ${ }^{2}$,Kieran Sheahan ${ }^{7}$. ${ }^{1}$ Dublin, ${ }^{2}$ St. Vincent's University Hospital, ${ }^{3}$ Dublin, Ireland, ${ }^{4}$ St. Lukes Hospital, Dublin, Ireland, ${ }^{5}$ Centre For Colorectal Disease, Dublin, Ireland, ${ }^{6}$ Centre for Colorectal Disease, St. Vincent's University Hospital, Elm Park, Dublin Ireland, ${ }^{7}$ St. Vincent Hospital, Dublin

Background: Caudal-related homeobox transcription factor 2 (CDX2) is an intestine-specific transcription factor. It is implicated in tumour differentiation, proliferation, cell-adhesion and migration, and has been shown to be of prognostic significance in colorectal cancer (CRC). Here we sought to investigate the counterintuitive association of mismatch repair deficient (dMMR) CRC, with its reported good prognosis and resistance to 5-fluorouracil based (5-FU) adjuvant chemotherapy with CDX2-negative CRC, a phenotype conferring a worse prognosis and a benefit from adjuvant 5-FU.

Design: We retrospectively investigated the prognostic and predictive value of CDX2 expression and other histological parameters on the presence of lymph node metastasis (LNM) and clinical outcome in a cohort of 238 dMMR CRCs. CDX2 status was determined via immunohistochemistry (IHC) using the Leica Bond CDX2 (clone EP25) ready to use primary antibody. MMR status was determined by IHC for the mismatch repair proteins hMLH1, hMSH2, hMSH6 and hPMS2.

Results: Assessment of CDX2 expression demonstrated excellent interobserver reliability $(\mathrm{k}=0.863 ; \mathrm{P}<0.000)$. CDX2-negativity was seen in $15.7 \% \quad(n=37)$ of cases. CDX2-negativity was associated with gender, size, stage, grade, poorly differentiated cluster (PDC) grade, mucinous type, peri-neural and lympho-vascular invasion, and pNstage ( $\mathrm{P} \leq 0.038$ ). Independent predictors of LNM were PDC grade (Odds ratio [OR] 4.116; 95\% confidence interval [CI]: $1.76-9.63$ $\mathrm{P}=0.001$ ) and extramural venous invasion (OR $3.79 ; 95 \% \mathrm{Cl}: 1.62$ 8.85; $\mathrm{P}=0.002$ ). Budding (Hazard ratio [HR] 2.79; $95 \% \mathrm{Cl}: 2.60-4.87$ $\mathrm{P}<0.000$ ), pTstage (HR 3.586; 95\% Cl: $1.29-10.01 ; \mathrm{P}=0.015$ ) and adjuvant chemotherapy (HR 2.07; 95\% Cl: $1.15-3.74 ; \mathrm{P}=0.016$ ) were independently associated with worse DFS.

Conclusions: CDX2 does not confer a negative prognosis in dMMR CRC. The MMR status of CRC should be determined prior to assessing CDX2 status.

\section{Sebaceous Glands in the Esophagus (Fordyce's Spots): A Rare, Benign and Enigmatic Lesion. Study of 9 cases and Review of Literature}

Sandra Gjorgova-Gjeorgjievski', Alia Gupta², Mihajlo Gjeorgjievski ${ }^{3}$ Mitchell Cappelß, Mitual Amin'. 'Beaumont Health, Royal Oak, MI ${ }^{2}$ Troy, MI, ${ }^{3}$ Beaumont Health, ${ }^{4}$ William Beaumont Hospital, Royal Oak, $\mathrm{Ml}$

Background: Ectopic sebaceal glands (ESG), also known as Fordyce spots, are well known histologic entity in mucosal surfaces. However ESG in esophagus is extremely rare with currently $<50$ cases described in English literature. We present 9 additional cases making this the largest case series.

Design: Retrospective review of 9 cases diagnosed over a 14 year period (2001-2015). Cases were searched for terms "sebaceal" and "sebaceous" in esophageal biopsies, resections and autopsy cases. Clinical history, endoscopic findings and pathologic features were analyzed. 
Results: The mean age was 55 years, and ESG was identified in similar frequency in both sexes. The patients underwent EGD for various reasons, including dyspepsia, epigastric pain, iron deficiency anemia and GERD. On the EGD, occasionally yellow-white patchy submucosal areas in the esophagus were identified. Biopsy pathology showed lobules of benign ectopic sebaceous glands within the squamous epithelium and in the underlying lamina propria. Concomittant esophagitis was identified in 4 cases. The ESG were composed of vacuolated, lipid-filled cells, and did not demonstrate any atypical features. The lobules were commonly grouped in groups of 2 up to 5 in one unit, and did not exceed more that $3 \mathrm{~mm}$ in size. One case had lobules associated with rudimentary hair follicle. We did not identifiy any sebaceous glands in other locations like the stoamch or duodenum.

Conclusions: Ectopic sebaceal glands (ESG) in the esophagus are a benign entity and usually are discovered incidentally, although a third of patients presented with failure to respond to GERD treatment. Due to the lack of upper gastrointestinal tract screening in the United States, the incidence in the general population cannot be determined. Therefore, ESG are most likely to found in association with dyspepsia, GERD and epigastric pain. A sngle Korean study reported a prevalence rate of of $0.05 \%$ in a systematically screened asymptomatic population. Histological confirmation is essential for definitive diagnosis, since it is endoscopically indistinguishable from other entities such as xanthomas, candidiasis, glycogenic acanthosis and papillomas. The etiology for this has been widely postulated with most authors beleiving this to be either benign metaplasia of salivary or submucus gands and others favoring this to be ectopic tissue. This difference between ectopic versus metaplastic cannot be definitively ascertained due to the endodermal origin of both and lack of supportive data.

\section{CDX2 Expression in Metastatic Colorectal Adenocarcinoma}

Amanda Gohlke1, Melissa Contos ${ }^{2}$, Rachel B Fielden ${ }^{3}$, Michael Idowu ${ }^{4}$. ${ }^{1}$ Chesterfield, VA, ${ }^{2}$ Richmond, VA, ${ }^{3}$ Virginia Commonwealth University Health System, Chesterfield, VA, ${ }^{4}$ Virginia Commonwealth Univ Health System, Richmond, VA

Background: Loss of CDX2, a transcription factor expressed in intestinal epithelial nuclei, has helped identify high risk stage II colon cancer patients that may benefit from additional chemotherapy. While CDX2 immunohistochemistry is often used to help distinguish primary site of a carcinoma, approximately $4 \%$ of colorectal carcinomas are CDX2 negative. The aim of this study is to correlate overall survival to race and $C D X 2$ reactivity.

Design: A computerized database search retrospectively identified cases of metastatic colorectal adenocarcinoma. Slides from the cases were reviewed and select paraffin embedded blocks were used to create a tissue microarray (TMA) with three $1 \mathrm{~mm}$ cores per block. Three authors (MOI, MC, AG) reviewed and scored the stain intensity (rated 0-3) to achieve consensus. For a site to be considered negative for CDX2, all sections were required to have a staining intensity of 0 , or less than $5 \%$ positive. Clinical information gathered from the electronic medical record was used to compare the groups.

Results: 117 sites of metastatic colorectal adenocarcinoma were identified from 97 patients. Of these, 6 sites were negative for CDX2 in 5 patients. Overall in both groups, 12 patients exhibited mucinous and/or signet ring cell morphology. Specifically, signet ring cell morphology was present in five patients, two of which were negative for CDX2. Tumors with CDX2 negative status metastasized to a variety of sites with no predilection for a particular tissue site (see Table). The CDX2 negative group had a median overall survival (from tissue diagnosis of metastasis) of 501 days with an interquartile range of 171-832 days. The CDX2 positive median overall survival (from tissue diagnosis of metastasis) was 334 days with an interquartile range of 101-936 days. Lastly, within the CDX2 positive group black patients had a median overall survival of 504 days while white patients had a median overall survival of 1200 days.

\begin{tabular}{|l|l|l|l|}
\hline Metastasis Site & Number of blocks & CDX2 Positive & CDX2 Negative \\
\hline Liver & 24 & 23 & 1 \\
\hline Omentum & 17 & 17 & \\
\hline Brain & 11 & 10 & 1 \\
\hline Ovary/pelvic mass & 10 & 10 & \\
\hline Abdominal wall & 9 & 9 & \\
\hline Lung, Bronchus & 9 & 8 & 1 \\
\hline Peritoneum & 9 & 8 & 1 \\
\hline Lymph Node & 4 & 4 & \\
\hline Bone & 3 & 3 & \\
\hline Diaphragm & 3 & 3 & \\
\hline Mesentery & 3 & 3 & \\
\hline Small bowel & 3 & 3 & \\
\hline Cervix & 2 & 2 & \\
\hline Pleura & 2 & 1 & 1 \\
\hline Retroperitoneum & 2 & 2 & \\
\hline Gonadal vein & 1 & 1 & \\
\hline Pericardium & 1 & 1 & \\
\hline Trachea & 1 & 1 & \\
\hline Uterus & 1 & 1 & \\
\hline Vagina & 1 & 1 & \\
\hline Vulva & 1 & 1 & \\
\hline
\end{tabular}

Conclusions: CDX2 negative status has been associated with highe risk stage II colorectal carcinoma. While we found no difference in overall survival time for CDX2 negative status in metastatic tissue, colorectal adenocarcinomas with mucinous and signet ring morphology may be more likely to show negative to focal CDX2 staining. Additional investigation is needed to correlate negative CDX2 staining with signet ring morphology and prognosis.

\section{Mesenteric Tumor Deposit Number, But Not Size, Affects Prognosis of Patients with Small Intestinal Well-Differentiated Neuroendocrine Tumors}

Raul S Gonzalez', Justin Cates ${ }^{2}$, Chanjuan Shỉ. 'University of Rochester Medical Center, Rochester, NY, ${ }^{2}$ Vanderbilt University Medical Center, Nashville, TN, ${ }^{3}$ Vanderbilt University, Nashville, TN

Background: We have previously shown that mesenteric tumor deposits (MTDs) are a significant adverse prognostic factor in small intestinal well-differentiated neuroendocrine tumors (SI-NETs), more so than lymph node metastasis. The American Joint Committee on Cancer (AJCC) 8th Edition incorporates MTDs into tumor staging, with any deposit larger than $2 \mathrm{~cm}$ classified as $\mathrm{pN} 2$. This size criterion has not been critically evaluated as a prognostic factor for SI-NET, nor have MTD multifocality or histologic findings within MTDs.

Design: We evaluated 70 cases of SI-NET with associated MTDs. Findings helpful to distinguish MTDs from replaced nodes included entrapped nerves and vessels and lack of peripheral inflammation or subcapsular sinuses. Proliferative indices of the largest MTD from each case were calculated from Ki67-stained sections. MTD number and largest MTD size were recorded. Each MTD was evaluated for lesional contour (rounded or irregular), sclerosis, inflammation, calcification, entrapped blood vessels, and perineural invasion. Associations between these factors (along with patient age, primary tumor Ki67 index, and AJCC stage) and development of liver metastases were assessed by logistic regression. Prognostic impact of these factors on disease-specific survival was assessed by stratified Cox proportional hazard regression.

Results: Median MTD size was $1.5 \mathrm{~cm}$ (range: $0.2-7.0 \mathrm{~cm}$ ), and median MTD number was 1 (range: 1-13). Correlation between primary and MTD Ki67 within a given patient was non-significant both impacted prognosis similarly. MTD size had a non-significant effect on prognosis, both for incremental size increase (hazard ratio [HR] 1.07, 95\% confidence interval [Cl] 0.67-1.69) and when comparing MTD $\leq 2 \mathrm{~cm}$ to $>2 \mathrm{~cm}$ (HR $0.85,95 \% \mathrm{Cl} 0.31-2.30$ ). In contrast increasing number of MTD did, with a $22 \%$ increase in HR for every additional MTD present (95\% Cl 1-47\%; $P=0.040$ ). MTD morphology had no significant prognostic impact. However, MTDs with irregular contours were associated with development of liver metastases (odds ratio $4.57 ; 95 \% \mathrm{Cl} 1.00-20.88 ; P=0.050$ ), suggesting that rounded MTDs may represent nodal metastasis otherwise mimicking MTDs microscopically.

Conclusions: Increasing number of MTDs is associated with shorter disease-specific survival in patients with SI-NETs. Size of MTDs and other histologic features do not appear to affect prognosis. Our findings also argue against the utility of performing Ki67 immunohistochemical stains on MTDs in lieu of primary lesions. 
738 What Remains of Appendiceal Adenocarcinoma After LAMN and Goblet Cell Neoplasms are Excluded?

Raul S Gonzalez', Joseph Misdraji', Rhonda Yantiss'. 'University of Rochester Medical Center, Rochester, NY, ${ }^{2}$ Massachusetts General Hospital, Boston, MA, ${ }^{3}$ Weill Cornell Medical College, New York, NY

Background: Most large series of appendiceal adenocarcinoma (AAC) predate the concept of "low-grade appendiceal mucinous neoplasm" (LAMN) as a tumor with pushing invasion, and the understanding of the serrated pathway of colorectal tumorigenesis. Also, many carcinomas with destructive invasion develop in association with goblet cell neoplasms, and their histogenesis is distinct from conventional adenocarcinomas. Once these groups are excluded, the clinicopathologic features of AAC are not well defined. This study assessed clinical and pathologic features in a large series of AACs.

Design: We identified 85 cases of AAC in our archives, after excluding LAMNs and tumors arising from goblet cell neoplasms. Patient age, sex, presentation, and outcome data were obtained from medica records. Tumors were assessed for size, histologic features, mucosal precursors, and stage.

Results: All patients were adults (mean age 61 years, range 30-90; male/female ratio 9:8). They usually presented with abdominal pain or symptoms of appendicitis $(65 \%)$, or a right lower quadrant mass (24\%). Tumors were usually large (mean $4.9 \mathrm{~cm}$ ), and $55 \%$ were associated with appendiceal perforation. Most AACs (94\%) had intestinal-type features and showed frequent mucinous differentiation $(55 \%)$ or signet ring cell morphology $(21 \%)$. Five tumors resembled pancreatobiliary carcinoma, with angulated glands lined by cuboidal cells that contained pale to clear cytoplasm embedded in abundant desmoplastic stroma. One case showed solid growth, and one displayed serrated features. Precursors included colonic-type tubulovillous or serrated adenoma $(n=43)$, LAMN $(n=10)$, and highgrade appendiceal mucinous neoplasm $(n=10)$; no precursor was identifiable in 22 cases. Regional lymph node disease was present in $16(19 \%)$ patients, and $32(38 \%)$ had distant metastases, usually involving peritoneal organs. Follow-up information was available for 69 patients; $46(67 \%)$ were alive without disease or died of other causes, $9(13 \%)$ were alive with disease, and $14(20 \%)$ died of disease (mean follow-up: 55 months, range: 0.5-155)

Conclusions: AACs generally show intestinal-type differentiation with frequent mucinous or signet ring cell features; a minority resemble pancreatobiliary adenocarcinomas. Serrated adenocarcinomas are rare in the appendix. Most AACs arise from colonic-type adenomas, similar to colorectal carcinoma. Unlike LAMNs, AACs occasionally metastasize to regional nodes, and like other appendiceal neoplasms, they often spread to the peritoneum.

\section{Relationships Among Histologic Characteristics, Molecular Phenotypes, and Patient Outcomes in Mucinous Colorectal Carcinoma}

Raul S Gonzalez', Justin Cates', Mary Kay Washington'. 'University of Rochester Medical Center, Rochester, NY, ${ }^{2}$ Vanderbilt University Medical Center, Nashville, TN, ${ }^{3}$ Vanderbilt Univ. Med. Ctr., Nashville, TN

Background: Mucinous colorectal carcinoma (mCRC) often shows microsatellite instability (MSI), but the impact of mucinous phenotype on prognosis remains unclear. This is partly due to molecular heterogeneity of $\mathrm{mCRC}$; recent research categorized $\mathrm{mCRC}$ into 3 subtypes, based partially on whether a tumor was KRAS-mutated $B R A F$-mutated, or neither. Using a large single-center cohort, we explored whether clinicopathologic or histologic characteristics impacted $\mathrm{mCRC}$ prognosis and whether histologic features suggested molecular phenotype.

Design: We evaluated $224 \mathrm{mCRC}$ ( $\geq 5 \%$ mucin production) for patient sex, age, race, and outcome; tumor size, location, grade, stage, MSI status, and molecular phenotype (when available); percent of glands producing mucin; percent of tumor bulk composed of mucin; whether tumoral epithelium floated in mucin (colloid pattern); tumor budding; signet ring cells (SRCs); and peritumoral inflammation (PI). Cases with neoadjuvant therapy or predominantly SRC morphology were excluded. We assessed relationship of these features to disease-specific survival by multivariate analysis and compared stage-matched mCRC outcomes with 499 colorectal carcinomas, not otherwise specified (CRC-NOS).

Results: Factors predicting worse outcome in $\mathrm{mCRC}$ on univariate analysis included MSI-high ( $P=0.0005)$, SRC in mucin pools $(P=0.0017)$, and lack of PI $(P=0.0034)$. Only chronic PI independently predicted decreased hazard on stage-adjusted multivariate analysis (hazard ratio $0.27,95 \%$ confidence interval $0.076-0.93, P=0.038$ ). Percent of glands producing mucin and percent tumor bulk composed of mucin did not affect prognosis, even after dichotomization at $50 \%$ cutoffs. Overall patient survival for mCRC (median follow-up, 35 months) and CRC-NOS (median follow-up, 50 months) did not significantly differ after accounting for stage $(P=0.95)$. Among $54 \mathrm{mCRC}$ stratified by mutation status (KRAS mutation / BRAF mutation / neither), BRAFmutated tumors were more often MSI-high $(76 \%$ vs. $33 \%$ and $25 \%, P=0.004)$, and $K R A S$-mutated tumors had higher rates of budding $(85 \%$ vs. $58 \%$ and $50 \%, P=0.043$ ) and tumor deposits $(45 \%$ vs. $28 \%$ and $10 \%, P=0.030)$; no significant survival differences were observed among these groups.

Conclusions: Our findings in a large cohort support the assertion that, stage for stage, $\mathrm{MCRC}$ and CRC-NOS have similar outcomes. Aside from chronic $\mathrm{PI}$, histologic characteristics in $\mathrm{mCRC}$ are not associated with clinical outcome. In addition, these histologic features are not specific for molecular phenotype in mCRC.

\section{ARID1A Loss In Small Intestinal Adenocarcinoma Is Rare In Western Cohort}

Iván González', Bella GoyaR', Reetesh Paỉ, Changqing Ma'. 'Washington University School of Medicine, St. Louis, MO, ${ }^{2}$ Washington University School of Medicine, Saint Louis, MO, 'UPMC-Presbyterian Hospital, Pittsburgh, PA, ${ }^{4}$ University of Pittsburgh, Pittsburgh, PA

Background: Primary small intestinal adenocarcinoma (SIC) is often diagnosed at an advanced disease stage and is associated with a poor clinical outcome. Identifying prognostic biomarkers may help to stratify patients. ARID1A has been identified as a tumor-suppresso gene in carcinomas of the gastrointestinal tract. A recent study from Asia has reported loss of AR1D1A in 20\% of SICs and implicated loss of or low ARID1A expression is associated with loss of MLH1 and MSH2 expression, high tumor grade and pathologic stage, and poo prognosis. In this pilot study, we sought to evaluate the prevalence of ARID1A loss and its significance in a cohort of surgically resected, primary SICs in Western population.

Design: Ninety-two surgically resected, primary SICs including 62 $(67 \%)$ duodenal and $30(33 \%)$ jejunal/ileal adenocarcinomas were collected from two tertiary care medical centers in the US. ARID1A immunohistochemistry (IHC) was performed on whole tissue sections. Loss of ARID1A expression was defined as the complete absence of nuclear staining. MSI was tested during clinical workup for 52 SICs. For the remaining $40 \mathrm{SICs}$, IHCs for DNA mismatch repair proteins were performed to evaluate MSI. ARID1A expression was correlated with demographics, MSI status, and clinicopathologic features.

Results: ARID1A loss was identified in $6(7 \%)$ of 92 SICs including 5 duodenal tumors and 1 jejunal tumor. MSI was detected in $18(20 \%$ of the 92 SICs. One-third (2/6) of the ARID1A loss SICs were MS while MSI was present in $19 \%(16 / 86)$ of SICs with retained ARID1A expression $(P=0.6)$. Interestingly, retained ARID1A expression was also seen in two (2\%) Epstein - Barr virus associated SICs. Onethird (2/6) of ARID1A loss SICs were poorly-differentiated tumors One $(17 \%)$ ARID1A loss SIC had medullary features but none had signet ring cell differentiation. In contrast, $40 \%(34 / 86)$ of SICs with retained ARID1A expression were poorly-differentiated, $11(13 \%)$ had medullarly features, and $9(11 \%)$ signet ring cell features $(P>0.05$ fo all). Four (66.7\%) ARID1A loss SICs were pathologic stage 4 tumors (pT4) while $35(41 \%)$ SICs with retained ARID1A were pT4 $(P=0.2)$.

Conclusions: Loss of ARID1A expression is a rare event in our cohort of primary small intestinal adenocarcinoma of the Western population. Furthermore, ARID1A loss is not significantly associated with MSI histologic grade, or pathologic tumor stage. Our results suggest the role of $A R I D 1 A$ in primary small intestinal adenocarcinoma should be further elucidated.

\section{Gene expression profile of appendiceal adenocarcinoma ex-goblet cell carcinoid, signet ring cell type: a review of 16 cases}

Jeenal A Gordhandas ${ }^{1}$, Grace Lin ${ }^{1}$, Mark Valasek ${ }^{1}$, Ann Tipps ${ }^{1}$, Vera Vavinskaya1, Mojgan Hosseini'. 'University of California San Diego, San Diego, CA

Background: Appendiceal goblet cell carcinoids (GCC) is a rare appendiceal neoplasm with dual glandular and neuroendocrine differentiation which can lead to diagnostic confusion. Occasionally, an additional component of adenocarcinoma might be present which will lead to a diagnosis of adenocarcinoma ex-goblet cell carcinoids (GCC-AC). In 2008, Tang et al proposed a classification system which divided cases with adenocarcinoma into two groups: GCC-AC, signe ring cell type and GCC-AC, poorly differentiated carcinoma type. The gene expression profile of GCC-ACs remains relatively unknown. We report the genetic abnormalities identified in our patient population.

Design: Mutational analysis was performed on 16 cases of GCC $\mathrm{AC}$, signet ring cell type. Any one or both of commercially available comprehensive genomic profiling panels by Foundation Medicine and Guardant were used in each case. Both use next generation sequencing-based assays that identify genomic alterations within hundreds of cancer-related genes in tissues. The reportable alterations include substitutions, amplifications, insertions, deletion and frameshift mutations.

Results: Genetic information was available on 16 cases of appendicea 
adenocarcinoma ex-goblet cell carcinoid with signet ring cell features. Previously reported pathogenic mutations are identified in tumor suppressor genes TP53 (5 cases), SMAD4 ( 2 cases), RUNX1 (1 case) and ATM (1 case) and oncogenic genes KRAS (2 cases), ERBB2 (1 case), CCNE1 (1 case), PIK3CA (1 case), MYCL1 (1 case), MDM2 (1 case), FRS2 (1 case). Alterations of unknown significance are seen in tumor suppressor genes PTEN (2 cases), SMAD4 (2 cases) and oncogenes PDGFRA(1 case), PIK3CA (1 case), MET (1 case), SOX9 (2 cases) and EGFR (1 case) as well as hormonally regulated genes MLL2 (1 case) and AR (1 case). Three cases of GCC-AC did not have any reportable alterations. MSI immunostain panel was performed on 12 cases which showed intact expression.

Conclusions: In our GCC-AC, signet ring cell type cases, we identified mutation in TP53 and SMAD4 which are classically associated with conventional colorectal and pancreatobiliary adenocarcinomas. Many tumor suppressor and oncogenes that play a role in the pathogenesis of other gastrointestinal and non-gastrointestinal tumors may play a role in development of GCC-AC as well.

\section{Histological Severity of Ulcerative Colitis Predicts Accelerated Progression to Pan-Colitis in a Subgroup of Patients with Limited Disease}

Yansheng Hao', Russell B McBride ${ }^{2}$, Giulia Roda ${ }^{3}$, Ryan Ungaro ${ }^{2}$, Jeanfrederic Colomber ${ }^{2}$, Alexandros D Polydorides ${ }^{4}$, Qingqing Liu, Hongfa $Z^{2} u^{5}$, Noam Harpaz ${ }^{6}$, Huaibin Mabel Ko ${ }^{4}$. ${ }^{1}$ The Mount Sinai Hospitial, New York, NY, ${ }^{2}$ The Mount Sinai Hospital, ${ }^{3}$ University of Bologna Medical Center, ${ }^{4}$ cahn School of Medicine at Mount Sinai, New York, NY, ${ }^{5}$ The Icahn School of Medicine at Mount Sinai, Paramus, NJ, ${ }^{6}$ Mount Sinai Med. Center, New York, NY

Background: Among patients with limited ulcerative colitis (UC), i.e. proctitis (E1 per Montreal classification) or left-sided UC (E2), 30\% ultimately extend to pan-colitis (E3) and typically have adverse clinical outcomes. The risk of extension to E3 has been found to correlate with clinical features such as early age of onset and more extensive disease. Since classification of extension is based on endoscopy, we sought to determine whether histologic features at initial examination correlate with disease extension.

Design: The study comprised 28 patients with limited UC diagnosed at a large academic center from 2006-2017. Eligible cases had $\geq 2$ consecutive endoscopic examinations with biopsies $\geq 12$ months apart, the index examination sampling both affected and unaffected mucosa. Non-extenders were defined as patients with absence of disease extension at a minimum of 5 years from the first colonoscopy. Clinical and demographic data were obtained from medical records. Patients were excluded if they were examined elsewhere or if any slides were unavailable. Severity of inflammation was scored in blinded fashion by a GI pathologist utilizing 2 different established methods, the Mount Sinai (Gupta RB, et al. Gastroenterology, 2007) and Nancy (Marchal-Bressenot A, et al. Gut, 2017) indices.

Results: 343 slides were scored in total. Of the 28 patients, $15(54 \%)$ progressed to E3 ("extenders") and 13 did not ("non-extenders") Based on the Mount Sinai index, the histologic scores in the affected mucosa did not differ between extenders and non-extenders $(p=0.97)$. However, the scores of the index biopsies in affected mucosa of the extenders correlated with the scores of the entire colon after extension $(r=0.602, p=0.025)$ and inversely with the time to extension $(r=-0.506, p=0.038)$. Interestingly, the presence of an "isolated cecal patch" showed a trend toward extension $(n=3, p=0.06)$. Among nonextenders, the scores of the index biopsies did not correlate with those of the subsequent biopsies. Similar results were obtained with the Nancy index.

Histological Scores: Mount Sinai Score and Nancy Index

\begin{tabular}{|c|c|c|c|c|}
\hline & \multicolumn{2}{|c|}{ Mount Sinai Score } & \multicolumn{2}{|l|}{ Nancy Index } \\
\hline & Affected area & Pancolonic & Affected area & Pancolonic \\
\hline $\begin{array}{l}\text { Severity at progression } \\
\text { in extenders }\end{array}$ & $\begin{array}{l}r=0.602, \\
p=0.025\end{array}$ & $\begin{array}{l}r=0.559 \\
p=0.04\end{array}$ & $\begin{array}{l}\mathrm{r}=0.612, \\
\mathrm{p}=0.022\end{array}$ & $\begin{array}{l}r=0.674 \\
p=0.01\end{array}$ \\
\hline $\begin{array}{l}\text { Severity at next biopsy } \\
\text { in non-extenders }\end{array}$ & $\begin{array}{l}r=0.197 \\
p=0.513\end{array}$ & $\begin{array}{l}\mathrm{r}=0.317 \\
\mathrm{p}=0.288\end{array}$ & $\begin{array}{l}r=0.223, \\
p=0.458\end{array}$ & $\begin{array}{l}\mathrm{r}=0.312 \\
\mathrm{p}=0.295\end{array}$ \\
\hline $\begin{array}{l}\text { Progression time in } \\
\text { extenders }\end{array}$ & $\begin{array}{l}r=0.506, \\
p=0.038\end{array}$ & $\begin{array}{l}\mathrm{r}=-0.495, \\
\mathrm{p}=0.057\end{array}$ & $\begin{array}{l}r=-0.571, \\
p=0.024\end{array}$ & $\begin{array}{l}\mathrm{r}=-0.577 \\
\mathrm{p}=0.021\end{array}$ \\
\hline $\begin{array}{l}\text { Next biopsy time in } \\
\text { non-extenders }\end{array}$ & $\begin{array}{l}r=0.326, \\
p=0.241\end{array}$ & $\begin{array}{l}\mathrm{r}=-0.177, \\
\mathrm{p}=0.541\end{array}$ & $\begin{array}{l}\mathrm{r}=-0.312, \\
\mathrm{p}=0.275\end{array}$ & $\begin{array}{l}\mathrm{r}=-0.16, \\
\mathrm{p}=0.593\end{array}$ \\
\hline
\end{tabular}

Conclusions: Histological severity of left sided disease does not predict which UC patients will extend. However, in the subgroup of extenders, severity of inflammation at the index examination correlates with shorter progression time and more severe inflammation when progression occurs. Patients with limited UC but severe histologic inflammation, and perhaps those with an isolated cecal patch, may warrant individualized therapy.

\section{Clinicopathological Prognostic Factors of Adenocarcinoma of the Ampulla of Vater in a Mexican Population}

Roberto Herrera-Goepfert ${ }^{1}$ Giovanny Soca-Chafre ${ }^{1}$ Samuel Almeida-Navarro', Norma Hernández-Pedro'. 'Instituto Nacional de Cancerología, Mexico City, México

Background: The ampulla of Vater is formed by the confluence of the common bile and pancreatic ducts and is separated from the duodenal papilla by the sphincter of Oddi. Carcinomas arising from this region are generally called carcinomas of the ampulla of Vater (CAV). They account for $0.5 \%$ of malignant neoplasms of the gastrointestinal tract and $6-20 \%$ of malignant peri-ampullary neoplasms. Only $20 \%$ of the patients are candidates for elective surgery. In non-operable cases, average survival is seven months. Our objective was to evaluate the clinicopathological prognostic factors of the CAV after surgical resection.

Design: From the records of the Department of Surgical Pathology at the Instituto Nacional de Cancerología, México, cases diagnosed as CAV were selected over a period of 11 years, from January 2005 to September 2015. Cases with a pancreatoduodenectomy report were included, and from each case, the demographic and pathological data of the surgical specimen, were obtained. Univariate and multivariate statistical analyses were performed using the Log-rank test and Cox regression.

Results: Of 157 cases diagnosed as CAV, 104 patients were excluded because they were not suitable for surgical treatment at the time of diagnosis. In the remaining 53 patients, a pancreatoduodenectomy was performed. The median age of the group was 57 years and the majority were male. Intestinal-type were more frequent than pancreatobiliary-type adenocarcinomas, and the lymph node metastasis status was the only variable that had an adverse impact on overall survival of the patients (18.1 vs. 55.9 months) (Figure). Most adenocarcinomas with intestinal phenotype were of low-grade, with no lymphovascular or perineural invasion, less than $3 \mathrm{~cm}$, showing a trend towards better overall survival (Table).

\begin{tabular}{|c|c|c|c|c|c|}
\hline Variable & & & & & $\begin{array}{l}P \\
\text { value }\end{array}$ \\
\hline \multicolumn{6}{|l|}{ TNM(N) vs. } \\
\hline Perineural Invasion & N0: $36 \%$ & N1: $64 \%$ & & & 0.054 \\
\hline \multicolumn{6}{|l|}{ Involved LN vs. } \\
\hline Whipple histological rade & $\begin{array}{l}\mathrm{G} 1 *: \\
5.3 \%\end{array}$ & $\begin{array}{l}\mathrm{G} 2 *: \\
84.2 \%\end{array}$ & $\begin{array}{l}\text { G3: } \\
10.5 \%\end{array}$ & & 0.016 \\
\hline \multicolumn{6}{|l|}{$\begin{array}{l}\text { Intestinal Biopsy Subtype } \\
\text { vs. }\end{array}$} \\
\hline Biopsy histological grade & $\begin{array}{l}\text { G1: } \\
33.3 \%\end{array}$ & $\begin{array}{l}\mathrm{G} 2 *: \\
63.0 \%\end{array}$ & $\begin{array}{l}\text { G3: } \\
3.7 \%\end{array}$ & $\begin{array}{l}\mathrm{G} 4^{*}: \\
0.0 \%\end{array}$ & $\begin{array}{l}< \\
0.001\end{array}$ \\
\hline Lymphatic permeation & $\begin{array}{l}\text { YES: } \\
3.7 \%\end{array}$ & NO: $96.3 \%$ & & & 0.001 \\
\hline \multicolumn{6}{|l|}{$\begin{array}{l}\text { Intestinal Whipple Sub- } \\
\text { type vs. }\end{array}$} \\
\hline \multirow[t]{2}{*}{ Tumor size } & $\begin{array}{l}\leq 2 \mathrm{~cm}: \\
64.3 \%\end{array}$ & $\begin{array}{l}>2 \mathrm{~cm}: \\
35.7 \%\end{array}$ & & & 0.039 \\
\hline & $\begin{array}{l}\text { <3 cm: } \\
89.3 \%\end{array}$ & $\begin{array}{l}>3 \mathrm{~cm}: \\
10.7 \%\end{array}$ & & & 0.007 \\
\hline TNM(T) & $\begin{array}{l}\mathrm{T} 1^{*}: \\
23.3 \%\end{array}$ & T2: $60.0 \%$ & $\begin{array}{l}\text { T3*: } \\
16.7 \%\end{array}$ & & 0.002 \\
\hline Lymphatic Permeation & $\begin{array}{l}\text { YES: } \\
6.7 \%\end{array}$ & NO: $93.3 \%$ & & & 0.002 \\
\hline Perineural invasion & $\begin{array}{l}\text { YES: } \\
13.3 \%\end{array}$ & NO: $86.7 \%$ & & & 0.026 \\
\hline
\end{tabular}

Chi squared or Fisher's Exact Test $(n<5)$

*Significant after post hoc test with Bonferroni correction 


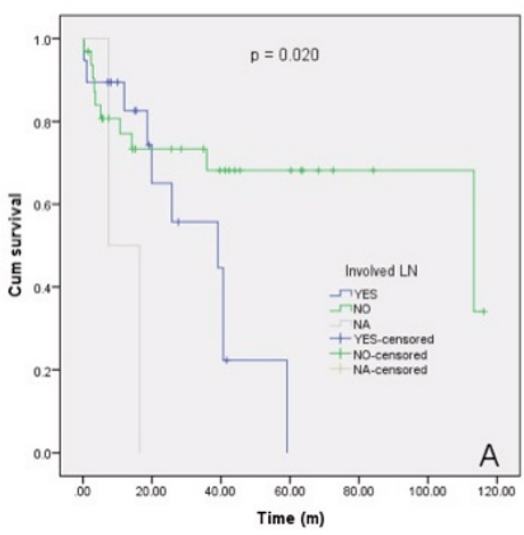

Conclusions: CAV can originate from intestinal or pancreatobiliary epithelia, and biological behavior depends largely on this condition. The intestinal phenotype showed a trend towards better overall survival and was associated with low-grade histology, absence of lymphovascular and perineural invasion, and smaller tumor size. In conclusion, lymph node metastasis and the pancreatobiliary phenotype, have an adverse effect on the prognosis of patients with CAV. We propose to simplify the classification according to the histopathological phenotype, as well as referring the anatomical site as the Vaterian system.

\section{Gastritis with Russell Bodies Is a Frequent} Inflammatory Phenotype Associated with Global Shifts of the Gastric Microbiome and Enrichment of Helicobacter and/ or Streptococcal Genera

Richard A Hickman', Liying Yang², Yuhan Hao ${ }^{3}$, Christopher J Schwartz', Azore-Dee Bradshaw ${ }^{4}$, Antonio Galvao-Neto ${ }^{2}$, Susan Kornacki', Cristina H Hajdu', Dervla Kelly', Stuart Brown ${ }^{3}$, Jonathan Melamed", Zhiheng Pei'. 'Columbia University, New York, NY, ${ }^{2}$ New York University School of Medicine, New York, NY, ${ }^{3}$ Applied Bioinformatics Center, New York University School of Medicine, NY, ${ }^{4} \mathrm{New}$ York University Medical Center, New York, NY, ${ }^{5}$ University of California San Diego Department of Pathology, ${ }^{6} \mathrm{New}$ York University School of Medicine, New York, NY, ${ }^{7}$ New York University Medical Center, New York, NY

Background: Russell Body Gastritis (RBG) is considered to be a rare histologic finding that has an unclear pathogenesis and an unpredictable clinical outcome. We sought to clarify the frequency and significance of RBG by assessing their associated histology and relationship with the local microbiome.

Design: We reviewed all 220 gastric biopsies over a 2-month period at 1 institution for the presence and density of Russell bodies (RB). In biopsies with RB, the quantity of RB was manually counted using light microscopy at 200x magnification in every biopsy level (3068 sections) and the sectional area was estimated using a $1 \times 1 \mathrm{~mm}$ grid overlay. RB density was calculated by dividing the total quantity of RB in all sections by the total sectional area. 48 additional patients, which corresponded to an extra 100 histologic biopsies, were consented at the same visit for an extra gastric biopsy for $16 \mathrm{~S}$ rRNA sequencing, and these microbiome profiles were correlated with the highest RB density per patient.

Results: Russell bodies (RB) were frequent in gastric biopsies (43\% of all gastritides) and the RB density significantly increased with more severe gastritides $(p<0.001, n=320)$. The gastric microbiome globally differed in beta diversity between RB-positive and RB-negative cases by unweighted and weighted principal component analysis $(p=0.03, p=0.007, n=48$, respectively). In particular, Helicobacter and Streptococcus were significantly enriched in gastritis with RB and their abundances correlated with RB density $(\mathrm{p}=0.0002, \mathrm{r}=0.51 ; \mathrm{p}=0.009, \mathrm{r}=0.37, \mathrm{n}=48$, respectively). Protonpump inhibitor (PPI) use reduced RB density per unit abundance of Streptococcus $(\mathrm{p}=0.0021, \mathrm{n}=48)$. H. pylori abundance significantly correlated with RB density and two Streptococcus species (an unclassified Streptococcal species and $S$. anginosus) significantly correlated with RB density in $H$. pylori-negative gastritis $(p=0.009$, $\mathrm{n}=36, \mathrm{r}=0.36 ; \mathrm{p}=0.0025, \mathrm{n}=36, \mathrm{r}=0.51$, respectively). $7 \mathrm{H}$. pylorinegative patients were followed for 1 year and variation in Streptococcus abundance reflected RB density $(p=0.085, n=7)$.

Conclusions: RB are common within the inflamed gastric mucosa and gastritis with RB is associated with Helicobacter and Streptococcus enrichment and consequent global shifts in the gastric microbiome. PPI dampens RB production, presumably through anti-inflammatory effects. Gastritis with RB might represent a reactive humoral response to bacteria within the gastric microbiome. Streptococcus species may influence chronic gastritis.
745 Dynamics of Genetic Aberrations in Crohn's Disease Associated Colorectal Carcinogenesis

Daniela Hirsch ${ }^{1}$, Darawalee Wangsa ${ }^{2}$, Jack Zhu' ${ }^{2}$, Daniel C Edelman ${ }^{2}$ Paul S Meltzer ${ }^{2}$, Kerstin Heselmeyer-Haddad ${ }^{3}$, Thomas Ried ${ }^{\beta}$, Timo Gaiser'. 'University Medical Center Mannheim, Mannheim, BadenWuerttemberg, ${ }^{2}$ National Institutes of Health, ${ }^{3}$ Section of Cance Genomics, Genetics Branch, National Cancer Institute, National Institutes of Health, Bethesda, MD, ${ }^{4}$ University Medical Center Mannheim, Mannheim, BW

Background: Chronic inflammation of the large intestine in patients with Crohn's disease (CD) increases the risk for colorectal cance (CRC), though only a subset of CD patients progresses to dysplasia and invasive carcinoma. CRCs in the setting of $C D$ are thought to arise in stepwise fashion from dysplastic precursor lesions, comparable to the sequential development of sporadic CRCs from adenomas. However molecular data on CRCs associated with CD and corresponding precursor lesions are limited and the underlying genomic alterations have not yet been resolved.

Design: We analyzed a total of 73 tissue samples from 28 patients with CD associated CRC, comprising primary CRCs, dysplastic lesions, inflamed mucosa, lymph node metastases and histologically normal colonic mucosa, by targeted next generation sequencing of 563 cancer related genes and array-based comparative genomic hybridization. The results were compared to 24 sporadic CRCs of comparable histomorphology, namely mucinous adenocarcinomas, and to data from The Cancer Genome Atlas (TCGA)

Results: The majority of CD associated CRCs (20 of 32, 63\%) was of mucinous and/or signet ring cell type. CD associated CRCs had a distinct mutation signature compared to sporadic mucinous CRCs : TP53 (76\% in CD CRCs versus $33 \%$ in sporadic mucinous CRCs), KRAS (24\% versus $50 \%)$, APC (17\% versus $75 \%)$, PIK $3 C A(17 \%$ versus $13 \%)$, SMAD4 $(12 \%$ versus $17 \%)$, SMAD2 (10\% versus $29 \%)$ and $B R A F(0 \%$ versus $13 \%)$. Our analysis of multiple lesions at different stages of development showed that TP53 mutations and copy number alterations occurred as early and frequent events in $C D$ progression while $A P C$, KRAS and $S M A D$ mutations occurred later, which is different from sporadic adenoma-carcinoma progression. In four of 28 patients with $C D$ associated CRC, genetic alterations including TP53 mutations and copy number alterations could already be detected in colonic mucosa without histologic evidence for dysplasia, pointing to an occult tumor evolution in CD patients.

Conclusions: Our comprehensive molecular profiling of CD associated CRCs and precursor lesions reveals an inflammationassociated landscape of genetic aberrations. Given the limitations of histopathology for the diagnosis of CD associated dysplasia and determination of progression risk, TP53 mutations and aneuploidy in non-dysplastic mucosa and dysplastic lesions may serve as predictive markers for disease progression and as diagnostic biomarkers indicating $C D$ associated, non-sporadic etiology of the neoplastic lesion.

\section{Evaluating for Lynch Syndrome: What Are We Doing and How Are We Doing It?}

Erika Hissong', Yao-Tseng Chen"2, Rhonda Yantiss'. 'New York, NY ${ }^{2}$ Weill Cornell Medical College, New York, NY

Background: As recently as 2012, only $38 \%$ of US institutions screened all colorectal cancers (CRC) for Lynch syndrome (LS). PCR for $\mathrm{MSI}$ and mismatch repair (MMR) protein immunohistochemistry (IHC) are two testing methods that are often used in combination due to reported $4 \%$ discordance rates between them. Several criteria have been proposed to guide testing, and the WHO has even incorporated MSI into tumor grade assignment. However, none recommend specific test modalities and, thus, institutional practices are variable. We performed this study to explore current practices regarding LS screening.

Design: An 11-item survey that queried clinical practices regarding MMR testing for LS screening was sent to 185 institutions, including 163 in the US. Topics included case selection criteria, screening test (IHC vs. PCR), follow-up studies for MLH1/PMS2 deficient tumors, and compliance with WHO recommendations. Given the financial costs of molecular tests, we asked respondents if they knew whether their institutions were reimbursed per test performed.

Results: 93 respondents described their practices as academic $(n=57)$ community $(n=28)$, and private laboratory $(n=8)$. Overall, $85 \%$ reported universal screening and $58 \%$ screened biopsy samples. Most $(78 \%$ utilized IHC alone, $18 \%$ performed both IHC and PCR, and $2 \%$ used PCR alone. $59 \%$ estimated $<1 \%$ discordance rates between $\mathrm{IHC}$ and PCR when dual testing was performed. When tumors showed loss of MLH1/PMS2, 63\% evaluated them for BRAF mutations, 13\% assessed MLH1-promoter hypermethylation, and 13\% performed both tests $53 \%$ of respondents performed $\mathrm{MLH}-1$ promoter hypermethylation when BRAF was wild-type. Only $24 \%$ of respondents complied with WHO recommendations to consider all MMR-deficient tumors to 
be low-grade. Respondents in academic settings were more likely to perform universal testing and use both IHC and PCR than those in community practice $(p=0.08$ and $p=0.006$, respectively). Despite widespread usage, most $(65 \%)$ respondents were not familiar with their own institutional reimbursement policies for molecular tests.

Conclusions: $85 \%$ respondents reported universal screening; most utilized IHC alone and evaluated biopsy material. Very low $(<1 \%)$ discordance rates between IHC and PCR were seen in most centers. Despite WHO recommendations, $<25 \%$ of practitioners assigned histologic grade based on MMR status. Of interest, $>60 \%$ of pathologists who routinely evaluate colorectal cancers for Lynch syndrome are unaware of whether molecular tests were reimbursed.

\section{CMV Reactivation Is Rare in the Era of Biologic Therapies for Inflammatory Bowel Disease}

Erika Hissong ${ }^{1}$, Rhonda Yantiss ${ }^{2} .{ }^{1} \mathrm{New}$ York, NY, ${ }^{2}$ Weill Cornell Medical College, New York, NY

Background: Cytomegalovirus (CMV) shows a predilection for inflamed mucosae in patients with inflammatory bowel disease (IBD). Detection rates of $10-25 \%$ have been reported among patients with refractory disease, leading many pathologists to obtain CMV immunostains whenever they encounter severe colitis. We suspect that CMV detection is heavily influenced by corticosteroid therapy and, thus, viral reactivation may be less common now that biologic agents are increasingly used to treat both ulcerative colitis (UC) and Crohn disease (CD). We performed this study to explore CMV detection rates over time and determine whether detection is related to type of therapy used to treat IBD.

Design: We collected cases of chronic colitis with ulcers from 2002 2017, and searched medical records to identify patients with well documented therapeutic interventions. Patients with new-onset IBD were excluded. Selected patients were placed in two groups based on medical regimen: corticosteroid-dependent and maintenance with biologic agents. Patients in the latter group were free of corticosteroid use for at least 6 months prior to colonoscopy. Histologic sections were reviewed and cases with viral inclusions were not further evaluated; the remainder were subjected to CMV immunohistochemical stains.

Results: There were 155 patients in the corticosteroid-dependent group (male/female: $49 / 29$, mean age: 46 years); most $(78 \%)$ of them had UC and were identified from the 2002-2009 period (77\%). 147 patients were maintained with biologic agents (male/female: 79/68, mean age: 43 years). Patients with UC (50\%) and CD (50\%) were equally represented in this group and most $(98 \%)$ of them were seen from 2010-2017. Cytomegalovirus was detected in 15 (10\%) patients; $67 \%$ were diagnosed between 2002-2009 and 33\% were diagnosed between 2010-2017. All IBD patients with CMV in colonic biopsies were corticosteroid dependent $(p=<0.001$ versus patients maintained with biologic agents).

Conclusions: CMV reactivation in IBD patients is strongly associated with corticosteroid dependence. As a result, the incidence of CMV detection in refractory IBD has decreased as treatment strategies have shifted away from maintenance corticosteroid therapy. Immunostains for CMV should be used to evaluate corticosteroid dependent patients with refractory disease, as they will detect CMV in approximately $10 \%$ of cases. However, they are of no value among patients who have disease flares while receiving biologic agents for long term management of IBD symptoms.

\section{Phospho-histone H3 in Gastrointestinal Stromal Tumors Risk Stratification Evaluated by Manual Counting and Computer-Assisted Image Analysis}

Yan Huang ${ }^{1}$, Mansoor Nasim ${ }^{1}$, Yihe Yang ${ }^{2}$, Lili Lee ${ }^{1} .{ }^{1}$ Northwell Health Hofstra Northwell School of Medicine, New Hyde Park, NY, ${ }^{2}$ Northwell Health Hofstra Northwell School of Medicine, Lake Success, NY

Background: Risk evaluation of gastrointestinal stromal tumors (GISTs) relies on mitotic index, size and location of the tumor. However, manual mitotic counting on H\&E stained slides (MMC-HE) is inefficient with low reproducibility. Phospho-histone $\mathrm{H} 3(\mathrm{PHH})$ is associated with mitotic chromatin condensation in late G2 and $\mathrm{M}$ phase of the cell cycle. Manual count of $\mathrm{PHH} 3$ positive cells on immunohistochemical stained slides (MC-PHH3) has been shown to have comparable reliability to MMC-HE. This study aims to confirm the reliability of MC-PHH3 in GISTs compared to MMC-HE and then to further compare MC-PHH3 to computer-assisted image analysis of $\mathrm{PHH} 3$ positive cells (Comp-PHH3)

Design: The study included 119 patients with GISTs from our institution from 2010 to 2017. We reviewed the tumor size, location, mitotic count, patient's sex, age, and other tumor parameters. $\mathrm{PHH} 3$ immunohistochemical stains were performed. MC-PHH3 was assessed by three pathologists as counts/50 HPF $\left(5 \mathrm{~mm}^{2}\right)$. Whole slide images were captured and the tumor area with greatest mitotic activity was manually identified. The PHH3-positive cells were automatically counted in $0.5 \mathrm{~mm}^{2}$ using Ventana Virtuoso software. Results were compared using Spearman correlation and Lin's concordance. The inter-observer agreement for MC-PHH3 was calculated using the interclass correlation coefficient.

Results: The median age of 119 patients was 67 years (35 to 83 years) and 64 patients were males $(53.8 \%)$. The locations of the GISTs included stomach $(n=89)$, small bowel $(n=21)$, large bowel $(n=3)$, and extra-Gl tract $(n=6)$. The tumor size ranged from 0.5 to $30 \mathrm{~cm}$. MMC $\mathrm{HE}$ ranged from 0 to $157 / 50 \mathrm{HPF}\left(5 \mathrm{~mm}^{2}\right)$. MC-PHH3 ranged from 0 to $35.6 / 50$ HPF $\left(5 \mathrm{~mm}^{2}\right)$. Comp-PHH3 ranged from 0 to $66 / 0.5 \mathrm{~mm}^{2}$. Interclass correlation coefficient indicates good agreement between the three pathologists for MC-PHH3 (ICC=0.74, $\mathrm{p}=0.42)$. There is a strong correlation between MMC-HE and MC-PHH3. The Spearman correlation coefficient was 0.63 ( $p<0.0001)$. Lin's concordance furthe indicated a moderate diagnostic agreement between MC-PHH3 and Comp-PHH3 (Figure 2).
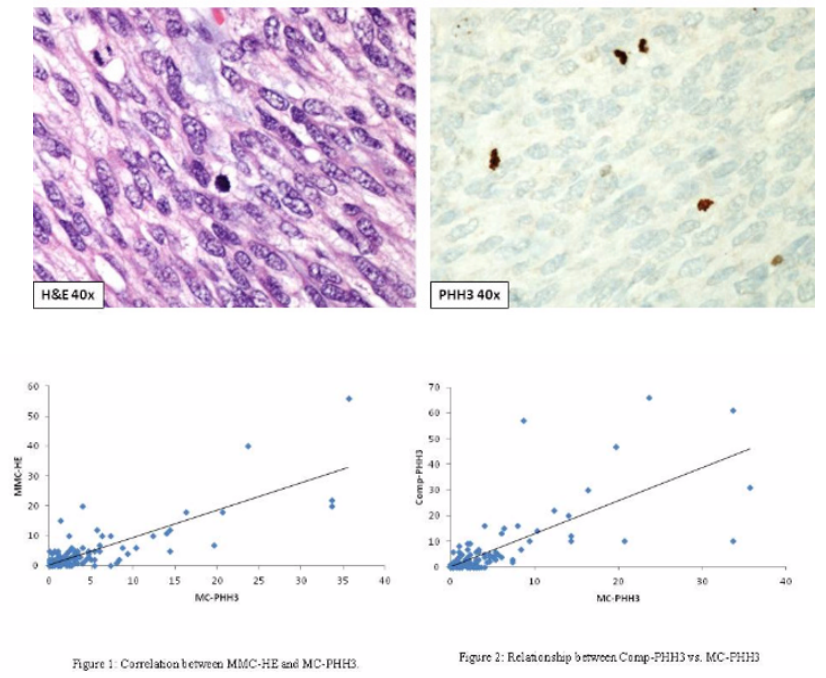

Conclusions: MC-PHH3 is proposed as a superior alternative to MMC $\mathrm{HE}$ with potential application in GIST reporting and prognostication. Furthermore, Comp-PHH3 is a valid alternative to MC-PHH3. Since GIST risk stratification relies heavily on mitotic count, we propose mitotic counting by $\mathrm{PHH} 3$ immunohistochemical stain slides as a more precise and reproducible method than manual mitotic counting by $H \& E$ stained slides alone.

\section{Review of the Minimum Immunostaining} Criteria for HER2 Determination in Gastric and Gastroesophageal Junction Cancer Endoscopic Biopsies: Is Membrane Expression in Five Cells Enough?

Mar Iglesias', Maria Teresa Rodrigo ${ }^{2}$, Ghita Tagmouti², Josep Sabate ${ }^{3}$ Lorena Diaz Sanchez, Ariadna Torner ${ }^{5}$, Marta Salido ${ }^{6}$, Dolores Naranjo-Hans ${ }^{2}$, Javier Gimeno ${ }^{5}$, Anna Puiggros ${ }^{7}$, Blanca Espinet $^{8}$ Belen Lloveras ${ }^{2}$. ${ }^{1}$ Hospital del Mar, Barcelona, ${ }^{2} \mathrm{Hospital}$ del Mar Barcelona, ${ }^{3}$ Hospital del Mar., ${ }^{4}$ Parc de Salut Mar, Hospital del Mar Barcelona, ${ }^{5}$ Hospital del Mar., ${ }^{6}$ Hospital del Mar, Barcelona, ${ }^{7}$ IMIMHospital del Mar, 8IMIM-Hospital del Mar, Barcelona, Catalonia

Background: HER2 overexpression/amplification should be determined in gastric (GC) or gastro esophageal junction cancer (GEJC) tributary for anti-HER2 treatment. In cases with a borderline immunohistochemistry (IHC) result (moderate intensity) an assessment of the amplification of the gene by in situ hybridization (ISH) analysis must be performed. The IHC cut-off varies between surgical and endoscopy samples. The minimal threshold of cellular membrane expression in biopsy is five cells. Our aim is to analyze the correlation of this threshold when expression is borderline with the amplification of the gene by ISH.

Design: Retrospective review of GC and GEJC cases in the Hospital del Mar of Barcelona between 2010 and 2016, with HER2 2+ studied by inmunohistochemistry. The exact number or percentage of cells with expression of HER2 and the status of the gene using in situ hybridization were recorded and compared.

Results: We have reviewed 86 cases (63 biopsies and 23 surgical specimens). From the 63 endoscopic biopsies, we selected 46 cases HER2 2+ with a homogeneous intensity of HER2. We found very weak staining of HER2 in 9 cases $(14.3 \%)$, weak in 30 cases $(47.6 \%)$, and moderate in 7 cases $(11.1 \%)$.

In terms of the number of cells and amplification, 2 cases with very weak expression depicted between 5 and $10 \%$ stained cells and were non-amplified by ISH and 7 cases had more than $10 \%$ positive cells and one of them were amplified. Fourteen cases with weak positivity presented $\geq 10 \%$ stained cells and 12 of them were non amplified, 5 cases with $\geq 5-<10 \%$ stained cells were non amplified and among 
11 cases with $<5 \%$ one case was amplified. All 7 moderately stained cases presented staining in $\geq 10 \%$ neoplastic cells and 3 of them were amplified.

Conclusions: We have proved that the minimal threshold criteria used nowadays in biopsies, for HER2 testing, detected some amplified cases by ISH, even when there are only very few positive cells with weak membrane expression. The real impact of this finding regarding response to therapy deserves further analysis.

\section{Expression of CD47 in Small Intestinal Neuroendocrine Tumors Is Strongly Associated with Lymph Node Metastasis, a Novel Potential Prognostic Predictor}

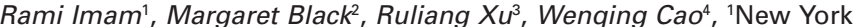
University, New York, NY, ${ }^{2}$ NYU Langone Medical Center, Long Island City, NY, ${ }^{3}$ New York University Medical Center, New York, NY, ${ }^{4} \mathrm{New}$ York University Langone Medical Center, New York, NY

Background: The incidence of small intestinal neuroendocrine tumors (SI-NETs) has significantly increased over the last several decades, with overall 5 -year survival rates varying from $56 \%$ to $79 \%$. Although tumor size, mitotic rate and $\mathrm{Ki}-67$ index provide relevant information related to tumor aggressiveness and prognosis, sensitive and specific biomarkers that can accurately predict prognosis are generally lacking. CD47 is an integrin-associated protein which inhibits macrophage phagocytosis. Overexpression olf CD47 has been associated with increased tumor growth and metastasis in a variety of malignancies and anti-CD47 treatments are being investigated in hepatocellular carcinoma and pancreatic neuroendocrine tumors (P-NETs). In this study, we examine the prognostic implications of CD47 expression in SI-NETs.

Design: 25 well differentiated SI-NET (13 G1 and 12 G2) resection specimens were selected and analyzed for CD47 expression using immunohistochemistry. The cohort included 9 females and 16 males, ranging from 21 to 77 years of age. $\mathrm{H}$-score method was used to evaluate both staining intensity (0-3) and percentage of positive cells. One-way analysis of variance or Student's t-test was utilized to correlate mean $\mathrm{H}$-score with various clinical parameters.

Results: Positive cytoplasmic and membranous CD47 staining was seen in all samples of SI-NETS, but not in adjacent noncancerous small intestinal epithelium. The staining pattern is diffuse. At the time of resection, 17 cases showed lymph node metastasis. The $\mathrm{H}$-score of CD47 does not relate to tumor size, stage, grade, mitotic rate or Ki67 proliferation index. Interestingly, the cases with lymph node metastasis have significant lower $\mathrm{H}$-score of CD47 than those without lymph node metastasis $(192.9 \pm 8.4$ vs $233.1+19.4, P=0.035)$. Overexpression of CD47 inversely associates with lymph node metastasis in SI-NETS.

Conclusions: This is the first study to investigate the expression of CD47 in human SI-NETs and its correlation with currently suggested prognostic parameters. We found CD47 overexpression in all SINETs, and CD47 expression significantly correlated with lymph node metastasis. Our data suggests CD47 might be a novel marker for predicting prognosis of SI-NETs.

\section{Immunostaining for Cytomegalovirus (CMV) in Gastrointestinal (GI) Biopsies: Significance of Rare Staining Cells}

Bogdan Isaila', Razvan Lapadat', Michael Moravek' ${ }^{1}$ Xianzhong Ding Stefan Pambuccian', Mohammed Atieh'. 'Loyola University Medical Center, Maywood, IL

Background: $\mathrm{CMV}$ can cause clinically significant GI injury especially in immunocompromised patients and patients with inflammatory bowel disease (IBD). CMV inclusions, when seen on $\mathrm{H} \& \mathrm{E}$ Gl biopsies are specific; however pathologists may perform CMV immunohistochemical (IHC) stains for increased sensitivity. CMV IHC stains usually confirm the inclusions identified or suspected on $\mathrm{H} \& \mathrm{E}$ examination and often show many more infected cells than initially suspected. Occasionally, however, very rare IHC-positive cells are encountered, the significance of which is unclear. The aim of this study was to determine the clinical significance of such rare IHC positive cells by correlating their presence with the clinical, histologic and serum CMV molecular studies.

Design: We searched our department's electronic database (Sunquest Copath v6) for all Gl biopsies with CMV immunostaining performed during a 10 year period (9/1/2007-8/31/2017). All cases that were diagnosed as positive were reviewed by a GI pathologist who recorded: the presence and number of inclusions on $\mathrm{H} \& \mathrm{E}$, number and type of $\mathrm{IHC}$ staining cells, and the presence, type and severity of inflammation and ulceration. CMV IHC was considered equivocal/not convincing if staining was not typical for CMV (faint staining/inappropriate localization, etc.). The EMR was searched for clinical diagnosis, concomitant molecula CMV tests, treatment and follow-up.

Results: $2988 / 62506 \mathrm{GI}$ biopsies (4.78\%) had CMV stains performed
(3.01\%) were diagnosed as CMV positive by the signout pathologist and were the subject of this study. The correlation of CMV staining patterns, clinical, histologic and serum CMV results is shown in Table1.

\begin{tabular}{|c|c|c|c|c|c|}
\hline & & $\begin{array}{l}\text { IHC not } \\
\text { convinc- } \\
\text { ing } \\
(n=18)\end{array}$ & $\begin{array}{l}\text { IHC rare } \\
(1-2 \text { cells }) \\
(n=30)\end{array}$ & $\begin{array}{l}\text { IHC few } \\
(3-5 \\
\text { cells }) \\
(n=12)\end{array}$ & $\begin{array}{l}\text { IHC } \\
\text { many (>5 } \\
\text { cells) } \\
(\mathrm{n}=30)\end{array}$ \\
\hline \multirow{3}{*}{ Clinical } & $\begin{array}{l}\text { Immunocompro- } \\
\text { mised patient }\end{array}$ & $\begin{array}{l}6 \\
(33.33 \%)\end{array}$ & $\begin{array}{l}13 \\
(43.33 \%)\end{array}$ & $\begin{array}{l}8 \\
(66.67 \%)\end{array}$ & $24(80 \%)$ \\
\hline & Patient with IBD & $\begin{array}{l}5 \\
(27.78 \%)\end{array}$ & $8(26.67 \%)$ & $\begin{array}{l}2 \\
(16.67 \%)\end{array}$ & 0 \\
\hline & $\begin{array}{l}\text { Patient without IBD } \\
\text { or known immuno- } \\
\text { compromise }\end{array}$ & $\begin{array}{l}7 \\
(38.89 \%)\end{array}$ & $9(30 \%)$ & $\begin{array}{l}2 \\
(16.67 \%)\end{array}$ & $6(20 \%)$ \\
\hline \multirow{6}{*}{ Histology } & $\begin{array}{l}\text { Cases with inclu- } \\
\text { sions seen on } \mathrm{H} \& \mathrm{E}\end{array}$ & 0 & $4(13.33 \%)$ & $\begin{array}{l}7 \\
(58.33 \%)\end{array}$ & $\begin{array}{l}28 \\
(93.33 \%)\end{array}$ \\
\hline & $\begin{array}{l}\text { Number of H\&E } \\
\text { inclusions seen } \\
\text { (mean, SD, range) }\end{array}$ & 0 & $\begin{array}{l}0.27 \pm 0.82 \\
(0-4)\end{array}$ & $\begin{array}{l}1.08 \pm \\
1.24 \\
(0-4)\end{array}$ & $\begin{array}{l}11.43 \pm \\
15.27 \\
(0-58)\end{array}$ \\
\hline & $\begin{array}{l}\text { No mucosal inflam- } \\
\text { mation }\end{array}$ & $\begin{array}{l}3 \\
(16.67 \%)\end{array}$ & $1(3.33 \%)$ & $\begin{array}{l}1 \\
(8.33 \%)\end{array}$ & 0 \\
\hline & $\begin{array}{l}\text { Mild mucosal } \\
\text { inflammation }\end{array}$ & $1(5.56 \%)$ & $3(10 \%)$ & 0 & $3(10 \%)$ \\
\hline & $\begin{array}{l}\text { Moderate mucosal } \\
\text { inflammation }\end{array}$ & $\begin{array}{l}7 \\
(38.89 \%)\end{array}$ & $15(50 \%)$ & $\begin{array}{l}1 \\
(8.33 \%)\end{array}$ & $\begin{array}{l}11 \\
(36.67 \%)\end{array}$ \\
\hline & $\begin{array}{l}\text { Severe mucosal } \\
\text { inflammation }\end{array}$ & $\begin{array}{l}7 \\
(38.89 \%)\end{array}$ & $\begin{array}{l}11 \\
(36.67 \%)\end{array}$ & $\begin{array}{l}10 \\
(83.33 \%)\end{array}$ & $\begin{array}{l}16 \\
(53.33 \%)\end{array}$ \\
\hline \multirow{4}{*}{ Molecular } & $\begin{array}{l}\text { Serum CMV Not } \\
\text { performed }(\%)\end{array}$ & $\begin{array}{l}11 \\
(61.11 \%)\end{array}$ & $15(50 \%)$ & $\begin{array}{l}2 \\
(16.67 \%)\end{array}$ & $\begin{array}{l}14 \\
(46.67 \%)\end{array}$ \\
\hline & $\begin{array}{l}\text { Serum CMV nega- } \\
\text { tive }(\%)\end{array}$ & $\begin{array}{l}6 \\
(33.33 \%)\end{array}$ & $6(20 \%)$ & $3(25 \%)$ & $2(6.67 \%)$ \\
\hline & $\begin{array}{l}\text { Serum CMV low } \\
\text { positive (<250 } \\
\text { copies } / \mathrm{ml})(\%)\end{array}$ & 0 & $6(20 \%)$ & $\begin{array}{l}4 \\
(33.33 \%)\end{array}$ & $3(10 \%)$ \\
\hline & $\begin{array}{l}\text { Serum CMV pos- } \\
\text { itive (>250copies/ } \\
\text { ml) }(\%)\end{array}$ & $1(5.56 \%)$ & $3(10 \%)$ & $3(25 \%)$ & $\begin{array}{l}11 \\
(36.67 \%)\end{array}$ \\
\hline
\end{tabular}

Conclusions: 1. Increasing numbers of IHC staining cells correlate with immunocompromise, the presence and number of inclusions seen on H\&E, severity of inflammation, and positivity of serum molecular tests for CMV.

2. Equivocal/not convincing CMV IHC staining should probably be considered as negative, or repeated.

3. Reporting of CMV IHC stain results in the absence of inclusions on H\&E could be reported semiquantitatively, since increasing numbers of staining cells correlate with increasing likelihood of clinically significant CMV infection.

752 IBD associated colorectal carcinomas are frequently associated with loss of SATB2 expression and an aberrant mucin profile

Mai Iwaya', Hiroyoshi Ota², Yoko Tateish ${ }^{3}$, Tomoyuki Nakajima ${ }^{4}$ Robert Riddell', James Conner'. 'Mount Sinai Hospital, Toronto, ON' ${ }^{2}$ Shinshu University School of Health Sciences, Matsumoto, Nagano, ${ }^{3}$ Mount Sinai Hospital, ${ }^{4}$ Shinshu University Hospital, Matsumoto, Nagano-ken

Background: SATB2 is a sensitive and specific diagnostic marker for colorectal cancer (CRC) and reduced expression of SATB2 in $\mathrm{CRC}$ is associated with a poor prognosis. IBD-associated CRC often shows distinct morphologic and molecular phenotypes compared to sporadic cases. However, the sensitivity of SATB2 expression has not been described in a large series of IBD-associated CRC.

Design: 53 consecutive IBD associated CRCs from 52 resections between 2010 and 2017 were examined and 20 sporadic CRCs were used as controls. Immunohistochemistry for SATB2, CDX2, MUC5AC, MUC6, and mismatch repair proteins was performed. The extent of staining for each marker was scored semiquantitatively (no staining; $<5 \%$; $5-25 \%$; $26-50 \%$; $51-75 \%$; $76-100 \%$ ), and the maximum intensity was graded as negative, weak, moderate, or strong.

Results: SATB2 had moderate or strong staining in $45.3 \%(24 / 53$ of IBD-associated CRC cases and $85.0 \%(17 / 20)$ of sporadic CRCs $(\mathrm{P}=0.003)$. In contrast, $\mathrm{CDX} 2$ was moderate or strong in $85.0 \%(45 / 53)$ of IBD-associated CRCs and $100 \%(20 / 20)$ sporadic CRCs ( $P=0.097)$. Six of the 8 IBD-associated CRCs with loss of CDX2 also had loss of SATB2. Absence of SATB2 expression was significantly more frequent in cases with mucinous histology $(p=0.015)$. Although the relationships between expression of SATB2 and MUC5AC or MUC6 did not reach statistical significance, MUC5AC was more frequently expressed in IBD-associated colorectal cancer compared to sporadic cases $(64.2 \%$ 
vs $35.0 \% ; p=0.048$ ). Cases with negative or weak SATB2 expression also had a higher ratio of nodal metastasis compared to cases with moderate or strong expression $(\mathrm{P}=0.033)$. There was no association between SATB2 expression and tumor grade, location, MMR protein expression status, age, and sex.

Conclusions: SATB2 is lost significantly more frequently in IBD associated CRC compared to sporadic CRC, especially in mucinous carcinomas. IBD associated CRC is also more frequently associated with aberrant expression of MUC5AC. While the exact mechanism for this effect remains undefined, these results raise the possibility that a significant proportion of IBD-associated CRC may lose intestinal differentiation, in favor of a gastric-like phenotype, and may be associated with more aggressive behavior given the increased rate of nodal metastasis.

\section{Relationship between carcinoma subtype and overlying dysplasia in IBD-associated colorectal carcinoma}

Mai Iwaya', Hiroyoshi Ota², Yoko Tateishi ${ }^{3}$, Mayada Basheer ${ }^{4}$, Robert Riddell', James Conner'. 'Mount Sinai Hospital, Toronto, ON, ${ }^{2}$ Shinshu University School of Health Sciences, Matsumoto, Nagano, ${ }^{3}$ Mount Sinai Hospital, ${ }^{4}$ University of Toronto, Toronto, ON

Background: A new classification system for subtypes of dysplasia in IBD was proposed in 2017 by Harpaz et.al. (Mod Pathol. Feb 2017 (30:supplement $2 \mathrm{~s})$ ), raising the question of the relationship between these subtypes and the morphology of invasive carcinomas to which they may give rise. The aim of this study was to investigate this relationship by characterizing tumor morphology along with adjacent or overlying dysplasia subtype in a large cohort of IBDassociated colorectal carcinomas (CRC).

Design: 92 consecutive IBD-associated CRCs (including synchronous tumors from a total of 88 colectomy specimens) resected between 2000 and 2017 were examined. 32 patients had Crohn's colitis, 55 patients had ulcerative colitis, and 1 had IBD-unclassified. $4 \mathrm{Gl}$ pathologists reviewed $\mathrm{H} \& \mathrm{E}$ slides containing carcinoma and dysplasia (when present). Subtypes of carcinoma were classified as: conventional, mucinous, serrated, low grade tubuloglandular, and others. Dysplasia was classified as: conventional, serrated, hypermucinous, terminally differentiated, goblet cell depleted, and others. Conventional dysplasia was further divided into tubular adenoma-like and villous adenomalike.

Results: Carcinoma subtypes were: 49 conventional, 20 mucinous, 14 low grade tubuloglandular, 7 serrated, and 2 of special subtype (1 hepatoid-enteroblastic, 1 carcinoma ex goblet cell carcinoid-like). Adjacent/overlying dysplasia was found in 77 cases, some of which had multiple morphologies, for a total of 111 dysplasia subtypes: 17 conventional tubular adenoma-like, 17 conventional villous adenomalike, 29 serrated, 11 hypermucinous, 15 terminally differentiated, 17 goblet cell depleted, and 5 unclassified. Conventional type CRC was significantly associated with conventional tubular adenoma-like dysplasia $(p=0.039)$; mucinous adenocarcinoma with conventional villous adenoma like dysplasia $(p=0.014)$; and low grade tubuloglandular adenocarcinoma with goblet cell depleted dysplasia $(p=0.002)$. There were no significant associations between CRC morphology and tumor location, IBD phenotype, age, or sex.

Conclusions: In a large series of IBD-associated CRC, approximately half of cases exhibited conventional morphology, with the next most common subtypes being mucinous, low-grade tubuloglandular, and serrated. The association between these groups and specific subtypes of dysplasia proposed by Harpaz et al. suggests that the genetic basis underlying variation in dysplasia morphology persists in the form of distinct carcinoma subtypes.

754 Diagnostic Usability of Peptide Nucleic Acid Probe (PNA) based MSI Test: A Comparison Study of Immunohistochemistry and Three Molecular Tests for Microsatellite Instability Status

Mi Jang ${ }^{1}$, Hoguen Kim ${ }^{2}$. ${ }^{Y}$ Yonsei University College of Medicine, Seoul, $\mathrm{SE},{ }^{2}$ Seoul

Background: A subset of colorectal carcinoma (CRC) shows high microsatellite instability (MSI-H) phenotype and the detection of MSI is important for evaluating prognosis and predicting outcome for the objective therapy. MSI has been performed by several standard methods including PCR fragment analysis, and immunohistochemistry (IHC). Although these methods show relatively high specificity and sensitivity, there are some substantial barriers to the use of these methods. Recently, we developed peptide nucleic acid probe (PNA)mediated real-time PCR-based MSI analysis test and tried to compare the accuracy of the variable MSI analysis test.

Design: MSI status of 166 samples were compared using four different MSI tests: PCR fragment analysis with five $\mathrm{NCl}$ markers (PCR-NCI), PCR fragment analysis with five quasi-monomorphic mononucleotide markers (PCR-MNR), peptide nucleic acid probe (PNA)-mediated real-time PCR-based sensing method (PNA-PCR), and IHC analysis with four MMR proteins. IHC was performed on the surgically resected specimen and the percent of nuclear expression was measured. We defined MSI-H as a diagnosis of MSI-H by at least two out of three standard tests. Based on determined MSI status, we evaluated the diagnostic sensitivity and specificity of the PNA-PCR PCR-MNR methods and IHC analysis with variable cut off value.

Results: Of the $166 \mathrm{CRCs}, \mathrm{MSI}-\mathrm{H}$ and microsatellite stable were determined in 76 and $90 \mathrm{CRCs}$, respectively. PCR-NCl and PNA PCR showed $100 \%$ sensitivity and $100 \%$ specificity. By contrast PCR-MNR showed $98.68 \%$ sensitivity and $100 \%$ specificity. When we set the cut-off value as less than $5 \%$ mismatch repair protein expression, immunohistochemistry showed $98.68 \%$ sensitivity and $100 \%$ specificity. When the complete loss of MMR expression was used as the cut off value, the sensitivity was $90.79 \%$. The performance comparison between PCR-MNR and PNA-PCR, which use the same five markers, showed that PNA-PCR could detect alterations in all five mononucleotide repeat markers in samples containing down to $5 \%$ MSI-H DNAs, whereas PCR-MNR required at least $20 \%$ MSI-H DNAs to achieve the same performance.

Conclusions: Combined interpretation using both $\mathrm{IHC}$ with appropriate cut-off value and PCR based MSI test will provide useful information about MSI status. We suggest that PNA-PCR can be used as a simple alternative with higher sensitivity and no limit on specimens containing a small proportion of tumor cells for the analysis of MSI status in cancers.

\section{Traditional serrated adenomas: pathological, endoscopical and molecular and epigenetic characteristics}

Sara Javidiparsijani', Lela Buckingham², Arlen Brickman', Shriram Jakate'. 'Rush University Medical Center, Chicago, IL

Background: Traditional serrated adenoma (TSA) is the rarest type of serrated polyps comprising for $<1 \%$ of all serrated polyps. The majority are located in the left colon. While their microscopic features are well documented, we observed distinctive endoscopic, morphologic and molecular characteristics. We reviewed 25 cases of TSA to determine these characteristics.

Design: From our Medical Center's pathology and gastroenterology databases of past four years (2014-2017), we identified 25 patients with TSA (ages 30-75, $\mathrm{F}: \mathrm{M}=0.7: 1$ ). We reviewed their endoscopic and microscopic features and performed Immunohistochemical studies for MMR (MLH1, PMS2, MSH2 and MSH6). BRAF V600E and KRAS mutations (codons 11, 12, 13) were assessed by PCR and pyrosequencing, respectively.Global methylation (5-methyl cytosine ELISA) was compared with our prior data on normal colon (30 cases) tubular adenomas (TA, 88 cases) and sessile serrated adenomas (SSA, 8 cases).

Results: All 25 TSAs were left-sided (7/25 rectum, 12/25 sigmoid 2/25 descending, 4/25 transverse). Endoscopically, TSAs were pedunculated or semi-sessile and amenable to endoscopic excision. Additionally, these were often congested and showed a characteristic "mosaic" or "spaghetti" surface pattern that has not been previously reported. Microscopically, there was at least low grade dysplasia, but it was patchy and not uniform unlike an adenomatous polyp Immunohistochemically, all MMR proteins showed wild type or norma staining pattern. BRAF V600E mutation was detected in 15/25 cases and KRAS mutation was present in 4/25 TSAs. Two cases showed both $K R A S$ and $B R A F$ mutations.TSAs had lower mean 5-mC methylation level compared to normal $(p=0.002)$ but their mean methylation level were not significantly different from TAs and SSAs. Among the TSAs, those with KRAS mutation had marginaly higher methylation level compared to TSAs with BRAF mutation ( $\mathrm{p}=0.058$ ).

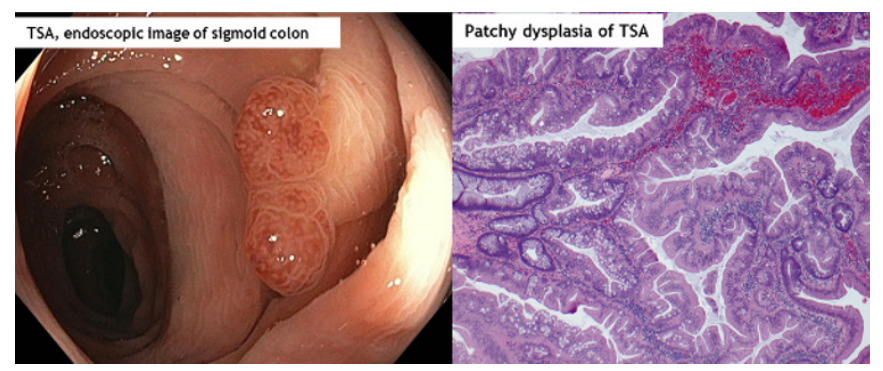




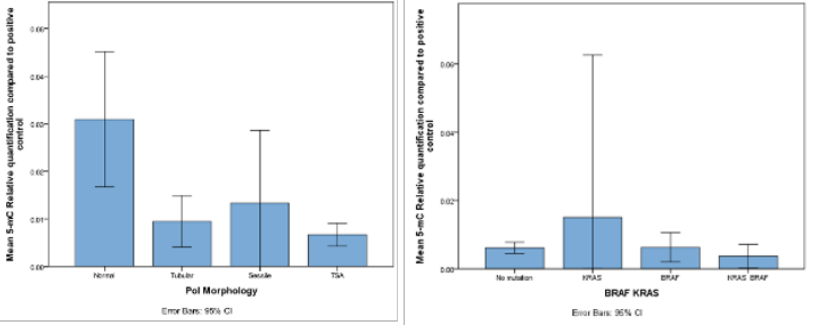

Conclusions: Endoscopically TSAs are left-sided, pedunculated or semi-sessile, resectable and show characteristic "spaghetti" surface pattern and congestion. The dysplastic glands show patchy rather than diffuse distribution. The BRAF mutation in TSA is well known but simultaneous BRAF and KRAS mutation has not been reported previously in TSA. Methylation studies showed lower mean methylation compared to normal. Higher methylation level in KRAS mutated TSA compared to BRAF mutated TSAs may reflect the possible lower malignant potential of these TSAs.

\section{Examination of Patient Risk and Histologic Features} in Esophageal Biopsies Indefinite for Dysplasia

William Jeck', Juliana Andrici ${ }^{2}$, Agoston (Tony) Agoston ${ }^{3}$, Martin Taylor $^{1}$, Matthew Stachler, Robert Odze ${ }^{3}$, Vikram Deshpande ${ }^{5}$. 'Boston, MA, ${ }^{2}$ Brigham and Women's Hospital, Boston, MA, ${ }^{3}$ Brigham \& Women's Hospital, Boston, MA, 'Brigham and Women's Hospital, East Boston, MA, ${ }^{5}$ Massachusetts General Hospital, Boston, MA

Background: Despite decades of experience with Barrett's related neoplasia, occasional esophageal biopsies must be labeled as "indefinite" for dysplasia. Reasons to call a biopsy "indefinite" are several, but include the presence of inflammation or, in the absence of inflammation, crypt changes similar to those in dysplasia that do not reach the mucosal surface. Uncertainties remain surrounding outcomes in these patients, as well as the important histologic features to assess in this situation.

Design:We identified all patients with esophageal, orgastroesophageal junction biopsy at two academic hospitals in a 25 year time interval. Cases were placed in 6 categories: negative for Barrett's or related neoplasia, Barrett's esophagus, indefinite for dysplasia, low-grade dysplasia, high-grade dysplasia, and adenocarcinoma. A set of patients with an explicit diagnosis of "indefinite for dysplasia", at least 5 years of follow-up biopsies, and no other prior evidence of dysplasia, were reviewed by an expert pathologist (VD) while blinded to patient outcome $(n=31)$. Cases were first categorized as inflamed or not inflamed. Uninflamed biopsies were then assessed for crypt dysplasia-like atypia and graded as mild, moderate, or severe.

Results: The cohort contained 61801 patients and 103236 unique cases. Patients with a first-time diagnosis of "indefinite" for dysplasia (581 patients) showed a 3.8-fold increased hazard of later conversion to high-grade dysplasia or adenocarcinoma as compared to Barrett's. In comparison, patients with a first-time diagnosis of low-grade dysplasia showed a 7.4-fold increased hazard compared to Barrett's, about twice that of the indefinite cases (see figure; gray areas show $95 \%$ confidence interval). In the cohort subjected to review, $19 \%$ of patients eventually showed definite dysplasia in follow-up. Of patients with inflammation, $12 \%$ showed later definite dysplasia. Patient's with uninflamed biopsies stratified in keeping with the grade of crypt dysplasia-like atypia; Mild, moderate, and severe grades progressed in $0 \%, 8 \%$, and $33 \%$ of patients, respectively.

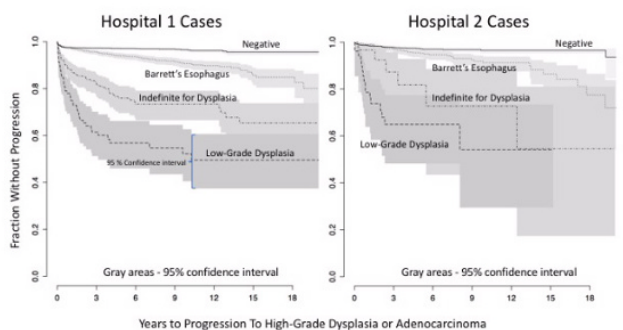

Conclusions: We describe an increased risk of later definite dysplasia in patients receiving a first-time diagnosis that is indefinite for dysplasia, substantiating the need for closer clinical follow-up in these patients. We also find that, for patients with indefinite biopsies without inflammation, the severity of crypt dysplasia-like atypia may be an important predictor of a patient outcome.
757 Impact of Referral Center Pathology Review on Diagnosis and Management of Patients with Appendiceal Neoplasms

Jolanta Jedrzkiewicz' ${ }^{1}$ Yoko Tateishi ${ }^{2}$, Richard Kirsch ${ }^{3}$, James Conner ${ }^{4}$ Emily L Taylor ${ }^{5}$, Anand Govindarajan 6 . 'University of Utah, Salt Lake City, UT, ${ }^{2}$ Toronto, ON, ${ }^{3}$ Mount Sinai Hospital, ${ }^{4}$ Mount Sina Hospital, Toronto, ON, ${ }^{5}$ Mount Sinai Hospital, Toronto, ON, Canada, ${ }^{6}$ Department of Surgery, Mount Sinai Hospital, University of Toronto, Toronto, ON, Canada

Background: Pathologic diagnosis and reporting of appendiceal lesions may be challenging because of their relative rarity. However data on the clinical impact of their review by specialized gastrointestinal pathologists are limited. The objective of this study was to determine whether pathology review at a referral center resulted in significant changes in diagnosis and clinical management of lesions originally evaluated at referring hospitals.

Design: We conducted a retrospective cohort study, including all patients with a diagnosis of appendiceal neoplasm seen at a single tertiary-care referral center in Ontario, Canada from 2010-2016. Pathology specimens from all patients underwent second review by experienced gastrointestinal pathologists. The rate of discordance between original and review diagnoses, the type of discordance, and the impact of any discordance on patient management were recorded. A change in nomenclature only was not categorized as a discordance.

Results: A total of 145 patients were included, with the following pathologic diagnoses: mucinous neoplasm $(n=91)$, colonic type adenocarcinoma $(n=12)$, goblet cell tumor $(n=24)$, adenoma $(n=9)$, neuroendocrine tumor $(\mathrm{n}=2)$ and non-neoplastic/benign lesion $(\mathrm{n}=7)$. One or more changes in diagnoses were found in 36 cases $(24.8 \%)$. The majority of the cases had one change $(n=32)$, three cases had 2 changes and one case had 3 changes. Overall, changes within the same category of interpretation were the most common $(24 \%)$, followed by change in stage $(17 \%)$, grade $(15 \%)$ and categorical interpretation $(12 \%)$. In $10 / 36$ patients $(27.7 \%)$, the diagnostic change led to a significant change in management, including recommendation for additional surveillance $(n=2)$, chemotherapy $(n=6)$, additional surgery $(n=2)$, or discontinuation of further surveillance $(n=3)$.

Conclusions: In patients with appendiceal neoplasms, we found that pathology review by gastrointestinal pathologists at a tertiary-care referral center resulted in a change in diagnosis in $25 \%$ of cases. In almost one-third of these patients, this led to a significant modification of the clinical management plan. Our findings suggest that routine specialist review of appendiceal neoplasms may be warranted.

\section{Histologic Grade Is Prognostically Important Among Colorectal Carcinomas Regardless of Microsatellite Status}

Melanie E Johncilla', Zhengming Chen', Rhonda Yantiss'. 'Weill Cornell Medical College, New York, NY, ${ }^{2}$ Weill Cornell Medicine

Background: Colorectal carcinomas (CRC) are graded based solely on extent of gland formation. Mucinous, signet ring cell, and solid cancers are, by convention, histologically high-grade. Microsatellite unstable (MSI-H) CRC frequently show mucinous or signet ring cell differentiation, and/or solid growth, but the WHO classifies them as low-grade neoplasms to reflect their favorable prognosis compared with microsatellite stable (MSS) cancers. However, some patients with MSI-H CRC present at advanced stage, or have aggressive disease. We performed this study to determine whether established prognostic criteria for MSS CRC have predictive value when applied to MSI-H tumors.

Design: We identified 119 CRC with mismatch repair deficiency by immunohistochemistry and MSI-H by PCR. Cases were evaluated for extent of gland formation, extracellular mucin, signet ring cell differentiation, and solid growth (including medullary features), as well as nuclear grade, tumor infiltrating lymphocytes (TILs) and tumo budding. Relationships between histologic features, pathologic stage, and disease-free survival were assessed.

Results: 116 cases showed a predominant ( $>50 \%$ of tumor volume) growth pattern: $61(53 \%)$ were gland-forming, $25(23 \%)$ were mucinous, $2(2 \%)$ were signet ring cell carcinomas, and $28(24 \%)$ showed solid growth. Three showed heterogeneous features. There were 78 Stage I-II and 41 Stage III-IV tumors. Mucinous and signet ring cell carcinomas were similarly represented among Stage I-II $(60 \%$ and $50 \%$, respectively) and Stage III-IV ( $40 \%$ and $50 \%$, respectively) tumors. $46(59 \%)$ Stage I-II tumors showed $>50 \%$ gland formation compared with $15(37 \%)$ Stage III-IV tumors $(\mathrm{p}=0.01)$. Solid tumors were overly represented among Stage III-IV CRC $(37 \%)$ compared with Stage I-II disease $(18 \%, \mathrm{p}=0.06)$. Overall, a dominant high-grade component (i.e. $>50 \%$ mucinous, signet ring cell, or solid growth), significantly correlated with Stage III-IV disease $(p=0.02)$. Not surprisingly, Stage III-IV disease predicted decreased survival $[p=0.01$, HR $12.99(2.92$ 57.73)]. Nuclear grade, TILs and tumor budding were not significantly associated with pathologic stage or outcome. 
Conclusions: Low-grade (i.e. $>50 \%$ gland-forming) MSI-H carcinomas present at an earlier stage and pursue a more favorable course than those with high $(>50 \%)$ proportions of mucinous, signet ring cell, or solid elements. Although MSI-H is prognostically important, this parameter should not supplant histologic grade in the assessment of colorectal cancers.

\section{A Combination of Histologic Features Differentiates PPI Responsive Esophageal Eosinophilia from Eosinophilic Esophagitis in Pediatric Patients}

Melanie E Johncilla', Jose Jessurun', Sari Kay', Elaine Barfield", Debra Beneck', Amanda Artis'. 'Weill Cornell Medical College, New York, NY, ${ }^{2}$ New York, NY

Background: PPI responsive esophageal eosinophilia (PPI-REE) shares many clinical and histologic features with eosinophilic esophagitis (EoE). Most studies have shown that there are no reliable clinical, endoscopic or histologic criteria to distinguish these conditions prior to PPI therapy. This study was designed to explore whether histologic features of targeted esophageal mucosa biopsies in pediatric patients not treated with PPI can be used to differentiate PPI-REE from EoE.

Design: Pediatric patients who underwent endoscopic esophagea biopsies were identified in a retrospective chart review from 2010 to 2016. Patients with eosinophil rich esophagitis ( $>15$ eosinophils per high power field (HPF)) who had follow-up biopsies after PP treatment were included in the study. Patients with prior PPI or steroid therapy or without follow-up biopsies were excluded. Those with $<5$ eosinophils/HPF on subsequent biopsies were defined as PPI-REE. Those with a persistence of $>15$ eosinophils/HPF were defined as EoE. Biopsy specimens from the distal, middle and proximal esophagus prior to PPI therapy were assessed by two blinded pathologists. The following features were evaluated: regenerative changes (basal zone hyperplasia, elongation of papillae), spongiosis, epithelial cell injury (balloon cells), characteristics of intraepithelial eosinophils (location within the mucosa, number per HPF, eosinophil microabscesses, degranulation), presence of intraepithelial lymphocytes, and lamina propria fibrosis.

Results: Of the 3286 patients with esophageal biopsies during the study period, only 25 fulfilled inclusion criteria; most excluded patients had received prior treatment. Based on response to treatment as assessed by follow-up biopsies, 13 were classified as EoE and 12 as PPI-REE. The following histologic features were significantly associated with EoE as compared with PPI-REE in distal and proximal biopsy specimens: basal zone hyperplasia $>50 \%$, >30 eosinophils/ HPF, eosinophil microabscesses, and lamina propria fibrosis. In midesophageal biopsies, the only significant difference between the two groups was basal cell hyperplasia.

\begin{tabular}{|l|l|l|l|l|}
\hline $\begin{array}{l}\text { Histologic } \\
\text { Feature }\end{array}$ & Site & EoE & PPI-REE & $\mathrm{p}$ \\
\hline \multirow{3}{*}{$\begin{array}{l}\text { Basal zone hy- } \\
\text { perplasia }>50 \%\end{array}$} & Distal & 11 & 2 & 0.001 \\
\cline { 2 - 5 } & Mid & 8 & 1 & 0.008 \\
\cline { 2 - 5 } & Proximal & 9 & 1 & 0.004 \\
\hline \multirow{2}{*}{$\begin{array}{l}\text { HPF eosinophils/ } \\
\text { HPF }\end{array}$} & Distal & 7 & 2 & 0.04 \\
\cline { 2 - 5 } & Mid & 2 & 1 & 0.10 \\
\cline { 2 - 5 } & Proximal & 3 & 0 & 0.006 \\
\hline \multirow{2}{*}{$\begin{array}{l}\text { Eosinophil mi- } \\
\text { croabscesses }\end{array}$} & Distal & 6 & 0 & 0.01 \\
\cline { 2 - 5 } & Mid & 5 & 1 & 0.16 \\
\cline { 2 - 5 } & Proximal & 6 & 0 & 0.01 \\
\hline \multirow{2}{*}{$\begin{array}{l}\text { Lamina propria } \\
\text { fibrosis }\end{array}$} & Distal & 10 & 4 & 0.01 \\
\cline { 2 - 5 } & Mid & 6 & 2 & 0.13 \\
\cline { 2 - 5 } & Proximal & 7 & 1 & 0.04 \\
\hline
\end{tabular}

Conclusions: This is the first study to find significant histologic differences between EoE and PPI-REE. Basal zone hyperplasia of $>50 \%$, numerous eosinophils, eosinophilic abscesses and lamina propria fibrosis of the esophagus are significantly associated with EoE and are especially suggestive of this condition if they are present in biopsies of the proximal esophagus.

\section{Are Enterocolic Mucosal Mast Cell Aggregates Clinically Relevant in Patients Without Suspected Systemic Mastocytosis?}

Melanie E Johncilla', Ian Brown ${ }^{2}$, Jinru Shia ${ }^{3}$, Jason L Hornick', Jose Jessurun ${ }^{5}$, Rhonda Yantiss. . 'Weill Cornell Medical College, New York, NY, ${ }^{2}$ Envoi Pathology, Brisbane, AUS, ${ }^{3}$ Memorial Sloan-Kettering CC, New York, NY, "BBigham and Women's Hospital, Boston, MA, ${ }^{5}$ Weill Cornell Medical College, New York, NY

Background: The WHO considers enterocolic mast cell aggregates that show atypical morphologic or immunohistochemical features sufficient for a diagnosis of systemic mastocytosis. However, most published data regarding atypical enterocolic mast cell aggregates are influenced by inclusion of patients with symptomatic cutaneous or bone marrow involvement. The clinical relevance of seemingly incidental enterocolic mast cell aggregates is unknown. This study aims to describe the clinicopathologic features and prognostic implications of this finding.

Design: We identified 14 patients who met WHO criteria for systemic mastocytosis based on enterocolic biopsy findings, none of whom had clinically suspected mast cell disease. Information regarding clinical signs and symptoms, endoscopic findings, and outcome was obtained. Cases were assessed for mast cell morphology, mucosal distribution, and associated inflammatory cells. Immunohistochemical stains for CD117 were performed on all cases and a subset was stained with CD25.

Results: Four patients were men and 10 were women (mean age: 58 years, range: 36-90); 13 underwent endoscopy. Indications included colon cancer screening $(n=5)$, abdominal pain $(n=3)$, diarrhea $(n=3)$, changing bowel habits $(n=1)$, and dysphagia $(n=1)$. One cance patient had mast cell aggregates in a colectomy specimen. 13 cases occurred in the colon and 1 involved the duodenum. The mucosa was polypoid $(n=9)$, edematous $(n=2)$, or normal $(n=3)$. All cases featured a diffuse proliferation of ovoid mast cells subjacent to the epithelium or involving the entire mucosal thickness. Eosinophils were present in high numbers, and obscured mast cells in $64 \%$ of cases. Mast cells were immunoreactive for CD117 in all cases; $5 / 5$ cases also showed aberrant CD25 staining. Spontaneous resolution of symptoms occurred in patients with diarrhea, pain, or changing bowel habits on follow-up (mean: 51 months). Asymptomatic patients remained asymptomatic on follow-up (mean: 10 months). Five patients had negative bone marrow biopsies and 1 lacked a KIT mutation on peripheral blood analysis.

Conclusions: We describe 14 patients with atypical mast cell infiltrates confined to the GI tract mucosa, none of whom developed evidence of progressive mast cell disease. Although carefu clinical assessment of such patients is reasonable, bone marrow and molecular studies are likely unnecessary as the results will not influence clinical management.

\section{Clinicopathologic Features of Anal and Peri-Anal Squamous Cell Carcinomas and Relationship to Human Papillomavirus}

Jennifer Ju', Edward Stelow². 'University of Virginia, Charlottesville, VA, ${ }^{2}$ Univ. of Virginia Health System, Charlottesville, VA

Background: While anal squamous cell carcinomas (ASCC) represen only $2.4 \%$ of all gastrointestinal malignancies, the incidence has been growing over the past few decades for both men and women. Here we use in situ hybridization (ISH) for high-risk and low-risk human papillomavirus (HR-HPV and LR-HPV) with p16 immunohistochemistry (IHC) to define HPV status for ASCC resected at our institution ove a 30-year period and correlate the findings with clinicopathologic features.

Design: 50 cases of ASCC diagnosed between 1992 and 2016 were used to create a tumor microarray. HR-HPV and LR-HPV ISH (RNAscope technique by Advanced Cell Diagnostics) and p16 IHC (CINtec by Ventana) were performed. Morphology was assessed as keratinizing infiltrative, non-keratinizing infiltrative and warty. Additional histologic features were reviewed for cases negative fo HPV or positive for LR-HPV. Patient demographics, history of other HPV-related genital diseases, and tumor locations were recorded. Presence of lymph node or other metastases identified pathologically or radiographically were noted.

Results: 39 ASCC were related to HR-HPV (all p16 positive; 36 HR HPV positive). 5 tumors were related to LR-HPV (all LR-HPV positive all p16 negative). 6 tumors were not related to HPV (all LR-HPV, HRHPV, and p16 negative). ASCC not secondary to HR-HPV were more likely to be keratinizing and were more likely to involve the peri-anal area $(p<0.05)$ (see table). Of those tumors not associated with HPV none were associated with lichen sclerosis. Tumors associated with LR-HPV were very well differentiated and had a verruco-cystic appearance. No cases associated with LR-HPV were found to have or to develop metastases.

\begin{tabular}{|l|l|l|l|}
\hline & HR-HPV (n=39) & LR-HPV (n=5) & No HPV (n=6) \\
\hline Sex (male:female) & $12: 27$ & $4: 1$ & $2: 4$ \\
\hline Age (mean; range) & $57 ; 31-88$ & $46 ; 37-51$ & $63 ; 46-77$ \\
\hline $\begin{array}{l}\text { History of HPV } \\
\text { lesion }\end{array}$ & 6 & 3 & 0 \\
\hline $\begin{array}{l}\text { Location } \\
\quad \text { Canal }\end{array}$ & 35 & 2 & 4 \\
Verge & 3 & 1 & 0 \\
$\quad$ Peri-Anal & 1 & 2 & 2 \\
\hline $\begin{array}{l}\text { Morphology } \\
\text { Keratinizing } \\
\text { Non-Keratinizing } \\
\text { Warty }\end{array}$ & 6 & 2 & 4 \\
\hline
\end{tabular}


Conclusions: Slightly more than $20 \%$ of ASCC are related to LR-HPV or not related to HPV. These tumors are more likely to be keratinizing and are more likely to involve the peri-anal area. Those associated with LR-HPV appear to have low metastatic potential. In our experience, p16 IHC works well to identify ASCC secondary to HR-HPV.

\section{Indoleamine 2,3 Dioxygenase Expression and Programed Death Ligand-1 in Mismatch Repair Deficient and Intact Colorectal Carcinomas}

Jennifer Ju', Anne Mills', Edward Stelow'. 'University of Virginia, Charlottesville, VA, ${ }^{2}$ Charlottesville, VA, ${ }^{3}$ Univ. of Virginia Health System, Charlottesville, VA

Background: Only a minority of colorectal carcinomas (CRC) respond to immunotherapies blocking the programmed cell death protein and ligand (PD-1/ PD-L1) pathway. Mismatch repair deficient (MMR-d) CRC have changes that impart vulnerability to PD-1/PD-L1 therapy, especially in patients with Lynch Syndrome (LS). However, responses are not uniform and PD-L1 positive staining by IHC does not correlate well with outcome. This may be due to the concomitant presence of other immune modulatory molecules in some cases of CRC. Analysis has shown that MMR-d CRC have a high expression of indoleamine 2,3-dioxygenase (IDO), a T-cell suppressive enzyme. It is possible that dual IDO and PD-1/PD-L1 immunotherapy would benefit MMR-d CRC. IDO inhibition may also be of utility in MMR-intact (MMR-i) CRC where PD-1/PD-L1 antagonism has shown little promise. To investigate this, we compared IDO and PD-L1 expression by IHC in MMR-d and MMR-i CRC.

Design: Tumor microarrays were made of 66 cases of MMR-i CRC and 48 cases of MMR-d CRC: 25 likely LS, 4 possible LS, and 19 likely sporadic cases (determined by MMR protein status and BRAF / MLH1 testing). Tumor morphology was recorded and lymphoplasmacytic infiltrate (LPI) and tumor cell infiltrating lymphocytes (TIL) were scored semi-quantitatively. Microarrays were immunostained for IDO and PD-L1. Staining in tumor cells (TC) and inflammatory cells (IC) were scored semi-quantitatively.

Results: There were no differences in tumor grade. MMR-d CRC were more likely to be mucinous (not significant). Robust LPI was more common for MMR-d CRC than MMR-i CRC [21\% (10/48) vs $12 \%(8 / 66)]$, but this difference was only statistically significant for LS-associated cases $[32 \%(8 / 25)$ vs $12 \%(8 / 66), p=.03]$. MMR-d cases were more likely to have TIL [77\% (37/48) vs $35 \%(23 / 66), p<0.0001]$ and to show IDO and/or PD-L1 staining in both TC and IC. Regarding TC: IDO-only or dual IDO/PD-L1 staining occurred in 10\% of MMR-d cases. Regarding IC: IDO-only or dual IDO/PD-L1 staining occurred in $50 \%$ of MMR-d cases (see figure).

\begin{tabular}{|c|c|c|c|c|}
\hline \multicolumn{5}{|c|}{ Tumor Cell Staining for IDO and PD-L1: > 10\% Staining } \\
\hline & IDO+ only & PD-L1+only & Dual IDO+/PD-L1+ & Dual IDO-/PD-L1- \\
\hline MMR-Deficient & $6 \%(3 / 48)$ & $10 \%(5 / 48)$ & $4 \%(2 / 48)$ & $79 \%(38 / 48)$ \\
\hline Likely LS & $12 \%(3 / 25)$ & $12 \%(3 / 25)$ & $0 \%(0 / 25)$ & $76 \%(19 / 25)$ \\
\hline Possible LS & $0 \%(0 / 4)$ & $0 \%(0 / 4)$ & $0 \%(0 / 4)$ & $100 \%(4 / 4)$ \\
\hline Likely Sporadic & $0 \%(0 / 19)$ & $11 \%(2 / 19)$ & $11 \%(2 / 19)$ & $79 \%(15 / 19)$ \\
\hline MMR-Intact & $2 \%(1 / 66)$ & $2 \%(1 / 66)$ & $5 \%(3 / 66)$ & $92 \%(61 / 66)$ \\
\hline \multicolumn{5}{|c|}{ Inflammatory Cell Staining for IDO and PD-L1: More Than Only Rare Cells Staining } \\
\hline & IDO+ only & PD-L1+ only & Dual IDO+/PD-L1+ & Dual IDO-/PD-L1- \\
\hline MMR-Deficient & $0 \%(0 / 48)$ & $42 \%(20 / 48)$ & $50 \%(24 / 48)$ & $8 \%(4 / 48)$ \\
\hline Likely LS & $0 \%(0 / 25)$ & $36 \%(9 / 25)$ & $52 \%(13 / 25)$ & $12 \%(3 / 25)$ \\
\hline Possible LS & $0 \%(0 / 4)$ & $25 \%(1 / 4)$ & $75 \%(3 / 4)$ & $0 \%(0 / 4)$ \\
\hline Likely Sporadic & $0 \%(0 / 19)$ & $53 \%(10 / 19)$ & $42 \%(8 / 19)$ & $5 \%(1 / 19)$ \\
\hline MMR-Intact & $6 \%(4 / 66)$ & $11 \%(7 / 66)$ & $52 \%(34 / 66)$ & $32 \%(21 / 66)$ \\
\hline
\end{tabular}

Conclusions: TC of a subset of MMR-d and, to a lesser extent, MMR-i CRC express IDO, and co-expression with PD-L1 is common. IC associated with both tumor types were likely to express IDO with a much smaller percentage of cases having only PD-L1 expression. This suggests that anti-IDO immunotherapy could be a useful adjuvant treatment to PD-1/PD-L1 inhibitors in CRC, and furthermore may be a valuable single-agent option for a proportion of PD-L1-negative MMR-d and MMR-i cancers.

\section{Updated American Joint Commission on Cancer Staging for Ileal Neuroendocrine Tumors Predicts Recurrence-Free Interval}

Rossana Kazemimood', Wai Chin Foo', Susan Abraham1, Asif Rashid", Melissa W. Taggart'. ' 'University Of Texas, MD Anderson Cancer Center, Houston, TX

Background: Neuroendocrine tumors are the most common tumor of the small intestine with incidence increasing over the past 30 years. Prognostic factors, however, are limited. Recent changes in the staging of ileal NET (iNET) by the American Joint Commission on Cancer (AJCC) include the segregation of ileal tumors from duodenal primaries, creation of the $\mathrm{N} 2$ category (mesenteric mass $>2 \mathrm{~cm}$ or $\geq 12$ lymph nodes with metastatic disease), separation of the $M$ category into those with liver (1a) or extrahepatic (1b) metastases or both (1c), and collapse of the overall stage subcategories (IIA/IIB and IIIA/IIIB into II and III). Here, we evaluate the effect of these changes on recurrencefree interval and overall survival.
Design: Patients with resected well-differentiated iNET (WHO G1/ G2) from 2010 to 2016 and followed for at least 6 months (mo) at one institution were included. Relevant clinicopathologic features were identified and parameters needed for staging by the 7th and 8th editons of the AJCC staging manual were evaluated. Statistica analyses were performed using SPSS software (version 20; SPSS, Armonk, NY). P $<0.05$ was statistically significant.

Results: Fifty patients were included with a mean follow-up of 34 mo (6-142 mo). Data are presented in Table 1. $25(50 \%)$ patients were alive without disease, $23(46 \%)$ alive with disease and $2(4 \%)$ died (only 1 due to iNET). There was no change in T category between the editions. $\mathrm{M}$ category and overall stage remained the same with the only change in the 8th ed being subcategorization for the $M$ category and the removal of subgroups in stages II and II in the more recent edition. These changes were not associated with differences in survival ( $p=N S$ ) 7 patients had NO disease and, using AJCC 7th ed 43 patients had $\mathrm{N} 1$ disease. Subcategorization of $\mathrm{N}$ disease by 8th ed criteria showed $28(56 \%)$ patients with $\mathrm{N} 1$ and $15(30 \%)$ patients with $\mathrm{N} 2$ disease. $\mathrm{N} 1$ patients had a significantly longer disease-free interval when compared with $\mathrm{N} 2$ patients $(\mathrm{P}=0.04)$. There was a trend toward longer overall survival in N1 vs. N2 patients $(P=0.08)$.

Table 1. Clinicopathologic characteristics of ileal neuroendocrine tumors

\begin{tabular}{|c|c|}
\hline Gender & $\begin{array}{l}\text { Male: } 29(58 \%) \\
\text { Female: } 21(42 \%) \\
\end{array}$ \\
\hline Age (years) & Mean: 59 (35-82) \\
\hline Grade & 1: $39(78 \%) 2: 11(22 \%)$ \\
\hline Size of tumor $(\mathrm{cm})$ & $2.5(0.1-7.0)$ \\
\hline Number of tumors & $\begin{array}{l}\text { 1: } 37(74 \%) \\
\text { 2: } 1(2 \%) \\
\geq 3: 12(24 \%)\end{array}$ \\
\hline Lymphovascular invasion & $42(84 \%)$ \\
\hline Perineural invasion & $34(68 \%)$ \\
\hline Number of positive lymph nodes & $\begin{array}{l}1-11: 37(74 \%) \\
\geq 12: 3(6 \%)\end{array}$ \\
\hline Mesenteric mass $>2 \mathrm{~cm}$ & $\begin{array}{l}\text { Absent: } 37(74 \%) \\
\text { Present: } 13(26 \%) \\
\end{array}$ \\
\hline Liver metastasis & $\begin{array}{l}\text { Present: } 18(36 \%) \\
\text { Synchronous: } 15(30 \%) \\
\text { Metachronous: } 3(6 \%) \\
\end{array}$ \\
\hline T category & $\begin{array}{ll}\text { 7th edition } & \text { 8th edition } \\
\text { T1 2 (4\%) } & \text { T1 2 (4\%) } \\
\text { T2 8 (16\%) } & \text { T2 8 (16\%) } \\
\text { T3 29 (58\%) } & \text { T3 29 (58\%) } \\
\text { T4 11(22\%) } & \text { T4 11(22\%) } \\
\end{array}$ \\
\hline $\mathrm{N}$ category & $\begin{array}{lc}\text { 7th edition } & \text { 8th edition } \\
\text { N0 7 (14\%) } & \text { N0 7 (14\%) } \\
\text { N1 43 (86\%) } & \text { N1 28 (56\%) } \\
& \text { N2 15 } 130 \%) \\
\end{array}$ \\
\hline M category & $\begin{array}{cc}\text { 7th edition } & \text { 8th edition } \\
\text { M0 29 (58\%) } & \text { M0 29 (58\%) } \\
\text { M1 21 (42\%) } & \text { M1a 18 (36\%) } \\
\text { M1b 1 (2\%) } \\
\text { M1c 2 (4\%) } \\
\end{array}$ \\
\hline Stage & $\begin{array}{ll}\text { 7th edition } & \text { 8th edition } \\
\text { I } 1(2 \%) & \text { I } 1(2 \%) \\
\text { IIA } 2(4 \%) & \text { II } 6(12 \%) \\
\text { IIB } 4(8 \%) & \\
\text { IIIA } 1(2 \%) & \text { III } 22(44 \%) \\
\text { IIIB 21(42\%) } & \\
\text { IV 21 } 21(42 \%) & \text { IV 21(42\%) } \\
\end{array}$ \\
\hline
\end{tabular}

Conclusions: Using new AJCC guidelines, patients with N2 disease have significantly shorter recurrence-free survival compared to the N1 group; thus, careful grossing practices will be required to accurately evaluate patients. The new parameters did not correlate with survival. Longer follow-up will be needed to determine if the new critieria show additional prognostic value.

\section{Expression of Stem Cell Markers in the Histologic Sub-Types of Ampullary Carcinomas}

Muhammad S Khurram', Won Jae Huh', Chanjuan Shỉ. 'Vanderbilt University Medical Center, Grosse Pointe Park, MI, ${ }^{2}$ Nashville, TN ${ }^{3}$ Vanderbilt University, Nashville, TN

Background: Ampullary carcinomas can arise from two types of mucosa: intestinal and pancreatobiliary type. Currently surgica resection is the only therapeutic option with curative potential. Adjuvant chemotherapy for cases with lymph node or distant metastases is offered, however response rates are usually low. Establishing the molecular profiles of different histologic subtypes of ampullary carcinoma could help identify potential molecular targets. In this study we explored stem cell marker expression in ampullary carcinomas. 
Design: Sixty-three subjects underwent radical resection for ampullary carcinoma at our institution between January 1, 1994 and January 31, 2013, with blocks available for immunohistochemical (IHC) studies. Histological slides were reviewed and tumor blocks were labeled with antibodies against stem cell markers, including CD24, CD44, OLFM4 and SOX9. IHC stains were scored based on the intensity of the staining (negative $=0$, weak $=1$, moderate $=2$, strong=3) and the percentage of positivity $(<1 \%=0,1-25 \%=1,>25-50 \%=2,>50$ $75 \%=3,>75 \%=4)$. Patient electronic medical records were reviewed for demographics, clinical characteristics and follow-up information.

Results: Sixty-three subjects included 33 males and 30 females, with mean age of 64 , ranging from 39 to 79 years. There were 38 intestinal-type and 23 pancreatobiliary-type tumors (Table 1). Other minor histologic variants included adenosquamous $(n=1)$ and undifferentiated carcinoma $(n=1)$. The overall percentage of stem cell marker expression in all ampullary carcinomas was: CD24 $(44 \%, n=28)$ : CD44 (60\%, $n=38)$; OLFM4 (54\%, $n=34)$; SOX9 (100\%, n=63). CD24 and OLFM 4 expression in intestinal type was significantly higher than the pancreatobiliary type $((p<0.05)$, whereas CD44 expression was more likely detected in pancreatobiliary tumors $(p<0.05)$. Expression of CD24, CD44 and OLFM4 did not affect patient survival ( $p>0.05$ ).

\begin{tabular}{|l|l|l|l|l|l|l|}
\hline & Female & Male & $\begin{array}{l}\text { Mean } \\
\text { age } \\
\text { (range) }\end{array}$ & $\begin{array}{l}\text { CD24* } \\
\text { (Mean } \pm \text { SEM) }\end{array}$ & $\begin{array}{l}\text { CD44* } \\
\text { (Mean } \pm \text { SEM) }\end{array}$ & $\begin{array}{l}\text { OLFM4* } \\
\text { (Mean } \pm \text { SEM) }\end{array}$ \\
\hline $\begin{array}{l}\mathrm{INT} \\
(\mathrm{n}=38)\end{array}$ & 14 & 24 & $\begin{array}{l}61(31- \\
79)\end{array}$ & $2.00 \pm 0.34$ & $1.13 \pm 0.27$ & $4.71 \pm 0.76$ \\
\hline $\begin{array}{l}\mathrm{PB} \\
(\mathrm{n}=23)\end{array}$ & 15 & 8 & $\begin{array}{l}66(44- \\
79)\end{array}$ & $0.93 \pm 0.33$ & $2.34 \pm 0.52$ & $2.59 \pm 0.74$ \\
\hline
\end{tabular}

\section{${ }^{*} \mathrm{p}<0.05$; INT=intestinal type; PB=pancreatobiliary type}

Conclusions: Intestinal-type and pancreatobiliary-type ampullary carcinoma had different stem cell marker expression profiles, suggesting that they have distinct underlying molecular mechanisms and thereby different molecular targets.

\section{Tumor Microenvironmental Factors Have Prognostic Significances in Advanced Gastric Cancer}

Joo Young Kim', Yang-Seok Chae', Chul Hwan Kim³, Youngseok Lee, Jeong Hyeon Lee', Bokyung Ahn'. 'Korea University Anam Hospital, Seoul, ${ }^{2}$ Korea Univ Med College, Seoul, Korea, 'Korea University Anam Hosp, Seoul, Korea, ${ }^{4}$ Seoul

Background: The importance of the tumor microenvironment has been emerged in the progression and survival of cancer cells. We evaluated the prognostic significance of tumor stroma percentage (TSP), Klintrup-Mäkinen (KM) grade, which reflects the density of inflammatory cells of the tumor, and Glasgow microenvironment score (GMS), a combination of TSP and KM grade, in advanced gastric cancers.

Design: A total of 196 pT3 and pT4 gastric cancers were retrieved and histologically evaluated using TSP, KM grade, and GMS and correlated with other clinicopathologic factors including patients' survival.

Results: High TSP was identified in 78 cases $(39.8 \%)$ and high KM grade was observed in 72 cases $(36.7 \%)$. And GMS 0,1 , and 2 were identified in $72(36.8 \%), 53(27.0 \%)$, and $71(36.2 \%)$ cases, respectively. High TSP, low KM grade, and high GMS were correlated with poor differentiation, diffuse type, presence of lymphovascular invasion, perineural invasion, and lymph node metastasis. High TSP was significantly correlated with low KM grade $(\mathrm{p}<0.001)$. High TSP $(H R$, $3.079 ; 95 \% \mathrm{Cl}, 1.612-5.883, \mathrm{p}=0.001)$, low KM grade (HR, 3.201; $95 \% \mathrm{Cl}$, 1.774-5.776, p<0.001), and high GMS (HR, $12.274 ; 95 \% \mathrm{Cl}, 3.684-40.895$, $\mathrm{p}<0.001)$ were significantly associated with poor overall survival, and independent poor prognostic factors in advanced gastric cancers.

Conclusions: The TSP, KM grade, and GMS are significantly correlated with clinical outcome of the gastric cancer patients. These parameters are feasible and cost-effective ways to identify tumor microenvironment having different biological features, prognosis and potential treatment options.

\section{Intestinal Metaplasia of the "Gastric" Cardia is Associated with Barrett Esophagus and Esophageal Dysplasia and Adenocarcinoma}

Tae Hun Kim', Parakrama Chandrasoma'. 'LAC + USC Medical Center, Los Angeles, CA

Background: Incidence of adenocarcinoma of the esophagus and the "gastric" cardia have been rising. There is controversy whether intestinal metaplasia (IM) in the "gastric" cardia represents a precursor lesion to Barrett esophagus (BE) and esophageal adenocarcinoma or is associated with Helicobacter pylori-related gastritis. This study evaluates the correlation between IM distal to the endoscopic GEJ with visible CLE (VCLE) without IM, BE, and esophageal dysplasia or adenocarcinoma.
Design: Patients undergoing endoscopy from 2008-2011 had biopsies routinely taken at the endoscopic gastro-esophageal junction (GEJ), immediately distal to the endoscopic GEJ, and if present in vCLE. The associations of histologic subtypes distal to the endoscopic GEJ and vCLE without IM, BE, dysplasia, and adenocarcinoma were evaluated.

Results: There were no statistical associations between the overal histology subtypes in biopsies immediately distal to the endoscopic GEJ and visible CLE without IM, BE, or dysplasia and adenocarcinoma $(\mathrm{p}=0.28$ and $\mathrm{p}=0.89$, respectively, Table 1 ). Only cardiac epithelium with IM was significantly higher in VCLE than without $\mathrm{VCLE}(20.9 \%$ versus $10.0 \% ; p=0.0057)$. There was no statistical association between vCLE and cardiac epithelium without IM $(p=0.15)$, oxyntocardiac epithelium $(p=0.78)$, or oxyntic epithelium $(p=1.00)$.

$\mathrm{BE}$ had a significantly higher prevalence of cardiac epithelium with IM than in patients without vCLE $(21.6 \%$ versus $10.0 \% ; p=0.0054)$ and significantly lower prevalence of cardiac epithelium without IM than in patients without $\mathrm{vCLE}(8.1 \%$ versus $19.7 \% ; \mathrm{p}=0.0126)$ (Table 2, Figure 1). There were no statistically significant associations between $B E$ and IM in either oxyntocardiac epithelium $(p=0.54)$ or oxyntic epithelium $(p=1.00)$.

The prevalence of cardiac epithelium with IM was significantly higher in dysplasia or adenocarcinoma of the cardia, squamocolumna junction, and tubular esophagus than those without adenocarcinoma or dysplasia ( $21.8 \%$ versus $10.4 \% ; p=0.0138$ ) (Table 2 , Figure 2 ). There was no statistical association between adenocarcinoma or dysplasia and cardiac epithelium without IM ( $p=0.16)$, oxyntocardiac epithelium $(p=0.72)$, or oxyntic epithelium $(p=1.00)$.

Table 1

\begin{tabular}{|c|c|c|c|c|}
\hline & CE & OCE & $\mathrm{OE}$ & Total \\
\hline No Visible CLE & $\begin{array}{l}261 \\
29.8 \%\end{array}$ & $\begin{array}{l}539 \\
61.5 \%\end{array}$ & $\begin{array}{l}77 \\
8.8 \%\end{array}$ & 877 \\
\hline $\begin{array}{l}\text { Visible CLE } \\
\text { without IM }\end{array}$ & $\begin{array}{l}7 \\
58.3 \%\end{array}$ & $\begin{array}{l}5 \\
41.7 \%\end{array}$ & $\begin{array}{l}0 \\
0.0 \%\end{array}$ & 12 \\
\hline $\mathrm{BE}$ & $\begin{array}{l}22 \\
29.7 \%\end{array}$ & $\begin{array}{l}45 \\
60.8 \%\end{array}$ & $\begin{array}{l}7 \\
9.5 \%\end{array}$ & 74 \\
\hline \multicolumn{5}{|l|}{$\mathrm{p}=0.28$} \\
\hline & CE & OCE & OE & Total \\
\hline $\begin{array}{l}\text { No Dysplasia } \\
\text { or Cancer }\end{array}$ & $\begin{array}{l}272 \\
30.0 \%\end{array}$ & $\begin{array}{l}557 \\
61.3 \%\end{array}$ & $\begin{array}{c}79 \\
8.7 \%\end{array}$ & 908 \\
\hline $\begin{array}{l}\text { Dysplasia or } \\
\text { Cancer }\end{array}$ & $\begin{array}{l}18 \\
32.7 \%\end{array}$ & $\begin{array}{l}32 \\
58.2 \%\end{array}$ & $\begin{array}{l}5 \\
9.1 \%\end{array}$ & 55 \\
\hline
\end{tabular}

Legend: Distribution of retrograde biopsy histologic subtypes with visible CLE and dysplasia or adenocarcinoma of the cardia squamocolumnar junction, and tubular esophagus. No statistical associations are identified. Key: CE - cardiac epithelium, CLE columnar-lined esophagus, OCE - oxyntocardiac epithelium, OE oxyntic epithelium

Table 2

\begin{tabular}{|c|c|c|c|c|c|c|c|}
\hline & $\begin{array}{l}\text { CE } \\
\text { with } \\
\text { IM }\end{array}$ & $\begin{array}{l}\text { CE } \\
\text { without } \\
\text { IM }\end{array}$ & $\begin{array}{l}\text { OCE } \\
\text { with } \\
\text { IM }\end{array}$ & $\begin{array}{l}\text { OCE } \\
\text { without } \\
\text { IM }\end{array}$ & $\begin{array}{l}\text { OM } \\
\text { with } \\
\text { IM }\end{array}$ & $\begin{array}{l}\text { OM } \\
\text { without } \\
\text { IM }\end{array}$ & Total \\
\hline $\begin{array}{l}\text { No Visible } \\
\text { CLE }\end{array}$ & $\begin{array}{l}88 \\
10.0 \%\end{array}$ & $\begin{array}{l}173 \\
19.7 \%\end{array}$ & $\begin{array}{l}36 \\
4.1 \%\end{array}$ & $\begin{array}{l}503 \\
57.4 \%\end{array}$ & $\begin{array}{l}3 \\
0.3 \%\end{array}$ & $\begin{array}{l}74 \\
8.4 \%\end{array}$ & 877 \\
\hline Visible CLE & $\begin{array}{l}18 \\
20.9 \%\end{array}$ & $\begin{array}{l}11 \\
12.8 \%\end{array}$ & $\begin{array}{l}4 \\
4.7 \%\end{array}$ & $\begin{array}{l}46 \\
53.5 \%\end{array}$ & $\begin{array}{l}0 \\
0.0 \%\end{array}$ & $\begin{array}{l}7 \\
8.1 \%\end{array}$ & 86 \\
\hline $\begin{array}{l}\text { Visible CLE } \\
\text { without IM }\end{array}$ & $\begin{array}{l}2 \\
16.7 \%\end{array}$ & $\begin{array}{l}5 \\
41.7 \%\end{array}$ & $\begin{array}{l}0 \\
0.0 \%\end{array}$ & $\begin{array}{l}5 \\
41.7 \%\end{array}$ & $\begin{array}{l}0 \\
0.0 \%\end{array}$ & $\begin{array}{l}0 \\
0.0 \%\end{array}$ & 12 \\
\hline BE & $\begin{array}{l}16 \\
21.6 \%\end{array}$ & $\begin{array}{l}6 \\
8.1 \%\end{array}$ & $\begin{array}{l}4 \\
5.4 \%\end{array}$ & $\begin{array}{l}41 \\
55.4 \%\end{array}$ & $\begin{array}{l}0 \\
0.0 \%\end{array}$ & $\begin{array}{l}7 \\
9.5 \%\end{array}$ & 74 \\
\hline $\begin{array}{l}\text { No Dysplasia } \\
\text { or Cancer }\end{array}$ & $\begin{array}{l}94 \\
10.4 \%\end{array}$ & $\begin{array}{l}178 \\
19.6 \%\end{array}$ & $\begin{array}{l}39 \\
4.3 \%\end{array}$ & $\begin{array}{l}518 \\
57.0 \%\end{array}$ & $\begin{array}{l}3 \\
0.3 \%\end{array}$ & $\begin{array}{l}76 \\
8.4 \%\end{array}$ & 908 \\
\hline $\begin{array}{l}\text { Dysplasia or } \\
\text { Cancer }\end{array}$ & $\begin{array}{l}12 \\
21.8 \%\end{array}$ & $\begin{array}{l}6 \\
10.9 \%\end{array}$ & $1.8 \%$ & $\begin{array}{l}31 \\
56.4 \%\end{array}$ & $\begin{array}{l}0 \\
0.0 \%\end{array}$ & $\begin{array}{l}5 \\
9.1 \%\end{array}$ & 55 \\
\hline
\end{tabular}


Legend: Distribution of IM in retrograde biopsy histologic subtypes with visible CLE and dysplasia or adenocarcinoma of the cardia, squamocolumnar junction, and tubular esophagus. Key: CE cardiac epithelium, CLE - columnar-lined esophagus, IM - intestinal metaplasia, OCE - oxyntocardiac epithelium, OE - oxyntic epithelium.
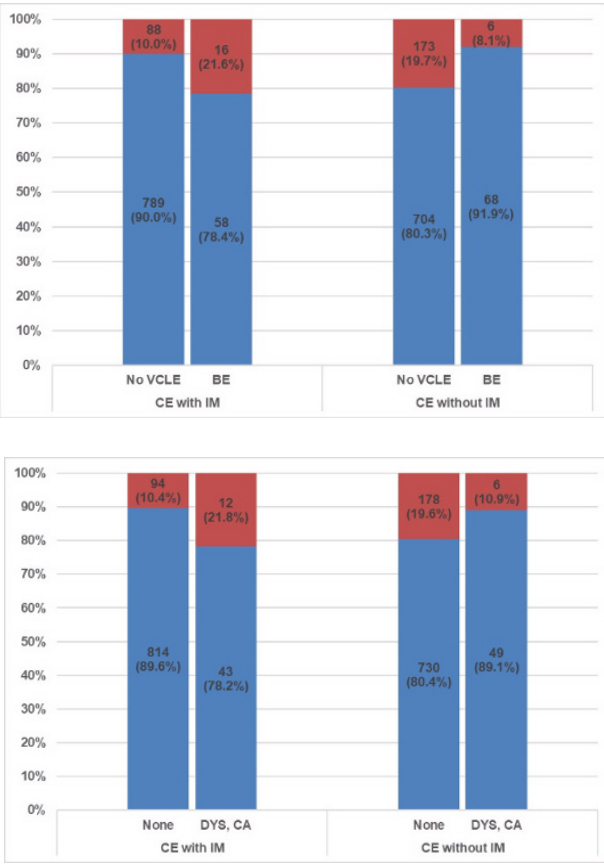

Conclusions: Cardiac epithelium with IM is associated with vCLE, BE, and esophageal dysplasia and adenocarcinoma. Cardiac epithelium without IM, oxyntocardiac epithelium, and oxyntic epithelium had no association with $\mathrm{vCLE}, \mathrm{BE}$, and esophageal dysplasia and adenocarcinoma.

\section{Different Prognostic Impacts of Fusobacterium Nucleatum Based on Tumor Location in Stage II/ III Colorectal Carcinomas Treated with Adjuvant} FOLFOX Chemotherapy

Jung Ho Kim ${ }^{1}$, Nam-Yun Cho' ${ }^{2}$ Jeong Mo Bae ${ }^{3}$, Gyeong Hoon Kang ${ }^{4}$. 'Seoul National University Hospital, Seoul, ${ }^{2}$ Cancer Research Institute, Seoul National University College of Medicine, 3Jongno-gu, Seoul, ${ }^{4}$ Seoul

Background: Recent experimental evidence has suggested an association between Fusobacterium nucleatum (Fn) and chemoresistance in colorectal carcinoma (CRC). Therefore, this study aimed to investigate the prognostic effects of intratumoral Fn in a large series of CRCs treated with adjuvant FOLFOX (leucovorin, 5-fluorouracil, and oxaliplatin) chemotherapy.

Design: Real-time polymerase chain reaction was used to quantitatively measure $16 \mathrm{~S}$ ribosomal RNA gene DNA sequence of Fn in tumor DNA extracted from 424 CRC samples. All samples were retrospectively collected from the pathology archive of surgically resected specimens of stage III or high-risk stage II CRC patients who had received radical surgery and subsequent adjuvant FOLFOX treatment at single institute from 2006 to 2012 .

Results: Among the $424 \mathrm{CRC}$ cases, 272 were determined to be Fnpositive. Fn-positive CRCs were further classified into two subgroups (Fn-high or Fn-low) using a cutoff median value of 2-ACt. Among the total 424 CRCs treated with adjuvant FOLFOX, a significant survival difference was not observed between the Fn-high and $F n$-low/negative subgroups $(P=0.725)$. In subgroup analysis using only the adjuvant FOLFOX-treated proximal colon carcinomas $(n=134)$, diseasefree survival was significantly better in Fn-high than that of Fn-low/ negative $(P=0.044)$. In contrast, survival analysis including only distal CRCs treated with adjuvant FOLFOX $(n=290)$ showed that Fn-high had worse prognostic effects in this group, although this finding was not statistically significant $(P=0.268)$.

Conclusions: In stage II/III CRCs treated with adjuvant FOLFOX prognostic impacts of intratumoral $F n$ may be different depending on tumor location.

768 Down regulation of Partitioning defective proteins is associated with poor prognosis in colorectal adenocarcinoma
Kyung-Hee Kim ${ }^{1}$, Min-kyung Yeo ${ }^{1}$, Jin Man Kim ${ }^{1}$, Kwang-Sun Suh ${ }^{1}$ Choong-Sik Lee1, Kyu Sang Song', 'Sing-Yi Choi'. 'Chungnam National University School of Medicine, Daejeon, Chungnam

Background: Partitioning defective (Par) proteins regulate cell polarity and differentiation. Par3, Par6 $\beta$, and protein kinase $\mathrm{C} \zeta$ (PKC $\zeta$ ), which are are PAR complex members, have been shown to be associated with oncogenesis and progression. Herein, the expression patterns and clinical relevance of Par3, Par6 $\beta$, and $\mathrm{PKC} \zeta$ in colorectal adenocarcinoma (CRAC) are reported.

Design: A total of 393 primary CRACs, 41 primary-metastatic CRAC pairs, 41 adenomas with low-grade dysplasia, and 41 non-tumo colorectal tissue samples were examined by immunohistochemistry and western blot assays for Par3, Par6 $\beta$, and $\mathrm{PKC} \zeta$ protein expressions. The association of Par3, Par6 $\beta$, and $\mathrm{PKC} \zeta$ expressions with clinica pathologic factors, including patient survival, was evaluated.

Results: Primary CRACs and adenomas demonstrated higher levels of Par3, Par6 $\beta$, and PKC $\zeta$ than in non-tumor colorectal epithelia $(p<0.001$, respectively). The expressions of Par3, Par6 $\beta$, and $\mathrm{PKC} \zeta$ were higher in primary CRACs as compared to adenomas $(p=0.005, p=0.005$ $p<0.001$, respectively) or in metastatic CRACs ( $<<0.001$, respectively). Among primary CRACs, decreased Par3 expression was found to correlate with a high proliferation rate and poor histologic differentiation ( $p=0.043$ and $p<0.001$, respectively), decreased $\mathrm{PKC}$ expression was correlated with pathologic TNM stage (I-II versus IIIIV) and lymph node metastasis ( $p=0.028$ and $p=0.039$, respectively) and decreased Par6 $\beta$ and $\mathrm{PKC}$ expressions were correlated with a shortened overall survival ( $p=0.008$ and $p=0.005$, respectively). In metastatic CRACs, decreased $\mathrm{PKC}$ expression was correlated with a shortened metastasis-free survival $(p=0.007)$.

Figure 1. Comparison of the expressions of Par3, Par6 $\beta$, and PKC $\zeta$ by immunohistochemistry among non-tumor colorectal mucosa, tubular adenoma, primary CRAC, and matched metastatic CRAC $(n=41)$.

Figure 2. Representative immunohistochemical expressions of Par3, Par6 $\beta$, and $\mathrm{PKC} \zeta$ in CRAC. Faint or weak expression on non-tumo colorectal mucosa $(A, D, G)$, moderate expression on tubular adenoma $(B, E, H)$, and marked high expression on primary CRAC (C, F, I).
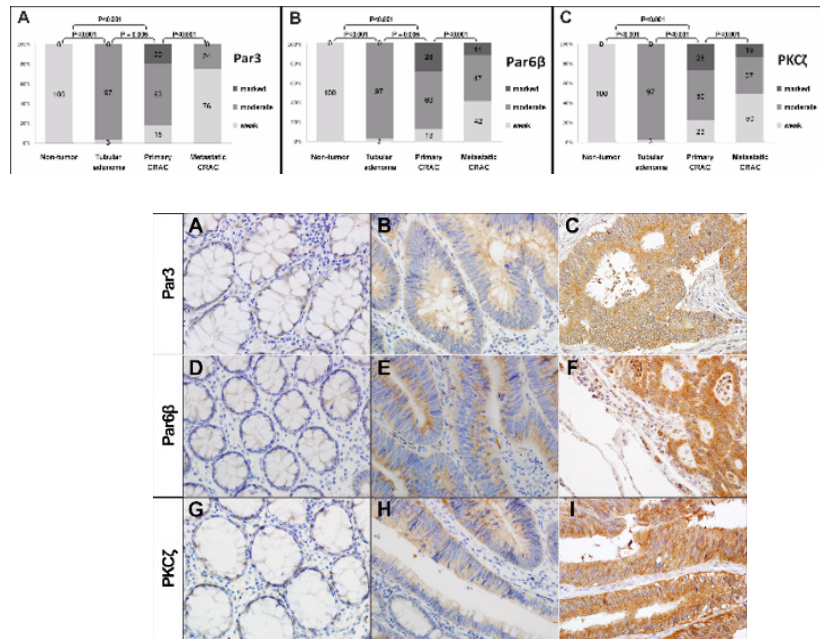

Conclusions: While increased Par3, Par6 $\beta$, and $\mathrm{PKC} \zeta$ expressions were implicated in tumorigenesis, decreased expressions of Par3, Par6 $\beta$ and $\mathrm{PKC}$ were found to be associated with worse clinicopathologic factors in CRAC. In particular, the results of the present study suggest that $\mathrm{PKC} \zeta$ down-expression is an independent poor prognostic and metastatic factor for CRAC.

\section{Reduced p16 Expression in Barrett's Intestinal Metaplasia Correlates with Somatic Genomic Alterations and is Associated with Progression to Dysplasia and Esophageal Adenocarcinoma}

Sarawut Kongkarnka1, Elena V Komissarova², Jorge L Sepulveda ${ }^{2}$ Antonia Sepulveda'. 'Department of Pathology \& Cell Biology, New York, NY, ${ }^{2}$ Columbia University Medical Center, New York, NY, ${ }^{3} \mathrm{New}$ York, NY

Background: The CDKN2A gene product, p16, is thought to play a role in progression of intestinal metaplasia of the esophagus (Barrett's intestinal metaplasia, BIM) to dysplasia and esophagea adenocarcinoma (EAC). Our aim was to evaluate for a correlation between p16 expression by immunohistochemistry (IHC) and mutational and somatic copy number alterations (SCNAs).

Design: We performed IHC for CDKN2A p16 (Ventana) in 48 FFPE endoscopic samples from 42 Barrett's patients, mean age $63.8+11.0$ $76.2 \%$ male: a) 22 from non-progressors to dysplasia/EAC (DAC) with 
never dysplastic BIM (BIM-NP); b) 26 from progressors to DAC: 9 baseline BIM (B-BIMP) obtained before progression to dysplasia (mean 54 months \pm 38 , range 13-143), 14 BIM with concurrent DAC elsewhere on the same endoscopy (C-BIMP) and 3 DAC lesions. P16 expression was evaluated using a score based on the stain intensity as a scale of 0 to 3 for both nuclear and cytoplasmic staining. Next generation sequencing (NGS) was performed with AmpliSeq (Ion Torrent) and TruSeq (Illumina) cancer panels and data were analyzed with NextGENe software (SoftGenetics) and with the Integrative Genomics Viewer (Broad Institute). Whole genome SCNAs were detected by OncoScan SNP microarrays (Affymetrix) and data were analyzed with the Chromosome Analysis Suite version 3.1 (Affymetrix).

Results: The proportions of samples with $\mathrm{p} 16$ nuclear expression decreased from BIM-NP to DAC: BIM-NP (55\%), B-BIMP (22\%), C-BIMP $(14 \%)$ and DAC $(0 \%)$. Nuclear p16 expression was significantly higher in BIM-NP (mean expressed score: $1.32+1.39$ ) than in non-dysplastic BIM (B-BIMP and C-BIMP), $\mathrm{p}=0.004$. P16 nuclear expression was significantly lower in B-BIMP (mean expressed score: $0.22 \pm 0.44$ ) than in BIM-NP, $\mathrm{p}=0.032$; whereas, $\mathrm{C}$-BIMP also showed lower $\mathrm{p} 16$ expression $(0.29 \pm 0.82)$ but did not reach significance $(p=0.054)$. Furthermore, p16 expression was significantly lower in Barrett's samples containing genomic alterations (point mutations or gene losses) involving the CDKN2A locus ( $p=0.004)$.

Conclusions: Immunohistochemical staining showed progressive loss of nuclear p16 during progression of Barrett's intestinal metaplasia to dysplasia and adenocarcinoma, correlating with accumulation of genomic alterations in the CDKN2A locus. P16 expression and genomic alterations are already detected in non-neoplastic intestinal metaplasia up to 12 years before development of dysplasia/EAC and may be useful in the workup of patient's surveillance biopsies.

\section{Concordance between an Automated, Ready-To-Use MMR IHC Panel and Next-Generation Sequencing for Analysis of Mismatch Repair and BRAF V600E Status in Colorectal Cancer}

Eric Konnick', Joel A Yambert ${ }^{2}$, Steven P Stratton ${ }^{2}$, Andrew Hannon ${ }^{2}$, Alyssa Jordan ${ }^{2}$, June Clements ${ }^{2}$, Shalini Singh ${ }^{2}$, Colin Pritchard ${ }^{3}$ Leigh Henricksen'. 'Seattle, WA, '2Ventana Medical Systems, Tucson, Arizona, ${ }^{3}$ University of Washington, Seattle, WA

\section{Disclosures:}

Eric Konnick: Research Support, Ventana Medical Systems, Inc. Joel Yambert: Employee, Ventana Medical Systems, Inc. Steven Stratton: Employee, Ventana Medical Systems, Inc. Andrew Hannon: Employee, Ventana Medical Systems, Inc. Alyssa Jordan: Employee, Ventana Medical Systems, Inc. June Clements: Employee, Ventana Medical System, Inc. Shalini Singh: Employee, Ventana Medical Systems, Inc. Colin Pritchard: Research Support, Roche/Ventana Leigh Henricksen: Employee, Ventana Medical Systems, Inc.

Background: This study verified concordance between a ready-touse immunohistochemistry (IHC) assay panel on a fully automated staining instrument, next-generation sequencing (NGS) and $\mathrm{MLH} 1$ promoter hypermethylation testing to identify colorectal cancer (CRC) cases with mismatch repair (MMR) deficiency and BRAF V600E mutation consistent with somatic or germline mutations (probable Lynch syndrome), or MLH1promoter hypermethylation.

Design: The study set included 111 sequentially selected CRC cases plus 15 enrichment cases to ensure representative loss of each MMR marker. These 15 cases were previously identified as deficient for MMR (dMMR) by IHC. The final study set (126 cases) was sequenced using the ColoSeq tumor sequencing assay (University of Washington) and stained by IHC using an MMR IHC panel including anti-MLH1 (M1) mouse, anti-PMS2 (A16-4) mouse, anti-MSH2 (G2191129) mouse, anti-MSH6 (SP93) rabbit, and anti-BRAF V600E (VE1) mouse monoclonal antibodies (Ventana Medical Systems, Inc.) on a fully automated instrument. NGS and IHC results were analyzed independently. Cases with loss of MLH1protein expression by IHC were tested for $M L H 1$ promoter hypermethylation. For analysis of concordance, mutations and hypermethylation status predictive of protein loss were compared to IHC.

Results: In the final analysis of 118 cases, point estimates for positive percent agreement and negative percent agreement were $99.4 \%$ and $93.5 \%$, respectively, between IHC and NGS/hypermethylation. The overall percent agreement for each marker exceeded $96 \%$. Twentythree cases were positive for BRAF V600E by IHC and confirmed by NGS. Twenty showed loss of MLH1 protein by IHC while positive for MLH1 promoter hypermethylation; and three were intact for all MMR markers by IHC.

Conclusions: This ready-to-use MMR IHC panel correctly identified MMR and BRAF V600E status based on concordance with ColoSeq and $M L H 1$ promoter hypermethylation. The IHC panel was able to identify CRC with $\mathrm{dMMR}$ resulting from mutations that may be consistent with germline mutations. In addition, the data support the use of BRAF V600E IHC as an aid to identify sporadic CRC, as all BRAF V600E positive cases were either positive for $M L H 1$ promoter hypermethylation or roficient for MMR and confirmed by sequencing. Thus, this MMR IHC panel can detect dMMR to identify individuals at risk for Lynch syndrome and, with BRAF V600E status, aid to differentiate between sporadic and probable Lynch syndrome CRC in the absence of MLH1 protein expression.

\section{P53 and RB-P16 are the Most Common Genetic Alterations, but No High-Risk Human Papillomavirus (HPV) is Detected in Esophageal Squamous Cell Carcinogenesis}

Katrina Krogh ${ }^{1}$, Andrew Bandy ${ }^{2}$, Audrey Deeken-Draisey ${ }^{2}$, Leyu Sun ${ }^{3}$ Jie Liao ${ }^{4}$ M. Sambasiva Rao', Maryam Kherad Pezhouh', Guang-Yú Yang ${ }^{2}$. 'Northwestern Memorial Hospital, Chicago, IL, ${ }^{2}$ Chicago, IL, ${ }^{3}$ Northwestern, Chicago, IL, ${ }^{4}$ Northwestern University, Chicago, IL, ${ }^{5}$ Northwestern University, Feinberg School of Medicine, Chicago, IL

Background: Esophageal squamous cell cancer (SCC) is one of the most common malignancies worldwide. P53 mutation is known to be a significant factor, mostly due to smoking and other environmental carcinogens. Recently, human papillomavirus (HPV) infection has become a significant etiology for head and neck SCC in the US, but is not well studied in esophageal SCC. In the present study, we aim to analyze the prevalence of high-risk HPV infection and the expression of P53 and P16 in a large cohort of esophageal SCC and its in situ lesions using an HPV-HR18 probe cocktail (HPV-ISH) and immunohistochemical (IHC) approaches.

Design: A total of 68 esophageal resection and biopsy cases with in situ and/or invasive esophageal SCC were retrieved from ou institution between 2009-2017. Histologic slides were reviewed, and slides with representative tumor were chosen. IHC for P53 and P16 were conducted, and slides with diffuse P16-positive tumor were subjected to HPV-ISH. Proper positive and negative controls were established. P53 nuclear positivity and P16 nuclear/cytoplasmic staining in epithelial cells or lesional areas were analyzed. P53 positive staining was calculated as positive in $>5 \%$ positive cells, and significant P16 nuclear/cytoplasmic staining was analyzed as $>80 \% /$ diffuse positive neoplastic cells. HPV-ISH was analyzed as positive brown-nuclear dot staining

Results: Among our cohort, the age of patients ranged from 34 to 92 years with a median age of 66 years, 1:1 female to male ratio, and 3:1 Caucasian to African American ratio. Diffuse P16 nuclear/cytoplasmic staining (Fig. A) was identified in $28 \% \operatorname{SCC}(n=68)$, but none of them $(0 \%)$ were positive for HPV-ISH. $75 \%$ SCC showed positive P53 nuclear accumulation, of which $15 \%$ were positive for both P53 and P16; only $12 \%$ displayed neither $(\mathrm{p}<0.008)$. For in situ SCC, $14 \%(3 / 22)$ showed diffuse P16 nuclear/cytoplasmic staining (Fig. A) but HPV-ISH was negative for all of these cases (Fig. C); 41\% (9/22) displayed P53 protein nuclear accumulation (Fig. B).

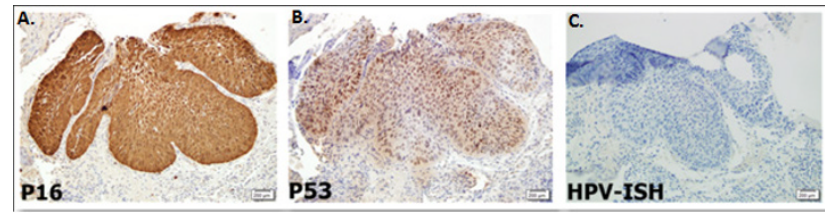

Conclusions: Our large cohort study demonstrated that 1) high-risk HPV is an extremely rare event in esophageal SCC, and 2) the findings of significant/diffuse P16 protein accumulation in SCC probably is due to genetic alterations in the Rb pathway (positive feedback regulation by mutant $R B$ gene). Our study further confirmed that mutant P53 nuclear accumulation is the most common genetic alteration in both in situ and invasive SCC, which is a practical biomarker for detecting early precancerous lesions.

\section{Biopsy Findings in Patients with Diarrhea Taking Oxcarbazepine}

Stephen Lagana', Aimee Kwak², Benjamin Lebwohß John Hart' ${ }^{1}$ Columbia University Medical Center, New York, NY, ${ }^{2}$ University of Chicago, ${ }^{3}$ Celiac Disease Center at CUMC, ${ }^{4}$ Univ. of Chicago, Chicago, IL

Background: Oxcarbazepine (OCB) is an anti-seizure drug. A case report exists which showed a convincing example of spruelike enteropathy associated with its use. We recently encountered a patient taking OCB being evaluated for celiac disease due to chronic diarrhea. Duodenal biopsies revealed marked increase in intraepithelial lymphocytes (IELs), but celiac serologies and HLA testing were negative and the patient did not respond to gluten free diet. Inflammation due to OCB was suggested. Our aim was to further investigate this possible association.

Design: The case files of a single medical center were queried for patients taking OCB and undergoing upper or lower endoscopy (from 2007-2013). Patients who had biopsies of the esophagus or intestines (excluding polyps) were considered cases. The index case was 
contributed by another center. Patients with inflammatory findings were investigated further.

Results: Including the index case, 4 of 16 patients taking OCB had uncommon patterns of inflammation on biopsy (25\%). All were adults (33-68) and $50 \%$ were female. The 4 histologically inflamed cases presented with diarrhea whereas only 2 of 12 patients with normal histology had diarrhea $(p=.008)$. The indications of the non-inflamed patients (12) were abdominal pain (7), screening (3), diarrhea (2), iron deficiency anemia (2), and reflux (1) (values include patients with more than 1 indication). Of the inflamed cases, 2 were suspected of having $C D$, but were seronegative. One had microscopic colitis and 1 had suspected irritable bowel syndrome. Histologic patterns of injury were variable, but were all somewhat unusual (Table 1).

\begin{tabular}{|l|l|l|l|}
\hline Patient & Age & Gender & Histologic finding(s) \\
\hline 1 & 33 & M & $\begin{array}{l}\text { Diffuse, marked intraepithelial lymphocytosis of duode- } \\
\text { num (IELd) with preserved villi. }\end{array}$ \\
\hline 2 & 47 & F & $\begin{array}{l}\text { Focal IELd, preserved villi. Numerous apoptotic bodies } \\
\text { in esophagus. Colonic eosinophilia with focal collagen } \\
\text { thickening and focal active colitis. }\end{array}$ \\
\hline 3 & 68 & M & $\begin{array}{l}\text { Marked lymphocytic colitis with focal collagen thick- } \\
\text { ening. }\end{array}$ \\
\hline 4 & 48 & F & Eosinophilic esophagitis (>100/hpf). \\
\hline
\end{tabular}

Conclusions: OCB exposed patients with diarrhea were more likely to have a histological abnormality than OCB exposed patients with indications other than diarrhea. OCB exposure may be associated with diarrhea and unusual (though protean) patterns of histologic inflammation, including a celiac-like enteropathy. A case control study is needed to determine if there is a true association. A multicenter study is currently underway.

\section{Gastric Plexiform Fibromyxoma: A Great Mimic of Gastrointestinal Stromal Tumor (GIST) and Diagnostic Pitfalls}

Jinping Lai', Jesse Kresak, Dengfeng Cao², Dongwei Zhang, Sharon Zhang ${ }^{3}$, David Hernandez Gonzalo4, Hanlin L Wang, Xiuli Liu, Xuemo (Sean) Fan ${ }^{5}$. 'University of Florida, Gainesville, FL, ${ }^{2}$ Washington University School of Medicine, Saint Louis, MO, ${ }^{3}$ David Geffen School of Medicine at UCLA, Los Angeles, CA, ${ }^{4}$ University of Florida-Shands, Gainesville, FL, ${ }^{5}$ Cedars-Sinai Medical Center, West Hollywood, CA

Background: Gastric plexiform fibromyxoma is a rarely reported benign mesenchymal neoplasm usually arising in the gastric antrum and is challenging for pre- and intraoperative diagnosis.

Design: Through a multicenter study, we collected seven cases of this rare entity. The clinical demographics, morphologic features and immunoprofile of the tumor were evaluated by more than three GI pathologists each case.

Results: The seven patients included four females and three males, and they were 11 to $79(46.1 \pm 10.1)$ year-old. All cases showed unilocular lesion arising from antrum or body. The tumor sizes ranged 1.6 to $17(6.1 \pm 2.4) \mathrm{cm}$ in greatest dimension. Six of the seven cases had EUS-FNA and/or intraoperative frozen sections, and all of them were preoperatively or intraoperatively diagnosed as GIST. Histologically, the tumor exhibited a plexform growth pattern with nodules in the muscularis propria and infiltrative borders. The tumors were composed of a rich myxoid stroma with cytological bland uniform spindle cells without mitotic figures identified except one case with nuclear pleomorphism and three mitotic figures per ten high power fields. Immunostains were performed in all of the cases. They were focally positive for SMA (6/6), focally and weakly positive for desmin (3/6) and caldesmon (2/3), negative for CD117 $(0 / 7), \operatorname{CD} 34(0 / 7), \operatorname{Dog} 1(0 / 4)$ and S100 (0/5). Six of the seven cases are morphologically benign including the $17 \mathrm{~cm}$ one. The tumor 13.7 $\mathrm{cm}$ ) with nuclear atypia and high mitotic activity also showed $30 \%$ of KI-67 index, but no lymphovascular invasion, perineural invasion or metastasis was identified. In this patient, there was a synchronous and immunostain-confirmed GIST $(0.7 \mathrm{~cm})$ incidentally identified from the partial gastrectomy specimen. No loss of SDHB immunoreactivity was identified in this tumor and the tumor $(1.6 \mathrm{~cm})$ from another case.

Conclusions: Plexiform fibromyxoma of the stomach can be underrecognized without immunostains. Although rare, it's easily misdiagnosed as GIST leading to inappropriate treatment. In addition to originating from the antrum, it can also arise from the body. No malignant cases have previously been reported in the English literature. One of our cases with nuclear polymorphism and high Ki67 index is still being followed up.

774 Knowledge Gaps in Upper Gastrointestinal Pathology: Multicenter Data from Academic Consultation Practices
Dora Lam-Himlin ${ }^{1}$, Michael Torbenson ${ }^{2}$, Rondell Graham ${ }^{2}$,Christina A Arnold $^{3}$, Dhanpat Jain ${ }^{4}$, Sanjay Kakar ${ }^{5}$, Bita V Naini ${ }^{6}$, Matthew M Yeh ${ }^{7}$, Tsung-Teh $W u^{2}$. ${ }^{1}$ Mayo Clinic, Scottsdale, AZ, ${ }^{2}$ Mayo Clinic, Rochester MN, ${ }^{3}$ The Ohio State University Wexner Medical Center, Columbus, $\mathrm{OH},{ }^{4}$ Yale Univ./Medicine, New Haven, CT, ${ }^{5}$ Univ. of California, San Francisco, San Francisco, CA, ${ }^{6}$ UCLA Medical Center, Santa Monica, CA, ${ }^{7}$ Univ of Washington Med Ctr, Seattle, WA

Background: There is little data on the knowledge gaps in post training understanding of upper gastrointestinal (GI) pathology. By looking at the types of cases submitted for external expert consultation at multiple academic centers, we can better understand the areas of diagnostic difficulty to improve residency training and CME course design.

Design: During 2016-17, 1203 consecutive consultative cases in GI, liver and pancreas pathology were collected at 6 academic centers by 9 pathologists with subspecialty interest in Gl pathology (range 75-250 cases per site).

Results: Upper GI cases accounted for $29 \%$ of all consults (354/1203) Contributors were the pathologist of record $(92 \%)$, clinician $(8 \%)$ and patient $(n=1)$. Specimen types included biopsies $(92 \%)$, resections $(6 \%)$, and polypectomy (1\%). Neoplastic conditions were 3 times more frequent than non-neoplastic. Esophagus, the most commonly consulted site $(56 \%)$, comprised mostly of Barrett-related consults $(84 \%)$ largely initiated by pathologists (93\%). Discordance with submitted diagnosis was $40 \%$, and downgrading of dysplasia was more common (67\%). Other esophageal consults included ulcers $(5 \%)$ other malignancy $(4 \%)$, squamous lesions (3\%), eosinophilia (1\%) and other $(3 \%)$. Stomach consults $(25 \%)$ were more heterogeneous with dysplasia grading (27\%), polyp classification (17\%), gastritis $(12 \%)$ lymphoid aggregates $(12 \%)$, carcinomas $(10 \%)$, other neoplasms $(8 \%)$, and other findings (17\%). Disagreement was $33 \%$, again with the largest disagreements among dysplasia grading (46\%) and downgrading more common (80\%). Small bowel consults were even more heterogeneous, with non-clustering non-neoplastic lesions (27\%), dysplasia grading (19\%), intraepithelial lymphocytes/ malabsorption (16\%), lymphoid aggregates (9\%), neuroendocrine tumors $(9 \%)$, other tumors $(9 \%)$, polyps $(7 \%)$, and carcinomas $(3 \%)$ Disagreements in small bowel diagnosis (30\%) were primarily in tumor classification (50\%). Additional stains were performed by the consultant in $43 \%$ of all cases.

Conclusions: Upper GI consultations are overwhelmingly contributed by the originating pathologists and the most common esophagea consultations are Barrett-related and show $40 \%$ discordance with contributors. Gastric consults are dominated by dysplasia grading classification of polyps and gastritis, while small bowel consults are far more heterogeneous with diverse non-neoplastic representation. These common challenges in upper GI pathology offer opportunity for improvement in pathology training and education.

\section{PREVIOUSLY PUBLISHED}

\section{The Use of dsDNA Derived Exosomes as a Readout of Mutational Status in Colorectal Cancer (CRC)}

Stefania Landolfi', Rosa Somoza², Josefa Domenech ${ }^{3}$, Douglas Sanchez $z^{4}$, Maria del Carmen Martin Ástorga ${ }^{5}$, Maria Eugenia Semidey Raven ${ }^{6}$, Santiago Ramon y CajaI, Javier Hernandez-Losa ${ }^{8}$. 'Hospital Universitari Vall d'Hebron, Barcelona, 2Vall d'Hebron Institute Research, Barcelona, Spain, 'Vall d'Hebron Institute Research Barcelona, Spain, "Vall d'Hebron Universitary Hospital, Barcelona Catalonia, ${ }^{5}$ Vall d'Hebron Research Institute (VHIR), Barcelona, ${ }^{6}$ Rubi, ${ }^{7}$ Hospital Universitari Vall d'Hebron, Barcelona, ${ }^{8} \mathrm{Hospital}$ Universitari Vall d'Hebron, VHIR, CIBERONC, Barcelona

Background: In pathology labs RAS mutations are routinely used as predictive biomarkers to assess anti-EGFR treatment response in CRC. However, analysis of tumor tissue are limited by genetic intratumoral heterogeneity (ITH) and tha availability of tumor content, leading to possible false negatives in the tested genes. Therefore, a biopsy may not adequately reflect the mutational status (MS) of the entire tumor In this context, the DNA contained into the exosomes (ExoDNA) obtained from tumor tissue opens an extraordinary opportunity to avoid the problem in predicting response to therapy caused by ITH. The aim of this study is to analyze the MS of different genes in ExoDNAs obtained by sampling CRC tissue and comparing the results with MS from DNA obtained in Formalin-Fixed-Paraffin Embedded (FFPE) and Frozen tissues in the same set of samples, establishing the potential role of mutational assessment in ExoDNA

Design: We have evaluated the MS of KRAS, BRAF, NRAS and PIK3CA, analyzing samples from 42 patients with CRC. In detail we have analyzed DNA from 42 fresh tumor tissues (FTT) (separated in 76 samples) and exosomes secreted by the tissue from patients with CRC ( $n=42$ samples). We also analyzed 23 FFPE tissues (performing 3 different punches that represent the whole tumor ( $n=69$ samples)). All mutational analysis were done using CLART-CMA technology and subsequently validated by other commercial methods. The detection of exosomes was performed by Western Blot method and Transmission Electron Microscopy (TEM). 
Results: Mutations in 42 FTT were: 19 KRAS mutated $(2 \mathrm{G} 12 \mathrm{~V}$, 1 G12A, 2 G12C, 2 G12D, 1 G12R, 6 G13D, 4 A146T and 1 Q61H ), and 2 cases BRAF (V600E) and 21 wild type. In ExoDNA we found the same mutational profile in $41 / 42$ frozen samples. In FFPE we observed that the MS of the three punches agreed in 23/24 cases (one sample was KRAS G12V in one punch and WT in others). Overall, there were high concordance between ExoDNA and FTT and the different areas from FFPE samples confirmed by different methods.

Conclusions: In this pilot study we demonstrate the potential value of ExoDNA in determining the MS in CRC. The data obtained provide an adequate reflection of the tumor as a whole, although we could not observed high ITH due to a reduced number of samples. These results must be validated in further studies including more samples and high sensitive technologies to assess the potential role of ExoDNA in determining MS of CRC

\section{Clinical Significance of Acellular Mucin in Lymph Nodes Isolated From Treatment-Naive Colorectal Cancer Resections}

James Lapinski', Alok Khorana', Amitabh Srivastava², Lisa Rybicki', Michael McNamara', Deepa T Patiß . 'Cleveland Clinic, ${ }^{2}$ Brigham \& Women's Hospital, Boston, MA, ${ }^{3}$ Cleveland Clinic, Cleveland, $\mathrm{OH}$

Background: Lymph nodes (LN) with acellular mucin (ACM) found in treated rectal cancer resections do not affect clinical outcome and are staged as pNO. However, there are no guidelines about staging LN with ACM found in treatment-naïve colorectal cancer (CRC) resections without any other nodal disease. It is also unclear if their clinical outcome is similar to patients with pN0 or pN1 disease. In an effort to address these questions, a case-control study was performed in a cohort of treatment-naïve CRC, and clinicopathologic features between patients with and without ACM in LN were compared.

Design: Twelve CRC with ACM (median age 68 yrs; range 54-84; 8 females, 4 males) resected from 1990-2016 were compared with 44 CRC controls (matched for age, tumor location, and stage) without ACM (median age 67; range: 50-89; 24 females, 20 males). The following clinical and pathologic features were recorded: type of surgery, gross appearance, number of tumors, grade, perineural invasion (PNI), lymph-vascular invasion (LVI), MSI status, post-op therapy, and follow-up. In all cases, multiple deeper sections with or without cytokeratin IHC were performed to exclude a viable neoplasm. Baseline parameters were compared with Fisher's exact test or Wilcoxon rank sum test; survival was compared with log-rank test.

Results: The grade distribution of 12 cases (9 right-sided, 3 left-sided) with ACM (1-2 LN per case) was low-grade - 7 (58\%), high-grade - 4 $(36 \%)$, and unknown - $1(8 \%)$. pT stage distribution was pT2 - $4(34 \%)$, pT3 - $7(58 \%)$, and pT4 - $1(8 \%)$. There were no differences in grade or PNI/LVI between the two groups. Cases were commonly associated with MSI-H status (all showed MLH1 loss) compared to controls $(100 \%$ vs $16 \% ; p<0.001)$. Of the 9 cases with follow-up, 7 (78\%) are alive with no evidence of disease (median: 31 mos; range:15-161) and 2 $(25 \%)$ died of causes unrelated to CRC. Only 1 patient received postop adjuvant therapy. Although not statistically significant, by staging cases with $A C M$ as $p N 0$, their survival outcome was found to be more similar to $p N 0$ controls $(p=0.48)$ compared to $p N 1$ controls $(p=0.11)$.

Conclusions: $A C M$ in $L N$ retrieved from treatment naïve CRC resections is a rare finding and needs to be assessed diligently to ensure lack of viable neoplasm. As patients with ACM within pericolonic LN, without any other nodal disease, are more likely to show survival outcome that is similar to $\mathrm{pNO}$ stage-matched CRC, we recommend that these patients should be clinically managed as nodenegative disease.

\section{Looking for a needle in a haystack, the search for ALK-, NTRK- and ROS1 fusion genes in colorectal carcinoma with immunohistochemistry and Archer Dx fusion assay}

Jerzy Lasota', Anna Felisiak-Golabek ${ }^{2}$, Zengfeng Wang, Artur Kowalik ${ }^{3}$ Piotr Waloszczyk, Rafal Peksa ${ }^{5}$ Krzysztof Okon ${ }^{6}$. Wojciech Biernat ${ }^{5}$, Jason Christiansen ${ }^{7}$ Markku Miettinen ${ }^{2}$. ${ }^{1}$ National Cancer Institute, Bethesda, MD, ${ }^{2}$ National Cancer Institute, ${ }^{3}$ Holycross Cancer Center Poland, ${ }^{4}$ Zdunomed, Poland, Szczecin, Zachodniopomorskie, ${ }^{5}$ Medica University of Gdansk, Poland, 'Jagiellonian University, Poland, ${ }^{7}$ Ignyta, San Diego

Background: Understanding of molecular genetic mechanisms underlying the initiation of colorectal carcinoma (CRC) is crucial for the development of rational targeted therapy. Genomic rearrangements of receptor tyrosine kinases (RTK), such as anaplastic lymphoma kinase (ALK), neurotrophic receptor tyrosine kinase 1, 2, and 3 (NTRKs), and ROS1, leading to the formation of oncogenic fusion genes were reported in a variety of human cancers. The detection of such fusion genes and their chimeric transcripts has immediate clinical implications by expanding therapeutic options, including treatment with specific tyrosine kinase inhibitors. Since CRCs driven by RTK fusion genes appear to be rare, the development of a diagnostic strategy allowing effective identification of such tumors is of major importance.

Design: A cohort of 1200 well characterized primary CRCs was evaluated for the expression of ALK, NTRKs, and ROS1 using Leica Bond-Max automated immunohistochemistry (IHC) and following antibodies: D5F3 for ALK, A7H6R for NTRKs (Pan-Trk antibody), and D4D6 for ROS1. DNA and RNA was extracted from formalin-fixed paraffin embedded tissues of CRCs with positive IHC. The presence of functional fusion gene transcripts was assessed via the Archer Dx fusion assay. Gene mutation profiles of RTK fusion tumors were characterized by the lon Torrent next-generation sequencing (NGS) of 409 genes panel.

Results: Strong membrane and cytoplasmic staining was detected in 3 and 2 CRCs with Pan-Trk and ALK antibody, respectively. No ROS 1 positivity was recorded. TPM3 (Tropomyosin 3)-NTRK1 fusions were detected in all Pan-Trk-positive tumors. ALK-positive CRCs revealed DIAPH2 (diaphanous related formin 2)-ALK and SPTBN1 (spectrin beta non-erythrocytic 1)-ALK fusion gene transcripts. NGS of BRAF, RAS, and PIK3CA, oncogenes typically mutated in CRCs revealed wild-type sequences. The latter confirmed biological significance of chimeric transcripts detected in $5(0.4 \%)$ of 1200 CRCs.TPM3-NTRK tumors were from cecum, transverse-, and sigmoid colon, while both ALK fusion CRCs derived from ascending colon. All NTRK- and ALKfusion CRCs developed in female patients.

Conclusions: Immunohistochemistry with ALK D5F3 and PanTrk antibodies validated by the Archer Dx fusion assay offers costeffective and specific detection of CRCs driven by ALK- or NTRKfusion genes. Although frequency of such tumors appears to be low $(<0.5 \%)$, implementation of this diagnostic strategy has a great clinical significance for the individual patient.

\section{HER2 Overexpression, Gene Amplification and Microsatellite Instability Status of Lymphoepithelioma-like Gastric Carcinomas: A Study of 320 Cases}

Sun Mi Lee', Jeong-hwa Kwon'. 'Asan Medical Center, Seoul

Background: Lymphoepithelioma-like gastric carcinoma (LELCs) is a rare variant of gastric carcinoma which is highly associated with Epstein-Barr virus (EBV) infection. Histologically, this tumor is characterized by nests of poorly differentiated or undifferentiated neoplastic cells within a lymphoid stroma. Clinicopathologic and molecular characteristics of EBV-associated LELCs are known to be different from those of conventional gastric adenocarcinomas (CGAs). Although clinicopathologic characteristics of LELCs have been relatively well known, HER2 amplification and microsatellite instability status of LELCs has not been fully elucidated.

Design: We analyzed 320 cases of surgically resected LELCs, consisting of 112 advanced and 208 early tumors between 2005 and 2017. All H\&E stained slides of 320 LELCs were reviewed. Immunohistochemical analysis of cerbB-2, c-MET, p53 and Epstein-Barr Virus (EBV) in situ hybridization was performed on 320 LELCs. Additionally, silver in situ hybridization for HER2 (SISH) was performed on LELCs with immunoreactivity for HER2 (2+ and $3+)$ for confirmation of HER2 amplification. Microsatellite instability (MSI) analysis was performed on 40 LELCs by multiplex polymerase chain reaction with five quasi-monomorphic mononucleotide repeat markers.

Results: Study patients comprised 252 (84\%) males and $68(16 \%$ females aged 56 yr. Fifty-six (18\%) LELCs showed lymphovascular invasion and seventy-four $(23 \%)$ tumors had lymph node metastasis. Only five $(2 \%)$ patients were found to have distant metastasis. Only one $(2.5 \%)$ of forty LELCs revealed high level of microsatellite instability by MSI testing. Seventeen LELCs demonstrated focal or diffuse membranous staining of cerbB2 $(2+$ or $3+)$ and all of them demonstrate amplification of HER2 revealed by $\mathrm{SISH}$.

Conclusions: In our study, EBV associated LELCs demonstrate a male predominance and predisposition to the proximal part of the stomach and have better histologic factors related with a favorable prognosis. Only one (2.5\%) tumor revealed high level of microsatellite instability, suggesting that most of EBV associated LELCs are microsatellite stable tumors. Although HER2 overexpression in gastric carcinomas was reported approximately $20 \%$, amplification of HER2 in LELCs is relative infrequent, occurred in $8.5 \%$ of 320 LELCs. In summary, EBV associated LELCs have distinct clinicopathologic features related with a favorable clinical outcome and low frequencies of HER2 amplification and microsatellite instability.

\section{Clinicopathologic and Immunohistochemical Characteristics of Twenty-Five Gastric Adenocarcinomas with Enteroblastic Differentiation}

Sun Mi Lee', Chang Ohk Sung ${ }^{2} .{ }^{1}$ Asan Medical Center, Seoul, ${ }^{2}$ Seoul

Background: Gastric adenocarcinoma with enteroblastic differentiation (GAED) is a rare but distinct histologic subtype of 
gastric adenocarcinoma. It is characterized by tubular glands lined by columnar cells with clear cytoplasm, mimicking a primitive intestinelike structures. This fetal type intestinal adenocarcinomas are usually labelled by oncofetal protein or embryonic markers such as alphafetoprotein, glypican-3, SALL4 and so on. As the behavior of GAED has been demonstrated in a few studies to be more aggressive than conventional gastric adenocarcinomas (CGAs). Our study investigates clinicopathologic and immunohistochemical features of GAEDs compared with those of GCAs.

Design: We present 25 patients with GAED consisting of 18 advanced and 7 early tumors between 2011 and 2017. For comparison, 100 CGAs with matched T stage were selected and their clinicopathologic features was also reviewed. Immunohistochemical analysis of alphafetoprotein, glypican-3, SALL-4, HepPar-1, cerbB-2, p53 and silver in situ hybridization for HER2 (SISH) was performed on 25 GAEDs.

Results: Study patients comprised $19(76 \%)$ males and 6(24\%) females aged 73yr. Lymphatic and venous invasion was more frequent in GAEDs $(80 \%$ and $89 \%)$ than CGAs $(38 \%$ and $30 \% ; \mathrm{p}<0.001)$. Lymph node metastasis and extracapsular invasion of lymph nodes $(71 \%$ and $14 \%$ ) was more commonly identified in GAEDs than CGAs $32 \%$ and $6 \% ; p=0.005)$. Three GAEDs also had synchronous GAED in different site of the stomach. $96 \%$ of GAEDs expressed SALL-4 and $68 \%$ of GAEDs expressed alpha-fetoprotein and were focally positive for HepPar-1. All GAEDs revealed aberrant p53 expression or its loss. Two GAEDs demonstrated strong and diffuse membranous expression of cerbB2 (3+) confirmed by SISH. One GAED had foci of teratoid carcinosarcoma and large cell neuroendocrine carcinoma.

Conclusions: GAEDs showed multiple distinct and worse pathologic factors which are associated with an aggressive biologic behavior of this subtype. In our study, SALL4 is the most sensitive and specific marker for GAED, which is matched well with their histologic characteristics. One case demonstrated sarcomatoid transformation of stroma within fetal type intestinal glandular component and also showed neuroendocrine differentiation, suggesting that potential dedifferentiation or multipotent differentiation of GAEDs. Although GAED is a rare histologic subtype but definite histologic distinction from CGAs is critical.

\section{The Expression of Markers of Site of Origin in Poorly Differentiated Neuroendocrine Carcinoma (PDNEC)}

Hwajeong Lee1, Zhiyan $\mathrm{Fu}^{2}$, Jingmei Lin ${ }^{3}$, Deepa T Patil ${ }^{4}$, Christine Sheehan', Zhaohai Yang ${ }^{5}$. 'Albany Medical College, Guilderland, NY, ${ }^{2}$ Albany Medical College, Albany, NY, ${ }^{3}$ Indiana University Department of Pathology, Indianapolis, IN, ${ }^{4}$ Cleveland Clinic, Cleveland, OH, ${ }^{5} \mathrm{Penn}$ State Hershey MC, Hershey, PA

Background: Immunohistochemical markers of origin are often used to identify primary sites of metastatic well differentiated neuroendocrine tumors, critical to the diagnosis and management of these tumors. The expression of such markers in PDNEC is less well studied. This study aimed to evaluate their utility in this aggressive entity.

Design: Representative tumor sections from 74 PDNECs from the GI tract (38 cases from 8 sites) and lung (36); including 48 large cell NEC (LCNEC) and 26 small cell carcinoma (SmCC) were subject to immunohistochemical stain for CDX2, TTF1, PAX8 and ISL1. Assessment for protein expression for each marker was based on intensity [1 (weak) to 3 (strong)] and percentage of positive tumor cells [0 (negative), $1(<50 \%)$ and $2(\geq 50 \%)$ ]. The multiplicative index (maximum 6) was calculated and the average total score (aTS) was determined for each primary site and histologic subtype.

Results: There were 43 males and 31 females, with a mean age of 67 years (range 43-87). In the $38 \mathrm{Gl}$ PDNECs, CDX2, TTF1, PAX8 and ISL1 staining was observed in $71 \%$ (aTS 3.1), $16 \%$ (aTS 0.4 ), $50 \%$ (aTS 0.9 ) and $63 \%$ (aTS 1.9) overall (breakdown by site tabulated below) and $22 \%$ (aTS 0.6 ), $72 \%$ (aTS 2.9), 47\% (aTS 0.9) and $94 \%$ (aTS 3.8) of the 36 pulmonary ones, respectively. GI PDNECs showed a higher aTS for CDX2 and lower aTS for TTF1 and ISL1, compared to that of lung tumors (student's t test, $\mathrm{p}<0.001$ ). No marker was specific for a particular GI site. SmCC had a higher aTS for TTF1 and ISL1 ( $p<0.001)$ and lower aTS for CDX2 $(p=0.004)$ than that of LCNEC. No difference in PAX8 was observed in either comparison.

\begin{tabular}{|l|l|l|l|l|l|}
\hline \multicolumn{2}{|l|}{$\begin{array}{l}\text { Number of cases } \\
\text { CDX2 }\end{array}$} & \multicolumn{4}{l|}{$\%(\mathrm{aTS})$} \\
\cline { 3 - 6 } & TTF1 & PAX8 & ISL1 & \\
\hline $\begin{array}{l}\text { Primary site } \\
\text { (n=74) }\end{array}$ & Colorectum (14) & $79(3.4)$ & $14(0.5)$ & $50(0.7)$ & $64(2.3)$ \\
\hline & $\begin{array}{l}\text { Esophagus/ } \\
\text { GEJ (9) }\end{array}$ & $56(2.6)$ & $11(0.3)$ & $44(1.1)$ & $78(2.3)$ \\
\hline & Ampulla (5) & $80(2.2)$ & $40(1.0)$ & $80(1.6)$ & $60(1.0)$ \\
\hline & Pancreas (4) & $75(2.0)$ & $25(0.3)$ & $25(0.3)$ & $75(2.0)$ \\
\hline & Bile duct (3) & $33(1.7)$ & $0(0)$ & $33(0.3)$ & $0(0)$ \\
\hline & Stomach (1) & $100(5.0)$ & $0(0)$ & $0)$ & $0(0)$ \\
\hline & Duodenum (1) & $100(2.0)$ & $0(0)$ & $100(1.0)$ & $100(1.0)$ \\
\hline & Anus (1) & $100(6.0)$ & $0(0)$ & $100(4.0)$ & $100(6.0)$ \\
\hline & Lung (36) & $22(0.6)$ & $72(2.9)$ & $47(0.9)$ & $94(3.8)$ \\
\hline & LCNEC (48) & $62(2.3)$ & $27(0.8)$ & $48(0.8)$ & $70(2.0)$ \\
\cline { 2 - 6 } & SmCC (26) & $23(0.8)$ & $73(3.2)$ & $50(1.0)$ & $92(4.2)$ \\
\hline $\begin{array}{l}\text { Histologic } \\
\text { type (n=74) }\end{array}$
\end{tabular}

GEJ=gastroesophageal junction

Conclusions: CDX2, TTF1 and ISL1 are differently expressed in GI versus pulmonary PDNEC and SmCC and LCNEC. While this finding has potential diagnostic utility, the ubiquitous expression regardless of site reflects the poor differentiation of this tumor and warrants caution in routine practice in order to avoid interpretation of immunoreactivity as specific and minimize false positives.

\section{What's Hiding In Your Hemorrhoids? A Review of 1,015 Hemorrhoids}

David R Levy', John Higgins', Christine Y Louie'. 'Stanford University School of Medicine, Stanford, CA, ${ }^{2}$ Stanford University Hospital, Stanford, CA, ${ }^{\mathrm{V}} \mathrm{VA}$ Palo Alto Health Care System, Palo Alto, CA

Background: Hemorrhoidectomy specimens are routinely submitted for histopathologic evaluation and while a vast majority of specimens submitted as "hemorrhoid" demonstrate the classic features of dilated vascular spaces with or without thrombosis, occasionally, incidental lesions are identified. All specimens submitted as "hemorrhoid" at two hospitals were retrospectively reviewed to examine the prevalence of unexpected findings in hemorrhoidectomy specimens.

Design: Our institutions' databases were searched from 1991-2017 for specimens submitted as "hemorrhoid" or "hemorrhoidectomy." Pathology reports and slides were reviewed.

Results: 1,015 specimens submitted/labeled as "hemorrhoid" were identified, out of which $798(78.6 \%)$ were diagnosed as hemorrhoid/ hemorrhoidal tissue or consistent with hemorrhoid. $127(12.5 \%)$ were found to be thrombosed hemorrhoids. In addition, $35(3.4 \%$ demonstrated features of a fibroepithelial polyp or skin tag. Notably, 34 cases $(3.3 \%)$ demonstrated proliferative, neoplastic, dysplastic or malignant findings, including condyloma acuminata (10), squamous cell carcinoma in-situ/AIN3 (9), invasive squamous cell carcinoma (2), invasive adenocarcinoma (2), tubular adenoma (2), hyperplastic polyp (2), granular cell tumor (1), small cell carcinoma (1), invasive melanoma (1), neurofibroma (1), tubulovillous adenoma (1), Paget's disease (1), and benign ectopic breast tissue (1). A single hemorrhoid case was finalized as a gross only diagnosis.

Conclusions: Unexpected findings are occasionally seen in hemorrhoidectomy specimens. Although a majority of cases demonstrated findings consistent with hemorrhoids or thrombosed hemorrhoids, $3.3 \%$ showed neoplastic, dysplastic or malignant findings. This study emphasizes the importance of submitting hemorrhoidectomy specimens for histopathologic review, as unexpected diagnostic findings may occur that require additional follow up and treatment.

\section{Hydroxysteroid 11-beta dehydrogenase 1 is an overexpressed lipid-catabolizing enzyme with pro- proliferative and anti-apoptotic functions to drive tumor progression in GISTs}

Chien-Feng Li', Ting-Ting Liu', Hsuan-Ying Huang ${ }^{3}$. 'Chi Mei Foundation Hospital, Tainan, ${ }^{2}$ Kaohsiung Chang Gung Memoria Hospital and Chang Gung University College of Medicine, Kaohsiung ${ }^{3}$ Chang Gung Memorial Hospital, Kaohsiung City, Taiwan

Background: Metabolic drivers remain largely unknown in gastrointestinal stromal tumors (GISTs), for which we aimed to identify and characterize aberrant lipid metabolism-regulating enzymes underpinning the progression in GISTs.

Design: Focusing on genes categorized into the lipid-catabolizing bioprocess, we reappraised published transcriptomes of GISTs and discovered differential upregulation of hydroxysteroid 11-beta dehydrogenase 1 (HSD11B1)-encoding gene in high-risk GISTs. In full sections of 70 GITSs, HSD11B1 mRNA abundance was assessed 
using QuantiGene assay. Tissue microarrays of an independent cohort were applied to analyze the associations between HSD11B1 immunoexpression and clincopathological variables, yielding 350 informative cases with KIT/PDGFRA/BRAF genotypes determined in 213. In vitro, GIST cell lines were stably transduced with one of the two efficient short-hairpin RNA clones targeting HSD11B1 (shHSD11B ) to evaluate the effects on cell proliferation and apoptosis.

Results: Compared with normal tissue, HSD11B1 mRNA abundance significantly increased across tumors of various risk levels ( $p<$ $0.001)$, as well as from the non-high-risk group to high-risk GISTs $(p=0.009)$. HSD11B1 overexpression was strongly associated with presence of epithelioid histology, non-gastric location, and increases in tumor size, mitosis and risk levels defined by both the NIH and NCCN schemes (all $p \leq 0.001$ ) and strongly predictive of worse shorter disease-free survival $(p<0.0001)$. In the multivariate analysis, HSD11B1 overexpression remained prognostically independent as an adverse factor. Compared with shLacZ, both shHSD11B1 clones significantly decreased the cell proliferation rate in the Brdu uptake assay and increased the percentage of early apoptotic cells in the Annexin V-stained flow cytometric assay.

Conclusions: In conclusion, HSD11B1 is a novel overexpressed lipid-catabolizing enzyme causatively implicated in GIST progression, given its association with unfavorable clincopathological factors, independent negative prognostic impact, and pro-proliferative and anti-apoptotic functions.

\section{Accuracy and Impact of Clinical Grading (cG) of Esophageal Adenocarcinoma Using a Modified Grading System}

Jingwei Li ${ }^{1}$, Siva Raja², John Goldblum², Lisa Rybicki², Sudish Murthy², Usman Ahmad ${ }^{2}$, Thomas Rice ${ }^{2}$, Eugene Blackstone ${ }^{2}$, Deepa T PatiR. ${ }^{1}$ Case Western Reserve University, Cleveland, $\mathrm{OH},{ }^{2} \mathrm{Cleveland}$ Clinic, Cleveland, $\mathrm{OH}$

Background: Histologic grade $(G)$ is a principle category for AJCC $8^{\text {th }}$ edition grouping of stage $\mathrm{I} / \mathrm{Il}$ esophageal adenocarcinomas (ADC). Grading biopsies is challenging, and therefore clinical grade (cG) is inconsistently reported in clinically diagnosed intramucosal ADC (cIMC). To this end, we developed a modified grading system for cIMC and evaluated the correlation between $\mathrm{CG}$ and corresponding resection grade (pG). In addition, we also assessed histologic features in biopsies that might be predictive of pG3 and Stage III/IV ADC

Design: From 2006 to 2015, 43 patients undergoing cADC resections without pre-operative therapy and with pre-operative biopsies were reviewed. None of the patients had any form of therapy between index biopsy and resection. Retrospective blind grading was performed using $8^{\text {th }}$ edition for $\mathrm{pG}$ and the following modified grading was used to determine cG: cG1-tubules or simplified glands infiltrating lamina propria (LP) or muscularis mucosae (MM), cG2-complex back to back or "never-ending glands" with budding and/or cribriform architecture, cG3- cords or sheets of atypical cells, signet ring cells, or anastomosing glands infiltrating LP or MM.

Results: The distribution of cG was: cG1-14\%, cG2-47\%, cG3-39\% and pG was: pG1-21\%, pG2-28\%, pG3-51\%. cG showed substantial correlation with pG $(0.837 ; p<0.001)$. None of the $\mathrm{cG}_{1}, 25 \% \mathrm{cG} 2$, and all cG3 cancers showed $p G 3$ in resection specimens $(p<0.001)$. A lower percentage of biopsies with simple abortive glands (12\% vs $60 \%$; $\mathrm{p}=0.02)$ or complex budding glands $(29 \%$ vs. $65 \% ; p=0.03)$ were $p G 3$ while all biopsies with cords or sheets of atypical cells were associated with pG3 (100\% vs 38\%; $p=0.001)$. Compared to all biopsies with signet ring cells, only $40 \%$ biopsies without signet ring cells showed pG3 $(p=0.004)$. Increasing cG ( $0 \%$ cG1 vs. $10 \%$ cG2 vs. $59 \%$ cG3; $p<0.001)$ and sheets of atypical cells on biopsies were significantly associated with pStage III/IV $(p<0.001)$

Conclusions: The modified grading system facilitates accurate determination of $\mathrm{cG}$ and significantly correlates with $\mathrm{pG}$ in treatment naïve ADC patients. $c G 1$ and $c G 3$ are distinct entities, and patients with cG2 are unlikely to harbor pG3 ADC. This is important in clinical decision-making and AJCC $9^{\text {th }}$ edition staging.

\section{Hypemutation in Colorectal Adenocarcinoma (CRC) detected by Ion AmpliSeq Cancer Hotspot Panel Is Highly Correlated with High Tumor Grade}

Xiaodong Li', Katherine Sun ${ }^{2}$, Xiaoyan Liao $^{3}$, Hongfa Zhu ${ }^{4}$, Naima Ismail ${ }^{5}$, Matija Snuderl ${ }^{6}$, Ruliang X $u^{7}$. ${ }^{1} \mathrm{NYU}$ Langone Medical Center. South Pasadena, CA, ${ }^{2}$ NYU Langone Medical Center, New York, NY, ${ }^{3}$ Icahn School of Medicine at Mount Sinai, New York, NY, ${ }^{4}$ Mount Sinai Hospital, ${ }^{5} \mathrm{NYU}$ Langone Medical Center, ${ }^{6} \mathrm{New}$ York University, ${ }^{7} \mathrm{New}$ York University Medical Center, New York, NY

Background: $\mathrm{CRC}$ is a heterogeneous and complex disease, harboring numerous genetic and epigenetic alterations acquired during cancer development. The genetics and epigenetics of CRCs may dictate their histology, biology, and clinical outcome. We reviewed the sequencing data generated from the next generation sequencing technology to analyze the relationship between the quantity of gene mutations and the biology of CRCs esign: 152 cases of resected CRCs without neoadjuvant therapy that ave sequencing data generated by Ion AmpliSeq Cancer Hotspot Panel (or 50 genes panel) were retrospectively identified from the department database. These 152 cases also had immunostaining results for mismatch repair proteins (surrogate markers for Microsatellite instability status, or MSI). The CRCs were divided into two groups: hypermutated (3 or more gene mutations) and hypomutated (2 or less gene mutations). Tumor grade, T stage, lymph node metastasis, and MSI status in the two groups were compared and the data analyzed using Fisher's exact test.

Results: Of the 152 cases, 93 (61.2\%) were classified into the hypomutation group and $59(38.8 \%)$ into the hypermutation groups. In the hypomutation group, $80(86.0 \%)$ were low (well and moderately differentiated) and $13(14.0 \%)$ were high grade (poorly differentiated) CRCs. In the hypermutation group, 37 (62.7\%) were diagnosed as low and $22(37.3 \%)$ as high grade CRCs. The hypermutation status is strongly associated with high tumor grade $(P=0.0014)$. High $T$ stages (stage 4) does not correlate with mutation status (25/93 in hypomutation group and 19/59 in hypermutation group, p>0.05). In addition, no correlation between hypermutation and positive lymph nodes or MSI-High was found (54/93 or 20/90 in hypomutation group and $29 / 59$ or $18 / 57$ in hypermutation group, $p>0.05$ ).

Conclusions: CRCs with hypermutation detected by Ion AmpliSeq Cancer Hotspot Panel are more likely to have high grade of histology, suggesting accumulation of gene mutations leading to worse biological behavior.

786 Dysregulation of Epidermal Growth Factor Receptor (EGFR) Signaling Pathway in Mucinous Colorectal Adenocarcinoma (CRC) and CRC with Mucinous Component as Evidenced by High Frequency of KRAS and BRAF Mutations

Xiaodong Li ${ }^{1}$, Katherine Sun ${ }^{2}$, Xiaoyan Liao ${ }^{3}$, Hongfa Zhu ${ }^{4}$, Naima Ismail/ ${ }^{5}$, Matija Snuderl', Ruliang X ${ }^{7}{ }^{7}$. ${ }^{1} \mathrm{NYU}$ Langone Medical Center South Pasadena, CA, ${ }^{2}$ NYU Langone Medical Center, New York, NY ${ }^{3}$ Icahn School of Medicine at Mount Sinai, New York, NY, ${ }^{4}$ Mount Sinai Hospital, ${ }^{5} \mathrm{NYU}$ Langone Medical Center, ${ }^{6} \mathrm{New}$ York University, ${ }^{7} \mathrm{New}$ York University Medical Center, New York, NY

Background: Mucinous adenocarcinoma is a special type of colorectal cancer (CRC) that has poor response to the treatment more aggressive behavior and poorer outcome than non-mucinous CRC. Its biological and clinical behavior is largely determined by its molecular genetics. However, the genetics of this group of CRC is yet to be defined.

Design: 152 cases of resected $\mathrm{CRC}$ with sequencing data generated by Ion AmpliSeq Cancer Hotspot Panel (or 50 genes) in the past two years were retrospectively retrieved from the departmenta databases. No neoadjuvant therapy was performed before surgery. CRCs were divided into two groups: 1). Mucinous CRC (MCRC) group ( 13 mucinous CRC and 19 CRC with at least $20 \%$ of mucinous component $(n=32)$ and 2). Non-mucinous CRC (NMCRC) group without mucinous component $(n=120)$. The type and frequency of gene mutations and microsatellite instability (MSI) status defined by immunohistochemistry for mismatch repair proteins were analyzed. Fisher exact test was employed for statistical analysis.

Results: In MCRC group, 31 of 32 (97\%) were positive for the mutation of either KRAS (15/32), BRAF (15/32) or double mutations (1/32). Only one case showed no mutation for KRAS or BRAF, but TP53. The highest frequent mutations in MCRC group were KRAS (16/32) and BRAF (16/32), PIK3CA (10/32), followed by APC (9/32) and TP53 (8/32) In NMCRC group, 64 of $120(53 \%)$ cases had either KRAS $(50 / 120$ $42 \%)$ or $B R A F(14 / 120,12 \%)$ mutation, and no double mutations. TP53 mutation $(65 / 120,54 \%)$ is most frequent mutation in this group followed by KRAS (50/120, 42\%), APC (39/120, 33\%), PIK3CA (26/120, $22 \%)$ and $B R A F(14 / 120,12 \%)$. MSI-high status is more frequently seen in BRAF mutated CRCs in MCRC group $(12 / 15,80 \%)$ than in BRAF mutated NMCRC group $(50 \%)(p<0.05)$, suggesting that MSI-high status is more commonly related with epigenetic effect in MCRCs.

Conclusions: The vast majority of mucinous CRC or CRC with mucinous component have the mutations either in KRAS or BRAF in EGFR signaling pathway, suggesting that dysregulation of EGFR pathway plays a critical role in the development of mucinous CRC or $\mathrm{CRC}$ with mucinous component. Poor response to the treatment of mucinous adenocarcinoma may be partially attributable to the unique genetics of this group of CRCs.

\section{Clinicopathological Characterization of SMAD4-} Mutated Intestinal Adenocarcinomas

Xiaoyan Liao' ${ }^{1}$, Xiaofei Zhang ${ }^{1}$, Min Cui', Qingqing Liu, Huaibin Mabel $K \mathrm{~K}^{1}$, Hongfa Zhu ${ }^{2}$, Noam Harpaz ${ }^{3}$, Alexandros D Polydorides'. ${ }^{1}$ Icahn School of Medicine at Mount Sinai, New York, NY, ${ }^{2}$ The Icahn Schoo of Medicine at Mount Sinai, Paramus, NJ, ${ }^{3}$ Mount Sinai Med. Center New York, NY

Background: SMAD4 is a tumor suppressor gene that inhibits the 
transforming growth factor- $\beta$ pathway and is sporadically mutated in up to $20 \%$ of colorectal carcinomas (CRC). We sought to evaluate the clinicopathologic associations of this mutation in intestinal adenocarcinomas.

Design: We identified all primary adenocarcinomas of small bowel (SBA) or colorectal (CRC) origin biopsied or resected at our institution and genetically sequenced between 2013-2017. Clinical and pathological data were obtained from the electronic medical records. Representative tumor sections had been analyzed by targeted next generation sequencing (NGS) of 50 genes using the Ion AmpliSeq Cancer Hotspot Panel (v2, Thermo Fisher). Chi square statistical analysis employed a significance threshold of $P \leq 0.05$.

Results: Twenty-four of $443(5.2 \%)$ intestinal adenocarcinomas harbored SMAD4 mutations, including 2 SBAs and 22 CRCs. The patients were of mean age 64 (range, 38-83 years) with a 1.3:1 male predominance. For each case, we selected as random wildtype SMAD4 controls the subsequent 2 specimens from the same anatomical site in the analysis stream. Age and sex distribution were statistically similar. A significantly higher proportion of SMAD4-mutated carcinomas had mucinous features compared with the control group ( $54 \%$ vs. $14 \%$, $\mathrm{P}<0.001)$. In the SMAD4-mutated group, mutations in the $\mathrm{MH} 2$ domain (c. $1081 \mathrm{C}>\mathrm{T}$ or c. $1082 \mathrm{G}>\mathrm{A}$ ) were significantly enriched in carcinomas with mucinous differentiation compared to nonmucinous tumors (69\% vs. $18 \%, P=0.01$ ). The proportion of Crohn's disease-associated carcinomas with SMAD4 mutations $(4 / 7,57 \%)$ was significantly higher than that in non-Crohn's tumors (20/436, 4.6\%; $\mathrm{P}<0.001)$. SMAD4 mutations were invariably accompanied by other genetic alterations including KRAS (68\%), APC (40\%), and TP53 (40\%).

Conclusions: In our series, SMAD4 mutations occurred in 5\% of intestinal adenocarcinomas and in a significantly higher proportion of Crohn's-associated carcinomas. They correlate with mucinous histology, especially in the subgroup with mutations in the $\mathrm{MH} 2$ domain.

\section{Clinicopathological and Molecular Characterization of Crohn's Disease-Associated Small Bowel Adenocarcinomas}

Xiaoyan Liao' ${ }^{1}$, Guangyuan Li ${ }^{2}$, Xiaofei Zhang ${ }^{1}$, Min Cui', Qingqing Liu'

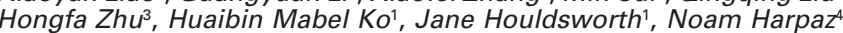
Alexandros D Polydorides'. 'Icahn School of Medicine at Mount Sinai, New York, NY ${ }^{2}$ Mount Sinai Hospital, ${ }^{3}$ The Icahn School of Medicine at Mount Sinai, Paramus, NJ, ${ }^{4}$ Mount Sinai Med. Center, New York, NY

Background: Crohn's disease (CD) is associated with a 60-fold increased risk of small bowel adenocarcinoma (SBA) but the absolute prevalence remains low, limiting opportunities for clinical, pathological, and molecular studies. We evaluated a large series of resected SBAs in Crohn's disease (CDSBA) and compared them to SBAs from non-IBD patients (NSBA).

Design: Primary jejunal and ileal SBAs resected at our institution from 2000-2017 were identified. Clinical and pathological data were obtained from electronic medical records. Representative sections (with at least $60 \%$ tumor cells) were analyzed by targeted next generation sequencing (NGS) of 50 genes using the Ion AmpliSeq Cancer Hotspot Panel (v2, Thermo Fisher). Mismatch repair (MMR) status was determined by immunohistochemistry for 4 MMR proteins. Chi square statistical analysis employed a significance threshold of $P$ $\leq 0.05$.

Results: We identified 45 patients with CDSBA (mean age 55, 69\% male) and 18 with NSBA (mean age 58,50\% male), the differences being nonsignificant (NS). Both CDSBAs and NSBAs occurred more frequently in the ileum ( $73 \%$ and $72 \%$, respectively) than the jejunum. Macroscopically, a significantly lower proportion of CDSBAs than NSBAs presented as a discrete mass $(31 \%$ vs. $94 \%$, respectively, $\mathrm{P}<0.001)$ and a significantly higher proportion as a stricture $(42 \%$ vs. $11 \%$, respectively, $\mathrm{P}=0.01)$. Ninety-three percent of CDSBAs were accompanied by adjacent chronic active enteritis and $67 \%$ by dysplasia. A significantly higher proportion of CDSBAs than NSBAs was stage T1/T2 N0 ( $36 \%$ vs. $6 \%, \mathrm{P}=0.01$ ), but the proportions with distant metastatic disease were similar (22\% vs. $28 \%$, respectively). The rates of signet ring cell, mucinous, neuroendocrine, or medullary differentiation were similar, however, well-differentiated tubuloglandular adenocarcinoma occurred uniquely in $16 \%$ of CDSBAs. Deficient MMR status was much less common in CDSBAs compared to NSBAs ( 6 vs. $44 \%, P=0.02$ ). The genes most frequently mutated in CDSBAs were TP53 $(80 \%)$, KRAS $(40 \%)$, SMAD4 $(20 \%)$, and IDH1 $(20 \%)$; no BRAF or APC gene mutations were detected.

Conclusions: This CDSBA case series, which is one of the largest reported to date, establishes that it is pathologically distinct from NSBA in certain important respects including a higher prevalence of stricturing rather than mass-forming gross configuration, tubuloglandular histology, lower tumor stage, MMR proficiency, and TP53 mutation.
789 In colorectal carcinoma (CRC), true tumor cell staining for PD-L1 is uncommon and occurs in sporadic microsatellite-instable tumors with loss of MHC-I

Sandy Liu' ${ }^{1}$, Monika Vyas ${ }^{2}, Z$ sofia stadler ${ }^{3}$, Martin Weiser ${ }^{3}$, Sarah King $^{3}$ Jaclyn Hechtman ${ }^{4}$, Efsevia Vakiani ${ }^{5}$, David Klimstra ${ }^{4}$, Jinru Shia ${ }^{4}$ ${ }^{1}$ Memorial Sloan Kettering Cancer Center, Ellicott City, MD, ${ }^{2} \mathrm{New}$ Haven, CT, ${ }^{3}$ Memorial Sloan Kettering Cancer Center, ${ }^{4}$ Memorial Sloan Kettering Cancer Center, New York, NY, ${ }^{5}$ New York, NY

Background: The use of immune checkpoint inhibitors is gaining increasing traction in the treatment of patients with microsatelliteinstability-high (MSI-H) CRC. It has been observed that PD-L1 mostly labels the tumor-stroma interface in CRC and it is often difficult to determine whether the cells stained are cancer cells or immune cells. Better characterization of the cellular components that label for PD-L is thus desirable; such data may not only allow better understanding of the tumor's immune environment, but may also shed light on how best to incorporate IHC into clinical studies.

Design: CRCs classified into subsets of MSI-H or microsatellitestable (MSS) tumors (based on mismatch-repair-protein IHC, PCR MS testing, MLH1 methylation testing, and germline mutation testing were further tested with PD-L1 IHC (E1L3N, Cell Signaling) as well as MHC-I (A4, Ebioscience) and B2M (Polyclonal, DAKO) IHC. Tumors found to have ambiguous tumor cell versus immune cell staining for PD-L1 were further examined by double IHC staining utilizing SATB2 (EP281, Cell Margue; nuclear staining in CRC cells) and PD-L (membranous staining).

Results: Study cases included $73 \mathrm{MSI}-\mathrm{H}$ and $39 \mathrm{MSS}$ CRCs. As outlined in the table, by single antibody IHC, some PD-L1 staining (5\% or more of the tumor area inclusive of both tumoral and non-tumora cells) was observed in the vast majority $(89 \%)$ of MSI-H cancers and a smaller but still significant subset (31\%) of MSS CRCs. When using $30 \%$ of the tumor area staining as the cutoff, the PD-L1 positive rate remained high $(70 \%)$ in $\mathrm{MSI}-\mathrm{H}$ tumors but dropped to only $3 \%$ in MSS tumors. Double staining showed that the PD-L1 positivity was present almost exclusively in the immune cells; only 2 tumors showed unequivocal tumor cell staining beyond scant individual cell positivity In both tumors, the staining was present in $>50 \%$ of the tumor cells Both tumors were MSI-H with abnormal MLH1 methylation, were from old females (70 and 83 yrs, respectively), were right-sided (cecum ascending colon), and had a solid/nested growth with medullary features. Both tumors also showed loss of MHC-I but retained B2M.

\begin{tabular}{|c|c|c|c|c|c|}
\hline PD-L1 IHC & $\begin{array}{c}\text { MSI-H } \\
\text { Total } \\
(\mathrm{n}=73)\end{array}$ & $\begin{array}{c}\text { MSI-H } \\
\text { Lynch } \\
\text { asso- } \\
\text { ciated } \\
(\mathrm{n}=23)\end{array}$ & $\begin{array}{c}\text { MSI-H Spo- } \\
\text { radic/MLH1 } \\
\text { methylated } \\
(\mathrm{n}=37)\end{array}$ & $\begin{array}{c}\text { MSI-H } \\
\text { Germline } \\
\text { mutation } \\
\text { neg./MLH1 } \\
\text { methylated } \\
\text { neg. }\end{array}$ & $\begin{array}{c}\text { MSS } \\
(\mathrm{n}=39)\end{array}$ \\
\hline $\begin{array}{c}>=5 \% \text { of tumor area } \\
\text { staining }\end{array}$ & $\begin{array}{c}65 \\
(89 \%)\end{array}$ & $21(91 \%)$ & $31(84 \%)$ & $13(100 \%)$ & $\begin{array}{c}12 \\
(31 \%)\end{array}$ \\
\hline $\begin{array}{c}>=30 \% \text { of tumor area } \\
\text { staining }\end{array}$ & $\begin{array}{c}51 \\
(70 \%)\end{array}$ & $17(74 \%)$ & $24(65 \%)$ & $10(77 \%)$ & $1(3 \%)$ \\
\hline $\begin{array}{c}>=30 \% \text { of tumor area } \\
\text { staining, positive } \\
\text { cells are recognizable } \\
\text { as immune cells on } \\
\text { single antibody IHC }\end{array}$ & $\begin{array}{c}35 \\
(48 \%)\end{array}$ & $15(65 \%)$ & $12(32 \%)$ & $8(62 \%)$ & $1(3 \%)$ \\
\hline $\begin{array}{c}\text { Ambiguous tumor } \\
\text { cell vs immune cell } \\
\text { staining }\end{array}$ & $\begin{array}{c}16 \\
(22 \%)\end{array}$ & $2(9 \%)$ & $12(32 \%)$ & $2(15 \%)$ & 0 \\
\hline $\begin{array}{c}\text { True tumor cell stain- } \\
\text { ing by double IHC }\end{array}$ & $2(3 \%)$ & 0 & $2(5 \%)$ & 0 & 0 \\
\hline
\end{tabular}

Conclusions: PD-L1 expression within CRC primarily occurs in tumorassociated immune cells; true tumor cell expression of this checkpoint receptor ligand is rare, and in our series, it was limited to sporadic $\mathrm{MSI}-\mathrm{H}$ tumors with loss of MHC-1. Our findings may help guide future research efforts and allow more informed PD-L1 IHC design in clinical trials.

\section{Immunopathologic Characterization of Immunotherapy Induced Colitis, with a Comparison to Inflammatory Bowel Disease: Phenotypic Differences May Reflect Drug Mechanisms and Disease Duration}

Ying-Chun Lo', Xuchen Zhang ${ }^{1}$, Marie E Robert'. 'Yale University School of Medicine, New Haven, CT

Background: Immune checkpoint inhibitors show efficacy in cancer but are associated with inflammatory side effects, including colitis The morphology of anti-CTLA4 and to a lesser extent, anti-PD-1/ PD-L1 associated colitis has been reported. However, underlying mechanisms are unknown. We characterized the morphologic and immune phenotype of immunotherapy associated colitis (IC), and 
contrasted the findings by type of therapy; with a comparison to ulcerative colitis (UC), and Crohn's disease (CD).

Design: Colon biopsies were retrieved from the pathology files in patients with colitis due to: anti-CTLA4 (10), anti-PD-1 (9), anti-PD-L1 (2), UC (5), and CD (5). Normal mucosa (5) and anti-CTLA4 without diarrhea (5) served as controls. H\&E and immunohistochemical (IHC) stains for CD4, CD8, CD68, FoxP3, PD-1 and PD-L1 were scored blindly by two pathologists on a $0-3+$ semi-quantitative scale. Histologic features of activity (neutrophils, cryptitis, crypt abscesses, epithelial injury), chronicity (crypt distortion, plasma cells, sub-cryptal space), apoptosis, crypt attenuation and increased intraepithelial lymphocytes were evaluated. FoxP3 positive cells were counted in 3 hot spots.

Results: Anti-CTLA4 biopsies had significantly higher scores for CD8, PD-1, PD-L1 and CD68 than anti-PD-1/PD-L1 biopsies, consistent with expected effects of the specific immunotherapy (Table 1, Figures 1-2). IBD biopsies had higher scores for CD8 (vs both drugs), CD4 and PD-1 (vs anti-PD-1/PD-L1 only), and FoxP3 (vs anti-CTLA4 only). The CD8/FoxP3 ratios were higher in anti-CTLA4 biopsies than in either IBD or anti-PD-1/PD-L1 groups, consistent with loss of inhibition by regulatory $\mathrm{T}$ cells in anti-CTLA4 therapy. With respect to morphology, chronicity scores were significantly higher in IBD compared to IC, while activity scores were similar. No differences in morphologic variables were found between the two types of immunotherapy, or between the anti-CTLA4 without diarrhea group and normal controls for any parameter

\begin{tabular}{|c|c|c|}
\hline Pathologic Features & $\begin{array}{l}\text { IBD vs. IC } \\
\text { ( } p \text { value) }\end{array}$ & $\begin{array}{l}\text { CTLA4 vs. PD-1/PD-L1 } \\
\text { (p value) }\end{array}$ \\
\hline Activity Score (0-12) & n.s. & n.s. \\
\hline Crypt Distortion (0-3) & $\begin{array}{l}\text { IBD }> \\
I C<0.0001\end{array}$ & n.s. \\
\hline $\begin{array}{l}\text { Subcrypt Inflamma- } \\
\text { tion }(0-3)\end{array}$ & $\begin{array}{l}\text { IBD > IC } \\
<0.001\end{array}$ & n.s. \\
\hline $\begin{array}{l}\text { Plasma Cells in LP } \\
(0-3)\end{array}$ & $\begin{array}{l}\text { IBD > IC } \\
<0.05\end{array}$ & n.s. \\
\hline Chronicity Score (0-9) & $\begin{array}{l}\text { IBD }> \\
I C<0.0001\end{array}$ & n.s. \\
\hline Apoptosis ( 0 or 1 ) & $0.11^{*}$ & n.s. \\
\hline $\begin{array}{l}\text { Crypt Attenuation (0 } \\
\text { or } 1 \text { ) }\end{array}$ & $0.11^{*}$ & n.s. \\
\hline Surface IEL (0 or 1 ) & n.s. & n.s. \\
\hline CD4 (0-3) & n.s. ${ }^{* *}$ & n.s. \\
\hline CD8 (0-3) & $\begin{array}{l}\mathrm{IBD}>\mathrm{IC} \\
<0.05\end{array}$ & $\begin{array}{l}\text { CTLA4 > PD-1/PD-L1 } \\
<0.01\end{array}$ \\
\hline PD-1 (0-3) & n.s. ${ }^{* *}$ & $\begin{array}{l}\text { CTLA4 > PD-1/PD-L1 } \\
<0.01\end{array}$ \\
\hline PD-L1 (0-3) & n.s. & $\begin{array}{l}\text { CTLA4 > PD-1/PD-L1 } \\
<0.01\end{array}$ \\
\hline CD68 (0-2) & n.s. & $\begin{array}{l}\text { CTLA4 > PD-1/PD-L1 } \\
<0.05\end{array}$ \\
\hline CD8/FoxP3 ratio & n.s. ${ }^{*}$ & $\begin{array}{l}\text { CTLA4 > PD-1/PD-L1 } \\
<0.01\end{array}$ \\
\hline
\end{tabular}

Student t-test and Fisher's exact test.

* There was a trend towards increased apoptosis and crypt attenuation in IC compared to UC that did not reach significance.

* See text for additional statistical comparisons between IBD and each drug separately.

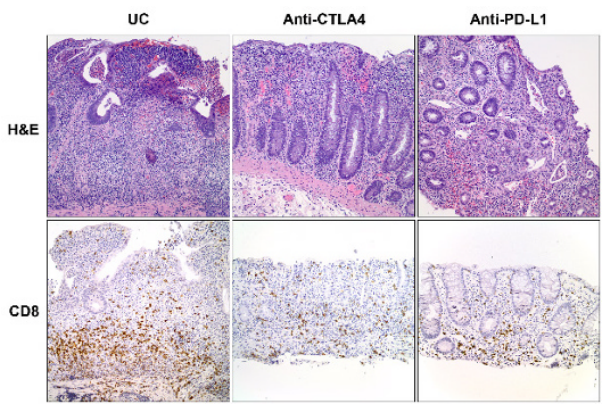

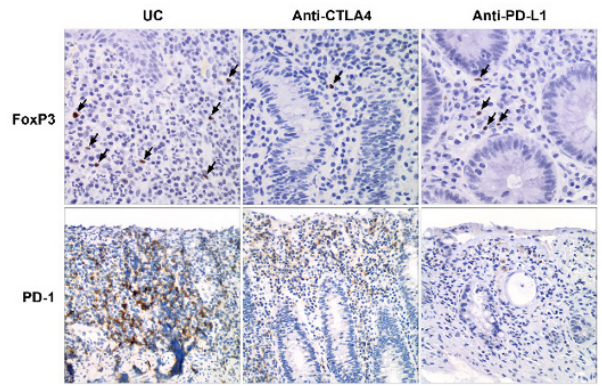

Conclusions: The number of mucosal CD8, CD4, FoxP3, and PD-1 positive cells, and histologic features of chronicity are significantly higher in IBD compared to IC, while acute inflammation and epithelia injury are similar. Whether these findings reflect mechanistic divergence or duration of disease is unknown. The differences in IHC found between drug groups likely reflect drug mechanisms. The possibility that mucosal inflammatory dynamics in IC mirror activity in the tumor environment could be explored.

\section{Gastric Mucosal Lymphoid Follicles: Location, Occupancy, Density, Size, and Etiology}

Nicolas Lopez-Hisijos ${ }^{1}$, Stefan Pambuccian², Michael Moravek², Daniel Shepherd?', Mohammed Atieh². 'Maywood, IL, ${ }^{2}$ Loyola University Medical Center, Maywood, IL

Background: It is thought that gastric mucosal lymphoid follicles do not occur in normal gastric tissue. However, we have noticed that they are present in otherwise normal partial gastrectomy specimens for obesity treatment. There are few studies on histological features of gastric mucosal lymphoid follicles. We sought to determine the normal baseline for size, location, and density of Iymphoid follicles. We also analyzed the relationship between these histologic findings and the pertinent clinical information.

Design: A search was performed in our department's electronic database (Sunquest Copath v6) for partial gastrectomy cases from 09/16 to 09/17. A single slide of each case was scanned (Aperio ScanScope CS2) and the images were analyzed digitally (Aperio ImageScope 12.2). The number, size, location, diameter, and occupancy were measured for all lymphoid follicles. Occupancy was defined as the ratio of the vertical length to the mucosal thickness. Location was documented from deep to superficial: muscularis mucosa, base, neck, and isthmus. The length of each tissue section was recorded. Statistical analysis was performed using R 3.4.1 software. Chi-square tests were used for categorical variables, two-sample t-tests and ANOVA were used for mean comparisons. The BMI and age of each patient was recorded.

Results: There were 94 partial gastrectomy specimens found. The vast majority were performed for morbid obesity (89\%). Most of them did not have any underlying pathology and were negative for $\mathrm{H}$. pylori in both the gastrectomy specimen and in the biopsy prior to surgery $(80 \%)$. The majority of cases were diagnosed as normal $(55 \%)$. There was no correlation between BMI or age with the histologic features of the follicles. There was a significant association between $\mathrm{H}$. pylori and a diagnosis of chronic active gastritis with the number and density of the follicles (Table 1). There was no significant difference for location, even when the lymphoid aggregates adjacent to the muscularis mucosa were considered confounding $(\mathrm{P}=0.11)$. There were 734 lymphoid aggregates measured. The vast majority of them $(71 \%)$ were small and adjacent to the muscularis mucosa (Figure 1).

FOR TABLE DATA, SEE PAGE 318, FIG. 791

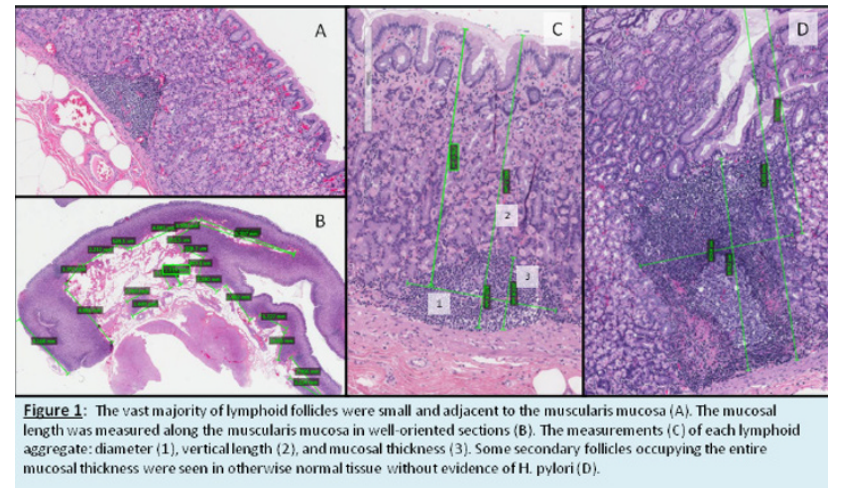

Conclusions: A baseline for lymphoid follicles has been established in this study, but their number, location, and size can be variable in normal gastric tissue. Therefore, the presence of lymphoid follicles 
in gastric tissue should not automatically be treated as pathologic without a known etiologic cause.

\section{Loss of ARID1A Expression Is Associated With Microsatellite Instability And Favorable Prognosis in Advanced Stage Surgically Resected Esophageal Adenocarcinoma}

Brett M. Lowenthal', Katie S. Nason ${ }^{2}$, James D Luketich ${ }^{2}$, Reetesh $\mathrm{Pal}^{3}$, Jon Davison ${ }^{4}$, Changqing $\mathrm{MA}^{4}$. 'UPMC-Presbyterian Hospital, San Diego, CA, 2UPMC, ${ }^{3}$ UPMC-Presbyterian Hospital, Pittsburgh, PA, ${ }^{4}$ University of Pittsburgh, Pittsburgh, PA

Background: $A R I D 1 A$ has been implicated as a tumor-suppressor gene in esophageal adenocarcinoma (EAC). Previous studies have shown loss of ARID1A protein expression is associated with microsatellite instability (MSI) and/or Epstein - Barr virus (EBV) infection in adenocarcinoma arising in stomach and colorectum. In this study, we sought to evaluate the prevalence of ARID1A loss and its prognostic significance in a large cohort of surgically resected, primary EACs.

Design: Tissue microarrays representing 316 EACs treated by esophagectomy without neoadjuvant treatment, including 5 (2\%) mid/proximal esophageal, 89 (28\%) distal esophageal, and $222(70 \%)$ gastroesophageal junction (GEJ) tumors were used to evaluate ARID1A loss and MSI by immunohistochemistry and EBV infection using in-situ hybridization. Loss of ARID1A was defined as the complete absence of nuclear staining with appropriate internal controls. ARID1A expression was correlated with demographics, clinicopathologic features, and clinical outcome.

Results: ARID1A loss was detected in 13\% (42/316) of EACs including 11 distal esophageal and $31 \mathrm{GEJ}$ tumors. MSI was detected in $5 \%$ (16/316) EACs but MSI was present in a significantly higher proportion of EACs with ARID1A loss compared with ARID1A retained EACs $(13 / 42,31 \%$ vs. $3 / 274,1 \%$ respectively; $P<0.001)$. ARID1A loss was also detected in the one EBV-associated EAC. In the entire cohort, ARID1A loss was not associated with overall survival (OS). However, in the subset of patients who had locally advanced or metastatic disease (stages III and IV, $\mathrm{n}=164$ ), patients with ARID1A loss EACs ( $\mathrm{n}$ $=22$ ) had significantly longer OS than patients with ARID1A retained EACs (36\% vs. $11 \%$ of patients alive at 5 years; $P=0.002)$. Importantly, in these patients, ARID1A loss was associated with a $48 \%$ reduction in mortality independent of other prognostic factors including MSI status, histologic grade, age, gender, and comorbidity index (hazard ratio: $0.52,95 \%$ confidence interval: $0.28-0.96, P=0.036$ )

Conclusions: ARID1A loss is frequently associated with MSI in EACs. Furthermore, loss of ARID1A expression is associated with improved overall survival in locally advanced or metastatic, surgically resected EAC.

\section{SATB2 is Useful in Distinguishing Signet Ring Cell Carcinoma of the Stomach and Lower Gastrointestinal Tract}

Brett M. Lowenthal', Changqing $\mathrm{Ma}^{2}$, Reetesh Pa ${ }^{3}$. 'UPMC-Presbyterian Hospital, San Diego, CA, 2University of Pittsburgh, Pittsburgh, PA, ${ }^{3}$ UPMC-Presbyterian Hospital, Pittsburgh, PA

Background: Signet ring cell carcinomas of the gastrointestinal (GI) tract are clinically aggressive diseases with frequent distant metastases at initial clinical presentation. Immunohistochemistry (IHC) analysis is often used to determine primary site of origin. Previous studies suggest SATB2 is a sensitive marker for colorectal adenocarcinoma. However, its diagnostic utility in distinguishing signet ring cell carcinomas of GI tract has not been examined. In this study, we evaluated the utility of SATB2 together with CDX2 IHC in establishing primary site of origin of signet ring cell carcinoma of the GI tract.

Design: SATB2 and CDX2 IHC was performed on whole tissue sections of 109 signet ring cell carcinomas of the Gl tract including 66 tumors from the lower $\mathrm{GI}$ tract (33 colorectal and 33 appendiceal) and 43 tumors primary to the stomach. A modified $\mathrm{H}$-score, defined as intensity $\mathrm{x}$ percentage of positive cells, was calculated. Positive expression was defined as a modified $\mathrm{H}$-score of $>5$.

Results: SATB2 was more frequently positive in signet ring cell carcinomas of the lower Gl tract compared to those primary to stomach $(82 \%$ vs. $12 \%, p<0.01)$. All 5 SATB2-positive gastric signet ring cell carcinomas demonstrated weak expression in only $5 \%$ of tumor cells (mean H-score: 0.5 ). In contrast, SATB2 was more strongly and diffusely expressed in signet ring cell carcinomas of the lower $\mathrm{Gl}$ tract (mean H-score: 91, p<0.01). Compared with SATB2, CDX2 IHC was more frequently positive in gastric signet ring cell carcinomas. However, CDX2 expression was significantly lower in gastric signet ring cell carcinoma than that of lower Gl tract tumors (44\% vs. $89 \%$ $\mathrm{p}<0.01$ ). Dual SATB2 and CDX2 positive expression (SATB2+/CDX2+) was seen in $74 \%$ of lower GI tract tumors but in only $7 \%$ of gastric tumors $(\mathrm{p}<0.01$ ) (Table 1). SATB2+/CDX2+ for predicting lower Gl tract origin for signet ring cell carcinoma has a sensitivity of $74 \% 195 \% \mathrm{Cl}$
$62-84 \%)$, specificity of $93 \%$ (95\% Cl 81-99\%), positive predictive value of $94 \%(95 \% \mathrm{Cl} 85-98 \%)$, and negative predictive value of $70 \%(95 \%$ $\mathrm{Cl} 61-78 \%)$.

Table 1. SATB2 and CDX2 staining profiles for lower GI tract and gastric signet ring cell carcinomas.

\begin{tabular}{|l|l|l|l|}
\hline $\begin{array}{l}\text { Immunohistochemical } \\
\text { staining profile }\end{array}$ & $\begin{array}{l}\text { Lower GI } \\
\text { Tract } \\
(\%)\end{array}$ & $\begin{array}{l}\text { Gastric } \\
(\%)\end{array}$ & p-value \\
\hline No. Cases & 66 & 43 & NA \\
\hline SATB2 + \& CDX2 + & $49(74)$ & $3(7)$ & $<0.01$ \\
\hline SATB2 - \& CDX2 + & $9(14)$ & $16(37)$ & $<0.01$ \\
\hline SATB2 + \& CDX2 - & $5(8)$ & $2(5)$ & 0.5 \\
\hline SATB2 - \& CDX2 - & $3(5)$ & $22(51)$ & $<0.01$ \\
\hline
\end{tabular}

Conclusions: SATB2 is frequently expressed in signet ring cell carcinomas of the appendix and colorectum but not stomach. Dua SATB2 and CDX2 positive expression is seen in 74\% of lower GI tract signet ring cell carcinomas but infrequently identified in gastric signet ring cell carcinoma. The addition of SATB2 to an immunohistochemical panel including CDX2 can help to determine primary site of origin in signet ring cell carcinomas.

\section{Gastric sarcoidosis and granulomatous Crohn's gastritis: similarities and differences}

Waqas Mahmud', Hussein Alnajar ${ }^{2}$, Shriram Jakate'. 'Rush University Medical Center, Chicago, IL, ${ }^{2}$ Rush University Medical Center Pathology Department, Chicago, IL

Background: Gastric sarcoidosis is quite rare and almost always incidentally detected on gastric biopsies performed for other reasons. Although the granulomas are microscopically identical to those in the lungs, lymph nodes and skin, the diagnosis is usually rendered only when there is pre-existent clinical knowledge of sarcoidosis and tissue diagnosis of sarcoidosis at another extra-gastrointestinal. Furthermore, granulomatous Crohn's gastritis is the main differentia diagnosis. We reviewed 36 cases of gastric sarcoidosis and 22 cases of granulomatous Crohn's gastritis for clinicopathological similarities and differences.

Design: From our Medical Center's pathology and clinical databases we identified 36 cases of gastric sarcoidosis $(27$ Females and 9 Males ages 29-68, mean 52) and 22 cases of granulomatous Crohn's gastritis (9 Females and 13 Males, ages 11-42, mean 18) from nearly 40,000 gastric biopsies during a period of 17 years (2000-2017). We reviewed the H\&E stains, clinical history and prior and subsequent biopsy diagnoses from other tissues.

Results: Gastric sarcoidosis is rarely $(<0.1 \%)$ encountered in gastric biopsies and most often (75\%) seen in middle-aged women. The compact epithelioid non-necrotizing granulomas occurred in clusters ( 3 or> clusters/biopsy fragment). 7/36 (20\%) had associated helicobacter gastritis. Only $1 / 36$ patients had concurrent sarcoid granulomas in random colonic biopsy. 29/36 (80\%) cases of gastric sarcoidosis had clinical diagnosis of sarcoidosis based upon either prior $(25 / 36)$ or subsequent $(4 / 36)$ extra-gastrointestinal sarcoidosis (lung, lymph nodes, skin, liver). Granulomatous Crohn's gastritis is also rare and often seen at a younger age. The granulomas are smaller than sarcoid, often isolated (non-clustered) and only seen as a part of dense focally enhancing gastritis. 20/22 (90\%) cases had associated intestinal granulomatous Crohn's enteritis and/or colitis.

Conclusions: Both gastric sarcoidosis and gastric granulomatous Crohn's are rare entities $(<0.1 \%$ of total gastric biopsies) Microscopically, gastric sarcoid granulomas are clustered and maybe associated with helicobacter gastritis $(20 \%)$, whereas gastric Crohn's granulomas are isolated. While the stomach is an almost exclusive gastrointestinal site for sarcoid, stomach is often a part of co-existen granulomatous intestinal Crohn's.Additionally, sarcoid gastritis predominantly occurs in older women (mean age 52) whereas granulomatous Crohn's gastritis in young patients (mean age 18).

\section{Gastric Heterotopia (GH) of the Colo-rectum and Anus - the Outlet Patch}

Rifat Mannan ${ }^{1}$, Armen Khararjian², Kathleen Byrnes ${ }^{3}$, Binny Khandakar', Songyang Yuan ${ }^{5}$, Feriyl Bhaijee ${ }^{6}$, Henry J Venbrux', David Heydt $^{8}$, Dora Lam-Himlin ${ }^{9}$, Kiyoko Oshima ${ }^{10}$, Lysandra Voltaggio ${ }^{3}$ Elizabeth Montgomery11. 'The Johns Hopkins Hospital, ${ }^{2}$ Baltimore MD, ${ }^{3} J o h n s$ Hopkins Hospital, Baltimore, MD, ${ }^{4} \mathrm{Mt}$ Sinai St Luke's Roosevelt Hospital, New York, NY, ${ }^{5}$ Mt Sinai Beth Israel, New York NY, ${ }^{6}$ AmeriPath Indiana, Indianapolis, IN, ${ }^{7}$ Jackson Siegelbaum Gastroenterology \&PAGI, Camp Hill, PA, ${ }^{8} \mathrm{C}$ apital Digestive Care, LLC Bowie, MD, ${ }^{9}$ Mayo Clinic, Scottsdale, AZ, ${ }^{10} \mathrm{Johns}$ Hopkins Hospital, 11 Johns Hopkins Medical Institutions, Baltimore, MD 
Background: Heterotopic gastric mucosa has been described throughout the gastrointestinal tract (GIT). However, the colorectal region is an extremely rare location for $\mathrm{GH}$. We describe the clinical and pathologic features of $\mathrm{GH}$ occurring in colon, rectum, and anus, highlighting its histologic features and its potential as a diagnostic pitfall.

Design: We identified 14 cases of $\mathrm{GH}$ involving the colon, rectum, and anus from in-house and consultation material. Demographic and clinical information were obtained from respective institutional records. H\&E sections were reviewed.

Results: There were 9 males and 5 females; with an average age of 51 years (range 4 months -73 years). Sites of involvement included the rectum $(n=9)$, anal canal $(n=2)$, ileocecal junction $(n=1)$, ascending colon $(n=1)$ and descending colon $(n=1)$. From the available information, 5 patients presented with hematochezia, 1 had altered bowel habits and another was asymptomatic. On endoscopy, GH appeared as a polyp $(n=8)$, raised erythematous patch $(n=1)$, or diverticulum $(n=1)$; the appearance was not known in 4 cases. Average size was $1.4 \mathrm{~cm}$ (range $0.2-5.5 \mathrm{~cm}$ ). All patients were initially treated by polypectomy. One with associated carcinoma in the area of $\mathrm{GH}$ underwent subsequent resection. No morbidity related to $\mathrm{GH}$ itself was reported for the patients with available follow up. Two patients died of unrelated diseases. Histologically, heterotopic gastric mucosa was characterized by oxyntic type mucosa $(n=10)$, mixed antral and oxyntic type $(n=1)$, mixed antral and cardiac type $(n=1)$, not specified $(n=2)$. One case had associated surface low grade dysplasia of the heterotopic oxyntic mucosa. In another case GH was associated with adenocarcinoma arising from the heterotopic mucosa (proven on subsequent resection). Associated abnormalities in the background mucosa included hemorrhoids $(n=2)$, diverticulosis $(n=1)$, and a duplication cyst $(n=1)$. No Helicobacter pylori organism was identified in any of the cases.

Conclusions: Our series highlights the features of GH in the distal GIT - the outlet patch. Association with adenocarcinoma in one of our cases and dysplasia in another raises concern for lower tract $\mathrm{GH}$ as a potential risk for malignancy, warranting follow-up.

\section{Reactive stromal pseudotumors of the gastrointetstinal tract (GIT): a potential diagnostic pitfall}

Rifat Mannan1, Lodewijk Brosens ${ }^{2}$, George Offerhaus ${ }^{3}$, Armen Khararjian ${ }^{4}$, Kathleen Byrnes ${ }^{5}$, Joy Snel/, Justin Poling ${ }^{7}$, Negar Rassaei ${ }^{8}$, Dora Lam-Himlin', Lysandra Voltaggio ${ }^{5}$. 'The Johns Hopkins Hospital, ${ }^{2}$ University Medical Center Utrecht, Utrecht ${ }^{3} \mathrm{AG}$ Bilthoven, NLD, ${ }^{4}$ Baltimore, MD, ${ }^{5}$ Johns Hopkins Hospital, Baltimore, MD, ${ }^{6}$ Advanced Pathology, Lawton, OK, 'Pathgroup, Nashville, TN, ${ }^{8}$ Hershey, PA, ${ }^{9}$ Mayo Clinic, Scottsdale, AZ

Background: Reactive spindle cell proliferations of GI tract (GIT) often pose diagnostic challenge and may mimic mesenchymal neoplasms. We sought to characterize helpful histologic features that could aid in diagnosis.

Design: We collected 11 cases from 10 patients and reviewed H\&E slides and available immunostains. Molecular analysis via next generation sequencing (NGS) and single nucleotide polymorphism (SNP) array was performed in 1 case. Demographic and clinical information were obtained from available records.

Results: Average age was 73 years (range, 29-84; $M=4, F=6$ ). $73 \%$ were consults sent with concern for a "spindle cell neoplasm". Patients presented with melena/hematochezia, diarrhea, anemia, and obstruction. Clinical associations included colon cancer, polyps, abdominal surgery/ irradiation, IBD, and ischemic colitis. All lesions were seen as colorectal polyps or ulcers. Average size was $2.2 \mathrm{~cm}$ (range 0.4-5.5). 1 lesion was located at an anastomotic site. A neoplastic diagnosis was entertained or adjudicated in 4 cases. 3 were associated with adenocarcinoma in adjoining mucosa. Most proliferations were moderately to highly cellular and featured myofibroblastic-like spindled or spindled/ epithelioid cells with abundant eosinophilic cytoplasm with hypochromatic nuclei and visible nucleoli; macronucleoli were seen in only 1 case. Mitoses were frequent in 4 cases ( 2 with atypical mitoses). Scattered large atypical cells were identified in 3 cases and multinucleated giant cells in 1 . The majority of the lesions featured an ulcerated surface with lesional cells arranged perpendicular to the ulcer. Prominent hemosiderin was seen in 1 case. All cases had an inflammatory backdrop of neutrophils variably admixed with lymphocytes, plasma cells, and eosinophils. Variable immunoreactivity was seen with SMA and desmin and all examples were negative for CD117, DOG1, ALK1, CK, CD34, and S100. NGS performed in one carcinoma-associated example showed KRAS mutation in the spindle cells, while the carcinoma featured KRAS, p53, and APC mutations. Whole genome SNP array revealed normal chromosomal copy number in the spindle cell component. Four patients had uneventful follow-up and 1 died of unrelated cause 2 years later.

Conclusions: Exuberant spindle cell proliferations of the GIT are important to recognize as cellularity and immunostaining pattern cy suggest a mesenchymal neoplasm. Attention to the cytologic features, inflammatory backdrop, and history of prior injury can help avoid a misdiagnosis.

\section{Basaloid Squamous Cell Carcinoma of the Anus: Histological Subtypes and HPV-Association}

Vicente Marco', Omar Clavero², Beatriz Quirós², Silvia de Sanjosée Laia Alemany ${ }^{2}$, Felip Hernandez ${ }^{3}$, FX Bosch Josét. ${ }^{1}$ Hospital Quiron Barcelona, Barcelona, Spain, ${ }^{2}$ Institut Catala d'Oncologia, ${ }^{3}$ Matadepera, ${ }^{4}$ Catalan Institute of Oncology; CIBERONC

Background: Basaloid Squamous Cell Carcinoma (BSCC) is a type of anal invasive squamous cell carcinoma (AISCC), histologically characterized by the presence of nests of basaloid cells, often showing comedonecrosis, and small foci of abrupt keratinization. There is very little data on morphologic variation within anal BSCC. HPV infection plays an important role in the development of AISCC. The aim of this study is to describe the different BSCC subtypes and their association with HPV.

Design: Formalin-fixed-paraffin-embedded biopsies with the diagnosis of AISCC, received from 22 countries in 5 continents, were reviewed. Cases were classified as BSCC, both pure basaloid or mixed basaloid (when combined with other AISCC diagnosis) into 6 subtyped groups: classic, transitional, papillary, adenoid cystic, with clear cells and mucoepidermoid-like. Samples were tested for HPV-DNA, and p16 INK4a immunochemistry was performed. HPV-DNA negative cases were assayed with tubulin to evaluate the quality of the specimens and samples containing HPV-DNA were further subjected to HPV E6* mRNA detection.

Results: From 426 AISCC with HPV-DNA, p16 ${ }^{\text {INK } 4 \mathrm{a}}$ and HPV E6*I mRNA available results, 177 were histologically diagnosed as BSCC $(41,5 \%)$, considering as basaloid related diagnosis pure basaloid and mixed basaloid-other AISCC cases. Histological subtyping of BSCC showed 118 classic $(66.7 \%), 35$ transitional $(19.8 \%), 12$ papillary $(6.8 \%)$, 7 adenoid cystic $(4 \%), 3$ clear cell $(1.7 \%)$, and 2 mucoepidermoid-like (1.1\%).

BSCC are more often HPV-DNA positive than other AISCC (96\% vs $81 \%$ p-value $<0.01$ ), and more often HPV-related, considering as HPV-related cases those with HPV-DNA+ and HPV E6*I mRNA+ or p16INK 4a+ $(96 \%$ vs $75 \%, p$-value $<0.01$ ). No significant differences were found between BSCC subtypes in HPV-DNA positivity, nor in HPV-related/HPV-non related cases, although transitional BSCC were more often HPV E6* mRNA negative than other BSCC subtypes ( $p$-value $<0.05$ ).

The most prevalent HPV-DNA type was HPV16 in all basaloid types. The second type detected in BSCC was HPV58 while in other AISCC was HPV18.

Conclusions: No differences in HPV-DNA positivity were found between different basaloid types classified cases, being HPV16 the most prevalent HPV-DNA type detected, followed by HPV58.

\section{PREVIOUSLY PUBLISHED}

\section{Immune-Profiling of Ipilumimab Induced Colitis}

Dipen Maru', Riham Katkhuda', Atin AgarwaP, Jorge Blando', Sumit Subudhi', Padmanee Sharma'. 'The University of Texas MD Anderson Cancer Center, Houston, TX, ${ }^{2}$ Irving, TX

Background: Ipilumimab is a monoclonal antibody that binds to cytotoxic T lymphocytes associated antigen-4 (CTLA-4), blocking the inhibitory signal resulting in immune-mediated tumor cell death. Because the amplified immune response is not restricted to the tumorspecific lymphocytes, Ipilumimab has been associated with potentially severe adverse effects including colitis in $27-50 \%$ of patients. The histopathologic features of Ipilumimab induced toxicity has been described. However, immunoprofile of inflammatory infiltrate is not known and needed to better understand the biology of Ipilumimab induced colitis.

Design: From the institutional database, we identified 17 patients (2010-2016) who were treated with Ipilimumb for different solid malignancies and developed colitis which was confirmed on colonoscopy biopsies and exclusion of other etiologies by correlation clinical and other laboratory findings. We also identified 5 patients (control cohort) who underwent colonoscopy screening and had normal colon on colonoscopy and biopsy. Quantitative analysis of IHC staining for lymphoid and macrophage markers and eosinophils were conducted using the image analysis software-ImageScopeAperio/Leica. Five random areas $\left(1 \mathrm{~mm}^{2}\right.$ each) were selected and a customized software algorithm for each immunologic marker was used to determine the density (number of positive cells per $1 \mathrm{~mm}^{2}$ field) and percentage of positive cells

Results: Number of positive cells $/ \mathrm{mm}^{2}$ for a marker (Density) and percentage of positve cells in colonic biopsies from control and colitis patients are shown in Table. 


\begin{tabular}{|c|c|c|c|c|c|}
\hline & \multicolumn{2}{|c|}{ Control, median (range) } & \multicolumn{2}{|c|}{ Colitis, median (range) } & \multirow[b]{2}{*}{$\begin{array}{l}p \text { value by } \\
\text { student } \\
\text { t-test for } \\
\text { percent } \\
\text { positive } \\
\text { cells }\end{array}$} \\
\hline Marker & $\begin{array}{l}\text { Density } \\
\text { (positive } \\
\text { cells per } \\
\mathrm{mm}^{2} \text { ) }\end{array}$ & $\begin{array}{l}\text { Percent of } \\
\text { positive } \\
\text { cells }\end{array}$ & $\begin{array}{l}\text { Density } \\
\text { (positive } \\
\text { cells per } \\
\mathrm{mm}^{2} \text { ) }\end{array}$ & $\begin{array}{l}\text { Percent of } \\
\text { positive } \\
\text { cells }\end{array}$ & \\
\hline CD3 & 865 & $\begin{array}{l}10 \\
14)\end{array}$ & 2,446 & $20(9-30)$ & $<0.001$ \\
\hline CD4 & 661 & $\begin{array}{l}8 \\
(4-24)\end{array}$ & 1,896 & $16(3-29)$ & 0.18 \\
\hline CD8 & 784 & $8(4-11)$ & 1,028 & $8(3-18)$ & 0.35 \\
\hline CD20 & 116 & $2(0-3)$ & 317 & $6(0.8-22)$ & 0.19 \\
\hline CD45RO & 2,549 & $29(19-30)$ & 3,584 & $24(8-38)$ & 0.15 \\
\hline CD68 & 1,662 & $22(15-37)$ & 2,359 & $23(3-49)$ & 0.44 \\
\hline FoxP3 & 201 & $2(2-4)$ & 716 & $6(0-12)$ & $<0.001$ \\
\hline Gr-B & 30 & $\begin{array}{l}0.33(0.05- \\
0.45)\end{array}$ & 187 & $1(0.35-7)$ & 0.05 \\
\hline ICOS & 81 & $\begin{array}{l}0.68 \\
(0.03-1)\end{array}$ & 384 & $3(0.04-19)$ & 0.00 \\
\hline LAG-3 & 34 & $\begin{array}{l}0.51 \\
(0.06-2)\end{array}$ & 246 & $\begin{array}{l}1.86 \\
(0.18-8)\end{array}$ & 0.01 \\
\hline OX40 & 240.64 & $3(2-5)$ & 1,328 & $10.32(3-22)$ & 0.00 \\
\hline PD-1 & 136 & $2(0.77-3)$ & 7 & $0.69(.05-3)$ & 0.03 \\
\hline PD-L1 & 27.36 & $\begin{array}{l}0.67(0.0- \\
0.78)\end{array}$ & 200 & $\begin{array}{l}2.42(0.16- \\
10)\end{array}$ & 0.00 \\
\hline Eosinophils & 41.92 & $\begin{array}{l}0.70(0.35- \\
1.70)\end{array}$ & 46 & $\begin{array}{l}0.45 \\
(0.01-3)\end{array}$ & 0.40 \\
\hline
\end{tabular}

801 CENP-A Immunohistochemistry Distinguishes Low Copy Number Alterations in Klatskin Tumors Versus Intrahepatic and Extrahepatic Cholangiocarcinomas

Daiva Mattis ${ }^{1}$, Tao Su${ }^{1}$, Aras Mattis'. ' University of California San Francisco, San Francisco, CA

Background: Cholangiocarcinomas arise in distinct locations including intrahepatic cholangiocarcinoma (ICC), extrahepatic cholangiocarcinoma (ECC), and Klatskin, but have poor outcomes due to late diagnosis and difficulty in resection. There are few therapeutic options beyond surgery. Recent molecular profiling by next generation sequencing identified alterations associated with ICC, ECC and Klatskin. Interestingly Klatskin tumors contain few chromosome copy alterations or common tumor drivers (in preparation for publication). We reasoned that Klatskin tumors contain fewer copy number alterations than ICCs or ECCs, making them a distinct entity that can be identified by immunostaining for centrosomal proteins that highlight chromosome copy number.

Design: 6 ICC, 6 ECC, and 7 Klatskin cases were identified within the institution archives. All slides and pathology reports were reviewed for consistency. Immunostains were used to quantify copy number alterations by detecting tumors with antibodies for Centromere Protein A (CENP-A), Centromere Protein C (CENP-C), Pericentrin and $\mathrm{Ki}-67$. Ki-67 tumor staining was assigned a percent and each centromere antibody staining was assigned a number (scaled 0-no staining to 5 -very strong positive staining), by a blinded pathologist. Strong CENP-A staining of focal individual cells was also recorded (scaled 1-strong to 3-very strong individual cell staining). Non-tumor cell staining was used as an internal control.

Results: CENP-A staining had a mean value in Klatskin tumors of $1.4 / 5$ (range $0-3.5$ ), ECC tumors $3 / 5$ (range $1.5-5$ ), and ICC tumors $2.25 / 5$ (range 0-3.5). Only ICC (3/6) and ECC (2/6) had strong individual cell staining present, while Klatskin had none (0/5). Ki-67 staining mean percent for Klatskin tumors was $11.8 \%$ (range $0-30 \%$ ), ECC tumors $58 \%$ (range $1.5-80 \%$ ), and ICC tumors $42 \%$ (range $2.5-90 \%$ ). CENP-C and Pericentrin results were equivocal. CENP-A and Ki-67 staining was significant ( $p$ values less than 0.05).

Conclusions: Our findings support the evidence of significant immune dysregulation and T cell activation with predominant immunophenotype of CD3+/CD4+ and OX40+ immune cells in colonic biopsies of patients with Ipilumimab induced colitis. Upregulation of PD-L1 after Ipilumimab treatment in Iymphocytes compared to control has not been shown before and should be explored in larger context of effects of Ipilumimab in normal and tumor cells.

\section{Helicobacter gastritis in renal transplant patients}

Karen Matsukuma', Kuang-Yu Jen². ${ }^{1}$ UC Davis Medical Center, Sacramento, CA, ${ }^{2}$ University of California, Davis, Sacramento, CA

Background: In the current era of solid organ transplant (tx), mycophenylate is a key component of immunosuppressive therapy. As mycophenylate has been shown to impede B-cell maturation, we investigated whether renal tx patients demonstrate an attenuated pattern of inflammation in the setting of Helicobacter gastritis (HG), thus potentially masking histologic clues to its presence.

Design: Gastric biopsies from all renal tx patients who underwent post-tx endoscopy from 2005-2017 were reviewed and correlated with clinical data. H. pylori immunohistochemistry (IHC) was performed on 26 biopsies which showed mild to moderate plasma cell infiltrates (and which were not initially evaluated for $\mathrm{H}$. pylori by IHC). As a control for $\mathrm{H}$. pylori prevalence, data from the pathology reports of 107 consecutive non-immunosuppressed, non-tx patients who underwent gastric biopsy were tabulated and their medication histories reviewed.

Results: Ninety-one post-tx gastric biopsies from 71 renal tx patients were identified. Of the 26 cases selected for $\mathrm{H}$. pylori IHC, 1 was positive. In total, HG was identified in 4 cases $(4 \%)$, of which 3 were identified during routine clinical service. Histologically, 3 of the HG cases were characterized by at least moderate superficial plasmacytic inflammation; one case showed only mild patchy plasma cell infiltrates in the deep mucosa. In all cases, only rare (and predominantly intracellular) $\mathrm{H}$. pylori organisms were identified. Just $20 \%$ of all gastric biopsies and $23 \%$ of $\mathrm{H}$. pylori IHC-stained cases met Sydney system recommendations for evaluation of gastritis (e.g., 2 antral, 2 body, 1 incisura angularis). In the tx group, 90\% were on mycophenylate and $77 \%$ were on a proton pump inhibitor (PPI) at the time of biopsy. All 4 patients with HG were on mycophenylate and a $\mathrm{PPI}$ at the time of biopsy. In the control group, $\mathrm{HG}$ was reported in 6 cases $(6 \%)$. Only $46 \%$ of controls were on PPI therapy.

Conclusions: The data suggest that the pattern of HG in renal tx patients is not qualitatively different from that seen in the setting of PPI therapy in general. However, a significantly higher rate of PPI use was identified in the tx group, suggesting additional vigilance in this cohort for assessment of $\mathrm{H}$. pylori by IHC (as PPIs can result in low volume infection). Additionally, the large number of gastric biopsies failing to meet Sydney system guidelines leaves open the possibility that it is sampling rather than absence of organisms that defines biopsies with a minimal inflammatory infiltrate.
Conclusions: Here we report for the first time the use of CENP-A as a marker for the differentiation of Klatskin from ICC and ECC cholangiocarcinomas. Klatskin tumors stain much weaker for CENP-A, suggesting low copy number alterations and maintain low Ki-67 proliferation. Both ECC and ICC tumors have significantly increased CENP-A staining, supporting increased copy number alterations with an elevated $\mathrm{Ki}-67$ ( $40 \%$ or greater). Overall our findings suggest that Klatskin tumors have fewer large chromosome alterations versus ICC

\section{Colorectal Serrated Neoplasia - A 12 Year Retrospective Review}

Aoife J McCarthy' ${ }^{1}$ Susanne O'Reilly², John Shanley ${ }^{3}$,Elizabeth Ryan ${ }^{4}$ Garret Cullen², Kieran Sheahan ${ }^{5}$. 'Laboratory Medicine Program, Department of Pathology, University Health Network and University of Toronto, Toronto, Canada, Cork, ${ }^{2}$ Centre for Colorectal Disease, St. Vincent's University Hospital, Elm Park, Dublin, Ireland, ${ }^{3}$ Department of Histopathology, St. Vincent's University Hospital, Elm Park, Dublin, Ireland, ${ }^{4}$ Centre For Colorectal Disease, Dublin, Ireland, ${ }^{5}$ St. Vincent Hospital, Dublin

Background: Colorectal cancer is a heterogeneous disorder that arises via multiple distinct pathways, including the serrated pathway, in which serrated polyps are the precursor lesions. As such, serrated polyps of the colorectum, including sessile serrated lesions/polyps (SSL/Ps) and traditional serrated adenomas (TSAs), have gained increased recognition in recent years.

Our aim was to characterize a series of serrated colorectal polyps, focusing on the clinicopathological features of SSL/Ps \& TSAs.

Design: A search was performed using the laboratory information system to identify all colorectal polyps assigned a 'serrated adenoma' suitable slides were reviewed by 1 pathologist, who was blinded to the original diagnosis and the site of the polyp. Subsequently discordant cases, SSL/Ps with dysplasia, and all TSAs were reviewed by a second pathologist.

Results: Over 149 months, 759 polyps were assigned a 'serrated adenoma' SNOMED code, with 686 (from 540 patients) available for review. $39.8 \%$ of these were reviewed by both pathologists, and $14.6 \%$ $(100 / 686)$ were reclassified, with the majority of these changes being from SSL/P to hyperplastic polyp (HYP) (66/686; $9.6 \%) .80 .3 \%$ of these HYP were located in the left colon and the majority exhibited prolapse effect.

There were 520 SSL/Ps (92.2\%) \& 40 TSAs $(7.1 \%) .86 .7 \%$ of SSL/Ps were in the right colon and $64.5 \%$ were small $(<1 \mathrm{~cm}) .6 .7 \%$ of SSL Ps exhibited dysplasia, and the majority of these $(66.7 \%)$ were large $(>/=1 \mathrm{~cm})$. Following consensus review, 13/520 (2.5\%) SSL/Ps were downgraded from SSL/P with dysplasia to SSL/P without dysplasia. or ECC tumors. SNOMED code between 01/01/2004 and 31/05/2016. All available and 
Detection of SSL/Ps peaked in the most recent years reviewed $(87.5 \%$ reported between 2013 and 2016, inclusive), coinciding with the introduction of 'BowelScreen' (the Irish colorectal cancer screening programme).

$85.7 \%$ of TSAs were in the left colon and $73.1 \%$ were large $(>/=1 \mathrm{~cm})$.

\begin{tabular}{|c|c|c|c|c|c|}
\hline \multicolumn{2}{|l|}{ Parameter } & All SSL/Ps & $\begin{array}{l}\text { SSL/Ps } \\
\text { without } \\
\text { dysplasia }\end{array}$ & $\begin{array}{l}\text { SSL/ } \\
\text { Ps with } \\
\text { dysplasia }\end{array}$ & TSAs \\
\hline \multicolumn{2}{|l|}{$\begin{array}{l}\text { Number } \\
(n, \%)\end{array}$} & 520 & 485 & 35 & 40 \\
\hline \multirow{3}{*}{$\begin{array}{l}\text { Site } \\
(n, \%)\end{array}$} & $\begin{array}{l}\text { Right } \\
\text { colon }\end{array}$ & $\begin{array}{l}451 \\
(86.7 \%)\end{array}$ & $\begin{array}{l}423 \\
(87.2 \%)\end{array}$ & $\begin{array}{l}28 \\
(80 \%)\end{array}$ & $\begin{array}{l}4 \\
(10 \%)\end{array}$ \\
\hline & Left colon & $\begin{array}{l}62 \\
(11.9 \%)\end{array}$ & $\begin{array}{l}55 \\
(11.3 \%)\end{array}$ & $\begin{array}{l}7 \\
(20 \%)\end{array}$ & $\begin{array}{l}34 \\
(85 \%)\end{array}$ \\
\hline & Colon NOS & $\begin{array}{l}7 \\
(1.4 \%)\end{array}$ & $\begin{array}{l}7 \\
(1.4 \%)\end{array}$ & - & $\begin{array}{l}2 \\
(5 \%)\end{array}$ \\
\hline \multirow[b]{3}{*}{$(\mathrm{mm})$} & Mean & 8.1 & 7.9 & 11.3 & 18.6 \\
\hline & Median & 8 & 8 & 10 & 13 \\
\hline & Range & $1-32$ & $1-32$ & $3-30$ & $2-60$ \\
\hline \multirow{2}{*}{ Size category } & $<1 \mathrm{~cm}$ & $\begin{array}{l}167 \\
(64.5 \%)\end{array}$ & $\begin{array}{l}162 \\
(66.4 \%)\end{array}$ & $\begin{array}{l}5 \\
(33.3 \%)\end{array}$ & $\begin{array}{l}7 \\
(26.9 \%)\end{array}$ \\
\hline & $>/=1 \mathrm{~cm}$ & $\begin{array}{l}92 \\
(35.5 \%)\end{array}$ & $\begin{array}{l}82 \\
(33.6 \%)\end{array}$ & $\begin{array}{l}10 \\
(66.7 \%)\end{array}$ & $\begin{array}{l}19 \\
(73.1 \%)\end{array}$ \\
\hline
\end{tabular}

adenomas; NOS, not otherwise specified.

Conclusions: It can be challenging to distinguish SSL/Ps from HYPs, as there are often only subtle differences. Following consensus review, 66/686 (9.6\%) polyps in our cohort were changed from SSL/P without dysplasia to HYP. A low rate of SSL/Ps with dysplasia $(6.7 \%)$ is confirmed in our institution, with over-diagnosis of dysplasia in $\mathrm{SSL} / \mathrm{P}$ being more common than under-diagnosis. As the malignant potential of SSL/Ps and TSAs has been clearly demonstrated, it is important that serrated polyps are identified and correctly classified histologically.

\section{Unusual Mismatch Repair Protein Immunohistochemical Patterns and Corresponding Microsatellite Instability and Molecular Patterns: More To It Than Meets The Eye}

Aoife J McCarthy', Tara Spence ${ }^{2}$, Sylvie Grenier ${ }^{2}$, Tracy Stockley ${ }^{2}$ Suzanne Kamel-Reid", Stefano Serra ${ }^{3}$, Runjan Chetty ${ }^{3}$. ' ${ }^{1}$ Laboratory Medicine Program, University Health Network and University of Toronto, Toronto, Canada, Cork, ${ }^{2}$ University Health Network and University of Toronto, ON, Canada, 3University Health Network, Toronto, ON

Background: Immunohistochemical (IHC) staining for mismatch repair (MMR) proteins is widely used to identify MMR deficient tumors, with tumors usually showing either diffuse positivity or uniform/widespread loss of staining. Typically, any nuclear staining in tumor cells is regarded as positive (pos), whereas negativity (neg) is reported only when nuclear staining is absent in all tumor cells. Focal \& geographic heterogeneity of protein expression is known.

Design: We identified 4 colonic adenocarcinomas with heterogenous staining patterns affecting at least 1 MMR protein: MLH1, PMS2 MSH2 \&/or MSH6.

A gastric adenocarcinoma with differing MMR IHC staining patterns between invasive tumor $\&$ adjacent dysplasia was also identified.

Based on alternating expression patterns, macro-dissected tumor/ dysplastic areas were separately analyzed for microsatellite instability (MSI) \& next generation sequencing (NGS).

Results: $\quad 3$ colonic adenocarcinomas each showed heterogeneous staining for 1 MMR protein (MLH1, PMS2 \& MSH6, respectively), with intact staining of the other 3 MMR proteins, while 1 other colonic adenocarcinoma showed heterogenous staining for MLH1/PMS2, with intact staining of the other 2 MMR proteins.

Heterogenous MMR staining correlated with differences in MSI status in 1 tumor (microsatellite stable (MSS) in IHC pos areas \& MSI-high (MSI-H) in IHC neg areas). Homogenous MSI status in a tumor with heterogenous MMR protein expression was observed in 3 cases (2 MSI-H \& 1 MSS)
In 1 case tested, NGS detected a TP53 c.524G>A variant in MMR IHC pos invasive tumour and absence of variants in IHC neg invasive tumour.

An invasive gastric adenocarcinoma showed complete loss of MLH1/ PMS2, with retention of $\mathrm{MSH} 2 / \mathrm{MSH} 6$, while the adjacent dysplasia showed complete loss of MLH1/PMS2/MSH6, with retention of MSH2

The invasion \& dysplasia were both MSI-H.

NGS detected an MSH6 c.3261delC variant in invasion \& both an MSH6 c.3261del C variant \& an MSH6 c.3261dupC variant in dysplasia, with neither variant detected in a concurrent pancreatic adenocarcinoma.

We postulate that the MSH6 c.3261delC \& MSH6 c.3261dupC variants in dysplasia resulted in negative MSH6 IHC, while MSH6 IHC expression was maintained in the invasion with only 1 variant.

\section{FOR TABLE DATA, SEE PAGE 321, FIG. 803}

Conclusions: Heterogenous MMR IHC can be seen in gastrointestinal cancers, \& can correspond to differences in MSI \& molecular status. Though rare, as seen in these 5 cases, attention to this phenomenon is necessary to prevent false-positive/-negative evaluations.

\section{Expression of PD-L1 in a Cohort of Stage III MSI Colorectal Cancer Patients}

Edwina Meade 1 Eanna Ryan ${ }^{2}$, Ben Creavin ${ }^{2}$, Elizabeth Ryan ${ }^{3}, Y$ Ling Khaw ${ }^{4}$, Robert Geraghty ${ }^{5}$, Aurelie Fabre, ${ }^{6}$, Des Winter ${ }^{2}$, Kieran Sheahan ${ }^{7}$. ${ }^{1}$ Centre for Colorectal Disease, St. Vincent's University Hospital, Elm Park, Dublin, Ireland, ${ }^{2}$ St. Vincent's University Hospital ${ }^{3}$ Centre For Colorectal Disease, Dublin, Ireland, ${ }^{4}$ Dublin, Ireland, ${ }^{5}$ Dublin, ${ }^{6}$ St. Vincent's Univ Hosp, Dublin, Ireland, ${ }^{7}$ St. Vincent Hospital, Dublin

Background: PD-L1 expression and its prognostic significance remains uncertain in MSI CRC patients. It is also unclear which detection system is optimal in this setting.

Design: The aim of this study was to analyse a stage III MSI CRC cohort $(n=61)$ using three different PD-L1 immunohistochemistry assays (Ventana SP263, Ventana SP142 and Dako 22C3), score both tumour cell (TC) and immune cell (IC) expression of PD-L1 and assess concordance between three antibody clones. This cohort were also assessed for the Klintrup-Mäkinen (K-M) immunoscore. Both tumour cells (TC) \& immune cell (IM) components were assessed using a recently described analytical methodology which allowed comparison between detection systems (El Jabbour, T et al. J Clin Path 2017)

Results: This study found that there was significant variation between the staining of the three PD-L1 antibody clones. The strongest correlation was between Ventana SP263 and Dako 22C3 in the assessment of tumour cell PD-L1 expression. These two PD-L1 antibody clones demonstrated similar IHC staining of PD-L1 positive tumour cells, with clearly defined (near-complete) membranous staining of positive cells aiding the microscopic assessment of PD L1 positivity. PD-L1 positivity of immune cells (IC) demonstrated a linear membrane, diffuse cytoplasmic, and/or punctate staining pattern. Overall Ventana SP263 identified the highest number of PDL1 positive cases, \& Ventana SP142 identified the lowest number of positive cases. There was a significant correlation between PD-L1 (IC) at the invasive margin and the K-M score for 2 of the three antibodies (Ventana SP263 \& Dako 22C3 $(\mathrm{p}<0.05))$. Preliminary survival analysis showed no correlation with outcome ( $p>0.05$ ).

Conclusions: PD-L1 TC expression is antibody-clone dependent, is heterogeneously expressed by tumour cells and will be an issue if biopsy material is examined. PD-L1 IC expression is also antibodyclone dependent \& correlated with the K-M immunoscore for two of the three antibodies tested.

\section{Simplification of Ryan Regression Grading into a 3-Tier System Provides Better Prognostic Stratification for Neoadjuvant Treated Esophageal Adenocarcinoma}

David Mendoza', Christopher Hartley², Catherine Hagen'. 'Medical College of Wisconsin, Milwaukee, WI, ${ }^{2}$ Milwaukee, WI

Background: Most cases of esophageal adenocarcinoma are treated with neoadjuvant chemotherapy and/or radiation. Tumor regression grading of resected specimens is a recommended component of synoptic reporting and the CAP guidelines suggest use of the modified Ryan system, which has been shown to have prognostic significance in neoadjuvant treated rectal cancer. While the Ryan system is modified from the 5-tier Mandard system, few studies have evaluated the prognostic significance of the 4-tier Ryan system in esophagea adenocarcinoma. The goal of this study was to assess the prognostic significance of the modified Ryan system for regression grading in neoadjuvant treated esophageal adenocarcinoma and compare to 
other commonly used regression grading systems.

Design: The pathology database at our institution was retrospectively searched from 2012-2017 for esophagectomy specimens performed for resection of adenocarcinoma of the esophagus or gastroesophageal junction. Cases not treated with neoadjuvant chemotherapy or radiation and cases with carcinomas other than adenocarcinoma were excluded. Slides were retrospectively reviewed and graded according to modified Ryan system $(0=$ complete response $(C R), 1=$ near $C R$, $2=$ partial response, $3=$ poor/no response) as well as the Becker system $(1 \mathrm{a}=\mathrm{CR}, 1 \mathrm{~b}=<10 \%$ residual tumor (RT), $2=10-50 \% \mathrm{RT}, 3=>50 \% \mathrm{RT})$. Clinicopathologic information was collected through chart review.

Results: 88 esophagectomy specimens from an equal number of patients ( $\mathrm{M}: \mathrm{F}=5.8: 1$, mean age $=62$ ) were included in the study. Overall, modified Ryan regression scoring correlated with disease specific survival $(p=0.02)$. However, there was no significant difference in survival curves for patients with tumor regression grades 1 and 2 (HR 1.4, 95\% Cl 0.4-5.1, $\mathrm{p}=0.96$ ) (Figure 1). Becker regression scoring showed essentially identical stratification for disease specific survival $(p=0.02)$. When regression grades 1 and 2 were combined for Ryan scoring, regression grading showed more significant association with disease specific survival $(p=0.006)$ (Figure 2$)$.

\section{Modified Ryan Score}

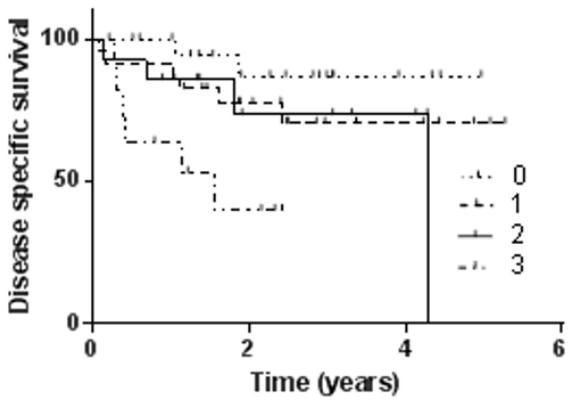

Three Tier System

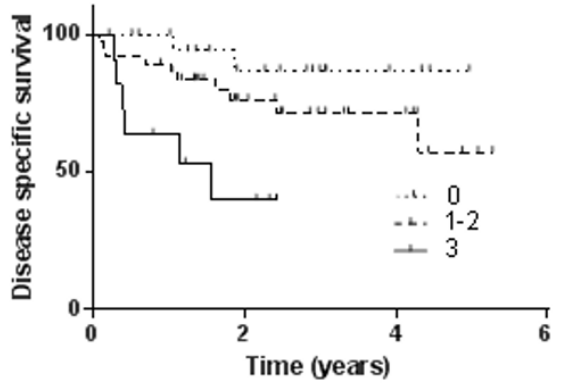

Conclusions: Both Ryan and Becker grading provide comparable prognostic stratification for neoadjuvant treated esophageal adenocarcinoma. Combining near complete and partial responders into a 3-tier scoring system simplifies regression grading and provides better prognostic stratification. These findings support the use of a 3-tier scoring system that has previously been reported in the literature.

806 The immune checkpoint protein PDL2 is more frequently co-expressed with PDL1 in EBV+ carcinomas compared to EBV- carcinomas in mismach repair proficient tumors

Salvatore Mignano1, Jeffrey Williams', Alysa Rud², Kimberly Thompson $^{2}$, Sandra Bohling ${ }^{2}$, Allen M. Gown ${ }^{2}$, Harry Hwang ${ }^{2}$. ${ }^{1}$ Tacoma, WA, ${ }^{2}$ PhenoPath Laboratories, PLLC, Seattle, WA

Background: Both PDL1 and PDL2 are important immune checkpoint proteins that are overexpressed in many human cancers. Although increased PD-L1 expression has been described in EBV+ carcinomas, the status of PDL2 in such tumors is unknown and is a potential biomarker for immunotherapy responsiveness. We therefore examined PDL2 and PDL1 by immunohistochemistry in a set of EBV+ and EBV- carcinomas. In addition, as deficient mismatch repair (MMR) staus has emerged as a predictor for immunotherapy responsiveness, we evaluated the MMR proteins MLH1 and MSH2 in these tumors to see if there is any association of MMR status with PDL1 and PDL2.

Design: We selected $6 \mathrm{EBV}+$ and $10 \mathrm{EBV}$ - carcinomas, as determined by EBER-ISH that were derived from gastric, nasopharyngeal, and lymphoid sites. The EBV- cases had variable histologies and variable lymphoid stroma. The EBV+ cases all had prominent lymphoid stroma. Each case was immunostained with MLH1 (ES05), MSH2 (FE11), PD-L1 (E1L3N), and PD-L2 (D7U8C). MSH1 and MSH2 stains were scored fo nuclear expression. For PDL1 and PDL2 any intensity of membranous staining within $\geq 1 \%$ cancer cells was scored positive. Staining intensity was graded on a $0-3$ scale: no staining 0 , weak staining $1+$, moderate $2+$, and strong $3+$.

Results: Of the EBV+ cases, 6 of $6(100 \%)$ showed any level of PDL (Figure 1), and $66.7 \%$ (4 of 6 ) showed any level of PDL2 (Figure 2). In contrast, 2 of $10(20 \%)$ of the EBV- cases showed any level of PDL1 and 5 of $10(50 \%)$ showed any level of PDL2. Overall, co-expression of PDL1 and PDL2 was seen in $66.7 \%$ (4 of 6) of the EBV+ cases and in 2 of $10(20 \%)$ of the EBV-cases. When PDL2 was positive, PDL1 was co-expressed in 4 of $4(100 \%)$ of the EBV+ cases and in 2 of $5(40 \%)$ of the EBV- cases. All examined cases had retained MLH1 and $\mathrm{MSH} 2$.
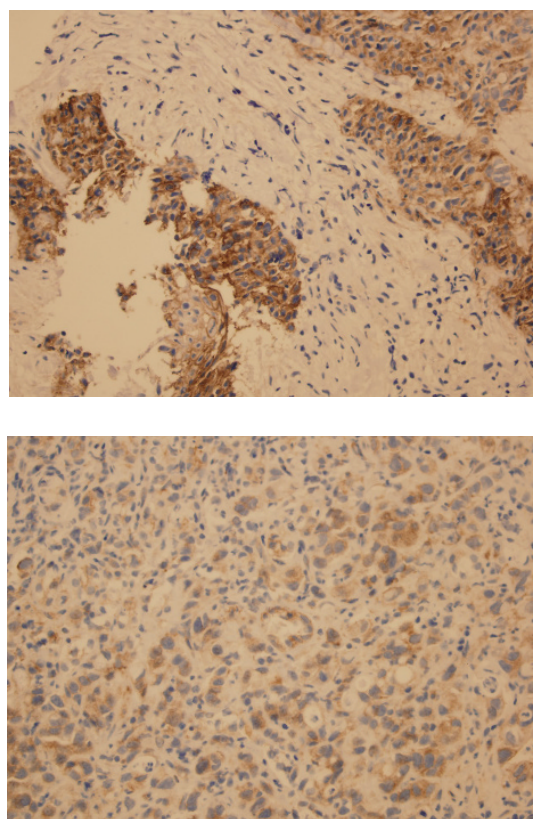

Conclusions: In this study, we found that PDL2 was positive in a significant fraction of EBV+ and EBV- carcinomas and more frequently co-expressed with PDL1 in EBV+ carcinomas. All tumors were proficient for MMR. These findings may be related to the tumors' prominent lymphoid stroma and tumor infiltrating lymphocytes, which tends to be seen in EBV+ carcinomas. In EBV- cases, half showed PDL2 expression, even in cases that showed no PDL1 expression or lymphoid stroma. PDL2 could be a possible therapeutic target in EBV+ and EBV- carcinomas.

\section{Clinicopathologic Features of Duodenal Pyloric Gland Adenoma - an Analysis of 20 Cases}

Gregory Miller', lan Brown², Gregory Y Lauwers ${ }^{3}$, Wajiha Sufyan ${ }^{4}$ 'Envoi Specialist Pathologists, Brisbane, QLD, ${ }^{2}$ Envoi Pathology, Brisbane, Australia, ${ }^{3} \mathrm{H}$. Lee Moffitt Cancer Center and Research Institute, ${ }^{4}$ Royal Darwin Hospital

Background: Duodenal pyloric gland adenomas (PGA) are rare lesions showing a gastric pyloric-type differentiation. PGA that arise in the duodenum are poorly characterised, with little known about their clinicopathological features, associations or potential for malignant transformation.

Design: We examined the clinical and pathologic features of 20 duodenal pyloric gland adenomas from 20 separate patients. All polyps were stained with Muc5AC and Muc6.

Results: Duodenal PGA occurred slightly more commonly in males (11/20 cases were male) at a mean age of $71.19 / 20$ presented as a polyp, most commonly in D1 (7/11 cases with location in duodenum documented). The mean size of the polyp was $13.0 \mathrm{~mm}$. No cases in this series were seen in association with a polyposis syndrome or Lynch syndrome. Four of the 20 cases $(20 \%)$ had evidence of gastric heterotopia in the adjacent mucosa.

The histological features in these cases were of lesions with eithe tubular (10/20) or a tubulopapillary (10/20) architecture. Low grade dysplasia (LGD) was seen in $55 \%(11 / 20)$, high grade dysplasia only (HGD) in $15 \%(3 / 20)$, and HGD associated with or transformed to intramucosal adenocarcinoma (IMC) in $25 \%(5 / 20)$ and submucosal invasive adenocarcinoma was present in 1 case only. When compared with PGA showing HGD/IMC, PGA with LGD were seen in older people (75y vs $65 y$ ) and were slightly smaller $(11 \mathrm{~mm}$ vs $15 \mathrm{~mm})$. There was no difference in sex distribution or location in the duodenum. PGA showing HGD and intramucosal carcinoma were more likely to show a 
tubulopapillary architecture (66\% vs $36 \%$ ). Only 1 case (showing LGD) recurred at 12 months follow up.

Immunohistochemical staining for MuC6 showed a diffuse staining pattern in all cases. Muc5AC showed staining confined to the superficial epithelium in 30\% (6/20 cases) while $70 \%$ (14/20 cases) showed staining of both superficial and deep tissue.

Conclusions: Duodenal PGA are uncommon lesions typically seen in older individuals as a polyp in D1. High grade dysplasia and intramucosal carcinoma is commonly seen and is associated with larger size and tubulopapillary architecture. Reassuringly, once removed, recurrence of PGA is unusual.

\section{KIFC1 as a novel therapeutic target for p53 mutant colorectal cancer}

Karuna Mittal', Guanhao Wei', Jaspreet Kaur ${ }^{1}$ Brian D Melton'1, Da H Choi', Michelle Reid", Padmashree Rida', Ritu Aneja'. 'Georgia State University, Atlanta, GA, ${ }^{2}$ Emory University Hospital, Atlanta, GA

Background: Loss or mutation of p53 gene is the most frequent genetic lesion in late-stage colon cancers. The optimal management of p53 mutant colorectal cancer (CRC) remains a significant therapeutic challenge owing to its resistance to 5-fluorouracil, the first-choice chemotherapy drug for CRC. Thus, the search for novel therapeutic targets for the treatment of p53 mutant CRC is highly warranted. We report here that p53 negatively regulates expression of KIFC1 (a centrosome clustering protein), which can serve as a potential therapeutic target for p53 mutant CRCs.

Design: We first immunohistochemically stained 203 CRC tissue samples for p53, KIFC1, and FOXM1 and calculated their weighted indices (WIs) for nuclear staining. To validate the clinical findings in silico analysis was performed on the publically available dataset (GSE41258). Further we quantitated the protein levels of KIFC1 and FOXM 1 by immunoblotting. For determining the mechanistic relationship of KIFC1 and p53, overexpression (OE) of p53 and ChiP assays were performed. Finally, inhibited KIFC1 by pharmacological and genetic methods to elucidate the role of the KIFC1 inhibition on the vitality of p53-/- CRC cells.

Results: p53-/- and p53 mutant CRC [p53 null $n=82$, p53mutant $n=40$ and WT $n=81$ ] exhibited significantly $(p<0.001)$ higher expression of KIFC1 and FOXM1 when compared with the p53 WT CRC tissue samples and was associated with worse overall survival $(H R=4.249$, $\mathrm{p}=<0.001)$ ). Similarly, our in silico analysis indicated significantly higher KIFC1 and FOXM1 mRNA expression in p53 mutant ( $n=93)$ CRC and was associated with poor overall survival $(H R=1.46, P=<0.05)$ p53-/- cells showed increased expression of KIFC1 and FOXM1. OE of p53 in p53-/- cells decreased the expression of FOXM1 and KIFC1 indicating the negative regulation of KIFC1 via FOXM1 which was further strengthened by ChiP assay (FOXM1 interaction with KIFC1 promoter was significantly higher $\mathrm{p}=0.037$ in $\mathrm{p} 53-/$ - cells when compared with p53 WT cells). Inhibition of KIFC1 using shRNA, CW069 (a known KIFC1 inhibitor) and griseofulvin (a known centrosome declustering drug), led to increased multipolarity followed by cell death only in p53-/- CRC cells.

Conclusions: Collectively our results indicate that p53 negatively regulates expression of KIFC1 via FOXM1 and contributes to poor outcomes in p53 mutant CRCs. Thus, KIFC1 may serve as a potential therapeutic target for p53 mutant CRCs.

\section{Are 'Eosinophilic Gastritis' and Collagenous Gastritis Related Conditions? A Pediatric Case Control Study}

Matthew K Morrow' ${ }^{1}$ Beate BeinvogR ${ }^{2}$, Helen Wang ${ }^{1}$, Menno Verhave ${ }^{2}$ Jeffrey D Goldsmith'. 'Beth Israel Deaconess Medical Center, Boston, MA, ${ }^{2}$ Boston Children's Hospital, Boston, MA

Background: 'Eosinophilic gastritis' (EG) is a pattern of gastric inflammation often associated with allergy; whereas collagenous gastritis (CG) is a poorly understood disease histologically characterized by thickened subepithelial collagen deposition and increased lamina propria inflammation, often including eosinophils. Due to the lamina propria eosinophilia that is common to both conditions, we sought to explore a potential association between these two diseases by performing a case control study.

Design: A retrospective case search was performed to identify gastric biopsies with increased lamina propria eosinophils collected between July 2012 and July 2017 that were not diagnosed as collagenous gastritis; the resultant cases were then reviewed for diagnostic features of CG. Diagnostic criteria for 'definitive' CG included subepithelial collagen thickness of at least $10 \mu \mathrm{m}$ spanning at 3 or more contiguous gastric pits. 'Equivocal' cases showed increased subepithelial collagen that spanned between 1-2 contiguous gastric pits. Positive and equivocal cases were each paired with two age and sex matched controls that had increased stomach lamina propria eosinophils but lacked features of CG. Sixty-four clinical and laboratory parameters, ascertained via medical record review, were correlated with histologic disease status using Mantel-Haenszel estimate of odds ratio.

Results: Of the 78 cases reviewed, five (6\%) were found to be 'definitive' for CG and four (5\%) cases were found to be 'equivocal.' The group of 9 patients designated as 'equivocal' and 'definitive' for CG were less likely to have food allergies $(p=0.03)$, were more likely to be treated with sucralfate before EGD $(p=0.01)$, and were more likely to be treated with proton pump inhibitors after EGD ( $p=0.009$ ) compared with the 18 age and sex matched EG controls. Notably, the hematocrit and the serum absolute eosinophil count at the time of presentation did not differ between the two groups.

Conclusions: Our preliminary analysis revealed that CG and EG are similar in almost all parameters tested with the exception of food allergies. Thus, our data generally support the hypothesis that CG and $E G$ are related conditions. Since $12 \%$ of cases with lamina propria eosinophilia were found to be positive or equivocal for CG, the finding of increased lamina propria eosinophils should prompt a search for diagnostic features of CG.

\section{Tumor deposits but not large nodal deposits are associated with poor prognosis in small bowe neuroendocrine tumors}

Mohamed Mostafa', Amrou Abdelkader ${ }^{2}$, Catherine Hagen ${ }^{3}$ Christopher Hartley4.' 'MCW, Wauwatosa, WI, 'Wauwatosa, WI, ${ }^{3}$ Medical College of Wisconsin, Milwaukee, WI, ${ }^{4}$ Milwaukee, WI

Background: Tumor deposits (TD) are a well-known prognostic factor for colorectal adenocarcinoma and recent studies have suggested TD may also be prognostically significant in small bowel neuroendocrine tumors (NETs). The new $8^{\text {th }}$ edition AJCC staging system for midgut NETs includes an N2 category which encompasses mesenteric "masses" $>2 \mathrm{~cm}$ and/or extensive nodal deposits. Criteria for what constitutes a mesenteric mass are not defined in the AJCC manual. The aim of this study was to evaluate the prognostic significance of TD and large nodal deposits $>2 \mathrm{~cm}$ (LND) in small bowel NETs to assess criteria used for categorization of the new N2 category.

Design: Our pathology database was retrospectively searched from 2006-2015 for resection specimens of small bowel well differentiated NETs. H\&E slides were reviewed for presence of TD, lymph node metastases, T stage, tumor grade (WHO 2010), perineural invasion (PNI), small vessel lymphovascular invasion, and venous invasion (VI). TD were defined as irregular foci of tumor $>1 \mathrm{~mm}$ in size located away from the main tumor in the mesenteric adipose tissue. Clinical data was collected through chart review.

Results: 45 small bowel resections from an equal number of patients (M:F 1:1.1; mean age 54.5) were included in the study. 20 patients $(44 \%)$ had at least one TD (size range $0.2-6.5 \mathrm{~cm}$ ). 15 patients (33\%) had large $(>2 \mathrm{~cm})$ mesenteric masses which showed areas of residua surrounding lymphoid tissue, most consistent with replaced lymph nodes. Compared to patients without TD, patients with TD were significantly more likely to have VI $(55 \%$ vs $12 \%, p=0.003)$ and PN $(90 \%$ vs.52\%, $p=0.009)$ and showed a trend of more frequent lymph node metastases $(90 \%$ vs. $64 \%, p=0.08)$ and distant metastases $(79 \%$ vs. $48 \%, p=0.06)$. Patients with TD also had worse progression free survival, although not statistically significant (figure 1) (HR 2.4, 95\% C $0.9-7.6, p=0.09)$. Patients with LND did not have an increased rate of VI $(p=0.17)$, $P N I(p=0.32)$, additional lymph nodes metastases $(p=1.0)$ or distant metastases $(p=1.0)$. Progression free survival in patients with TD compared to those with LND is shown in figure 2 (HR 2.5, 95\% Cl $0.6-10.1, p=0.21$ )
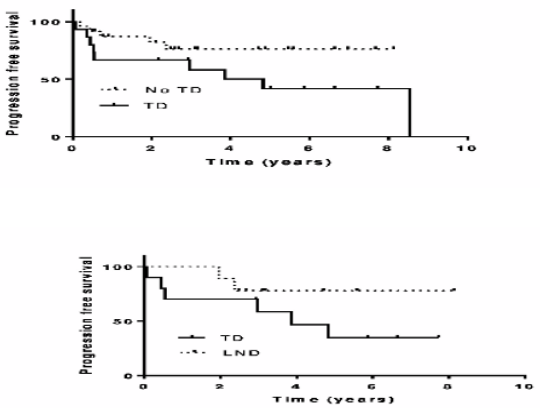

Conclusions: TD are associated with more aggressive histologic features and worse prognosis compared to LND. LND should be differentiated from true TD and should not be used as criteria for the new N2 AJCC staging category. 
811 Clinical and Histologic Correlates of the FilmArray BioFire Gastrointestinal Pane

Jonathan Mowers ${ }^{1}$, Laura W Lamps', Joel Greenson'1, Michael Bachman'. 'University of Michigan Hospitals, Ann Arbor, MI

Background: The FilmArray Gastrointestinal Panel (BioFire Diagnostics, Salt Lake City, Utah) is a multiplex PCR assay that tests stool for 22 different Gl pathogens, including Campylobacter, Salmonella, Shigella, Yersinia, Vibrio, pathogenic E. coli subtypes, P. shigelloides, Cryptosporidium, Cyclospora, Entamoeba, Giardia, adenovirus, astrovirus, norovirus, rotavirus and sapovirus. Although the use of this technology is increasing in frequency, the significance of a positive BioFire result is often confusing for clinicians. The goal of this study was to correlate positive BioFire results with histology on Gl biopsy and clinical findings.

Design: 3291 stool PCR assays were performed between April 2016 and June 2017. Of those, $1192(36.2 \%)$ assays were positive for one or more of the 22 pathogens, and 79 of the 1192 had a colon biopsy within one week of the stool PCR test. The 79 biopsies were reviewed by two Gl pathologists, and the electronic medical records were reviewed for clinical findings.

Results: The top three pathogens detected in the 79 patients who had biopsies were C. difficile (51\%), enteropathogenic E. coli $(20 \%)$, and Norovirus $(10 \%) .59$ patients $(75 \%)$ had histologic abnormalities, while $20(25 \%)$ patients had a positive stool PCR test but no significant histopathologic abnormality. Of the 59 patients with histologic findings, 31 had previous diagnoses of chronic idiopathic inflammatory bowel disease (10 Crohn's, 21 ulcerative colitis). Of the 28 patients with no prior history of IBD, patterns of inflammation included acute infectious-type colitis $(19,68 \%)$, pseudomembranes $(3,11 \%)$, mucosal necrosis $(3,11 \%)$, and granulomas $(2,7 \%)$.

Conclusions: The majority of patients in our study with a positive stool PCR assay had histologic findings on biopsy, although approximately half of these had previous diagnoses of IBD. In patients without IBD, the most common histologic pattern was acute infectious-type colitis. Of note, $68 \%$ of patients with both a positive PCR test and histologic abnormalities presented with a diarrheal illness, suggesting that the pathogens detected by the BioFire assay are either true primary pathogens or a possible cause of an IBD flare. However, the clinical significance of a positive BioFire assay in a patient with a negative biopsy remains unclear. Evaluation of the clinical significance of a positive stool PCR test, as well as subsequent therapeutic decisions, therefore, require correlation between clinical, molecular, and histologic data.

\section{Norovirus Colitis: Histopathology and Clinica Correlates}

Jonathan Mowers ${ }^{1}$, Laura W Lamps ${ }^{1}$, Joel Greenson ${ }^{1}$, Michael Bachman'. 'University of Michigan Hospitals, Ann Arbor, MI

Background: Norovirus infection is the most common infectious cause of diarrhea worldwide, and is an important cause of food-borne illness; however, little is known about the histopathology. This study describes the pathologic features and clinical correlates in 8 cases of confirmed Norovirus infection, which to our knowledge is the first series described in humans.

Design: Of 3291 FilmArray gastrointestinal panel (BioFire Diagnostics, Salt Lake City, Utah) stool PCR assays conducted during a 14 month period, 1192 were positive for one of 22 organisms. Eight of these were positive for Norovirus, and also had a colon biopsy within a week of the positive BioFire assay. Cases were reviewed for histologic features, and electronic medical records were reviewed for pertinent clinical and laboratory features at the time of the positive PCR test and biopsy.

Results: Patient age ranged from 9-79 years (median 43); gender distribution was equal. All presented with acute onset of diarrhea. Two patients had transplants (1 liver/kidney and 1 stem cell); 2 had chronic idiopathic inflammatory bowel disease; and 4 had no underlying condition. Colonoscopy showed colitis in 5 patients, and was normal in 3. Four biopsies showed focal or diffuse active colitis with surface epithelial damage and/or increased apoptotic epithelial cells, consistent with infectious colitis. Three biopsies were normal. One (with ulcerative colitis) showed nonspecific ulceration. The Crohn disease patient showed focal granulomatous inflammation in addition to active colitis. Follow-up ranged from 2-11 months; 3 patients (none of whom had underlying conditions) recovered spontaneously. Five patients were treated empirically wtih NSAIDS, steroids, and/or antibiotics, with full recovery or return to baseline.

Conclusions: Both the macroscopic and histologic features of Norovirus colitis are extremely variable, but it does appear to be associated with an infectious-type colitis pattern in some patients. Although the number of cases in our series is small, the data suggest that Norovirus may be an important pathogen in immunocompromised patients, as well as those suffering from chronic idiopathic inflammatory bowel disease. It is likely that complex host/ virus interactions determine the extent of damage to the bowel. Larger studies are needed to further elucidate the pathology of this common but poorly understood disease, and the clinicopathologic differences between immunocompromised and immunocompetent patients with Norovirus infection.

\section{The Clinical Significance of Enteropathogenic E. coli detected in Stool Multiplex PCR Assays}

Jonathan Mowers ${ }^{1}$, Laura W Lamps ${ }^{1}$, Joel Greenson ${ }^{1}$, Michael Bachman'. 'University of Michigan Hospitals, Ann Arbor, MI

Background: Enteropathogenic E. coli (EPEC) have been associated with diarrhea in infants and children in developing countries however, little is known about the prevalence and clinical features in industrialized nations, and the pathologic features have not been well described. We reviewed the clinicopathologic features of 18 patients with EPEC to determine the clinical significance and pathologic correlation.

Design: The FilmArray Gastrointestinal Panel (BioFire Diagnostics, Salt Lake City, Utah) is a multiplex PCR assay that detects 22 different $\mathrm{GI}$ pathogens in stool, including pathogenic E. coli subtypes. Between April 2016 and June 2017, 18 patients were positive for EPEC and also had a lower GI biopsy within one week of the positive stool PCR panel. All $\mathrm{GI}$ biopsies were blindly reviewed by $2 \mathrm{Gl}$ pathologists.

Results: 16 patients were positive for EPEC, and 2 were positive for EPEC and enteroaggregative E. coli (EAEC). Of the 16 EPEC patients, 5 had ulcerative colitis, 2 had Crohn's disease, 1 had indeterminate IBD, and 1 had celiac disease. Four were transplant patients, and 1 was being treated for melanoma with checkpoint inhibitors. One patient had an eating disorder and 1 had a perforated colon cancer. Of the 2 patients positive for both EPEC and EAEC, 1 had recurrent colorectal cancer and the other had a BMT. Biopsies were indicative of the patients known underlying disease process in 12 cases and were normal in 3 . Two BMT patients had ulcers (one with pseudomembranes) and both responded to antibiotictreatment for EPEC. A kidney transplant patien had colonic lymphocytosis and also responded to antibiotics. The 3 patients with normal biopsies were all treated with antibiotics and none improved. Three of 5 ulcerative colitis patients ultimately underwent colectomies for intractable disease, 2 of which had been treated with antibiotics.

Conclusions: All 18 patients who were positive for EPEC had a pre existing GI or systemic disease and most were immunocompromised suggesting EPEC may be a significant pathogen in these populations. As all patients had co-existent diseases for which they were treated, it is difficult to assess the response to antibiotics. Patients with normal biopsies seem to have been colonized rather than infected while some transplant patients had true infections. The presence of EPEC in IBD flares requires further study.

\section{Clinicopathological Features of Early Phase Fundic Gland Type Adenoma: Tumors Can Occurr At The Bottom of The Gland, And Even Minute Lesions May Show a Mixed Phenotype}

Koji Nagata', Michio Shimizu². 'Saitama Medical University, Hidaka, Saitama, Japan, ${ }^{2}$ Hakujikai Memorial Hospital, Tokyo

Background: So far it is known that at least two types of gastric adenoma, namely intestinal type and pyloric gland type, are present. Recently, tumors arising from the fundic gland have been recognized, and cases of fundic gland type adenomas or fundic gland adenocarcinoma have been reported. However, much remains unclear. Here, we investigated clinicopathological findings of such lesions and clarified the tumor progression.

Design: We found three cases (1 male, 2 females) excised by endoscopic resection or surgery among the cases of gastric cance between 2015 and 2016 in our hospital. To determine the phenotype of the tumor, we performed immunohistochemistry of CD10, MUC2 CDX-2, MUC5AC, MUC6, HGM, ATPase, pepsinogen I, and Mist1. We also examined the expression of $\mathrm{Ki}-67$ and p53.

Results: Regarding the size, all three lesions were less than $5 \mathrm{~mm}$. All three patients were in the 70's. Two lesions were located in the posterior wall of the upper part of the body, and one lesion in the anterior wall of the upper part of the body. Regarding the phenotype of the lesion, one lesion was pepsinogen I positive, MUC 6 negative (chief cell type), and one lesion pepsinogen I positive, MUC 6 positive with mosaic pattern (mucous neck cell type). Another lesion showed both chief cell type area (pepsinogen positive, MUC6 negative) and mucous neck cell type area (pepsinogen positive, MUC6 positive with mixed mosaic pattern and diffuse pattern). All lesions were Mist positive, and non-neoplastic fundic glands with parietal cells or chief cells were observed adjacent to the lesion. All cases were negative fo p53, and no cases showed Ki-67 labeling index more than $3 \%$, and no obvious malignant findings were found

Conclusions: Our study indicated that fundic gland type adenomas were mainly located at the bottom of the gland suggesting its origin Regarding mucous neck cell type adenoma, two types of MUC6 
positivity, namely mosaic pattern and diffuse pattern, were observed. It may be due to phenotypic difference. Coexistence of both chief cell type and mucous neck cell type in a small lesion (less than $5 \mathrm{~mm}$ ) suggested the possibility of immediate transformation of phenotype even in a small lesion.

\section{HIV Enteropathy: Real or Myth? A Histologic Review}

ILKe Nalbantoglu', Raul S Gonzalez'. 'Washington University in St. Louis, Saint Louis, MO, 2University of Rochester Medical Center, Rochester, NY

Background: Human immunodeficiency virus (HIV) infection has been reported to cause primary disease in the human gastrointestinal tract, termed "HIV enteropathy." Most of the previously described histologic features ascribed to this diagnosis are not specific. This study aimed to elucidate the histologic changes, if any, in a large series of gastrointestinal specimens from HIV patients (pts).

Design: We identified 369 GI biopsies (bxs) from 172 HIV pts. Clinical data was obtained from medical records. Tissue slides were re-examined, and histologic features were recorded. Two pts with concomitant inflammatory bowel disease were excluded. Relevant clinical parameters were compared using Fisher's exact test.

Results: Average pt age was 48 years (range 20-75); male/female ratio was roughly $2: 1$. HIV viral load was high (>1000 copies $/ \mathrm{ml}$ ) in $40(23 \%)$, low in $69(40 \%)$, and not available in $63(37 \%)$. Fifty-one pts $(30 \%)$ had CD4 counts of $<200$ ("advanced HIV"). Roughly two-thirds of pts (113) received treatment; one-fourth (43) did not, and treatment status was unavailable in 16 . Common presenting symptoms included diarrhea (60 pts, 35\%), abdominal pain (30 pts, 17\%) and dysphagia (27 pts, 16\%). There was no relationship between diarrhea and CD4 counts $(P=0.75)$ or HIV viral load $(P=0.13)$, or between treatment status and diarrhea $(P=0.30)$. Endoscopic reports were available for 156 pts: 106 were abnormal. Esophageal bxs $(n=52)$ showed refluxtype change (56\%), acute inflammation (33\%), and infectious agents (13\%; 6 Candida, 1 CMV). Stomach bxs ( $n=92)$ showed chronic inflammation (80\%), increased intraepithelial lymphocytes (34\%), acute inflammation (29\%), and Helicobacter pylori (14\%). Duodenal bxs $(n=46)$ showed increased lamina propria inflammation $(59 \%)$ acute inflammation (20\%), and villous blunting (13\%). Terminal ileum bxs $(n=24)$ were mostly normal $(88 \%)$, though acute inflammation was noted in $12 \%$. Colon bxs $(n=155)$ mostly showed normal architecture $(66 \%)$, though $31 \%$ showed increased lamina propria inflammation and $6 \%$ showed increased crypt apoptosis. Lymphoid aggregates were seen in $51 \%$, two pts had CMV, and five had spirochetosis.

Conclusions: Gastrointestinal bx findings in HIV-positive pts do not show features of distinct enteritis that can be described as "HIV enteropathy" by light microscopy. Although a direct relationship with treatment could not be established, any gastrointestinal symptoms these pts experience are likely due to medication side effects or possibly ultrastructural changes.

\section{Diverticular disease-associated colitis as a potentia form of IBD: Results of a gene expression profiling study}

Pooja Navale ${ }^{1}$, Qingqing Liu ${ }^{1}$, Huaibin Mabel Ko', Mena Mansour ${ }^{1}$ Alexandros D Polydorides ${ }^{1}$, Hongfa Zhu', Noam Harpaz ${ }^{2}$. ${ }^{1}$ Icahn School of Medicine at Mount Sinai Medical Center, New York, NY, ${ }^{2}$ Mount Sinai Med Center, New York, NY

Background: Diverticular disease-associated colitis (DDAC) is a chronic colitis that affects diverticular colonic segments, typically the sigmoid colon. Most patients are over 60 and present with LLQ pain and rectal bleeding. Microscopically, DDAC features mucosal-based chronic inflammation that closely mimics IBD, the distinction requiring awareness of the endoscopic findings and histologic sparing of nondiverticular segments. The histological similarity of DDAC to IBD, as well as case reports of progression from DDAC to classical IBD, has led to suggestions that DDAC might be considered a form of IBD. We addressed this issue by means of comparative gene expression profiling.

Design: Pooled mRNA isolated from paraffin-embedded sections of sigmoid colon from patients with DDAC $(N=9), U C(N=10)$, Crohn's disease $(\mathrm{N}=10)$ and normal controls $(\mathrm{N}=10)$ were analyzed in triplicate with the NanoString nCounter gene expression assay. The UC, CD and DDAC tissues were selected for mild to moderate intensity of histologic inflammation. The assays targeted 778 human genes, 594 and 184 of which are differentially expressed in immunological disorders and inflammatory conditions, respectively. The raw expression data were normalized using nSolver Analysis Software 3.0 and a dataset of gene expression ratios was generated.

Results: The expression of multiple genes previously known to be elevated in IBD was similarly increased in all 3 groups, some examples being tumor necrosis factor (UC 3-fold, CD 4-fold, DDAC 2-fold) interleukin 23 (UC 39-fold, CD 21-fold, DDAC 21-fold), interleukin 26 (UC 10-fold, CD 11-fold, DDAC 4-fold) and CXCL-1 (UC 13-fold, CD 7-fold, DDAC 7-fold). Isolated differences between DDAC and IBD ere observed, including disproportionately increased expression of CCAAT/enhancer-binding protein beta (CEBPB, UC 1.6-fold, CD 1.4 fold, DDAC 2.2-fold) and its downstream gene CLEC4E (UC 2-fold, CD 3-fold, DDAC 10-fold) in DDAC compared with UC and CD. Conversely, expression of defensin beta 4A (DEFB4A) was disproportionately increased in IBD compared with DDAC (UC 25-fold, CD 13-fold, DDAC 3-fold).

Conclusions: Colonic mucosa involved by DDAC showed substantia similarity to IBD with respect to the expression of genes that are known to be increased in the latter, lending additional support to the proposition that DDAC be classified as a particular form of IBD. The individual differences that we identified between the disorders might provide insight into the unique aspects of the pathogenesis of DDAC.

\section{The Cost of the 12th Lymph Node}

Bushra Nazir ${ }^{1}$, Nikolina Dioufa', Anu Peter ${ }^{1}$, Temple University Program ${ }^{1}$, Jasvir Khurana', Nirag Jhala'. 'Temple University Hospital, Philadelphia, PA

Background: Obtaining 12 lymph nodes in colectomies performed for colorectal adenocarcinoma is considered a standard requirement and is often used as a measure of the quality of Pathology reports. This however, may not be important for patients in today's personalized therapy. Our objective was to determine the performance of detecting 12 lymph nodes from colectomies performed at our institution and compared to other institutions. Secondarily, determine additional time and cost involved to consistently detect 12 lymph nodes.

Design: Utilizing our lab information system, we retrospectively looked at the reports of all colectomies done from 2010-2012 for colorectal adenocarcinoma. We noted the number of nodes obtained nodes positive, when the pericolonic fat was entirely sampled and whether the patient had metastatic disease at presentation. We also calculated the cost of obtaining lymph nodes using pathologist time in additional grossing, reviewing and adding processing costs.

Results: Other institutions' data taken together reported being able to identify 12 lymph nodes $80-90 \%$ of the time. In comparison at our institution in 8 of 13 quarters, 12 lymph nodes were found on an average of $100 \%$. In 5 quarters we did not have 12 lymph nodes $100 \%$ of the time. On an average 22 lymph nodes were submitted per case (range 3-74 lymph nodes per case). In $92 \%$ of cases greater than 12 lymph nodes were detected. In $8 \%$ (9/110) of cases, less than 12 lymph nodes were identified. In 6 of the 9 cases the patients had metastatic disease and submitting the extra lymph nodes would not have changed the management. In an effort to detect additiona lymph nodes on average 25 cassettes (range 18-32 cassettes) worth of pericolonic adipose tissue had to be submitted.

Conclusions: The guidelines are important but should be followed with rationale.

\section{Risk Stratification of Gastrointestinal Stromal Tumors Based on Gene Expression Profiling}

Klaudia Nowak', Benjamin Adam¹, Iyare Izevbaye ${ }^{1}$, Kim Formenti' 'University of Alberta, Edmonton, ÁB

Background: Gastrointestinal stromal tumor (GIST) is the most common maignant mesenchymal tumor of the gastrointestinal tract. Current models for predicting risk of recurrence include site, tumor size and mitotic count. However, up to $80-85 \%$ of patients currently classified as high risk may in fact be at minimal to no risk of recurrence, questioning the value of these models. Gene expression profiling has been demonstrated to be a powerful tool for risk recurrence in severa other cancers, most notably breast carcinoma. Previous studies have identified molecular targets associated with worse prognosis in GIST patients. The aim of this study is to identify gene expression signatures that can potentially provide more precise risk stratification for GIST patients.

Design: 56 GIST cases (confirmed by CD117/DOG1) with available clinicopathologic data were retrieved. NanoString ${ }^{\circledR}$ gene expression analysis was performed using mRNA from representative archiva formalin-fixed paraffin-embedded blocks. The NanoString ${ }^{\circledR} \mathrm{GX}$ Human Cancer panel, including 231 genes previously associated with human cancer, was used. Gene expression results were correlated with clinical and pathologic data. Analysis was performed with nSolver and $\mathrm{R}$

Results: Eight genes demonstrated statistically significant differential expression (FDR $<0.05$ ) between high risk and non-high risk tumors: CHEK1, BIRC5, PCNA, E2F1, CCNA2, TOP2A, CDC2, and TYMS (Figure 1). All had relatively greater expression in high risk vs. non-high risk tumors. Receiver operating characteristic curve analysis demonstrated the geometric mean of these 8 genes to be superior to all individual genes for differentiating high risk from non-risk tumors ( $A U C=0.861 \mathrm{vs}$ $A \cup C=0.715-0.858$ ). Aggregate 8-gene set expression was significantly higher in high risk tumors compared with intermediate $(\mathrm{p}<0.001)$, low $(p<0.001)$ and very low $(p=0.010)$ risk tumors (Figure 2$)$. 
Figure 1: Volcano plot showing statistically significant differential expression (FDR<0.05) for 8 out of 231 genes between high risk and non-high risk tumors. All DE genes have increased expression in high risk vs. non-high risk tumors.

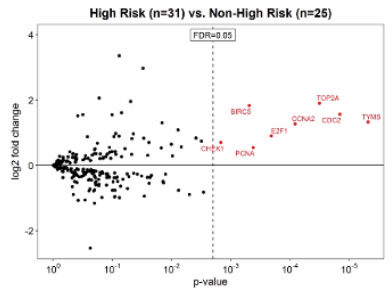

Figure 2: Gene expression box plot showing significantly higher expression of aggregate 8 gene set in high risk tumors vs. all other risk groups (Mann-Whitney U-test).

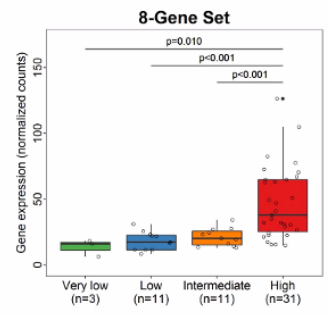

Conclusions: These results suggest that gene expression profiling can be used to assist risk classification in GIST patients. Future work including correlation with clinical outcomes will further clarify the utility of this approach in relation to the existing standard of practice.

\section{Should Ki67 Immunohistochemistry Be Performed on All Lesions in Multifocal Small Intestinal Neuroendocrine Tumors?}

Numbereye Numbere ${ }^{1}$, Aaron Huber ${ }^{2}$, Chanjuan Shi ${ }^{3}$, Justin Cates ${ }^{4}$ Raul S Gonzalez'. 'University of Rochester Medical Center, Pittsford NY, ${ }^{2}$ Rochester, NY, ${ }^{3}$ Vanderbilt University, Nashville, TN, ${ }^{4}$ Vanderbilt University Medical Center, Nashville, TN, ${ }^{5}$ University of Rochester Medical Center, Rochester, NY

Background: Well-differentiated small intestinal neuroendocrine tumors (SI-NETs) are often multifocal, and this has been suggested to impart worse disease-free survival. Practice guidelines have not been established for World Health Organization (WHO) grading of multiple primary lesions, including whether all lesions should undergo immunohistochemical staining for Ki67. In the absence of a scientifically supported approach, most pathologists likely stain the single largest lesion. We evaluated the link between SINET multifocality and other clinicopathologic features, and the link between SI-NET size, multifocality, and Ki67 index.

Design: We identified 68 cases of ileal or jejunal SI-NET, with a combined total of 207 primary lesions. Each case was evaluated for patient age and sex; size of all tumors; presence of lymph node metastases, mesenteric tumor deposits, and distant metastases; and disease-specific outcome. Ki67 immunohistochemical staining was performed on all 207 primary lesions, and a proliferation index was manually counted from one photographed hot-spot per tumor. The relationship between focality and clinicopathologic factors was compared using Fisher's exact test. Outcomes were compared by Cox proportional hazard regression.

Results: Forty-one patients had unifocal disease, and 27 had multifocal disease (median 5 lesions, range 2-32). Most tumors were WHO grade 1 (201/207,97\%), five were grade 2 , and 1 was grade 3 . Five patients had grade 2/3 tumors: Three had multifocal disease, one had two subcentimeter grade 2 lesions (including the largest) and eight subcentimeter grade 1 lesions, and one had one 1.6-cm grade 3 lesion and one subcentimeter grade 1 lesion. Ki67 index and tumor size had a positive correlation (coefficient 0.28 ; 95\% confidence interval [Cl] $0.05-0.52, P=0.017$ ) (Image). Multifocality was more common in male patients $(P=0.047)$ but was unrelated to age $(P=0.97)$. There was no significant association between disease focality and nodal metastases $(P=0.19)$, tumor deposits $(P=1.0)$, or distant metastases $(P=0.43)$. Multifocality conferred a hazard ratio of 2.07 with regard to overall survival on univariate analysis, but this was not statistically significant (95\% Cl 0.53-8.09, $P=0.30)$.

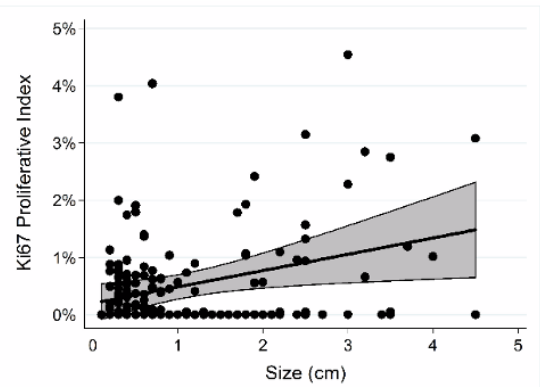

Conclusions: SI-NET multifocality does not appear to impact patient survival significantly. In patients with multifocal SI-NET, unless a particular lesion has a high mitotic rate, staining only the largest lesion for Ki67 appears to accurately grade essentially all cases.

\section{Correlation of Immunohistochemical Mismatch Repair Protein Status in Colorectal Carcinoma Biopsy and Resection Specimens}

Odharnaith O'Brien', Eanna Ryan², Ben Creavin², Robert Geraghty ${ }^{3}$ Des Winter ${ }^{2}$, Kieran Sheahan ${ }^{4}$. ${ }^{1}$ Clonmel, ${ }^{2}$ St. Vincent's University Hospital, ${ }^{3}$ Dublin, ${ }^{4}$ St. Vincent Hospital, Dublin

Background: Microsatellite instability (MSI) occurs in approximately $15 \%$ of all colorectal carcinomas (CRC), equating to loss of activity of one or more DNA mismatch repair (MMR) genes. This can be due to either a sporadic or germline mutation in the relevant mismatch repair gene. Initial evaluation of MMR status is determined by performing immunohistochemistry (IHC) on a patient's CRC resection specimen. We aim to evaluate the reliability of MMR IHC staining in CRC on preoperative endoscopic biopsy compared to matched surgical resection specimens. The small size of the biopsy materia allows for better fixation and easier interpretation of staining when compared to resection specimens. In patients with rectal carcinoma who receive neoadjuvant chemotherapy, the biopsy may be the only source of testing material if a complete response is obtained. It would also allow for earlier initiation of hypermethylation/BRAF/MSI testing to determine whether the patient has sporadic or germline MSI.

Design: We performed a retrospective search of ou laboratory's histopathology LIS database for all patients with colorectal carcinoma who had MMR IHC performed on both their preoperative endoscopic biopsy and subsequent resection specimens from January 2010 to January 2016. We then determined the concordance of MMR staining between the biopsy and resection specimens, in both MMR proficient (pMMR) and MMR deficient (dMMR) cases.

Results: From 2000 to 2016 we identified 53 patients who had IHC for MMR proteins performed on both their preoperative colorecta endoscopic biopsies and their resection specimens; 10 patients $(10 / 53,18.87 \%)$ demonstrated loss of one or more mismatch repair proteins by immunohistochemical analysis on their initial endoscopic tumour biopsy. The remainder $(43 / 50,81.13 \%)$ showed no loss of staining of any mismatch repair protein. There was complete agreement in MMR IHC status between the preoperative endoscopic biopsies and the corresponding surgical resection specimens in all dMMR and pMMR cases (sensitivity 100\%, specificity 100\%). 3 of the 10 patients $(33.3 \%)$ who demonstrated a dMMR phenotype on their endoscopic tumour biopsy received neoadjuvant chemoradiation prior to surgical resection; no difference in staining was observed between the biopsies and post neoadjuvant resection specimens.

\begin{tabular}{|l|l|l|l|l|l|l|l|l|l|}
\hline Case & Age & Sex & $\begin{array}{l}\text { Site of } \\
\text { tumour }\end{array}$ & $\begin{array}{l}\text { MMRP } \\
\text { loss } \\
\text { endo- } \\
\text { scopic } \\
\text { biopsy }\end{array}$ & $\begin{array}{l}\text { Neoad- } \\
\text { juvant } \\
\text { Ther- } \\
\text { apy }\end{array}$ & $\begin{array}{l}\text { MMRP } \\
\text { loss } \\
\text { col- } \\
\text { orectal } \\
\text { resec- } \\
\text { tion }\end{array}$ & $\begin{array}{l}\text { Cor- } \\
\text { related } \\
\text { Speci- } \\
\text { mens }\end{array}$ & $\begin{array}{l}\text { Mo- } \\
\text { lecular } \\
\text { Testing }\end{array}$ & $\begin{array}{l}\text { Constitutional } \\
\text { Testing }\end{array}$ \\
\hline 1 & 85 & F & $\begin{array}{l}\text { He- } \\
\text { patic } \\
\text { flexure }\end{array}$ & $\begin{array}{l}\text { MLH1+ } \\
\text { PMS2 }\end{array}$ & No & $\begin{array}{l}\text { MLH1+ } \\
\text { PMS2 }\end{array}$ & Yes & $\begin{array}{l}\text { BRAF } \\
\text { Mut (rs) }\end{array}$ & Not indicated \\
\hline 2 & 55 & F & $\begin{array}{l}\text { Rec- } \\
\text { tum }\end{array}$ & $\begin{array}{l}\text { MSH2 } \\
+ \\
\text { MSH6 }\end{array}$ & No & $\begin{array}{l}\text { MSH2 } \\
+ \\
\text { MSH6 }\end{array}$ & Yes & $\begin{array}{l}\text { None in- } \\
\text { dicated }\end{array}$ & $\begin{array}{l}\text { Awaiting Ger- } \\
\text { mline Testing }\end{array}$ \\
\hline 3 & 40 & F & $\begin{array}{l}\text { Rec- } \\
\text { tum }\end{array}$ & $\begin{array}{l}\text { MLH1+ } \\
\text { PMS2 }\end{array}$ & Yes & $\begin{array}{l}\text { MLH1+ } \\
\text { PMS2 }\end{array}$ & Yes & $\begin{array}{l}\text { Mut (bx) } \\
\text { KRAS } \\
\text { WT (bx) }\end{array}$ & $\begin{array}{l}\text { Awaiting Ger- } \\
\text { mline Testing } \\
\text { Rectal) }\end{array}$ \\
\hline 4 & 39 & M & $\begin{array}{l}\text { Rec- } \\
\text { tum }\end{array}$ & MSH6 & Yes & MSH6 & Yes & $\begin{array}{l}\text { None in- } \\
\text { dicated }\end{array}$ & $\begin{array}{l}\text { MSH6 VUS } \\
\text { c.3725G }>\text { A A }\end{array}$ \\
\hline 5 & 80 & F & $\begin{array}{l}\text { Trans- } \\
\text { verse } \\
\text { colon }\end{array}$ & $\begin{array}{l}\text { MSH2 } \\
+ \\
\text { MSH6 }\end{array}$ & No & $\begin{array}{l}\text { MSH2 } \\
+ \\
\text { MSH6 }\end{array}$ & Yes & $\begin{array}{l}\text { None in- } \\
\text { dicated }\end{array}$ & $\begin{array}{l}\text { Awaiting Ger- } \\
\text { mline Testing }\end{array}$ \\
\hline
\end{tabular}




\begin{tabular}{|c|c|c|c|c|c|c|c|c|c|}
\hline 6 & 43 & M & $\begin{array}{l}\text { As- } \\
\text { cend- } \\
\text { ing } \\
\text { colon }\end{array}$ & PMS2 & No & PMS2 & Yes & $\begin{array}{l}\begin{array}{l}\text { BRAF WT } \\
\text { (bx) }\end{array} \\
\text { KRAS } \\
\text { Mut (rs) } \\
\\
\text { MLH1 } \\
\text { Hyper- } \\
\text { meth- } \\
\text { ylation } \\
\text { status } \\
\text { - normal } \\
\text { (bx) }\end{array}$ & $\begin{array}{l}\text { PMS2 Mutation } \\
\text { c. } 137 \mathrm{G}>\mathrm{T}\end{array}$ \\
\hline 7 & 81 & M & $\begin{array}{l}\text { Cae- } \\
\text { cum }\end{array}$ & $\begin{array}{l}\text { MSH6 } \\
+ \\
\text { PMS2 }\end{array}$ & No & $\begin{array}{l}\text { MSH6 } \\
+ \text { PMS2 }\end{array}$ & Yes & $\begin{array}{l}\text { BRAF WT } \\
\\
\text { MLH1 } \\
\text { Hyper- } \\
\text { meth- } \\
\text { ylation } \\
\text { status- } \\
\text { normal }\end{array}$ & $\begin{array}{l}\text { Awaiting Ger- } \\
\text { mline Testing }\end{array}$ \\
\hline 8 & 30 & M & $\begin{array}{l}\text { Rec- } \\
\text { tum }\end{array}$ & $\begin{array}{l}\text { MLH1+ } \\
\text { PMS2 }\end{array}$ & Yes & $\begin{array}{l}\text { MLH1+ } \\
\text { PMS2 }\end{array}$ & Yes & $\begin{array}{l}\text { BRAF WT } \\
\text { (rs) } \\
\\
\text { Hyper- } \\
\text { meth- } \\
\text { ylation } \\
\text { status } \\
\text { - normal } \\
\text { (rs) }\end{array}$ & $\begin{array}{l}\text { MLH1 Mutation } \\
\text { c. } 1489 \text { dupC }\end{array}$ \\
\hline 9 & 28 & $\mathrm{~F}$ & $\begin{array}{l}\text { As- } \\
\text { cend- } \\
\text { ing } \\
\text { colon }\end{array}$ & $\begin{array}{l}\text { MLH1+ } \\
\text { PMS2 }\end{array}$ & No & $\begin{array}{l}\text { MLH1+ } \\
\text { PMS2 }\end{array}$ & Yes & $\begin{array}{l}\text { BRAF WT } \\
\text { (rs) } \\
\\
\text { Hyper- } \\
\text { meth- } \\
\text { ylation } \\
\text { status } \\
\text { - normal } \\
\text { (rs) }\end{array}$ & $\begin{array}{l}\text { MLH1 Mutation } \\
\text { c. } 1731+1 \mathrm{G}>\mathrm{A}\end{array}$ \\
\hline 10 & 86 & $\mathrm{~F}$ & $\begin{array}{l}\text { As- } \\
\text { cend- } \\
\text { ing } \\
\text { colon }\end{array}$ & $\begin{array}{l}\text { MLH1+ } \\
\text { PMS2 }\end{array}$ & No & $\begin{array}{l}\text { MLH1+ } \\
\text { PMS2 }\end{array}$ & Yes & $\begin{array}{l}\begin{array}{l}\text { BRAF WT } \\
\text { (bx + rs) }\end{array} \\
\text { KRAS } \\
\text { Mut (bx) } \\
\\
\text { Hyper- } \\
\text { meth- } \\
\text { yeation } \\
\text { status } \\
\text { - normal } \\
\text { (rs) }\end{array}$ & Not indicated \\
\hline
\end{tabular}

Conclusions: We demonstrated $100 \%$ concordance of MMR IHC status in 53 cases of dMMR and pMMR tumours. These results indicate endoscopic biopsies are a suitable source of tissue for MMR IHC analysis.

\section{Loss of SATB2 Expression in Colorectal Carcinoma Is Associated with DNA Mismatch Repair Protein Deficiency and Poor Survival}

Dane Olevian', Reetesh Pai'. 'Pittsburgh, PA, ${ }^{2}$ UPMC-Presbyterian Hospital, Pittsburgh, PA

Background: SATB2 is a sensitive marker for colorectal carcinoma (CRC), but correlation of expression with histologic features and molecular alterations has not been performed. This study examines the immunohistochemical (IHC) expression of SATB2 in gastrointestinal adenocarcinoma to assess its utility in diagnosis and analyzes the clinicopathologic, molecular, and survival characteristics of CRC stratified by SATB2 expression.

Design: SATB2 IHC was performed using tissue microarrays of $545 \mathrm{Gl}$ adenocarcinomas (241 CRC, 72 esophagus, 103 stomach, 20 appendix, and 109 pancreas). SATB2 was scored for intensity (0, absent; $1+$, weak; $2+$, strong) and percent of tumor cells staining. A modified $\mathrm{H}$-score (intensity multiplied by percent of positive cells) was calculated, with positive expression equal to score $>5$. SATB2 in CRC was correlated with clinicopathologic features; CK7, CK20, and CDX2 expression; KRAS mutation; BRAF mutation; DNA mismatch repair protein status by IHC; and overall survival.

Results: SATB2 expression was most frequent in CRC (201/241, 84\%, mean $\mathrm{H}$-score of 130$)$ and appendiceal adenocarcinoma $(15 / 20,75 \%$, mean $\mathrm{H}$-score of 61$)$, but infrequent in other $\mathrm{Gl}$ adenocarcinomas $(21 \%$ esophageal, $5 \%$ gastric, and $0 \%$ pancreatic) where it was weak and/ or focal. In CRC, loss of expression was more frequent in tumors with MMR protein deficiency (MMRD) (13/42,31\%) compared to proficiency $(4 / 199,2 \%)(p<0.01)$. CRC with loss of SATB2 expression more often harbored BRAF mutation than CRC with positive expression $(5 / 17$, $29 \%$ vs. $3 / 116,3 \%)(p<0.01)$, and more frequently displayed mucinous or signet ring cell differentiation and tumor infiltrating lymphocytes compared to CRCs with positive expression (all with $\mathrm{p}<0.01$ ). CRCs with loss of SATB2 more frequently expressed CK7 ( $26 \%$ vs. $2 \%$ ) and more frequently lacked CK20 and CDX2 expression (18\% vs. 4\%) (all with $p<0.01)$. Loss of SATB2 in CRC was associated with worse overall survival compared to preserved expression (median survival of 48 months vs. 54 months, $\mathrm{p}=0.02$ ), including when analysis was limited to MMRD CRC, although this is limited by small sample size $(n=42)$.

Conclusions: SATB2 is most commonly expressed in CRC and appendiceal adenocarcinoma and is useful in distinguishing CRC from upper Gl and pancreatic adenocarcinomas. In CRC SATB2 expression is associated with histologic features of MMRD, MMRD by $I H C$, and BRAF mutation. Loss of SATB2 expression is associated with decreased overall survival, even in patients with MMRD CRC.

\section{Microsatellite Stable Colorectal Cancer Harboring Mutations in Epigenetic Modifiers is Associated with Increased Tumor Infiltrating Lymphocytes and Mucinous Features}

Madhu Ouseph ${ }^{1}$, Shaolei Lu², Shamlal Mangray ${ }^{3}$,Kara A Lombardo ${ }^{3}$ Kyle Gowen ${ }^{4}$, James Sun ${ }^{4}$, Alexa B Schrock ${ }^{5}$, Jeffrey S Ross ${ }^{6}$, Sira Ali ${ }^{7}$ Alexander S Brodsky', Murray Resnick, Evgeny Yakirevich ${ }^{3}$ 1Johnston, $\mathrm{Rl}^{2}{ }^{2}$ Brown University, Providence, $\mathrm{Rl}$, ${ }^{3}$ Rhode Island Hospital, Providence, RI, ${ }^{4}$ Foundation Medicine, ${ }^{5}$ Foundation Medicine Cambridge, MA ${ }^{6}$ Foundation Medicine, Cambridge, MA, ${ }^{7}$ Cambridge, MA, ${ }^{8}$ Brown University

\section{Disclosures:}

Kyle Gowen: Employee, Foundation Medicine, Inc. James Sun: Employee, Foundation Medicine, Inc. Alexa Schrock: Employee, Foundation Medicine, Inc. Jeffrey Ross: Employee, Foundation Medicine, Inc. Siraj Ali: Employee, Foundation Medicine, Inc.

Background: Alterations in epigenetic processes, including chromatin remodeling, DNA methylation, and histone modifications are involved in tumorigenesis. Genomic alterations (GAs) in epigenetic regulators have been described in microsatellite unstable (MSI) colorectal cance (CRC); however, their spectrum and significance in microsatellite stable (MSS) CRC are yet to be unraveled. The aim of this study was to identify GAs in epigenetic regulators in samples of MSS CRC and correlate these with clinicopathologic characteristics.

Design: Sixty-five samples of MSS CRC were assayed by hybrid capture-based comprehensive genomic profiling (FoundationOne), including up to 315 cancer-related genes and introns from 28 genes frequently rearranged in cancer. GAs were correlated with clinicopathologic data. Tumor-infiltrating lymphocytes (TILs) were evaluated by CD3 IHC.

Results: There were 38 males and 27 females with a median age of 56 years (range $35-92$ years), all with stage IV CRC. A total of 368 GAs were identified (mean 5.66; range $0-15$ ) involving 129/315 tested genes. The most common GAs involved TP53 $(89 \%)$, APC $(80 \%)$ and KRAS (46\%). Fifty-six mutations were identified in 23 epigenetic regulators ARID1B, ARID1A, ARID2, ASXL1, ATM, ATR, DOT1L, MYST3, PBRM1, PRKDC, MLL, CREBBP, NSD1, SETD2, IDH1, EZH2, SMARCA4 EP300, MLL3, DNMT3A, TET2, KDM5A, and MLL2 (mean 0.86, range 0 - 4). Thirty-five tumors $(53.8 \%)$ harbored GAs in at least one epigenetic regulator. The mean number of mutations was significantly higher in tumors with GAs in epigenetic regulators (6.4) as opposed to those without (4.8), $\mathrm{p}=0.0087$. The most commonly affected epigenetic regulators were chromatin remodeling genes ARID1B (13.8\%), MLL3 $(12.3 \%)$ and the histone modifier ARID1A (9.2\%). Tumors with GAs in epigenetic regulators were associated with significantly increased TILs as compared to tumors without GAs (5.7 vs 2.5 CD3+ TILs/200 tumor cells, $\mathrm{p}=0.01$ ). In addition, tumors with $\mathrm{GAs}$ in epigenetic regulators were associated with mucinous differentiation ( $p=0.0011)$ There were no differences in age, sex, tumor grade, budding, or peritumoral lymphocytic response between cases with and withou GAs in epigenetic regulators.

Conclusions: MSS CRCs harboring GAs in epigenetic modifiers are associated with increased TILs and mucinous phenotype, akin to MSI tumors. These results identify a subset of MSS CRC patients who could potentially benefit from immunotherapy with checkpoint inhibitors as well as therapies targeting epigenetic alterations.

\section{MMR-deficient Normal Crypts in Patients with Lynch Syndrome}

Rish Pai', Amy French ${ }^{2}$, Neil O'Callaghan ${ }^{3}$, Christophe Rosty ${ }^{4}$, Steven Thibodeau ${ }^{2}$, Daniel D Buchanan ${ }^{5}$, Noralane M Lindor ${ }^{6}$, Reetesh Pai ${ }^{7}$. ${ }^{1}$ Mayo Clinic Arizona, Scottsdale, AZ, ${ }^{2}$ Mayo Clinic, ${ }^{3}$ University of Melbourne, ${ }^{4}$ Brisbane, 5 University of Melbourne, Parkville, Victoria, ${ }^{6}$ Mayo Clinic, Scottsdale, AZ, ${ }^{7}$ UPMC-Presbyterian Hospital, Pittsburgh, PA

Background: Lynch Syndrome (LS) is the most common form of hereditary colorectal carcinoma (CRC). CRC in LS patients is characterized by microsatellite instability as a result of germline mutations in mismatch repair (MMR) genes. Two recent publications from the same institution (Lancet Oncol. 2012 Jun;13(6):598-606; PLoS One. 2015 Mar 27;10(3):e0121980) have demonstrated that histologically normal intestinal crypts in patients with LS can lose 
expression of MMR proteins. This finding has not been independently verified by other groups. In this preliminary study, we evaluated expression of MMR proteins in normal mucosa from patients with LS-associated CRC, Lynch-like CRC, MSS CRC, and sporadic MMRdeficient CRC.

Design: Histologically normal mucosa obtained from colorectal cancer resection specimens ( $n=122$ ) was evaluated by immunohistochemistry for expression of various MMR proteins. Normal mucosa adjacent to the following CRC types was evaluated: LS $(n=52)$, Lynch-like $(n=10$, defined by immunohistochemistry and/or molecular testing suggestive of LS but negative germline testing), MSS ( $n=30)$, and sporadic MMRdeficient ( $\mathrm{n}=30$, defined by loss of MLH1/PMS2 with BRAF V600E mutation and/or MLH1 promoter hypermethylation). The presence or absence of MMR protein expression in normal mucosa was determined. The number of crypts with loss of expression as well as the total length of normal mucosa evaluated was recorded.

Results: No loss of MMR protein expression was identified in normal mucosa from the $30 \mathrm{MSS}$ and 30 sporadic MMR-deficient CRC specimens evaluated. Loss of MMR protein expression was seen in normal mucosa in 15/52 LS patients with a median of 3 crypts with loss (range 1-42) (Figure 1: Loss of MSH2 expression in normal colonic crypts in an LS patient with a germline MSH2 mutation). More normal mucosa was evaluated in LS cases with loss of MMR expression in normal crypts compared to those cases without loss $(117 \mathrm{~mm}$ vs. 70 $\mathrm{mm}, \mathrm{p}<0.003)$. In all cases, the loss of MMR protein expression was congruent with the germline results. One 47 yo patient with Lynchlike CRC demonstrated loss of MMR protein expression in normal mucosa.

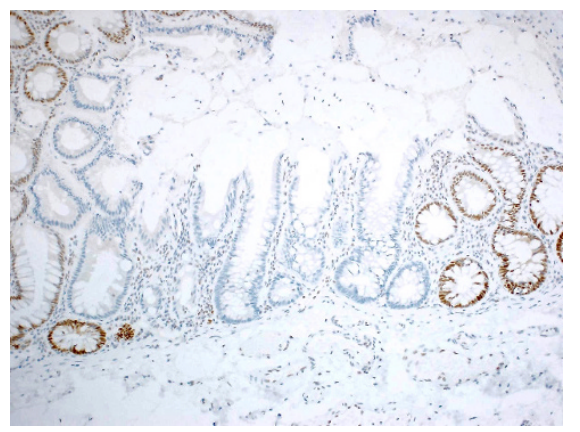

Conclusions: Loss of MMR protein expression in normal mucosa occurs in LS patients and not in patients with MSS and sporadic MSI-H tumors. The etiology of the loss of MMR protein expression in normal mucosa in one patient with Lynch-like carcinoma needs further study to exclude the possibility that this patient has an undetected germline mutation.

\section{A Novel Next Generation Sequencing Approach to Detecting Mismatch Repair Protein Deficiency in Colorectal Adenocarcinoma}

David J Papke', Jonathan A Nowak', Matthew B Yurgelun², Amitabh Srivastava', Priyanka Shivdasani", Alexander Frieden", Matthew D Ducar', Frank Kuo', Neal Lindeman', Lynette Sholl', Laura E MacConaill', Fei Dong1. 'Brigham and Women's Hospital, Boston, MA, 2Dana Farber Cancer Institute

Background: Mismatch repair protein deficiency (MMR-D) is a hallmark of Lynch syndrome, and immunohistochemical (IHC) staining is commonly used in clinical screening for MMR-D. Previously, we have utilized a 298-gene targeted next generation sequencing panel to demonstrate $92 \%$ sensitivity and $99 \%$ specificity for MMR-D in colorectal adenocarcinomas using a combination of total mutational burden and single-base insertions/deletions per megabase pair (indels/Mbp).

Design: Here, we refine our approach to use a single criterion of indels/Mbp in mononucleotide repeat regions for detection of MMR-D status as defined by IHC staining, based on a training cohort of 248 cases, of which 22 are MMR-D. We assess the validity of the criterion using a challenge cohort of 436 additional cases, of which 46 are MMR-D.

Results: Colorectal adenocarcinomas with MMR-D are enriched for indels at mononucleotide repeats of 4 or more nucleotides. Based on a refined criterion of 3 or more indels/Mbp to detect MMR-D, we find that sequencing achieves $95.5 \%$ sensitivity and $100 \%$ specificity for MMR-D in the training set and $95.7 \%$ sensitivity and $99.5 \%$ specificity in the challenge set. True-positive cases in the challenge set show an average of 13.9 indels/Mbp, with a standard deviation of 7.2 indels/ Mbp. The challenge set had 2 false-negative cases and 2 false-positive cases. In one false-negative case, there was weak IHC staining of MLH1 associated with an MLH1 missense variant, suggesting the deficiency was not complete and, hence, the mutational burden could be lower than in a typical MMR-D carcinoma. In both false-positive cases, IHC staining was intact but PCR testing demonstrated high microsatellite instability; we conclude that direct sequencing accurately detected functional mismatch-repair protein deficiencies in these false-positive cases that were not detected by IHC staining.

Conclusions: These findings demonstrate the efficacy of our nove approach for using targeted next generation sequencing to detect MMR-D in colorectal adenocarcinomas. They also provide support for using next generation sequencing to detect microsatellite instability in cases with intact mismatch repair protein IHC staining.

\section{Clinicopathologic Predictors of Long-term Responders to Trastuzumab-based therapy in Stage IV Esophageal Adenocarcinoma}

Viren R Patel', Rashmi T Samdani', Susan Abraham', Jeannelyn Estrella ${ }^{2}$, Melissa W. Taggart ${ }^{1}$, Dongfeng Tan ${ }^{1}$, Huamin Wang ${ }^{1}$, Asif Rashid", Wai Chin Foo', Dipen Maru'. 'The University of Texas MD Anderson Cancer Center, Houston, TX, ${ }^{2}$ Houston, TX

Background: Trastuzumab is a standard of care treatment option for esophageal and gastroesophageal junction adenocarcinoma (EAC) with distant metastases (stage IV). However, clinical and/or pathologic features which can predict response to Trastuzumab based therapy in stage IV EAC are unknown.

Design: We retrospectively searched institutional databases from 2010-2017 for patients who presented with or progressed to stage IV EAC and subsequently treated with Trastuzumab based therapy. Tumor histologic features, clinical parameters, Her2/neu immunohistochemistry $(\mathrm{IHC})$ and fluorescent in situ hybridization (FISH) scoring as per the guidelines described in ToGA trial were reviewed by one of the gastrointestinal pathologist. Based on response to Trastuzumab based therapy, each patient was grouped in one of the 3 categories: Long term responders; defined as disease improvement with progression free interval $>24$ months, stable disease; defined as stable disease or improvement up to 24 months or progression on treatment; defined as progression on first and second post-Trastuzumab imaging CT or PET-CT scans.

Results: We identified 310 patients with stage IV EAC tested for Her2/neu by IHC and/or FISH. Fifty of those patients had Her2/neu overexpression (IHC score $=3$ ) and/or amplification by FISH (Her2/neu/ Cep17 >2) and were treated with Transtuzumab in combination with chemotherapy at our institution. Clinicopathologic characteristics of long term responders and patients with stable or progressive disease are shown in Table

\begin{tabular}{|c|c|c|c|}
\hline & $\begin{array}{l}\text { Long Term } \\
\text { Responders } \\
(\mathrm{n}=15)\end{array}$ & $\begin{array}{l}\text { Stable or progres- } \\
\text { sion of disease } \\
\quad(n=35)\end{array}$ & $\begin{array}{l}\mathrm{p} \text { (by Chi- } \\
\text { square test) }\end{array}$ \\
\hline Location of tumor & & & $\begin{array}{l}\text { Not } \\
\text { significant }\end{array}$ \\
\hline Distal Esophagus & 7 & 18 & \\
\hline GE junction & 8 & 17 & \\
\hline Stage at first time diagnosis & & & 0.05 \\
\hline Distant Metastasis (Stage IV) & 13 & 21 & \\
\hline Locoregional (Stage II-III) & 2 & 14 & \\
\hline Histologic Grade & & & 0.03 \\
\hline Low Grade & 14 & 22 & \\
\hline High grade & 1 & 13 & \\
\hline Her2 IHC score & & & 0.06 \\
\hline$<3$ & 4 & 19 & \\
\hline 3 & 11 & 16 & \\
\hline Her2/Neu signals by FISH & & & 0.04 \\
\hline$\leq 6$ & 0 & 6 & \\
\hline$>6$ & 7 & 18 & \\
\hline $\begin{array}{l}\text { Type of adjuvant chemo- } \\
\text { therapy }\end{array}$ & & & $\begin{array}{l}\text { Not signifi- } \\
\text { cant }\end{array}$ \\
\hline $\begin{array}{l}\text { Platinum based adjuvant } \\
\text { chemotherapy therapy }\end{array}$ & 7 & 18 & \\
\hline $\begin{array}{l}\text { Taxol + Paltinum based } \\
\text { adjuvant chemotherapy }\end{array}$ & 8 & 8 & \\
\hline $\begin{array}{l}\text { Irinotecan based adjuvant } \\
\text { chemotherapy }\end{array}$ & 0 & 5 & $<0.05^{*}$ \\
\hline $\begin{array}{l}\text { Irinotecan + Taxol based } \\
\text { adjuvant chemotherapy }\end{array}$ & 0 & 4 & \\
\hline $\begin{array}{l}\text { Progression free survival, } \\
\text { median (range), months }\end{array}$ & $41(9-67)$ & $6(<1-20)$ & $<0.01$ \\
\hline Overall survival & $56(9-100)$ & $15(<1-65)$ & $<0.01$ \\
\hline
\end{tabular}

* p value comparing patients who received Irinotecan based chemotherapy vs. other chemotherapy. 
Conclusions: Stage IV disease at diagnosis, low-grade histology and Her2/Neu signals higher than 6 by FISH predict long term response to Trastuzumab based therapy in EAC. A validation of these findings in prospective and/or multi-institutional studies is warranted.

\section{Nuclear Translocation of $\beta$-Catenin After Neoadjuvant Chemoradiation Indicates Therapy Induced Activation of Wnt Pathway in Esophageal Adenocarcinoma}

Viren R Patel', Riham Katkhuda', Zhimin Tong1, Omkara Lakshmi M Veeranki Omkara', Alicia Mejia', Dipen Maru'. 'The University of Texas MD Anderson Cancer Center, Houston, TX

Background: Till to date no biomarker has been identified which can predict response to neoadjuvant therapy in locoregional Esophageal Adenocarcinoma (EAC). Aberrant nuclear translocation of $\beta$-Catenin is a marker of activation of Wnt pathway and is a major mechanism through which $\beta$-Catenin regulates cell processes critical to therapy resistance. However, status of $\beta$-catenin after neoadjuvant chemoradiation in EAC is unknown.

Design: We performed immunohistochemistry staining of $\beta$-catenin (Clone 14, BD Biosciences ${ }^{\mathrm{TM}}$ ) on matched pretreatment endoscopic biopsies and post neoadjuvant treatment resections specimens from 34 patients with EAC. $\mathrm{H}$ score $(\%$ of tumor cells with 0 intensity $\times 0+\%$ of tumor cells with intensity $1 \times 1+\%$ of tumor cells with intensity $2 \times 2+\%$ of tumor cells with intensity $3 \times 3$ ) was assessed for $\beta$-catenin staining of tumor cell membrane and nuclei in biopsy and resection samples. Hematoxylin \& Eosin stained slides from biopsies and resection specimens were reviewed for tumor histopathologic features, ypTNM stage and features indicative of epithelial mesenchymal transition like tumor budding or pleomorphic/spindle cells with dense cytoplasm and bizarre nuclei.

Results: Study population included 30 men and 4 women with average (range) age $62(42-83)$ years. Pretreatment biopsies from 33 patients were negative for nuclear staining of $b$-catenin $(H$-score $=0)$. One pretreatment biopsy showed nuclear staining $\beta$-catenin $(\mathrm{H}$ score $=150$ ). Nuclear staining for $B$-catenin with $3+$ intensity of at least $10 \%$ tumor cells was observed in resection specimens of 19 patients $(\mathrm{H}$-score median [range] $=170[30-300]$. Resection specimens from other 15 patients did not show any nuclear staining for 8 -catenin $(p<0.001)$. Type of neoadjuvant chemotherapy regimen, tumor stage, histologic grade on pretreatment biopsy, amount of residual tumor in resection specimens did not significantly differ in distribution between patients with and without nuclear $B$-catenin on resection specimens. However, nuclear labeling of $B$-catenin on resection specimens was significantly correlated with poorly differentiated histology $(p=0.032)$ and histologic features suggestive of EMT $(p=0.04)$ in resection specimens.

Conclusions: Nuclear translocation of $\beta$-catenin is observed in neoadjuvant treatment resistant residual EAC cells indicating activation of Wnt pathway. These findings support exploration of this marker in understanding biology of treatment response and assess its clinical relevance as a biomarker in locoregional EAC.

\section{Utility of a Limited Immunohistochemistry Pane for Predicting Site of Origin in Neuroendocrine Tumors: Focus on High-Grade Neoplasms}

Priyanka Patil', Qiang Liu', Nicole Panarelli. 'Montefiore Medical Center, Bronx, New York, Bronx, NY, ${ }^{2}$ Montefiore Medical Center, Princeton Junction, NJ, ${ }^{3}$ Montefiore Medical Center, Scarsdale, NY

Background: Neuroendocrine tumors (NET) frequently present as metastatic malignancies of unknown primary site. Immunohistochemistry (IHC) is increasingly employed to determine their sites of origin as targeted therapies become available. Data regarding the staining properties of high-grade neuroendocrine tumors are scarce. The aim of this study was to assess the utility of a limited IHC panel (TTF1, CDX2, Islet1, and PAX8) for determining the primary site of metastatic neuroendocrine tumors, particularly those of high histologic grade.

Design: Tissue blocks from primary and metastatic neuroendocrine tumors were retrieved from the pathology archives. Tumors were graded according to the most current WHO classifications. The origin of metastases was determined by review of medical records and imaging studies. Stains for TTF1, CDX2, Islet1, and polyclonal PAX8 were performed on formalin-fixed paraffin-embedded tissue sections, according to the manufacturers' instructions. Nuclear staining in at least $5 \%$ of tumor cells was considered a positive result.

Results: The study cases included 51 primary and 30 metastatic NETs from the lung and various digestive organs. Among primary welldifferentiated NETs, gastric tumors showed weak Islet1 expression $(n=12 / 13,92 \%)$, but were negative for other markers. Duodenal tumors only stained for Islet1 $(n=6,100 \%)$ and PAX8 $(n=4,80 \%)$. Small intestinal $(n=3)$, appendiceal $(n=2)$, and colonic tumors $(n=1)$ were uniformly positive for CDX2 only. Pancreatic $(n=5)$ and rectal $(\mathrm{n}=7)$ tumors only expressed Islet $1(100 \%$ and $86 \%$, respectively) and PAX8 $(50 \%$ and $29 \%$, respectively). Primary poorly-differentiated neuroendocrine carcinomas (PDNEC) from the lung $(n=8)$ were positive for TTF1 and Islet1 (both 63\%) and those from esophagus $(n=3)$ and stomach $(n=2)$ expressed Islet $1(100 \%$ and $50 \%$, respectively). The staining patterns of metastatic WDNETs were similar to primary tumors ( 13 of 16 cases, $81 \%$ ), regardless of grade, whereas metastatic PDNEC exhibited heterogeneous IHC profiles, but were frequently positive for Islet1 $(9 / 14,64 \%)$ (Table 1$)$.

\begin{tabular}{|c|c|c|c|c|c|c|}
\hline Site & Tumor Type & $\begin{array}{l}\text { Number } \\
\text { of cases }\end{array}$ & $\begin{array}{l}\text { TTF1 } \\
(n ; \%)\end{array}$ & $\begin{array}{l}\mathrm{CDX2} \\
(\mathrm{n} ; \%)\end{array}$ & $\begin{array}{l}\text { Islet1 } \\
(n ; \%)\end{array}$ & $\begin{array}{l}\text { PAX8 } \\
(n ; \%)\end{array}$ \\
\hline Stomach & WDNET G2 & 1 & 0 & 0 & 0 & 0 \\
\hline \multirow{3}{*}{ Ileum } & WDNET G1 & 3 & 0 & $3(100)$ & 0 & 0 \\
\hline & WDNET G2 & 3 & 0 & $3(100)$ & 0 & 0 \\
\hline & WDNET G3 & 2 & 0 & $\begin{array}{l}2 \\
(100 \%)\end{array}$ & 0 & 0 \\
\hline Colon & PDNEC G3 & 2 & 0 & $1(50)$ & $1(50)$ & 0 \\
\hline \multirow{4}{*}{ Pancreas } & WDNET G1 & 1 & 0 & 0 & $1(100)$ & $1(100)$ \\
\hline & WDNET G2 & 3 & 0 & 0 & $3(100)$ & $2(67)$ \\
\hline & WDNET G3 & 2 & $1(50)$ & 0 & 0 & 0 \\
\hline & PDNEC G3 & 2 & $1(50)$ & $1(50)$ & $1(50)$ & 0 \\
\hline \multirow{2}{*}{ Lung } & Atypical carcinoid & 1 & 0 & 0 & 0 & 0 \\
\hline & PDNEC G3 & 10 & $10(100)$ & 0 & $7(70)$ & $7(70)$ \\
\hline
\end{tabular}

Conclusions: A limited IHC panel helps to predict the origin of metastatic WDNET in the majority of cases. Co-expression of PAX8 favors pancreatic origin in Islet1-positive metastases. PDNEC do not show lineage-specific IHC expression; Islet1 was a marker of most types of PDNEC in biopsy samples of poorly-differentiated tumors, in our series.

\section{8 "Brunner Gland/Duct Cysts" with Dysplasia: Further Characterization of a Distinctive Lesion in the Duodenum of Probable Precursor Nature}

Burcin Pehlivanoglu', Yue Xue ${ }^{2}$,Michelle Reid ${ }^{2}$, Alyssa Krasinskas' Maria Westerhoff ${ }^{3}$ Juan M Sarmiento ${ }^{4}$, Jeanette D Cheng ${ }^{5}, N$. Volkan Adsay ${ }^{6}$. 'Emory University, Atlanta, GA, ${ }^{2} E$ mory University Hospital, Atlanta, GA, ${ }^{3}$ University of Michigan, Ann Arbor, MI, ${ }^{4}$ Emory University, ${ }^{5}$ Piedmont Atlanta Hospital, Atlanta, GA, ${ }^{6}$ Medical College of Wisconsin, Milwaukee, WI

Background: Recently, a distinctive lesion of Brunner glands/"ducts" with cystic dilatation and papillary mucinous changes, described as "IPMN-like", is being recognized but many of the clinicopathologic characteristics of this peculiar lesion remain to be disclosed.

Design: Twelve cases of Brunner gland/duct cyst were analyzed.

Results: Female: male ratio was 1.75 and median age, 65 (range 45-82). The mean size was $0.55 \mathrm{~cm}$ (range 0.2-0.9) with multiple lesions in 5 cases (42\%). Six cases were identified in resections with neighboring malignancy [ 4 pancreatic head adenocarcinoma, and 2 ampullary carcinoma]. 1 patient was diagnosed with pancreatic head adenocarcinoma in the follow-up. Five cases $(42 \%)$ showed only foveolar cell lineage, 6 displayed admixed intestinal characteristics (highlighted by CDX2/MUC2 as well) in addition, and 1 case $(8 \%)$ had multiple lesions with a spectrum of phenotypes. Two-thirds (8/12) had papilla formation, 7 with atypia that could be qualified as low-grade dysplasia (with features described in low-grade PanIN (previously PanlN-1A)/BillN-1), and 1 with high grade. Two cases without papilla formation also had flat (non-papillary) low-grade dysplasia. All cases with papillae showed S100P and MUC5AC positivity as well as loss of MUC6 in the papillae. Additionally, S100p was found to be a highly sensitive and specific highlighter of the dysplastic areas including the dysplastic foci in 2 cases without papilla formation; while it was not expressed in any of the non-neoplastic areas of any cases. Focal apical membranous MUC1 staining was seen in $83 \%$. None had p53 overexpression. SMAD4 was retained in all. 1 patient with numerous lesions were sent to genetic consultation by the authors based on the diagnosis, and was found to have germline deletion in all 9 exons of the STK11 gene, a highly uncommon abnormality of this gene.

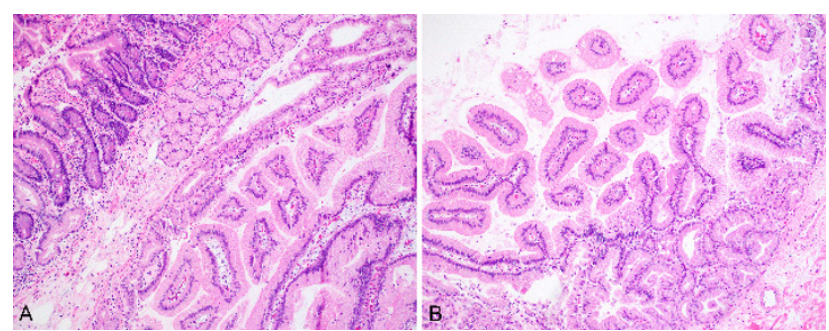




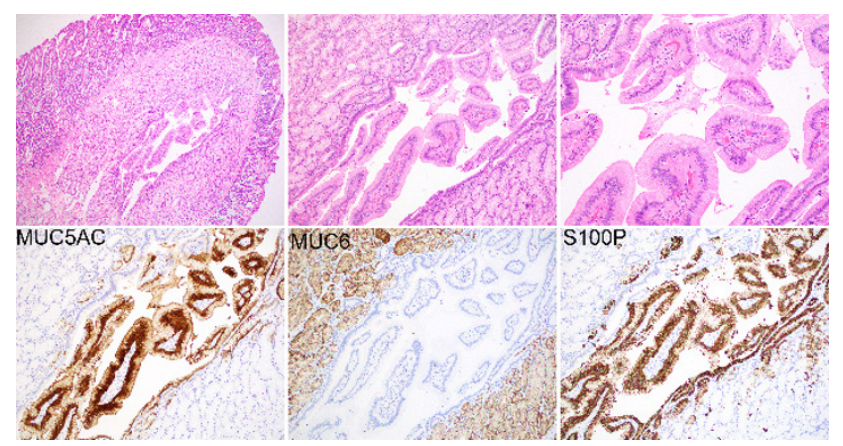

Conclusions: Brunner gland/duct cyst with papillary proliferation and atypia (dysplasia) is a distinctive lesion with possible precursor properties. The pattern of atypia and the acquisition of S100p (which has been shown to be consistently expressed in low-grade PanINs but not in reactive lesions), in areas with atypia in this lesion support its early neoplastic nature. The occurrence of HGD, albeit rare, also goes along with this. Some of the cases were identified in association with carcinomas in the PB tract; however, whether this is a co-incidence of case selection or a true association needs further investigation.

\section{Genomic profiles of mucinous versus non-mucinous colorectal adenocarcinoma}

Zheng Ping1, Virginia Duncan' ${ }^{2}$, Dejun Shen ${ }^{3}$, Rongjun Guo 4 . 'University of Alabama at Birmingham, Vestavia, AL, 'Seattle Children's Hospital, ${ }^{3}$ Kaiser Permanente, ${ }^{4}$ University of Alabama at Birmingham, Birmingham, AL

Background: Mucinous colorectal adenocarcinoma comprises about $10-15 \%$ of colorectal cancer and is characterized by abundant extracellular mucin production ( $>50 \%$ of tumor volume). However, molecular characterization of this histological subtype is not well established, and the relationship of molecular events to pathogenesis and clinical behavior remains to be elucidated.

Design: From The Cancer Genome Atlas (TCGA) database whole genome sequencing data were obtained in 217 cases of colorectal adenocarcinoma with complete mutation profiles and clinicopathological information. We excluded 35 cases with microsatellite instability (MSI) phenotype. Among the remaining cases, 19 cases $(10 \%)$ were mucinous and 163 cases $(90 \%)$ were nonmucinous adenocarcinomas. Individual genetic abnormalities were compared between these two subcategories. The data were analyzed via cBioPortal bioinformatic tools.

Results: There were no significant differences for demographic (patient age, gender) or pathologic (tumor depth, lymph node status, metastasis, and lymphovascular invasion) characteristics between mucinous vs. non-mucinous adenocarcinoma, suggesting minimal confounding bias for further genomic analysis. APC gene had the highest but similar mutation rates in these two subgroups $175 \%$ vs. $79 \%, p>0.05)$. Sixteen of the top 30 commonly mutated genes showed distinct frequencies between mucinous vs. non-mucinous adenocarcinomas. TP53 mutations occurred more frequently in nonmucinous adenocarcinomas (64\% vs. 37\%, p<0.05). The remaining 15 genes including BRAF and PIK3CA involved in RAS/RAF/MAPK and $\mathrm{PI}$ KK/AKT pathways showed significantly higher mutation rates in mucinous adenocarcinomas. Kaplan-Meier survival analysis indicated that mutation of $A P C$ was associated with better disease-free survival only in the non-mucinous subgroup, and mutation of SMAD4 associated with worse disease-free survival only in the mucinous subgroup (log-rank test, $\mathrm{p}<0.05$, Figure 1 and 2).
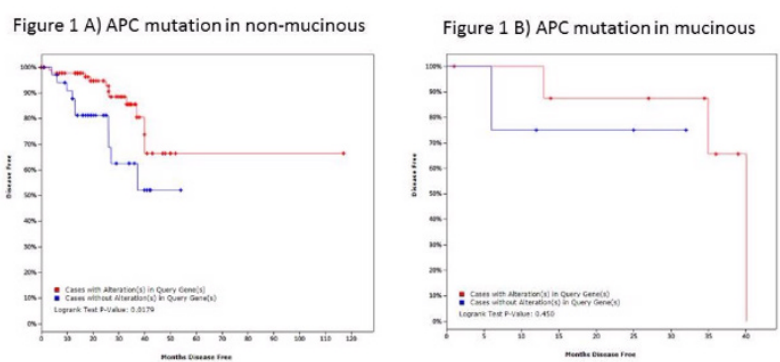

Figure 2B) SMAD4 mutation in mucinous
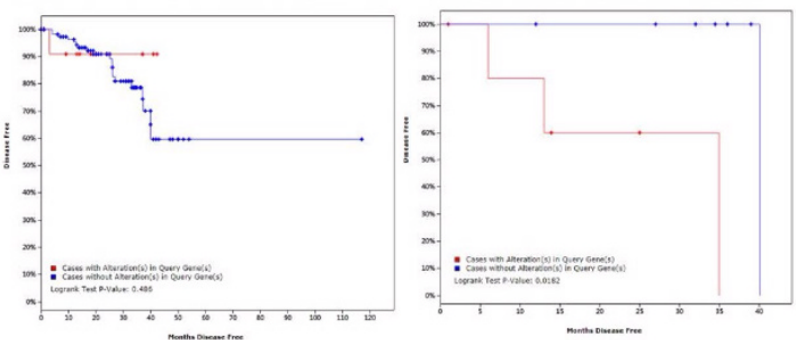

Conclusions: These data suggest that distinct molecular pathways may underlie the pathogenesis of mucinous versus non-mucinous colorectal adenocarcinomas, in which TP53 inactivation may play a critical role in non-mucinous carcinoma, while dysregulation of RAS/ RAF/MAPK and PI3K/AKT pathways may drive the pathogenesis of mucinous adenocarcinoma. Mutations of APC and SAMD4 appear associated with different disease-free survival rates in colorectal mucinous vs non-mucinous adenocarcinomas, and could thus be useful prognostic markers in clinical management.

\section{Therapy-Induced Epidermal Changes Can Mimic Recurrent Perianal Paget Disease}

Meredith Pittman', Rhonda Yantiss'. 'Weill Cornell Medical College, New York, NY

Background: The histologic differential diagnosis of primary perianal Paget disease includes secondary involvement by colorectal carcinoma, malignant melanoma, and intraepithelial squamous cell carcinoma, although none of these entities simulate its clinical features or is relevant when evaluating surveillance biopsies in patients with an established diagnosis. In fact, we have found that treatment of periana Paget disease by surgical excision and skin grafting combined with topical chemotherapeutic agents produces a variety of alterations in benign squamous cells that can simulate recurrent Paget disease. The purpose of this study is to describe epidermal changes that occur as a result of treatment for Paget disease, and highlight potential benign mimics of this entity.

Design: We reviewed surveillance biopsy samples from three patients with Paget disease, all of whom had been treated with wide excision skin grafting, and topical 5-fluorouracil. Samples were evaluated for the presence and distribution of atypical intraepidermal cells which were further characterized with immunostains directed against cytokeratin 7 and polyclonal CEA.

Results: We reviewed 372 samples from three women (mean age: 65 years) who underwent surveillance biopsy procedures every six months or less for four to six years. Biopsy samples from engrafted skin displayed single and clustered atypical cells with hyperchromatic nuclei dispersed among basal keratinocytes. These cells were surrounded by vacuoles and lacked intercellular bridges or othe features of squamous differentiation (Figure 1A, arrows). Topical chemotherapy elicited more striking epithelial cell changes. Cells with hyperchromatic, enlarged nuclei were scattered along the basement membrane. Large cells with eccentrically located, hyperchromatic nuclei and voluminous cytoplasm without intercellular bridges were present at all levels in the epidermis (Figure 1A, circles). Mitotic figures were readily identified and were not necessarily limited to the basa layers of the epithelium. Immunostains for CK7 and polyclonal CEA were negative in the atypical cells, although both markers showed limited staining of benign epithelial cells in some samples.

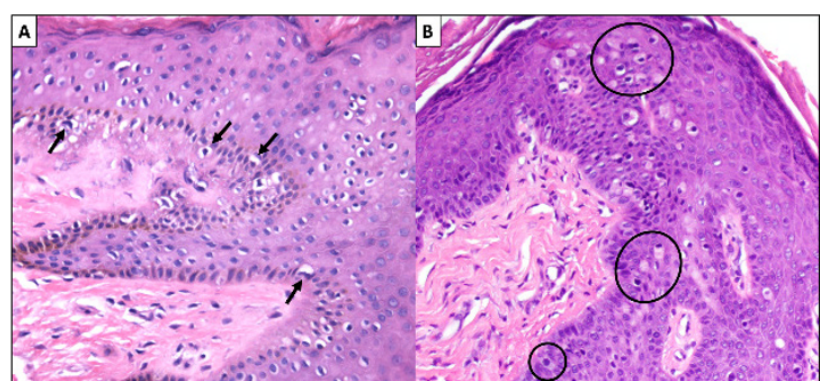

Conclusions: Topical chemotherapy in combination with skin grafting elicits degenerative changes in squamous cells that simulate the cytologic features of perianal Paget disease. Immunohistochemical stains for CK7 and/or polyclonal CEA can be helpful when evaluating surveillance samples from these patients. 
831 Barrett's Esophagus/Intestinal Metaplasia of the Gastroesophageal Junction in Children: A Clinicopathologic and Follow-Up Study

Juan Putra', Jeffrey D Goldsmith'. 'Boston Children's Hospital, Boston, MA

Background: Barrett's esophagus $(B E)$ is a rare condition in the pediatric population, and is most often seen in those with altered esophageal motility. A large prospective multicenter study reported that only $0.12 \%$ of children who presented for upper endoscopy had BE (Nguyen et al. Gastrointest Endosc 2011). Because of the paucity of literature on this subject, we aimed to evaluate the clinicopathologic characteristics of BE in children at our institution.

Design: Pediatric patients $(\leq 18$ years) with biopsy-proven intestinal metaplasia at the gastroesophageal junction were identified through pathology database search (2005-2017). The demographic information, clinical and endoscopic findings were evaluated. Followup endoscopic and biopsy results were documented when available. Data is presented as mean \pm standard deviation.

Results: Twenty patients (6 F) with an average age of $14 \pm 2.7$ years at presentation were evaluated in the study. Three obese $(15 \%)$, two overweight $(10 \%)$, and two underweight patients $(10 \%)$ were noted based on the age and sex adjusted body mass index at the time of presentation. Predisposing conditions for BE in these patients included cerebral palsy $(n=2)$, familial visceral myopathy $(n=2)$, systemic scleroderma $(n=1)$, esophageal atresia and tracheoesophageal fistula $(n=1)$, velocardiofacial syndrome $(n=1)$, cystic fibrosis $(n=1)$, Cornelia de Lange syndrome $(n=1)$, Gillespie syndrome $(n=1)$, and McCuneAlbright syndrome $(n=1)$. Nine patients did not have underlying disorders associated with esophageal dysmotility. Patients without predisposing conditions were significantly more likely to be overweight or obese compared to those with predisposing conditions ( $p=0.008$; Fisher's exact test). Endoscopic findings consistent with BE were noted in the majority of biopsy-proven BE cases $(83.3 \%$; mean length of BE segment $=3.0 \pm 1.2 \mathrm{~cm}$ ). Thirteen patients had follow-up biopsies (follow-up interval of $20 \pm 14.3$ months, total follow-up of 47 patient years). Persistent BE was identified in eleven patients (84.6\%) despite appropriate management. Dysplasia and malignancy were not seen in any of the initial or follow-up biopsies.

Conclusions: In our study, BE was predominantly seen in adolescent males and was associated with neurologic and motor disorders affecting esophageal motility. As in adults, increased body mass index seems to be associated with BE in those without predisposing conditions. Persistent disease was common, but evidence of neoplastic progression was not seen in this cohort.

\section{Adenocarcinoma Ex-Goblet Cell Carcinoid Is Not aNeuroendocrine Neoplasm: Immunoprofiling of 60 Cases Further Establishes Its Identity As a Distinct (Appendiceal Type) of Adenocarcinoma}

Michelle Reid ${ }^{1}$, Burcin Pehlivanoglu ${ }^{2}$, Yue Xue ${ }^{1}$, Bahar Memis ${ }^{3}$,Brian Robinson"2, M. Andrew Toussaint ${ }^{4}$, Alyssa Krasinskas², Charles Staley ${ }^{3}$, Bassel El-Rayes ${ }^{3}$.Harveshp Moga ${ }^{5}$, Douglas Evans ${ }^{5}$, N. Volkan Adsay ${ }^{5}$ ${ }^{1}$ Emory University Hospital, Atlanta, GA, ${ }^{2}$ Emory University, Atlanta GA, ${ }^{3}$ Emory University, ${ }^{4}$ Emory University, Decatur, GA, ${ }^{5}$ Medical College of Wisconsin, Milwaukee, WI

Background: "Adenocarcinoma ex-GCC" (XGC) still carries the name "carcinoid" and is currently regarded a form of "mixed adenoneuroendocrine carcinoma"(PMID:26030247). Thus, most pathologists expect substantial NE marker positivity, and oncologists expect them to secrete and behave like carcinoids.

Design: In this study, 17 cell lineage markers were investigated in 60 XGCs.

Results: I. No significant NE differentiation. Only a minority of cases showed any respectable NE marker expression. For chromogranin, $46 \%$ of cases were negative, and $31 \%$ showed only < $10 \%$ of the cells staining; diffuse positivity (> $75 \%$ of the cells) that is typical of carcinoids (WDNETs) was seen in 4\%. For synaptophysin, $36 \%$ were negative, $36 \%$ had $<10 \%$ of cells, and only $6 \%$ were diffuse. For CD56, these figures were $71 \%, 22 \%$ and $2 \%$, respectively. In-built controls stained appropriately. ATRX/DAXX, which is lost in many WDNETs was retained in $97 \%$ and $100 \%$. None of the cases showed loss of Retinoblastoma seen in > half of poorly-diff NECs. NKX6.1 (reported to be mostly negative in WDNETs of appendiceal origin though often positive in duodenal and rectal), was positive in $90 \%$. Il. High grade carcinoma. Ki67 index was high (median $44 \%)$. P53, which is seldom altered in WDNETs was over-expressed in $30 \%$. III. Evidence of adenocarcinoma lineage (with a profile distinct from other GI carcinomas). Although a histomorphologic differential was gastric carcinoma due to signet-ring like cells as well as poorly-cohesive-cell patterns and small tubular configuration, the cell lineage was more of lower-GI type (CDX2 - 98\%, MUC2 -76\%; CK20 -90\%; while upper GI markers were mostly negative: (MUC1 $11 \%$, MUC5AC 20\%, MUC6 2\%; CK716\%). CEA was expressed in $97 \%$.
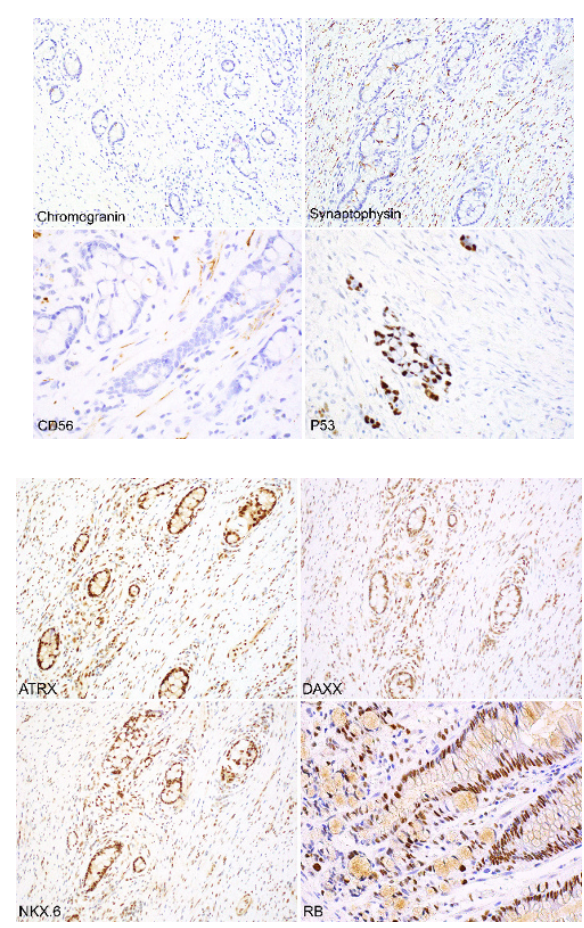

Conclusions: There is no evidence that "adenoca-ex-GCC" has substantial NE differentiation. In contrast, they show abundant expression of mucin-related oncoproteins, highlighting that they are adenocarcinomas, some driven by $\mathrm{p} 53$ and are high-grade by behavior and proliferation index. Although morphologic features show some overlap with upper-GI cancers, the immunoprofile is that of a lower-G adenocarcinoma, which, in combination with distinctive morphology (PMID: 27338636), warrants its classification as an appendiceal type adenocarcinoma with crypt cell lineage.

\section{Increased Paired Basic Amino Acid-Cleaving Enzyme 4 (PACE4) Expression Correlates with Better Survival in Primary and Metastatic Colorectal Cancer (CRC)}

Kevin Yi Mi Ren ${ }^{1}$, Alina Bocicariu ${ }^{2}$, Christine Orr ${ }^{3}$, Kevin Song ${ }^{3}$, Sarah $M$ Maritan $^{4}$,Yingwei Peng ${ }^{3}$, Chris $\mathrm{O}^{\prime}$ Callaghan ${ }^{3}$, Ravi Ramjeesingh ${ }^{5}$ Nazik Hammad ${ }^{3}$, Jim Biagi ${ }^{3}$, Christopher J Nicol' ${ }^{4}$ Lois M Mulligan ${ }^{4}$ Robert Day ${ }^{6}$, Harriet Feilotter ${ }^{7}$, David Hurlbut ${ }^{1}$, Scott Davey ${ }^{1}$. 'Queen's University, Kingston, ON, ${ }^{2}$ University of Toronto, University Health Network, Toronto, ON, ${ }^{3}$ Queen's University, ${ }^{4}$ Queen's University, Kingston, Ontario, ${ }^{5}$ Dalhousie University, 6 University of Sherbrooke, ${ }^{7}$ Richardson Laboratory

Background: Paired Basic Amino Acid-Cleaving Enzyme 4 (PACE4) is a member of the proprotein convertases family of endoproteases. Secretion of PACE4 is known for increasing matrix metalloproteinase 9 (MMP-9) activity and consequently plays a role in tumour invasion proliferation and progression. Recent studies in human prostate and breast cancer cell lines have shown the potential of PACE4 inhibition as a new therapeutic target. We present the first study investigating PACE4 expression in human primary colorectal carcinoma (CRC) and its correlation with clinicopathologic features.

Design: Cytoplasmic expression level of the consensus $(\mathrm{C})$ and alternate (A) versions of PACE4 was assessed by immunohistochemistry using tissue microarrays in 193 cases of primary CRC, including 60 cases with matched primary and metastatic tumors. Intensity and extent of PACE staining were independently assessed by 2 pathologists using a scale from 0 to 3 . PACE4 staining scores were calculated as a weighted average from 3 core samples per tumour. Univariate analysis of average scores and clinicopathological outcome measures from patient chart review was completed.

Results: High PACE4(C) immunostaining in both primary tumour and metastatic samples is associated with better 2-year survival ( $n=189$ $\mathrm{p}=0.02$ and $\mathrm{n}=57, \mathrm{p}=0.02$, respectively). In addition, high PACE4(C) immunostaining in primary $\mathrm{CRC}$ is associated with decreased perineural invasion $(n=185, p=0.02)$. Furthermore, high PACE4(C) to PACE4(A) ratio is associated with longer minimal survival time $(n=160$ $p=0.01$ ). PACE4 $(C)$ average immunostaining levels remained constant in matched normal epithelial cells $(a v=0.7)$ versus primary ( $a v=0.7$ $\mathrm{n}=157, \mathrm{p}=0.8$ ) and metastatic ( $a v=0.8, \mathrm{n}=45, \mathrm{p}=0.2$ ) tumour samples By contrast, the cytoplasmic immunostaining of PACE4(A) showed a significant overall decrease between non-neoplastic colorectal epithelium ( $a v=1.4)$ and primary tumor $(a v=1.0, n=157, p<0.01)$, and also between matched primary and metastatic ( $a v=0.7, n=57, p=0.04$ CRC. 
Conclusions: Our findings indicate that PACE4(C) levels in CRC may be associated with less aggressive tumor behaviour and better prognosis. Furthermore, our results suggest that levels of cytoplasmic PACE4(A) are significantly decreased in the transition from normal to primary to metastatic tumour; determining whether this is a result of decreased expression, or potentially increased secretion to the extracellular matrix will be an important next step in understanding the significance of this finding.

\section{Muscularis Mucosae Based Agrin Reactivity Distinguishes Hyperplastic Polyp from Sessile Serrated Adenoma/Polyp}

Steffen Rickelt ${ }^{1}$, Omer Yilmaz ${ }^{2}$, Richard O Hynes ${ }^{1}$, Vikram Deshpande ${ }^{3}$ 'David H. Koch Institute for Integrative Cancer Research, Cambridge, MA, ${ }^{2}$ Massachusetts General Hospital, ${ }^{3}$ Massachusetts General Hospital, Boston, MA

Background: Sessile serrated adenoma/polyps are precancerous colonic lesions, and their distinction from hyperplastic polyps can present a challenge in routine pathology. In a prior study using proteomics we were able to catalogue the ensembles of extracellular matrix (ECM) and ECM-associated molecules during colon cancer progression. We identified agrin, a basement membrane protein, in colonic carcinomas and associated liver metastases. To find new biomarkers for early detection of colorectal cancer we evaluated the expression pattern of agrin in hamartomatous, serrated polyps and tubular adenomas, and determined whether agrin immunostaining could discriminate between serrated polyps.

Design: Ninety-one colonic polyps were evaluated, including hyperplastic polyps (HP; $n=13$ ), sessile serrated adenoma/polyps (SSA/P) with and without dysplasia $(n=19)$, traditional serrated adenomas (TSA; $n=5)$, tubular adenomas $(T A ; n=36)$, juvenile polyps $(J P ; n=10)$ and Peutz-Jeghers polyps (PJP; $n=8)$. Immunohistochemistry for agrin was performed and the localization of agrin reactivity evaluated by two gastrointestinal pathologists and consensus was recorded in all cases.

Results: Agrin was not expressed in the normal colonic mucosa, however, basement membrane agrin localization was noted in all TA TSA, HP and SSA/P polyps. The expression in the latter two categories was higher in the basal half of the polyp, with a distinct decrescendo pattern. JP and PJP lacked basement membrane agrin reactivity. Additionally, all SSA/P and SSA/P with dysplasia showed diffuse agrin reactivity in the muscularis mucosae. Notably, muscularis mucosa localization of agrin was absent in all TA, HP, TSA, JP and PJP polyps.

Conclusions: Our study suggests that agrin immunostaining of the muscularis mucosae could be used as a novel marker to differentiate $\mathrm{SSA} / \mathrm{P}$ from HP, and assist with morphologically challenging cases.

\section{Mesenteric Tumor Deposits Arising from Small Intestinal Neuroendocrine Tumors are Frequently Associated with Sclerosis and IgG4-Expressing Plasma Cells}

Jordan Andrew Roberts 1 , Raul S Gonzalez², Frank Revetta ${ }^{3}$ Chanjuan $\mathrm{Shi}^{4}$. ${ }^{1}$ Nashville, TN, ${ }^{2}$ University of Rochester Medical Center, Rochester, NY, ${ }^{3}$ Vanderbilt University Medical Center, ${ }^{4}$ Vanderbilt University, Nashville, TN

Background: Mesenteric tumor deposits (MTDs) are frequently associated with small intestinal neuroendocrine tumors (SI-NETs) and radiographically simulate IgG4-related sclerosing mesenteritis. There is typically a prominent sclerosing fibrotic stromal response with associated foci of lymphoplasmacytic inflammation in these instances which, on core biopsy, can histologically mimic sclerosing mesenteritis when the tumor is not sampled. Our study investigates whether there are IgG4 positive plasma cells (IgG+ PCs) adjacent to MTDs that could lead to misdiagnosis on core biopsy.

Design: Fifty-two MTDs from 52 patients with SI-NET were evaluated by $H \& E$ and IgG4 immunohistochemical stains. The patient age, sex and clinical history were reviewed. IgG4+ PCs were quantified by finding the highest concentration of positive cells in one high-power field (HPF) in areas of sclerotic stroma adjacent to tumor. Cases were divided into 4 groups based on the number of $\mathrm{lgG}+\mathrm{PCs}$ per HPF (Group A: 0 per HPF, Group B: 1-20 per HPF, Group C: $21-40$ per HPF, Group D: $>40$ per HPF).

Results: Of the 52 patients, there were 29 males and 23 females with a mean age of 58 years (range: 19-83). Of the primary SI-NETs, 39 were WHO grade 1 and 13 were WHO grade 2. All 52 MTDs showed dense sclerotic stroma with associated foci of lymphoplasmacytic inflammation adjacent to tumor by H\&E evaluation. Sixteen MTDs $(30.8 \%)$ showed greater than $40 \mathrm{IgG}+\mathrm{PCs}$ (Group D) and $3(5.8 \%)$ showed 21-40 (Group C). Groups A and B had $18(34.6 \%)$ and 15 $(28.8 \%)$ cases, respectively. The majority of cases $(65.4 \%)$ showed at least some staining of IgG4+ PCs. Group C-D MTDs tended to be larger $(\mathrm{P}=0.06)$, and patients with Group A MTDs were the most likely to have multifocal, not unifocal, MTDs $(P=0.012)$. No obliterative thrombophlebitis was seen in any of the cases.

Conclusions: SI-NET-associated MTDs demonstrate dense sclerotic stroma and increased numbers of IgG4+ PCs. Our study demonstrates up to $36.5 \%$ (Groups C and D) of MTDs show 21 or more IgG4+ PCs per HPF. Therefore, the presence of IgG4-positive plasma cells should not be used as a sole diagnostic criterion for sclerosing mesenteritis, especially on core biopsy

\section{PD-L1 Expression Patterns in Histologic and Molecular Variants of Microsatellite Instability-High Intestinal Adenocarcinomas}

Jordan Andrew Roberts', Jordan Berlin', Chanjuan Shi ${ }^{3} .{ }^{1}$ Nashville, TN, ${ }^{2}$ Vanderbilt University Medical Center, Nashville, TN, ${ }^{2}$ Vanderbilt University, Nashville, TN

Background: The programmed death 1 (PD-1) pathway serves a crucial role in the host immune response to cancer. Microsatellite instability-high (MSI-H) colorectal cancers (CRCs) have been shown to highly express PD-L1. We aimed to explore PD-L1 expression patterns in different histologic and molecular (BRAF wild type versus mutant) variants of intestinal adenocarcinomas.

Design: Ninety-three MSI-H CRCs were identified from 93 patients Patient medical records, pathology reports, and pathology slides were reviewed. Histologic variants were recorded. BRAF mutation status was available in 68 tumors. Immunohistochemistry for PD-L1 was performed on all cases. PD-L1 positivity was defined as $\geq 1 \%$ cell surface expression of PD-L1 by tumor cells or intra- and peri-tumoral immune cells (lymphocytes and histiocytes).

Results: There were 49 females and 45 males, with a mean age of 65 years, ranging from 30 to 98 . There was no difference in tumo cell PD-L1 expression between adenocarcinoma, NOS (well to poorly differentiated) and mucinous/signet ring cell carcinomas; however, tumor cells in medullary carcinomas were more likely to express PD-L1 than adenocarcinoma, NOS and mucinous/signet ring cell carcinoma ( $p<0.001$, Table 1). PD-L1 was more likely expressed by immune cells in medullary carcinomas than those in mucinous/signe ring cell carcinoma $(p=0.03)$, but no difference was observed between medullary carcinomas and adenocarcinomas, NOS $(p=0.5)$. BRAF mutant tumors tended to express PD-L 1 in tumor cells more frequently than BRAF wild type tumors $(6 / 29$ versus $2 / 39, p=0.06)$. There was no difference in PD-L1 expression by immune cells between BRAF mutant and wild type tumors.

\begin{tabular}{|l|l|l|l|l|l|}
\hline & $\begin{array}{l}\text { Well/moderate } \\
\text { differentiated }\end{array}$ & $\begin{array}{l}\text { Poorly } \\
\text { differenti- } \\
\text { ated }\end{array}$ & $\begin{array}{l}\text { Mucin /sig- } \\
\text { net cell }\end{array}$ & $\begin{array}{l}\text { Medul- } \\
\text { lary }\end{array}$ & $\begin{array}{l}\text { To- } \\
\text { tal }\end{array}$ \\
\hline $\begin{array}{l}\text { Tumor cells + / } \\
\text { Immune cells + }\end{array}$ & 3 & 1 & 0 & 5 & 9 \\
\hline $\begin{array}{l}\text { Tumor cells + / } \\
\text { Immune cells - }\end{array}$ & 1 & 1 & 0 & 3 & 5 \\
\hline $\begin{array}{l}\text { Tumor cells- / } \\
\text { Immune cells+ }\end{array}$ & 24 & 3 & 2 & 1 & 30 \\
\hline $\begin{array}{l}\text { Tumor cells- / } \\
\text { Immune cells- }\end{array}$ & 31 & 3 & 14 & 1 & 49 \\
\hline Total & 59 & 8 & 16 & 10 & 93 \\
\hline
\end{tabular}

Conclusions: PD-L1 expression is different among histologic variants of MSI-H intestinal adenocarcinoma. Further studies correlating between morphology and efficacy of anti-PD-L1/PD-1 therapies may clarify the significance of different PD-L1 expression in these tumors.

\section{Helicobacter Pylori Detection: Correlation Between Tissue Biopsy and Non-Histologic Methods}

Scott Robertson ${ }^{1}$, Toby Cornish ${ }^{2}$. ${ }^{1}$ Cleveland Clinic, Cleveland, $\mathrm{OH}$ ${ }^{2}$ University of Colorado School of Medicine, Aurora, CO

Background: Several methods exist to detect Helicobacter pylor (HP) infection. Non-histologic tests have a range of sensitivity/ specificity values: IgG serology $(85 \%, 79 \%)$, stool antigen $(94 \%, 97 \%)$ tissue urease $(90 \%, 95 \%)$ and urea breath test $(88-95 \%, 95-100 \%)$. With histologic analysis, use of $\mathrm{IHC}$ is increasing, but the optimal use of $\mathrm{IHC}$ is not clear. In our practice, we do not stain histologically norma biopsies. Cases with gastritis are immunostained, provided organisms are not seen on H\&E. This study aims to assess the performance of non-histologic tests in our institution, explore the role of ancillary testing in inflamed HP negative biopsies, and to determine if our use of HP IHC is adequate to detect HP with high sensitivity.

Design: The results of all stomach biopsies $(n=38,751)$ and non histologic tests were collected $(n=52,646)$ from 2009-2016. 1162 patients received both tissue biopsy and a non-histologic HP test less than 1 month apart. From this group, sensitivity and specificity for each test were calculated using biopsy as gold standard. Biopsies called HP negative based on H\&E alone within 1 year of any positive 
non-histologic test were retrospectively were stained with IHC to detect any false negatives.

Results: HP status was determined on H\&E alone in $30 \%$ of cases $(18 \%$ HP positive); the remaining used IHC (11\% HP positive). Overall, $13 \%$ of cases were HP positive. Sensitivity and specificity for non-histologic tests were: IgG serology $(85 \%, 80 \%)$, stool antigen $(15 \%, 97 \%)$, tissue urease $(65 \%, 99 \%)$, urea breath test $(69 \%, 85 \%)$. For HP negative biopsies, there was no correlation between active inflammation and positive non-histologic test. Of the $84 \mathrm{HP}$ negative cases that were retrospectively immunostained, a single biopsy was found to be false negative based on H\&E; this biopsy showed very mild chronic inactive gastritis with small plasma cell collections.

Conclusions: Compared to published values, stool antigen testing in our institution showed extremely poor sensitivity. Tissue urease testing also showed suboptimal sensitivity, demonstrating the added value of histological analysis. There was no correlation between active inflammation in HP negative cases and positive non-histologic tests, arguing against the presence of an underlying HP infection in these cases. Finally, even with targeted retrospective $\mathrm{HP} \mathrm{IHC,} \mathrm{we}$ found no histologically normal stomach biopsies with HP organisms and, therefore, no evidence supporting the use of HP IHC in normal gastric biopsies.

\section{Clinical and Pathologic Characteristics of}

\section{Malakoplakia in the Gastrointestinal Tract}

Anthony Rubino ${ }^{1}$, Huaibin Mabel Ko ${ }^{2}$, Benjamin Lebwoh $\beta$, Stephen Lagana ${ }^{4}$. ${ }^{1} \mathrm{New}$ York Presbyterian Hospital/CUMC, New York, NY, ${ }^{2}$ Icahn School of Medicine at Mount Sinai, New York, NY, ${ }^{3}$ Celiac Disease Center at CUMC, ${ }^{4}$ Columbia University Medical Center, New York, NY

Background: Malakoplakia is a rare histiocyte mediated chronic inflammatory condition. It is most strongly associated with Rhodococcus equi infection, though other pathogens have also been implicated. The most common sites of involvement are in the genitourinary system, however there have been isolated case reports in the gastrointestinal tract (GIT) over the years. No systematic effort to describe malakoplakia in the GIT has been published to our knowledge.

Design: The case files of two large medical centers were searched for malakoplakia in the GIT. Clinical associations and pathologic presentations were recorded.

Results: 19 cases were identified. Cases ranged in age from 3.5 months to 92 years with $58 \%$ of the cases identified in female patients (11 of 19 patients). Lower GI involvement was much more frequent than upper (16 vs. 3). The most common indication for intervention was Diarrhea ( 3 of 7 cases for which data was available) and most common endoscopic finding was polypoid mucosa (9 of 18 cases for which data was available). Common clinical associations included cancer (7), solid organ transplant (3), and inflammatory bowel disease (3). See Table 1.

Table 1. Clinical and Pathologic Characteristics of Malakoplakia in GI Tract.

\begin{tabular}{|l|l|l|l|}
\hline Age & $\begin{array}{l}\text { Past Medical } \\
\text { History }\end{array}$ & Location & $\begin{array}{l}\text { Gross Find- } \\
\text { ings }\end{array}$ \\
\hline $\begin{array}{l}3.5 \text { mo--92 } \\
\text { years }\end{array}$ & Malignancy (7) & $\begin{array}{l}\text { Descending/Sig- } \\
\text { moid (7) }\end{array}$ & $\begin{array}{l}\text { Polyps/Nod- } \\
\text { ules (9) }\end{array}$ \\
\hline & Other (4) & Rectum (5) & Mass (5) \\
\hline & Transplant (3) & Right Colon (3) & Erythema (2) \\
\hline & IBD (3) & Stomach (3) & $\begin{array}{l}\text { Thickened } \\
\text { Wall (2) }\end{array}$ \\
\hline & HIV (1) & $\begin{array}{l}\text { Transverse Colon } \\
(1)\end{array}$ & Unknown (1) \\
\hline & No data (2) & Small Bowel (0) & \\
\hline
\end{tabular}

Conclusions: Malakoplakia effects the GIT tract rarely. It most commonly presents as polyps in patients who are immunocompromised either due to solid organ transplantation or cancer. Its recognition is of clinical importance as these patients must be treated with antibiotics.

\section{Significance of Increased Crypt Apoptotic Body Count in Colonic Allograft Biopsies}

Natalia Rush ${ }^{1}$, Chandrashekhar KubaR, Richard Mangus ${ }^{2}$, Romil Saxena ${ }^{3}$. Indianapolis, IN, ${ }^{2}$ Indiana University, ${ }^{3}$ Indiana Univ/Medicine, Indianapolis, IN

Background: Increased crypt apoptotic body count is one of the criteria used to diagnose acute cellular rejection (ACR) in colonic allografts. Increased crypt apoptotic body count alone does not define ACR but is a necessary feature. We perform clinicopathologic correlation of increased crypt apoptotic body count findings in biopsy specimens from colonic grafts utilizing findings of detailed histopathologic evaluation and colonoscopy data.

Design: We reviewed biopsy specimens obtained from patients that underwent intestinal transplantation procedure at our medical cente between 2013 and 2016. Cases with increased apoptotic body count ( 6 or more) in 10 consecutive crypts were further studied for association with colonoscopic and histopathologic findings.

Results: There were 235 colon allograft biopsy specimens available for review from 36 patients (17 male : 19 female). Signficantly increased crypt apoptotic body count was detected in 36 cases (15\%). Colonoscopy data was available for 34 out of 36 cases. Clinical indication for colonoscopy in these cases was either suspicion of graft malfunctioning $(n=24)$ or surveillance $(n=12$ cases). Nonsurveillance indications included diarrhea $(n=13)$, gastrointestinal bleeding $(n=4)$, increased ostomy output $(n=2)$, generalized abdominal pain $(n=2)$, imaging abnormality suggestive of allograft pneumatosis $(n=1)$. Out of 10 cases diagnosed with acute cellular rejection (28\%), 9 cases had colonoscopy data available for review, with 8 of which had indication other than surveillance $(89 \%)$ to include diarrhea, $\mathrm{n}=5$; increased ostomy output, $\mathrm{n}=2 ; \mathrm{Gl}$ bleed, $\mathrm{n}=1$. Colonoscopic findings were abnormal only in $56 \%$ of cases that demonstrated ACR. Correlation with other histopathlogic findings showed that the most frequently associated features with ACR were crypt loss (90\%), lamina propria inflammation (90\%), and crypt distortion (70\%)

Conclusions: 1. Less than a third of colonic allografts with increased crypt apoptotic body count demonstrated ACR (28\%). Most of the cases with ACR were associated with clinical indications for colonoscopy other than surveillance $(89 \%)$

2. Colonoscopic impression does not associate well with histopathologic findings of ACR since $44 \%$ of our cases with ACR showed no abnormalitites. Histopathologic evaluation remains an important diagnostic tool in managing patients with transplanted colons.

\section{Acute Cryptitis in Colonic Allografts and Infections: An Imperfect Alliance}

Natalia Rush ${ }^{1}$, Chandrashekhar Kubap, Richard Mangus², Romil Saxena ${ }^{3}$. Indianapolis, IN, ${ }^{2}$ Indiana University, ${ }^{3}$ Indiana Univ/Medicine, Indianapolis, IN

Background: Acute cryptitis in biopsies of colon allografts transplanted as part of isolated small intestinal (IST) or multiviscera (MVT) transplantation may be due to infection or other noninfectious inflammatory processes. This study utilizes colonoscopic and microbiologic findings to correlate presence of cryptitis with an infectious process.

Design: Patients who had received colon allografts were identified from the transplantation database. All colonic biopsies from these patients were reviewed to identify those that showed acute cryptitis. Colonocopic and microscopic findings concurrent to the biopsy were obtained from the medical records.

Results: There were 236 colonic biopsy specimens from 36 patients between 2013 and 2016, of which 26 (11\%) demonstrated acute cryptitis. Colonoscopic data was available for 25 biopsies; 12 biopsies were obtained during routine surveillance and 13 from symptomatic patients. The most frequent symptom was diarrhea Abnormal colonoscopic findings (congestion, erythema, ulceration pseudoemembranes) were present in 11 cases. Associated histopathologic findings included lamina propria inflammation (all cases), crypt loss (69\%), crypt distortion (58\%), ulceration (43\%), and $>6$ apoptosis in 10 crypts (35\%).

Microbiology studies were positive in 7 cases (27\%): C. difficile toxin was detected by PCR of stool samples in 4 cases; CMV DNA in blood in 1 case; E.coli in blood and urine in 1 case; and Stenotrophomonas maltophila in blood with Candida glabrata in urine in 1 case. Of these 7 cases, 4 were asymptomatic and 4 had no colonoscopic findings (fig 1).

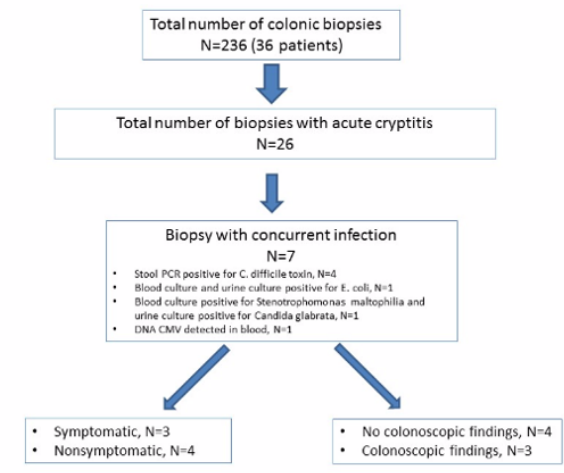




\section{Conclusions:}

1. Acute cryptitis was present in $11 \%$ of colonic allografts.

2. Almost half the patients with acute cryptitis were asymptomatic.

3. Approximately a quarter of the patients had a gastrointestinal or systemic infection. The most frequent culprit was C. difficile. More than half the patients were asymptomatic and more than half had no colonocpic findings, validating the utility of surveillance biopsies.

4. Although considered a cardinal feature for diagnosis of rejection, increased apoptoses are seen in gastrointestinal and systemic infections.

\section{Next Generation Sequencing Sheds Light on Lynch Syndrome Screening Using Immunohistochemistry vs. Microsatellite Instability by Polymerase Chain} Reaction

Taha Sachak', Rachel Pearlman², Heather Hampel2, Dan Jones ${ }^{2}$ Weiqiang Zhao', Debbie Knight², Wei Chen², Wendy L Franke? ${ }^{1}$ Columbus, $\mathrm{OH},{ }^{2}$ The Ohio State University Wexner Medical Center, Columbus, $\mathrm{OH}$

\section{Disclosures:}

Heather Hampel: Research Support, Myriad Genetic Laboratories; Advisory Board, InVitae Genetics

Background: The prevalence of Lynch Syndrome (LS) among colorectal carcinoma (CRC) patients is 3 to $4 \%$. Universal Tumor Screening for LS is feasible and recommended by many professional societies. Centers screen for mismatch repair (MMR) deficiency using immunohistochemistry (IHC) for MMR proteins or polymerase chain reaction (PCR) for microsatellite instability (MSI). Our goal was to compare IHC and MSI for the detection of LS as defined by a germline mutation found by next generation sequencing (NGS).

Design: 3312 adults with newly diagnosed CRC from 1/1/2013 to $12 / 31 / 2016$ were evaluated in our statewide initiative. Tumors were screened for MMR deficiency by MSI testing and IHC. The two-stain method was used (PMS2 and MSH6 with reflex to partner stain, MLH1 and $\mathrm{MSH} 2$, if absent), unless 4 stains were done clinically. If MSI-high or absent MLH1, MLH1 methylation was analyzed by pyrosequencing. Select patients underwent germline NGS testing of 25 to 66 cancer genes including those patients with defective MMR without methylation ( $n=224$; ColoSeq or BROCA, University of Washington) and those with proficient MMR or MLH1 methylated, if diagnosed $<50$ years, personal history of synchronous or metachronous CRC and/or endometrial cancer, or a first-degree relative with CRC or endometria cancer ( $n=1239$; MyRisk, Myriad Genetics Laboratories).

Results: A total of 1463 patients underwent germline NGS and 139 cases of LS were identified (9.5\%). Of the cases with LS, screening using $\mathrm{MSI}$ alone would have missed at least 8 (1 MSI-low; MSH6 mutation: 7 MSS; 2 MSH6, 5 PMS2 mutations) with an additional 18 cases having insufficient tissue for analysis. Therefore, up to 26 cases may have been missed. In contrast, using IHC alone, 10 cases would have been missed (6 PMS2 and 4 MSH6 mutations). In addition, 4 had equivocal staining ( $3 \mathrm{MSH} 6$ and $1 \mathrm{MSH} 2$ mutations). If both MSI and IHC were used for screening all, 5 cases ( 3 PMS2 and 2 MSH6 mutations) would have been missed; all were MSS by IHC and PCR (1 case had insufficient tissue for MSI).

Conclusions: MSI and IHC testing work well to identify most LS patients, and similar numbers of cases were diagnosed with each; however, MSI testing is more often limited due to insufficient tissue and does not suggest the most likely defective gene. Screening with both $\mathrm{MSI}$ and IHC is the most sensitive way to detect LS but is not as cost-effective and not recommended. Cases missed by both tests could be due to sporadic tumors in a LS patient, or limitations of screening methods.

\section{CDX2 protein expression in esophageal biopsies} without histological evidence of intestinal metaplasia may predict the presence of undetected Barrett's esophagus

James Saller ${ }^{1}$, Sameer Al Diffalha ${ }^{2}$, Kevin Neilß ${ }^{3}$ Cecilia Oliveri ${ }^{4}$, Hank Levine ${ }^{5}$, David Boulware ${ }^{6}$, Domenico Coppola ${ }^{7} .{ }^{1} \mathrm{H}$. Lee Moffitt Cancer Center \& Research Institute, Tampa, FL, ${ }^{2} \mathrm{UAB}$, Birmingham, $\mathrm{AL}$, ${ }^{3}$ Moffitt Cancer Center, Lutz, FL, ${ }^{4} \mathrm{CBL}$ Path, Orlando, FL, ${ }^{5}$ Center for Digestive Health, Orlando, FL, ${ }^{6}$ Moffitt Cancer Center, ${ }^{7} \mathrm{H}$. Lee Moffitt Cancer Ctr, Tampa, FL

Background: CDX2 is a nuclear homeobox transcription factor that is not expressed in normal esophageal and gastric epithelia, however CDX2 has been reported to highlight intestinal metaplasia of the esophagus. We sought to investigate whether the IHC positivity of CDX2 in the absence of goblet cells may have a predictive value in determining the presence of histologically undetected intestinal metaplasia in patients at risk for developing BE. In this study, we performed follow up on patients with GERD that had gastroesophageal junction biopsies with no histological evidence of goblet cells, as assessed by an Alcian blue (AB)/PAS staining as well as with IHC CDX2 positivity.

Design: This study was conducted under an IRB-approved protocol. A search of the surgical pathology electronic database (Pathnet) and the patients' electronic medical records (Powerchart) had revealed there were 171 patients between 2012-2016 who underwent upper endoscopy to rule out BE, as assessed by H\&E, ABPAS and CDX2 IHC The patients' age, gender, AB/PAS special stain and CDX2 IHC results were extracted and entered into a spreadsheet (Excel 2007, Microsoft).

Results: Out of 171 patients overall, there were 134 patients with initial CDX2 positivity and 47 patients with initial CDX2 negativity. Follow up of the 134 patients with CDX2 positivity had demonstrated that within this population, there were124 patients that subsequently developed intestinal metaplasia within 3 years $(79 \%)$. Follow up of the 47 patients with initial CDX2 negativity demonstrated that 11 patients had developed intestinal metaplasia within 3 years follow (23\%). The difference between these 2 cohorts is statistically significant (p-value $=0.06$ )

Conclusions: This study demonstrates the utility of CDX2 IHC for determining patients with a higher likelihood of harboring intestina metaplasia undetected by the ABPAS stain at their first biopsy. Ou findings show that patients with biopsies that are ABPAS negative and CDX2 positive at their first presentation may later present with frank goblet cell intestinal metaplasia.

\section{Gastric Carcinoma Post Pancreaticoduodenectom for Pancreatic Ductal Adenocarcinoma: A Case} Series

Rashmi T Samdani', DK Mizrak' ${ }^{1}, M$ Blum-Murphy' ${ }^{1}, B D$ Badgwell', MH Katz', Asif Rashid', Jeannelyn Estrella². 'The University of Texas MD Anderson Cancer Center, Houston, TX ${ }^{2}$ Houston, TX

Background: Survival in pancreatic ductal adenocarcinoma (PDAC) is low with a 2-year, 5-year and 10 -year survival of $20 \%, 7 \%$ and $1 \%$ respectively. Among PDAC survivors, gastric carcinoma (GC) arising at the anastomosis post pancreaticoduodenectomy (Whipple) is a possible complication. However, no reports describing this have been published. Our study is a retrospective analysis documenting clinicopathologic characteristics of patients with PDAC who underwent Whipple resection and subsequently developed GC. This information will help lay foundation for future management of these patients.

Design: A retrospective search in surgical pathology files and clinical and surgical databases was performed between 1994 to 2017 at MD Anderson Cancer Center to identify patients with PDAC who underwent Whipple resection and subsequently developed GC. Medical records and pathology material were reviewed and clinicopathologic characteristics were recorded.

Results: We identified 5 patients [mean age $=64$ years (range: 63 71), male:female ratio $=3: 2]$. One patient had BRCA2 mutation, had HNPCC (MSH6 mutation) and the remaining 3 were sporadic. Mean PDAC size was $2.3 \mathrm{~cm}$ (range: 1.5-3.5) with pathologic stage (AJCC 7th ed.) pT3N1 in 3 cases and pT3N0 in 2 cases. All developed diffuse type GC with focal $(<50 \%)$ signet ring cells (SRC) in 2 cases and $>50 \%$ SRC in 3 cases. One patient developed GC 23 months post Whipple (sporadic case) while the rest developed GC after 8 years, specifically at 107, 114, 176 and 190 months. Three underwent resection of gastrojejunal anastomosis with negative margins. Among resected GC, mean size was $1.7 \mathrm{~cm}$ (range: 0.5-2.8), with pathologic stage of pT1bN0 in 2 cases and pT1aNx in 1 case. Among the 2 patients who did not undergo resection, one was clinically cT3NxM1 with peritoneal disease (BRCA2 patient) while the other was cT1N0 (sporadic case). At last follow-up (mean = 38 months, range: $8-82$, time from Whipple to death or last follow-up), two died of GC, one died of sepsis (unknown etiology) and two were alive.

Conclusions: While rare, GC occurs in patients with PDAC status post Whipple resection. In our series, GC developed in both sporadic and genetically susceptible patients, as early as 23 months and as late as 190 months. All were diffuse type with at least focal SRC and the majority were low stage. In patients with PDAC who underwent Whipple resection, active and long-term surveillance with endoscopy is important in the early detection and treatment of GC.

\section{Therapeutic targeting of NOTCH and MAPK} signaling in colorectal cancer

Eva Marina Schmidt ${ }^{1}$, Sebastian Lamprecht ${ }^{2}$, Cristina Blaj ${ }^{3}$, Stefan Krebs ${ }^{1}$, Helmut Blum ${ }^{1}$, Christian Schaaf', Heiko Hermeking ${ }^{1}$, 'Andreas Jung ${ }^{1}$, Thomas Kirchner', David Horst ${ }^{2}$. 'Ludwig-MaximiliansUniversity, ${ }^{2}$ Ludwig-Maximilians-University, Munich, Bavaria, ${ }^{3}$ University of California

Background: Signaling pathways driving tumor progression are promising targets for systemic therapy in colorectal cancer. Besides WNT and MAPK signaling, upregulation of NOTCH signaling has been found in most tumors. Here, we examined the distribution, clinica significance and targetability of NOTCH signaling in colon cancer. 
Design: Phenotypes of tumor cells with differential pathway activity and clinical associations were characterized in tissue specimens of 225 stage II primary colorectal cancer cases. Effects of therapeutic targeting of MAPK and $\mathrm{NOTCH}$ signaling were evaluated in colon cancer xenografts. Changes in gene expression profiles were assessed by RNA-Sequencing of xenograft tumors. Phenotypic plasticity of tumor cell subpopulations was gauged by lineage-tracing experiments.

Results: In colon cancer, high NOTCH activity marked distinct tumor cell subpopulations with low levels of WNT and MAPK activity. MAPK and NOTCH activity indicated poor prognosis, especially upon combined evaluation. Therapeutic targeting of MAPK signaling had limited effects and caused expansion of tumor cells with high NOTCH activity. When targeting NOTCH signaling, tumor cells with high MAPK activity undergoing epithelial-mesenchymal transition (EMT) prevailed. Lineage tracing experiments revealed high plasticity between both tumor cell subpopulations. Combined targeting of MAPK and NOTCH had superior therapeutic effects on colon cancer growth in vivo.

Conclusions: Here, we propose a new concept for colon cancer therapy which advocates specific and simultaneous targeting of different tumor cell subpopulations with high MAPK and NOTCH activity to overcome treatment resistance by tumor cell plasticity.

845 Poorly Cohesive Gastric Carcinoma with Retained E-Cadherin Expression: A Less Aggressive Subgroup

Namrata Setia', Sangjeong Ahn ${ }^{2}$, Rebeca Rocha ${ }^{3}$, Do Youn Park', Gregory $Y$ Lauwers ${ }^{5}$. ${ }^{1}$ University of Chicago, Chicago, IL, ${ }^{2}$ Kangbuk Samsung Medical Cencer, Seoul, ${ }^{3}$ University of Chicago, ${ }^{4}$ Busan, ${ }^{5} \mathrm{H}$. Lee Moffitt Cancer Center and Research Institute

Background: The identification of high-risk biologic features of gastric cancer (GC) has become cardinally important for selection of modern chemotherapy. Tumor histology is a validated independent prognostic factor in GC with poor prognosis associated with poorly cohesive (PC, i.e., diffuse) GC. The study aims to explore the possibility of stratifying PC-GC into biologically relevant groups which may impact management.

Design: GC tissue microarrays from two institutions, annotated with clinical and pathologic information comprising of 84 PC-GCs formed the case cohort. All TMAs were scored for the retained or aberrant immunohistochemical expression of p53 and E-cadherin. Association between the expression of biomarkers and clinicopathological variables (age, gender, site, $\mathrm{T}$ and $\mathrm{N}$ stage, lymphovascular (LVI) and perineural (PNI) invasion) and overall survival was performed. According to WHO classification, PC histology was further subdivided into PC-SRC (signet ring cells) and PC-others (i.e., lymphocytic, eosinophilic, NOS).

Results: The clinicopathologic features are summarized in Figure 1. Of the 84 cases, $35.7 \%(30 / 84)$ retained $E$-cadherin expression and $41.7 \%(35 / 84)$ expressed normal p53. The expression of E-cadherin stratified the cases into clinically significant groups. In comparison to $\mathrm{PC}-\mathrm{GC}$ with loss of $\mathrm{E}$-cadherin, $\mathrm{PC}-\mathrm{GC}$ with retained $\mathrm{E}$-cadherin presented with a lower T stage (T1 vs. others, p 0.0009), lower N stage (N3 vs. others, $p$ 0.03) and lack of PC-others histotype (p 0.0009). Kaplan-Meier survival analysis for each group was performed as shown in Figure 1 (95\% Cl).

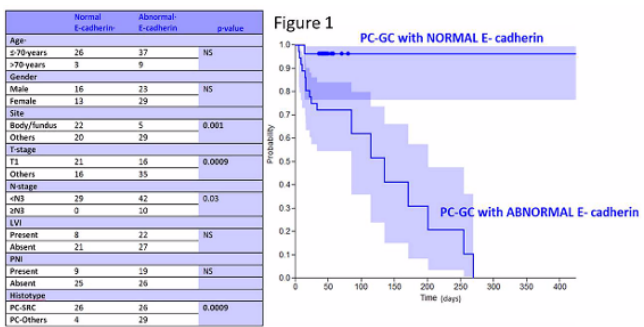

Conclusions: Retained E-cadherin expression in PC-GC identified a less aggressive subgroup of patients likely reflecting that downstream loss of E-cadherin is necessary for acquisition of aggressive biologic behavior in PC-GCs.

\section{Genomic Profiling of Colorectal Neuroendocrine Carcinoma (NEC) Reveals Multiple Mechanisms of RB1 Inactivation}

Eliah Shamir ${ }^{1}$, Walter $P$ Devine ${ }^{1}$, Kirk Jones ${ }^{1}$, Tara Saunders ${ }^{1}$, Sarah Umetsu', Soo-Jin Cho', Emily Bergsland", Grace Kim ${ }^{1}$, Sanjay Kakar', Nancy Joseph'. 'University of California, San Francisco, San Francisco, CA
Background: Colorectal NEC, both small cell (SC) and large cell (LC), are highly aggressive compared with colorectal adenocarcinoma (AdCa). NEC of various sites share immunomorphologic features. The molecular drivers are best defined in SC lung cancer, which show near universal genomic alteration of TP53 and RB1. Limited studies observed high-risk human papillomavirus (HR-HPV) infection in SC of cervix, oropharynx, and anorectum. Genetics of colorectal NEC are poorly understood, with recent work demonstrating frequent $A P C, K R A S, B R A F$, and TP53 mutation but infrequent RB1 mutation, suggesting that colorectal NEC more closely resemble colorecta AdCa. We aimed to determine whether colorectal NEC require $R B 1$ inactivation and if this is sometimes mediated by HR-HPV.

Design: Review of our archives identified 23 colorectal NEC (9 SC,14 LC; 8 right-sided, 15 left-sided with 12 rectal, 1 anal), al synaptophysin positive. Immunohistochemistry (IHC) and HR-HPV insitu hybridization (ISH) and PCR were performed on all cases and on 9 rectal neuroendocrine tumors (NET). Capture based next-generation sequencing (NGS) targeting 479 cancer genes was performed on 21/23 NEC.

Results: Right colon NEC (mean age 56) had an adenoma or AdCa component in $75 \%$. All NEC, but not glandular components, demonstrated $\mathrm{Rb}$ protein loss, accompanied by diffuse strong p16 in 7/8 (Fig 1A). HR-HPV ISH and PCR were negative in all. Genomic analysis (summarized in Fig 2) revealed frequent alterations in $R B 1$ (8/8), PI3K/Ras pathway genes (6/8), APC (5/8), and TP53 (3/8).

Left colon NEC (mean age 49) had an adenoma, AdCa, or squamous cell carcinoma component in 53\%. 5/13 NEC demonstrated Rb protein loss; 12/13 had diffuse strong p16 (Fig 1B). HR-HPV ISH and PCR were positive in 6/12 rectal (5 SC, 1 LC) and $1 / 1$ anal (SC). Of HPV+ cases, none had RB1 or TP53 alterations; $4 / 6$ had PI3K/Ras pathway alterations. Of HPV- cases, 5/7 had $R B 1$ alterations and the other 2 (both with ulcerative colitis) had $C C N E 1$ and $M Y C / M Y C N$ amplifications other frequent alterations were in TP53 (6/7), PI3K/Ras pathway genes (3/7), and $A P C(4 / 7)$.

Rectal NET (mean age 55) all had intact Rb, wild-type/negative p16, wild-type p53, and negative HR-HPV ISH and PCR.
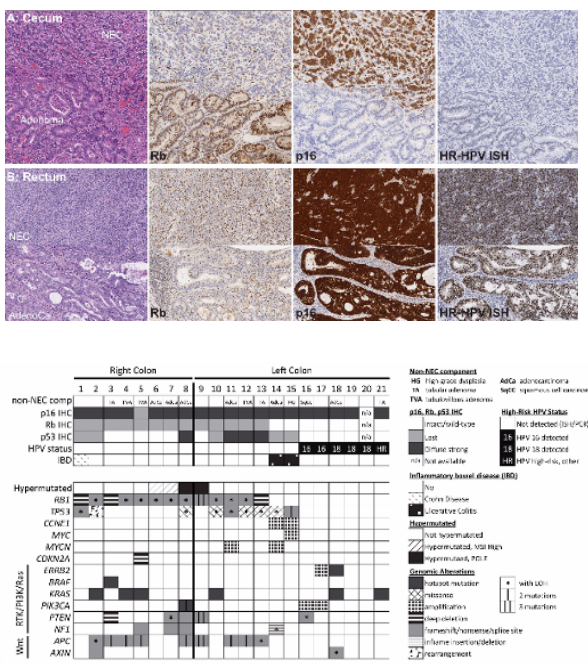

Conclusions: Like SC lung, Rb pathway inactivation is universa in colorectal NEC (21/21 sequenced cases). $R B 1$ genomic alteration was identified in all right colon NECs. In the left colon, Rb pathway disruption occurs by 2 additional mechanisms: CCNE1/MYC amplification in ulcerative colitis or HR-HPV infection.

\section{Lymphoproliferative Disorders in Patients with Crohn's Disease}

Yulei Shen', Scott Owens², Sarah M Choi ${ }^{3}$, Lauren Smith². ${ }^{1}$ University of Michigan, Ann Arbor, MI, ${ }^{2}$ University of Michigan, Ann Arbor, MI ${ }^{3}$ Department of Pathology, University of Michigan, Ann Arbor, MI

Background: Crohn's disease (CD) is a form of chronic idiopathic inflammatory bowel disease (IBD) estimated to affect up to 700,000 Americans. It is often treated with immunosuppression. IBD patients on thiopurines and antitumor necrosis factor (anti-TNF) agents are thought to be at increased risk for lymphoproliferative disorders (LPD).

Design: The database of the Department of Pathology was searched at the University of Michigan from June 2014-August 2017 in order to identify patients (both consultation and in-house) with a diagnosis of CD and lymphoproliferative disorder(s).

Results: Nine patients with CD were identified. The patients had a median age of 59 years (range 22-72 years) and included five men and four women. Sites of involvement included colon (2), small 
intestine (1), stomach (1), liver (2), lymph node (1), adenoid (1), and skin (2). The types of lymphoproliferative disorders included mycosis fungoides (MF) in two patients (one CD8 positive), peripheral T-cell lymphoma, NOS, diffuse large B-cell lymphoma (DLBCL) in two patients, EBV positive mucocutaneous ulcer (MCU), MALT lymphoma, hepatosplenic T-cell lymphoma (HSTCL), T-cell histiocyte-rich large B-cell lymphoma (THRLBCL), and chronic lymphocytic leukemia/ small lymphocytic lymphoma (CLL/SLL). One patient had two types of lymphoma (DLBCL and MF). Data on therapy was available for $6 / 9$ patients. Therapies included infliximab (3 patients), adalimumab (3 patients), methotrexate (1 patient), azathioprine (1 patient), and mesalamine (1 patient). EBER (in situ hybridization for Epstein-Barr virus) was performed in $6 / 9$ patients and two cases were positive. See table for summary of results.

\begin{tabular}{|c|c|c|c|c|c|}
\hline 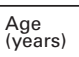 & Sex & Site & LPD Subtype & Medications & EBER \\
\hline 42 & M & $\begin{array}{l}\text { Rectosigmoid } \\
\text { colon }\end{array}$ & MALT & Unknown & Not done \\
\hline 72 & $\mathrm{~F}$ & Small intestine & $\mathrm{EBV}+\mathrm{MCU}$ & $\begin{array}{l}\text { Infliximab, } \\
\text { adalimumab }\end{array}$ & Positive \\
\hline 24 & $\mathrm{M}$ & Liver & THRLBCL & Infliximab & Negative \\
\hline 70 & $\mathrm{~F}$ & Skin & MF, CD8 positive & Unknown & Not done \\
\hline 45 & M & Liver & HSTCL & Unknown & Negative \\
\hline 22 & M & Adenoid & DLBCL, non-GCB & $\begin{array}{l}\text { Adalimumab, } \\
\text { methotrexate }\end{array}$ & Positive \\
\hline 59 & M & Stomach & PTCL, NOS & $\begin{array}{l}\text { Infliximab, } \\
\text { adalimumab, } \\
\text { azathioprine }\end{array}$ & Negative \\
\hline 68 & $\mathrm{~F}$ & $\begin{array}{l}\text { Right axillary } \\
\text { lymph node }\end{array}$ & CLL/SLL & No therapy & Not done \\
\hline 62 & $\mathrm{~F}$ & $\begin{array}{l}\text { Colon, } \\
\text { transverse and } \\
\text { sigmoid } \\
\text { Skin }\end{array}$ & $\begin{array}{l}\text { DLBCL, non-GCB } \\
\text { MF }\end{array}$ & Mesalamine & $\begin{array}{l}\text { Negative in } \\
\text { DLBCL } \\
\text { Not done } \\
\text { in MF }\end{array}$ \\
\hline
\end{tabular}

Conclusions: A variety of lymphoproliferative disorders can be seen in patients with $C D$, as demonstrated in this small series. Both T-cell and B-cell lymphoproliferative disorders were present. Only two patients $(2 / 6)$ were positive for EBER. Extranodal sites were frequent, with the gastrointestinal tract being involved in $6 / 9$ patients. A subset of patients was on immunosuppressive therapy, with some having a history of use of more than one agent. This small pilot study suggests that patients with $C D$ can have a variety of lymphoproliferative disorders and presentation in the gastrointestinal tract is common.

\section{Genomic Characterization and Tumor Mutational Burden in Esophageal Adenocarcinoma with a 467- gene Targeted Next-Generation Sequencing Panel}

Maryam Shirazi', Helen Fernandes², Susan Hsiao ${ }^{3}$, Armando Del Portillo ${ }^{1}$, Anthony Sireci ${ }^{2}$, Mahesh Mansukhani ${ }^{2}$, Jorge L Sepulveda ${ }^{2}$, Joshua $R$ Sonett ${ }^{4}$, Antonia Sepulveda'. ${ }^{1}$ New York, NY, ${ }^{2}$ Columbia University Medical Center, New York, NY ${ }^{3}$ Flushing, NY, ${ }^{4}$ Department of Thoracic Surgery

Background: Esophageal adenocarcinomas (EAC) and gastroesophageal junction adenocarcinomas (GEJAC) have dismal survival rates and limited precision therapy options are available. There has been an ongoing effort for comprehensive molecular analysis of these tumors to find new therapy targets. We describe our institution experience in applying a targeted Next Generation Sequencing (NGS) panel to 33 patients with EAC or GEJAC. We also assessed Tumor Mutational Burden (TMB) is these patients since immunotherapy blockade (ITB) may be effective in the treatment of some tumors with a higher TMB.

Design: 33 patients with EAC or GEJAC had targeted NGS panel testing. The median age was 63 , and there were 27 men. DNA was isolated from tumor paraffin-embedded sections and sequencing was performed using Agilent Sureselect capture and the Illumina Hiseq2500 NGS platform to evaluate for single nucleotide variants (SNVs) and small insertions and deletions. Copy number alterations (CNA) were analyzed in a subset of 12 patients. TMB was calculated as the number of somatic, SNVs and indels per megabase (Mb) of genome interrogated.

Results: The most frequently mutated genes were TP53 (57.6\%), CDKN2A (15.2\%), APC (12.1\%), and ARID1A (9.1\%) consistent with reported genomic findings in EAC (Fig 1). CNAs were detected in 11 of the 12 patients interrogated (Fig 2). The most commonly amplified gene was KRAS followed by CDK6, ERBB2, and PIK3CA. ERBB2 amplification was identified by the panel in one patient with $3+$ HER2 score by immunostain and amplification by in-situ hybridization. Gene losses were detected in CDKN2A and PMAIP1. The 25 $5^{\text {th }}$, median, and $75^{\text {th }}$ percentile thresholds for TMB were $3.8,6.4$, and 8.4 per $\mathrm{Mb}$, respectively. 5 patients had $\mathrm{TMB}<25^{\text {th }}$ percentile $(15.2 \%), 15(45.5 \%)$ were between $25^{\text {th }}-50^{\text {th }}, 4(12.1 \%)$ were between $50^{\text {th }}-75^{\text {th }}$, and $9(27.3 \%)$ had $>75^{\text {th }}$ percentile. 8 of 9 cases with $\mathrm{TMB}>75^{\text {th }}$ percentile had at least one frameshift, loss of function, and/or nonsense mutation, significantly higher than tumors with lower TMB. The highest TMB (23) was detected in a tumor with $\mathrm{MLH1}$ loss and microsatellite instability-high level (MSI-H).

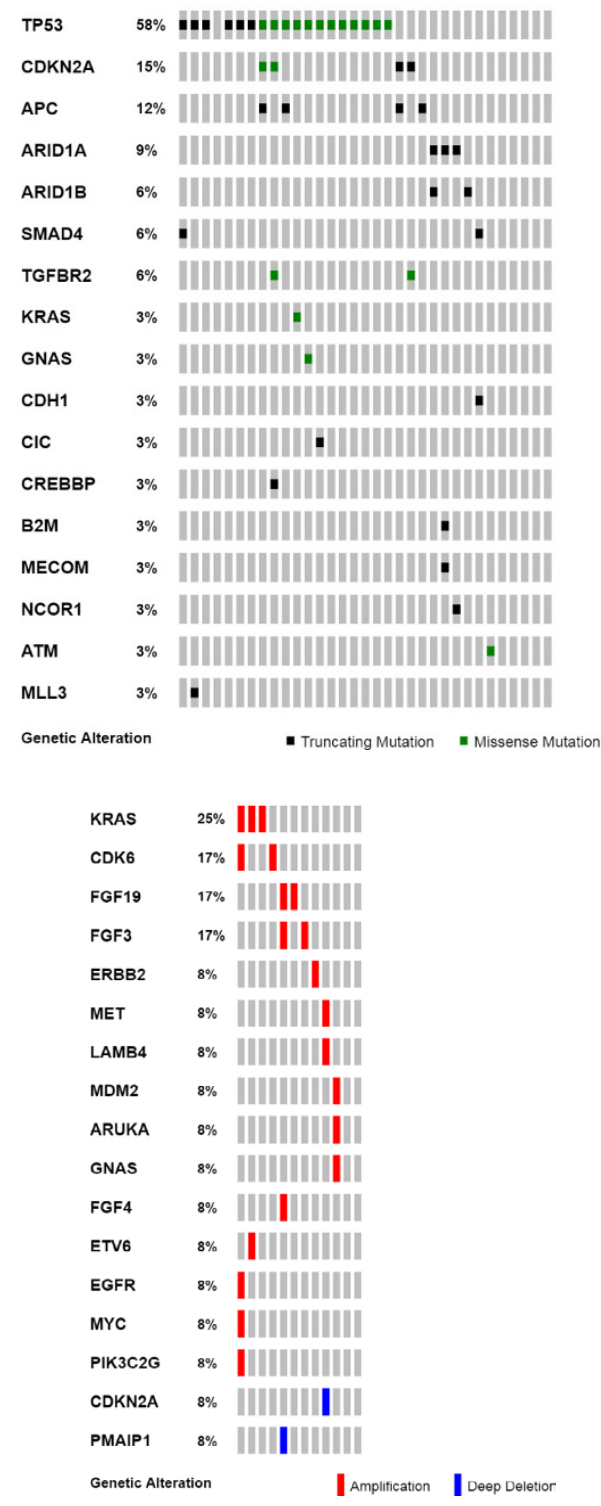

Conclusions: The mutation profile identified by our NGS gene panel is in concordance with recently reported data in EAC. Gene amplification and higher TMB were associated with poorly-differentiated tumors with higher clinical stages. These data may become useful, as nove combination therapies for EAC are uncovered. The role of TMB for patient selection for ITB warrants further studies.

\section{Helicobacter Pylori Negative Mucosa-Associated Lymphoid Tissues (MALT Iymphoma) of the Stomach: A Clinicopathologic Analysis}

Alexa Siddon', Scott Huntington", Dhanpat Jain ${ }^{3}$. 'VA Connecticut West Haven, CT, ${ }^{2}$ Yale University School of Medicine, ${ }^{3}$ Yale Univ./ Medicine, New Haven, CT

Background: Extranodal marginal zone lymphoma of mucosaassociated lymphoid tissue (MALT lymphoma) is known to be associated with Helicobacter pylori (HP) infections in $>80 \%$ of patients. However, in our experience the proportion of HP-negative MALT Iymphoma has been steadily increasing over the years. The clinicopathologic features of these lymphomas have not been well studied and the optimal management remains unclear.

Design: The pathology database was searched for primary gastric MALT Iymphomas diagnosed from 2000 to 2017. Cases with any high grade lymphoma were excluded. The clinical data and the pathology slides were reviewed, especially with regards to additional methods of HP testing, chronic gastritis, and treatment. The cases were grouped into those with a background of chronic gastritis with HP, 
chronic gastritis without HP, and those without either a background of chronic gastritis or HP.

Results: Seventy-two patients (24 males and 48 females) with primary gastric MALT Iymphomas were identified, of which 26 (36\% of total) had a background of chronic gastritis and were positive for HP histologically $(n=23)$ or by additional testing $(n=3)$. Of the 46 HP negative cases 29 had available slides which on review revealed no background gastritis in 18 cases $(62 \%)$, only mild gastritis in $4(14 \%)$ and moderate HP-like gastritis in $1(3 \%)$. In the remaining 6 cases the uninvolved background mucosa was never sampled. Of the total cases, 47 cases including 29 HP negative cases had B cell gene studies performed, with the majority ( $83 \%$ of the tested cases) being positive for a clonal population. The treatment modalities in the HP-negative group varied from antibiotic therapy, to radiation or chemotherapy, and 13 patients $(28 \%)$ showed disease recurrence or progression, but none died of the disease to date. The $t(11 \cdot 18)$ was evaluated in 5 patients of which 2 included HP negative patients of which 1 was positive.

Conclusions: The proportion of HP-negative MALT Iymphoma is increasing and in our practice is more common than HP associated MALT lymphomas. While those with a background of chronic gastritis may still be HP associated, the pathophysiology of HP negative MALT Iymphoma without chronic gastritis remains unclear. Follow up data in our study suggests that the prognosis of these cases is excellent irrespective of management modality.

\section{Surface Lewis Glycans: Potential Biomarkers to Distinguish Inflammatory Bowel Disease-Associated Neoplasia from Sporadic Colorectal Neoplasia}

Jordan Sim 1 , Goo Lee ${ }^{2}$, Sergey Pyatibrat ${ }^{3}$, Alton B Farris ${ }^{4}$, Charles Parkos ${ }^{5}$, Jennifer Brazil'. 'University of Ottawa, Ottawa, ON, ${ }^{2}$ The Ottawa Hospital, Ottawa, ON, ${ }^{3}$ The Ottawa Hospital, Ottawa, ON, ${ }^{4}$ Emory University, Atlanta, GA, ${ }^{5}$ University of Michigan Medical School, Ann Arbor, MI, ${ }^{6}$ University of Michigan

Background: IBD-associated neoplasia (IBDN) is known to have different carcinogensis from that of sporadic colorectal neoplasia (SN) in some ways. However, the search for effective biomarkers to distinguish IBDN from SN has to date been challenging. Aberrant transepithelial migration of neutrophils (TEM) as well as changes in surface glycosylation is associated with pathogenesis of IBD. Recent data have shown that epithelial and neutrophil glycans, in particular, Lewis $X\left(L^{x}\right)$ plays a role in TEM. In addition, Sialyl Lewis A (sLe ${ }^{a}$ ), is expressed in IBD and its blockade in murine models interferes with TEM, suggestive of new therapeutic target. The aim of this study is to evaluate surface Lewis glycans as biomarkers to distinguish IBDN from SN both in patients with and without IBD.

Design: 55 biopsies and resections from 54 patients were included in the study [Normal $(n=6)$, active IBD $(n=5)$, inactive IBD $(n=8)$, IBDdysplasia $(n=6)$, IBD-carcinoma $(n=6)$, sporadic adenoma (SA) $(n=5)$, SA-high grade dysplasia $(n=5)$, Sporadic carcinoma $(n=5)$, SA in IBD $(n=7)$, and dysplasia, indistinguishable (IBDN vs SA) in IBD $(n=2)]$. All tissues were immunostained with anti- $\operatorname{Le}^{x}(\lg G \& \lg M), \mathrm{sLe}^{\mathrm{a}}$ and blood group Lewis A (BGA). All immunostains were assessed by epithelial (membranous/cytoplasmic) staining as positive or negative $(<10 \%$ of lesion staining). Statistical analysis was calculated using chi squaretest.

Results: There is significant difference of $L^{x}$ and $s L e^{a}$ expressions between IBDN (dysplasia+ carcinoma) and SN (adenoma+carcinoma) $(p<0.01)$. Le $e^{x} \lg M$ and $\lg G$ antibodies stained significantly more cases of SN than IBDN (Le ${ }^{x} \lg M: 95.6 \%$ vs $20 \%$; IgG: $87.0 \%$ vs $6.7 \%$ respectively). In contrast, sLe ${ }^{a}$ stained significantly more cases of IBDN than SN (86.7\% vs $13.0 \%)$. BGA was not significant different between $\mathrm{SN}$ and IBDN ( $80 \%$ vs $60.9 \%, \mathrm{p}=0.108)$. In addition, there was no significant difference in the staining of SAs without or with IBD ( $>0.05)$ for Le $\operatorname{lgM}(93.3 \%$ vs $100 \%)$, Le $\mathrm{I}^{\mathrm{IgG}}(86.7 \%$ vs $87.5 \%)$, sLe $(13.3 \%$ vs $12.5 \%)$ or BGA $(46.7 \%$ vs $87.5 \%)$. The staining patterns are illustrated in Figure 1.

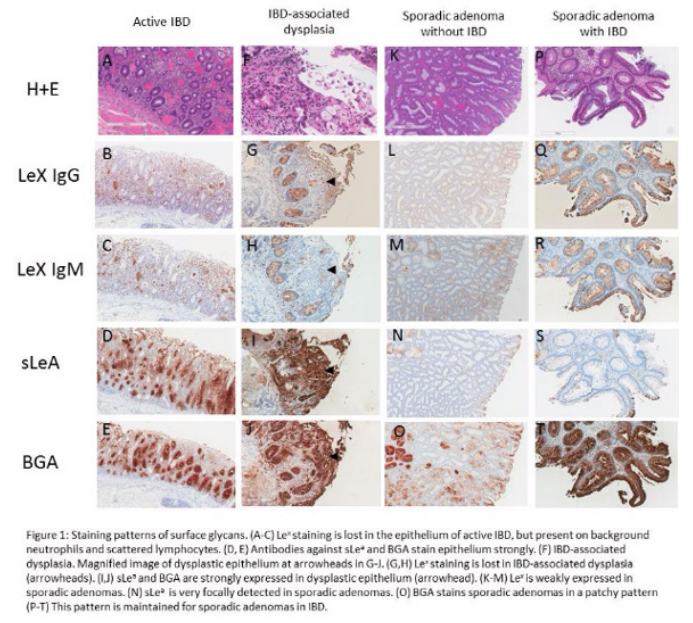

Conclusions: This study demonstrates that there is a striking difference in the expressions of surface Lewis glycans between IBDdysplasia and SA in IBD. SA in patients with or without IBD have similar staining patterns. This suggests that these glycans may serve as biomarkers to distinguish IBD-associated dysplasia from sporadic adenomas.

\section{Detection of TP53 and Other Somatic Mutations in Barrett's Esophagus that Later Progresses to High Grade Dysplasia or Adenocarcinoma}

Matthew Stachler ${ }^{1}$, Nicholas D Camarda $^{2}$, Christopher Deitrick ${ }^{3}$ Agoston (Tony) Agoston ${ }^{4}$, Robert Odze ${ }^{4}$, Jason L Hornick ${ }^{4}$, Anwesha $\mathrm{Nag}^{5}$, Aaron R Thorner 6 , Matthew D Ducar 4 , Scott L Carter ${ }^{8}$, Adam J Bass $^{5}$, Jon Davison ${ }^{3}$. 'Brigham and Women's Hospital, East Boston MA, ${ }^{2}$ Dana-Farber Cancer Institute, Boston, Massachusetts, ${ }^{3}$ University of Pittsburgh, Pittsburgh, PA, ${ }^{4}$ Brigham and Women's Hospital, Boston, MA, ${ }^{5}$ Dana Farber Cancer Institute, ${ }^{6}$ Dana Farber Cancer Institute, Boston, MA, ${ }^{8}$ Eli and Edythe L. Broad Institute

Background: Although Barrett's esophagus (BE) is the major risk factor for esophageal adenocarcinoma, only a small fraction of patients with BE ultimately progress to cancer. The aim of this study was to assess genomic differences in surveillance biopsies from patients who subsequently progressed to high grade dysplasia (HGD) or adenocarcinoma (EAC) compared to surveillance biopsies from those who had stable, non-progressive disease.

Design: We retrospectively identified 24 patients who progressed to HGD $(n=14)$ or EAC $(n=10)$ more than 1 year after their index BE diagnosis; we also selected a group of 73 controls with at least 5 years of total endoscopic biopsy surveillance without progression to HGD or EAC matched to cases on history of LGD/IFD. From each patient we selected a tissue sample obtained $>1$ year before first HGD/EAC diagnosis (cases) or $>2$ years prior to end of follow up (controls) that was representative of the highest grade of (pre-progression) dysplasia at that time point. The tissue underwent blinded review by 3 pathologists and sequencing using a custom 243 gene panel. Pathogenic mutations, copy number analysis, and ploidy were compared between progressors and non-progressors.

Results: On pathologic review, $88 \%$ of progressor samples and $90 \%$ of matched control samples were downgraded or confirmed as non-dysplastic BE. TP53 mutations and other pathogenic somatic alterations were recurrently present in BE tissue samples obtained 1.4-9.0 years before a diagnosis of HGD or EAC (Figure 1a). Most significantly, TP53 mutations were detected in $46 \%$ of samples from progressors, compared with $5 \%$ of controls (Figure $1 \mathrm{~b}$ ), representing a 13.8 -fold $(95 \% \mathrm{Cl}, 3.2-61)$ risk of progression (adjusted for age, sex and BE segment length). Eleven of 12 (92\%) of TP53 mutations in progressors were truncating or previously reported in EAC compared to only 1 of 4 TP53 mutations detected in non-progressors (Figure 1c). Levels of aneuploidy were low in these predominantly non-dysplastic, pre-progression BE samples, even in samples with TP53 and other somatic mutations.

Conclusions: Our findings challenge the conventional belief that TP53 mutations occur at the time of HGD or EAC and underscore the potential of genomic profiling as an early indicator of progression risk in patients with $B E$, even in the absence of dysplasia.

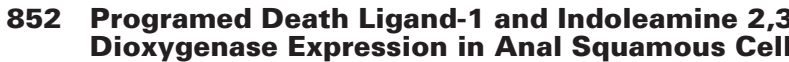
Carcinoma

Edward Stelow', Jennifer $\mathrm{Ju}^{2}$, Anne Mills ${ }^{3}$. 'Univ. of Virginia Health System, Charlottesville, VA, ${ }^{2}$ University of Virginia, Charlottesville, VA ${ }^{3}$ Charlottesville, VA 
Background: Approximately $68-88 \%$ of anal squamous cell carcinomas (ASCC) are associated with high-risk human papillomavirus (HR-HPV). The presence of the virus creates a strong immune environment in the tumor. Many cancers evade host immune response through the upregulation of programmed death ligand-1 (PD-L1), which interacts with programmed death-1 (PD-1) receptors on T-cells, inactivating the T-cell. PD-L1 has been shown to be expressed in high rates in ASCC. However, only a subset of patients with PD-L1 positive tumors on immunohistochemistry (IHC) experience clinical benefit to monoclonal antibodies blocking the PD-1/PD-L1 pathway. We hypothesize that the resistant subset expresses an additional T-cell suppressor to continue to evade immunotherapy. Our study compares the expression of PD-L1 and indoleamine 2,3-dioxygenase (IDO), a T-cell suppressor through tryptophan depletion, in ASCC.

Design: 49 cases of ASCC were used to create a tumor microarray. HPV status was determined with in situ hybridization (ISH) (RNAscope technique by Advanced Cell Diagnostics) and p16 IHC (CINtec by Ventana). The cases were then stained for PD-L1 (Spring Biosciences) and IDO (Sigma Prestige) by IHC. The tumor cells (TC) and inflammatory cells (IC) were scored for PD-L1 and IDO staining semi-quantitatively.

Results: 39 cases of ASCC (80\%) were positive for HR-HPV. Using a cut-off of $>1 \%$ staining, 32 cases showed PD-L1 TC immunoreactivity, of which 15 showed concomitant IDO positivity. 33 cases had IC that were positive for PD-L1, of which 19 cases co-expressed IDO. Only rare cases showed IDO expression only within the TC and / or IC. TC staining remained robust at a $>10 \%$ threshold $(2$ positive for IDO only, 15 positive for PD-L1 only, and 9 positive for both PD-L1 and IDO). All 5 cases not related to HPV showed TC PD-L1 staining, only one of which showed concomitant IDO staining. 2 of 5 cases related to LR-HPV showed TC PD-L1 staining only. $60 \%$ of cases not related to HR-HPV showed no IC staining for either PD-L1 or IDO.

Conclusions: At cut-offs of $>1-10 \%$, IDO expression is seen in 38$47 \%$ of PD-L1 positive ASCC. This suggests that anti-IDO therapy may be beneficial in a subset of PD-L1 positive ASCC as an addition to anti-PD-1/PD-L1 therapy. The results seem to be independent of HPV status. Given the infrequency of these cases, however, our results are not conclusive, and further studies to increase the power may be warranted.

\section{Expression of PD-L1 in Anal Squamous Cell Carcinoma: Association with HIV Status and Histologic Type}

Diana Sung 1 , Theresa Scognamiglio ${ }^{2}$, Yao-Tseng Chen 1 , Edyta Pirog ${ }^{3}$ 'Weill Cornell Medical College, New York, NY, ${ }^{2}$ New York Presbyterian Hosp, New York, NY, ${ }^{3}$ Cornell Univ. Medical College, New York, NY

Background: PD-L1 has been shown in previous studies to frequently be expressed in squamous cell carcinomas of various sites. Two distinctive expression patterns, induced and constitutive, have been described; the former is limited to low percentages of tumor cells at the tumor-stroma interface, a result of induction by cytokines secreted by tumor infiltrating immunocytes, while the latter represents innate PD-L 1 expression by the tumor, often in $>50 \%$ of tumor cells. In limited studies of anal squamous cell carcinoma (ASCC), PD-L1 expression in tumor cells has been reported in 56 to 74 percent of cases. The aim of this study is to further characterize PD-L1 expression frequency amongst different histologic types of ASCCs as well as to correlate PDL1 expression with HIV status and rate of treatment failure.

Design: Thirty-seven primary ASCCs were evaluated for membranous PD-L1 expression. Expression of PD-L1 in $>1 \%$ of tumor cells was considered positive. PD-L1 expression in immunocytes at the tumor-stroma interface was also noted and recorded separately. Immunohistochemistry for p16 was used as a surrogate marker for HPV status.

Results: Thirty-six of 37 ASCC cases were associated with HPV, and 10 cases were from HIV-positive patients. Sixteen (43\%) of 37 tumors were PD-L1-positive, which rises to 26 cases (70\%) if PD-L1 expression in tumor-infiltrating immunocytes is also included as positive. Basaloid ASCC cases more frequently showed PD-L1 expression in tumor cells than keratinizing ASCC cases $(13 / 23$ vs. $3 / 14,57 \%$ vs. $21 \%, \mathrm{p}<0.05)$. PD-L1 expression did not correlate with HIV status, age, or failure of standard therapy. ASCC cases from female patients more frequently expressed PD-L 1 than cases from male patients $(12 / 18$ vs $4 / 19,67 \%$ vs. $21 \%, p<0.05)$, and female cases were more likely to show an induced rather than a constitutive pattern of PD-L1 expression.

Conclusions: PD-L1 expression in tumor cells of ASCC is common and is more frequently associated with the basaloid histologic type. Interestingly, ASCC cases from female patients were more likely to show an induced pattern of PD-L1 expression, possibly suggesting a hormonally related mechanism. These findings are important in possible immunotherapeutic considerations of ASCC.

854 Immunoprofiling of Mucosal Lymphocytes in Patients with Newly Diagnosed Inflammatory Bowel
Disease

Jacob Sweeney ${ }^{1}$, Patrick McIntire ${ }^{2}$, Rhonda Yantiss ${ }^{3}$, Yao-Tseng Chen ${ }^{3}$. ${ }^{1}$ Weill Cornell Medicine, New York, NY, ${ }^{2}$ Weill Cornell Medicine - New York Presbyterian Hospital, New York, NY, ${ }^{3}$ Weill Cornell Medical College, New York, NY

Background: Ulcerative colitis (UC) and Crohn disease (CD) are immune-mediated disorders presumably triggered by a heightened response to enteric bacteria. Some data indicate that selective activation of Th1 and Th17 helper T-lymphocytes promotes inflammation in $C D$, whereas Th2 cells play a more important role in UC. Recent reports suggest that innate lymphoid cells (ILC1, ILC2, ILC3) may also contribute to the pathogenesis of both diseases. However most of the above knowledge was derived from animal models, or human subjects with established diagnoses who had already received immunomodulatory therapy. We performed this study to define immune cell profiles in colonic biopsy samples from untreated $C D$ and UC patients.

Design: The study group included 17 colonic biopsy samples from patients with newly diagnosed UC $(n=10)$ and $C D(n=7)$. Lymphoid infiltrates were assessed using antibodies directed against CD8 (cytotoxic T cells), T-bet (Th1 and ILC1 cells), GATA3 (Th2 and ILC2), RORYT (Th17 and ILC3) and FoxP3 (Treg). Immunopositive cells in the mucosa were quantified using $\mathrm{HALO}^{\mathrm{TM}}$ imaging analysis software and expressed as cell counts and densities (cells $/ \mathrm{mm}^{2}$ ). Positive cells in crypt epithelium were manually counted, normalized, and recorded as cells per 100 crypts.

Results: We did not observe significant differences with respect to any T-cell subsets when comparing newly diagnosed UC and CD. GATA-3 positive cells, representing Th2 and ILC2 cells, were more abundant than other subsets of immune cells; these cells were diffusely present in the lamina propria in both UC (mean: $1498 \mathrm{cells} / \mathrm{mm}^{2}$, range 961 2075) and CD (mean: 1449 cells $/ \mathrm{mm}^{2}$, range 930-2196). In contrast, RORYT-positive cells, reflecting Th17 and ILC3 cells, were more abundant than other subsets among intraepithelial lymphocytes, and were present in similar numbers in UC and CD (mean: 99 vs. 68/100 crypts).

Conclusions: Colonic biopsies from patients with untreated UC and $C D$ patients contain abundant GATA3-positive lymphocytes in the lamina propria, suggesting a role for Th2 immunity in both UC and CD. On the other hand, RORyT-positive cells predominate among intraepithelial lymphocytes, suggesting that Th17 and/or ILC3 cells might play a role in triggering inflammation following microbial exposure. Our results do not support the view that UC and CD can be distinguished based on relative numbers of Th1 and Th2 cells; reported differences may reflect the modulatory effects of therapy among patients with longstanding disease.

\section{Next-Generation Sequencing in a Mixed Neuroendocrine-Nonneuroendocrine Neoplasm}

Keith J Sweeney ${ }^{1}$, Daniel Coldren ${ }^{2}$, Faheem Farooq ${ }^{2}$, Kevin Zarrabi ${ }^{2}$, Joseph Kim², Minsig Choi ${ }^{2}$, Jela Bandovic'. 'Stony Brook University Hospital, Port Jefferson Station, NY, ${ }^{2}$ Stony Brook University Medical Center, Stony Brook, NY

Background: Mixed neuroendocrine-nonneuroendocrine neoplasms (MiNENs) are a group of rare tumors previously known as mixed adenoneuroendocrine carcinomas (MANECs) by the 2010 WHO classification. In addition to a neuroendocrine component, a nonneuroendocrine component may consist of adenocarcinoma or squamous cell carcinoma, with both elements rarely coinciding. The exact phenotypic behavior of these tumors with all three components remains unknown given the varied tumor composition. Moreover, little is known regarding histogenesis, cellular genomics, or responsiveness to current treatment options. In our study, we sough to further classify MiNEN tumors with squamous cell differentiation with next-generation sequencing genomic data and a review of the current literature to highlight the importance of recognizing this entity.

Design: We performed a review of the literature on PubMed and a natural language search using Sunquest's CoPath Plus $®$ laboratory information system at our institution for all cases with "MiNEN" and "MANEC" from 1/1/1981 until 9/1/2017 and identified all cases of MiNEN/MANEC with squamous cell differentiation.

Results: The literature review yielded five reported cases of gastric MiNEN with squamous cell differentiation in the literature and there has been one case of gastric MiNEN diagnosed at our institution. The five reported cases of gastric MiNEN do not provide genomic data and we provide the first genetic analysis of this tumor type. The histology, immunohistochemistry, and next-generation sequencing data of the case from our institution are reported in Figures 1 and 2 . 


\begin{tabular}{|l|}
\hline Detected Genomic Finding \\
\hline KRAS \\
D154del \\
\hline NF1 \\
Y1625 \\
\hline PTEN \\
Loss exon 2 \\
\hline CDKN2A/B \\
Loss \\
\hline Microsatellite status \\
MS-stable \\
\hline TP53 \\
Splice site 375G $>$ A \\
\hline Tumor Mutation Burden \\
TMB-Low; 2 Muts/Mb \\
\hline
\end{tabular}

subtype gold standard.

Results: Inter-observer concordance for subtype was fair (kappa 0.31). Percent correctness for subtype against IHC-established subtype was $50 \%$ for a majority ( 3 or more) of pathologists. In all 11 cases of 4 or 5 pathologists being concordant, the subtype was also correctly identified. Inter-observer concordance for morphologic parameters was also overall fair, with an average kappa 0.25 (0.11-0.39 for each parameter)

Conclusions: Despite 11 cases with strong concordance and correctness, overall subtyping concordance was only fair, and a majority of the five pathologists correctly identified the subtype in only $50 \%$ of cases. Thus, while some AC cases yield clear-cut subtyping significant discrepancy among pathologists remains. Concordance of individual morphologic parameters was also only fair, underscoring the ambiguous morphologic features of AC. It appears that utilization of IHC to identify subtype in AC cases is sometimes necessary to provide accurate, reliable prognostic and therapeutic information.

857 LINE-1 RNA In-Situ Hybridization and ORF1p Immunohistochemistry are useful adjuncts in diagnosing dysplasia in Barrett's Esophagus and LINE-1 up-regulation appears to be an early event in esophageal carcinogenesis

Martin Taylor ${ }^{1}$, Vikram Deshpande ${ }^{2}$, Kshitij S Arora ${ }^{3}$, Lawrence Zukerberg ${ }^{4}$ David T Ting ${ }^{5}$ Anthony Mattia ${ }^{6}$. ${ }^{1}$ Boston, MA ${ }^{2}$ Massachusetts General Hospital, Boston, MA, ${ }^{3}$ Jackson Memorial Hospital/University of Maryland Hospital, Miami, FL, ${ }^{4}$ Auburndale, MA, ${ }^{5}$ Massachusetts General Hospital, ${ }^{6}$ North Shore Medical Center Sudbury, MA

Background: Barrett's esophagus (BE) is the earliest histologic precursor to esophageal adenocarcinoma (EAC) and is highly prevalent, however, biopsy screening is ineffective in preventing progression to adenocarcinoma. This is in part due to sampling, but is also due to difficulty in diagnosis of dysplasia and subjective criteria, with poor agreement even among expert pathologists. TP53 mutations are an early molecular event in dysplasia, but the penetrance of TP53 abnormalities in EAC is under $70 \%$, limiting negative predictive value. Tests such as gene expression profiling have utility in research settings but are impractical and expensive for routine practice; better biomarkers are needed. One potential biomarker is the LINE-1 transposon (L1), a polymorphic, parasitic genetic element that comprises $\sim 18 \%$ of the genome, has recently been shown to be repressed by TP53, and is broadly upregulated in human epithelial malignancies including EAC.

Conclusions: MiNENs are highly variable in composition and information regarding the genomic characterization of these tumors is sparse. Our results highlight that this a rare disease process where a squamous cell carcinoma develops as a component of a MiNEN. As multiple genomic aberrancies were identified in this tumor variant, targeted therapy remains a viable option for future treatments. Going forward, genomic analysis will likely play a key role in understanding the histogenesis of this entity and help guide therapy.

856 Can Pathologists Reliably Establish Ampulla of Vater Carcinoma Histologic Subtype on H\&E Alone? Concordance of Subtype and Morphologic Parameters in Comparison to Immunohistochemistry-Established Subtype

Kevin S Tanager ${ }^{1}$, Lindsay Alpert', Namrata Setia', Christopher $R$ Weber', John Hart', Shu-Yuan Xiao'. 'University of Chicago, Chicago, IL, ${ }^{2}$ Univ. of Chicago Med Ctr, Chicago, IL

Background: Histologic subtyping of ampulla of Vater carcinoma (ampullary carcinoma, AC) may guide choice of adjuvant chemotherapy for advanced disease, and intestinal (INT) subtype may have a better treatment response and survival over the pancreatobiliary (PB) and mixed (M) subtypes. Unfortunately, ambiguous morphologic features can result in inconsistent subtyping among pathologists. Recent subtyping efforts with immunohistochemistry (IHC) panels have included the markers CK7, CK20, CDX2, and the MUC family proteins. Given that IHC is costly, it is important to determine whether accurate subtyping can be achieved based on hematoxylin and eosin (H\&E) evaluation alone, or if $\mathrm{IHC}$ is required.

Design: Five gastrointestinal pathologists evaluated 34 cases of $A C$ for histologic subtype (PB, INT, or M) based on H\&E slides alone. M subtype was defined as cases with a secondary subtype comprising $30 \%$ or more of the tumor. IHC studies (CK7, CK20, and CDX2) were used to determine a gold standard diagnosis for comparison (strong CK7 with weak CK20 and CDX2 = PB; strong CK20 or CDX2 and weak CK7 $=$ INT; strong CK7 and CK20 or CDX2 $=$ M). Morphologic parameters (goblet cells, brush border, non-goblet intracytoplasmic mucin, extracellular mucin, and foveolar-type epithelium) were also separately evaluated. Inter-observer concordance (kappa) was calculated for subtype and the individual parameters. Percent correctness of subtyping was calculated in relation to IHC-established

Design: Cross-sectional study of 109 esophageal biopsies with BE at a single center. All samples were re-analyzed for histological dysplasia by three experienced gastrointestinal pathologists and independently stained for LINE-1 ORF1 protein (IHC) and RNA (ISH). Follow-up with an endpoint of dysplasia or carcinoma was pursued in cases that were indefinite or negative for dysplasia.

Results: A consensus diagnosis was reached in 96 cases, with moderate agreement (kappa 0.43-0.51). The RNA-ISH results are shown in Table 1: L1 RNA-ISH and L1 ORF1p IHC demonstrated $84 \%$ concordance. L1 RNA-ISH distinguished dysplastic from neverdysplastic BE with a sensitivity and specificity of $91 \%$ and $88 \%$, respectively. $60 \%$ of the morphologically negative or indefinite cases that were L1 RNA-high had a prior or subsequent diagnosis of high grade dysplasia, but no similar L1 RNA-low case developed dysplasia in follow-up. These findings suggest an epigenetic "field effect" in at-risk tissue. Supporting this, in cases with a consensus diagnosis of dysplasia or carcinoma with areas of non-dysplastic mucosa, $46 \%$ (10/22), had at least one fragment of L1 RNA-high non-dysplastic mucosa.

L1 RNA ISH Results.

\begin{tabular}{|c|c|c|c|c|c|c|}
\hline$n=96$ & $\begin{array}{l}\text { Neg- } \\
\text { ative } \\
\text { (29) }\end{array}$ & $\begin{array}{l}\text { Indef- } \\
\text { inite } \\
\text { (9) }\end{array}$ & $\begin{array}{c}\text { Low } \\
\text { Grade } \\
\text { (17) }\end{array}$ & $\begin{array}{c}\text { High } \\
\text { Grade } \\
\text { (17) }\end{array}$ & $\begin{array}{l}\text { Intramucosal } \\
\text { Carcinoma (5) }\end{array}$ & $\begin{array}{c}\text { Invasive } \\
\text { Carcinoma } \\
\text { (19) }\end{array}$ \\
\hline $\begin{array}{l}\text { L1 } \\
\text { Low }\end{array}$ & $\begin{array}{c}24 \\
(83 \%)\end{array}$ & $\begin{array}{c}4(44 \%) \\
\quad \#\end{array}$ & $0(0 \%)$ & $1(6 \%)$ & $0(0 \%)$ & $2(11 \%)$ \\
\hline $\begin{array}{l}\text { L1 } \\
\text { High }\end{array}$ & $\begin{array}{c}5 \\
(17 \%)^{*}\end{array}$ & $\begin{array}{c}5 \\
(56 \%)^{*}\end{array}$ & $\begin{array}{c}17 \\
(100 \%)\end{array}$ & 16 (94\%) & $5(100 \%)$ & 17 (89\%) \\
\hline
\end{tabular}

\# No case developed dysplasia during followup. ${ }^{*} 60 \%$ had a previous or subsequent biopsy demonstrating dysplasia (both columns).

Conclusions: We identify two distinct L1-derived biomarkers that appear to have utility in diagnosing Barrett's Esophagus with dysplasia. These markers may have potential in identifying patients at risk for progression but with morphologically negative biopsies. Furthermore, the high prevalence of L1 expression indicates that L1 derepression may precede TP53 mutation. 
858 Immune-based Stratification of Colon Cancer Predicts Survival

Martin Taylor ${ }^{1}$, Vikram Deshpande ${ }^{2}$, Kshitij S Arora ${ }^{3}$, David T Ting $^{4}$, Cristina Ferrone, ${ }^{4}$ Anne Dinaux ${ }^{4}$, Lieve Leijssen ${ }^{4}$. ${ }^{1}$ Boston, MA ${ }^{2}$ Massachusetts General Hospital, Boston, MA, ${ }^{3}$ Jackson Memoria Hospital/University of Maryland Hospital, Miami, FL, ${ }^{4}$ Massachusetts General Hospital

Background: Immunotherapy provides a durable response in subsets of patients, and recently, checkpoint inhibitor therapy was approved for any tumor with microsatellite instability (MSI). Since a significant proportion of tumors show primary resistance to therapy and a proportion of responsive tumors relapse, there is an urgent need to identify the mechanisms underlying resistance. Although response correlates with mutational burden, expression of checkpoint proteins, and other immunologic regulators, there are an exponential number of potential combinations of markers and therapies. A more rational approach calls for a better understanding of the immunological milieu; most reports only evaluate one or a small number of components. Herein, we provide a detailed characterization of a broad panel of immune-related markers in mismatch repair (MMR)-proficient and -deficient colon cancers.

Design: We evaluated 210 consecutive colonic carcinomas with known $\mathrm{MSI}$ using a tissue microarray platform. All tumors were stained for PD-L1, PD1, IDO1, LAG3, Foxp3, CD4, CD8, CD163, PU.1, B2M, CTLA4 , CD28, CD80, Axin 2, and HLA class I and II using an automated platform. IDO and PD-L1 were quantified on both tumor and macrophages. Statistics were performed using commercially available software.

Results: Unsupervised hierarchical clustering of the results but blinded to MMR status resulted in five classes of colon cancers. Broadly, the subgroups seem to be anchored by expression level of HLA Class I, B2M, PD-L1, and IDO1 on tumor cells, along with PDL1 and IDO1 on macrophages. 90\% of MMR-deficient stratified into three classes; these also included $17 \%$ of MMR-proficient tumors. The remaining tumors are divided into immunologically "silent" class $(60 \%)$ and a class defined by strong PD-L1 and IDO1 on macrophages $(6 \%)$. There was a statistically significant difference in survival between the 5 classes $(p=0.001)$, and classes stratify survival in both MMRproficient and MMR-deficient tumors. Several statistically significant individual correlations and localization patterns were identified for subsets markers; no marker individually demonstrated a significant correlation with survival.

Conclusions: Immune-based stratification of colon cancers predicts survival in both MMR-proficient and MMR-deficient cases, whereas no individual marker was statistically significant. A subset of MMRproficient cancers cluster with MMR-deficient cases. This stratification may be useful in patient selection for clinical trials.

\section{Clinical Significance Of FXR Expression In Colon Cancer}

Stamatios Theocharis ${ }^{1}$ Chrysovalantou Mihailidou², loannis $D$ Kostakis ${ }^{3}$, Stavroula Papadodima ${ }^{4}$, Eugene Danas ${ }^{5}$, Michalis V Karamouzis ${ }^{6}$ Gerasimos Tsourouflis?. ${ }^{1}$ Athens, ${ }^{2}$ Medical School, University of Athens, Athens, Greece, ${ }^{3}$ National and Kapodistrian University of Athens, Medical School, Athens, Attica, ${ }^{4}$ Medical School, University of Athens, Athens, Greece, ${ }^{5}$ Medical School, University of Athens, Athens, Greece, ${ }^{6}$ National and Kapodistrian University of Athens, Laikon General Hospital, Athens, Greece, ${ }^{7}$ Athens Medical School, Athens, Attiki

Background: Several studies suggest that Farnesoid $X$ receptor (FXR) participates in and modulates tumorigenesis. The role of FXR expression in human colon cancer was investigated in this study.

Design: FXR nuclear and cytoplasmic immunohistochemical (IHC) expression on paraffin-embedded colon adenocarcinoma cases $(n=77)$, was correlated with clinico-pathological parameters and patients' survival. FXR levels were also assessed in a panel of colon cancer cell lines [ $\mathrm{CaCo} 2, \mathrm{HT} 29, \mathrm{HCT} 116$ (stage II), RKO (stage III), DLD-1 (stage high) SW480 (stage III-IV), Colo205 (stage IV)] with a differential tumor grade. Proteins from the whole cell, as well as, cytoplasmic and nuclear fractions were blotted for Western analysis.

Results: Nuclear FXR expression was found in $53(68.8 \%)$ whereas cytoplasmic in $23(29.9 \%)$ cases, respectively. High nuclear FXR expression was correlated with larger tumor size $(p=0.0037)$, presence of lymph node metastases $(p=0.0037)$ and advanced disease stage (III-IV) $(p=0.041)$, whereas high cytoplasmic FXR expression was correlated with locally advanced tumors (T2-T4) $(p=0.028)$. High nuclear FXR expression proved as an independent prognostic factor of worse relapsed-free $(p=0.001)$ and disease-specific $(p=0.001)$, survival. Western Blot analysis revealed high FXR levels in a stagedependent manner. FXR in colon cell lines showed mainly nuclear and barely cytoplasmic expression patterns.

Conclusions: Overall, our results indicate that FXR expression is correlated with an advanced colon cancer phenotype. Further studies are needed in order to verify FXRs' potential involvement in colon cancer patients' prognosis and management.

\section{Abnormal P53 Expression in Pre-Progression Barrett's Esophagus is a Risk Factor for Subsequent High Grade Dysplasia or Adenocarcinoma}

Minami Tokuyama ${ }^{1}$, Christopher Deitrick ${ }^{2}$, Reetesh Paỉ ${ }^{3}$ Jon Davison ${ }^{4}$ ${ }^{1}$ Icahn School of Medicine at Mount Sinai, New York, NY, ${ }^{2}$ University of Pittsburgh, ${ }^{3}$ UPMC-Presbyterian Hospital, Pittsburgh, PA, ${ }^{4}$ University of Pittsburgh, Pittsburgh, PA

Background: Although Barrett's esophagus (BE) is associated with a large increase in the relative risk of esophageal adenocarcinoma (EAC), only a small percentage of those diagnosed with BE progress to EAC. Abnormal p53 immunoexpression is reported to be a prognostic biomarker for progression to high grade dysplasia (HGD) or EAC in patients with BE. We sought to validate scoring criteria for abnormal p53 expression in pre-progression biopsy samples using a retrospective case-cohort study design.

Design: We retrospectively identified patients $(n=17)$ with $B E$ who progressed to HGD or EAC $>1$ year from their index biopsy and nonprogressor control patients $(n=76)$ who were never diagnosed with HGD or EAC during at least 5 years of total endoscopic surveillance. Non-progressors were matched with progressors based on the highest grade of pre-progression dysplasia (Table 1). For each patient a representative paraffin-embedded tissue sample taken at one time point $>6$ months before the first diagnosis of $\mathrm{HGD} / \mathrm{EAC}$ was used for p53 immunohistochemistry (IHC). Based on criteria proposed by Kastelein [PMID 23256952] and Younes [PMID 28226185], P53 expression was defined as abnormal if there was strong nuclear overexpression or complete loss of expression in at least one gland. IHC was scored by 2 independent pathologists blinded to outcomes; agreement was required for p53 to be scored as abnormal. Fo comparison, independent samples diagnosed with HGD $(n=10)$ and non-dysplastic BE adjacent to HGD $(n=8)$ were also tested.

Results: Abnormal p53 expression was observed in $7.1 \%(5 / 70)$ of surveillance biopsy samples originally diagnosed as non-dysplastic $\mathrm{BE}$ (negative for dysplasia or indefinite for dysplasia) and $30.4 \%(7 / 23)$ of surveillance biopsies originally diagnosed with low grade dysplasia. Non-dysplastic BE adjacent to HGD showed abnormal p53 expression in $25 \%$ (2/8); all samples (10/10) originally diagnosed as HGD had abnormal p53 expression. Abnormal p53 expression was detected in surveillance biopsies of $35.3 \%(6 / 17)$ progressors vs. $7.9 \%(6 / 76)$ nonprogressors $(P=0.007)$, a 6.3 -fold increased risk of progression to HGD or $\mathrm{EAC}(95 \% \mathrm{Cl} 1.7-23.3)$, adjusted for age, sex and $\mathrm{BE}$ segment length.

Table 1. Clinicopathologic Characteristics of Progressors and Non-progressors

\begin{tabular}{|c|l|l|l|}
\hline & $\begin{array}{l}\text { Non-progressors } \\
\mathrm{n}=76\end{array}$ & $\begin{array}{l}\text { Progressors } \\
\mathrm{n}=17\end{array}$ & $\mathrm{p}$-value \\
\hline Age in years, mean (range) & $59(36-81)$ & $63(52-83)$ & 0.105 \\
\hline $\begin{array}{c}\text { Sex, } \mathrm{n}(\%) \\
\text { Female }\end{array}$ & $\begin{array}{l}20(26.3 \%) \\
56(73.7 \%)\end{array}$ & $\begin{array}{l}1(5.9 \%) \\
16(94.1 \%)\end{array}$ & 0.107 \\
\hline $\begin{array}{c}\text { Male length, } \mathrm{n}(\%) \\
\text { Short segment }(<3 \mathrm{~cm}) \\
\text { Long segment }(\geq 3 \mathrm{~cm}) \\
\text { unknown }\end{array}$ & $30(39.5 \%)$ & $3(17.6 \%)$ & \\
\hline $\begin{array}{c}\text { Original dysplasia diagnosis, } \\
\mathrm{n} \text { (\%) }\end{array}$ & $39(51.3 \%)$ & $11(64.7 \%)$ & 0.162 \\
$\begin{array}{l}\text { Negative/indefinite } \\
\text { Low grade }\end{array}$ & $7(9.2 \%)$ & $3(17.6 \%)$ & \\
\hline $\begin{array}{c}\text { P53 IHC result, } \mathrm{n} \text { (\%) } \\
\text { Wild type } \\
\text { Abnormal }\end{array}$ & $20(26.3 \%)$ & $3(17.6 \%)$ & 0.549 \\
\hline $\begin{array}{c}\text { Duration of follow-up* } \\
\text { Years, mean (range) } \\
\text { Endoscopic exams, mean (range) }\end{array}$ & $4.7(1-20)$ & $2.2(1-7)$ & $<0.001$ \\
\hline
\end{tabular}


* Interval from the endoscopic biopsy tested for p53 expression to the end of follow up. End of follow up was defined as the date of first diagnosis of HGD/EAC (progressors) or last endoscopic exam/first ablation treatment (non-progressors).

Conclusions: Abnormal p53 expression is detected in HGD, BE adjacent to HGD and in BE samples obtained during surveillance, up to 3.5 years before the first diagnosis of HGD/EAC. Abnormal p53 expression in pre-progression surveillance biopsies is associated with an increased risk of progression to HGD or EAC.

\section{Comprehensive Analysis of the Molecular and Clinicopathological Features in Esophageal Carcinosarcoma by Next Generation Sequencing}

Sho Tsuyama1', Tsuyoshi Saito², Youichi Akazawa³, Yuka Yanai, Aiko Kurisaki ${ }^{3}$ Keisuke Akaike ${ }^{3}$, Takuo Hayashi ${ }^{4}$, Yuki Fukumura ${ }^{3}$, Atsushi Arakawa ${ }^{3}$, Takashi $\mathrm{YaO}^{3}$. '1Juntendo University, Graduate School of Medicine, Bunkyoku, Tokyo, ${ }^{2}$ Tokyo, ${ }^{3}$ Juntendo University, Bunkyo-ku, Tokyo, ${ }^{4}$ Juntendo University, Graduate School of Medicine, Tokyo,

Background: Esophageal carcinosarcoma (ECS) is a rare entity in the esophageal cancer, accounting for $0.5-2.8 \%$ of esophageal cancers. The molecular features including molecular pathogenesis of ECS have not been fully investigated. This study was performed to elucidate the differentiation mechanisms in ECS from the viewpoint of genetic alterations.

Design: Fifteen cases of esophageal carcinosarcoma were collected from pathological files at Juntendo University Hospital from 2003 to 2016. In addition to the clinicopathological analysis, we extracted DNA from carcinoma component and sarcomatous component, respectively. Four cases of them were analyzed by next generation sequencing (NGS) and the remaining cases were examined by DNA target sequences for the identified hot spot genes. The p53 immunohistochemistry was performed to correlate the TP53 mutation and overexpression. Ki-67 immunohistochemistry was also performed to evaluate the proliferative ability of each component of carcinosarcoma.

Results: Five-year overall survival rate in this series was $53.8 \%$. Lymph node metastasis was observed in 12 out of 15 cases, and histology of the metastatic lymph nodes were squamous cell carcinoma in all cases. NGS revealed that TP53 was the most frequently mutated gene in ECS, and the TP53 mutation patterns were almost consistent throughout the two components within the same tumors. Several gains and losses were commonly observed both in carcinoma/ sarcoma components and the several differences were also observed between these components. We found concordance with the p53 IHC staining and TP53 missense mutations. Proliferative index assessed by Ki-67 differed each component within the same tumor in most cases, however, sarcomatous component did not always show higher index and it was rather higher in carcinoma component.

Conclusions: Generally, dedifferentiated tumors have highly malignant potential which could be attributed to the dedifferentiated component. However, this is not always the case for ECS. Each carcinomatous and sarcomatous component showed relatively coincidence in TP53 mutation status and p53 overexpression. Thus, TP53 mutations seemed to occur as an early event of tumorigenesis in ECS, and two components could be considered as the same origin. NGS study revealed several copy number variations including the amplification of genes encoding tyrosine kinase receptors in carcinomatous and sarcomatous components, so these could be potential therapeutic target in ECS.

\section{Colorectal Carcinoma Incidence in Patients Less Than 50 Years-Old; A Retrospective Study}

Tayler Van Denakker ${ }^{1}$, Jason Rubinov ${ }^{2}$, Ammar Matloob $^{3}$, Ari Young ${ }^{4}$, Steven Tsistrakis ${ }^{5}$, Gabriel Levi ${ }^{6}$, Ilan Weisberg ${ }^{5}$. ${ }^{1}$ Mount Sinai, New York, NY, ${ }^{2}$ Mount Sinai St. Luke's-Roosevelt, ${ }^{3}$ Mount Sinai St. Luke's -West Hospital, New York, NY, ${ }^{4}$ Mount Sinai Beth Israel, New York, NY, ${ }^{5}$ Mount Sinai, ${ }^{6}$ Mount Sinai Beth Israel Medical Center

Background: The increasing incidence of colorectal cancer (CRC) in young adults in the United States has been a recent topic of interest. Our interest is in microsatellite instability (MSI), a hypermutable phenotype caused by the loss of DNA mismatch repair expression. $\mathrm{MSI}$ is detected in about $15 \%$ of all colorectal cancers; in both hereditary Lynch Syndrome and sporadic acquired CRC, most commonly associated with the MLH1 gene. The purpose of this study is to evaluate CRC in patients less than 50 years of age, and elucidate if the increasing incidence of $\mathrm{CRC}$ in young patients is attributed to microsatellite instability.

Design: The pathology reports of $C R C$ in patients less than 50 years old between 2008 and $2017(n=96)$ were retrieved from the Mount Sinai Beth Israel Medical Center electronic medical records. The CRC CAP protocol was utilized as a comparison tool.

Results: The average patient age was 42.875 years old (range; 20 49 years). There was no gender predominance observed between male $(n=47)$ and female $(n=49)$ patients. The rectum was the most common primary site $(n=30)$ followed by sigmoid $(n=23)$, then rectosigmoid $(n=10)$. Other sites involved were descending colon ascending colon, transverse colon, cecum, hepatic flexure, and splenic flexure. The most common CRC diagnosis was low grade adenocarcinoma $(n=69)$, followed by high grade adenocarcinoma $(n=13)$ and low grade mucinous adenocarcinoma $(n=13)$, as well as diagnoses of high grade mucinous adenocarcinoma, mixed low grade and high grade adenocarcinoma, neuroendocrine carcinoma and signet ring carcinoma, with the remaining 2 cases unable to be assessed. According to current CAP guidelines, CRC patients under 50 years should be tested for microsatellite instability. Of the cases tested $(n=63), 5$ cases $(7.94 \%)$ showed loss of mismatch repair (MMR) nuclear expression, with the majority of cases $(n=3)$ showing a loss of expression in the MLH-1gene. The remaining genes tested MSH1, MSH6, and PMS-2, showed loss of nuclear expression in equal distribution.

Conclusions: CRC incidence is increasing in younger patient populations despite an overall decrease in the general population. MS testing demonstrated that $60 \%(3 / 5)$ of the CRC cases studied were attributed to loss of nuclear expression in the MLH-1 gene. However with only $7.94 \%$ of cases demonstrating suggested association with microsatellite instability, it raises concern about sporadic tumors arising independently of MSI. Therefore, should screening for CRC start earlier?

\section{What Makes an Expert Pathologist? - Histopathologist Features Predictive of Diagnostic Concordance at Expert Level Amongst a Large International Sample of Pathologists Diagnosing Barrett's Dysplasia}

Myrtle van der Wel', Helen G Coleman², Marnix Jansen³, Sybren Meijer'. ${ }^{1}$ Academic Medical Center Amsterdam, Amsterdam, ${ }^{2}$ Queen's University Belfast, ${ }^{3}$ UCL Cancer Institute, London, ${ }^{4}$ Academic Medical Center, Amsterdam, the Netherlands

Background: Histopathological diagnosis of dysplasia in Barrett's esophagus (BE) is the gold standard for patient risk stratification but is subject to significant interobserver variation. For this reason guidelines recommend expert histopathologist review of all BE neoplasia diagnoses. We investigated features that predict diagnostic concordance amongst a large international cohort of gastro-intestinal (GI) pathologists.

Design: An online scoring environment was developed for GIpathologists ( $n=55$ ) from over 20 countries to grade a case set of 55 digitized BE biopsies encompassing the complete spectrum from non-dysplastic Barrett's esophagus (NDBE) to high-grade dysplasia (HGD). Detailed histopathologist demographic data (experience, center volume, etc.) was obtained through an online questionnaire. We also quantified the impact of p53 immunohistochemistry (IHC) on diagnostic concordance. Finally low-pass whole genome sequencing (WGS) was carried out to correlate molecular complexity to diagnostic concordance.

Results: We recorded over 6000 individual case diagnoses. We found excellent concordance for NDBE (643 of 816 diagnoses; $79 \%$ ) and HGD (544 of 765 diagnoses: $71 \%$ ) and intermediate concordance for LGD (382 of $918 ; 42 \%)$ and IND $(70$ of $306 ; 23 \%)$. Significant misdiagnoses (i.e. NDBE overstaged as HGD, or HGD understaged as NDBE) were rare (9 of 816 diagnoses; $1.1 \%$; and 17 of 765 diagnoses; $0.6 \%$ ). Addition of p53 IHC significantly increased diagnostic concordance. Regression analyses revealed histopathologist predictors of diagnostic concordance at expert level and allowed us to model optimal revision strategies based on individual case and pathologists characteristics.

Conclusions: We quantify pathologist dependent factors, which predict diagnostic concordance for BE dysplasia diagnosis at expert level. These data will allow rational formulation of quality assurance criteria and revision strategies for guideline development. Our study method is highly scalable and broadly applicable to any area of histopathologic diagnostic uncertainty.

\section{Distinguishing Crohn's Disease from Ulcerative Colitis in Terminal Ileal Biopsy Specimens}

Taisia Vitkovski', Robert Odze ${ }^{2}$, John Goldblum¹, Bo Shen ${ }^{1}$, Deepa Patil'. 'Cleveland Clinic, Cleveland, OH, ${ }^{2}$ Brigham \& Women's Hospital, Boston, MA

Background: A substantial (5-35\%) proportion of ulcerative colitis (UC) patients develop ileal inflammation during the course of their disease. Recent data suggest that ileitis in UC may simply represent a primary manifestation of UC in the ileum rather than secondary to "backwash". The purpose of this study was to perform a systematic analysis of histologic features in ileal biopsies from clinically confirmed UC or Crohn's disease (CD) patients in an effort to determine if there are features that help distinguish these disorders in biopsies.

Design: Eighty-seven ileal biopsies from 37 and 33 clinically confirmed previously treated CD (18 ileal, 19 ileocolonic) and UC (17 
pancolitis, 13 left sided, 3 subtotal colitis), respectively, were reviewed for histologic features such as villous atrophy, granulomas, muscularis mucosae (MM) abnormalities (thickening, duplication), pyloric gland metaplasia (PGM), and focality, grade (scored on a 4-point scale), and type of inflammation. Age, sex, disease distribution, imaging findings, endoscopic findings, primary sclerosing cholangitis (PSC), NSAID and alcohol use were also recorded.

Results: Clinically, PSC was only noted in UC patients $(18 \%$ vs $0 \% ; p=0.008$ ). Radiologic abnormalities (mural thickening, enhancement, narrowing/strictures) were significantly more common in CD compared to UC patients $(40 \%$ vs $10 \% ; p=0.04)$. Endoscopic abnormalities (ulcers, erythema, strictures) were more frequent in CD compared to UC patients $(81 \%$ vs $46 \% ; \mathrm{p}<0.001)$. Strictures were only present in CD patients ( $18 \%$ vs $0 \% ; p=0.015)$. Pancolitis $(51 \%$ vs $26 \%)$ and left colonic disease (39\% vs $26 \%$ ) were significantly more common in UC compared to CD patients $(p=0.011)$. Histologically, granulomas were only present in CD patients (15\% vs $0 \%$; $p=0.015)$. Abnormal MM $(21 \%$ vs $8 \%)$ and PGM $(25 \%$ vs $10 \%)$ were more common in CD compared to UC patients, but were of borderline significance $(p=0.09)$. There were no differences in the type, extent, or grade of inflammation between $C D$ and UC ileal biopsies. None of the histologic features correlated with radiologic or endoscopic abnormalities as a group or with specific subtype of abnormality.

Conclusions: Granulomas, unrelated to crypt injury, when present, are the best histologic indicator of CD in ileal biopsies. While CD patients are more likely to show radiologic and endoscopic abnormalities, especially strictures, the significant overlap in histologic findings makes it difficult to distinguish UC from CD on the basis of inflamed ileal biopsy specimens.

\section{Density of Neuroendocrine Cells Can Distinguish Hyperplastic Polyps from Small Sessile Serrated Polyps}

Taisia Vitkovski', Rahul Jawale², John Goldblum¹, Thomas Plesec ${ }^{1}$ Daniela Allende ${ }^{1}$, Michael Cruise ${ }^{3}$, Ilyssa Gordon ${ }^{1}$, Walter Henricks ${ }^{1}$ Jennifer Jeung ${ }^{4}$, Keith Lai ${ }^{1}$, James Lapinski ${ }^{5}$, Scott Robertson ${ }^{1}$, Erica Savage ${ }^{1}$, Rocio Lopez ${ }^{5}$, Carol Burke ${ }^{5}$, Deepa T Patil'. 'Cleveland Clinic, Cleveland, $\mathrm{OH},{ }^{2}$ Baystate Health, Springfield, MA, ${ }^{3} \mathrm{Cleveland}$ Clinic Solon, $\mathrm{OH},{ }^{4} \mathrm{Cleveland}$ Clinic, Willoughby Hills, $\mathrm{OH},{ }^{5} \mathrm{Cleveland}$ Clinic

Background: Despite the recommended simplified criteria where $\geq 1$ unequivocal abnormal shaped crypt is sufficient to diagnose sessile serrated adenoma/polyps (SSP), it has been shown that distinguishing hyperplastic polyps (HP) from small SSPs $(6-9 \mathrm{~mm})$ is challenging An underappreciated feature of SSPs is the reduced number of neuroendocrine (NE) cells in serrated crypts compared to HPs, which show normal or increased NE cells. The aim of our study was to systematically compare the NE cell density in a cohort of HPs and small SSPs with a consensus diagnosis (agreement by at least 7/11 pathologists), and assess the diagnostic utility of this finding.

Design: A total of 24 HPs (18 left-sided, 6 right-sided) and 25 SSPs (7 left-sided,18 right-sided) were assessed using chromogranin $\mathrm{A}(\mathrm{CgA})$ (DAKO, 1:100) and reviewed by 2 pathologists. The total number of CgA-positive cells in all serrated crypts (regardless of orientation) were counted, a CgA-positive cells/serrated crypt count (CgA/crypt) was generated for each polyp, and differences between HPs and SSP were analyzed using Kruskall-Wallis test. In addition, CgA/crypt in 6 normal right colon and 4 normal left colon biopsies was assessed and compared to that of serrated polyps using $\mathrm{Chi}^{2}$ test.

Results: Left-sided polyps had a significantly higher $\mathrm{CgA} / \mathrm{crypt}$ compared to right-sided polyps ( 1.4 vs. $0.12 ; \mathrm{p}=0.005)$. Compared to SSPS, HPs were associated with a significantly higher total number of $\mathrm{CgA}$-positive cells (2.0 vs. $27 ; \mathrm{p}=0.02)$ and $\mathrm{CgA} /$ crypt $(0.12$ vs.1.3; $\mathrm{p}=0.02)$. Compared to normal right colon (2.0), all right-sided polyps $(0.12 ; \mathrm{p}<0.001)$, right-sided SSPs $(0.08 ; \mathrm{p}<0.001)$ and right-sided HPs $(0.49 ; p=0.006)$ had significantly lower CgA/crypt. Compared to normal left colon (3.3), all left-sided polyps (1.4; $p=0.05)$ and left-sided SSPs (0.15; $p=0.014)$ had significantly lower CgA/crypt. There was no significant difference in $\mathrm{CgA} /$ crypt between normal left colon and left-sided HPs. A higher $\mathrm{CgA}$ /crypt was significantly associated with left-sided HPs compared to left-sided SSPs (1.9 vs.0.15; $p=0.049$ ). CgA/ crypt did not vary between right vs. left-sided SSPs, right vs. left-sided HPs, and right-sided SSPs vs. right-sided HPs.

Conclusions: Right-sided serrated polyps harbor fewer NE cells compared to left-sided polyps. Significant reduction in number of $\mathrm{CgA}$-positive NE cells in SSPs and normal or increased numbers of NE cells in HPs can serve as a helpful ancillary diagnostic tool to separate small SSPs from HPs, particularly in the left colon.

866 Immunohistochemical (IHC) and In-Situ Hybridization (ISH) Analysis of Common Hepatocellular Markers in Gastrointestinal Adenocarcinomas with Hepatoid Differentiation: A Pilot Assessment

Monika Vyas', Olca Basturk², Achim Jungbluth², Gokce Askan², David
Klimstra ${ }^{2}$ Jinru Shia ${ }^{1}$ New Haven, CT, ${ }^{2}$ Memorial Sloan Kettering Cancer Center, New York, NY

Background: Hepatoid differentiation (HD) can occur in non-hepatic neoplasms, including adenocarcinoma of the tubular gastrointestina tract (GIT). Whether this phenomenon carries specific biological or clinical implications remains to be determined. Previous studies on this entity suffer lack of consistent or objective criteria to define HD. In this analysis, we evaluated the utility of various commonly used hepatocellular immunohistochemistry (IHC) markers in the detection of HD in GIT adenocarcinomas.

Design: GIT adenocarcinomas with HD (solid/trabecular architecture and polygonal cells resembling hepatocellular carcinoma) were evaluated for IHC expression of HepPar-1, Arginase, Glypican-3 (GPC 3 ), and AFP, as well as albumin in situ hybridization (ISH). IHC was scored focal versus diffuse based on staining in greater or less than $50 \%$ of the tumor. Patients' clinical information (including serum AFP) was obtained form medical records. Study was approved by IRB.

Results: We collected 8 adenocarcinomas (6 gastric, 2 colonic) exhibiting distinct HD in at least $30 \%$ of the tumor. There were 6 males and 2 females with a mean age of 65.6 years (range $48-79$ years). 4 of 5 patients tested had elevated serum AFP levels (670-115,484 mg/ dL). 4 patients had stage IV disease at presentation; 3 died of disease within 14 months of diagnosis and 1 alive-with-disease at 24 months The remaining 4 patients presented at stage II (3) or Stage I (1); 3 were disease-free at an average of 17 months follow-up and 1 was lost to follow up. The IHC results are summarized in table 1. HepPar-1 was the most sensitive marker in detecting HD, but least specific as it was positive in the glandular component in $80 \%$ of the cases (albeit only focally). AFP positivity correlated with 1) serum AFP levels, 2) positivity of GPC-3 (also an oncofetal protein like AFP), and 3) Albumin ISH. A combination of AFP/GPC-3 with HepPar-1 was successful in capturing all the HD cases.

\begin{tabular}{|l|l|l|}
\hline ISH and IHC & \multicolumn{2}{|c|}{ Tumor components } \\
\hline & Hepatoid $(\mathrm{n}=8)$ & Glandular $(\mathrm{n}=5)$ \\
\hline Albumin ISH & $5 / 8(3$ focal, 2 diffuse $)$ & 0 \\
\hline AFP & $6 / 8(6$ diffuse $)$ & $2 / 5$ (2 focal) \\
\hline HepPar-1 & $6 / 8(4$ focal, 2 diffuse) & $4 / 5$ (4 focal) \\
\hline Arginase & $1 / 8$ (focal) & 0 \\
\hline Glyoican-3 & $6 / 8(2$ focal, 4 diffuse) & $2 / 5$ (2 focal) \\
\hline
\end{tabular}

Conclusions: An IHC panel combining AFP/GPC-3 with HepPar-1 showed a perfect sensitivity in capturing HD in our series. Our observations shed light on the potential utility of common hepatocellular markers in categorizing this uncommon tumor variant, and may enhance the detection of this variant that in our series exhibited a stage-dependent prognosis. The detection of this variant may also open novel targeted treatment options like use of kinase inhibitors like sorafenib which has shown good response in lung adenocarcinoma with HD.

\section{Actin Dynamics Arrest by Loss of Villin and Gelsolin Perturb Intestinal Epithelium Mitochondria Homeostasis--Link to Crohn's Disease}

Yaohong Wang ${ }^{1}$, Swati Roy ${ }^{2}$, George Sudeep ${ }^{2}$, Srinivas Patnaik ${ }^{3}$, Amin Esmaeilniakooshkghazi', Afzal Ahrorov' ${ }^{2}$ Seema Khurana'. ${ }^{1}$ University of Tennessee Health Science Center, Memphis, TN, ${ }^{2} \mathrm{U}$ of Houston, ${ }^{3} \mathrm{Kiit}$ University, ${ }^{4}$ Baylor College of Medicine

Background: Crohn's disease (CD) is a chronic, relapsing inflammatory disease. Autophagy-related gene, namely immununity related guanosine triphosphatase family member M (IRGM) play a vital role in the $C D$ pathogenesis. By acting on mitochondria, IRGM confers autophagic protection or cell death, that may act both in defense against bacterial infection and in damaging inflammation in CD. Villin and gelsolin are actin binding protein expressed in significant amount in the intestinal epithelium. Dynamic assembly and disassembly of actin filament by actin binding protein are crucial to maintain cellular homeostasis and normal function. Additionally studies from our lab and other groups have proved that both villin and gelsolin are involved in the regulation of apoptosis and autophagy.

Design: $14 C D$ patients and 6 non-CD patients were included in the study. Double knockout (DKO) mice were generated by crossing villin and gelsolin knockout mice. Animal study was performed on 6 DKO mice and 6 wild type mice. Histology, immunohistochemistry and ultrastructure analysis by transmission electron microscopy (TEM) were performed on distal ileum tissue from patients and mice.

Results: Immunohistochemistry staining showed a significant decrease including complete loss of villin and gelsolin expression in both the uninflamed and inflamed ileum epithelium from the CD patients but not in the non-CD controls. Villin and gelsolin levels decreased in response to TNF- $\alpha$ and pathogenic E. Coli challenging on isolated intestine epithelium. Genetic deletion of villin and 
gelsolin in mice associated with spontaneous ileocolitis resembling human $C D$ and showed segmental injury with focal inflammation, villus architectural distortions and transmural inflammation. TEM demonstrated abnormally excessive apoptosis and autophagy, defective mitochondria and increased mitochondrial fission on both CD patients and DKO mice. Furthermore, our studies found that villin/gelsolin regulated IRGM targeting and therefore impacted on mitochondrial membrane potential, mitochondrial fission, apoptosis and autophagy.

Conclusions: Our findings provide a novel molecular basis for the regulation of mitochondria homeostasis by villin/gelsolin, perturbing the regulation links to the $C D$ pathogenesis. The results of the newly identified targets (villin/gelsolin) and an understanding of the molecular pathophysiology of IRGM activation could guide, in the future, the successful development of targeted, individualized therapy to prevent and/or treat CD.

\section{Refined Criteria for Low Grade Dysplasia (LGD) and Nondysplastic Barrett Esophagus (BE) Reduce Equivocal Diagnoses (Indefinite for Dysplasia) and Improve Prediction of Patient Outcome: A 10 Year Review}

Kevin M Waters', Kevan Salimian ${ }^{2}$, Lysandra Voltaggio ${ }^{3}$, Elizabeth Montgomery $4 .{ }^{1}$ Cedars-Sinai Medical Center, Los Angeles, CA, ${ }^{2}$ Johns

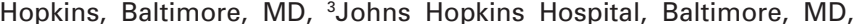
${ }^{4}$ Johns Hopkins Medical Institutions, Baltimore, MD

Background: The indefinite for dysplasia (IFD) category in BE was created for cases that are neither unequivocally dysplastic nor clearly negative for dysplasia (NFD). In 2012, we refined our criteria in our large urban center, thereby reducing the fraction of IFD cases; BE with maintained nuclear polarity and surface gastric-type mucin vacuoles is now considered NFD even with mild to moderate nuclear atypia. We examined changes in the proportion and short-term followup of cases classified as IFD over a 10-year span.

Design: 1,705 cases from 1,167 patients with biopsies of BE were identified from 2007-2016 after eliminating those from a clinic in our institution specializing in treatment of dysplasia. These cases were categorized based on the biopsy with the highest grade of dysplasia (NFD, IFD, LGD, high-grade dysplasia (HGD), or at least intramucosal carcinoma (IMC)). The highest grade of dysplasia was determined for a subsequent biopsy if available. The distribution of dysplasia categories from 2007-2011 was compared to that from 2012-2016. Follow-up on patients with IFD biopsies was obtained to learn if the new thresholds better defined risk of progression.

Results: The mean age of patients was 63 years-old, $70 \%$ were men, and $83 \%$ were Caucasian. Earlier cases (2007-2011) were less likely than later cases $(2012-2016)$ to be NFD $(81.0 \%$ vs. $88.5 \%)$ but more likely to be IFD $(9.2 \%$ vs. $4.5 \%)$. The proportions of LGD ( $4.9 \%$ vs. $3.3 \%$, HGD $(2.4 \%$ vs. $1.8 \%)$, and IMC $(2.4 \%$ vs. $1.9 \%)$ were similar between the earlier and later cases respectively. Subsequent biopsy was more likely to be available for early $(60 \%)$ than later cases $(37 \%)$. Later IFD cases more frequently had dysplasia (3/26, $12 \%)$ on subsequent biopsies than earlier cases $(3 / 61,5 \%)$. The rate of dysplasia on subsequent biopsies for NFD cases was not higher in the later cases $(8 / 249,3 \%)$ than the earlier cases $(22 / 390,5.6 \%)$. A later NFD case did have a subsequent biopsy showing IMC, but this subject had a prior history of IMC.

Conclusions: At one academic institution, improved diagnostic criteria reduced the proportion of cases categorized as IFD by over $50 \%$ from 2007-2016. Although long-term follow-up is necessary, the increased diagnostic threshold for IFD has coincided with a higher proportion of IFD patients having dysplasia on subsequent biopsy. More importantly, NFD patients had no increase in dysplasia on subsequent biopsies, providing evidence that refined criteria that reduce IFD diagnoses do not miss cases with dyplasia.

\section{9 "Indefinite for Dysplasia" in Inflammatory Bowel Disease: Aneuploidy as a Diagnostic and Prognostic Marker of High-Grade Dysplasia or Colorectal Cancer}

Kwun Wah Wen ${ }^{1}$, Peter S Rabinovitch ${ }^{2}$, Danning Huang ${ }^{3}$, Aras Mattis Won-Tak Choi'. 'University of California San Francisco, San Francisco, CA, ${ }^{2}$ University of Washington, ${ }^{3}$ SUNY Upstate Medical University

Background: The clinical significance and natural history of "indefinite for dysplasia" (IND) in inflammatory bowel disease (IBD) remain unclear. To date, there is no reliable biomarker that can differentiate between dysplastic and non-dysplastic epithelium, and facilitate risk stratification of IND patients. This study examines the utility of DNA content analysis in the diagnosis of dysplasia and risk stratification of IND using formalin-fixed paraffin-embedded (FFPE) tissue.

Design: DNA flow cytometry was performed on 52 IND colon samples (51 biopsies and 1 resection) from 36 IBD patients. Forty-three samples of adjacent non-dysplastic background mucosa from the same cohort were utilized as controls. When aneuploidy was detected in the background mucosa (which can rarely occur in IBD), the IND sample was considered to have aneuploidy, only when it shows a distinct aneuploid peak different from that of the background mucosa. Three to six 60-micron thick sections were cut from each block, and the area of IND was dissected for analysis.

Results: The overall 1-, 3-, 5-, and 7-year detection rates of highgrade dysplasia (HGD) or colorectal cancer (CRC) in all IND patients were $4.6 \%, 17.9 \%, 25.3 \%$, and $30 \%$, respectively. More interestingly $12 \%$ of IND cases with aneuploidy were found to have HGD or CRC within 1 year $(p=0.136)$, with $34 \%(p=0.029), 50.5 \%(p=0.002)$, and $58.8 \%$ ( $p=<0.001$ ) detected within 3,5 , and 7 years, respectively. By comparison, 1-, 3-, 5-, and 7-year detection rates of HGD or CRC for IND patients with normal DNA content were $0 \%, 4.8 \%, 4.8 \%$, and $4.8 \%$, respectively. The presence of aneuploidy was a significant risk factor for subsequent detection of HGD or CRC with the hazard ratios of $12.7(p=0.017)$ and $12.0(p=0.019)$ based on the univariate and multivariate Cox models, respectively. No other risk factors, including age, gender, ethnicity, type of IBD, disease duration, endoscopic appearance, and primary sclerosing cholangitis, were associated with an increased risk for HGD or CRC.

Conclusions: IND may not be a low-risk condition for HGD or CRC as $30 \%$ of IND cases were found to have HGD or CRC within 7 years. In this regard, flow cytometric analysis of FFPE tissue can facilitate risk stratification of IND patients, as the finding of aneuploidy was highly predictive of subsequent detection of HGD or CRC. IND diagnosis in the setting of aneuploidy may even support a diagnosis of dysplasia and warrant more frequent follow-up surveillance intervals.

\section{DNA Flow Cytometric Analysis of Gastric Epithelial Dysplasia: Association of DNA Content Abnormality in Gastric Dysplasia with Development of High-Grade Dysplasia and Gastric Adenocarcinoma}

Kwun Wah Wen ${ }^{1}$, Peter S Rabinovitch ${ }^{2}$, Danning Huang ${ }^{3}$, Aras Mattis ${ }^{1}$ Gregory $Y$ Lauwers ${ }^{4}$, Won-Tak Choi'. 'University of California San Francisco, San Francisco, CA, ${ }^{2}$ University of Washington, ${ }^{3}$ SUNY Upstate Medical University, ${ }^{4} \mathrm{H}$. Lee Moffitt Cancer Center and Research Institute

Background: The diagnosis of gastric epithelial dysplasia (GED) can be challenging. To date, there is no reliable immunohistochemical or molecular technique that can reproducibly diagnose and/or risk stratify GED. Yet, such a tool would be helpful in designing optimal surveillance strategies. This study examines the utility of DNA content analysis in the diagnosis and risk stratification of GED using formalinfixed paraffin-embedded (FFPE) tissue.

Design: DNA flow cytometry was performed using FFPE gastric samples from 23 high-grade dysplasia (HGD; 17 biopsies and 6 resections) and 38 low-grade dysplasia (LGD; 35 biopsies and 3 resections). Twenty-four benign background mucosal samples (20 biopsies and 4 resections) from the same cohort (15 LGD and 6 HGD patients) were utilized as controls. Three to four 60-micron thick sections were cut from each block, and the dysplastic areas were dissected for analysis.

Results: DNA content abnormality (aneuploidy or elevated 4N fraction) was identified in $18(78 \%)$ of HGD, 5 (13\%) of LGD, and none in the non-dysplastic mucosa. One- and 4-year detection rates of HGD or gastric adenocarcinoma (GAC) for LGD patients with DNA content abnormality were $80 \%(p=0.003)$ and $100 \%(p=0.005)$, respectively whereas LGD patients in the setting of normal DNA content had 1$4-$, and 12 -year detection rates of $23 \%, 32 \%$, and $54 \%$, respectively. The univariate hazard ratio (HR) for subsequent detection of HGD or GAC for LGD patients with DNA content abnormality was 6.9 (p $=0.001)$. Patients with familial adenomatous polyposis $[n=12]$ or Lynch syndrome [ $n=2$ ] also showed an increased risk for HGD or GAC (HR $=9.7, p=0.029$ ), but only DNA content abnormality remained as a significant risk factor in the multivariate analysis $(H R=5.9, p=$ 0.005 ). No other risk factors, including age, gender, race, endoscopic appearance, intestinal metaplasia, and Helicobacter pylori infection, were associated with an increased risk for HGD or GAC. Among 18 HGD cases with DNA content abnormality, 13 cases (72\%) developed GAC within a mean follow-up time of 9 months (range: 0-109).

Conclusions: The presence of DNA content abnormality can identify a subset of LGD patients who may have greatest risk for subsequent detection of HGD or GAC. It can also provide confirmatory evidence of a morphologic suspicion of HGD. The majority of GED patients with DNA content abnormality developed HGD or GAC within a yea and thus may benefit from more thorough and frequent endoscopic surveillance.

\section{Histologic Predictors of Pouch Outcome: A Case- Control Study of Ulcerative Colitis Patients Status- Post Ileal Pouch-Anal Anastomosis}

Eric Willis ${ }^{1}$, Luca Stocchi ${ }^{2}$, Robert Odze ${ }^{3}$, Mariane G Camargo ${ }^{2}$ 
Alexandra Aiello², John Goldblum², Tracy HulR, Deepa T PatiR. ${ }^{1}$ Cleveland Clinic, East Cleveland, $\mathrm{OH},{ }^{2} \mathrm{Cleveland}$ Clinic, Cleveland, $\mathrm{OH},{ }^{3}$ Brigham and Women's Hospital, Boston, MA

Background: Pouch complications, such as pouchitis and pouch failure can cause significant morbidity in UC patients undergoing ileal pouch-anal anastomosis procedure. Unfortunately, there are no reliable clinical or histologic predictors of pouch outcome. Furthermore, a detailed analysis of ileal inflammation in UC patients and its impact on pouch outcome has not been performed. The goal of our study was to determine if histologic features in the colon and ileum from resections are predictive of pouch outcome.

Design: lleocolonic resections from 33 clinically confirmed UC patients (17 male, 16 female; mean age $36 \mathrm{yrs}$ ) who developed pouch complications [obstruction (33\%), chronic pouchitis $(36 \%)$, pouch failure $(6 \%)$, fistulae $(12 \%)$, anastomotic stricture/leak $(36 \%)$ ] upon follow-up (mean:15 mos) were compared to $42 \mathrm{UC}$ patients who did not develop pouch complications (controls; 28 male 14 female; mean age: 39 yrs) over a similar follow-up duration (mean:14 mos). A variety of histologic features including type, extent and degree of colonic and ileal inflammation (graded from 0-4), appendiceal involvement, pyloric gland metaplasia, Paneth cell metaplasia, granulomas and muscularis mucosae (MM) abnormalities (thickening, duplication) were recorded. Features only evaluated in the ileum were villous atrophy, length and extent of involvement. Duration of disease, history of primary sclerosing cholangitis, pre-surgery medications, and type of complication were noted. Colonic inflammation was graded in each anatomic segment (cecum, ascending, transverse, left colon and rectum) and the data was analyzed by anatomic segment and by the worse and average score for entire colon.

Results: There were no significant clinical differences between the cases and controls. There were also no significant differences in any of the histologic features, including the extent, type, or degree of inflammation within the colon or terminal ileum between the two groups, when correlated with pouch outcome as a group. However, when analyzed by type of complication, presence and severity of ileal inflammation $(p=0.05)$, villous atrophy $(p=0.006)$, ileal ulcers $(p=0.08)$ and abnormal MM ( $p=0.08)$ were significantly associated with anastomotic complications.

Conclusions: Although there are no histologic features that predict overall pouch outcome, presence and grade of ileal inflammation, including ulcers, villous atrophy, and MM abnormalities are specific histologic predictors of pouch anastomotic complications.

\section{Colon Cancer Molecular Profiling and Clinicopathological Features in a Large Urban Population}

Joseph Willis', Zhenghe Wang ${ }^{2}$, Harry Reynolds', Smitha Krishnamurthi', Lan Zhou', Sanford Markowitz', Kishore Guda², Navid Sadri', Li Li'. ' 'Cleveland Medical Center/ Case Western Reserve University, Cleveland, $\mathrm{OH},{ }^{2}$ Case Western Reserve University

Background: Colon cancer is one the most common lethal malignancies in the US. Mismatch repair status, KRAS, NRAS, BRAF are recognized as important prognostic and predictive markers, and are commonly obtained for routine patient care, frequently through NGS panels that include PIK3CA and TP53. This study explores the relationship of these molecular features with clinical outcomes in a large single institution.

Design: A retrospective analysis combining results from two multigene NGS studies as well as standard KRAS, Extended RAS [KRAS and NRAS] panels was performed. Adverse outcome was defined as Stage IV or recurrence. MSI cancers were excluded.

Results: 571 patients were included in the study - 76\% White, $23 \%$ Black; 403 had adverse outcomes, 118 were disease free at 5 yrs. Mutations results were: KRAS 51\%; NRAS 3\%; BRAF V600E 5\%; TP53 $70 \%$; PIK3CA $20 \%$. TP53mt was associated with left-sided vs rightsided cancer $[p<0.001]$, Ext RASmt $[p<0.01]$, PIK3CAmt $[p<0.01]$ but not BRAFmt. TP53mt was associated with overall adverse outcome $[p=0.01]$; in Stage II patients $[p=0.03]$ and the presence of $>8+$ lymph nodes [vs. $<3+$ nodes] $[p<0.001]$. Patients with BRAFmt/TP53mt cancers had worse outcomes than with BRAFmt/TP53wt cancers $[p=0.01]$. KRAS $G 12 \mathrm{~V} / \mathrm{G} 12 \mathrm{~A}$ mutations were significantly associated with adverse patient outcome [vs. all other KRAS mutations] [ $p=0.001]$. Overall ExtRAS results, even with stratification for TP53 status, were not associated with patient outcomes. Interestingly TP53mt/PIK3CAwt status was linked to adverse outcome [vs. TP53mt/PIK3CAmt] $[p=<0.01]$. No mutation/race associations were found.

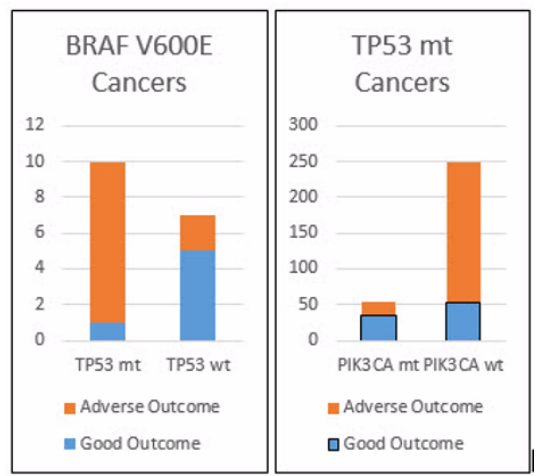

Conclusions: Analysis of routinely sequenced colon cancer molecular markers identifies unique biological subsets with significantly disparate clinical outcomes. Use of these markers in prognostic algorithms likely would be useful in identifying high-risk patients.

\section{Striking Heterogeneity of Driver Gene Mutations in Colon Adenomas}

Joseph Willis', Martina VeigR ${ }^{2}$, Alexander Miron'2, Kishore Guda², Salendra Singh ${ }^{2}$, Sanford Markowitz', Vinay 'Varadan', Harry Reynolds'. 'Cleveland Medical Center/ Case Western Reserve University, Cleveland, $\mathrm{OH},{ }^{2}$ Case Western Reserve University

Background: The driver gene mutational pathway of colon carcinogenesis from adenomas to high grade dysplasia [HGD] to cancer is well established. Our group and others have identified the major driver gene mutations found in colon cancers. Though the majo genomic components of the progression of adenomas to cancer have been identified, the mutational landscape of the driver gene mutation spectrum in colon adenomas has not been delineated.

Design: A 234 gene hybrid capture panel [Illumina TruSeq] of known and postulated colon cancer driver genes was constructed based on literature review. Colon adenomas with matching normal tissues were micro dissected, tested for microsatellite status and submitted for NGS using a validated informatics pipeline.

Results: 60 samples from 54 MSS polyps from 43 patients were successfully analyzed. 18 samples were from regions of HGD. 412 mutations in 149 genes were identified. The median mutation burden was 5 [range 1:63]. After APC and KRAS, only 8 genes, GNAS, GPR112, TCF7L2, CSMD3, HECW1, ADAMTSL3, TP53 and ZNF208 were mutated in $10 \%$ or more adenomas and 81 genes were mutated only once. 55 genes were only mutated in adenomas and 42 genes were only mutated in HGD. HGD was not associated with increased mutation burden. Four of 5 polyps with a TP53 mutation had HGD. Of the four samples harboring SMAD4 mutations, three were HGD. 12 adenomas from which cancer arose averaged 7 mutated genes with 2 having TP53 mutations. No clinicopathological associations, including polyp size, correlated with mutation burden. 117 colon cancers were also sequenced in the same manner and an average of 8 mutations per cancer were identified.

Conclusions: Colon adenomas contain a wide variety of mutations in known driver genes of diverse function. The overall mutation burden in many adenomas, both routine and HGD, is comparable to colon cancers. Similar to colon cancer, apart from a relatively well defined set of approximately $10-12$ genes, the vast majority of driver genes in colon adenomas are rarely mutated. These less frequently mutated genes encompass a broad array of cellular functions. The mutational profile of colon cancers mostly is reflective of the genomic events derived from its precursor adenoma.

\section{Lymphocytic Esophagitis in Adult Crohn's Disease is Characterized by Younger Age, Lower Incidence of Reflux Symptoms, and a CD4 Predominant Infiltrate}

Elizabeth Yiru Wu' ${ }^{1}$, Deepa T PatiR, Michael Drage ${ }^{3}$, Amitabh Srivastava ${ }^{4}$ ${ }^{1}$ Brigham and Women's Hospital, Brookline, MA, ${ }^{2}$ Cleveland Clinic Cleveland, $\mathrm{OH},{ }^{3}$ University of Rochester, Rochester, NY, ${ }^{4}$ Brigham and Women's Hospital, Boston, MA

Background: Lymphocytic esophagitis (LE) is characterized by increased intraepithelial lymphocytes in a predominantly peripapillary distribution and shows diverse etiological associations. A CD4 predominant lymphocytic infiltrate has been reported to be a useful marker for LE in the setting of motility disorders. LE may also be associated with Crohn's disease (CD) but the CD4:CD8 ratios have not been systematically evaluated in this setting and was the purpose of this study.

Design: Biopsies diagnosed as LE in patients with CD between 2011 2017 were used as cases while those diagnosed as LE in patients 
without a history of CD, over the same time period, comprised the control group. H\&E slides from all biopsies were reviewed again and CD4 and CD8 immunohistochemistry performed in all cases. Each case was recorded as CD4 or CD8 predominant or equivocal based on the immunohistochemical findings. Clinical parameters, including age at diagnosis (of LE and CD), gender, endoscopic findings, and the presence of co-morbid conditions (motility disorders, food allergies, and reflux symptoms) were collected by medical chart review. For patients with $C D$, data was also collected for any extraintestinal manifestations, disease distribution, treatment history and remission status.

Results: Demographic characteristics of LE patients with and without Crohn's disease are described in Table 1. Median age of Crohn's diagnosis was 23 (range 7-41 years); $16.7 \%$ of these patients had isolated small or large bowel involvement, $50 \%$ had ileocolonic involvement, and $33.3 \%$ had upper Gl involvement. A minority of patients $(26.9 \%)$ were in remission, and $33.3 \%$ of patients had extraintestinal manifestations. Patients presenting with LE in Crohn's disease were younger (median age 30; 18-64 years) compared to LE in non-CD controls (median $58 ; 18-83$ years, $\mathrm{p}<0.001$ ). Control LE patients were more likely to have a history of reflux symptoms $(55.2 \%)$ compared to those with CD $(33.3 \%), p=0.02$. The difference between the proportion of LE cases with and without CD that showed a CD4 predominant infiltrate was not statistically significant $(61.1 \%$ vs $41.1 \%$; $\mathrm{p}=0.15) .20 \%$ of controls, in comparison with $5.6 \%$ of CD patients had established diagnoses of motility disorders $(p=0.15)$

Table 1. Clinicopathologic characteristics of LE in Crohn's versus controls.

\begin{tabular}{|l|l|l|l|}
\hline & LE in Crohn's & LE controls & $\mathrm{p}$ value \\
\hline Sex $(\%$ male) & $44.4 \%$ & $35.7 \%$ & \\
\hline $\begin{array}{l}\text { Age at diagnosis of } \\
\text { lymphocytic esopha- } \\
\text { gitis (median, range } \\
\text { [yrs]) }\end{array}$ & $30(18-64)$ & $58(18-83)$ & $\mathrm{p}<0.001$ \\
\hline Biopsy location & $33.3 \%$ distal & $66.1 \%$ distal & $\mathrm{p}=0.006$ \\
\hline & $22.2 \%$ mid & $25 \%$ mid & \\
\hline & $22.2 \%$ proximal & $3.6 \%$ proximal & \\
\hline & $22.2 \%$ unspecified & $5.4 \%$ unspecified & \\
\hline $\begin{array}{l}\text { Symptoms at presen- } \\
\text { tation }\end{array}$ & $44.4 \%$ dysphagia & $53.6 \%$ dysphagia & $\mathrm{p}=0.29$ \\
\hline & $33.3 \%$ reflux & $35.7 \%$ reflux & \\
\hline & $16.7 \%$ none & $3.6 \%$ none & $\mathrm{p}=0.15$ \\
\hline $\begin{array}{l}\text { Positive history of } \\
\text { reflux }\end{array}$ & $5.6 \%$ other & $7.1 \%$ other & \\
\hline $\begin{array}{l}\text { Positive esophageal } \\
\text { dysmotility }\end{array}$ & $5.6 \%$ & $55.2 \%$ & $20 \%$ \\
\hline
\end{tabular}

Conclusions: Our findings show that LE in CD is characterized by a younger age at presentation, lower frequency of reflux, and a CD4predominant lymphoid infiltrate similar to what has been described in association with esophageal motility disorders.

\section{The age variation of HER2 immunohistochemistry positive rate in gastric cancer}

Yangfeng $X i^{1}$, Chen $X u^{2}$, Yiqiang Liu ${ }^{3}$, Xiaochu Yan ${ }^{4}$, Chuansheng Huang ${ }^{5}$, Yueping Liu $^{6}$, Jinhong Mei ${ }^{7}$, Zhe Wang ${ }^{8}$, Bin Liu ${ }^{9}$, Xiaoming $\mathrm{Li}^{10}$, Wencai Li1 $i^{11}$ Jianyun Lan ${ }^{12}$, Peng $\mathrm{GaO}^{13}$, Jifeng $\mathrm{Wu}^{14}$, Jianming Zheng $^{15}$, Guimei Qu ${ }^{16}$, Qi Sun ${ }^{17}$, Jingshu Geng ${ }^{18}$, Yejun Qin ${ }^{19}$, Jianping Liu $^{20}$, Daiqiang $\mathrm{Li}^{21}$, Guoping Wang ${ }^{22}$, Xiu Nie ${ }^{23}$, Yong Zhao ${ }^{24}$, Fan Zhang ${ }^{25}$, Yujun Li ${ }^{26}$, Xiaojun Zhou ${ }^{27}$, Jianghua Zhou ${ }^{28}$, Enshan Han ${ }^{29}$, Wenyong Sun ${ }^{30}$, Yinghong Yang ${ }^{31}$, Yinghao $Y u^{32}$, Qingkai $Y u^{33}$, Qing $\mathrm{L}^{34}$, Jianguo Źhang ${ }^{35}$, Rongge X'Xing ${ }^{36}$, Xiaowei Q ${ }^{37}$, Oiang' Liu ${ }^{38}$ Guoping Ren ${ }^{39}$, Lingfei Kong ${ }^{40}$, Xiaoyan Chen ${ }^{41}$, Hongyu Yu ${ }^{42}$, Meifu Gan $^{43}$, Hua Chen ${ }^{44}$, Guo-rong Chen ${ }^{45}$, Zhihong Zhang ${ }^{46}$, Mei Jin ${ }^{47}$, Hongwei Guan ${ }^{48}$, Yúfei Jiao ${ }^{49}$, Gang Chen ${ }^{50}$, Baocun Sun ${ }^{51}$, Chao Pan ${ }^{52}$, Lingchuan Guo ${ }^{53}$, Hualiang Xiao ${ }^{54}$, Xiaolong Jin ${ }^{55}$, Jianrong Wang ${ }^{56}$, Jiannong $W u^{57}$, Xuenong $L{ }^{58}$, Sheng Zhang ${ }^{59}$, Rong Ying ${ }^{60}$, Yonghong Shi ${ }^{61}$, Dianbin $\mathrm{Mu}^{62}$, Wenbin Huang ${ }^{63}$, Haiping Zhang ${ }^{64}$, Jinhui Shen ${ }^{65}$ Aijing Sun ${ }^{66}$, Lifeng Wang ${ }^{67}$, Xiaobing Li $i^{68}$, Lirong Chen ${ }^{69}$, Feng Tang ${ }^{70}$, Gang $X u^{71}$, Guoqing Pan ${ }^{72}$, Zhenzhu Sun ${ }^{73}$, Jingping Yun ${ }^{74}$, Guoping Zhong ${ }^{75}$, Zongkai Zou ${ }^{76}$, Ying Wang ${ }^{77}$, Jiping Da ${ }^{78}$, Jing Yuan ${ }^{79}$, Guifang Yang ${ }^{80}$, Xianghong Yang' ${ }^{81}$, Jinliang Ping ${ }^{82}$, Zhuo Wang ${ }^{83}$, Xiaofeng $L^{84}{ }^{84}$,

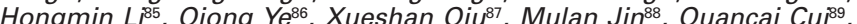
Wei Y ${ }^{90}$, Haiyan Zhang ${ }^{91}$, Bing Liao ${ }^{92}$, Xiaolan Xiao ${ }^{93}$, Dawei Ma Ma , Yuan $L_{u o}^{95}$, Hao Chen ${ }^{96}$, Hongwen Gao ${ }^{97}$, Yulan L98, Wanxin Wu ${ }^{99}$, Song $\mathrm{He}^{100}$, Zhihong Liu ${ }^{101}$, Yunjie Zeng ${ }^{102}$, Lifen Wang ${ }^{103}$, Yingyong Hou ${ }^{104}$. 'Shanxi Cancer Hospital, ${ }^{2} Z$ hongshan Hospital, Fudan University, Shanghai, ${ }^{3}$ Department of Pathology, Beijing Cancer Hospital, Beijing, China, ${ }^{4}$ Southwest Hospital, ${ }^{5}$ Jiangxi Cancer Hospital, ${ }^{6}$ Department of Pathology, The Fourth Hospital of Hebei Medical University, ${ }^{7}$ The First Affiliated Hospital of Nanchang University, Nanchang, Jiangxi,
${ }^{8}$ Fourth Military Medical University, Xi'an, Shaanxi, China, ${ }^{9}$ Lanzhou General Hospital of PLA, ${ }^{10}$ Lanzhou University Second Hospital, ${ }^{11}$ The First Affiliated Hospital of Zhengzhou University, ${ }^{12}$ Yancheng City No. 1 People's Hospital, ${ }^{13}$ Qilu Hospital of Shandong University, ${ }^{14}$ The First Affiliated Hospital of Anhui Medical University, ${ }^{15}$ Changhai Hospital ${ }^{16}$ Yuhuangding Hospital of Yantai, Shandong Province, ${ }^{17}$ Nanjing Drum Tower Hospital The Affiliated Hospital of Nanjing University Medical School, ${ }^{18} \mathrm{Harbin}$ Medical University Cancer Hospital, ${ }^{19} \mathrm{JiNan}$ ${ }^{20}$ West China Hospital, Sichuan University, ${ }^{21}$ The Second Xiangya Hospital of Central South University, ${ }^{22}$ Tongji Hospital Tongji Medical College of HUST, ${ }^{23}$ Union Hospital Tongji Medical College Huazhong University of Science and Technology, ${ }^{24}$ The First Affiliated Hospita of Chongqing Medical University, ${ }^{25}$ The First Affiliated Hospital of Wannan Medical College, ${ }^{26}$ The Affiliated Hospital of Qingdao University, ${ }^{27}$ Nanjing General Hospital of Nanjing Military Command ${ }^{28}$ Xiangya Hospital Central South University, ${ }^{29}$ General Hospital of Ningxia Medical University, ${ }^{30}$ Zhejiang Cancer Hospital, ${ }^{31}$ Department of Pathology, Fujian Medical University Union Hospital, ${ }^{32}$ Fuzhou General Hospital of Nanjing Military Command, ${ }^{33}$ Henan Cance Hospital, ${ }^{34}$ The First People's Hospital of Changzhou, Changzhou Jiangsu, ${ }^{35}$ Affiliated Hospital of Nantong University, ${ }^{36}$ Cangzhou Central Hospital, ${ }^{37}$ Affiliated Hospital, Jiangnan University, ${ }^{38}$ Renji Hospital Shanghai Jiaotong University School of Medicine, ${ }^{39}$ Zhejiang University, Hangzhou, ${ }^{40} \mathrm{Henan}$ Provincial People's Hospital, ${ }^{41} \mathrm{Fujian}$ Provincial Hospital, ${ }^{42}$ Changzheng Hospital, ${ }^{43}$ Taizhou Hospital of Zhejiang Province, ${ }^{44}$ Qingdao Municipal Hospital, ${ }^{45}$ Dept. of Pathology Wenzhou, Zhejiang, ${ }^{46}$ Jiangsu Province Hospital, ${ }^{47}$ Sir Run Run Shaw Hospital School of Medicine, Zhejiang University, ${ }^{48}$ First Affiliated Hospital of Dalian Medical University, ${ }^{49}$ The 2nd Affiliated Hospital of Harbin Medical University, ${ }^{50}$ Fujian Provincial Cancer Hospital, ${ }^{51}$ Tianjin Medical University Cancer Institute \& Hospital, 52Zhongshan Hospital Xiamen University, ${ }^{53}$ The First Affiliated Hospital of Soochow University, ${ }^{54}$ Daping Hospital, Research Institute of Surgery Third Military Medical University, ${ }^{55}$ Rui Jin Hospital Shanghai Jiao Tong University School of Medicine, ${ }^{56} \mathrm{Jiangsu}$ Province Hospital of TCM, ${ }^{57}$ Affiliated Hospital of Jiangsu University, ${ }^{58}$ Nan Fang Hospital, Southern Medical University, ${ }^{59}$ Fuzhou City, ${ }^{60}$ Gansu Provincial Cancer Hospital, ${ }^{61}$ The First Affiliated Hospital of Inner Mongolia Medical University, ${ }^{62}$ Shandong Cancer Hospital and Institute, ${ }^{63} \mathrm{Nanjing}$ First Hospital, ${ }^{64}$ The First Affiliated Hospital of Xaimen University, ${ }^{65}$ Shantou Central Hospital, ${ }^{66}$ Shaoxing People's Hospital, ${ }^{67}$ Xin Hua Hospital Affiliated to Shanghai Jiao Tong University School of Medicine, ${ }^{68}$ The 307th Hospital of Chinese People's Liberation Army, ${ }^{69}$ The Second Affiliated Hospital of Zhejiang University School of Medicine, ${ }^{70}$ Shanghai Huashan Hospital, ${ }^{71}$ Sichuan Academy of Medical Sciences \& Sichuan Provincial People's Hospital, ${ }^{72}$ First Affiliated Hospital of Kunming Medical University ${ }^{73}$ People's Hospital of Xinjiang Uygur Autonomous Region, ${ }^{74}$ Sun Yatsen University Cancer Center, ${ }^{75}$ Yinzhou Hospital Affiliated to Medical School of Ningbo University, ${ }^{76}$ Zhangzhou Municipal Hospital of Fujian Province, ${ }^{77}$ Sichuan Provincial Cancer Hospital, ${ }^{78}$ China-Japan Friendship Hospital, ${ }^{79}$ The General Hospital of People's Liberation Army(301 Hospital), ${ }^{80}$ Wuhan, ${ }^{81}$ Shengjing Hospital of China Medical University, ${ }^{82}$ Huzhou Central Hospital, ${ }^{83}$ Shaanxi Provincial People's Hospital, ${ }^{84}$ The First Affiliated Hospital of Xi'an Jiaotong University ${ }^{85}$ Tangshan People's Hospital, ${ }^{86}$ Wenzhou Central Hospital, ${ }^{87}$ The First Hospital of China Medical University, ${ }^{88} \mathrm{Beijing}$ Chao-yang Hospital ${ }^{89}$ Peking Union Medical College Hospital, ${ }^{90}$ Guizhou Provincial People's Hospital, ${ }^{91}$ The 2nd Affiliated Hospital \& Yuying Children's Hospital of Wenzhou Medical University, ${ }^{92}$ The First Affiliated Hospital, Sun Yat-sen University, Guangzhou, Guangdong Province, ${ }^{93}$ Hainan General Hospital, ${ }^{94}$ Jiangsu Cancer Hospital, ${ }^{95}$ Affiliated Cancer Hospital of Guangxi Medical University, ${ }^{96}$ The First People's Hospital of Lianyungang, ${ }^{97}$ The Second Hospital of Jilin University ${ }^{98}$ Affiliated Hospital of Hebei University, ${ }^{99}$ The First Hospital of Jiaxing, ${ }^{100}$ Nantong Tumor Hospital, ${ }^{101}$ Hunan Cancer Hospital, ${ }^{102}$ Guangzhou, ${ }^{103}$ The Second Hospital of Dalian Medical University, ${ }^{104}$ Department of Pathology, Zhongshan Hospital, Fudan University

Background: The HER2 positive rate of gastric cancer (GC) varied in different regions around the world. In China, studies with large sample number in this field were lacking. By exploring HER2 status and characteristics of GC in Chinese population in this large scale multicenter study, we hope to find new features of HER2 in GC.

Design: A total of 27787 biopsy specimens of GC from 103 hospitals were retrospectively obtained. Immunohistochemistry (IHC) staining of HER2 was performed on all the cases. HER2 status and its associations with key clinicopathological factors were analyzed.

Results: Overall HER2 IHC positive rate was $11.2 \%$. HER2 positive rate elevated with the increase of age in total patients and both genders (Figure.1A). The rates were $7.1 \%, 8.1 \%, 9.0 \%, 10.9 \%, 11.8 \%, 12.6 \%$ and $12.1 \%$ when patient age was $\leq 30,31-40,41-50,51-60,61-70,71-80$ and $>80$, respectively $(P<0.001)$. In male, the rates were $6.5 \%, 8.4 \%$ $9.6 \%, 11.5 \%, 12.4 \%, 13.3 \%$, and $12.1 \%(P<0.001)$. In female, the rates were $7.4 \%, 7.9 \%, 8.0 \%, 9.0 \%, 9.6 \%, 10.6 \%$, and $11.9 \%(P=0.128)$. The changes in male was more dramatic than in female $(P<0.001)$. Cases with $\geq 4$ tumor containing fragments demonstrated a higher HER2 IHC positive rate than those with $<4$ fragments, but with similar age variation in both sets $(P<0.001)$ (Figure. $1 \mathrm{~B})$. The intestinal type and diffuse type instead of the mixed type demonstrated the age variation(Figure. 2A, B , C). The proportion of the intestinal type GCs increased with age in total patients and both genders $(P<0.001)$, and 
in male the changes were more dramatic $(P<0.001)$ (Figure. $2 A)$. While the proportion of the diffuse type showed the opposite tendency to that of the intestinal type $(P<0.001)$ (Figure. 2B). HER2 IHC positive rate showed a positive correlation with the proportion of the intestinal type $(r=0.986, P<0.001)$ (Figure.2D), and a negative correlation with the proportion of the diffuse type $(r=0.984, P<0.001)$ (Figure. $2 E$ ).
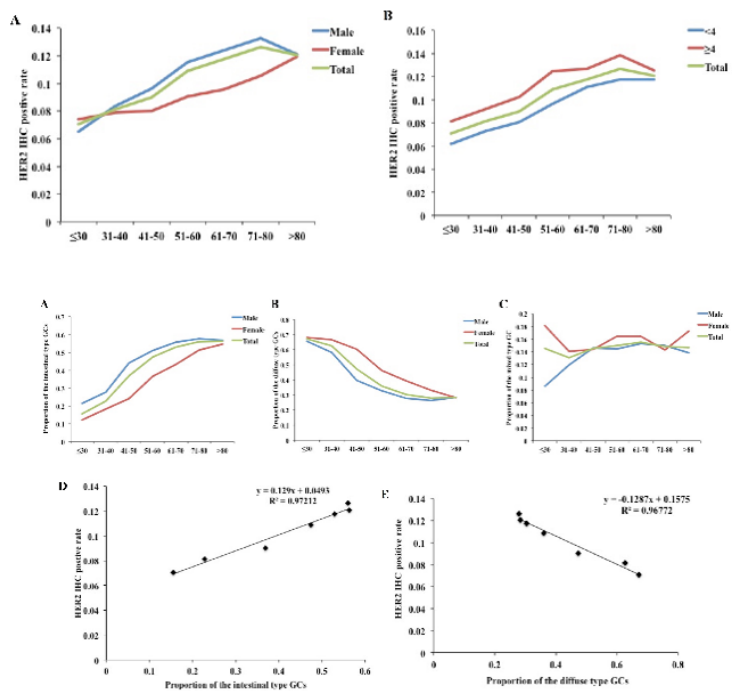

Conclusions: The HER2 IHC positive rate showed age variation in biopsy specimens of GC. In male the variation was more dramatic than in female. The variation of HER2 positive rate can be attributed to the age variation of the Lauren subtypes.

\section{Pathologic Patterns of Anti-PD-1 Induced Colitis}

Dongmei Xing ${ }^{1}$, James Kim², Robin Mendelsohn², Jaclyn Hechtman², Efsevia Vakiani', David Klimstra', Jinru Shia'. ' 'George Washington University, Washington, DC, ${ }^{2}$ Memorial Sloan Kettering Cancer Center, New York, NY

Background: Pembrolizumab and nivolumab are anti-PD1 monoclonal antibodies that show remarkable clinical activity across a number of different tumor types with less adverse events compared to CTLA-4 inhibitors such as ipilimumab. Ipilimumab-related colitis has been well described with established treatment using steroids and infliximab. The pattern and severity of anti-PD-1 induced colitis have not been well described. Here we characterize the pathologic findings of 25 patients with anti-PD-1 induced colitis from our institute.

Design: We performed a retrospective review of all patients at MSKCC who received nivolumab or pembrolizumab and underwent a colonoscopy or flexible sigmoidoscopy for evaluation of diarrhea or colitis during 2011-2017. We identified 28 biopsies from 25 patients with anti-PD-1 induced colitis. Clinical presentation, medical history, endoscopic findings, microbiology, pathologic findings, treatment and outcomes were recorded.

Results: The median age of subjects at the time of biopsy was 62 and $56 \%$ of the subjects were male. Of the patients included in this cohort, 18 received nivolumab and 7 received pembrolizumab. Primary cancers included melanoma $(10,40 \%)$, non small cell lung cancer $(4,16 \%)$, and renal cell carcinoma $(3,12 \%)$ etc. Onset of diarrhea occurred 7.7 months after drug initiation. $92 \%$ of the patients received steroids, with $25 \%$ requiring infliximab for steroid-refractory colitis. All patients had resolution of their symptoms after intervention. Upon histologic review, $32 \%$ of the patients had normal histology. Four pathologic patterns were identified: active colitis with cryptitis, chronic active colitis, collagenous colitis and lymphocytosis. Among them, acute colitis with cryptitis pattern has the highest frequency $(46.4 \%)$. There is no significant difference in pathologic findings between Nivolumab and Pembrolizumab; however the two cases of collagenous colitis were associated with pembrolizumab use only.

Conclusions: A wide range of histologic patterns can be seen with anti-PD-1 colitis including normal, active colitis, chronic active colitis, collagenous colitis and lymphocytosis. The differential diagnoses include inflammatory bowel disease, infection and other immunemediated disease processes. It is critical for pathologists to recognize these patterns because unlike other entities on the differential, all patients experienced resolution of their symptoms with either discontinuation of nivolumab/pembrolizumab or use of steroids/ infliximab.

877 Dual block HER2 assessment increased HER2 immunohistochemistry positive rate in resected specimens of gastric cancer: a prospective multicenter clinical trial from China
Rong Qin 6 , Lijuan Luan 7 Yingyong Hou ${ }^{7}$. 'Zhongshan Hospital, Fudan University, Shanghai, Shanghai, ${ }^{2}$ Cancer Hospital, Henan Province, ${ }^{3}$ Sir Run Run Shaw Hospital School of Medicine, Zhejiang University ${ }^{4}$ The first affiliated Hospital, Zhejiang University, ${ }^{5}$ The second affiliated Hospital, Zhejiang University, ${ }^{6}$ The First Affiliated Hospital of Anhui Medical University, 'Zhongshan Hospital, Fudan University

Background: HER2 assessment with two primary tumor blocks (dual block HER2 assessment) has been indicated to be an efficient and practical approach to increase HER2 positive rate in gastric cancer (GC) by our former single center studies. To further verify its value, the multicenter prospective clinical trial was conducted.

Design: The multicenter prospective clinical trial (NCT 02843412) was conducted at 6 Chinese hospitals from August 3,2016. A total of 2213 participants with primary GCs have been collected. Two primary tumor blocks were selected and recorded as block 1 and block 2 after histological evaluation. Blocks with the following features were given priority in the selection, containing an intestinal component and rich in tumor cells. An HER2 (4B5) rabbit monoclonal antibody was used for the immunohistochemistry (IHC) analysis.

Results: HER2 IHC positive (3+) rate with dual block assessment $(10.2 \%)$ was higher than that with single block assessment (block 1: $8.4 \%$, block 2: $8.5 \%)(\mathrm{P}<0.001)$. Compared with single-block assessment, dual-block assessment increased the positive rate by approximate $20 \%$. Similarly, HER2 equivocal $(2+)$ rate was increased in dual block assessment $(29.3 \%)$, which was higher than that in single block assessment (block 1: $23.2 \%$, block 2: $24.0 \%)(P<0.001)$. Conversely, dual block assessment demonstrated a lower HER2 negative $(0 / 1+)$ rate $(60.5 \%)$ than single block assessment (block1: $68.4 \%$, block 2: $67.1 \%)(P<0.001)$.

Conclusions: Dual block HER2 assessment effectively increased HER2 IHC positive rate in resected specimens of GC. We recommended dual block assessment be used routinely HER2 analysis in GC.

\section{Gene Expression Profiling in Gastric Cancer: Toward a Better Understanding of the Disease from the perspective of metabolic rearrangement}

Midie $\mathrm{Xu}^{1}$, Shujuan $\mathrm{Ni}^{2}$, Weiwei Weng', Hui Sun ${ }^{3}$, Qiongyan Zhang ${ }^{4}$ Weiqi Sheng ${ }^{5}$, Xiang $D u^{5}$. ${ }^{1}$ Shanghai, ${ }^{2}$ Shanghai, China, ${ }^{3}$ Fudan University Shanghai Cancer Center, Shanghai, "'Fudan University Shanghai Cancer Center, Shanghai, ${ }^{5}$ Fudan University Shanghai Cancer Center

Background: Metabolic rearrangement has been shown to be an important characteristic for stomach adenocarcinomas (STAD). Here we aimed to improve our understanding of the tumorigenesis of gastric cancer by means of gene and protein expression profile analysis with a focus on metabolic genes and pathways.

Design: Array-based gene expression profiling of fresh frozen cancer tissues and adjacent normal tissues were obtained from 8 patients with gastric cancer at early stage by using Affymetrix oligonucleotide microarray. Assays targeted 179 unique genes related to cance metabolism. The raw expression data were normalized using nSolver Analysis Software 3.0 and a dataset of gene expression ratios for GC vs. controls was generated. The $\mathrm{p}$ values were calculated using a paired t-test, and the threshold for up- and down-regulated genes was set at a fold change $>2.0$ and a $p$ value $<0.05$. Hierarchical clustering was performed based on differentially expressed gene using Multi Experiment Viewer (Dana-Farber Cancer Institute, MA). The protein expression of the dysregualted genes were detected by immunohistochemistry $(\mathrm{IHC})$ in the formalin fixed paraffin embedded (FFPE) tissue blocks obtained from the aforementioned 8 cases. The signal was quantified by the Allred score system which represented the estimated intensity and proportion of positive-staining cells.

Results: Our results showed increased expression of 9 metabolic genes (SLC2A3, HK3, PDGFRB, LDHA, CAD, G6PD, HIF1A, ENO1 and GLS) and decreased expression of 2 metabolic genes (ALDOB and JUN) in all cases of GC. Consistently, the protein expression leve of SLC2A3, HK3, PDGFRB, LDHA, CAD, G6PD, HIF1A, ENO1 and GLS are higher in GC tissues than normal gastric mucous, while ALDOB and JUN expression levels are lower in GC tissues than norma gastric mucous. Additionally, we noted most of dysregulated genes (7/11) in our GC population are implicated in Carbon Metabolism, a pivotal metabolic approach involving in nucleic acid biosynthesis. Furthermore, between these 11 altered genes, HIF1A and LDHA are cancer metabolism drivers, which means that both genes may induce the oncogenesis of gastric cancer. 

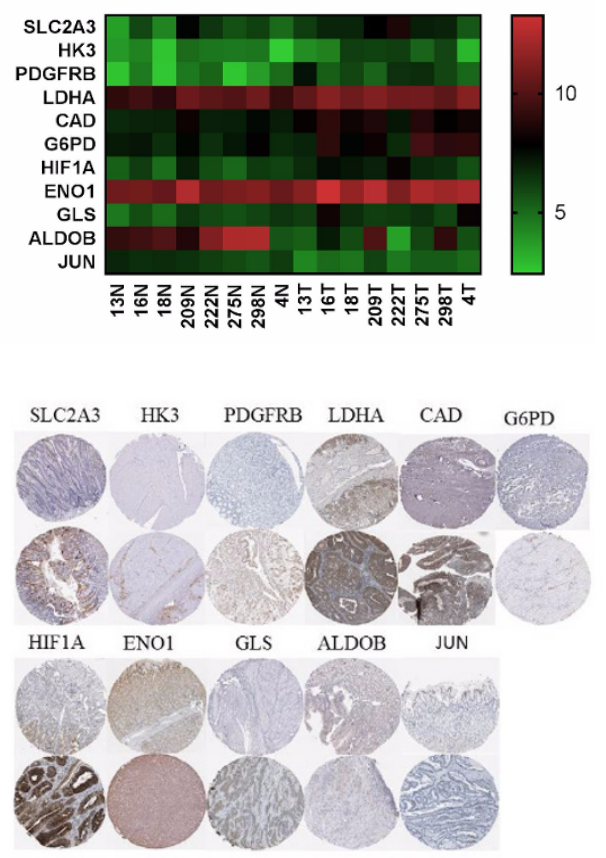

Conclusions: The gastric mucosa in GC at early stage is characterized by dysregulated expression of a limited repertoire of metabolic genes. The nature of the corresponding metabolic rearrangement and pathways may help guide further investigations into its etiology.

\section{Is Tumor Grade Associated with Lymph Node Metastasis in Small Intestinal Neuroendocrine Tumor?}

Liyan $X u^{1}$, Xuefeng Zhang'. ${ }^{1}$ Duke University, Durham, NC, ${ }^{2}$ Duke University Medical Center, Durham, NC

Background: The incidence of small intestinal neuroendocrine tumors (NET) has been increasing over the last several decades. In clinical practice, it is not uncommon to encounter low grade NET of small size that already developed lymph node (LN) metastasis at the time of being diagnosed. In this study, we aimed to find out whether there is an association between tumor grade and LN metastasis, and seek other clinicopathologic parameters that may be associated with LN metastasis in small intestinal NET.

Design: We used the data registries between 2004 and 2014 from Surveillance, Epidemiology and End Results (SEER) Program. Adult patients with small intestinal NET were screened. We excluded patients with no LN summited, no information of tumor size or extension, or undifferentiated NET. Positive lymph node metastasis was defined as metastatic NET in at least one lymph node. Tumor grade was based on variable "GRADE" in SEER data. Tumor extension was defined as below:

T1: tumor invades submucosa;

T2: tumor invades muscularis propria;

T3: Tumor invades into the subserosa;

T4: tumor perforates the visceral peritoneum or invades other organs or structures.

Results: Nine hundred and four cases were included in this study. Among these, $81 \%$ had at least one positive LN. In univariate analysis, $82.0 \%$ of well differentiated NET (568/693), $78.5 \%$ of moderate differentiated NET $(157 / 200)$, and $81.8 \%$ of poorly differentiated NET $(9 / 11)$ had LN metastasis, and there was no statistical difference (Fisher exact test, $\mathrm{p}=0.5$ ). In multivariate analysis, after other factors being adjusted, the status of positive LN was not associated with tumor grade $(p=0.28)$. The factors associated with LN metastasis included tumor size (>1 cm vs $\leq 1.0 \mathrm{~cm}$, adjusted odds ratio [aOR]: $4.4,95 \% \mathrm{Cl}$ : 2.77 to 7.03 . $\mathrm{P}<0.01$ ), advanced tumor extension (T2 vs T1, aOR: 1.3 , $95 \% \mathrm{Cl} 0.74$ to 2.43 ; T3 vs T1 aOR: $2.77,95 \% \mathrm{Cl}: 1.58$ to 4.87 ; T4 vs T1 aOR: $3.84,95 \% \mathrm{Cl}: 1.94$ to $7.60 . \mathrm{p}<0.01$ ), younger age (age decrease by one year, aOR: $1.02,95 \% \mathrm{Cl}: 1.00$ to $1.03, \mathrm{p}=0.03$ ) and number of sampled LN ( $\geq 12$ vs. $<12$, aOR: $3.5,95 \% \mathrm{Cl}: 2.37$ to 5.30 . $\mathrm{p}<0.01$ ).

Tumor grade is not associated with LN metastasis in small intestinal NET in either univariate or multivariate analysis after other factors being adjusted. Adequate LN sampling $(\geq 12)$ is important for detecting LN metastasis.
880 Growth Pattern Of Invasive Ampullary Carcinomas As Demarcated Versus InfitIrative Has Significant Prognostic Correlation; A Clinicopathologic Analysis Of 257 Cases

Yue Xue 1 Burcin Pehlivanoglu², Michelle Reid", Alyssa Krasinskas ${ }^{2}$ Serdar Balci ${ }^{3}$ Grace Kim ${ }^{4}$ Drew Davis ${ }^{5}$. Huimin Liu ${ }^{6}$, Limin Peng ${ }^{7}$ Takuma Tajiri ${ }^{8}$, Nobuyuki Ohike ${ }^{9}$, Juan M Sarmiento ${ }^{7}$, Bassel El-Rayes ${ }^{7}$, Susan Tsai ${ }^{10}$, Kathleen K Christians ${ }^{10}$, Douglas Evans ${ }^{10}, N$. Volkan Adsay ${ }^{10}$. ${ }^{1}$ Emory University Hospital, Atlanta, GA, ${ }^{2}$ Emory University, Atlanta, GA, ${ }^{3}$ Ankara, Turkey, ${ }^{4}$ Univ. of California, San Francisco, San Francisco, CA, ${ }^{5}$ Clarkston, GA, ${ }^{6} \mathrm{Emory}$, ${ }^{7} \mathrm{Emory}$ University, ${ }^{8}$ Decatur, GA, ${ }^{9}$ SHOWA University, ${ }^{10}$ Medical College of Wisconsin, Milwaukee, $\mathrm{WI}$

Background: It is becoming increasingly clear that infiltration patterns of carcinomas, not only in the advancing edge (budding, micropapillary, etc), but also of the overall growth pattern is an important reflection of the tumor biology, and classifications proposed accordingly have been found to have significant prognostic value in some organs.

Design: Parallel to the classification schemes proposed in colon (PMID: 24600585: PMID: 25648412), 257 invasive ampullary carcinomas (selected by the recently refined criteria) were analyzed based on the growth pattern as " $A$ " for demarcated tumors with only limited (if any) small cluster spreading away from the boundaries of the main tumor, and " $\mathrm{C}$ " highly infiltrative pattern with several carcinoma units away from the tumor and dissection of normal tissue planes. Cases with borderline characteristics, or those with both $\mathrm{A}$ and $\mathrm{C}$ patterns in different areas of the tumor were classified as " $\mathrm{B}$ ".

Results: The clinicopathologic associations of the three growth patterns are documented in the Table 1. Circumscribed carcinomas had significantly larger overall tumor size but were significantly less likely to show vascular and perineural invasion, were lower stage tumors ( $T 1 / 2 \mathrm{vs} 3 / 4)$, and had a tendency to have lower incidence of lymph node metastasis. They also had a tendency to occur more in women. No surprisingly, circumscribed tumors were more commonly intestinal type and infiltrative ones, pancreatobiliary. The circumscribed tumors also appeared to have significantly better behavior (statistically significant in univariate analysis) although this was not independent of the other parameters in the multivariate analysis in which only LVI, age, LN status were found to be independent (along with T stage, but only when evaluated as $T 1 / 2$ vs $3 / 4$ ). Histologic typing was also not independent, and of note, its prognostic relevance seemed to show slightly weaker marginal association with survival as compared to growth pattern (histologic: $\mathrm{HR}, 1.6, \mathrm{Cl} 1.0-2.5, \mathrm{p}=0.02$; growth: HR, $1.8,95 \% \mathrm{Cl} 1.2-2.6, \mathrm{p}=0.001)$. Histologically borderline/hybrid cases appeared to have in-between features although they seemed to be closer to the circumscribed category in many aspects. 
TABLE 1. Univariate Analysis of Correlation of growth patterns A, B, C with the clinicopathologic features of invasive ampullary carcinomas

\begin{tabular}{|c|c|c|c|c|}
\hline & $\begin{array}{l}\text { Pattern A } \\
(n=14)\end{array}$ & $\begin{array}{l}\text { Pattern B } \\
(n=94)\end{array}$ & $\begin{array}{l}\text { Pattern C } \\
(n=149)\end{array}$ & $P$ value \\
\hline $\begin{array}{l}\text { Age } \\
\text { (Mean) }\end{array}$ & 65 & 65 & 65 & 0.57 \\
\hline $\begin{array}{l}\text { Gender } \\
\text { M }\end{array}$ & $\begin{array}{l}3(15 \%) \\
11(85 \%)\end{array}$ & $\begin{array}{l}34(35 \%) \\
60(65 \%)\end{array}$ & $\begin{array}{l}66(43 \%) \\
83(57 \%)\end{array}$ & 0.09 \\
\hline $\begin{array}{l}\text { Histologic } \\
\text { classification } \\
(\%) \\
\text { PB } \\
\text { INT }\end{array}$ & $\begin{array}{l}0 \\
10(100 \%)\end{array}$ & $\begin{array}{l}34(45 \%) \\
42(55 \%)\end{array}$ & $\begin{array}{l}110(89 \%) \\
13(11 \%)\end{array}$ & $<.001$ \\
\hline $\begin{array}{l}\text { Invasive size } \\
(\mathrm{mm}) \\
(\text { Mean } \pm \mathrm{SD} \text { ) }\end{array}$ & $21.8 \pm 12.3$ & $22.6 \pm 16.8$ & $20.8 \pm 9.5$ & 0.50 \\
\hline $\begin{array}{l}\begin{array}{l}\text { Overall tumor } \\
\text { size }(\mathrm{mm})\end{array} \\
(\text { Mean } \pm \mathrm{SD})\end{array}$ & $40.8 \pm 14.9$ & $31.9 \pm 19.2$ & $24.7 \pm 13.0$ & $<0.001$ \\
\hline $\begin{array}{l}\text { T stage } \\
1+2 \\
3+4\end{array}$ & $\begin{array}{l}10(83 \%) \\
2(17 \%)\end{array}$ & $\begin{array}{l}64(70 \%) \\
28(30 \%)\end{array}$ & $\begin{array}{l}51(35 \%) \\
95(65 \%)\end{array}$ & $<0.001$ \\
\hline $\begin{array}{l}\text { Positive LNs } \\
0 \\
1\end{array}$ & $\begin{array}{l}7(64 \%) \\
4(36 \%)\end{array}$ & $\begin{array}{l}52(60 \%) \\
35(40 \%)\end{array}$ & $\begin{array}{l}62(45 \%) \\
76(55 \%)\end{array}$ & 0.06 \\
\hline $\begin{array}{l}\mathrm{PNI} \\
0 \\
1\end{array}$ & $\begin{array}{l}12(92 \%) \\
1(8 \%)\end{array}$ & $\begin{array}{l}68(74 \%) \\
24(26 \%)\end{array}$ & $\begin{array}{l}72(49 \%) \\
74(51 \%)\end{array}$ & $<0.001$ \\
\hline LVI & $\begin{array}{l}7(54 \%) \\
6(46 \%)\end{array}$ & $\begin{array}{l}35(38 \%) \\
57(62 \%)\end{array}$ & $\begin{array}{l}34(23 \%) \\
112(77 \%)\end{array}$ & 0.008 \\
\hline $\begin{array}{l}\text { Survival rate } \\
\text { 3-year } \\
\text { 5-year }\end{array}$ & $\begin{array}{l}71 \% \\
50 \%\end{array}$ & $\begin{array}{l}71 \% \\
62 \%\end{array}$ & $\begin{array}{l}51 \% \\
38 \%\end{array}$ & 0.006 \\
\hline
\end{tabular}

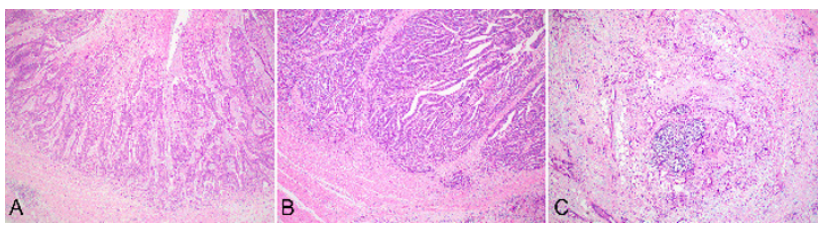

Conclusions: Similar to other organs, tumor growth pattern (circumscribed versus infiltrative) has significant clinicopathologic associations in invasive ampullary carcinomas, and appears to have prognostic implications. This characteristic needs to be considered in prognostic evaluation of ampullary cancers.

\section{Genomic Profiling of EBV-Positive and EBV- Negative Gastric Carcinoma Using Targeted Next Generation Sequencing}

Nour Yadak', Joshua Routh ${ }^{2}$, Kassandra Jiron³, Mohammad Vasef, 'University of Pittsburgh Medical Center, Homestead, PA, ${ }^{2}$ University of New Mexico, Albuquerque, NM, ${ }^{3}$ TriCore Reference Laboratories

Background: Gastric carcinoma (GC) remains one of the most common cancer type worldwide. About $10 \%$ of GC harbors EBV. EBV-associated GC (EBV-GC) accounts for one of 4 major molecular classes per The Cancer Genome Atlas (TCGA). Given the unique demographics in New Mexico, we analyzed the incidence of EBV in $\mathrm{GC}$ and performed targeted next generation sequencing (NGS) of 50 common oncogenes and tumor suppressor genes in both EBV-GC and selected EBV-negative GC

Design: Three tissue microarrays (TMAs) composed of $2 \mathrm{~mm}$ in diameter cores were constructed using archived formalin fixed paraffin embedded tissue (FFPE) of previously confirmed gastric carcinoma cases. In situ hybridization using EBV-encoded small RNA probes (EBER) was performed on TMAs. For targeted pane sequencing analysis, all EBV-positive cases along with 7 EBV-negative cases were selected and the corresponding FFPE tissue blocks were pulled. DNA was extracted from the recuts of tissue blocks and was subjected to multiplex PCR followed by library preparation using lon AmpliSeq Library Kit. The libraries underwent emulsion PCR after an equalization step and were loaded on a 318 Chip for sequencing on Ion Torrent PGM system. The sequence data were then analyzed using the Ion Torrent Suite and NextGENe software.

Results: Abundant expression of EBER was identified in 4 of $60(7 \%)$ of GC cases including in 3 patients of Hispanic origin. Targeted gene panel sequencing demonstrated 1-2 pathogenic mutations in 7 of 11 $(60 \%)$ cases including PIK3CA in 4, TP53 in 3, CTNNB1 in 2, and ERBB2 (HER2) in 1. The results are summarized in Table.

\begin{tabular}{|l|l|l|l|l|l|l|l|}
\hline Cases & $\begin{array}{l}\text { Histo- } \\
\text { logic } \\
\text { Subtype }\end{array}$ & Ethnicity & EBER & PIK3CA & CTNNB1 & TP53 & ERBB2 \\
\hline 1 & Intestinal & Asian & Positive & - & S37A & - & - \\
\hline 2 & Intestinal & Hispanic & Positive & R880 & G34E & & \\
\hline 3 & Intestinal & Hispanic & Positive & - & - & R175H & D769Y \\
\hline 4 & Mixed & Hispanic & Positive & - & - & - & - \\
\hline 5 & mixed & Hispanic & $\begin{array}{l}\text { Nega- } \\
\text { tive }\end{array}$ & H1047R & - & - & - \\
\hline 6 & Intestinal & Hispanic & $\begin{array}{l}\text { Nega- } \\
\text { tive }\end{array}$ & E545K & - & - & - \\
\hline 7 & Diffuse & $\begin{array}{l}\text { Native } \\
\text { American }\end{array}$ & $\begin{array}{l}\text { Nega- } \\
\text { tive }\end{array}$ & E545K & - & R175L & \\
\hline 8 & Intestinal & $\begin{array}{l}\text { Native } \\
\text { American }\end{array}$ & $\begin{array}{l}\text { Nega- } \\
\text { tive }\end{array}$ & - & - & R2480 & - \\
\hline 9 & Diffuse & $\begin{array}{l}\text { Native } \\
\text { American }\end{array}$ & $\begin{array}{l}\text { Nega- } \\
\text { tive }\end{array}$ & - & - & - & - \\
\hline 10 & Diffuse & $\begin{array}{l}\text { Native } \\
\text { American }\end{array}$ & $\begin{array}{l}\text { Nega- } \\
\text { tive }\end{array}$ & - & - & - & - \\
\hline 11 & Diffuse & Hispanic & $\begin{array}{l}\text { Nega- } \\
\text { tive }\end{array}$ & - & - & - & - \\
\hline
\end{tabular}

Conclusions: The results of our study indicate that EBV-GC accounts for approximately $7 \%$ of GC in our patient cohort similar to the one reported previously. The most frequently mutated genes in our GC cohort identified by NGS included PIK3CA (36\%) TP53 (27\%) and CTNNB1 (18\%). The frequently mutated PIK3CA and TP53 as identified in our study are in keeping with those of the TCGA published data. However, the frequency of PIK3CA mutations in EBV-GC in our limited EBER-positive cases appears less compared to that previously reported in TCGA data. In addition, the presence of CTNNB1 mutations in half of our EBV-GC appears to be a novel finding and not previously reported. Furthermore, we identified an actionable ERBB2 mutation in one of our 11 cases. The latter finding suggests that ERBB2 mutationa testing may prove useful in identifying a subset of GC cases with ERBB2 mutation for potential anti-HER2 targeted therapy.

\section{An IHC Evaluation of Multiple Immunocheckpoint Marker and Mismatch Repair Gene Expression in Colorectal Carcinoma}

George Yang', Sara L Figueroa ${ }^{2}$. $S a n$ Francisco, CA, ${ }^{2}$ Biocare Medical, Pacheco, CA

Background: The recent success of checkpoint inhibitor therapies in clinical oncology practice has generated overwhelming enthusiasm for immunotherapeutics. Recent studies have suggested colorectal carcinoma (CRC) patients with mismatch repair deficiency (dMMR)/ microsatellite-instable high (MSI-H) may benefit from anti-PD-1/ PD-L1 inhibitors. In this study, we have evaluated a large panel of immunocheckpoint markers on FFPE colorectal carcinoma tissues.

Design: A panel of dMMR protein markers and immunocheckpoint antibodies (PD-L1, PD-1, LAG-3, IDO1, CTLA-4, FOXP3, CD4, CD8, CD137 and T-bet) were tested by IHC on 21 CRC cases consisting of MSI-H CRCs $(n=11)$ and microsatellite-stable (MSS) CRCs $(n=10)$. Sections were reviewed via H\&E to confirm the presence of adequate pathological features including histology, grade and the abundance of tumor-infiltrating lymphocytes (TILs). Loss of dMMR protein expression and thus MSI-H classification was determined by MLH1, MSH2, MSH6 and PMS2 IHC panel result. Expression of immunocheckpoint markers in TILs was categorized in the following manner: 0 (negative, no expression), 1 (scattered, $<5$ cells/200X), (weak, 5-20 cells/200X), 3 (moderate, 21-50 cells/200X) and 4 (high, $>50$ cells/200X) 
Results: Only two cases of MSI-H showed $10-30 \%$ positive PD-L1 tumor cell expression and the remaining nine cases were negative. $50 \%$ of the MSS CRCs exhibited $10-30 \%$ positive PD-L1 tumor cell expression. All cases of MSI-H and MSS demonstrated high expression of CD4, CD8, CTLA-4 and ID01 in TILs whereas CD137, FOXP3 and T-Bet were variable, from scattered to high expression. $50 \%$ of MSS cases were negative for LAG-3 and $50 \%$ exhibited a range of scattered to weak expression. For MSI-H CRCs ( $\mathrm{n}=11)$, two cases were negative for LAG3 , three cases exhibited scattered expression, five were weak and one case showed high expression of LAG-3. PD-1 expression in MSI-H was high in seven cases, moderate in two cases, weak in one case and scattered in one case. Similar PD-1 expression occurred in MSS CRCs $(n=10)$ with high expression in five cases, moderate expression in three cases, weak in one and scat

Conclusions: In this study, more MSS expressed PD-L1 than MSI-H. TIL PD-1 expression showed little to no difference in either MSI-H or MSS CRCs. There appears no significant difference in TIL expression of immunocheckpoint markers in MSI-H CRCs versus MSS CRCs. Further study of expression patterns of immunocheckpoint markers may provide useful information for clinical patient management.

\section{Molecular Pathogenesis and Genomic Evolution of Pyloric Gland Adenomas by Next Generation Sequencing}

Lindsay Yassan', Sabah Kadri', Sushant A Pati ', Lauren L Ritterhouse ${ }^{1}$ Lindsay Alpert', Christopher $R$ Weber ${ }^{2}$, Shu-Yuan Xiao ${ }^{3}$, John Hart ${ }^{1}$ Jeremy Segal', Namrata Setia'. 'University of Chicago, Chicago, IL, ${ }^{2}$ The University of Chicago, Oak Park, IL, ${ }^{3}$ Univ of Chicago Med Ctr, Chicago, IL

Background: Pyloric gland adenoma (PGA) is an under-recognized differentiated gastric dysplasia exhibiting a pyloric phenotype. These adenomas frequently exhibit low- and high-grade dysplasia and are rarely associated with invasive carcinoma. Autoimmune gastritis and hereditary cancer syndromes including familial adenomatous polyposis and Lynch syndrome are risk factors for the development of PGAs. Targeted mutation analysis shows characteristic activating GNAS and KRAS mutations in these tumors. In this study, we investigated molecular alterations in PGAs by next-generation sequencing (NGS).

Design: Cases with the following diagnoses were collected from the pathology archives between 2000 and 2017: PGAs with lowgrade dysplasia (PGA-L), PGAs with high-grade dysplasia (PGA-H) and invasive carcinoma in the setting of PGA (PGA-C). A clinical targeted NGS panel was employed to detect mutations, insertions, and deletions in 147 genes as well as copy number variations (CNVs) for 136 autosomal genes. Molecular data was appropriately analyzed, and molecular analysis of microsatellite instability was performed in select cases.

Results: The average patient age at the time of diagnosis was 74.4 years (range 53 to 83), and the average PGA size was $39 \mathrm{~mm}$ (range 5 to $100 \mathrm{~mm}$ ). All PGAs were gastric in origin (43\% gastroesophageal junction, $43 \%$ cardia, $14 \%$ body). Relevant clinicopathologic features are summarized in Figure 1 (top). Mutations and CNVs are demonstrated in Figure 1 (bottom). The study identified multiple CNVs not previously described in PGAs. Several new mutations were seen (Figure 1) while previously described GNAS, KRAS, and APC mutations were confirmed. A variant mutation of uncertain significance was seen in MSH6 in 4 paired specimens from 2 patients, although the adenomas were microsatellite stable. Increased CNVs and gain of MDM2 were seen in $\mathrm{HG}$ adenomas from paired specimens (PGA-LG and -HGD and PGA-H and PGA-C). PGA-C was seen to have a KRAS mutation absent in the associated HG adenoma.

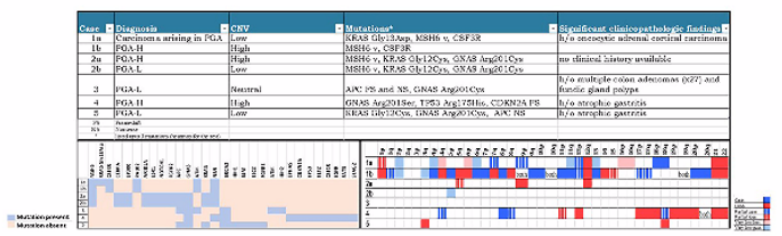

Conclusions: NGS confirms the importance of GNAS and KRAS mutations in the pathogenesis of PGA but also potentially implicates additional pathogenic pathways based on new mutations and copy number alterations. Validation of these findings in a larger cohort is needed to determine their significance.
884 Topography, Morphology and Etiology of Lymphocytic Gastritis, A Single Institution Experience

Raymond H Yip ${ }^{1}$, Lawrence Lee ${ }^{2}$, Lik Hang Lee ${ }^{3}$, David Schaeffer ${ }^{4}$ Hui-Min Yang'. 'Vancouver General Hospital, University of British Columbia, Vancouver, BC, ${ }^{2}$ University of British Columbia, Vancouver, BC, ${ }^{3}$ St. Paul's Hospital, University of British Columbia, Vancouver, BC, ${ }^{4}$ Vancouver General Hospital, Vancouver, BC

Background: Lymphocytic gastritis (LG) is an uncommon reaction pattern of injury characterized by increased intraepithelia lymphocytosis of the surface epithelium and lamina propria chronic inflammation of the stomach. LG most commonly occurs in association with gluten-sensitive enteropathy (GSE), H. Pylori gastritis, NSAID use and microscopic colitis. While the topography of LG has been described in GSE and $\mathrm{H}$. Pylori infection, no morphologic features have been used to subcategorize LG based on possible etiologies. In particular, while LG with atrophy has been observed in clinical practice, few reports of this finding are available. Furthermore, new immunotherapy agents have been associated with lymphocytic infiltrate in the gastrointestinal tract, but their association with LG is unknown.

Design: Cases of lymphocytic gastritis diagnosed at our institution in the recent six year period were confirmed via histologic assessment by two study pathologists, following approval by the research ethics board. Topography and morphologic features (atrophy, intestina metaplasia, endocrine cell hyperplasia, H. Pylori colonization) were assessed. Findings of GSE ( $\geq$ Marsh 3 ) were recorded in concomitant duodenal biopsies. Additional clinical information (GSE serology, pernicious anemia, history of $\mathrm{H}$. Pylori, microscopic colitis, lymphoma, Menetrier's disease, medication) was obtained from medical records.

Results: Of 27 total cases of LG, GSE was identified in 33\% (8/27) 3 of 4 GSE cases with both antral and body sampling showed antral predominant LG without atrophy, intestinal metaplasia or endocrine cell hyperplasia. $19 \%(5 / 27)$ of cases were attributed to NSAID injury, and showed antral predominant, body predominant and pan-gastritis pattern of injury. One case occurred in the setting of previous $\mathrm{H}$. Pylori infection, and one case in association with melanoma immunotherapy. In $44 \%$ of cases, the underlying cause of LG could not be identified. Of four cases with oxyntic atrophy, the cause was identified for only one case (H. pylori infection). Of note, no cases were associated with macrocytic anemia or B12 deficiency.

Conclusions: GSE was the predominant cause of lymphocytic gastritis, followed by NSAID injury. H. pylori infection is an uncommon cause for the diagnosis of LG. In addition, a previously unreported possible association with melanoma immunotherapy was found Topography and morphology of lymphocytic gastritis may point to the cause of injury, allowing for proper treatment of underlying disease.

885 Five-stain-based approximation of molecular subtyping in gastric adenocarcinoma and its prognostic significance in a single institution cohort

Ju-Yoon Yoon', Catherine Streutker2, ${ }^{1}$ University of Toronto, Toronto, ON, ${ }^{2}$ St. Michael's Hospital, Toronto, ON

Background: The cancer genome atlas (TCGA) study identified four molecular subtypes of gastric carcinoma, namely Epstein-Bar virus (EBV)-positive, microsatellite instability (MSI), chromosome instability (CIN) and genomically stable (GS) subtypes. The TCGA clustering was robust, reflecting the biological differences between the subtypes. Despite the robust clustering seen in the TCGA dataset, the molecular subtyping did not show a significant prognostic value In contrast, a number of studies from other groups have proposed different molecular subtypes, which were prognostically significant in large cohorts.

Design: We accrued a cohort of patients treated with gastrectomy or endoscopic mucosal resection (EMR). Cases of gastric and gastroesophageal junction carcinoma from 2001 to 2011 were identified, and a tissue microarray containing 107 cases was constructed.

Results: We employed a subtyping algorithm, based on the TCGA algorithm, first identifying the EBV-CIMP cases, identified by EBER positivity. The MSI subtypes were identified through immunohistochemistry (IHC) for mismatch repair (MMR) pathway proteins, MLH1, PMS2, MSH2 and MSH6. Among the remaining cases, the distinction between CIN and GS subtypes was made based on combined assessment of the histology (diffuse vs. others).

Using our simplified algorithm, our cohort of 107 cases of gastric carcinoma was divided into 3 cases of EBV-positive, 23 cases of MSI, 27 cases of GS and 54 of CIN tumours. $87 \%$ of the tumours with diffuse histology were classified as GS subtype, which was notable for younger age at diagnosis (median 64 vs. 70.5 years for others) Examining for characteristic molecular features of the TCGA subtypes, aberrant p53 immunostaining (null or aberrantly high) was seen most frequently in the CIN subtype (46\% in CIN vs. $8 \%$ in others), whereas ARID1A loss was rarely seen ( $6 \%$ vs. $35 \%$ in others). HER2 overexpression (3+ immunostaining) was seen exclusively in CIN 
tumours, seen in $17 \%$ of CIN tumours.

When we compared overall survival (OS) in our cohort with a mean follow-up period of 34 months, 9 deaths occurred among GS cases $(9 / 27,33 \%)$ compared to $17 / 80(21 \%)$ for other subtypes. This difference was not statistically significant by log-rank analysis.

Conclusions: Taken together, our modified algorithm categorizes gastric carcinomas into subtypes that reflect a number of clinical and molecular features of the TCGA subtypes. Worse OS was seen with the GS subtypes, although the difference was not statistically significant.

\section{Loss of Expression of MLH1 in Non-Dysplastic Crypts of Colonic Sessile Serrated Polyps is a Harbinger of the Development of Cytologic Dysplasia in these Polyps}

Masato Yozu1, Odise Cenaj², Robert Odze ${ }^{2}$, Joseph Misdraji!. ${ }^{1}$ Massachusetts General Hospital, Boston, MA, 'Brigham and Women's Hospital, Boston, MA

Background: Sessile serrated polyps (SSPs) with cytologic dysplasia (CD) have been shown to have loss of MLH1 in the CD component in up to $70 \%$ of patients. We have noted loss of MLH1 expression in non-dysplastic crypts (NDCs) in some SSPs but the significance of this finding is currently unknown. We hypothesized that loss of MLH1 in NDCs is an early change and maybe a harbinger for the development of CD. The purpose of this study is to evaluate the prevalence, associations, and biological significance of MLH1-deficient NDCs in SSPs with and without CD.

Design: Four hundred SSPs including 158 SSPs without CD (SSPND) and 242 SSPs with CD (SSP-CD) from 288 patients (M:F=38:62, mean age 66) were evaluated immunohistochemically for MLH1 loss in both non-dysplastic and dysplastic portions of the polyps. The number of foci and their location with respect to areas of CD were noted. Endoscopic parameters (polyp size and location) were also collected.

Results: Overall, 71 of 400 (18\%) SSPs showed MLH1-deficient NDCs. 41 of $166(25 \%)$ SSPs $\geq 10 \mathrm{~mm}$ showed MLH1-deficient NDCs compared to 30 of $234(13 \%)$ in SSAs $<10 \mathrm{~mm}(\mathrm{p}<0.003)$. SSPs with and without MLH1 loss in NDCs were equally likely to be right-sided $194 \%$ vs $90 \%, p=0.12)$. SSP-ND in patients who had a concomitant colorectal carcinoma were more likely to have at least one focus of MLH1 loss in NDCs than patients without colorectal carcinoma $(7.7 \%$ vs. $1.3 \%$, $\mathrm{p}=0.02$ ). MLH1-deficient NDCs were significantly more common in SSP-CD compared to SSP-ND (25\% vs $7 \%$, p<0.0001). Among SSPCD, polyps with MLH1-deficient CD were more likely to show MLH1 loss in NDCs than were SSP-CD in which the CD had preserved MLH1 expression ( $66 \%$ vs. $8.1 \%, p<0.0001)$, and the former had more foci of MLH1-deficient NDCs (3.6 foci vs 1.1, p<0.008). Interestingly, MLH1deficient NDCs were significantly more likely to be contiguous with CD when the dysplasia was also MLH1-deficient: in SSPs with MLH1deficient CD, 43/164 (26\%) foci of NDCs with MLH1 loss were adjacent to CD.

Conclusions: Our results suggest that MLH1 loss in SSPs is an early change, precedes the development of overt CD and predicts the development of MLH1-deficient CD. Prospective studies are warranted to determine if SSPs with MLH1-deficient NDCs should be surveilled with the same frequency as SSPs showing overt CD.

\section{Clinical Outcome of Perineal Carcinoma Cuniculatum in a Cohort of 38 Cases}

Dongwei Zhang1, Raul S Gonzalez², Michael Feely', Kavita Umrau ${ }^{3}$, Hwajeong Lee ${ }^{4}$, Daniela Allende ${ }^{5}$, Dipti Karamchandaní, Michael Ṕ Zaleski ${ }^{7}$, Jingmei Lin ${ }^{8}$, Maria Westerhoff, Xuchen Zhang' ${ }^{10}$, Lindsay Alpert11, Jinping Lai, Xiuli Liu'. 'University of Florida, Gainesville, FL, ${ }^{2}$ University of Rochester Medical Center, Rochester, NY, ${ }^{3}$ Albany Medical College, ${ }^{4}$ Albany Medical College, Guilderland, NY, ${ }^{5}$ Cleveland Clinic, Cleveland, $\mathrm{OH},{ }^{6}$ Penn State Hershey Medical Center, Hershey, PA, ${ }^{7}$ Penn State Health Hershey Medical Center, Hershey, PA, ${ }^{8}$ Indiana University Department of Pathology, Indianapolis, IN, 'Úniversity of Michigan, Ann Arbor, MI, ${ }^{10}$ Yale University School of Medicine, Orange, CT, ${ }^{11}$ University of Chicago, Chicago, IL

Background: Perineal carcinoma cuniculatum (PCC, also known as Buschke-Lowenstein tumor or giant condyloma acuminculatum) is a rare sexually-transmitted disease (STD), mostly described in clinical literature as case reports or small series. This study aims to investigate the clinical features and outcome of PCC in a reasonably sized cohort.

Design: We identified 38 cases of PCC in our institutional archives. Clinical information and demographics were obtained by chart review. All cases were reviewed and confirmed histologically.

Results: Patients had a mean age of $47.6( \pm 12.8)$ years (range $19-80)$ at diagnosis and a male to female ratio of 4.4 . Presenting symptoms included pain and discomfort $(42.1 \%)$, bleeding $(31.6 \%)$, mass $(28.9 \%)$, and discharge $(26.3 \%)$. Twenty-nine $(76.3 \%)$ patients were smokers or ex-smokers and $21 \%$ reported alcohol use. At least $13.2 \%(5 / 38)$ patients were homosexual. One patient $(2.6 \%)$ had a history of heart transplant. Other significant medical histories included HIV (26.3\%) STDs $(10.5 \%)$, condyloma or other human papillomavirus (HPV)related disease $(23.7 \%)$, and other malignancies $(15.8 \%)$. Two $(5.3 \%)$ patients had Crohn's disease, and one $(2.6 \%)$ had chronic perianal abscess. All 36 cases with available physical examination information had a perineal lesion/mass. Clinical impression was condyloma in $27(71 \%)$, verrucous carcinoma in $3(7.8 \%)$, suspicious for cancer squamous cell carcinoma (SCC), peri-anal mass, and abscess/fistula in $2(5.2 \%)$ for each. Mean tumor size and thickness were $8.5( \pm 6.6) \mathrm{cm}$ (range 1.2-26) and $1.5( \pm 1.3) \mathrm{cm}$ (range 0.2-5.5), respectively. Twenty two $(57.9 \%)$ had a positive resection margin. Nineteen $(50 \%)$ cases had an invasive squamous cell carcinoma (SCC) component. Four patients with and 2 without invasive SCC received chemoradiation therapy, and one with invasive SCC was treated with imiquimod. Median follow-up was 24 months (range 1-207, 35 patients). Nine $(23.7 \%)$ patients had recurrence of disease, including 4 PCC with invasive SCC and 5 without invasive SCC, with a median interval of 21 months (range: 1-207). Four (10.5\%) patients died; one died from invasive SCC with nodal metastasis 2 years after an initial diagnosis of non-invasive PCC. <

Conclusions: PCC has unique clinicodemographic features, including male predilection and frequent links to smoking and positive HIV and HPV status. Common symptoms are pain/discomfort, bleeding, mass and discharge. PCC can recur and can transform into invasive SCC. We found a recurrence rate of $23.7 \%$

\section{GNAS Activating Mutation is Correlated with Mucinous Morphology in Colorectal/Appendiceal Adenocarcinoma}

Xiaofei Zhang', Min Cui', Xiaoyan Liao', Jia Qin', Huaibin Mabel Ko ${ }^{1}$ Qingqing Liu, Hongfa Zhu' ${ }^{2}$, Alexandros D Polydorides' ${ }^{1}$, Noam Harpaz ${ }^{3}$ ${ }^{1}$ Icahn School of Medicine at Mount Sinai, New York, NY, ${ }^{2}$ Mount Sinai Hospital, ${ }^{3}$ Mount Sinai Med. Center, New York, NY

Background: GNAS is an oncogene encoding the $\alpha$-subunit of the stimulatory $\mathrm{G}$ protein. Mutations of this gene occur in various neoplasms. Recently, GNAS mutations have been reported in colorectal cancer (CRC) by targeted next generation sequencing (NGS), however, their role in tumorigenesis is unknown. We determined the rate of GNAS mutation in colorectal/appendiceal adenocarcinomas and sought to determine any associated clinical or pathological features.

Design: All colorectal and appendiceal carcinomas biopsied or resected between 2013 and 2016 were analyzed by targeted next generation sequencing (NGS) of 50 genes using the Ion AmpliSeq Cancer Hotspot Panel (v2, Thermo Fisher). The corresponding pathologic features were determined from the medical records Continuous variables were compared with the Student $t$ test and categorical variable by the $\chi^{2}$ method with $p<0.05$ as the significance threshold.

Results: Of 423 tumors analyzed, GNAS mutations were detected in 16 patients $(3.8 \%)$. The patients were 7 males and 9 females with mean age of $59.2 \pm 2.7 y$. Of the 16 tumors, 6 were located in the appendix $(37.5 \%)$. Of the other 10,6 were in the right colon and 4 in the rectosigmoid. All 16 tumors were classified as mucinous, including one with signet ring features. Fifteen of the 16 mutations $(93.8 \%$ were activating in codon 201 (9 pR201H, 5 pR201C and 1 pR201S and one in codon $227(\mathrm{pQ} 227 \mathrm{H})$. Further analysis showed a positive correlation with BRAF mutations $(31.3 \%$ in GNAS-mutated tumors vs $11.5 \%$ in GNAS-wt tumors, $\mathrm{p}=0.038$ ) and negative correlation with APC mutation (0/16 in GNAS-mutated tumors vs. $31.4 \%$ in GNAS-wt tumors, $\mathrm{p}=0.017)$. There was also a trend toward negative association with TP53 mutation $(p=0.07)$. There was no significant association between GNAS mutation and mutations of the other genes tested including KRAS, NRAS and PIK3CA.

Conclusions: Based on this cohort, which is the largest published to date targeting GNAS, we conclude that activating GNAS mutations in colorectal adenocarcinomas are infrequent, involve a codon p201 hotspot, and are strongly correlated with mucinous histologic phenotype and BRAF mutation. They are enriched in appendicea adenocarcinomas, but once that subgroup is excluded there is only a weak predilection for right-sided location.

\section{Submucosal Vascular Remodeling in Crohn's Disease-Associated Ileal Strictures}

Xiaofei Zhang ${ }^{1}$, Zhenjian Cai ${ }^{2}$, Huaibin Mabel Ko' ${ }^{1}$, Min Cui ${ }^{1}$, Xiaoyan Liao $^{1}$, Qingqing Liu, Hongfa Zhu ${ }^{3}$, Alexandros D Polydorides ${ }^{1}$, Noam Harpaz'. 'Icahn School of Medicine at Mount Sinai, New York, NY, ${ }^{2}$ The University of Texas Health Science Center at Houston, Houston, TX, ${ }^{3}$ Mount Sinai Hospital, ${ }^{4}$ Mount Sinai Med. Center, New York, NY

Background: The pathogenesis of intestinal strictures in Crohn's disease is poorly understood, impeding progress toward their prevention and management. Pathologically, stricturing has been 
associated with mural thickening caused by fibromuscular proliferation, especially of the submucosa and muscularis mucosae, however, there has been little attention to corresponding vascular alterations. We studied submucosal vascular remodeling in resected strictures associated with Crohn's ileitis by means of immunohistochemistry and quantitative morphometry.

Design: Fifteen surgically resected ileal strictures from $15 \mathrm{CD}$ patients (7 males, 8 females, age $45.3 \pm 4.6 y$ ) were formalin fixed and crosssectioned along with adjoining non-strictured intestine as control tissue. The histologic sections were stained for collagen (trichrome, Sirius red (SR); collagen types I, III and V (immunofluorescence); and smooth muscle actin, desmin, CD31, CD34, CD45, CD68, CD117, D240, S100 and Ki-67 (immunoperoxidase). The slides were digitally scanned at $20 \mathrm{X}$ and evaluated morphometrically with Halo software (v. 4, Indica Labs). Continuous variables associated with the strictures and adjacent control tissue were compared using the Student t test with $p<0.05$ as significance threshold.

Results: Total submucosal vascular density was significantly increased from $16 \pm 2.0$ to $34 \pm 6.4$ vessels per cross section, $p=0.017$ ). The most prominent microscopic change was a perivasculopathy involving the muscular submucosal arteries and veins. This was characterized by the formation of a crescent-shaped adventitial mantle that consistently faced toward the intestinal lumen. The vasculopathy was significantly more common in strictures than controls $(41.4 \pm 4.5 \%$ vs. $8.0 \pm 2.1 \%$ of vessels, respectively, $P<0.001$ ). The mantles consisted of myocytes, interstitial type $\mathrm{V}$ collagen and newly-formed capillaries. Myofibroblasts and histiocytes were rare or absent. The mantles did not contain collagen types I or III or cells expressing CD117. Ki-67 expression was minimal to absent.

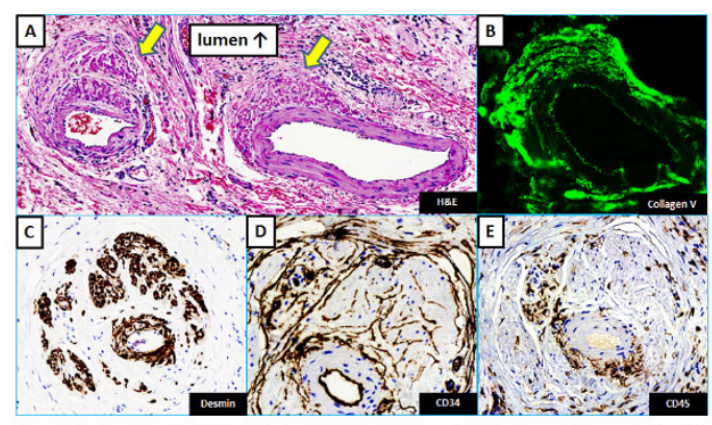
Figure 1. Perivasculopathy in Crohn's-associated ileal stricture. (A) Submucosal artery and vein with
adventitial mantles (arrows). The mantles are consistently oriented towards the intestinal lumen. (B) Fibrous component of mantle consists of type $\mathrm{V}$ collagen (anti-collagen $\mathrm{V}$, immunofluorescence). (C) Bulk of the mantle consists of smooth muscle (desmin, immunoperoxidase). (D) Other components (iclude newy-formed capiliaries (CD34, immunoperoxidase). (E) Sparse lymphocytic infiltration between muscle bundles ( $C D 45$, immunoperoxidase).

Conclusions: Vascular remodeling in Crohn's-associated ileal strictures results in a hitherto under-recognized perivasculopathy that is characterized by adventitial proliferation of smooth muscle and type $\mathrm{V}$ collagen and by polarization toward the intestinal lumen. The histogenesis of this phenomenon and its pathophysiological implications with respect to vascular perfusion and reactivity remain to be explored.

\section{Colonic Mucosal Eosinophilia: Clinical and Pathologic Implications}

Wei Zhang', Kathryn Tanaka', Sun M Chung ${ }^{1}$, Nicole Panarelli ${ }^{2}$. ${ }^{1}$ Montefiore Medical Center, Bronx, NY, ${ }^{2}$ Montefiore Medical Center, Scarsdale, NY

Background: Increased eosinophils in colonic mucosal biopsy samples are a common source of confusion among pathologists and clinicians. Established etiologies include immune-mediated disorders, infections, and drug reactions; however, many cases remain unexplained. The purpose of this study was to correlate the number and distribution of eosinophils in colon biopsy specimens with clinical findings.

Design: We retrospectively identified 20 normal biopsy samples each from the right and left colon to serve as controls. Cases with increased eosinophils were identified by searching the pathology database, and clinical information was retrieved from the electronic medical record. We estimated the normal range of tissue eosinophilia, in our population, by calculating the mean number and standard deviation (SD) of eosinophils in the most eosinophil-rich high power field (hpf) in each sample. Intraepithelial eosinophils were also counted, when present. The cutoff for increased lamina propria eosinophils was set at more than $1 \mathrm{SD}$ above the mean. Eosinophils numbering 2- and 3- fold above the cutoff were considered moderately and markedly increased, respectively.

Results: Controls included 10 men and 14 women with a mean age of 45 years. A mean of $21 \pm 12$ and $16 \pm 11$ eosinophils were present in the right and left colon, respectively. Control cases contained $\leq 5$ intraepithelial eosinophils per hpf. The study group included 28 men and 31 women with a mean age of 51 years. Increased eosinophils were present in the right colon only $(n=44,75 \%)$, left colon only $(n=6$, $10 \%)$ or at both sites $(n=9,15 \%)$. Increased intraepithelial eosinophils were seen in 9 cases $(15 \%)$. Increased intramucosal mast cells (ckit immunostain) were seen in 4 of 22 cases with markedly increased eosinophils. The findings are summarized in Table 1.

Table 1. Clinical and pathologic features of cases with colonic eosinophils

\begin{tabular}{|l|l|l|l|l|}
\hline Etiology & $\begin{array}{l}\text { Mildly } \\
\text { Increased } \\
(\mathrm{n}=19)\end{array}$ & $\begin{array}{l}\text { Moderately } \\
\text { increased } \\
(\mathrm{n}=18)\end{array}$ & $\begin{array}{l}\text { Markedly } \\
\text { increased } \\
(\mathrm{n}=22)\end{array}$ & $\begin{array}{l}\text { Intraepithelial } \\
\text { eosinophils } \\
(\mathrm{n}=9)\end{array}$ \\
\hline Prior procedure site & 0 & $3(17 \%)$ & $6(27 \%)$ & $1(11 \%)$ \\
\hline Crohn disease & $6(32 \%)$ & $2(11 \%)$ & $1(5 \%)$ & $1(11 \%)$ \\
\hline Ulcerative colitis & $2(11 \%)$ & $5(28 \%)$ & $1(5 \%)$ & 0 \\
\hline Irritable bowel syndrome & $2(11 \%)$ & 0 & 0 & 0 \\
\hline Parasites & 0 & 0 & $3(14 \%)$ & $2(22 \%)$ \\
\hline Peripheral eosinophilia & 0 & 0 & $2(9 \%)$ & $2(22 \%)$ \\
\hline Medication & 0 & $2(11 \%)$ & $1(5 \%)$ & $1(11 \%)$ \\
\hline Diverticular disease & $1(5 \%)$ & $1(6 \%)$ & $1(5 \%)$ & 0 \\
\hline Eosinophilic gastroenteritis & 0 & $1(6 \%)$ & 0 & 0 \\
\hline $\begin{array}{l}\text { Increased intramucosal mast } \\
\text { cells/mastocytic colopathy } \\
\text { (>40/hpf) }\end{array}$ & 0 & 0 & $4(18 \%)$ & 0 \\
\hline Microscopic colitits & $1(5 \%)$ & 0 & $1(5 \%)$ & 0 \\
\hline Food allergy & $1(5 \%)$ & 0 & 0 & 0 \\
\hline Asthma & 0 & $1(6 \%)$ & 0 & 0 \\
\hline Interval appendicitis & 0 & 0 & $1(5 \%)$ & 0 \\
\hline Unknown/loss of follow up & $6(32 \%)$ & $3(17 \%)$ & $1(5 \%)$ & $2(22 \%)$ \\
\hline
\end{tabular}

Conclusions: A mild or moderate increase in eosinophils was often associated with a known underlying inflammatory condition or medication-induced injury in our series (e.g. inflammatory bowel disease, irritable bowel syndrome, and diverticular disease). Notably, cases of parasitic infection and mastocytic colopathy that were previously clinically unsuspected were detected by colonic mucosal biopsy, particularly in cases with markedly increased eosinophils. Pathologists should consider this differential diagnosis when markedly increased eosinophils are present in order to prompt appropriate ancillary studies and clinical follow-up.

\section{Perineal Carcinoma Cuniculatum: Histology Review of 38 Case}

Dongwei Zhang1, Raul S Gonzalez², Michael Feely', Hwajeong Lee ${ }^{3}$ Kavita Umrau ${ }^{4}$, Daniela Allende ${ }^{5}$, Dipti Karamchandani6, Michael $P$ Zaleski ${ }^{7}$, Jingmei Lin ${ }^{8}$, Maria Westerhoff ${ }^{2}$, Xuchen Zhang ${ }^{10}$, Lindsay Alpert'11, Jinping Lai, Xiuli Liu'. 'University of Florida, Gainesville, FL, ${ }^{2}$ University of Rochester Medical Center, Rochester, NY ${ }^{3}$ Albany Medical College, Guilderland, NY, ${ }^{4}$ Albany Medical College, ${ }^{5}$ Cleveland Clinic, Cleveland, $\mathrm{OH},{ }^{6} \mathrm{Penn}$ State Hershey Medical Center, Hershey, PA, ${ }^{7}$ Penn State Health Hershey Medical Center, Hershey, PA, 8 Indiana University Department of Pathology, Indianapolis, IN, 'University of Michigan, Ann Arbor, MI, ${ }^{10}$ Yale University School of Medicine, Orange, CT, "' $U$ niversity of Chicago, Chicago, IL

Background: Perineal carcinoma cuniculatum (PCC, also known as Buschke-Lowenstein tumor or giant condyloma acuminculatum) is a rare sexually-transmitted disease, mostly described in clinical literature in case reports or small series. Histology of the mass lesions has not been well characterized in a reasonably sized cohort.

Design: We identified 38 cases of PCC in our institutional archives. Slides from all cases were reviewed for histological features described previously [Zidar $\mathrm{N}$ et al., 2017; Landau M et al., 2012]. Cases were further divided into PCC with and without invasive squamous cell carcinoma (SCC).

Results: Nineteen $(50 \%)$ cases had an invasive SCC component manifested as individual cells or small nest infiltrating stroma and/ or desmoplasia. All invasive components were well differentiated except one which was moderately differentiated. None of the cases had lymphovascular or perineural invasion. Four cases had lymph nodes in the resection, but none showed nodal disease. The tumor size and thickness between PCC with or without invasive component were not significantly different between PCC with or without invasive $\operatorname{SCC}(7.4 \pm 5.8 \mathrm{~cm}$ vs. $9.7 \pm 7.3 \mathrm{~cm}, \mathrm{p}=0.29 ; 1.3 \pm 1.4 \mathrm{~cm}$ vs. $1.6 \pm 1.2 \mathrm{~cm}$ $\mathrm{p}=0.37$ ). PCCs with invasive SCC had higher frequency of dyskeratosis abnormal keratosis, intraepithelial neutrophilic microabscesses, and abnormal mitoses in the non-invasive component and they were less likely to have a pushing border [see Table 1].

\section{FOR TABLE DATA, SEE PAGE 322, FIG. 891}

Conclusions: Invasive SCC-harboring PCC tends to have higher rates of dyskeratosis, abnormal keratosis, neutrophilic microabscesses, and abnormal mitoses in the non-invasive portion. The features may be 
important on lesional biopsy to predict the presence of underlying invasive SCC.

\section{MCM7 Expression Correlates With Tumor Size and Ki67 Index in Well-Differentiated Small Intestinal Neuroendocrine Tumors}

Zhongren (David) Zhou', Numbereye Numbere ${ }^{2}$, Aaron Huber ${ }^{1}$ Chanjuan Shi3, Raul S Gonzalez. 'Rochester, NY, '2University of Rochester Medical Center, Pittsford, NY, ${ }^{3}$ Vanderbilt University, Nashville, TN, ${ }^{4}$ University of Rochester Medical Center, Rochester, NY

Background: Minichromosomal maintenance proteins, such as MCM7, are involved in DNA replication and have been reported as potential proliferative markers. We have previously shown that MCM7 expression correlates with Ki67 immunohistochemical index in esophageal malignancies. In addition to indicating rate of tumor proliferation, Ki67 index is one of two grading criteria (along with mitotic rate) for well-differentiated neuroendocrine tumors, including those in the small intestine (SI-NETs). In this study, we evaluated MCM7 expression in SI-NETs and its link to Ki67 index and other clinicopathologic factors.

Design: We performed MCM7 immunohistochemical staining on 58 cases of ileal or jejunal SI-NET, from 33 patients. Each case was also evaluated for patient age and sex; tumor size and Ki67 index (manually counted from a photographed hot-spot); lymph node metastases, mesenteric tumor deposits, and distant metastases; and diseasespecific outcome. The relationships between MCM7 expression and these clinicopathologic factors were compared using Spearman's rank correlation coefficient $(\rho)$ or Fisher's exact test as appropriate.

Results: MCM7 expression positively correlated with lesion size $(\rho=0.49 ; P=0.0001)$ and Ki67 index $(\rho=0.74 ; P<0.0001)$. MCM7 index $(1.8 \pm 2.7)$ was significantly higher than Ki67 index $(0.7 \pm 1.0)$ by Wilcoxon signed-rank test $(P<0.0001)$. MCM7 index was not significantly associated with patient age $(P=0.07)$, sex $(P=0.47)$, nodal disease $(P=0.40)$, distant metastases $(P=0.11)$, or tumor deposits $(P=0.15)$. No patients died of disease at last follow-up, precluding comparison between MCM7 and disease-specific survival.

Conclusions: MCM7 index is correlated with the Ki67 proliferative index in SI-NET and is generally higher. As Ki67 proliferative index is a key component of grading SI-NETs but is often relatively low, MCM7 expression could serve as a more sensitive marker to differentiate among grade 1 and 2 SI-NETs.

\section{Dynamic Changes of Genomic Mutational Profiles in Disseminated Appendiceal Mucinous Tumors}

Xiaoqin Zhu', Xiuling Meng², Keith Tomaszewicz ${ }^{3}$ Bradley Switzer ${ }^{4}$, Laura Lambert ${ }^{5}$, Ediz F Cosar ${ }^{6}$, Xiaofei Wang ${ }^{7}$, Lloyd Hutchinson ${ }^{7}$. ${ }^{1}$ University of Massachusetts Memorial Medical Center, Shrewsbury, MA, ' $U$ Mass Medical School, Worcester, MA, ${ }^{3}$ UMass Medical School, ${ }^{4}$ University of Massachusetts Memorial Medical Center, Worcester, MA, 5 University of Massachusetts Memorial Medical Center, Worcester, MA, ${ }^{6}$ University of Massachusetts Memorial Medical Center, Worcester, MA, 7 UMass Memorial Healthcare, Worcester, MA

Background: Dissemination of appendiceal mucinous tumors may lead to peritoneal mucinous carcinomatosis (pseudomyxoma peritonei; PMP). Patients with PMP are at risk of intraperitoneal recurrence. Recent studies and our data suggested molecular heterogeneity among epithelial tumors of appendix at different histopathological grades. However, it is unclear whether the mutational profiles of tumor cells stay constant or change by time of recurrence. Evidence is also lacking whether the tumor cells of different morphologies from one specimen possess the same or distinct mutations.

Design: Ten patients with recurrent disseminated appendiceal mucinous tumors in our institute from 2006 to 2015 were studied. The tumors were classified into 3 grades (low-grade, high-grade, and signet ring cells), as advocated by the AJCC. All patients underwent hyperthermic intraperitoneal chemotherapy (HIPEC) except one patient in case \#1. They also received chemotherapy after the first tumor debulking except 3 patients (case \#1, \#4, and \#6). Two specimens obtained from each individual at different time points of debulking surgery were tested by next-generation sequencing (NGS) of 50 cancer genes using the Ampliseq Cancer Hotpost Panel v2. NGS testing was also performed on tumor cells with different morphology in each of the "mixed morphology" specimens obtained at one time point.

Results: The morphologies of recurrent tumors remained the same as those of original tumors. Among the 10 patients analyzed, three (cases \#1-3) acquired additional mutations, and three (cases \#4-6) had loss of one mutation in recurrent tumors, compared to original profiles. In 4 patients the mutations found in the recurrent tumors remained identical to original tumors (cases \#7-10). The detailed information was assembled in table 1. Of the four cases composed of two morphology types (Table 2), two cases showed distinct but related mutation profiles in high-grade and signet ring cell components. The remaining two cases carried the same mutational profile in the components with different morphologies.

FOR TABLE DATA, SEE PAGE 322, FIG. 893

Table 2: Comparison of genomic mutations in distinct components of morphology in the specimens at one time point.

\begin{tabular}{|c|c|c|c|c|c|c|c|}
\hline Case & Gender & Age & Location & Pathology & \multicolumn{3}{|c|}{ Mutations } \\
\hline \multirow[b]{2}{*}{11} & \multirow[b]{2}{*}{$\mathrm{F}$} & \multirow[b]{2}{*}{68} & Appendix & HG & $\begin{array}{l}\text { KRAS } \\
\text { p.G12V }\end{array}$ & $\begin{array}{l}\text { SMAD4 } \\
\text { p.RQ256L }\end{array}$ & \\
\hline & & & Omentum & SRC & $\begin{array}{l}\text { KRAS } \\
\text { p.G12V }\end{array}$ & $\begin{array}{l}\text { GNAS } \\
\text { p.Q227R }\end{array}$ & $\begin{array}{l}\text { TP53 } \\
\text { c.580in- } \\
\text { sA }\end{array}$ \\
\hline \multirow{2}{*}{12} & \multirow{2}{*}{$\mathrm{M}$} & \multirow{2}{*}{63} & Appendix & HG & $\begin{array}{l}\text { KRAS } \\
\text { p.G12V }\end{array}$ & $\begin{array}{l}\text { GNAS } \\
\text { p.R201C }\end{array}$ & \\
\hline & & & $\begin{array}{l}\text { mesoap- } \\
\text { pendix }\end{array}$ & $\mathrm{HG}+\mathrm{SRC}$ & $\begin{array}{l}\text { KRAS } \\
\text { p.G12V }\end{array}$ & $\begin{array}{l}\text { GNAS } \\
\text { p.R201C }\end{array}$ & $\begin{array}{l}\text { FBXW7 } \\
\text { p.R465H }\end{array}$ \\
\hline \multirow{2}{*}{13} & \multirow{2}{*}{$\mathrm{F}$} & \multirow{2}{*}{71} & $\begin{array}{l}\text { Peritone- } \\
\text { um }\end{array}$ & LG & $\begin{array}{l}\text { KRAS } \\
\text { p.G12D }\end{array}$ & $\begin{array}{l}\text { GNAS } \\
\text { p.R201LR }\end{array}$ & \\
\hline & & & Omentum & $\mathrm{LG}+\mathrm{HG}$ & $\begin{array}{l}\text { KRAS } \\
\text { p.G12D }\end{array}$ & $\begin{array}{l}\text { GNAS } \\
\text { p.R201LR }\end{array}$ & \\
\hline \multirow{2}{*}{14} & \multirow{2}{*}{$\mathrm{F}$} & \multirow{2}{*}{71} & Appendix & HG & $\begin{array}{l}\text { KRAS } \\
\text { p.061L }\end{array}$ & $\begin{array}{l}\text { TP53 } \\
\text { p.R273H }\end{array}$ & $\begin{array}{l}\text { APC } \\
\text { p.R1450X }\end{array}$ \\
\hline & & & $\begin{array}{l}\text { mesoap- } \\
\text { pendix }\end{array}$ & SRC & $\begin{array}{l}\text { KRAS } \\
\text { p.061L }\end{array}$ & $\begin{array}{l}\text { TP53 } \\
\text { p.R273H }\end{array}$ & $\begin{array}{l}\text { APC } \\
\text { p.R1450X }\end{array}$ \\
\hline
\end{tabular}

Conclusions: The mutational profiles of disseminated appendicea mucinous tumors can remain stable or can undergo dynamic changes prior to recurrence, regardless of chemotherapeutic treatment. Similarly, appendiceal tumor cells showing different morphologies in the same specimen may exhibit overlapping but distinct mutations suggesting morphological association with mutational heterogeneity. 


\begin{tabular}{|c|c|c|c|c|c|c|c|}
\hline & & \multicolumn{2}{|l|}{ Tumor } & \multirow{2}{*}{$P$} & \multicolumn{2}{|l|}{ TILS } & \multirow{2}{*}{$P$} \\
\hline & & Pos & $\mathrm{Neg}$ & & Pos & $\mathrm{Neg}$ & \\
\hline \multirow{2}{*}{ Gender } & $M$ & 20 & 127 & \multirow{2}{*}{0.863} & 66 & 81 & \multirow{2}{*}{0.185} \\
\hline & $\mathrm{F}$ & 8 & 47 & & 19 & 36 & \\
\hline \multicolumn{2}{|l|}{ Age } & $66.64 \pm 10.67$ & $61.76 \pm 12.57$ & 0.053 & $63.11 \pm 12.38$ & $61.95 \pm 12.47$ & 0.514 \\
\hline \multirow{2}{*}{ Location } & Proximal & 11 & 70 & \multirow{2}{*}{0.925} & 40 & 45 & \multirow{2}{*}{0.085} \\
\hline & Distal & 17 & 104 & & 41 & 76 & \\
\hline \multirow{2}{*}{$\mathrm{T}$} & $1-2$ & 5 & 51 & \multirow{2}{*}{0.208} & 37 & 19 & \multirow{2}{*}{0.341} \\
\hline & $3-4$ & 23 & 123 & & 87 & 61 & \\
\hline \multirow{2}{*}{$N$} & 0 & 9 & 51 & \multirow{2}{*}{0.737} & 36 & 24 & \multirow{2}{*}{0.882} \\
\hline & $1-3$ & 19 & 125 & & 88 & 56 & \\
\hline \multirow{2}{*}{ M } & 0 & 28 & 168 & \multirow{2}{*}{0.249} & 120 & 76 & \multirow{2}{*}{0.523} \\
\hline & 1 & 0 & 8 & & 4 & 4 & \\
\hline \multirow{2}{*}{ S } & $1-2$ & 12 & 77 & \multirow{2}{*}{0.929} & 58 & 31 & \multirow{2}{*}{0.259} \\
\hline & $3-4$ & 16 & 99 & & 66 & 49 & \\
\hline \multirow{3}{*}{ Grade } & 1 & 0 & 9 & \multirow{3}{*}{0.430} & 4 & 5 & \multirow{3}{*}{0.188} \\
\hline & 2 & 12 & 64 & & 38 & 38 & \\
\hline & 3 & 16 & 101 & & 43 & 74 & \\
\hline \multirow{3}{*}{ Lauren } & Intestinal & 12 & 70 & \multirow{3}{*}{0.936} & 45 & 37 & \multirow{3}{*}{0.009} \\
\hline & Diffuse & 9 & 62 & & 23 & 48 & \\
\hline & Mixed & 7 & 42 & & 17 & 32 & \\
\hline \multirow{4}{*}{$\begin{array}{l}\text { Histological } \\
\text { subtypes }\end{array}$} & $\begin{array}{l}\text { Poorly } \\
\text { cohesive }\end{array}$ & 6 & 51 & \multirow{4}{*}{0.257} & 16 & 41 & \multirow{4}{*}{0.005} \\
\hline & Tubular & 15 & 85 & & 43 & 57 & \\
\hline & Papillary & 7 & 23 & & 21 & 9 & \\
\hline & Mucinous & 0 & 15 & & 5 & 10 & \\
\hline
\end{tabular}


Table 1: CAG: chronic active gastritis; CIG: chronic inactive gastritis; N: Normal; Pos: Positive; Neg: Negative; ()=standard deviation

\begin{tabular}{|c|c|c|c|c|c|c|c|c|c|}
\hline & \multirow{2}{*}{ Totals } & \multicolumn{4}{|c|}{ Partial Gastrectomy Diagnosis } & \multirow[b]{2}{*}{$P$} & \multicolumn{2}{|c|}{ H. pylori (biopsy or partial gastrectomy) } & \multirow[b]{2}{*}{$P$} \\
\hline & & CAG* & $\mathrm{CIG}$ & $\mathrm{N}$ & Other ${ }^{\wedge}$ & & Pos & Neg & \\
\hline $\begin{array}{l}\text { Number of } \\
\text { Cases }\end{array}$ & 94 & 3 & 29 & 52 & 10 & $<0.001$ & 19 & 75 & $<0.001$ \\
\hline $\begin{array}{l}\text { Number of } \\
\text { Follicles }\end{array}$ & 734 & 122 & 220 & 355 & 37 & $<0.001$ & 294 & 440 & $<0.001$ \\
\hline $\begin{array}{l}\text { Average } \\
\text { Number } \\
\text { of Follicles } \\
\text { per Case }\end{array}$ & $7.8(10.9)$ & $40.6(39.0)$ & $7.5(8.8)$ & $6.8(6.5)$ & $3.7(5.5)$ & $<0.001$ & $15.5(18.5)$ & $5.9(6.7)$ & 0.03 \\
\hline $\begin{array}{l}\text { Average } \\
\text { Density of } \\
\text { Follicles (\#/ } \\
\mathrm{mm} \text { )per } \\
\text { case }\end{array}$ & $0.14(0.16)$ & $0.56(0.48)$ & $0.13(0.14)$ & $0.12(0.11)$ & $0.10(0.21)$ & $<0.001$ & $0.24(0.24)$ & $0.11(0.13)$ & 0.02 \\
\hline $\begin{array}{l}\text { Average } \\
\text { Number } \\
\text { of Primary } \\
\text { Follicles } \\
\text { per Case }\end{array}$ & 7.2(9.7) & $34.3(31.6)$ & $7.1(8.6)$ & $6.4(6.5)$ & $3.7(5.5)$ & $<0.001$ & $13.6(15.6)$ & $5.5(6.9)$ & 0.04 \\
\hline $\begin{array}{l}\text { Average } \\
\text { Number of } \\
\text { Secondary } \\
\text { Follicles } \\
\text { per Case }\end{array}$ & $0.59(1.88)$ & $6.3(7.8)$ & $0.45(0.82)$ & $0.46(1.3)$ & $0(0)$ & $<0.001$ & $1.8(3.4)$ & $0.29(1.0)$ & 0.08 \\
\hline $\begin{array}{l}\text { Average } \\
\text { Follicle } \\
\text { Diameter } \\
(\mathrm{mm})\end{array}$ & $0.39(0.18)$ & $0.50(0.18)$ & $0.36(0.17)$ & $0.37(0.19)$ & $0.34(0.16)$ & $<0.001$ & $0.40(0.20)$ & $0.38(0.17)$ & 0.17 \\
\hline $\begin{array}{l}\text { Average } \\
\text { Occupancy } \\
(\%)^{* *}\end{array}$ & $0.23(0.15)$ & $0.23(0.16)$ & $0.23(0.17)$ & $0.22(0.14)$ & $0.28(0.13)$ & 0.23 & $0.25(0.18)$ & $0.22(0.13)$ & 0.04 \\
\hline
\end{tabular}

*Paired P-values are only significant in combinations with CAG (CAG-CIG, CAG-N, CAG-Other). The remaining combinations are not independently significant. **Average Occupancy was measured in 505/734 lymphoid follicles due to lack of orientation in the histologic sections

${ }^{\wedge}$ Other includes parietal cell hyperplasia(3), Hernia (3), GIST (2), and ulcer without chronic active gastritis (2) 
FIG. 803

\begin{tabular}{|c|c|c|c|c|c|}
\hline \multicolumn{2}{|l|}{ Parameter } & Case 1 & Case 2 & Case 3 & Case 4 \\
\hline \multicolumn{2}{|l|}{ Gender } & M & $\mathrm{F}$ & $\mathrm{F}$ & M \\
\hline \multicolumn{2}{|l|}{ Age (years) } & 85 & 84 & 45 & 70 \\
\hline \multicolumn{2}{|l|}{ Site of tumor in colon } & Caecum & Ascending & Transverse & Caecum \\
\hline \multicolumn{2}{|l|}{ Tumor type } & Adenocarcinoma & Adenocarcinoma & Adenocarcinoma & Adenocarcinoma \\
\hline \multicolumn{2}{|l|}{ Differentiation } & Moderate & Poor & Moderate & Moderate \\
\hline \multicolumn{2}{|l|}{ Special type } & $\begin{array}{l}\text { Mucinous differentiation } \\
(<50 \%)\end{array}$ & NST & NST & Mucinous differentiation $(<50 \%)$ \\
\hline \multicolumn{2}{|l|}{$\mathrm{pT}$} & 2 & $4 a$ & 3 & 3 \\
\hline \multicolumn{2}{|l|}{$\mathrm{pN}$} & 0 & $1 \mathrm{a}$ & 0 & $1 \mathrm{a}$ \\
\hline \multirow{4}{*}{ MMR IHC in invasive tumor } & MLH1 & MLH1 heterogeneity & MLH1 - & MLH1 heterogeneity & MLH1 - \\
\hline & PMS2 & PMS2 heterogeneity & PMS2 heterogeneity & PMS2 + & PMS2 - \\
\hline & MSH2 & $\begin{array}{l}\mathrm{MSH} 2+ \\
\mathrm{MSH} 2+\end{array}$ & $\mathrm{MSH} 2+$ & $\mathrm{MSH} 2+$ & $\mathrm{MSH} 2+$ \\
\hline & MSH6 & $\mathrm{MSH} 6+$ & $\mathrm{MSH} 6+$ & MSH6 + & MSH6 heterogeneity \\
\hline \multicolumn{2}{|l|}{ Pattern of IHC loss } & Intra-glandular \& zonal & Intra-glandular \& zonal & Intra-glandular \& zonal & Intra-glandular \& zonal \\
\hline \multirow{2}{*}{ MSI in heterogeneous areas } & $\begin{array}{l}\text { Invasive tumor } \\
- \text { MMR IHC + }\end{array}$ & MSS & MSI-H & MSS & MSI-H \\
\hline & $\begin{array}{l}\text { Invasive tumor } \\
\text { - MMR IHC - }\end{array}$ & MSI-H & MSI-H & MSS & MSI-H \\
\hline \multirow{2}{*}{ NGS in heterogeneous areas } & $\begin{array}{l}\text { Invasive tumor } \\
- \text { MMR IHC + }\end{array}$ & TP53 c.524G>A & - & No variants* & - \\
\hline & $\begin{array}{l}\text { Invasive tumor } \\
\text { - MMR IHC - }\end{array}$ & No variants* & - & No variants* & - \\
\hline \multicolumn{6}{|c|}{$\begin{array}{l}\text { Abbreviations: M, male; F, female; NST, no special type; MMR, mismatch repair; IHC, immunohistochemis } \\
\text { MSS, microsatellite stable; MSI-H, microsatellite instability high; NGS, next-generation sequencing; * no Ly }\end{array}$} \\
\hline \multicolumn{6}{|c|}{ Case with heterogeneous mismatch repair immunohistochemistry between invasive tumor and dysplasia. } \\
\hline \multicolumn{4}{|l|}{ Parameter } & \multicolumn{2}{|l|}{ Case 5} \\
\hline \multicolumn{4}{|l|}{ Gender } & \multicolumn{2}{|l|}{ M } \\
\hline \multicolumn{4}{|l|}{ Age (years) } & \multicolumn{2}{|l|}{62} \\
\hline \multicolumn{4}{|l|}{ Site } & \multicolumn{2}{|l|}{ Stomach, antrum } \\
\hline \multicolumn{4}{|l|}{ Tumor type } & \multicolumn{2}{|l|}{ Adenocarcinoma } \\
\hline \multicolumn{4}{|l|}{ Differentiation } & Poor & \\
\hline Special type & & & & Intestinal & \\
\hline pT & & & & $1 b$ & \\
\hline $\mathrm{pN}$ & & & & 0 & \\
\hline Invasive tumor - MMR & & Invasive tumor - MSH2 & & $\mathrm{MSH} 2+$ & \\
\hline & Invasive tumor - & MSH6 & $\mathrm{MSH} 6+$ & & \\
\hline & Invasive tumor - & MLH1 & MLH1 - & & \\
\hline & Invasive tumor - & PMS2 & PMS2 - & & \\
\hline Dysplasia - MMR IHC & & Dysplasia - MSH2 & & $\mathrm{MSH} 2+$ & \\
\hline & Dysplasia-MSH & & MSH6 - & & \\
\hline & Dysplasia - MLH & & MLH1 - & & \\
\hline & Dysplasia-PMS & & PMS2 - & & \\
\hline MSI & & Invasive tumor - MSI & & MSI high & \\
\hline & Dysplasia - MSI & & MSI high & & \\
\hline NGS & & Invasive tumor - NGS & & MSH6 c.3261delC va & \\
\hline & Dysplasia-NGS & & $\begin{array}{l}\text { MSH6 c.3261del C variant \& } \\
\text { MSH6 c.3261dupC variant }\end{array}$ & & \\
\hline
\end{tabular}


Table 1: Histology of PCC from the non-invasive area

\begin{tabular}{|c|c|c|c|c|c|c|c|c|c|c|c|c|c|c|c|}
\hline & HPK (\%) & $A C(\%)$ & DK $(\%)$ & $\begin{array}{l}\text { AK } \\
(\%)\end{array}$ & $\begin{array}{l}\text { IEN } \\
(\%)\end{array}$ & $\begin{array}{l}\text { NM } \\
(\%)\end{array}$ & Atypia (\%) & KC (\%) & $\begin{array}{c}\text { Koilocyte } \\
(\%)\end{array}$ & $\begin{array}{l}\text { AM } \\
(\%)\end{array}$ & INF (\%) & \multicolumn{4}{|c|}{ Interface of tumor with stroma } \\
\hline & & & & & & & & & & & & $\mathrm{P}(\%)$ & $\mathrm{P} / \mathrm{l}(\%)$ & I (\%) & $\mathrm{O}(\%)$ \\
\hline PCC w/ INV & 100 & 94.7 & 94.7 & 94.7 & 83.3 & 66.6 & 84.2 & 94.4 & 94.4 & 50 & 89.4 & 21.1 & 63.2 & 5.2 & 10.5 \\
\hline $\begin{array}{l}\text { PCC w/o } \\
\text { INV }\end{array}$ & 94.7 & 100 & 68.4 & 57.9 & 57.9 & 15.8 & 68.4 & 68.4 & 73.7 & 10.5 & 78.9 & 100 & 0 & 0 & 0 \\
\hline$P$ value & NS & NS & $<0.0001$ & 0.018 & NS & 0.003 & NS & 0.09 & 0.18 & 0.007 & NS & \multicolumn{4}{|l|}{$<0.000$} \\
\hline
\end{tabular}

Note: PCC: perineal carcinoma cuniculatum; w/: with; w/o: without; INV: invasive squamous cell carcinoma; HPK: hyperkeratosis; AC: acanthosis; DK: dyskeratosis; AK; abnormal keratosis; IEN: intraepithelial neutrophilic inflammation; NM: intraepithelial neutrophilic microabscess; KC: keratotic cyst; AM: abnormal mitotic figure; INF: intra- and peri-tumoral inflammation; P: pushing; P/l: pushing/infiltrative; I: infiltrative; O: others

FIG. 893

Table 1: Comparisons of the genomic mutations in disseminated appendiceal mucinous tumors at two different time points in each individual.

\begin{tabular}{|c|c|c|c|c|c|c|c|c|c|c|c|}
\hline $\begin{array}{c}\text { CASE } \\
\#\end{array}$ & Gender & Age & $\begin{array}{l}\text { Location of } \\
\text { tumor }\end{array}$ & $\begin{array}{l}\text { Surgical } \\
\text { procedure } \\
\text { debulking } \\
\text { (1), HIPEC } \$ \\
\text { (2) }\end{array}$ & $\begin{array}{l}\text { Pathology } \\
\text { features }\end{array}$ & $\begin{array}{l}\text { Chemo- } \\
\text { therapy }\end{array}$ & & & Mutations & & \\
\hline \multirow{2}{*}{1} & \multirow{2}{*}{$\mathrm{F}$} & 76 & Uterus & (1) & LG* & \multirow{2}{*}{ No } & $\begin{array}{l}\text { KRAS } \\
\text { p.G12V }\end{array}$ & GNAS p.R201C & & & \\
\hline & & 78 & Intraabdomen & (1) & LG & & $\begin{array}{l}\text { GNAS } \\
\text { p.R201C }\end{array}$ & $\begin{array}{l}\text { TP53 p.R283C } \\
\text { \& p.R280K }\end{array}$ & CDKN2A p.R80X & STK11 p.R332X & $\begin{array}{l}\text { HRAS } \\
\text { p.G12D }\end{array}$ \\
\hline \multirow{2}{*}{2} & \multirow{2}{*}{$\mathrm{F}$} & 43 & Diaphragm & $(1)+(2)$ & LG & \multirow{2}{*}{ Yes } & \begin{tabular}{|l} 
KRAS \\
p.G12VG
\end{tabular} & & & & \\
\hline & & 47 & Intraabdomen & $(1)+(2)$ & LG & & \begin{tabular}{|l|} 
KRAS \\
p.G12VG
\end{tabular} & GNAS p.R201C & & & \\
\hline \multirow{2}{*}{3} & \multirow{2}{*}{$\mathrm{F}$} & 43 & Peritoneum & $(1)+(2)$ & $\mathrm{SRC}^{*}$ & \multirow{2}{*}{ Yes } & \begin{tabular}{|l|} 
TP53 \\
p.R248W \\
\end{tabular} & $\begin{array}{l}\text { HNF1A } \\
\text { p.R263H }\end{array}$ & & & \\
\hline & & 49 & \begin{tabular}{|l|}
$\begin{array}{l}\text { Peritoneum } \\
\text { Kidney }\end{array}$ \\
\end{tabular} & $(1)+(2)$ & SRC & & \begin{tabular}{|l|} 
TP53 \\
p.R248W
\end{tabular} & \begin{tabular}{|l} 
HNF1A \\
p.R263H
\end{tabular} & KRAS p.G12D & GNAS p.R201C & $\begin{array}{l}\text { HRAS } \\
\text { p.A18VA }\end{array}$ \\
\hline \multirow{2}{*}{4} & \multirow{2}{*}{$\mathrm{F}$} & 59 & Spleen & $(1)+(2)$ & LG & \multirow{2}{*}{ No } & $\begin{array}{l}\text { KRAS } \\
\text { p.G12D }\end{array}$ & GNAS p.R201C & PIK3-CA p.E545L & & \\
\hline & & 64 & Stomach & (1) & LG & & \begin{tabular}{|l|} 
KRAS \\
p.G12D \\
\end{tabular} & & PIK3-CA p.E545L & & \\
\hline \multirow{2}{*}{5} & \multirow{2}{*}{$F$} & 67 & Spleen & $(1)+(2)$ & $\mathrm{HG}^{*}$ & \multirow{2}{*}{ Yes } & \begin{tabular}{|l|} 
KRAS \\
p.G12VG \\
\end{tabular} & TP53 p.R273RH & PTEN 2450>OX & & \\
\hline & & 69 & Pelvis & (1) & HG & & \begin{tabular}{|l|} 
KRAS \\
p.G12VG
\end{tabular} & TP53 p.R273RH & & & \\
\hline \multirow{2}{*}{6} & \multirow{2}{*}{ M } & 53 & Peritoneum & $(1)+(2)$ & LG & \multirow{2}{*}{ No } & \begin{tabular}{|l|} 
KRAS \\
p.G12C \\
\end{tabular} & \begin{tabular}{|l|} 
GNAS \\
p.R201HR
\end{tabular} & TP53 p.R2820 & & \\
\hline & & 57 & Abdominal wall & $(1)+(2)$ & LG & & \begin{tabular}{|l|} 
KRAS \\
p.G12C
\end{tabular} & GNAS p.R201H & & & \\
\hline \multirow[b]{2}{*}{7} & \multirow[b]{2}{*}{$F$} & 64 & Peritoneum & $(1)+(2)$ & LG & \multirow[b]{2}{*}{ Yes } & \begin{tabular}{|l|} 
KRAS \\
p.G12VG \\
\end{tabular} & & & & \\
\hline & & 68 & $\begin{array}{l}\text { Ileocolonic } \\
\text { anastomosis }\end{array}$ & $(1)+(2)$ & LG & & $\begin{array}{l}\text { KRAS } \\
\text { p.G12VG }\end{array}$ & & & & \\
\hline \multirow{2}{*}{8} & \multirow{2}{*}{$F$} & 47 & Peritoneum & $(1)+(2)$ & LG & \multirow{2}{*}{ Yes } & \begin{tabular}{|l|} 
KRAS \\
p.G12VG \\
\end{tabular} & \begin{tabular}{|l|} 
GNAS \\
p.R201HR
\end{tabular} & & & \\
\hline & & 51 & Peritoneum & $(1)+(2)$ & LG & & $\begin{array}{l}\text { KRAS } \\
\text { p.G12VG }\end{array}$ & GNAS p.R201H & & & \\
\hline \multirow{2}{*}{9} & \multirow{2}{*}{ M } & 42 & Omentum & $(1)+(2)$ & LG & \multirow{2}{*}{ Yes } & \begin{tabular}{|l|} 
KRAS \\
p.G13D \\
\end{tabular} & \begin{tabular}{|l|} 
GNAS \\
p.R201HR
\end{tabular} & & & \\
\hline & & 44 & Peritoneum & $(1)+(2)$ & LG & & \begin{tabular}{|l|} 
KRAS \\
p.G12S \\
\end{tabular} & \begin{tabular}{|l|} 
GNAS \\
p.R201HR \\
\end{tabular} & & & \\
\hline \multirow{2}{*}{10} & \multirow{2}{*}{$F$} & 44 & Omentum & $(1)+(2)$ & LG & \multirow{2}{*}{ Yes } & $\begin{array}{l}\text { KRAS } \\
\text { p.G12V }\end{array}$ & GNAS p.R201H & & & \\
\hline & & 48 & Peritoneum & $(1)+(2)$ & LG & & \begin{tabular}{|l|} 
KRAS \\
p.G12V
\end{tabular} & GNAS p.R201H & & & \\
\hline
\end{tabular}

* LG: Low-grade epithelium; HG: High-grade epithelium; SRC: Signet-ring cells

\$ HIPEC: Hyperthermic Intraperitoneal Chemotherap 